\title{
Modeling small business growth, migration behavior, local public services and household income in Appalachia: A spatial simultaneous equations approach
}

Gebremeskel H. Gebremariam

West Virginia University

Follow this and additional works at: https://researchrepository.wvu.edu/etd

\section{Recommended Citation}

Gebremariam, Gebremeskel H., "Modeling small business growth, migration behavior, local public services and household income in Appalachia: A spatial simultaneous equations approach" (2006). Graduate Theses, Dissertations, and Problem Reports. 2515.

https://researchrepository.wvu.edu/etd/2515

This Dissertation is protected by copyright and/or related rights. It has been brought to you by the The Research Repository @ WVU with permission from the rights-holder(s). You are free to use this Dissertation in any way that is permitted by the copyright and related rights legislation that applies to your use. For other uses you must obtain permission from the rights-holder(s) directly, unless additional rights are indicated by a Creative Commons license in the record and/ or on the work itself. This Dissertation has been accepted for inclusion in WVU Graduate Theses, Dissertations, and Problem Reports collection by an authorized administrator of The Research Repository @ WVU.

For more information, please contact researchrepository@mail.wvu.edu. 


\title{
MODELING SMALL BUSINESS GROWTH, MIGRATION \\ BEHAVIOR, LOCAL PUBLIC SERVICES AND \\ HOUSEHOLD INCOME IN APPALACHIA: A SPATIAL \\ SIMULTANEOUS EQUATIONS APPROACH
}

Gebremeskel H. Gebremariam

Dissertation Submitted to the Davis College of Agriculture, Forestry and Consumer Sciences at West Virginia University in partial fulfillment of the requirements for the Degree of

\author{
Doctor of Philosophy \\ in \\ Natural Resource Economics \\ Tesfa G. Gebremedhin, Ph.D. Chair \\ Peter V. Schaeffer, Ph.D. \\ Gerard E. D'Souza, Ph.D. \\ Timothy T. Phipps, Ph.D. \\ Stratford M. Douglas, Ph.D.
}

Agricultural and Resource Economics Program

Division of Resource Management

Morgantown, West Virginia

2006

Keyword: Spatial Regression Models, Moran's I, Simultaneous equations, Small Business Growth, Spatial Simultaneous Panel Model, GMM, Appalachia, Spatial Lag, FGS3SLS, Autocorrelation, Programs

Copyright 2006 Gebremeskel H. Gebremariam 


\title{
ABSTRACT \\ Modeling Small Business Growth, Migration Behavior, Local \\ Public Services and Household Income in Appalachia: A Spatial Simultaneous Equations Approach
}

\author{
Gebremeskel H. Gebremariam
}

In an effort to analyze the interdependences among small business growth, migration behavior, local public services, and median household income, this study developed a simultaneous-equation system under the assumptions of profit maximization of firm and utility maximization of households as well as the neoclassical assumption of equilibrium growth in a partial lag-adjustment growth-equilibrium framework. This model is an extension of the "jobs follow people or people follow jobs" literature and it improved previous models in the growth-equilibrium tradition by explicitly modeling local government and regional income in the growth process. It also explicitly modeled gross in-migration and gross out-migration separately in order to spell out the differential effects, which used to be glossed over under net population change in previous studies.

Test for spatial effects showed that the underlying data generating process includes spatial dimension. To incorporate these spatial spillover effects, the standard model is also further extended both in the cross sectional and panel data setting. Apart from the feedback simultaneities, the models now include spatial autoregressive lag and spatial cross-regressive lag simultaneities. The models are also tested for the presence of spatial autocorrelation in the error terms using Moran's I test. The existence of both types of spatial dependences in all equations of the system led to the specification of the system in terms of spatial cross-sectional and spatial panel data models that incorporate both spatial autoregressive dependent variables and spatial autoregressive process in the error terms. The spatial models are estimated by Feasible Generalized Spatial Three-Stage Least Squares (FGS3SLS) Estimator. Detailed separate computer programs are written in TSP to run the five-equation spatial simultaneous equations model in cross-sectional and panel data setting. Both the modeling and the estimation strategies are significant improvements and contributions to the existing literature in spatial econometrics. The simultaneous spatial panel data model estimation is a new addition in empirical work. The implementation of the model with five-equations even in a single cross-sectional data set is a major improvement over previous efforts.

The empirical implementation of the model used county-level data from the 418 Appalachian counties for 1980-2000. Both single equations and system of equations methods of estimation are employed to estimate the standard as well as the spatial simultaneous equations models. In the standard (non-spatial) simultaneous equations model, the estimation for cross-sectional analyses is carried in EViews using standard built-in functionalities. The estimation of the standard simultaneous panel data model and both the spatial cross-sectional and spatial panel simultaneous equations models, however, required the development of special programs. The codes for these programs 
are written in TSP. The spatial regression analyses are preceded by exploratory data analyses which aimed at identifying spatial pattern/or spatial clustering in the data sets. In this respect, ArcGIS and GeoDa are used to calculate Moran's I of Global Spatial Autocorrelation and Local Indicators of Spatial Association (LISA) for the endogenous variables of the models.

Generally, the results from these model estimations are consistent with the theoretical expectations and empirical findings in the equilibrium growth literature and provide support to the basic hypotheses of this study. First, both the spatial and nonspatial models estimates showed the existence of feedback simultaneities among the endogenous variables of the models. This is especially true for the spatial panel model where the coefficients on the endogenous variables in almost all equations of the model are statistically significant atleast at the five percent levels. This indicates that the interdependences among employment growth rate, gross in-migration growth rate, gross out-migration growth rate, median household income growth rate and direct local government expenditures growth rate are very strong. The directions of causation as indicated by the signs of the coefficients are also consistent with the theoretical expectations.

Second, the results from both the spatial and the non-spatial model estimations also showed the existence of conditional convergence with respect to the respective endogenous variable of each equation of the models. This is indicated by the negative and statistically significant coefficients on the lagged dependent variables of the models. This implied that the rates of growth of employment, gross in-migration, gross out-migration, median household income, and direct local government expenditures were higher in counties that had low initial levels of employment, gross in-migration, gross outmigration, median household income, and direct local government expenditures, respectively compared to counties with high initial levels of the same.

Third, the results from the parameter estimation of spatial models and from the exploratory spatial data analysis indicated the existence of spatial autoregressive lag effects and spatial cross-regressive lag effects with respect to the endogenous variables of the models. Besides, the results for Global Moran's I statistics indicated the existence of spatial spillover effect with respect to the error terms of the spatial models. These results would imply that employment growth rate, gross in-migration growth rate, gross outmigration growth rate, median household income growth rate, and direct local government growth rate in a given county are dependent on the averages of employment growth rates, gross in-migration growth rates, gross in-migration growth rates, median household income growth rates, and direct local government growth rate of neighboring counties in the study area. These results are also important from the economic and policy perspectives because they indicate that each of the dependent variables in the model is not only dependent on the characteristics of that county but also on the characteristics of those of its neighbors. Thus, spatial effects should be tested for in empirical works involving growth rate of employment (EMPR), growth rate of gross in-migration (INMGR), growth rate of gross out-migration (OTMGR), growth rate of median household income (MHYR), and growth rate of direct local government expenditures per capita (DGEXR). The existence of spatial dependences in the error terms is an indication that random shocks into the system with respect to each of these endogenous variables do not only affect the county/counties where the shock originated and its/their neighbors, but 
also create shock waves across the study area (Appalachia). This is possible because of the structure of the autoregressive error model.

The existence of spatial dependences in the dependent variables and the error terms of the models would mean, retroactively, the spatial estimation methods which account for such spatial spillover effect tend to give more consistent, efficient and unbiased coefficient estimates compared to the non-spatial methods that are considered in this study. 


\section{ACKNOWLEDGEMENTS}

Undertaking this research study has been one of the most challenging yet productive and rewarding experiences of my life. This was possible because of the unfettered financial, intellectual, moral and leadership support that I have received from a number of institutions, families, friends and relatives. First, I would like to express my deepest gratitude to the Division of Resource Management, Davis College of Agriculture, Forestry and Consumer Sciences for granting me the graduate research assistantship. I also gratefully acknowledge the institutional services and tremendous support received from the faculty and staff of the Division of Resource Management. In this respect, I would like to extend my utmost respect and great appreciation to the Division Director, Dr. Peter V. Schaeffer, the Department Chair, Dr. Alan R. Collins, and the Program Coordinator, Dr. Tesfa G. Gebremedhin for their caring and understanding characters as well as for their outstanding leaderships.

I would also like to express my immense gratitude and thanks to my committee members: Dr. Tesfa G. Gebremedhin, Dr. Peter V. Schaeffer, Dr. Gerard E. D’Souza, Dr. Timothy T. Phipps, and Dr. Stratford M. Douglas for generously sharing their knowledge, experience, insights, and perceptions and for their unreserved support, encouragements and intellectual advice and guidance. Special thanks and appreciation go to my committee chair, Dr. Tesfa G. Gebremedhin and to Dr. Peter V. Schaeffer who provided insightful, practical, theoretical and conceptual knowledge of the subject and professional guidance throughout my study period. I am also grateful to Dr. Alan R. Collins, Dr. Ge Lin and Dr. Ingram R. Prucha for generously sharing their expertise and for their willingness to help. Without the generous and free availability of the resources and works of Professor Prucha this work could not have 
been a success. Special thanks also go to all staff and faculty of the Department of Economics, College of Business and Economics, WVU for their course work related services. Especially, I owe special thanks to Dr. Stratford M. Douglas, Dr. Santiago M. Pinto, Dr. Ronald Balvers, Dr. Brain Cushing and Dr. Subhaya Bandyopadhyay for their comments on my research and for being exemplary instructors.

Equally important, my special thanks go to Ellen Hartley-Smith, Melanie Jimmie, Lisa A. Lewis, and Alice Compton for their wonderful and all rounded services, and above all for their caring, willingness to help and support. I am also grateful to Dr. Tim M. Phipps, for his critical role during my enrolment and for being exemplary instructor, Dr. Fletcher for his technical supports, Dr. Walter C. Labys for expressing his willingness to allow me to use EViews software in his computer, Jacquelyn Strager for providing me with GIS shape files, Gloria Nestor for helping me in printing large sized maps, Mark Aronhalt, John.J. Kilionski and Ben Groover for their computer technical services, Denise Hunnell and Dr. Dennis K. Smith for their outstanding leadership, Office of the Associate Director for granting me Doctoral Travel Assistants, Dr. Peter Li and Deborah Jacques for their unreserved help and understanding, Dr. Walter Graeme Donovan for being a nice friend and for his willingness to help, Dr. John E. Saymansky, Dr. Mark Sperow, and Dr. Chery Lynn Brown for their encouragements, and all staff of West Virginia University Libraries for their services.

The successful completion of this study owes credit to the unfailing support of many individuals and families. Most important and with utmost gratitude, I would like to thank Dr. Tesfa and his family: Mehret Gebremeskel, Adam Gebremedhin, Luwam Gebremedhin and Abnet Gebremedhin, Mussie Futur and their family. This is a very kind, generous and caring family. Their continuous and unfailing all rounded help and encouragements made my study 
most pleasant. This family demonstrated its affection and respect to me and to my family by standing with me during the bad and good times. Special thanks go to Aday Sembetu Tesfay, Tesfamariam, Alganesh, Mebrat, Milete, Yacob, Andeab, Yicalo, Adane Gebremeskel and their families. Special thanks also go to Etiopia, Haimanot, Andemariam and Algnesh Gebremedhiin and their families. Each member of these families has been supportive in one form or another. I would also like to gratefully acknowledge the support, respect, and care I have received from Dr. Kiflay Gebremedhin, Tsedal Issak and their family. This family is uniquely special to me in many ways.

Tesfahunei Tecle, Almaz and their family, Dr. Semere and his family, Dr. Kesete Y. Ghebreyesus, Lemlem Tecle and their daughter Rahwa, Dr. Gebre Hiwet Tedfaghiorgis and his family, Habteyesus Kifleyesus and his family, Tseggai Haile and his family, my cousins Mehret Gebremichael and her family, Haile, Nigisti and Semere Tseggai and their families, Tseggai Ogube, Kifle'ab, Saba, Habte Ghirma, Aster Ghebrezghiabher and her siblings, Habtemichael Woldemichael's family, and Micheal Saare, Terhas Fessehatsion and their son Manna.

I am also grateful to all my friends from "Alem Bekagn", my colleagues from University of Asmara, and all my former students for their friendship, intellectual and moral supports throughout my intellectual and educational developments. Special thanks go to Dr. Ghebreberhan Ogubazghi, Dr. Tadesse Mehary, Dr. Melake Tewolde, Dr. Giorgis Tekle, Dr. Tesfayesus Mehary, Dr. Woldesellasie Ogubazghi, Kiflemariam Zerom, Dr. Abel Berhe and his family and to all staff and faculty of University of Asmara.

I would like also to thank all my friends who made my study at WVU easy and enjoyable. Special thanks go to my office mate Semoa D'sousa-Brown, to my friends Mulugeta 
Saare, Yodit Asser, Dr. Xiaobing Zhao, Dr. Tatiana Borisova, Dr. Xaixiao Huang, Dr. Doolarie Singh-Knights, Dr. Anura Amarasinghe, Dr. Kamar Ali, Dr. James Bukenya, and to my former students Dr. Yohaness G. Hailu and Ahadu T. Tekle. I am also very much grateful for the moral support received from the Greek Community in Morgantown. Especially, special thanks go to Jack, Mary and Grand Ma Helen for their constant moral support and their concerns about my family.

I am greatly indebted to my brothers (Gebru, Amanuel, Woldeabizghi, Kidane), my cousins, and my in-laws for being wonderful loving families and for their constant and unfettered supports. Most important and with utmost gratitude I would like to thank my cousin Ocubamariam (Hiwet) Gebreselassie for her unparalleled financial and moral supports throughout my undergraduate and graduated studies. She also shares with me the burden of extended family responsibilities. I also thank Lemlem Haile for her important role in easing for me this extended family responsibility. I have also received love and support from my cousins Atsedemariam Gebreselassie, and Rigbe Araya and my brother in-law Woldegebriel Tareke and their families. I thank them all.

Last, but by no means least, I extend my immense gratitude and appreciation to my dear wife, Luul Yehdego Beraki and to my beautiful daughter Ellen Gebremeskel for their unwavering love, patience and inspiration that surrounded me on a daily basis and for the stimulus they have provided me throughout the preparation of this dissertation. Unfortunately and very sadly, my family was denied exit visa from Eritrea to the United States to come to join me here Thus, Luul not only had to take the burden of family responsibility so that I could concentrate on my study, but also sacrificed her plan of having more one child in order to allow me to finish my study. The cost of pursuing my study to my little beautiful daughter is 
also exceptionally very high. She has to miss her father's love and caring at the time when she needs it most. 


\section{DEDICATION}

It is with utmost respect that I dedicate this work to my wife Luul Yehdego Beraki and to my daughter Ellen Gebremeskel who paid the most - love and caring - for the successful completion of this study; to my late parents, my father Habteyonas Gebremariam and my mother Tsedal Beyene and to many Eritrean parents like them who sent their sons and daughters to the liberation fronts for freedom and independence but never be fortunate enough to see Independence Day, May 24, 1991; to my late brother Samuel Habteyons and my late only sister Ellen Habteyonas who unfortunately are not survived by any children; to my late mentors and friends Asmelash Fessehatsion and Abraha Ghirmatsion; and to all those who languished in prisons or being martyred in the fight for freedom, justice and democracy in Eritrea. 


\section{TABLE OF CONTENTS}

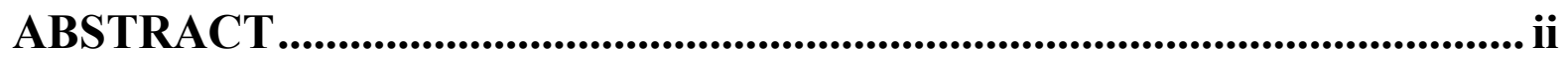

ACKNOWLEDGEMENTS .................................................................. v

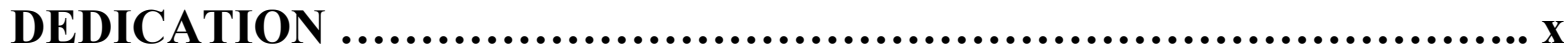

TABLE OF CONTENTS ............................................................................

LIST OF TABLES ..................................................................................

LIST OF FIGURES .............................................................................................. xvi

CHAPTER I - INTRODUCTION .................................................................. 1

1.1 Background and Problem Statement ......................................................................1

1.2 Study Area Profile........................................................................................................... 5

1.3 Justification. ..........................................................................................................17

1.4 Objective of the Study ......................................................................................20

1.5 Methodology ........................................................................................................................21

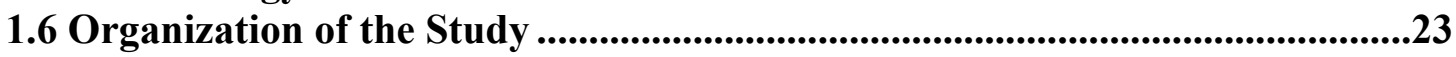

CHAPTER II - LITERATURE REVIEW ........................................................... 25

2.1 Introduction.....................................................................................................25

2.2 The Role of Small Business in Economic Development and Poverty Alleviation .........................................................................................27

2.3 County-Level Determinants of Small Business Growth....................................31

2.4 County-Level Determinants of Migration Behavior ...........................................42

2.5 County-Level Determinants of Local Public Services ...........................................47

2.6 County-Level Determinants of Median Household Income...............................52

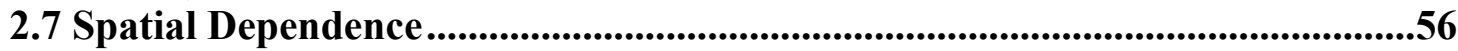

CHAPTER III - THEORETICAL FRAMEWORK ......................................62 62

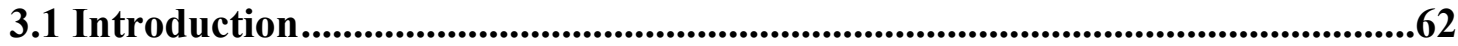

3.2 Fundamental Issues In Simultaneous Models ...................................................65

3.3 The Identification Problem .....................................................................................69

3.3.1 Identification through Restrictions on the Structural Parameters.........................................................................73

3.4 Method of Estimation .......................................................................................78

3.4.1 Single-Equation Method.................................................................80

3.4.1.1 Ordinary Least Squares ..........................................................81 
3.4.1.2 Indirect Least Squares .................................................................84

3.4.1.3 Two-Stage Least Squares ...............................................................88

3.4.2 System of Equations Method .......................................................................99

3.4.2.1 Three-Stage Least Squares .........................................................99

3.4.2.2 Full-Information Maximum-Likelihood ....................................107

3.4.2.3 Generalized Method of Moments (GMM)...........................113

3.5 Estimating Simultaneous-Equations Models Using Panel Data .......................118

3.5.1 Single-Equation Estimation ..........................................................................118

3.5.2 System of Equations Method .............................................................123

3.6 Spatial Analysis .................................................................................................127

3.6.1 Spatial Weight Matrix .................................................................................128

3.6.2 Spatial Autocorrelation ...............................................................................132

3.6.2.1 Global Indicators of Spatial Autocorrelation ..........................133

3.6.2.2 Local Indicators of Spatial Association (LISA)......................139

3.7 Spatial Autoregressive Process Models...................................................................147

3.7.1 Spatial Regression Models .........................................................................148

3.7.1.1 Spatial Dependence in Cross-Sectional Models ......................150

3.7.1.2 Spatial Dependence in Simultaneous-

Equestions Panel Data Models...............................................162

3.8 Estimation Issues in Spatial Simultaneous Equations Models..........................167

3.8.1 Cross-Sectional Data Setting................................................................168

3.8.2 Panel Data Setting.......................................................................................175

3.9 Specification Tests............................................................................................................185

CHAPTER IV - METHODOLOGY AND DATA......................................189

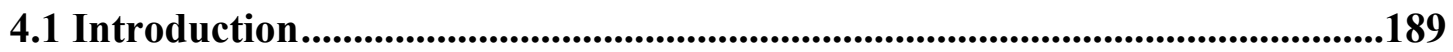

4.2 Theoretical Model Development...............................................................................190

4.3 Spatial Models .............................................................................................................212

4.4 Empirical Models.........................................................................................................217

4.5 Definition of Variables, Sources of Data and Descriptive Statistics................221

4.5.1 Definition of Variables...............................................221

4.5.2 Sources of Data.................................................225

4.5.3 Descriptive Statistics..............................................227

CHAPTER V - EMPIRICAL RESULTS AND ANALYSES......................231

5.1 Introduction..............................................................................................................231

5.2 Empirical Estimation: Standard (Non-Spatial) Simultaneous

Equations Growth Model..........................................................................................233

5.2.1 Cross-Sectional Results Analysis: 1900-2000 .......................................234

5.2.1.1 Employment (Business) Growth Rate ......................................234

5.2.1.2 Gross In-Migration Growth Rate ...........................................241

5.2.1.3 Gross Out-Migration Growth Rate...........................................245

5.2.1.3 Median Household Income Growth Rate ..............................247

5.2.1.3 Direct Government Expenditures Growth Rate.....................250

5.2.3 Panel Results Analysis ...............................................................................254 
5.3 Empirical Estimation: Spatial Simultaneous-Equations

Equilibrium Growth Model .................................................................259

5.3.1 Exploratory Spatial Data Analysis ..................................................260

5.3.2 Spatial Regression Estimation Results and Discussion ..................263

5.3.2.1 Estimation Issues ..........................................................263

5.3.2.2 Results and Discussion ......................................................265

CHAPTER VI - SUMMARY AND CONCLUSIONS ................................297

6.1 Introduction......................................................................................................................297

6.2 Concluding Summaries of Results..................................................................299

6.3 Policy Implications.....................................................................................................310

6.4 Contributions and Limitations .........................................................................313

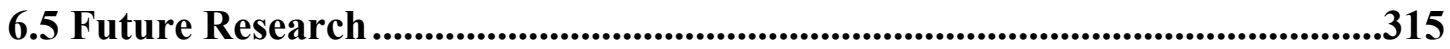

REFERENCES..........................................................................316

APPENDICES ...............................................................................................................340

APPENDIX 1: Cross Sectional and Panel Date Non-Spatial

Models Estimation Results for Appalachian

Counties, Appalachian States' Counties and

US Counties.

APPENDIX 2: Estimation Results of the Spatial

Simultaneous Equation Models............................................379

APPENDIX 3: Global and Local Spatial Auto-

correlation (Moran's I and LISA)........................................419

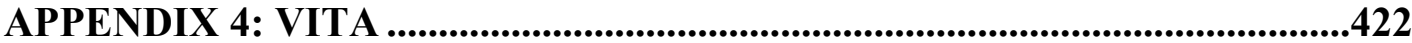




\section{LIST OF TABLES}

$\begin{array}{lll}\text { Table Title } & \text { Page }\end{array}$

County Economic Indicators ..................................................

1.2 Income and Poverty Measures in

U.S. and Appalachia - 1999 ..........................................................10

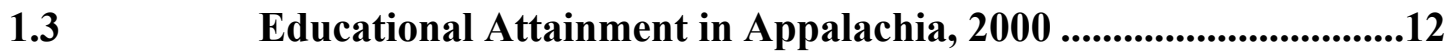

1.4 County-to-county Migration for Appalachia

and its Sub-regions, 1990, 2000...........................................................14

4.5.2a Variable Descriptions and Data Sources ....................................226

4.5.2b Descriptive Statistics for Appalachia

Counties, 1980-1990 ..............................................................227

4.5.2c Descriptive Statistics for Appalachia

Counties, 1990-2000 .......................................................................228

4.5.2d Descriptive Statistics for Appalachia

Counties, 1980-2000 .............................................................229

5.2.1a GMM Estimation Results, APPALACHIA,

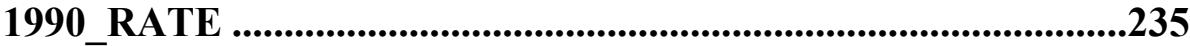

5.2.1a GMM Estimation Results, APPALACHIA, 1990_RATE (continued) ............................................................238

5.2.3a E3SLS Estimation Results, APPALACHIA,

PANEL_RATE .................................................................256

5.2.3a E3SLS Estimation Results, APPALACHIA,

PANEL_RATE (continued) ........................................................257

5.3.1a Global Moran's I Statistics of Spatial

Autocorrelation: Appalachia, 1990-2000 ........................................261

5.3.1b Global Moran's I Statistics of Spatial

Autocorrelation: Appalachia, 1980-1990 .....................................262 
5.3.1c Tests for Spatial Autocorrelation in the

Error Terms, Appalachia .......................................................262

5.3.2a Feasible Generalized Spatial Three-Stage

Least Squares (FGS3565) Estimated

Results, PANEL_RATE

5.3.2a Feasible Generalized Spatial Three-Stage

Least Squares (FGS3SLS) Estimated

Results, PANEL_RATE (continued) .............................................2275

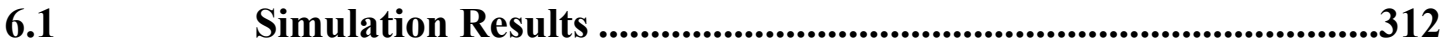




\section{LIST OF FIGURES}

Figure Title Page

Appalachian Counties and Sub-regions............................................6

Economic Categories of Appalachia..............................................9

$1.3 \quad$ Organization of the Study ....................................................................24

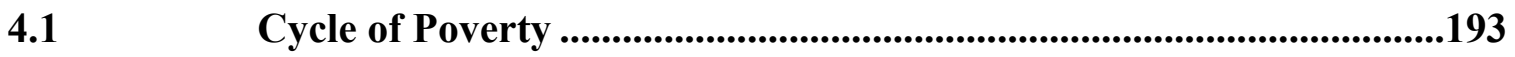

$5.1 \quad$ Empirical Models Estimation Strategies ........................................232 


\section{CHAPTER I}

\section{INTRODUCTION}

\subsection{Background and Problem Statement}

Persistent rural poverty is one of the most stubborn social problems facing policy makers in the United States. Despite decades of intervention, and the spending of billions of public dollars, many rural communities remain mired in poverty. The economic boom of the 1990s not only failed to reduce poverty in all counties, but it was associated with rising poverty rates in certain counties (Rupasingha and Goetz, 2003). Counties in Appalachia, for example, had above average poverty rates in 1990s. Thus, after a decade of unprecedented expansion of the economy of the United States, many regions in Appalachia are still suffering from high unemployment, shrinking economic base, deeply rooted poverty, low human capital formation, and out migration (Deavers and Hoppe, 1992; Haynes, 1997; Dilger and Witt, 1994; Maggar, 1990). The slow growth of income and employment in the region, out-migration and the disappearance of rural households are both causes and effects of persistent high rates of poverty. This lagging economic development negatively affect the economic and social well-being of the rural population, the health of local businesses, and the ability of local governments to provide basic human services (Cushing and Rogers, 1996).

The changing structure of traditional rural industries and the impact of those changes on rural communities have been sources of concern to many groups interested in the welfare of rural areas. State policy makers and local leaders have been placing a high priority on rural economic development (Pulver, 1989; Ekstrom and Leistritz, 1988). 
Consequently, a better understanding of factors that influence the local employment earning capacity and rural quality of life issues has become important from county, state and regional policy perspectives with respect to designing human capital development programs needed for rural community development. Since many of the forces responsible for past economic and social changes in the rural communities will continue to affect rural families, it becomes necessary to study the rural economy and evaluate alternative policy measures to promote diverse and resilient local communities.

Rural development is moving away from the agricultural sector and is increasingly linked to enterprise development. Since national economies are getting more and more globalized and competition is intensifying at unprecedented pace, affecting not only industry but any economic activity, it is not surprising that rural entrepreneurship is gaining in its importance as a force of economic change that must take place if many rural communities are to survive. Widely dispersed small communities with relatively small local and regional markets dominate in Appalachia. The businesses that serve these markets also tend to be small. Thus, considering one-by-one count, it is therefore tempting to dismiss small businesses as unimportant. Collectively, however, they make a large contribution to the economic diversity of small communities. However, small business has not been the traditional approach to rural development. While entrepreneurship is not a panacea, it should be considered as an important part of any comprehensive rural development strategy.

Improving a region's economic basis requires an economic environment where business can prosper. Appalachia, however, despite efforts of multilateral, national and local policy programs to induce economic prosperity and ameliorate poverty, has many 
economically depressed areas and regions. To strengthen and diversify the economy, policy makers and local leaders need to know the characteristics and impact of small businesses on the local economy. Understanding the characteristics of poverty and the contribution of small businesses to economic growth of the local economy is crucial in designing specific and appropriate development policies. The targets of such policies are to improve and expand community-based capabilities and initiatives to assist small communities to retain and expand local small businesses.

The potential role of Small and Medium Enterprises (SMEs) includes: generating employment and thus contributing to absorbing any surplus labor which results from economic restructuring; contributing to the development of a diversified economic structure (including their role as suppliers to large firms); contributing to trade balance through export earnings or import substitution; sources of government revenue; and as sources of innovative activity, thereby acting as source of change in the market (Acs and Audretsch, 1993). In addition, within the context of local and regional development, the growth of SMEs, especially in rural areas, has a number of non-economic benefits. Some of such benefits include: countering out-migration; protection of rural culture; and enhancing local facilities and infrastructure benefiting all.

Studies have shown that employment, occupation and salary as well as the quality and quantity of local public services are major considerations in the decision to migrate. The direction and magnitude of migration depends on the salary differential across regions. High earnings are associated with net in-migration and low earnings with net out-migration. Since low-income states are dominated by occupations with relatively low earning at the national level, and the earnings within particular occupation in low-income 
states tend to be lower than the national average, low-income regions face a net outmigration. The opposite relationships characterize the high-income states. By promoting the growth and development of small firms, low-income regions can, however, reduce and ultimately reverse the net out-migration of skilled labor.

In the absence of vibrant small firms, communities are devoid of the capability to provide job opportunities for skilled labor and college graduates that result in their outmigration. The out-migration of these educated people from such communities, in turn, erodes the income and property tax base that provides the major source of revenue to finance local public services such as schools, infrastructure, health, etc. This increases the tax price per remaining persons for any level of public spending. Consequently, the cost of providing local public services for the community at large increases. Over time, the quality and quantity of local public services in the community deteriorates, and further out-migration results. The out-migration of skilled labor and the declining population, in turn, not only increases the cost of providing local public services and consequently, a decline in the quality and quantity of these services but also constrains the expansion and growth of small business by limiting the supply of labor and demand for small business products. Low quality and quantity of public services (such as education, health, etc.) reduces the earning capacity of residents and discourages business formation and growth. But, this fuels back into the cycle and ultimately results in the perpetuation of poverty and underdevelopment in the region.

Although understanding the interconnections between small businesses growth, migration behavior, local public services and the incidence of rural poverty has been the interest of many researchers, there have not been much attempts made to explain their 
interdependence in a simultaneous equations approach. This study first, develops a standard (non-spatial) five-equation simultaneous-equations model with small business growth (using employment growth rate as a proxy), gross in-migration, gross outmigration, local public services (measured by local public expenditures) and incidence of poverty (using median household income as a proxy) as endogenous variables of the model. The implementation of the empirical model uses data from 418 Appalachian counties. The underlying data generating process of regional data such as county-level data, however, are prone to contain spatial dimensions. Since ignoring such spatial spillover effects leads to biased, inconsistent and inefficient estimates, the standard model is expanded to accommodate such concerns. Consequently, five-equation spatial simultaneous equation models (for both cross-sectional and panel data setting) have been developed. This effort is preceded by an extensive exploratory spatial data analysis and specification tests in order to detect the existence and form of spatial dependences in the data set.

\subsection{Study Area Profile}

Differential rate of economic growth has become a process that characterized the US economy. Thus, despite decades of unprecedented expansion of the economy of the United States, many regions in Appalachia are still suffering from high unemployment, shrinking economic base, deeply rooted poverty, low human capital formation, and out migration. This characterization of Appalachia has become a basis for regional development policy that aims at revitalizing the local economy. However, understanding the determinants of regional growth variation is important from a local economic 
development policy perspective. In recognition of this perspective, this dissertation examines the determinants of growth in Appalachia during the 1990s.

Geographically, Appalachia is about 200,000 square miles area that stretches from southern New York State to northeast Mississippi along Appalachian Mountains. Appalachia as defined by Appalachia Regional Commission (ARC) covers 418 counties, including 8 independent cities in Virginia, in thirteen states- Alabama (37 counties); Georgia (37 counties); Kentucky (51 counties); Maryland (3 counties); Mississippi (24 counties) New York (14 counties); North Carolina (24 counties); Ohio (29 counties); Pennsylvania (52 counties); Tennessee (50 counties); Virginia (31 counties, including 8 independent cities); and the whole 55 counties of West Virginia. The region is also divided into the Northern, Central and Southern sub-regions as indicated in Figure 1.1.

Figure 1. 1: Appalachian Counties and Sub-regions

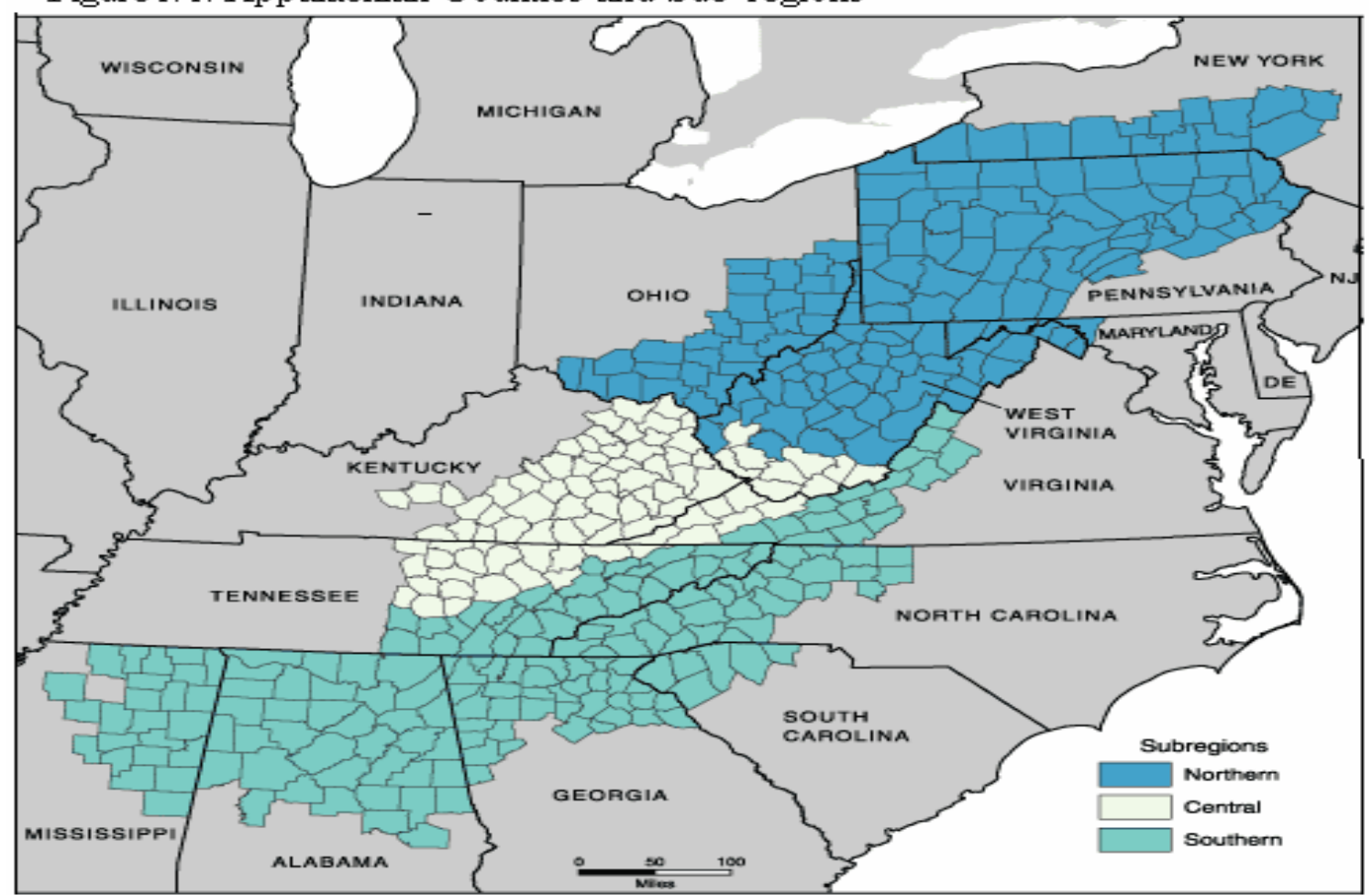

Source: Appalachian Regional Commission, 2002 
The Northern Sub-region contains every Appalachian county in New York, Pennsylvania, Maryland, and Ohio, as well as 46 of West Virginia's 55 counties. The Central Subregion consists of nine southernmost counties of West Virginia, all of Appalachia Kentucky, the southwestern tip of Virginia and the northwest part of Appalachian Tennessee.

Finally, most of Appalachian parts of Virginia and Tennessee, as well as all of Appalachian North and South Carolina, Georgia, Alabama and Mississippi constitute Southern Appalachia Sub-region. Nearly two-third of Appalachian counties are classified as rural, and Central Appalachia is the most rural of the three sub-regions.

The ARC also classifies Appalachia into four categories of economic development: distressed, transitional, competitive, and attainment. This system of classification is based on the comparison of three county indicators of economic viability - per capita market income, poverty, and three-year average unemployment- to their respective national average.

Table 1.1: County Economic Indicators

\begin{tabular}{|c|c|c|c|}
\hline $\begin{array}{l}\text { County } \\
\text { Economic level }\end{array}$ & $\begin{array}{l}\text { Per Capita } \\
\text { Market Income }\end{array}$ & Poverty Rate & $\begin{array}{l}\text { Three-Year Average } \\
\text { Unemployment Rate }\end{array}$ \\
\hline Distressed & $\begin{array}{l}67 \% \text { or less of } \\
\text { the U.S. average }\end{array}$ & $\begin{array}{l}150 \% \text { or more of } \\
\text { U.S. average }\end{array}$ & $\begin{array}{l}150 \% \text { or more of United } \\
\text { States average }\end{array}$ \\
\hline Transitional & \multicolumn{3}{|c|}{ All counties not in other classes. Individual indicators vary } \\
\hline Competitive & $\begin{array}{l}80 \% \text { or more of } \\
\text { U.S. average }\end{array}$ & $\begin{array}{l}100 \% \text { or less of } \\
\text { U. S. average }\end{array}$ & $\begin{array}{l}100 \% \text { or less of United } \\
\text { States average }\end{array}$ \\
\hline Attainment & $\begin{array}{l}100 \% \text { or more of } \\
\text { U.S. average }\end{array}$ & $\begin{array}{l}100 \% \text { or less of } \\
\text { U. S. average }\end{array}$ & $\begin{array}{l}100 \% \text { or less of United } \\
\text { States average }\end{array}$ \\
\hline
\end{tabular}

Source: Appalachia Regional Commission, 2002 
As indicate in Figure 2, distressed counties are generally found clustered in central Appalachia coalfields, and in the deeper southwestern portions of Appalachian Mississippi and Alabama. On the other side of the continuum are the attainment counties which are few and are found either in or near major metropolitan areas. More than twothird of Appalachian counties (70\%), however, are in transitional stage and are found in every Appalachian State (ARC, 2002).

Historically, the average socioeconomic statuses of Appalachians have been lower than the status of the rest of the country. Appalachia remained for many a symbol of poverty and underdevelopment in the midst of America's global power and prosperity. Actually, it was Appalachia's income and poverty levels in the 1960s that helped get the federal government to pay attention to the region in the first place- which ultimately led to the formation of Appalachian Regional Commission (Pollard, 2003). Per capita market income in Appalachian was about 77 percent of the U. S. average in 1960 and 31.1 percent of the region's residents lived in poverty, compared to 22.1 percent of all Americans (Wood and Bischak, 2000). The gap has narrowed since then and per capita income in Appalachia reached 81 percent and 84 percent of the U. S. income in 1989 and 1999, respectively. Despite these improvements, income in Appalachia did not reach parity with the rest of country by 2000. Similarly, although there have been improvements, Appalachian poverty rates remain higher than the country as a whole (see Table 1.2). 
Figure 1.2: Economic Categories of Appalachia

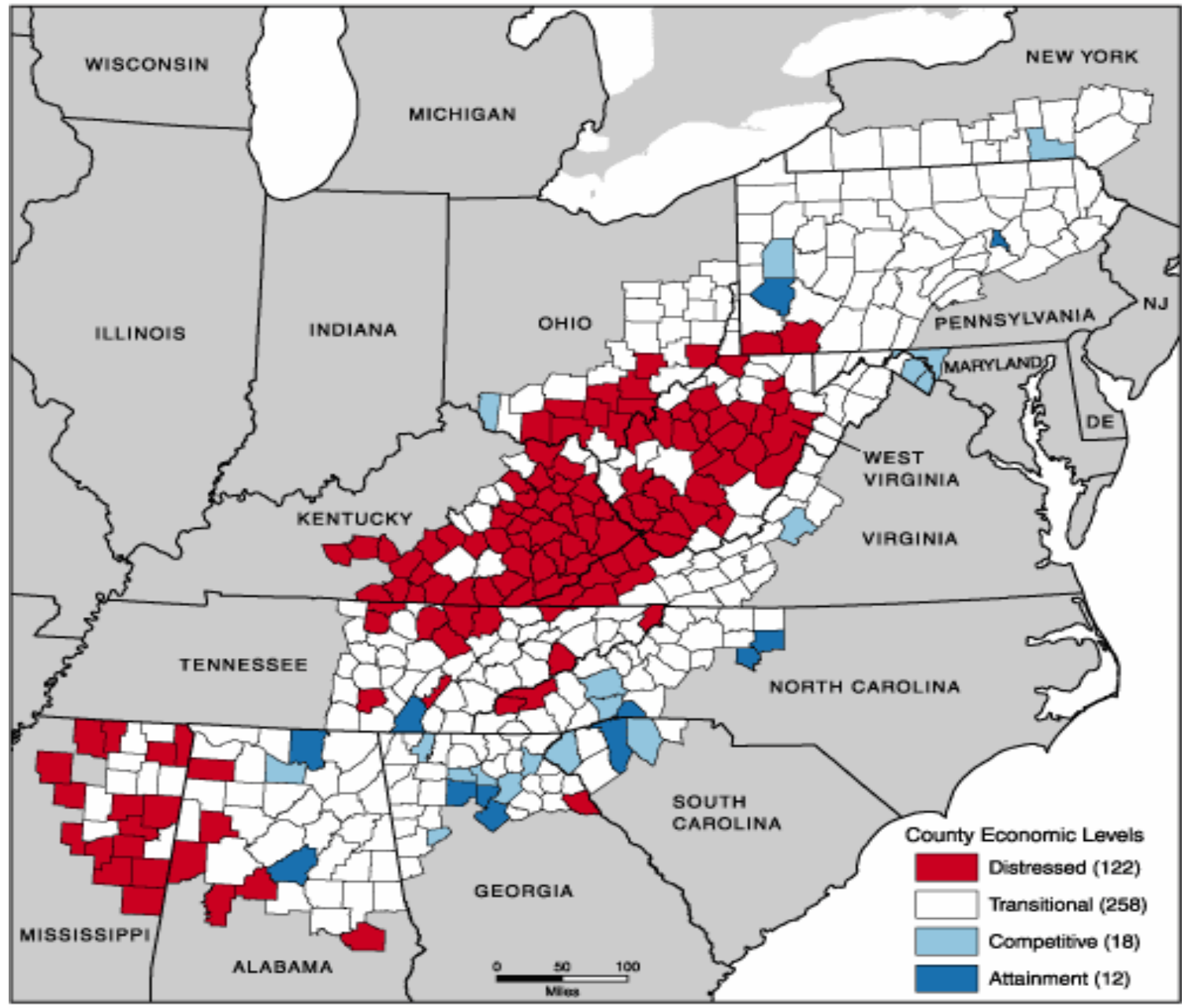

Source: Appalachian Regional Commission, 2002

As indicated in Table 1.2, there is also regional variation within Appalachia with respect to socio-economic status. The lowest per capita income is found in Central and rural Appalachia. Central Appalachia, for example, had a per capita income of 66 percent of the national average compared to southern Appalachia which had per capita income of 89 percent of the national average. The majority of the distressed counties (84 percent) are found in Central Appalachia and rural areas. Thus, it is not surprising to find that per capital income levels in Central Appalachia were significantly lower than in any other categories. 
Table 1.2: Income and Poverty Measures in U. S. and Appalachia, 1999

\begin{tabular}{|c|c|c|c|c|}
\hline & $\begin{array}{l}\text { Per Capita } \\
\text { Income }\end{array}$ & $\begin{array}{l}\text { Median Family } \\
\text { Household Income }\end{array}$ & $\begin{array}{l}\text { Individuals } \\
\text { in Poverty }\end{array}$ & $\begin{array}{l}\text { Families in } \\
\text { Poverty }\end{array}$ \\
\hline Area & \multicolumn{2}{|c|}{ In 1999 U. S. Dollars } & \multicolumn{2}{|c|}{ Percent } \\
\hline United States & 21,587 & 41,994 & 12.4 & 9.2 \\
\hline Appalachia & 18,218 & 35,234 & 13.7 & 10.2 \\
\hline Northern & 18,055 & 34,728 & 12.8 & 9.2 \\
\hline Central & 14,315 & 26,028 & 22.1 & 18.1 \\
\hline Southern & 19,167 & 37,617 & 12.8 & 9.5 \\
\hline Metro Counties & 19,733 & 38,064 & 12.0 & 8.6 \\
\hline Urban Counties & 16,404 & 32,178 & 14.8 & 11.0 \\
\hline Rural Counties & 14,428 & 27,082 & 20.0 & 16.3 \\
\hline Distressed & 13,287 & 24,220 & 25.3 & 20.7 \\
\hline Transitional & 17,471 & 33,805 & 13.6 & 9.9 \\
\hline Competitive & 20,613 & 40,240 & 10.3 & 7.5 \\
\hline Attainment & 23,704 & 46,393 & 9.1 & 6.5 \\
\hline
\end{tabular}

Source: Appalachia Regional Commission, 2002

It is also important to note that income from Social security makes up a larger portion of income in Appalachia than in the United States, with Central, rural, and distressed counties having the highest values. The higher rates of public assistance in Appalachia indicate the general low level of income and the high rate of poverty in the region.

Previous studies have shown that characteristics related to the working world such as labor force participation, occupation and industry are crucial in assessing the overall economic vitality of a locality. These measures summarize the information on the 
region's human capital endowment, the nature of and composition the working force, and the nature of the industry mix in the region. In the past, Appalachian economy was heavily dependent on natural resource extraction and manufacturing. More recently, there has been some diversification, with high emphasis on services. However, coal mining still remains to be an important economic activity for many Appalachian communities. With the shift to a more service based industry, Appalachian female labor force participation rate has increased from 51 percent in 1990 up to 53 percent in 2000 while labor force participation rate for men declined from 70 per cent down to 67 per cent during the same period. Labor force participation rate was higher for both sexes- 71 per cent for men, 58 percent for women- outside Appalachia in 2000 (Pollard, 2003).

Human capital in the form of education and skilled labor force plays a critical role in the development of a community. A well-educated person is best prepared to meet the demands of a complex and changing society, as well as to participate fully in today's global economy. In fact, education beyond high school has become a requirement to secure a high paying job. Educational attainment in Appalachia is lower compared to the rest of the country. Nevertheless, there has been an improvement in educational attainments more recently. In 2000, 77 per cent of Appalachian residents age 25 and older have completed high school, up from 68 percent in 1990. The proportion of the population with at least bachelor's degree has also grown from 14 per cent in 1990 to 18 percent in 2000. The corresponding figures in non-Appalachian United States for the year 2000 are 81 and 25 percent respectively. As indicated in Table 1.3, there was also wide gap in educational attainment within Appalachia in 2000. Both high school and college education are higher in northern and southern Appalachia than in central Appalachia. 
Overall, there is positive correlation between the level of educational attainment and the socio-economic status.

Table1.3: Educational Attainment in Appalachia, 2000

\begin{tabular}{|c|c|c|c|c|}
\hline \multirow[b]{3}{*}{ AREA } & \multicolumn{2}{|c|}{ HIGH SCHOOL GRADUATES } & \multicolumn{2}{|c|}{ COLLEGE GRADUATES } \\
\hline & $\begin{array}{c}\text { NUMBER } \\
(, 000)\end{array}$ & PERCENT & $\begin{array}{l}\text { NUMBE } \\
\text { R (,000s) }\end{array}$ & PERCENT \\
\hline & & & & \\
\hline NON-APPALACHIA U.S. & 13,4752 & 80.7 & 41,750 & 25.0 \\
\hline APPALACHIA & 11,744 & 76.8 & 2,712 & 17.7 \\
\hline Northern Appalachia & 5,523 & 81.2 & 1,204 & 17.7 \\
\hline Central Appalachia & 926 & 64.1 & 154 & 10.7 \\
\hline Southern Appalachia & 5,295 & 75.2 & 1,354 & 19.2 \\
\hline Distressed Counties & 1,191 & 65.3 & 186 & 10.2 \\
\hline Transitional Counties & 7,500 & 76.8 & 1,561 & 16.0 \\
\hline Competitive Counties & 1,016 & 78.4 & 259 & 20.0 \\
\hline Attainment Counties & 2,037 & 84.6 & 707 & 29.4 \\
\hline Appalachian Section of: & & & & \\
\hline ALABAMA & 1,408 & 75.4 & 360 & 19.3 \\
\hline GEORGIA & 1,088 & 76.7 & 298 & 21.0 \\
\hline KENTUCKY & 470 & 62.5 & 79 & 10.5 \\
\hline MARYLAND & 127 & 78.6 & 23 & 14.4 \\
\hline MISSISSIPPI & 262 & 68.1 & 54 & 14.0 \\
\hline NEW YORK & 574 & 83.1 & 144 & 20.8 \\
\hline NORTH CAROLINA & 795 & 75.8 & 201 & 19.1 \\
\hline OHIO & 743 & 78.2 & 117 & 12.3 \\
\hline PENNSYLVANIA & 3,293 & 82.7 & 759 & 19.1 \\
\hline SOUTH CAROLINA & 509 & 75.3 & 139 & 20.6 \\
\hline TENNESSEE & 1,234 & 73.4 & 290 & 17.2 \\
\hline VIRGINIA & 312 & 69.8 & 66 & 14.8 \\
\hline WEST VIRGINIA & 928 & 75.2 & 183 & 14.8 \\
\hline
\end{tabular}

Source: Appalachia Regional Commission, 2002 
The socio-economic statuses of counties are also significantly influenced by the population dynamics of the region. Appalachia had 22.9 million people in 2000, which is about 8 percent of the total U. S. population at that time. Central Appalachia is the least populated sub-region with a population of 2.2 million in 2000 , followed by northern Appalachia with 10.1 million and southern Appalachia with 10.7 million populations. The pattern of population concentration is along the metro-non-metro and on the economic categories (distressed, transitional, competitive, and attainment) divide. In 2000, for example, about 58 percent or 13.2 million of Appalachians lived in the region's 109 metropolitan counties and the 9 attainment counties had 3.2 million persons, nearly 400,000 more than the region's 121 distressed counties (Pollard, 2003).

The growth in population during the 1990s was not even within Appalachia. Appalachia as a whole grew by 9 percent, which is far lower than the 14 percent for the rest of the nation. Most of this growth, however, occurred in southern Appalachia, which grew by 18 percent. The corresponding figures for central Appalachia and northern Appalachia are less than 6 percent and less than 2 percent, respectively. About 83 Appalachian counties, mostly in northern Appalachia, also lost population during the 1990's. Metropolitan Appalachia grew faster than non-metropolitan Appalachia (9.5 percent versus 8.6 percent) and competitive and attainment counties grew faster than distressed and transitional counties during the 1990s (Pollard, 2003).

One of the determinants of change in population is migration. A study by Obermiller and Howe (2002) found that Appalachian migration patterns during the 1970s and 1980s were changing from long-range flows into northern, southern, and western states outside the region to short-range, urban-suburban exchanges principally centered 
around cities in and immediately adjacent to the region. Their study showed that on net, migrants entering the region had lower-status jobs, lower incomes, less education, and were more likely to live in poverty than the people who migrated from the region. The same study also showed that Northern and Central Appalachia lost population while at the same time became refuge for low-income blue-collar workers with relatively little education. Southern Appalachia, on the other hand, gained population and attracted migrants with better education, with higher-status jobs and with diverse social and ethnic background.

These migration patterns also continued into the 1990s. As a percentage of its population, Northern Appalachia had net migration rate of 1.0 percent, -1.1 percent for 1990 and 2000, respectively. The corresponding figures for Southern Appalachia, on the other hand, are 4.6 percent and 3.9 percent. Central Appalachia lost population in 1990 (2.5 percent) and gained population in 2000 (0.6 percent) as shown in Table1.4.

Table 1.4: County-to-county Migration for Appalachia and its Sub-regions, 1990, 2000

\begin{tabular}{|c|c|c|c|c|}
\hline Area & Year & $\begin{array}{c}\text { Total } \\
\text { In-migration } \\
\end{array}$ & $\begin{array}{c}\text { Total } \\
\text { Out-migration } \\
\end{array}$ & $\begin{array}{c}\text { Net } \\
\text { Migration }\end{array}$ \\
\hline Appalachia & $\begin{array}{l}1990 \\
2000\end{array}$ & $\begin{array}{l}1,776,982 \\
2,057,900\end{array}$ & $\begin{array}{l}1,633,732 \\
1,732,484\end{array}$ & $\begin{array}{l}143,250 \\
325,416\end{array}$ \\
\hline \multicolumn{5}{|c|}{ Sub-regions of Appalachia } \\
\hline Northern & $\begin{array}{l}1990 \\
2000\end{array}$ & $\begin{array}{l}699,965 \\
700,388\end{array}$ & $\begin{array}{l}828,316 \\
795,510\end{array}$ & $\begin{array}{l}-128351 \\
-94,122\end{array}$ \\
\hline Central & $\begin{array}{l}1990 \\
2000\end{array}$ & $\begin{array}{l}119,498 \\
153,518\end{array}$ & $\begin{array}{l}150,578 \\
129,319\end{array}$ & $\begin{array}{l}-31,080 \\
24,199\end{array}$ \\
\hline Southern & $\begin{array}{l}1990 \\
2000\end{array}$ & $\begin{array}{l}957,519 \\
1,202,994\end{array}$ & $\begin{array}{l}654,838 \\
807,655\end{array}$ & $\begin{array}{l}302,681 \\
395,339\end{array}$ \\
\hline
\end{tabular}

Source: Appalachia Regional Commission, 2002 
The largest Appalachia migration flows during the 1990s were principally between counties with major cities and the counties that surround them. A study by Obermiller and Howe (2004) showed that, on net, metro and urban counties gained population while rural counties lost population through migration in 2000. Rural counties gained population from outside the region but lost population to metro and urban counties in the region. Thus, the over all direction of internal migration in Appalachia remained rural to urban. The region as whole also continues to be the destination for low-income population with little education, and low-occupational status. Obermiller and Howe (2004) concluded their study by stating that more people in poverty moved into Appalachia, while those with higher incomes, more education and higher job status moved out during the second half of 1990s. This pattern was specifically true for Northern and Central Appalachian sub-regions.

It is not hard to imagine the long-run consequences of this pattern of population dynamics on the socio-economic condition of Appalachia. If this pattern continues, the human resource stock of the region will deteriorate and its long-run capacity of supporting decent living will diminish. To address this problem, it is necessary first to understand the reasons for whys Appalachia is facing this pattern of population dynamics. Economic theory teaches us that individuals migrate to maximize their utility, which varies over space. Households evaluate the earning opportunities, cost of living, local amenities or dis-amenities, local taxes, and local public services associated with alternative residential locations and move freely to reside and seek for employment they want. Appalachian migration patterns, hence, are reflections of the pattern of the 
distribution of employment opportunities and other characteristics that affect quality of life in the region.

The distribution of employment opportunities and the general economic health of a region are dependent upon the distribution and nature of businesses that create jobs. Compared to the rest of the U. S. economy, in Appalachia, there were lower establishment formation and attrition rates, as well as lower job creation and destruction rates during the last decade A study by Brandow (2001), for example, shows that Appalachia although able to maintain existing firms, remains caught in a cycle of low levels of entrepreneurship, low growth among existing firms, and a continued overreliance on branch activities. Not only the availability of jobs were lower but also the quality of jobs, as measured by the average wages paid at the establishment, were about 10 percent lower in Appalachia compared to those in the rest of the nation.

In summary, Appalachia continues to be distinct from the rest of the United States. It is less diverse in terms of racial and ethnicity and with a higher median age and disproportionately fewer children and more elderly; its population is aging earlier than the rest of the nation. Appalachia is also lagging behind the rest of the nation on a variety of economic, labor force, and education measures despite some improvements in some areas. Appalachia has many economically distressed areas that suffer a shortage of skilled, well-educated workers who need to take advantage of a growing post-industrial economy. 


\subsection{Justification}

The issue whether regional development can be associated with population driving employment changes or employment driving population changes has attracted the attention of many researchers during the last three decades. Many of the studies that have tried to investigate the direction of causation in this 'people follow jobs or jobs follow people' literature begin with the two-equation model developed by Steinnes and Fisher (1994) to investigate population and employment changes within an urban area: Chicago and its suburbs (see Carlino and Mills, 1987, Dietz, 1998). One of the shortcomings of these growth equilibrium models, however, is that they do not explicitly incorporate the role of space in explaining variation in economic growth. Subsequent efforts to develop spatial extension of these models are also limited (see Boarnet, 1994, Henery, Schmitt, Kreistensen, Barkley and Bao, 1999; Henry, Schmitt, and Piguet, 2001). The limited empirical literature on the efforts to expand these models so that they can incorporate the role of space in explaining variation in economic growth is also mostly limited to cross sectional data only. Spatial panel data models are not very well documented in the spatial econometrics literature (Elhorst, 2003). A second shortcoming of the Carlino-Mills type models as well as their spatial extensions is their assumptions about in-migrants and outmigrants. The endogenous variable "population change" includes both (1) natural population increase and (2) the difference between in-migration and out-migration. Unless the characteristics of in-migrants and out-migrants are assumed to be the same (with respect to their effects to regional economy), taking "population change" as a net figure will gloss over the differential effects of in-migrants and out-migrants. This is even certain for Appalachia where in-migrants and out-migrants are markedly different. 
Another shortcoming of these models is, although local governments, through their taxation and spending actions, affect the economy and are being affected by it, the role of government is not explicitly captured by these models. The government sector is generally considered exogenous to the system. Besides, the level of per capita regional income is also treated as exogenously determined.

This study develops a methodology that addresses these shortcomings. First, a five-equation standard simultaneous equation model that explains the interdependences among small business growth, migration behavior, household income, local public services at the county-level is developed in a growth equilibrium framework. The model, spells out the 'feed-back simultaneities among these five endogenous variables conditional on a set of regional socio-economic variables. The rationale for this type of modeling is because estimating the coefficients of each equation of the model without considering the feed-backs would lead to biased, inconsistent and inefficient estimates. Consequently, this leads to wrong inferences and policy recommendations.

Second, the model is expanded to incorporate spatial spillover effects in a cross sectional setting. This is done after a spatial exploratory data analysis showed the existence of spatial interdependences in the data set. When the underlying data generating process includes a spatial dimension, and if the effect is ignored, regression could give inconsistent, inefficient and biased coefficient estimates (see Anselin, 1988, 2001; Anselin Bera, 1998). Thus, the inclusion of spatial effects is important from an econometric perspective. Besides, the inclusion of spatial spillover effects is important from and economic policy perspective because it answers whether and if so to what extent each of the dependent variables of the model in a given county depends on the 
characteristics of neighboring counties (spatial correlation). Such information is important to design appropriate policies that account for and give room for cross-border effects.

This study also further develops the spatial cross-sectional data model into spatial panel data model. This is important in the sense that panel data are generally more informative, and they contain more variation and less collinearity among variables. The greater availability of degree of freedom that results from the use of panel data increases estimation efficiency. Specifications of more complicated behavioral relationships that cannot normally be addressed using pure cross-sectional or time-series data are possible with the use of panel data (Elhorst, 2003). Thus, the rationale for the development and implementation of the spatial panel data model is the improvement in the accuracy of hypothesis testing and the subsequent inferences about the interdependences among the core variables of the basic model.

This study, generally, develops standard (non-spatial) cross-sectional, standard (non-spatial) panel data, spatial cross-sectional and spatial panel data simultaneousequation models. The empirical implementations of these model use data on 418 Appalachian counties. Although Appalachia is far from being homogenous, the region remains a distinct part of America. Appalachia lags the rest of the nation in every measure of socio-economic indicator (see the section on 'Study Area Profile' for details). Thus, Appalachia defines a good study area to test the hypotheses set in this study (see chapter IV for the specific hypotheses). 


\subsection{Objectives of the Study}

The overall objective of this study is to develop and implement empirical models that explain the interdependences among small business growth, migration behavior, local public services and median household income in order to generate detailed information for regional policy makers. The empirical implementations of the models use data primarily from 418 Appalachian counties. Data from the whole Appalachian State counties and the whole US counties are also used to make some comparative analyses. The estimated models are also solved (simulated) in order to compute the effects of possible changes in the policy variables. On the bases of these exercises, this study aims at developing a set of specific policy recommendations for small community development. The specific objectives of this study are to:

1. Examine the interdependences and the possible direction of causation among small business growth, migration behavior, local public services and median household income in Appalachia using a standard simultaneous equations models;

2. Test for spatial effects in the data set and prepare detailed exploratory spatial data analysis with respect to small business growth, migration behavior, local public services and median household income.

3. Expand the standard simultaneous models by incorporating spatial spillover effects and implement the resulting models with both cross sectional and panel data in order to generate the strength and direction of spatial spillover effects among Appalachian counties. 
4. Simulate (solve) the estimated simultaneous equation models (both spatial and non-spatial) for different scenarios of the exogenous or policy variables of the models.

5. Develop policy recommendations that aim at tackling poverty and underdevelopment in small communities in Appalachia. Specifically, policies that target at reversing the out-migration of the skilled, more educated, and the wealthy from Appalachia; improving the performance of small business; encouraging local governments to play their important roles in community development.

6. Develop research agenda for possible future extension and research in the area of small community development.

\subsection{Methodology}

The methodology followed in this study is an extension of the "jobs follow people, or people follow jobs" literature. A simultaneous-equation system that expresses the interdependences among small business growth, migration behavior, local public services and median household income is developed in a partial lag-adjustment growthequilibrium framework. This model improves previous models in the growth-equilibrium tradition by explicitly modeling the role of local government and regional income in the growth process. It is obvious that local governments through their spending and taxation actions affect and being affected by the local economy. Regional income is not also something that is exogenously determined. It also affects and being affected by the other regional factors. The model developed in this study is thus more realistic compared to previous models. 
The models in this study also explicitly modeled in-migration and out-migration separately in order to spell out their differential effects, which used to be glossed-over under net population change in previous models. This is significantly important because migration is treated as population equilibrating process in the growth-equilibrium models. Taking net population change as a variable of interest has a potential effect of hiding any differential effect between in-migration and out-migration on the local economy, unless in-migrants and out-migrants are characteristically similar. In-migrants and out-migrants in Appalachian counties, however, are characteristically different. Appalachia tends to be the destination for low-income people with little education, and low-occupational status. During the second half of the 1990s, for example, more people in poverty moved into Appalachia, while those with higher incomes, more education and higher job status moved out (Obermiller and Howe, 2004).

Test for spatial effects showed that the underlying data generating process includes spatial dimension. To incorporate these spatial spillover effects, the standard models were extended both in the cross sectional and panel data setting. Apart from the feed-back simultaneities, the models now include spatial autoregressive lag and spatial cross-regressive lag simultaneities. The models were also tested for the presence of spatial autocorrelation in the error terms using Moran's I test as suggested in Anselin and Kelejian (1997). The existence of both types of spatial dependences in all equations of the system led to the specification of the system in terms of spatial cross-sectional and spatial panel data models that incorporate both spatially autoregressive dependent variables and spatial autoregressive process in the error terms. The spatial models are estimated by Generalized Spatial Three-Stage Least Squares (GS3SLS) Estimator. This is 
an extension (interims of number of equation and number of cross-sections) of the threestep Generalized Method of Moments Estimator suggested by Kelejian and Prucha (1998). Detailed separate computer programs are written in TSP to run the five-equation spatial simultaneous equations model in cross-sectional and panel data setting. Both the modeling and the estimation strategies are significant improvements and contributions to the existing literature in spatial econometrics. The simultaneous spatial data model estimation is new addition. There is no paper or work that I know of so far which uses this technique in empirical work. The implementation of the model with five-equations even in a single cross-sectional data set is a major improvement over previous efforts.

\subsection{Organization of the Study}

The study is organized into six chapters including chapter I as the introduction. A detailed review of the literature is given in chapter II. Chapter III is devoted to the development of the theoretical framework. A detailed description of the technical issues that involve in simultaneous equations (both non-spatial and spatial) modeling, testing and estimation are presented in this chapter. The development of the methodology and the description of data are explained in chapter IV. The basic theoretical model of the study and its empirical extensions are developed in the first subsection of this chapter. The other subsection of the chapter is devoted to the description of the data set used in the implementations of the empirical models. Chapter V presents the empirical results of study. This chapter has three major subsections. The first subsection is devoted to the presentation of the non-spatial (both cross-sectional and panel) data analysis. In the second subsection, exploratory spatial data analysis (data visualization) is given. The 
third subsection finalizes chapter $\mathrm{V}$ by presenting the results of the spatial (panel) data analysis. Finally, conclusions and recommendations are illustrated in chapter VI..

Figure1.3. Organization of the Study

Chapter I

Chapter II

Chapter III

Chapter IV

Chapter V

EMPIRICAL RESULTS AND ANALYSES

Chapter VI

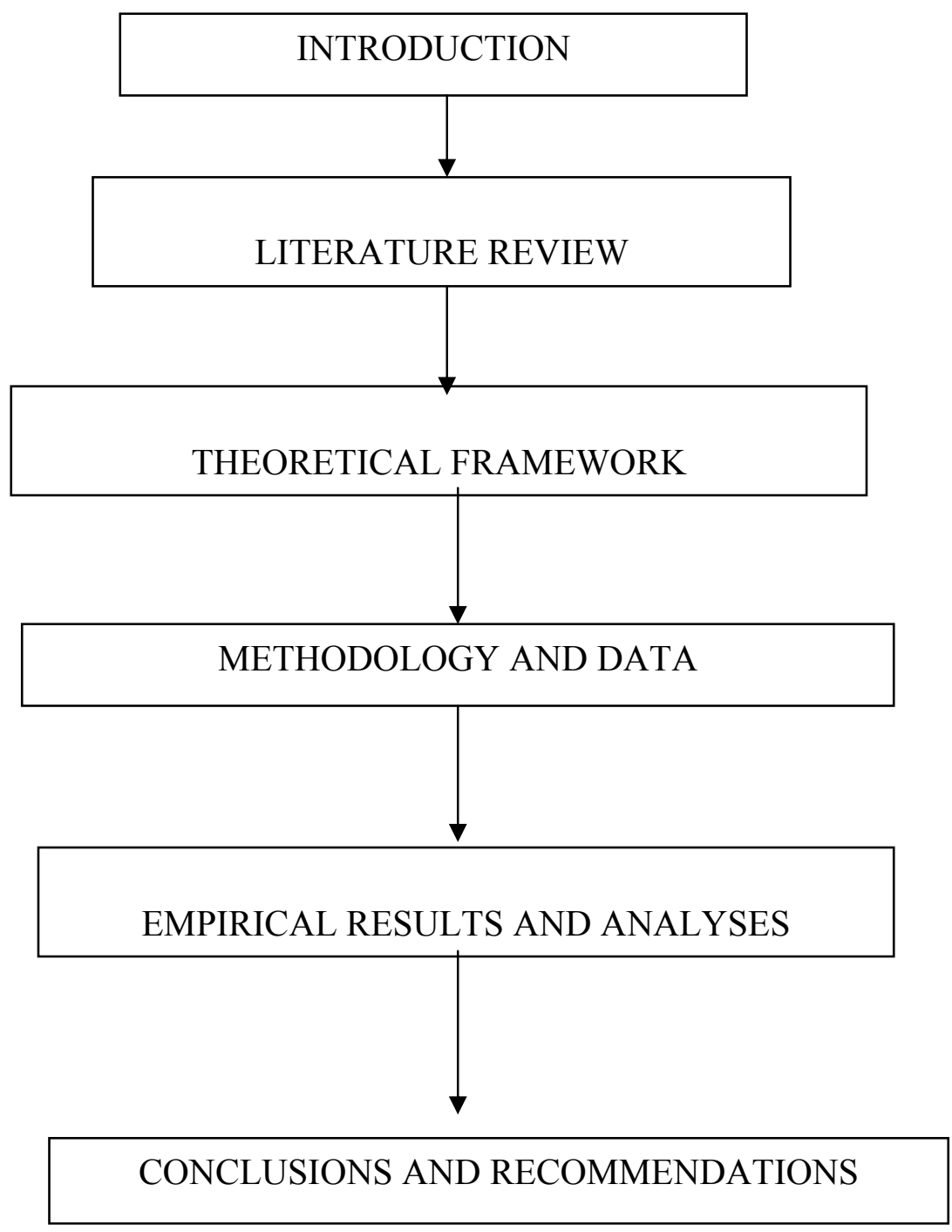




\section{CHAPTER II}

\section{LITERATURE REVIEW}

\subsection{Introduction}

Although a lot of knowledge has been gained through research and experience, the question of how to generate entrepreneurship and sustainable economic development remains unanswered (Voslee, 1994). The traditional approach to rural development was 'top-down'. Federal development authorities designed programs to provide infrastructure, human capital and investment from outside the rural community. While the investment in infrastructure was beneficial in attracting and supporting commercial activities and enhancing the rural quality of life, it did not necessarily provide a long term growing economic base (Petrin, 1992), and many rural areas were excluded because the cost of such schemes were too high to implement them in all rural areas. Because of such and other shortcoming, rural areas throughout the U.S. are still suffering from a lack of job opportunities, poverty, inadequate public infrastructure, and, as a result, the negative effects of out-migration. Therefore, new ideas were sought, and one that appear promising to many policy makers and scholars, is the development of small business and entrepreneurship. This approach is still fairly new, as theories of economic growth have traditionally largely ignored the role of small businesses and entrepreneurship, and the few existing theories are somewhat fragmented (Christy, Wenner and Dassie, 2000). By focusing on small, usually local, businesses, rural communities capture a greater share of the existing local income, and the focus on entrepreneurship has the potential of increasing the efficiency of existing local establishments and forming new businesses (Woods, Frye and Ralstin 1999). The focus on small business is particularly relevant in 
rural states of Appalachia, because small firms play a comparatively larger role in rural than in urban regions (U.S. Small Business Administration 1999).

In the absence of vibrant small firms, communities are devoid of the capability to provide job opportunities for skilled labor and college graduates that result in their outmigration. The out-migration of these educated people from such communities, in turn, erodes the income and property tax base that provides the major source of revenue to finance local public services such as education, infrastructure, health, etc. This increases the tax price per remaining persons for any level of public spending. Consequently, the cost of providing local public services for the community at large increases. Over time, the quality and quantity of local public services in the community deteriorates, and further out-migration results. The out-migration of skilled labor and the declining population, in turn, not only increases the cost of providing local public services and consequently, a decline in the quality and quantity of these services but also constrains the expansion and growth of small business by limiting the supply of labor and demand for small business products. Low quality and quantity of public services (such as education, health, etc.) reduces the earning capacity of residents and discourages business formation and growth. But, this fuels back into the cycle and ultimately results in the perpetuation of poverty and underdevelopment in the region. The current study is aimed at investigating the strengths and direction of causality in the interdependence among small business growth, migration behavior, local public services and median household income. This chapter reviews previous empirical works on the determinants of regional variations in each of these variables separately. 
The rest of this chapter is organized as follows. Section 2 reviews the literature on the role of small business in economic development and poverty alleviation. This is followed by section 3 which reviews previous empirical works on the county-level determinants of small business growth. The emphasis is on factors that lead to regional variation in small business growth. Section 4 reviews the literature on the determinants of regional variation in population migration behavior. Section 5 reviews the literature on county-level determinants of local public services. The literature on the determinants of regional variation in median household income is reviewed in section 6. Finally, section 7 reviews the literature on spatial analysis and application of spatial econometrics in simultaneous equation systems.

\subsection{The Role of Small Business in Economic Development and Poverty Alleviation}

The history of small business has been one of the most controversial stories in economic development in the world. The role of small business in an economy has frequently been undermined and even misinterpreted. In the past small businesses were believed to impede economic growth by attracting scarce resources from their larger counterparts (Audretsch, Carree, Stel and Thurik, 2000). Large corporations capitalizing on economies of scale were rather considered as the deriving force of growth and development.

The emergence of computer-based technology in production, administration and information has, however, reduced the role of economies of scale in many sectors. Studies by Loveman and Sengenberger (1991) and Acs and Audretsch (1993), for example, have shown a shift in industry structure away from greater concentration and 
centralization towards less concentration and decentralization - a shift towards an increased role for small firms. This was mainly due to changes in production technology, in consumer demand, labor supply, the pursuit of flexibility and efficiency. These factors, in turn, led to the restructuring and downsizing of large enterprises and the entry of new firms. More and more evidences became available to indicate that economic activity moved away from large firms to small, predominantly young firms. Brock and Evans (1989), for example, also provided an extensive documentation of the changing role of small businesses in the U.S. economy.

Parallel with this literature, the changing patterns of consumer expenditure and demand patterns that resulted from rising living standards has contributed to the emergence of fragmented consumer markets. Moreover, many new business opportunities in small and medium size enterprises have been created as many large firms downsized their activities in an attempt to reduce costs. Thus, more recently, the alternative view is that small business is the key element and deriving force in generating employment and realizing economic development. This paradigm shift has, in turn, brought a revival in the promotion of small businesses and entrepreneurial initiative at local, national and international levels.

It is now well accepted both among researchers and policy makers that small businesses play a vital role in contributing to overall economic performance of countries (Dean, Holmes and Smith, 1996; Karlsson, Lindmark and Olofsson, 1993). Small businesses play an important role in community development by enticing private investment back into lagging areas and spread the benefits of economic growth to people and places too often left behind. Through their capital investments private small 
businesses and micro-enterprises create jobs and new opportunities that promote community-building and social activities in the rural and small towns.

Hence, the economic contribution of small business to economic growth and job creation is now well recognized and established in the literature (Birch, 1979; Markusen and Teitz, 1985; Storey, 1994; O’Neill, 1993; Karlsson et al., 1993). In his initial study, David Birch (1979), for example, reported that 80 percent of the jobs created between 1969 and 1976 in the U.S. economy were in firms employing less than 100 workers. Firms employing fewer than 20 workers generated 88.1percent of net job growth and start-ups generated nearly as twice as many jobs as expansion of existing firms between 1980 and 1985 (Birch, 1987). Miller (1990) also found net employment growth in existing small rural firms to be much faster than in large firms over the period 1980-1986. Studies of the US economy in the 1990 showed that new firm births and small enterprise expansion were the major sources of job creation that played a significant positive role in regional economic change (Karlsson et al., 1993). In most U.S. industries, small firms account for much of the capital stock, employment, and a large fraction of innovation (Acs and Audretsch, 1988, 1990). Research by the U.S. Small Business Administration showed that job creation capacity in the U.S. is inversely related to the size of the business. Between 1991 and 1995, the net job created in enterprises employing 1-4, 5-19, 20-99, 100-499 people were 3.843 million, 3.446 million, 2.546 million, and 1.011 million jobs respectively; whereas enterprises employing more than 500 people lost 3.182 million net jobs (U.S. Small Business Administration, 1999).

By creating jobs and promoting economic growth, small businesses play critical role in poverty alleviation. Understanding the connection between small businesses, 
economic growth and the incidence of poverty has been the interest of many researchers and there have been many attempts to establish statistical relationships between official poverty rates and overall macroeconomic performance on the basis of aggregate timeseries data (Freeman, 2002; Haveman and Schwabish, 2000; Blank, 2000; Cain, 1998; Powers, 1995;Blank and Card, 1993; Cutler and Katz, 1991; Blank and Blinder, 1986; Gottschalk and Danziger, 1985). The results from these studies show an inverse relationship between economic growth and poverty rates. Blank and Blinder (1986), for example, found that both the unemployment rate and the inflation rate to be positively related to poverty rate, with a high quantitative effect of unemployment. Cutler and Katz (1991), Blank (1993) and Powers (1995) also found similar results apart from the post recession period of the 1980s where unemployment rate was found to be inversely related to poverty rate. Using GDP growth rate as explanatory variable, Haveman and Schwabish (2000) tested the differential effect of macroeconomic performance on the poverty rate for various periods. Their result shows a strong inverse relationship between economic growth and poverty rate. They also showed that a one-percentage decrease in unemployment rate was associated with a 0.43 percentage point decrease in poverty rate between 1993 and 1998.

However, a number of research have not only indicated contradictory evidence about the role of small businesses but also produced results that rejected the view that small businesses are the engines of job creation and economic growth (Armington and Odle, 1982; Dunne, Roberts and Samuelson., 1989; Brown, Medoff and Hamilton, 1990; Acs and Audretch, 1993; Duncan and Handler, 1994; Harrison, 1994). Such studies show that although small firms exhibit higher growth rate in percentage terms, most new firms 
don't grow at all, and large start-ups account for the larger share of new firm growth. Besides, while the gross rate of job creation and lose are higher in small firms, there is no systematic relationship between net job creation and firm size (Davis, Haltiwanger and Schuh, 1993). Small businesses provide low quality jobs to their employees compared to large businesses. Empirical evidence indicates that large firms provide more stable employment, higher wages, and more non-wage benefits than small businesses (Rosenzweig, 1988; Brown et al., 1990). In addition, average firm size distribution does not indicate a growing dominance of small firms. Many small firms are established as last resort rather than as first choice and have limited growth potential (Liedholm and Mead, 1987). Recent research evidence also shows that small firms are not more innovative than large firms. Using a sample of European industries, Pagano and Schivardi (2001), for example, concluded that larger firm size is associated with faster rates of innovation. Much of the empirical evidence on the relationship between small business and economic growth is derived from firm-level and cross-country studies.

\subsection{County-Level Determinants of Small Business Growth}

Confronted with rising concerns about unemployment, job creation, economic growth and international competitiveness in global markets, policy makers at local, state, and national levels have responded to this new evidence with a new mandate to promote the creation of new businesses (see Reynolds, 2000). The results of empirical studies show that the new business phenomenon in most cases implies a small business phenomenon, since most of the new businesses start small and more importantly, most of

the newly created jobs are generated by new businesses that start small (Acs and 
Audretsch, 2001; Audretsch et al., 2000, 2001; Carree and Thurik, 1998, 1999; Wennekers and Thurik, 1999; Fritsch and Falck, 2003). These studies indicate that there has been a structural shift in the industrial sector towards a higher dependence on flexibility and knowledge-intensive production. This is considered to have made the small business sector as a more important feature of both the regional and the national economies. The recognition of the importance of new business formation for regional development raised the interest to further investigate the reasons why some economic spaces show high rates of new business formation while others do not.

A long tradition of studies of the determinants of new plant entry has focused on tax rates, transportation costs and economies of scale at the plant level (Bartik, 1989; Kieschnick, 1981; Harrison and Kanter, 1978). More recently, a growing literature has sought the determinants of variation in new business formation on regional basis (see Reynolds, 1994 and Acs and Armington, 2002 for the United States; Fritsch, 1992 and Audretsch and Fritsch, 1994 for West Germany; Hart and Gudgin, 1994 for the Republic of Ireland; Keeble and Walker, 1994 and Johnson and Paker ,1996 for United Kingdom; Davidson et al., 1994 for Sweden; Guesnier, 1994 for France; Garofoli, 1994 for Italy; Kangasharju, 2000 for Finland; Fotopoulos and Spence, 1999 for Greece; and Callejon and Segarra, 2001 for Spain). Each of these studies attempted to identify the most important influences underpinning spatial variations in new firm (business) formation. In these studies a set of regional characteristics concerning socioeconomic structure of the region are examined in order to explain the variations in new business formation. These include demand-side, supply-side and policy variables. 
On the demand-side, most of these researches suggest that new and small businesses tend to serve restricted geographical markets, and are therefore influenced by local variations in level and growth of market demand as measured by variables such as family median income, GDP and resident population statistics. Increases in the demand for goods and services that results from increases in per capita income or GDP per capita is associated with higher business formation (Armington and Acs, 2002). As wealth increases consumer demand for a variety of products and services increases and small businesses are well equipped to supply these new and specialized goods and services (Carree, 2000). Besides, the employment-share of the service sector which is characterized by intensive presence of small business increases with increases in per capita income (Wennekers, Uhlaner and Thurik, 2002). A growing population increases the demand for consumer goods and services and it is positively related to business formation (Acs and Armington, 2004a).

In addition to their demand-side influences, both population growth and net migration measures incorporate supply-side influences. This is because population growth, which often includes in net migration, also increases the local pool of potential entrepreneurs. Entrepreneurship and small business formation is strongly associated with previous population in-migration, itself powerfully stimulated by residential amenities and preference considerations (Keeble, Broom and Lewis, 1992).

Supply-side variables include the variables that reflect the supply of resources required setting up new business. These include measures of aggregation/externalities, of unemployment, of the structure of production, of availability of capital and entrepreneurial culture. 
Concentration of people and firms in certain areas decreases both the cost of access to customers and cost of access to suppliers (Reynolds, 1994). Both the consumer and the producer benefit from the easy availability of pooled services in such areas. This encourages new firm formation as a result of the agglomeration effects that come from either the demand effect, such as increase in population, or from regional spillovers, such as labor market characteristics. Krugman (1991a and 1991b) identified three types of spillovers within a region that may lead to the localization of economic activities. The first emanates from the observation by Marshall (1920) that a pooled labor market most commonly associated with agglomerations yields increasing returns at a spatial level. Agglomerations enable the production and provision of non-traded specialized inputs at a greater variety and lower cost. The third source of spillovers emanates from economics in information flows, or what Jaffe (1989) and Acs, Audretsch and Feldman, (1992, 1994) term it as technological spillovers. Technological spillovers are more beneficial to new small firms than to incumbent large enterprises (Acs et al., 1994). Thus, regions where such spillovers are greatest are more conducive for new business locations.

Regional spillovers are more likely to be most prevalent in areas with high population density because the infrastructure of services and inputs is more developed in densely populated regions. The concentration of several firms in a single location, for example, offers a pooled market for workers with industry-specific skills, ensuring both a lower probability of unemployment and a lower probability of labor shortage (Krugman, 1991a). Localized industries can also support the production of non-tradable specialized inputs. Besides, the informational spillovers that associate agglomeration can give cluster firms a better production function than isolated firms. That is, economies of localization 
and urbanization yield reduced cost of making transactions. This would suggest that both population density and population growth be positively related to new firm start-ups (Reynolds, 1991). Such agglomerations would also tend to exist where output per capita is relatively high.

The agglomeration effects that contribute to new firm formation can also come from supply factors related to the quality of the local labor market and business climate. Regions with similar demand and business climate patterns still differ in the rates of new firm formation, survival, and growth as a result of differences in their human capital endowment, and the propensity of locally available knowledge to spill over and stimulate new firm formation and growth. More educated population provide more human capital, embodied in their general and specific skills, for implementing new ideas for creating and growing new businesses (Acs and Armington 2004b). A number of empirical researches have found a strong connection between human capital and new firm formation and growth. Cross (1981), for example, argues that the availability of specialized labor influences the birth of new firms because there is a larger supply of potential entrepreneurs. Specialized workers are better prepared than non-specialized workers to create their own businesses, and workers with management skills favor the creation of new firms (Lloyd and Mason, 1984).

Human capital studies have found that entrepreneurship is related to educational attainment and work experience. People with more educational attainment tend to found business more often than those with less educational attainment (Evans and Leighton, 1990). In the 1990s, there were increases in the incidences of highly educated people stating new businesses, especially in the highly advanced sectors of the economy, like 
computers, biotechnology, and internet-dependent businesses. Guesneir (1994) finds that the propensity to create a new firm is positively associated with adults with bachelor degree. Highly educated people in most cases have easier access to research and development facilities, and perhaps a good insight into the business world and thus a clear idea about the present and the future needs of the market. Entrepreneurs with good education are also likely to know how to transform innovative ideas into marketable products (Christensen, 2000). People in regions that have a high percentage of college graduates are much more likely to start business than those in regions with high concentration of less skilled workers (Armington and Acs, 2001). Regions with higher average share of adults with college degrees are associated with higher new firm formation rates. Although the actual knowledge acquired with a college degree seldom suffices as the basis for a successful new business, the analytical methods learned in college facilitates both future acquisition of knowledge and openness to new ideas received as spillover from other activities in the region (Acs and Armington 2004b). However, studies by Hart and Gudgin (1994) have shown that the percentage of population with a university degree is inversely associated with the rate of new firm formation. A comparative study by Uhlaner, Thurik and Hutjes, 2002) in fourteen OECD countries has also shown that countries with higher level of education tend to have a smaller proportion self-employed entrepreneurs. While the educational level of the entrepreneurs may not, however, play a specific role in the survival of individual firms, the general consensus is that education more broadly influences the overall probability of survival of new firms in a region (Storey, 1994). 
Past research has found conflicting evidence about whether higher unemployment leads to more new firm formation, or the contrary. Traditionally, regional unemployment rate has been used as a measure of regional economic distress; with high unemployment rates would indicate slack growth, thereby dampening the incentives for new firms to locate within the region. Higher levels of unemployment might also indicate a reduction in aggregate demand throughout a regional economy, thereby putting downward pressure on the rate of new firm formation (Storey and Johnson, 1987). Moreover, unemployed individuals may not have the capital necessary to start their own business (Storey and Jones, 1987; Audretsch and Fritsch, 1994; Garofoli, 1994). Nevertheless, there is substantial literature, which indicates that higher levels of unemployment may lead to higher levels of firm formation. Actually, in many studies of new firm formation in the 1980s, there was a heavy emphasis on the possible positive explanatory power of unemployment (Evans and Leighton, 1990; Storey, 1991). A higher rate of unemployment may mean lower labor costs for firms and, therefore, favoring the creation of new firms (Highfield and Smiley, 1987). A higher rate of unemployment also indicates that more people have reason to search for alternative ways to make a living. In the absence of alternative job opportunities, some workers take the steps to start their own businesses (Davidsson, Lindmark and Olofsson, 1994; Beesley and Hamilton, 1994; Storey, 1994). This activity, in turn, reduces the unemployment rate as the resulting new firm employs not only the owners, but also others.

The empirical evidence provided at best depends on the methods it is followed to calculate the rate of new firm formation and on the data type used. If the rate of new firm formation is calculated with respect to the number of existing firms/establishments in the 
region, then higher rates of unemployment are positively associated with new firm formation. However, it is negatively associated with the rate of new firm formation if the latter is calculated with respect to number of employees in the region. Time series analyses point to unemployment being, ceteris paribus, positively associated with new firm formation, whereas studies using cross sectional, or pooled cross sectional analysis appear to indicate the reverse (Storey, 1991). Cross sectional studies by Armington and Acs (2001), however, indicate that unemployment rate is positively related to new firm formation in US in the 1990s. Acs and Armington (2004b) also found that the unemployment rate is positively associated with the rate of new firm formation during recession and negatively associated during growth periods. The impact of unemployment rate on the rate of new firm formation also depends on the type of the sector of activity, with industries that require small capital being more suitable for new firm formation during periods of higher unemployment (Armington and Acs, 2001). Thus, the direction of the effect of a region's unemployment rate on new firm formation is indeterminate.

Higher personal household wealth can provide either the financial resources, as equity or loans to finance new business, that is required to start new firm or it reflects wealth and income that can create demand for goods and services that encourages entrepreneurship. In order to capture the availability of finance, several variables have been used in the empirical studies. These include variables such as the distribution of wealth at regional level (Fotopoulos and Spence, 1999); percentage of homes owned by their occupants (Storey, 1982; Ashcrof, Love and Maloy, 1991; Reynolds, 1994; Reynolds, Miller and Maki, 1994; Keeble and Walker, 1994; Garofoli, 1994; Whittington, 1984; Guesnier, 1994), per capita saving deposits in the banking system 
(Fotopoulos and Spence, 1999; and annual growth rate of bank deposits (Gaygisiz and Koksal, 2003).

The percentage of home owned by their occupants is the variable that is frequently used in the empirical analysis and captures two different effects. A higher percentage of homes owned by their occupants may be an indication that there is a capacity to finance new business by potential entrepreneurs. It could also be a sign that at a regional level there is a demand for new business. Besides, a higher proportion of home ownership influences positively the formation of new firms because homes may be used as collateral for loans to start new business. In his study of the United States, Reynolds (1994) has found that personal household wealth is associated with higher new firm formation in the traditional rural regions. The local availability of personal finance, epitomized and embodied in the value of local owner-occupied housing, appears to play an important role in enabling or inhibiting new business creation (Keeble and Walker, 1994).

Guesnier (1994) and Garofoli (1994) have, however, found a negative relationship between home ownership and new firm formation. If houses already serve as collateral of bank loans and the burden imposed by those loans is too heavy for families, it may happen that the ability to finance a new business is limited. Besides, the consumption of other goods is lower, influencing therefore the rate of new firm formation through the demand side. The other possibility where a negative relationship between homeownership and the rate of new firm formation can be obtained is when the young with the higher probability of becoming entrepreneurs tend to live in rented homes more than older individuals. This effect may be captured in the variable related with property 
ownership if we do not control for the percentage of the young individuals in our regression (Guesnier, 1994).

The size structure of existing enterprises can be a factor influencing the rate of new business formation. The shift from manufacturing to services that has resulted from industrial restructuring in the 1980s increased the rate of new firm formation. And many researchers suggest that areas having many small firms are likely to have high rates of new firm formation (Cross, 1981; Storey, 1982; Lloyd and Mason, 1984; O'Farrel and Crouchley, 1984; Garofoli, 1994; Keeble and Walker, 1994; Audretsch and Fritsch, 1994; Hart and Gudgin, 1994; Reynolds, 1994; Armington and Acs, 2002; Acs and Armington 2004b). A local business structure with no dominant large firms may offer fewer barriers to entry of new firms. In a region dominated by small firms there is a much broader population of business owners and more individuals may visualize their own careers as leading to the founding of independent new firms (Acs and Armington 2004b). Whereas regions that are dominated by large branch plants or firms will have less new firm formation (Gudgin, 1978; Mason, 1994, Garofoli, 1994; Keeble and Walker, 1994; Audretsch and Fritsch, 1994; Hart and Gudgin, 1994; Reynolds, 1994; Armington and Acs, 2002; Acs and Armington 2004b). This is because large firms both provide employment for highly skilled workers in the economy but they fail to provide a suitable training ground for new entrepreneurs. Cross (1981) argues that the small firm is the best incubator of entrepreneurial capacity. A large proportion of entrepreneurs usually spring from having had prior experience in small firms.

The importance of public services for regional growth stems from their effect on production and location decisions of private firms. Public services such as education, 
highways, public safety, sewer and, water treatment services can be viewed as unpaid inputs in the process of production of private businesses that contribute independently to output.

Many studies have shown that public services have positive and statistically significant effects on business location and growth (Fox, 1979; Charney, 1983; Bartik, 1985, 1989; Helms, 1985; Newman, 1983; Papke, 1991; Deich, 1989; Fisher, 1997; Gaygisiz and Koksal, 2003; Gabe and Bell, 2004). Fox (1979), for example, found a positive location effect for local public services consumed by firms as measured by the expenditures for police and fire protection. A study by Charney (1983) also shows significant positive effects of the availability of water and sanitation infrastructure on location decision by firms. Similarly, Bartik (1991) found that fire protection services and local school spending have the strongest positive effects on small business start-ups. Out of the 19 studies reviewed by Fisher (1997), education spending has a positive effect on business activities in 12 of them, and a positive and significant effect in 6 of them. More recently, a study by Gabe and Bell (2004) shows a positive and significant effect of local public spending on business location. Besides, Gabe and Bell (2004) find that the benefits of tax-financed public services are more important than the costs (taxes) as determinants of business location. Helms (1985) also found that local tax revenues used to fund transfer payments tend to reduce economic growth, whereas local tax revenues used to finance improvement in public services such as highways, education and public health tend to have a positive growth impact and concluded that a high public service level attracts businesses and economic activity, whereas transfer payments do not have the 
same positive effect on economic growth. Besides, Helms study shows that the net impact of tax-financed increases in government services is positive.

Studies by Reynolds (1994) Keeble and Walker (1994) and Audretsch and Fritsch, (1994), however, show that there is little evidence that variations in local government spending (on education, highways, public safety) have statistically significant effect on business growth.

\subsection{County-Level Determinants of Migration Behavior}

In the United States, 3 percent of people make inter-state moves annually, and almost 10 percent relocate to a different state over a 5-year period (Borjas, 1996). Both workers and jobs have been involved in self-enforcing movements. But, do workers follow jobs or jobs follow workers? The literature on migration has grown phenomenally as researchers tried to answer this question and better understand the movements in population and employment that the United States has experienced during the last four decades. Much of the research has focused on the search for causal factors or determinants that explain migration behavior. Several researchers, for example, have studied the role of regional characteristics and amenities on interregional migration using aggregate data.

There can be little doubt that economic conditions affect migration behavior. One obvious way to see this is through the human capital model. As Milne (1991) asserts, there is overwhelming empirical support for the human capital model using both crosssectional data and time series data. Sjaastad (1962) was the first researcher to use the human capital theory in the context of migration. These models view migration as an 
investment. Migrants are motivated by the perceived interregional differences in factors that influence their economic opportunity. The results from a number of human-capitalbased researches have shown that real income and the probability of employment as important determinants of interregional migration (Pack, 1973; Fields, 1979; Greenwood, Hunt, and McDowell, 1986; Greenwood and Hunt, 1989; Clark and Hunter, 1992; Lundberg, 2003; Glaeser, Scheinkman and Shleifer, 1995; Aronsson, Lundberg and Wikstrom, 2001). Greenwood and Hunt (1989), for example, using migration data from 1958 to 1975 and a simultaneous equation model found that jobs and wages to be the primary determinants of interregional migration. Williams (1981) reviewed a number of studies and found that the price of labor is the primary determinant of interregional migration in most of the studies. Different studies, which use aggregate measures of income, wages and earnings, also show similar results. Muth (1968), for example, has examined the relationship between interregional migration and average wage growth, and Greenwood $(1975,1976)$ has analyzed the relationship between interregional migration and the growth of median income. Muth $(1968,1972)$, Olvey (1972), Persky and Kain (1970), and Greenwood $(1975,1976)$ have also found a significant mutual dependence between interregional migration and employment growth.

Macroeconomic theory considers migration as an equilibrating factor in regional labor markets. In other wards, job seekers are expected to move away from highunemployment regions where they cannot find a job to low-unemployment regions where the prospects of finding employment are more favorable. Many research results support this proposition (Sommers and Suits, 1973; Molho, 1986; Carlino and Mills, 1987; Cook, 1987; Herzog, Schlottman and Boehm, 1993; Charney, 1993; Hunt, 1993; Raimondos, 
1993; Gabriel, Mattey and Wascher, 1995). Gabriel et al. (1995), for example, found a very high correlation between unemployment and net migration in California for the 1982-1995 periods. A number of other studies, however, found unanticipated signs or insignificant coefficients on an unemployment rate variable (Greenwood, 1975, 1993; Pissarides and Wadsworth, 1989). One possible explanation for these results is the observation that many of these studies are subjected to simultaneous-equation bias due to the use of an end-of-period employment rate to explain migration over the period. Another possible reason lies in the observation that unemployment tends to be highest in the least mobile groups in the labor force (Lansing and Mueller, 1967). Blanco (1964) also argues that prospective unemployment rather than the level of unemployment rate, is a major determinant of migration. Generally, labor market opportunities have been found to influence migration (Ezzet-Lofstrom, 2003).

Net interregional migration also affects regional unemployment. Blanchard and Katz (1992), and Decressin and Fatas (1995), show that interregional net migration can decrease regional unemployment disparities. The study by Bilger, Genosko and Hirte (1991), for example, show negative effect of net interregional migration on regional unemployment rate.

Migration behavior is also affected by the site characteristics of alternative locations. Humans migrate in order to consume non-traded or location-specific goods. Some of these site attributes are associated with the public sector, typically measures of hospital care, education, fire protection, crime prevention, etc.. Several researchers have studied the role of regionally variable public sector attributes on migration decisions (see Carlino and Mills, 1987; Fox, Herzog Jr. and Schlottmann, 1989; Helms, 1985; Glaeser 
et al., 1995; Aronsson et al., 2001; and Lundberg 2003). The local government expenditure per capita and local government investment per capita, for example, are likely to provide indicators of the present and the expected future service levels, which makes them potential determinants of net migration. The local income tax rate is also one factor that might influence migration between regions located in densely populated areas near big cities, where the decision to move does not necessarily mean that the individual changes his/her place of work.

Tiebout (1956), in his seminal work, recognizes the importance of local government spending and local tax prices on interregional migration of households. He holds that the establishment of a large number of jurisdictions, which assess local preferences and provide public output efficiently, will enable individuals to migrate to a stable equilibrium of optimal communities. Wheaton (1975), on the other hand, demonstrates theoretically that the critical factor that determines migration preferences is the method by which each community finances its public consumption. Wheaton's most relevant results for Sweden concern migration incentives under proportional income taxation and his results show that the per capita tax base is an important fiscal variable worth examining as a potential determinant of migration behavior.

High taxes, especially property taxes, have a negative effect on interregional net migration (Pack, 1973; Cebula, 1974; Mead, 1982; Fox et al., 1989; Clark and Hunter, 1992). Mead (1982), for example, found that property tax has an indirect impact on migration behavior that operates through change in employment. Schachter and Althaus (1989) also concluded that high taxes tend to deter in-migration and to encourage outmigration. In a logit estimation of labor force migration, Herzog and Schlottmann (1986), 
concluded that tax rates, recreational accessibility and education significantly reduce the probability of out-migration while high crime rates increases the chance of moving out. Day (1992) used aggregate data in a multinomial legit model of migration to test whether interprovincial migration is influenced by tax and expenditure policies in Canada. He found that interprovincial differences in the level of public sector spending influence interprovincial migration. His results also show that the magnitude and direction of the effect depends on the type of government spending. Higher per capita spending in education and health induces in-migration. Earlier studies by Pack (1973) and Liu (1977) also show that higher expenditures on education attract migrants. Provincial income tax rates, unemployment insurance benefits and provincial transfer policies are found to have major impacts on migration behavior (Day, 1992). Poorer communities tend to levy higher tax rates to pay for a particular level of public service compared to their richer counterparties. Generally, the implication of studies that find significant public sector influence on migration behavior is that regions with desirable fiscal packages attract migrants.

The line of causation in the migration-public sector linkage goes in both directions. Not only net interregional migration is affected by the fiscal structures but also the fiscal structures themselves are affected by net interregional migration. Haurin and Haurin (1988), for example, have found that migration affects a location's ability to generate revenue, which in turn changes public sector attributes. Migration influences the net income and public good provision attainable at a location, which both have further implications for location choice (White and Knapp, 1994). 
Another non-traded goods model of migration builds upon the hedonic wage approach of Rosen (1979). In the interregional hedonic model, migration only eliminates only wage differentials that result from spatial disequilibrium, while differentials resulting from the relative amenity mix are capitalized into the local wage and land rent (Graves and Linneman, 1979; Mathur and Stein, 1991). Empirical results from various researches in this field suggest that amenities tend to be priced out through local labor /or land markets, whereby wage and rent gradients capture, at least in part, local demand for amenities (Rosen, 1979; Roback, 1982; Hoehn, Berger and Blomquist, 1987; Blomquist, Berger and Hoeln, 1988; Izraeli, 1987; Beeson and Eberts, 1989; Clark and Hunter, 1992; Herzog and Schlottman, 1993, Ezzet-Lofstrom, 2003). Thus, residents pay for amenities with lower wages and higher land rents. Migration is motivated by the altered demand for amenities that are site-specific. Ezzet-Lofstrom (2003), for example, found that crime rates, school quality, pollution, open space, housing prices and access to health care all affect out-migration in the expected directions.

\subsection{County-Level Determinants of Local Public Services}

The public sector interacts with the private sector and affects the economic well being of individuals in many ways. The distribution of income, the overall price level, and the quality and quantity of public goods and services such as highways, education, health and other local public services are affected by such local government activities as taxes, and public expenditure. The level of public expenditure and tax revenue in turn are determined by the economic, demographic and political characteristics of the local economy. 
Many cross-sectional studies exist in the literature trying to explain regional variations in per capital local public expenditures (Hawley, 1957; Brazer, 1959; Hirsch, 1959; Hansen, 196; Henderson, 1968; Borcherding and Deacon, 1972; Ohls and Wales, 1972; Bergstrom and Goodman, 1973; Bergstrom, Rubinfeld and Shapiro, 1982; Fisher and Navin, 1992). Hawley (1957), Brazer (1959), Hirsch (1959), and Hansen (1965), for example, employed a one-equation multiple-regression model to express per capita local public expenditure as a function of selected explanatory variables using cross-sectional data. Henderson (1968) also used a multiple-regression analysis of per capita crosssectional county data for the United States with two equations. Borcherding and Deacon (1972) estimated demand functions for eight specific public services: local education, higher education, highways, health and hospitals, police, fire, sewers and sanitation using cross-sectional data aggregated at state level. Using cross-sectional expenditure data for 1968, Ohls and Wales (1972) also estimated the demand and cost functions for three broad categories of state and local public expenditure: expenditures on highways per capita, education expenditures per school-age population and local service expenditures per capita (including fire, police, sanitation, health and hospitals, and local utility expenditure).

Similarly, Bergstrom and Goodman (1973) employed multiple-regression analysis to estimate the demand functions for three categories of municipal services: police, parks and recreation, and total municipal expenditure excluding education and welfare. These studies are based on the median voter theory where individual demand functions are inferred from cross-sectional studies in which actual public expenditure by local governments are regressed on indicators of economic and social composition of the 
jurisdiction's population. Bergstrom et al. (1982), however, devised and applied a method for estimating demand for local public goods, which does not require the median voter assumption. By combining individual's responses from survey data to questions about whether they want more or less of various public goods with observations of their incomes, tax rates, and of actual spending in their home communities to obtain estimates of demand functions.

The standard model in the literature assumes that differences in local public expenditures across regions are explained by differences in per capita incomes, population density, tax base, tax rates, population size, age structure of the population, grants in-aid from higher levels of governments, labor market characteristics, and schoolage population as well as other socio-economic and institutional factors.

The results from the various studies show that the income elasticity of local public expenditure is positive and significant whereas the estimates of tax price elasticity are negative and significant (Henderson, 1968; Borcherding and Deacon, 1972; Ohls and Wales, 1972; Bergstrom and Goodman, 1973; Bergstrom et al., 1982; Sanz and Velazquez, 2002; Painter and Bae, 2001). Studies by Randolph, Bogetic and Hefley, (1996), Canning and Pedroni (1999) and Fay (2000) also found that spending on economic services such as those relating to transport and communications respond primarily and directly to per capita income changes. Similarly, wide varieties of studies show that estimates of income elasticity greater than one for merit goods such as health, education and housing (Lue, 1986; Newhouse, 1987; Gertham, Sogaard, Jonsson and Andersson, 1992; Falch and Rattso, 1997; Snyder and Yachovlev, 2002; Hashmati, 2001). Duffy-Deno and Eberts (1991) analyzed the linkage between public infrastructure 
and regional development in a system of two equations and found that per capita real personal income has a positive and statistically significant contemporaneous effect on local public investment.

The findings from the study by Painter and Bae (2001) indicates that income per capita, total long-term debt, the unemployment rate, and the proportion of students of college age have a positive and statistically significant impact on state government expenditure. The results from this study and others (Randolph et al., 1996; Gertham et al., 1992; Falch and Rattso, 1997; Fay, 2000; Hashmati, 2001) also show that population density has negative coefficient. Population and its density play a highly important role in per capita spending on the purest or non-rival goods such as transportation and communications as well as merit goods and other economic services. A negative coefficient, thus, indicates the advantage of economies of scale in the provision of these public services. A small community must provide many public services such as education, hospitals, policy and sewage removal at relatively high per capita costs, which decline as its population increases. The reverse also holds true, large expenditures for public services result in places with declining population (Bergstrom and Goodman, 1973). This is one of the significant problems that small rural communities often face. Larger communities usually have better taxable capacity, which can provide a broader range of services that a small community cannot or need not provide (Henderson, 1968).

Since net migration changes the size and the density of population of a region, it has an impact on the demand of locally provided public goods and services as well as on the revenues that support the provision of these public goods and services. The mix of migrants or the mix of individuals who choose not to migrate may have profound 
consequences on the local public sector. A high-income in-migrant family, for example, may provide more tax revenue to the local economy than a low-income in-migrant family. The type and the quantity of public services they demand, however, are likely to be different. Similarly, growth in population of children that results from in-migrant families with children or women likely to have children creates big pressure on schools because they will be faced not only by the need to expand services but also they will be faced with the costs of expanding capacity. At the same time excess capacity and very high costs associated with maintaining overstock of buildings in the areas of origin where school enrolment declined will be created. The problems are exacerbated if outmigration is severe to impact property value and overall fiscal health (Charney, 1993).

The population age structure is also a significant determinant of local public services and goods. An increase in the proportion of the old and the young in a community increase spending in health, housing and social security (Heller, Hemming and Kalvert, 1986; Hagmann and Nicolleti, 1989; Di Matteo and Di Matteo 1998; Curie and Yelowtz, 2000). An increase in the proportion of young people will also generate pressure for increases in public spending on education (Marlow and Shiers, 1999; Alhin and Johansson, 2001).

Local public expenditure per capita is positively related to grants in-aid from higher-level governments (Fisher and Navin, 1992; Henderson, 1968). 


\subsection{County-Level Determinants of Median Household Income}

The literature on economic growth at the regional level has focused attention on the so-called convergence hypothesis predicted by neoclassical growth theory: poorer regions grow faster than richer regions, that is, poorer regions tend to catch up with the richer regions in per capita income as time passes. Barro and Sala-i-Martin (1992, 2004), for example, found such convergence for U.S. states, Japanese prefectures and between European countries. The results from the studies by Persson (1997) and by Aronsson et al. (2001) also show income convergence across Swedish counties. The study by Glaeser et al. (1995), however, does not show significant evidence of income convergence between U.S. cities. There are also other studies on regional/local income growth which focused attention on a broader set of possible average income growth determinants, which include among others, geographic characteristics, initial conditions describing the regions (such as the average income, regional/local public expenditure, regional/local income tax rates, educational status of the population, resource endowment, etc.) and national policies directed towards the regional level (see, for example, Helms, 1985; Glaeser et al., 1995; Fagerberg, et al., 1997; Aronsson et, al., 2001; Lundberg, 2003).

The initial average level of income is negatively related to the subsequent average income growth rate (Aronsson et, al., 2001; Lundberg, 2003). Capital mobility across regions tends to make regions more homogeneous over time, which leads to convergences in the sense that regions with low initial income levels tend to grow faster than regions with high initial income (Barro and Sala-i-Martin, 1992, 2004; Persson, 1997; Aronsson et al., 2001). Subsequent income growth is also positively related with initial unemployment rate. Unemployment causes out-migration, which decreases labor 
supply and increases wages during a subsequent period. The out-migration of unemployed persons changes the population composition such that average income increase for a given structure of wage among the employed (Aronsson et al., 2001). A rise in regional or area unemployment rate, indicating a slack labor market, however, leads to low average per capita income, primarily through the depressing effect on wages (Duffy-Deno and Eberts, 1991; Bilger, Genosko and Hirte, 1991; Chalmers and Greenwood, 1980, 1984).

The level of human capital and physical capital and the underlying level of productivity in the long run determine per capita incomes. Particularly, the role of education and human capital externalities has been emphasized as a key variable in recent theories of economic growth. Romer (1986), Lucas (1993), and Krugman (1991), for example developed models that link such externalities within geographically bounded region to higher rates of growth in per capita income. Rauch (1993) also finds that cities with higher average levels of human capital also have higher wages. Similarly, Glaeser et al. (1995) finds that for a cross section of cities a key economic determinant of growth is the initial level of schooling of the population. Simon and Nardinelli $(2002,1996)$ also found historical evidence for both United States and the United Kingdom that cities with more knowledgeable people grow faster in the long run. Duffy-Deno and Eberts (1991) found that the average year of education is positively related to the average per capita personal income. A study by Aronsson et al. (2001), however, shows that human capital, measured as the initial percentage of the population with a degree from university or college, has no effect on subsequent growth in per capita income. But, Aronsson et al. 
(2001) found that counties adjacent to regions where the major city areas are located tend to have higher growth rates of average income than other counties.

The size of the population of a region is positively correlated with real per capita personal income due to the beneficial effects of agglomeration economies of firm location (Duffy-Deno and Eberts, 1991). Population growth captures the extent to which regions are relatively attractive to migrants and a growing population increases the demand for consumer services which in turn leads to growth in business and employment. Incremental employment opportunities in turn provide a strong attraction for migrants that lead to net in-migration. Net in-migration not only increases local labor supply but it also increases local labor demand. If migrants possess differential endowments of human capital in the form of education, accumulated skills, or entrepreneurial talent compared to the receiving population, then their skills, inventiveness, and innovativeness will contribute to local productivity. Migrants may also own physical and financial capital that they bring with them and invest them in the receiving area. Moreover, migrants may contribute to the growth of markets and to the achievement of scale and agglomeration economies. Such demand effects of net inmigration are sources of growth in per capita personal income. Greenwood et al. (1986), for example, found results that are consistent with a migrant-induced labor demand shift that offsets the migrant-induced labor supply shift. Bilger et al., (1991), and Chalmers and Greenwood, (1984) also found that regional net migration rates positively and significantly influences the change in regional median earnings. Similarly, Duffy-Deno and Eberts (1991) found that the proportion of manufacturing employment in a metropolitan area is positively correlated with per capita personal income. 
There also exists evidence in the literature that local public expenditures on public health and hospitals, highways, local schools, higher education, police/fire protection, transfer payments/welfare, and other public services affect economic development as measured by different indicators such as net business establishments created (lost), net employment gains (losses), change in personal income, or/and change in per capita personal income (Duffy-Deno and Eberts, 1991; Jones, 1990). Government expenditures for certain aspects of the physical and human infrastructure such as expenditures on highways, education, and health can promote growth (Dye, 1980; Helms, 1985; Blair and Premus, 1987; Erickson, 1987; Schneider, 1987; Jones and Vedlitz, 1988, Jones, 1990; Glaeser et al., 1995). Dye's (1980) early study, for example, found that increases in highway spending in the 1960s yielded relatively greater economic growth in the 1970s. Highway expenditures add to the capital investment in the local transportation system, whether these expenditures are for construction or maintenance. Public investment increases personal income by increasing employment and wages in the construction industry (Duffy-Deno and Eberts, 1991). The studies by Helms (1985) and Jones (1990), for example, reported a significant and positive impact of highway expenditure on per capita personal income. Helms and Jones also found similar results for the impacts of public expenditures on education and health services on per capital personal income growth. Education expenditures add to the quality of the labor force. Health service expenditure is growth-enhancing due to the externalities associated with preventive and primary care, which increases labor productivity and reduce the lose of working hours due to illness. Using a panel of 260 US cities, Glaeser et al. (1995) found that economic growth is positively affected by public expenditures on sanitation, infrastructure, housing 
and urbanization, and transport services. Public expenditures on police and fire protection are positively related to per capita personal income growth (Jones, 1990). The impact of welfare spending on per capita personal income growth, however, is negative in most studies (Dye, 1980; Helms, 1985; Jones, 1990).

\subsection{Spatial Dependence}

Parallel to the rapid development of Geographic Information System (GIS) in recent years, a growing body of international research is developing new ways to think about the role of space or geography. Regional disparities have received renewed emphasis in the emerging growth theory and in new economic geography, starting with Romer (1986, 1990), Lucas (1988), and Krugman (1991a). These theories aim at explaining the location behavior of firms and their agglomerative processes. They give several theoretical information and principles that help us understand the uneven spatial repartition of economic activities between regions. The emphasis of the theories of new economic geography upon the effects of the uneven spatial distribution of economic activities on the economic growth of regions led to renewed interest in models of social interaction and dependence among economic agents and spatial spillovers (Anselin, 2002). Thus, decisions and transactions of economic agents may depend upon present and past behavior of neighboring economic agents, which can yield spatial or spatiotemporal dependence.

In the past, models that explicitly incorporate space or geography and therefore applications of spatial econometrics were primarily found in specialized fields such as regional science, urban and real estate economics and economic geography (Anselin, 1999). More recently, however, the technique of spatial econometrics is increasingly 
being applied in a wide range of empirical investigations in more traditional fields of economics, such as public economics and finance (Case, Rosen and Hines, 1993; Bruenkner, 1998), agricultural and environmental economics (Benirschka and Binkley, 1994), labor economics (Topa, 1996). There is also a growing spatial econometric literature that focus on methodological issues that deal with alternative model specifications, test statistics and estimators of models that use spatial data (the literature include, among others, Anselin, 1988, 1999, 2001, 2003; Anselin and Bera, 1998; Anselin and Kelejian, 1997; Conley, 1999; Driscoll and Kraay, 1998; Elhorst, 2003; Kelejian and Prucha, 1998, 1999, 2002, 2004; Pinkse and Slade, 1998). The development of the spatial econometric techniques further helped researchers to use models that are corrected to misspecifications which result from spatial dependence and heterogeneity. This is significant improvement because spatial dependence, if unaccounted for, can create either inefficient estimates (when the spatial dependence is in the error term) or biased and inconsistent estimates (when the spatial dependence is in the dependent variable). Inefficient regression estimates result when spatial dependence in the error terms is ignored because, in the presence of positive spatial autocorrelation the standard errors of regression are inflated, making the t-values lower and statistical significance more difficult to achieve, and in the presence of negative spatial autocorrelation the standard errors of regression become deflated, giving increased potential for a Type 1 statistical error. When the spatial dependence is in the dependent variable of the model, it is referred as spatial lag and if ignored it leads to biased and inconsistent regression estimates because of omitted variable bias. Ignoring spatial dependence in the dependent variable (spatial lag) is considered to be more serious than ignoring spatial dependence in 
the error terms (spatial error) (Anselin, 1988). Spatial dependence is particularly problematic in research with politically constructed geographical units of analysis, such as counties (Doreian, 1981; Land and Deane, 1992).

Although advances in spatial econometrics provide researchers with new avenues to address regression problems that are associated with the existence of spatial dependence in regional data sets, most of the applications have been in single-equation frame-works. Yet for many economic problems there are both multiple endogenous variables and data on observations that interact across space. Until recently, researchers have been in the undesirable position of having to choose between modeling spatial interactions in a single equation frame-work, or using multiple equations but losing the advantage of a spatial econometric approach (Rey and Boarnet, 2004). Although not explicitly spatial econometric approach, Steinnes and Fisher's (1974) model of population and employment levels was the first application that tried to incorporate spatial interactions in a simultaneous equations framework. To provide some degree of spatial interaction, they innovated the model by developing potential variables that aggregated community area population and employment into larger units. This enabled them to express community area population and community area employment as functions of a weighted average of employment in all community areas, and a weighted average of population in all community areas in the data set, respectively. Thus, both population and employment were endogenous variables and by use of lagged population and (instrumented) employment as regressors in the population equation and lagged employment and (instrumented) population in the employment equation, Steinnes and Fisher were able to show the direction of causality between population and employment 
change. Actually, empirical work on identification of the direction of causality in the 'jobs follow people or people follow jobs' literature and empirical models of small regional development often begin with this two-equation model. Carlino and Mills, 1987 and Dietz, 1998, for example, used this simultaneous system without incorporating spatial effects.

Recognizing the shortcoming of the Carlino-Mills model, Boarnet (1994) proposed a model which integrated the use of potential variables and spatial econometrics in a two-equation model of population and employment growth in New Jersey municipalities. In order to adjust for the difference in the place of residence and the place of work at the community level, he added spatial lags of the endogenous variables to the Carlino-Mills model. Since Boarnet thought that New Jersey municipalities are too small to be their own labor markets, he used a spatial cross-regressive lag model, in the sense that the right-hand side of each equation contains spatial lag of the endogenous variable from the other equation, creating spatial links across equations. Community population change depends on the change in employment aggregated over all communities within commuting distance. In the same token, community employment change depends on population change within commuting distance of the given community.

The Boarnet model was subsequently extended by Henry, Barkley, and Bao (1997) in their efforts to analyze population and employment changes in rural areas and to reveal which kinds of forces are dominant. This model contains interaction terms between urban growth rates and the spatial lag variables as regressors. These linkages enabled them to examine how urban growth affects rural hinterland population and employment change. The parameter estimates on the interaction variables reveal if faster 
urban growth has a spread or backwash effect on proximate rural communities. Using southern Functional Economic Areas, Henry et al.(1997) found a mix of spillover and backwash effects from urban core and fringe areas to their rural hinterlands. Henry, Schmitt, Kristensen, Bakley, and Bao (1999) also extended the work of Henry et al. (1997) by comparing empirical results across three countries (Denmark, France, and the United States) in order to evaluate how country differences in the local socio-economic conditions affect the linkage between urban growth and rural change. Their results indicate that rural population and employment changes in the regions of the three countries under study are sensitive to the performance of the urban core/fringe that is nearby. The general trends that emerge are of urban spread to rural places that have average or large labor market and population.

Herny, Schmitt, and Piguet (2001) also estimated the Carlino and Mills (1987), Boarnet (1994), and the Herny et al.(1997) models for six French regions and compared the results for several related spatial econometric models for simultaneous equation systems defined in the taxonomy developed in Rey and Boarnet (2004). Their results indicates that adding the spatial cross-regressive terms to the Carlino-Mills model provides an important correction that results in empirical results consistent with the theory in the Carlino-Mills and Boarnet models. Besides, comparing the strength and direction of population effects on employment and vice versa, their results show that people follow jobs in rural France. Moreover, their results suggested general tendency of local spread masking both urban backwash and spread effects, depending on the pattern of urban growth between the core and the fringe. 
The literature on the application of spatial econometrics to simultaneous systems in the context of panel data set is yet to develop.

In summary, this chapter surveyed the empirical literature on the role of small business in economic growth, the regional determinants of small business growth, migration behavior, local public services and median household income. Literature on the role of space and the application of spatial econometrics to simultaneous equation system, which is relevant to the present study, has also been reviewed. As an extension to this review of previous empirical works, the next chapter discusses the conceptual and methodological frameworks that guide the empirical analyses in this study. The literature review is the basis for the development of the theoretical and empirical models of this study which are given in Chapter IV. These theoretical and empirical models are also developed upon the backdrops of conceptual and methodological frameworks that are discussed in Chapter III. 


\section{CHAPTER III}

\section{THEORETICAL FRAMEWORK}

\subsection{Introduction}

This chapter presents the methodological and conceptual framework and discusses the theoretical and estimation issues underlying the use of standard (nonspatial) and spatial simultaneous-equations models, both in cross-sectional and panel data setting, of relevance to this study. The first half of this chapter is devoted to the discussions of the theoretical and estimation issues with regards to the standard (nonspatial) simultaneous models. Much of these discussions are built on the works of Greene (2003), Wooldridge (2002), Davidson and Mackinnon (1993), Maddala (1986), Johnston (1984), Intriligator (1978), Hausman (1978), Baltagi (1980, 1981, 1995), Hsiao (1985, 1986). The second half of this chapter is devoted to the discussions of the theoretical and estimation issues which are relevant to the extension of the standard (non-spatial) model to account for spatial spillover effects. Various researches by Luc Anselin on exploratory spatial data analyses and spatial regression provided a general framework for these discussions. The research work by Kelejian and Prucha (2004) was also provided important basis for the discussions on extensions of the non-spatial simultaneous equations model to a spatial simultaneous equations cross-sectional model. The further extension of the model to spatial simultaneous equations panel model, however, is altogether new addition to the existing literature

Previous studies show that much of economic data comes from an economic system that can be described by a set of economic relations that are stochastic, dynamic and simultaneous and the theories that try to explain economic relations are built on sets, 
or systems, of relationships (Hausman, 1978). Thus, single equation models are not sufficient to determine the economic meaning of the statistical relationships between the variables of the economic system. Instead, a simultaneous equation model in which all the endogenous variables of the economic system can be determined simultaneously should be considered.

Simultaneous equation models are fundamentally different from single equation models. In simultaneous equation models, there are a number of exogenous variables and many endogenous or jointly determined variables. The endogenous variables are the object of the explanation sought by the simultaneous equation system and typically, lefthand-side variables of some equations in the system will appear on the right-hand-side of other equations in the system. In a true simultaneous system, all of the left-hand-side variables are jointly determined and the system is complete when the number of equations is equal to the number of endogenous variables.

The model of the entire date set of endogenous variables is conditional on the exogenous variables. In this sense, exogenous variables are causal, characterizing the environment in which the endogenous variables are determined. That is, exogenous variables enter the system and affect the endogenous variables, but are not affected by them. Some endogenous variables may also affect the jointly determined variables with a lag, in which case they are called lagged endogenous variables. Lagged values of endogenous variables are treated as exogenous variables because for determination of the current period's values of the endogenous variables, they are pre in the sense of being prior in time or as given outside the model. Exogenous (whether current or lagged) and lagged endogenous variables constitute predetermined variables. The simultaneous 
equation system conditions the behavior of each observation of the endogenous variables on the predetermined variables.

Whether the interest is in only a particular part of the system or in the system as whole, the interaction of the variables in the model will have important implications for the estimation and interpretation of the model's parameters (Greene, 2003). The interpretations of the model parameters depend on the parametric (structural or reduced) form of the equations of the system. The estimation principle that leads to consistent and efficient parameter estimates also depends on whether the simultaneous equation system is in structural or reduced-form. But even before the question of estimation is considered, the problem of identification should be resolved. This deals with the fundamental question of whether the parameters of interest in the model are estimable. Besides, the models are estimated for cross-sectional observations on aggregate spatial unities such as counties. Such datasets, however, are likely to exhibit a lack of independence in the form of spatial autocorrelation. Thus, model estimation should also be preceded by exploratory spatial data analysis to test for the presence of spatial interdependences. The presence of spatial interdependence in the data set would necessitate the application of spatial econometrics in the context of multi-equation systems. This is a significant departure and improvement over previous modeling efforts. Researchers in this area have often been in the undesirable position of having to choose between modeling spatial interactions in a single-equation framework or using multiple equations but losing the advantage of a spatial econometric approach (Rey and Boarnet, 2004). Extending the analysis to panel data setting also involves major methodological issues. A discussion of the difficulties 
involved in estimating panel data models using spatial econometrics is given in Elhorst (2003) and Baltagi, Song and Koh (2003).

The chapter is organized as follows. Section two presents the fundamental issues in simultaneous equation modeling. This deals with the specification of the model or the parametric-structural/reduced- form of the equations. The problem of identification is

given in section three. Section four presents estimation techniques and estimation principles in standard (non-spatial) simultaneous equations models in cross-sectional data setting. The corresponding estimation techniques and estimation principles in a panel data setting is given in section five. Issues related to the implementation of diagnostic tests for the presence of spatial interdependence in the data set is given in section six. Extending the standard (non-spatial) model to accommodate for the presence of spatial dependence in the data set involves a number of methodological issues. Section seven presents such methodological issues both in cross-sectional and panel data setting. Section eight discusses the estimation issues in spatial simultaneous-equations models in both crosssectional and panel data setting. Finally, section nine discusses issues related to specification tests in spatial and non-spatial simultaneous-equations models.

\subsection{Fundamental Issues in Simultaneous-Equations Models}

The structural and the reduced-form models are the two basic specifications that have been used in the interpretation of linear simultaneous equation models. The structural form has stochastic (behavioral) equations, which describe empirical relations between variables, and sometimes accounting identities which correspond to the basic economic theory underlying the model. It typically contains all the economic knowledge 
that can be included in the model. The equations in the model are derived from theory and each purports to describe a particular aspect of the economy.

The linear simultaneous equation model in structural form consisting of $G$ endogenous variables (denoted by $\mathbf{y}$ ) and $\mathrm{k}$ exogenous variables ( $\mathbf{x})$ can be written as:

$$
\left[y_{1} y_{2} \ldots y_{G}\right]_{i}\left[\begin{array}{c}
\beta_{11} \beta_{12} \ldots \beta_{1 G} \\
\beta_{21} \beta_{22} \ldots \beta_{2 G} \\
\vdots \\
\beta_{G 1} \beta_{G 2} \ldots \beta_{G G}
\end{array}\right]+\left[x_{1} x_{2} \ldots x_{k}\right]_{i}\left[\begin{array}{c}
\gamma_{11} \gamma_{12} \ldots \gamma_{1 G} \\
\gamma_{21} \gamma_{22} \ldots \gamma_{2 G} \\
\vdots \\
\gamma_{k 1} \gamma_{k 2} \ldots \gamma_{k G}
\end{array}\right]=\left[u_{1} u_{2} \ldots u_{G}\right]_{i}
$$

or

$$
\mathbf{y}_{i}^{\prime} \mathbf{B}+\mathbf{x}_{i}^{\prime} \boldsymbol{\Gamma}=\mathbf{u}_{i}^{\prime} \quad \mathrm{i}=1, \ldots, \mathrm{n}
$$

Where:

$\mathbf{B}=\mathrm{G} \times \mathrm{G}$ matrix of coefficients of the endogenous variables $\Gamma=\mathrm{G} \times \mathrm{k}$ matrix of coefficient of the predetermined/exogenous variables $\mathbf{y}_{\mathrm{i}}=\mathrm{G} \times 1$ vector of observation on the endogenous variables $\mathbf{x}_{\mathrm{i}}=\mathrm{k} \times 1$ vector of observation on the predetermined /exogenous variables $\mathbf{u}_{\mathrm{i}}=\mathrm{G} \times 1$ vector of disturbances, independent across i

The disturbances $\mathbf{u}_{\mathrm{i}}$ are assumed to be randomly drawn from a multivariate distribution with:

$\mathrm{E}\left[\mathbf{u}_{\mathrm{i}}\right]=\mathbf{0}$ and $\mathrm{E}\left[\mathbf{u}_{\mathrm{i}} \mathbf{u}_{\mathrm{i}}^{\prime}\right]=\boldsymbol{\Sigma}$ where $\boldsymbol{\Sigma}$ is a nonsingular covariance matrix

Thus, $(\mathbf{B} \Gamma \mathbf{\Sigma})$ are matrices containing the structural form parameters which are subjected to linear restrictions, normalizations and exclusions. The underlying economic theory will impose a number of restrictions on these matrices of parameters. It is 
necessary to impose some sort of normalization on each of the equations of the model. For instance, since one of the variables in each equation is labeled as dependent variable, the diagonal elements of $\mathbf{B}$ should be either one (1) or minus one (-1) so that the coefficient of the dependent variable be one. Multiplying every coefficient in the equation by the same constant will not change the relationship defined for a given equation. This indeterminacy can be eliminated simply by choosing a dependent variable (making its coefficient one through normalization). Some of the parameters will also be zero because all variables do not appear in each equation of the model.

The formulation $\quad \mathbf{y}_{i}^{\prime} \mathbf{B}+\mathbf{x}^{\prime} \boldsymbol{\Gamma}_{i}=\mathbf{u}_{i}^{\prime}$ applies to an observation $\left[\mathbf{y}^{\prime}, \mathbf{x}^{\prime}, \mathbf{u}^{\prime}\right]_{i}$ in a cross section. In a sample of data, each joint observation will be one row in a data matrix.

$$
\left[\begin{array}{lll}
\mathbf{Y} & \mathbf{X} & \mathbf{U}
\end{array}\right]=\left[\begin{array}{c}
\mathbf{y}_{1}^{\prime} \mathbf{x}_{1}^{\prime} \ldots \mathbf{u}_{1}^{\prime} \\
\mathbf{y}_{2}^{\prime} \mathbf{x}_{2}^{\prime} \ldots \mathbf{u}_{2}^{\prime} \\
\vdots \\
\mathbf{y}_{n}^{\prime} \mathbf{x}_{n}^{\prime} \ldots \\
\mathbf{u}_{n}^{\prime}
\end{array}\right] .
$$

Now it is possible to write the structure of the model in terms of the full set of $n$ observations as follows:

$$
\mathbf{Y B}+\mathbf{X} \boldsymbol{\Gamma}=\mathbf{U}
$$

with

$$
\mathrm{E}(\mathbf{U})=\mathbf{0} \text { and } \mathrm{E}\left[(1 / n) \mathbf{U}^{\prime} \mathbf{U}\right]=\boldsymbol{\Sigma} .
$$

The equations that describe how the endogenous variables are really determined are known as reduced-form equations. The reduced-form model can be obtained from the structural model by a non-singular linear transformation. The joint endogeneity is eliminated from the model by the reduced form transformation as each endogenous 
variable can be written as a linear function of only exogenous variables and a disturbance term.

Since $\mathbf{B}$ is assumed to be non-singular, $\mathbf{B}^{-1}$ exists. By post-multiplying the structural model (equation 3.2) with $\mathbf{B}^{-1}$, we can obtain the reduced-form of the linear simultaneous model.

$$
\begin{aligned}
& \mathbf{y}_{i}^{\prime}+\mathbf{x}_{i}^{\prime} \mathbf{\Gamma} \mathbf{B}^{-1}=\mathbf{u}_{i}^{\prime} \mathbf{B}^{-1} \\
& \mathbf{y}_{i}^{\prime}=\mathbf{x}_{i}^{\prime} \boldsymbol{\Pi}+\mathbf{v}_{i}^{\prime}
\end{aligned}
$$

where $\boldsymbol{\Pi}=-\boldsymbol{\Gamma} \mathbf{B}^{-1}$ and $\mathbf{v}_{i}^{\prime}=\mathbf{u}_{i}^{\prime} \mathbf{B}^{-1} . \quad \mathbf{v}_{i}^{\prime}$ is the reduced form disturbances which have $\mathrm{E}\left[\mathbf{v}_{\mathrm{i}}\right]=\mathbf{0}$ and $\mathrm{E}\left[\mathbf{v}_{\mathrm{i}} \mathbf{v}_{\mathrm{i}}^{\prime}\right]=\left(\mathbf{B}^{-1}\right)^{\prime} \mathbf{\Sigma} \mathbf{B}^{-1}=\boldsymbol{\Omega}(\mathrm{G} \times \mathrm{G})$ matrix. $\boldsymbol{\Pi}, \boldsymbol{\Omega}$ are the matrices of the reduced-form parameters. The reduced-form equations form a multivariate regression mode, provided there are no restriction on $\boldsymbol{\Pi}$ and $\boldsymbol{\Omega}$, and hence $\boldsymbol{\Pi}$ can be estimated by least squares. In that case

$$
\hat{\boldsymbol{\Omega}}=1 / n \sum\left(\mathbf{y}_{i}^{\prime}-\mathbf{x}_{i}^{\prime} \hat{\boldsymbol{\Pi}}_{L S}\right)\left(\mathbf{y}_{i}^{\prime}-\mathbf{x}_{i}^{\prime} \hat{\boldsymbol{\Pi}}_{L S}\right)^{\prime} .
$$

While we can always recover the reduced-form parameters from the structural form parameters, the reverse is not always true. We can only obtain the structural form parameters from the reduced-form parameters only if there are additional restrictions on the parameters. The problem of identification arises from this necessity. But, if the reduced-form equations and their coefficients (parameters) can tell us all we need to know about the economic process under examination, since they determine the set of outcomes for the current endogenous variables given any set of values for the predetermined variables, why there is a need to estimate the structural form parameters? Knowledge of the reduced-form parameters can tell us the outcome of the simultaneous 
system but not about the relationships and interdependences that operate within the system. If the purpose of econometric analysis were only forecasting and policy analysis, then knowledge of the reduced-form parameters is enough. But, in the long-run even these purposes are dependent on the further development of economic theory through hypothesis testing in an effort to discriminate among alternative explanations of the way in which institutions and economic processes interact and behave. Structural model provide a crucial inductive method to increase our knowledge about economic relationships and to test hypotheses about economic behavior. Besides, structural equations have the autonomous characteristics that if any one equation is subjected to a change of specification, this need not affect the specification of any other structural equation. All the reduced-form equations, however, may be affected by a change in the parameters of one structural form equation. Structural parameters are much more stable than the composite reduced-form parameters due to the autonomous nature of structural relationships. They are thus, the subject of a priori or non-sample knowledge concerning their signs and magnitudes. Besides, shifts in structural parameters are much more readily the subject of rational explanations and interpretation than are the associated shifts in reduced-form parameters.

\subsection{The Identification Problem}

The identification problem is a mathematical problem associated with simultaneous equations system. It is concerned with the question of the possibility or impossibility of obtaining meaningful estimates of the structural parameters. In general, it deals with the question of recovering the structural parameters from the reduced-form 
parameters. The relevant question here is: Can the reduced-form parameters be used to deduce unique values of the structural parameters? This assumes that the reduced-form parameters, however, are observable.

Following Green (2003), consider the reduced-form model, $\mathbf{y}_{i}^{\prime}=\mathbf{x}_{i}^{\prime} \boldsymbol{\Pi}+\mathbf{v}_{i}^{\prime}$, where $\mathrm{E}\left[\mathbf{v}_{i} \mathbf{v}_{i}^{\prime}\right]=\boldsymbol{\Omega}$ and $\mathrm{E}\left[\mathbf{v}_{i} \mid \mathbf{x}_{i}\right]=\mathbf{0}$. Given an infinite-sized sample data of observations on the variables in the model, we can observe the followings:

$$
\begin{aligned}
& \operatorname{plim}(1 / \mathrm{n}) \mathbf{X}^{\prime} \mathbf{X}=\mathbf{Q}, \text { where } \mathbf{Q} \text { is a finite positive definite matrix, } \\
& \operatorname{plim}(1 / \mathrm{n}) \mathbf{X}^{\prime} \mathbf{Y}=\operatorname{plim}(1 / \mathrm{n}) \mathbf{X}^{\prime}(\mathbf{X} \boldsymbol{\Pi}+\mathbf{V})=\mathbf{Q} \boldsymbol{\Pi}, \\
& \operatorname{plim}(1 / \mathrm{n}) \mathbf{Y}^{\prime} \mathbf{Y}=\operatorname{plim}(1 / \mathrm{n})\left(\boldsymbol{\Pi}^{\prime} \mathbf{X}^{\prime}+\mathbf{V}^{\prime}\right)(\mathbf{X} \boldsymbol{\Pi}+\mathbf{V})=\boldsymbol{\Pi}^{\prime} \mathbf{Q} \boldsymbol{\Pi}+\mathbf{\Omega} .
\end{aligned}
$$

Hence, $\Pi$, the matrix of the reduced-form coefficients, is observable:

$$
\boldsymbol{\Pi}=\left[\operatorname{plim}\left(\frac{\mathbf{X}^{\prime} \mathbf{X}}{n}\right)\right]^{-1}\left[\operatorname{plim}\left(\frac{\mathbf{X}^{\prime} \mathbf{Y}}{n}\right)\right]
$$

$\boldsymbol{\Pi}$ is the equation by equation least squares regression of $\mathbf{Y}$ on $\mathbf{X}$.

$\boldsymbol{\Omega}$, the matrix of least squares residual variances and co-variances is also observable and can be calculated as follows:

$$
\Omega=\operatorname{plim}\left[\frac{\mathbf{Y}^{\prime} \mathbf{Y}}{n}\right]-\operatorname{plim}\left[\frac{\mathbf{Y}^{\prime} \mathbf{X}}{n}\right]\left[\frac{\mathbf{X}^{\prime} \mathbf{X}}{n}\right]^{-1}\left[\frac{\mathbf{X}^{\prime} \mathbf{Y}}{n}\right]
$$

Thus, $\boldsymbol{\Pi}$ and $\boldsymbol{\Omega}$ can be calculated consistently by least squares regression of $\mathrm{Y}$ on $\mathrm{X}$.

The relevant question now is: Can we deduce $\mathbf{B}, \boldsymbol{\Gamma}$ and $\boldsymbol{\Sigma}$ from our knowledge of $\boldsymbol{\Pi}$ and $\mathbf{\Omega}$ A correspondence between the reduced-form and the structural form parameters can be established as:

$$
\boldsymbol{\Pi}=-\boldsymbol{\Gamma} \mathbf{B}^{-1} \text { and } \boldsymbol{\Omega}=\left(\mathbf{B}^{-1}\right)^{\prime} \boldsymbol{\Sigma} \mathbf{B}^{-1}
$$


If, $\mathbf{B}$ were known, we could deduce $\boldsymbol{\Gamma}$ as $-\boldsymbol{\Pi B}$ and $\boldsymbol{\Sigma}$ as $\mathbf{B}$ ' $\boldsymbol{\Omega} \mathbf{B}$. The problem, however, is that $\mathbf{B}$ is a matrix of parameters that should be estimated. The problem of identification, in fact, arises because the most that can be determined from the observational data on the variables of the model is the knowledge of $\boldsymbol{\Pi}$ and $\boldsymbol{\Omega}$. Thus, knowledge of $\mathbf{B}$ and $\boldsymbol{\Gamma}$ only comes from knowledge of $\boldsymbol{\Pi}$. The $\boldsymbol{\Pi}$ matrix is the order of $\mathrm{G}$ $\mathrm{x}$ K and contains GK elements. The $\mathbf{B}$ and $\boldsymbol{\Gamma}$ matrices contain at most $\mathrm{G}^{2}+\mathrm{GK}$ elements. There is thus an infinity of $\mathbf{B}$ and $\boldsymbol{\Gamma}$ structures corresponding to any given $\boldsymbol{\Pi}$ matrix. In general, different sets of structural parameter values can give rise to the same set of reduced-form parameters, so that knowledge of the reduced-form parameters does not allow the correct set of structural parameter values to be identified. Let us see how this arises in the likelihood context.

Since $\mathbf{u}_{\mathrm{i}}$ are assumed to be independent over individual $\mathrm{i}$, the joint distribution can be written as $\prod_{i=1}^{n} p\left(\mathbf{u}_{i}\right)$. Because $\mathbf{u}_{i}$ are not observable, $\prod_{i=1}^{n} p\left(\mathbf{u}_{i}\right)$ should be converted into the density function of the observables $y_{i}$. Then we have:

$$
p\left(\mathbf{y}_{i} \mid \mathbf{x}_{i}\right)=p\left(\mathbf{u}_{i}\right) . J
$$

where $J$ is the Jacobian of the transformation, $\left|\partial \mathbf{u}_{i} / \partial \mathbf{y}_{i}\right|=|\mathbf{B}|$, from equation (3.1). Hence the density function of the endogenous variables given the exogenous variables is:

$$
p\left(\mathbf{y}_{1} \mathbf{y}_{2} \ldots \mathbf{y}_{n} \mid \mathbf{x}_{1} \mathbf{x}_{2} \ldots \mathbf{x}_{n}\right)=\|\mathbf{B}\|^{n} \prod_{i=1}^{n} p\left(\mathbf{u}_{i}\right)
$$

where $\|\mathbf{B}\|$ denotes the absolute value of $|\mathbf{B}|$ (the determinant of matrix $\mathbf{B}$ ). 
This turn out to be the likelihood function when it is written in terms of the unknown parameters $\mathbf{B}, \boldsymbol{\Gamma}$ and $\boldsymbol{\Sigma}$. Thus

$$
\begin{gathered}
L(\mathbf{B}, \boldsymbol{\Gamma}, \boldsymbol{\Sigma})=|\mathbf{B}|^{n} \prod_{i=1}^{n} p\left(\mathbf{u}_{i}\right) \\
L=(2 \pi)^{-n G / 2}\|\mathbf{B}\|^{n}|\boldsymbol{\Gamma}|^{-n / 2} \exp \left(-\frac{1}{2} \sum_{i=1}^{n} \mathbf{u}_{i}^{\prime} \mathbf{\Sigma}^{-1} \mathbf{u}_{i}\right) \\
=(2 \pi)^{-n G / 2}\|\mathbf{B}\|^{n}|\boldsymbol{\Gamma}|^{-n / 2} \exp \left[-\frac{1}{2} \sum_{i=1}^{n}\left(\mathbf{y}_{i}^{\prime} \mathbf{B}+\mathbf{x}_{i}^{\prime} \boldsymbol{\Gamma}\right)^{\prime} \mathbf{\Sigma}^{-1}\left(\mathbf{y}_{i}^{\prime} \mathbf{B}+\mathbf{x}_{i}^{\prime} \boldsymbol{\Gamma}\right)\right]
\end{gathered}
$$

Alternatively, the likelihood function can be set up in terms of the reduced-form equations. This gives

$$
\begin{aligned}
& L=p\left(\mathbf{y}_{i} \mid \mathbf{x}_{i}\right)=(2 \pi)^{-n G / 2}|\boldsymbol{\Omega}|^{-n / 2} \exp \left(-\frac{1}{2} \sum_{i=1}^{n} \mathbf{v}_{i}^{\prime} \mathbf{\Omega}^{-1} \mathbf{v}_{i}\right) \\
& =(2 \pi)^{-n G / 2}|\boldsymbol{\Omega}|^{-n / 2} \exp \left[-\frac{1}{2} \sum_{i=1}^{n}\left(\mathbf{y}_{i}-\mathbf{x}_{i}^{\prime} \boldsymbol{\Pi}\right)^{\prime} \boldsymbol{\Omega}^{-1}\left(\mathbf{y}_{i}-\mathbf{x}_{i}^{\prime} \boldsymbol{\Pi}\right)\right]
\end{aligned}
$$

Now, consider the structure given in equation (3.1). A different structure can be obtained by post-multiplying this structure by a nonsingular matrix, say, $\mathbf{F}$.

$$
\mathbf{y}^{\prime} \mathbf{B F}+\mathbf{x}_{i}^{\prime} \mathbf{\Gamma F}=\mathbf{u}_{i}^{\prime} \mathbf{F}
$$

which can be written as:

$$
\mathbf{y}_{i}^{\prime} \mathbf{B}^{*}+\mathbf{x}_{i}^{\prime} \boldsymbol{\Gamma}^{*}=\mathbf{u}_{i}^{\prime *}
$$

where $\mathbf{B}^{*}=\mathbf{B F}, \Gamma^{*}=\boldsymbol{\Gamma} \mathbf{F}$ and $\mathbf{u}_{i}^{\prime *}=\mathbf{u}_{i}^{\prime} \mathbf{F}$

$$
p\left(\mathbf{u}_{i}^{\prime *}\right)=p\left(\mathbf{u}_{i}^{\prime}\right) \frac{\partial \mathbf{u}_{i}^{\prime}}{\partial \mathbf{u}_{i}^{\prime *}}=\mathbf{F}^{-1} p\left(\mathbf{u}_{i}^{\prime}\right)
$$

The joint density of the endogenous variables given the exogenous variables is now:

$$
\|\mathbf{B} \mathbf{F}\|^{n}\left|\mathbf{F}^{-1}\right|^{n} \prod_{i=1}^{n} p\left(\mathbf{u}_{i}^{\prime}\right)=\|\mathbf{B}\|^{n} \prod_{i=1}^{n} p\left(\mathbf{u}_{i}^{\prime}\right)
$$

This is the likelihood function for the new set of parameters $\mathbf{B}^{*}, \Gamma^{*}$ and $\Sigma^{*}$. Thus, we have: 


$$
L\left(\mathbf{B}^{*}, \Gamma^{*}, \boldsymbol{\Sigma}^{*}\right)=L(\mathbf{B}, \boldsymbol{\Gamma}, \boldsymbol{\Sigma})
$$

In this case, if there are no restrictions on $(\mathbf{B}, \boldsymbol{\Gamma}, \boldsymbol{\Sigma}),\left(\mathbf{B}^{*}, \Gamma^{*}, \boldsymbol{\Sigma}^{*}\right)$ and $(\mathbf{B}, \boldsymbol{\Gamma}, \boldsymbol{\Sigma})$ are said to be indistinguishable structures, because they both lead to the same likelihood function. The two structures are observationally equivalent and there is no way we can tell them apart. This situation is expressed by saying the $(\mathbf{B}, \boldsymbol{\Gamma}, \boldsymbol{\Sigma})$ are not identified. However, if there are some restrictions on $(\mathbf{B}, \boldsymbol{\Gamma}, \boldsymbol{\Sigma})$, this and the transformed structures $\left(\mathbf{B}^{*}, \Gamma^{*}, \boldsymbol{\Sigma}^{*}\right)$ will be indistinguishable only if the transformed structure also satisfies the same restrictions. If it is not possible to find $\mathbf{F}$ such that $\left(\mathbf{B}^{*}, \Gamma^{*}, \boldsymbol{\Sigma}^{*}\right)$ also satisfies the same restrictions as those on $(\mathbf{B}, \boldsymbol{\Gamma}, \boldsymbol{\Sigma})$, then the structure $(\mathbf{B}, \boldsymbol{\Gamma}, \boldsymbol{\Sigma})$ is said to be identified. The fact that $\mathbf{F}$ was chosen arbitrarily means that any nonsingular transformation of the original structure has the same reduced form. Hence, if the only information we have is the reduced-form parameters, then the structural model is not estimable. Identification of the structural parameters in $\mathbf{B}, \boldsymbol{\Gamma}$, and $\boldsymbol{\Sigma}$ thus depends on the addition of further information (non-sample information) to the model. Economic theory and extraneous information can be used to place restrictions on the set of simultaneous equations. These restrictions can take a variety of forms.

\subsubsection{Identification through Restrictions on the Structural Parameters}

Although additional information for identification comes in several forms, the most used ones take the form of specifying that certain structural parameters are zero. Placing a restriction on the structural parameters makes it more difficult to find a 
transformation of the structural equations that corresponds to the same reduced form, since that transformation must maintain the restriction. Besides, it becomes more difficult to find a linear combination of the equations that is indistinguishable from an original equation when there is a restriction placed on the structural parameters.

Since the structure $(\mathbf{B}, \boldsymbol{\Gamma}$, and $\boldsymbol{\Sigma})$ is said to be identified if each equation in the model is identified, the identification criteria is formalized by considering a single equation and then generalize the method derived to any structural equation in the model.

Consider the jth equation of the structural form model (3.1). The coefficients of the jth equation are in the jth columns of the $\mathbf{B}$ and $\boldsymbol{\Gamma}$ matrices. By dropping the subscript for convenience, this equation is written as:

$$
\mathbf{y}^{\prime} \mathbf{B}_{j}+\mathbf{x}^{\prime} \Gamma_{j}=\mathbf{u}_{j}^{\prime}
$$

Partitioning $\mathbf{B}_{\mathbf{j}}$ and $\boldsymbol{\Gamma}_{\mathbf{j}}$ each into two components corresponding to the included and excluded variable in this equation gives:

$$
\mathbf{B}_{j}^{\prime}=\left[\underline{\boldsymbol{\beta}}_{j}^{\prime} \boldsymbol{\beta}_{j}^{\prime *}\right] \text { and } \Gamma_{\mathrm{j}}^{\prime}=\left[\boldsymbol{\gamma}_{j}^{\prime} \boldsymbol{\gamma}_{j}^{\prime *}\right]
$$

where $\boldsymbol{\beta}_{j}^{\prime}$ corresponds to $\mathrm{G}_{1}$ included and $\boldsymbol{\beta}_{j}^{\prime *}$ corresponds to $\mathrm{G}_{2}$ excluded endogenous variables. Similarly, $\boldsymbol{\gamma}_{j}^{\prime}$ corresponds to $\mathrm{K}_{1}$ included and $\boldsymbol{\gamma}_{j}^{\prime *}$ corresponds to $\mathrm{K}_{2}$ included exogenous variables

The whole system contains $\mathrm{G} 1+\mathrm{G} 2=\mathrm{G}$ equations and $\mathrm{K} 1+\mathrm{K} 2=\mathrm{K}$ exogenous variables. One of the elements of $\underline{\boldsymbol{\beta}}_{j}^{\prime}$ is one and the exclusions imply that $\boldsymbol{\beta}_{j}^{\prime *}=\boldsymbol{\gamma}_{j}^{\prime *}=\mathbf{0}$. Portioning the $\mathrm{G} \times \mathrm{K}$ reduced-form coefficient matrix $\boldsymbol{\Pi}$ conformably gives the following: 


$$
\left[\begin{array}{ll}
\boldsymbol{\Pi}_{j} & \overline{\boldsymbol{\Pi}}_{j} \\
\boldsymbol{\Pi}_{j}^{*} & \overline{\boldsymbol{\Pi}}_{j}^{*}
\end{array}\right]
$$

Note that $\boldsymbol{\Pi}_{j}$ is G1 x K1, $\overline{\boldsymbol{\Pi}}_{j}$ is G1 x K2, $\boldsymbol{\Pi}_{j}^{*}$ is G2 $\times \mathrm{K} 1$, and $\overline{\boldsymbol{\Pi}}_{j}^{*}$ is G2 x K2 matrix.

The reduced-form coefficient matrix is $\Pi=-\boldsymbol{\Gamma B}^{-1}$ (equation 3.7), which implies that $\Pi \mathbf{B}=-\boldsymbol{\Gamma}$. The $\mathrm{jth}$ column of this matrix equation applies to the $\mathrm{jth}$ equation, $\boldsymbol{\Pi B}_{j}=-\boldsymbol{\Gamma}_{j}$ Then, using equations (3.13) and (3.14) we have:

$$
\left[\begin{array}{ll}
\underline{\boldsymbol{\beta}}_{j}^{\prime} & \mathbf{0}
\end{array}\right]\left[\begin{array}{cc}
\boldsymbol{\Pi}_{j} & \overline{\boldsymbol{\Pi}}_{j} \\
\boldsymbol{\Pi}_{j}^{*} & \overline{\boldsymbol{\Pi}}_{j}^{*}
\end{array}\right]=-\left[\begin{array}{ll}
\boldsymbol{\gamma}_{j}^{\prime} & \mathbf{0}
\end{array}\right]
$$

Hence

$$
-\underline{\boldsymbol{\beta}}_{j}^{\prime} \boldsymbol{\Pi}_{j}=\boldsymbol{\gamma}_{j}^{\prime}
$$

and

$$
\underline{\boldsymbol{\beta}}_{j}^{\prime} \overline{\boldsymbol{\Pi}}_{j}=\mathbf{0}
$$

Equation (3.16) is a system of $\mathrm{K} 2$ homogenous equations in G1 unknowns. If they can be solved for $\boldsymbol{\gamma}_{j}^{\prime}$, then equation (3.15) gives the solution for $\underline{\boldsymbol{\beta}}_{j}^{\prime}$ and the equation is identified. There will be a solution only if $\mathrm{K} 2 \geq \mathrm{G} 1-1$. This is known as the order condition of identification. It states that the number of exogenous variables excluded from the equation (in this case equation $\mathrm{j}$ ) must be at least as large as the number of endogenous variables included in the right-hand-side of equation $\mathrm{j}$. The order condition can also alternatively be written as:

$$
\mathrm{G} 2+\mathrm{K} 2 \geq \mathrm{G} 2+\mathrm{G} 1-1 \text { or } \quad \mathrm{G} 2+\mathrm{K} 2 \geq \mathrm{G}-1 .
$$


Note that G2 + K2 is the number of variables, both endogenous and exogenous, excluded from the equation. Hence this alternative order condition states that the number of excluded variables should be greater than or equal to the number of equations minus one. The order condition is a necessary but not sufficient condition for identification in that it can only ensure equations such as equation (3.16) has at least one solution, but cannot ensure that it has only one solution. It is merely a counting rule. The sufficient condition for identification is the rank condition and it states that:

$$
\operatorname{Rank}\left(\overline{\boldsymbol{\Pi}}_{j}\right)=\mathrm{G} 1-1 .
$$

This rank condition ensures that there is only one solution for the structural parameters given the reduced-form parameters. It is, however, difficult to apply since it requires the construction of the $\Pi$ matrix, which is complicated even in small models.

An alternative and easier method to apply is a rank condition which involves only the structural form parameters. This can be derived by using the a priori restrictions on $[\mathbf{B}, \boldsymbol{\Gamma}]$ to eliminate all false structures. Let:

$$
\mathbf{A}=\left[\begin{array}{l}
\mathbf{B} \\
\mathbf{\Gamma}
\end{array}\right]
$$

denote an original set of coefficients, and let $\mathbf{A F}$ denote a new structure obtained from $\mathbf{A}$ by post-multiplication with an arbitrary $\mathrm{G} \times \mathrm{G}$ nonsingular transformation matrix $\mathbf{F}$. The new structure is said to be admissible, or equivalently $\mathbf{F}$ is said to be an admissible transformation matrix, if $\mathbf{A F}$ satisfies all the restriction on $\mathbf{A}$. Rearranging matrix A in the partition form gives: 


$$
\mathbf{A}=\left[\begin{array}{l}
\mathbf{B} \\
\boldsymbol{\Gamma}
\end{array}\right]=\left[\begin{array}{ll}
\underline{\boldsymbol{\beta}}_{j} & \mathbf{A}_{1} \\
\mathbf{0} & \mathbf{A}_{2} \\
\boldsymbol{\gamma}_{j} & \mathbf{A}_{3} \\
\mathbf{0} & \mathbf{A}_{4}
\end{array}\right]=\left[\begin{array}{ll}
\mathbf{a}_{j} & \mathbf{A}_{j}
\end{array}\right]
$$

Then $\left[\begin{array}{ll}\mathbf{B} \mathbf{f}_{j} & \boldsymbol{\Gamma} \mathbf{f}_{j}\end{array}\right]$ would be the jth column in the false structure $\mathbf{A F}$, where $\mathbf{f}_{\mathbf{j}}$ is the $\mathbf{j t h}$ column of $\mathbf{F}$. The new jth equation is to be built up as a linear combination of the old one and the other equations in the model. Thus,

$$
\tilde{\mathbf{a}}_{j}=\left[\begin{array}{cc}
\underline{\boldsymbol{\beta}}_{j} & \mathbf{A}_{1} \\
\mathbf{0} & \mathbf{A}_{2} \\
\boldsymbol{\gamma}_{j} & \mathbf{A}_{3} \\
\mathbf{0} & \mathbf{A}_{4}
\end{array}\right]\left[\begin{array}{l}
\mathbf{f}_{0} \\
\mathbf{f}_{1}
\end{array}\right]=\left[\begin{array}{l}
\tilde{\boldsymbol{\beta}}_{j} \\
\mathbf{0} \\
\tilde{\boldsymbol{\gamma}}_{j} \\
\mathbf{0}
\end{array}\right]
$$

Identification of the jth equation requires that the jth equation of every admissible structure be some scalar multiple of the true jth equation. For this to happen $\tilde{\mathbf{a}}_{j}$ must have nonzero elements in the same places, which can be ensured by taking $f_{0}=1$, and zeros in the same positions as the original $\mathbf{a}_{j}$. If $\tilde{\mathbf{a}}_{j}$ is to be admissible, then it must meet the requirement:

$$
\left[\begin{array}{l}
\mathbf{A}_{2} \\
\mathbf{A}_{4}
\end{array}\right] \mathbf{f}_{1}=\mathbf{0}
$$

But, if $\left[\begin{array}{l}\mathbf{A}_{2} \\ \mathbf{A}_{4}\end{array}\right]$ has a full column rank, this equality will not hold and hence $\tilde{\mathbf{a}}_{j}$ is not admissible. This which would mean the new structure and the original structure are not equivalent and in that case the equation is said to be identified. Note that $\mathbf{A}_{2}$ and $\mathbf{A}_{4}$ are the structural coefficients in the other equations on the variables that are excluded from 
equation j. Thus, $\left[\begin{array}{l}\mathbf{A}_{2} \\ \mathbf{A}_{4}\end{array}\right]$ is $(\mathrm{G} 2+\mathrm{K} 2) \times(\mathrm{G}-1)$ matrix and if it has full column rank, the equivalent rank condition for identification is given by:

$$
\operatorname{rank}\left[\begin{array}{c}
\mathbf{A}_{2} \\
\mathbf{A}_{4}
\end{array}\right]=\mathrm{G}-1
$$

\subsection{Method of Estimation}

Once questions of model specification and identification are considered, one has to deal with the estimation of the model parameters. Although it is possible to estimate the reduced-form parameters, $\boldsymbol{\Pi}$ and $\boldsymbol{\Omega}$, consistently by ordinary least squares, generally the parameter of interest are $\mathbf{B}, \boldsymbol{\Gamma}$, and $\boldsymbol{\Sigma}$, and not $\boldsymbol{\Pi}$ and $\boldsymbol{\Omega}$ except for forecasting $\mathbf{y}$ given $\mathbf{x}$ (Greene, 2003). When the system of simultaneous equations is exactly identified, the number of unrestricted reduced-form parameters equals the number of structural form parameters, and hence it is possible to recover the structural coefficients from the reduced form coefficients. In this special case, the reduced-form and the structural form are alternative forms of parameterization of the model, but estimation is convenient in the reduced-form parameterization. First, the reduced-form coefficients are estimated by OLS, and then the structural parameters are estimated using the relationships between these parameters and the reduced-form parameters and the identifying restrictions. Although these estimates are consistent, they are biased and inefficient. If the system of simultaneous equations is over identified, this method does not work either. The transformation of the reduced-form parameters become ambiguous, it gives multiple solutions and it is not clear which is the best. Ordinary Least squares (OLS) method of estimation applied to structural equations of simultaneous equation system also in general 
leads to inconsistent estimates, because the included endogenous variables in each equation are correlated with the disturbances (Greene, 2003; Kmenta, 1997). Thus, in order to obtain consistent estimates of the structural coefficients, alternative methods should be developed.

The simultaneous equation system to be estimated, which is also given in equation (3.1) is:

$$
\mathbf{y}_{i}^{\prime} \mathbf{B}+\mathbf{x}_{i}^{\prime} \boldsymbol{\Gamma}=\mathbf{u}_{i}^{\prime} \quad \mathrm{i}=1,2, \ldots, \mathrm{n}
$$

In terms of full set of $\mathrm{n}$ observations, this simultaneous equation system can be written as:

$$
\underset{n \times G}{\mathbf{Y}} \underset{G \times G}{\mathbf{B}}+\underset{n \times K}{\mathbf{X}} \underset{K \times G}{\boldsymbol{\Gamma}}=\underset{n \times G}{\mathbf{U}} .
$$

The problem of simultaneous equation estimation is then that of using the matrices of $\mathbf{Y}$ and $\mathbf{X}$ to estimate the coefficient matrices $\mathbf{B}, \boldsymbol{\Gamma}$, and the variance-covariance matrix $\boldsymbol{\Sigma}$. Using equation (3.2), the jth equation of the system in (3.19) can be written as:

$$
\underline{\mathbf{Y}}_{j} \underline{\boldsymbol{\beta}}_{j}+\mathbf{Y}_{j}^{*} \boldsymbol{\beta}_{j}^{*}+\mathbf{X}_{j} \gamma_{j}+\mathbf{X}_{j}^{*} \gamma_{j}^{*}=\mathbf{u}_{j}
$$

and when it is normalized( the jth element of $\underline{\boldsymbol{\beta}}_{j}=1$ )

$$
\mathbf{y}_{j}=\mathbf{Y}_{j} \boldsymbol{\beta}_{j}+\mathbf{Y}_{j}^{*} \boldsymbol{\beta}_{j}^{*}+\mathbf{X}_{j} \gamma_{j}+\mathbf{X}_{j}^{*} \gamma_{j}^{*}+\mathbf{u}_{j}
$$

In the case of zero restrictions $\left(\boldsymbol{\beta}_{j}^{\prime *}=\boldsymbol{\gamma}_{j}^{\prime *}=\mathbf{0}\right)$ this becomes:

$$
\mathbf{y}_{j}=\mathbf{Y}_{j} \boldsymbol{\beta}_{j}+\mathbf{X}_{j} \gamma_{j}+\mathbf{u}_{j}
$$

Where $\mathbf{y}_{j}$ is $\mathrm{n} \times 1$ column vector of data on the dependent endogenous variable (the one on which this equation has been normalized), $\mathbf{Y}_{j}$ is the $\mathrm{n} \times(\mathrm{G} 1-1)$ matrix of data on the 
G1 - 1 include right-hand side endogenous variable, $\boldsymbol{\beta}_{j}$ is $(\mathrm{G} 1-1)$ x 1 column vector of non-zero coefficients on $\mathbf{Y}_{j}$ and the rest as define above. Equation (3.20) is the basic equation upon which several estimators of the simultaneous equation system will be developed.

There are two basic approaches to estimating simultaneous equations system. The first one is the Single-Equation or Limited Information methods in which a method is designed to estimate a single structural equation at a time without using the information contained in the model. The second one is the Full Information methods in which all the equations of the structural model are estimated simultaneously.

\subsubsection{Single-Equation Method}

The single-equation or limited information method estimates one equation at a time and uses information as to which endogenous as well as exogenous variables are included in the other equations of the system but excluded from the equation being estimated. Thus, it uses all identifying restrictions pertaining to the equation. The information required, however, are limited to the variables included in or excluded from the equation being estimated. This approach does not require information on the specification of the other equations in the system. The identifying restrictions on the other equations are not used for estimation purposes although they should be used to check the identifiability of that particular equation.

The most commonly used single-equation methods are ordinary least squares

(OLS), indirect least squares (ILS), two-stage least squares (2SLS), and limitedinformation maximum likelihood (LIML). The OLS method does not give consistent 
estimates of the parameters because of the correlation between the residuals and the regressors, whereas the other methods give consistent estimates (Maddala, 1986; Johnston, 1984, Intriligator, 1978, Kmenta, 1997; Greene, 2003)

\subsubsection{Ordinary Least Squares}

This method applies least squares to each equation of the model separately, ignoring the distinction between explanatory endogenous variables and included exogenous variables. It also ignores all information available concerning variables not included in the estimated equation. Although it gives inconsistent estimates, it has been found that the OLS method is more robust against specification error than many of the simultaneous equation methods and also that the predictions from equation estimated by OLS often compare favorably with those obtained from equation estimated by the simultaneous equations methods ( Maddala, 1986; Johnston, 1984). Thus, it is useful to report OLS estimates of the structural equations along with those from the other methods that give consistent estimates.

Suppose the structural equation that we are interested to estimate is the jth equation of the simultaneous equation system in (3.19), that is:

$$
\begin{aligned}
& \begin{array}{l}
\mathbf{y}_{j}=\mathbf{Y}_{j} \boldsymbol{\beta}_{j}+\mathbf{X}_{j} \boldsymbol{\gamma}_{j}+\mathbf{u}_{j} \\
=\mathbf{Z}_{j} \boldsymbol{\delta}_{j}+\mathbf{u}_{j}
\end{array} \\
& \text { where } \mathbf{Z}_{j}=\left[\begin{array}{ll}
\mathbf{Y}_{j} & \mathbf{X}_{j}
\end{array}\right] \text { and } \boldsymbol{\delta}_{j}=\left[\begin{array}{l}
\boldsymbol{\beta}_{j} \\
\boldsymbol{\gamma}_{j}
\end{array}\right] .
\end{aligned}
$$

The OLS estimators of the coefficient are obtained in the same way they are used in the case of single equation models: 


$$
\hat{\boldsymbol{\delta}}_{j O L S}=\left[\mathbf{Z}_{j}^{\prime} \mathbf{Z}_{j}\right]^{-1} \mathbf{Z}_{j}^{\prime} \mathbf{y}_{j}=\left[\begin{array}{ll}
\mathbf{Y}_{j}^{\prime} \mathbf{Y}_{j} & \mathbf{Y}_{j}^{\prime} \mathbf{X}_{j} \\
\mathbf{X}_{j}^{\prime} \mathbf{Y}_{j} & \mathbf{X}_{j}^{\prime} \mathbf{X}_{j}
\end{array}\right]^{-1}\left[\begin{array}{c}
\mathbf{Y}_{j}^{\prime} \\
\mathbf{X}_{j}^{\prime}
\end{array}\right] \mathbf{y}_{j},
$$

where the inverse exists if $\mathbf{Z}_{j}$ has a rank of $\mathrm{G} 1-1+\mathrm{K} 1$.

Since the $\mathbf{Y}_{j}$ in equation (3.21) are endogenous variables, which are not statistically independent of the stochastic disturbance terms, even in the probability term, the OLS estimators of the simultaneous equation system are biased and also generally inconsistent estimators. This can easily be demonstrated by substituting $\mathbf{Z}_{j} \boldsymbol{\delta}_{j}+\mathbf{u}_{j}$ from equation (3.21) for $\mathbf{y}_{j}$ in equation (3.22), which gives:

$$
\begin{aligned}
\hat{\boldsymbol{\delta}}_{j O L S} & =\left[\mathbf{Z}_{j}^{\prime} \mathbf{Z}_{j}\right]^{-1} \mathbf{Z}_{j}^{\prime}\left(\mathbf{Z}_{j} \boldsymbol{\delta}_{j}+\mathbf{u}_{j}\right)=\left[\begin{array}{ll}
\mathbf{Y}_{j}^{\prime} \mathbf{Y}_{j} & \mathbf{Y}_{j}^{\prime} \mathbf{X}_{j} \\
\mathbf{X}_{j}^{\prime} \mathbf{Y}_{j} & \mathbf{X}_{j}^{\prime} \mathbf{X}_{j}
\end{array}\right]^{-1}\left[\begin{array}{c}
\mathbf{Y}_{j}^{\prime} \\
\mathbf{X}_{j}^{\prime}
\end{array}\right]\left(\mathbf{Z}_{j} \boldsymbol{\delta}_{j}+\mathbf{u}_{j}\right) \\
& =\boldsymbol{\delta}_{j}+\left[\mathbf{Z}_{j}^{\prime} \mathbf{Z}_{j}\right]^{-1} \mathbf{Z}^{\prime} \mathbf{u}_{j}=\boldsymbol{\delta}_{j}+\left[\begin{array}{ll}
\mathbf{Y}_{j}^{\prime} \mathbf{Y}_{j} \mathbf{Y}_{j}^{\prime} \mathbf{X}_{j} \\
\mathbf{X}_{j}^{\prime} \mathbf{Y}_{j} \mathbf{X}_{j}^{\prime} \mathbf{X}_{j}
\end{array}\right]^{-1}\left[\begin{array}{l}
\mathbf{Y}_{j}^{\prime} \mathbf{u}_{j} \\
\mathbf{X}_{j}^{\prime} \mathbf{u}_{j}
\end{array}\right]
\end{aligned}
$$

Takinge expectations,

$$
\mathrm{E}\left(\hat{\boldsymbol{\delta}}_{j O L S}\right)=\boldsymbol{\delta}_{j}+\mathrm{E}\left(\left[\mathbf{Z}_{j}^{\prime} \mathbf{Z}_{j}\right]^{-1} \mathbf{Z}^{\prime} \mathbf{u}_{j}\right)=\boldsymbol{\delta}_{j}+\mathrm{E}\left(\left[\begin{array}{cc}
\mathbf{Y}_{j}^{\prime} \mathbf{Y}_{j} & \mathbf{Y}_{j}^{\prime} \mathbf{X}_{j} \\
\mathbf{X}_{j}^{\prime} \mathbf{Y}_{j} \mathbf{X}_{j}^{\prime} \mathbf{X}_{j}
\end{array}\right]^{-1}\left[\begin{array}{l}
\mathbf{Y}_{j}^{\prime} \mathbf{u}_{j} \\
\mathbf{X}_{j}^{\prime} \mathbf{u}_{j}
\end{array}\right]\right)
$$

Since $\mathbf{Y}_{j}$ are stochastic and not independent of the stochastic disturbance term, none of the terms in the inverse matrices converges to $\mathbf{0}$. Hence, $\mathrm{E}\left(\left[\mathbf{Z}_{j}^{\prime} \mathbf{Z}_{j}\right]^{-1} \mathbf{Z}^{\prime} \mathbf{u}_{j}\right)$ or $\mathrm{E}\left(\left[\begin{array}{c}\mathbf{Y}_{j}^{\prime} \mathbf{Y}_{j} \mathbf{Y}_{j}^{\prime} \mathbf{X}_{j} \\ \mathbf{X}_{j}^{\prime} \mathbf{Y}_{j}\end{array} \mathbf{X}_{j}^{\prime} \mathbf{X}_{j}^{-1}\left[\begin{array}{c}\mathbf{Y}_{j}^{\prime} \mathbf{u}_{j} \\ \mathbf{X}_{j}^{\prime} \mathbf{u}_{j}\end{array}\right]\right)\right.$ does not disappear, and that would mean $\hat{\boldsymbol{\delta}}_{\text {jOLS }} \neq \boldsymbol{\delta}_{j}$, implying that the OLS estimators are biased. The OLS bias is give by: 


$$
\begin{aligned}
\operatorname{Bias}\left(\hat{\boldsymbol{\delta}}_{j O L S}\right)= & \mathrm{E}\left(\hat{\boldsymbol{\delta}}_{j O L S}-\boldsymbol{\delta}_{j}\right) \\
& =\mathrm{E}\left(\left[\mathbf{Z}_{j}^{\prime} \mathbf{Z}_{j}\right]^{-1} \mathbf{Z}^{\prime} \mathbf{u}_{j}\right) \\
& =\mathrm{E}\left(\left[\begin{array}{c}
\mathbf{Y}_{j}^{\prime} \mathbf{Y}_{j} \mathbf{Y}_{j}^{\prime} \mathbf{X}_{j} \\
\mathbf{X}_{j}^{\prime} \mathbf{Y}_{j} \mathbf{X}_{j}^{\prime} \mathbf{X}_{j}
\end{array}\right]^{-1}\left[\begin{array}{l}
\mathbf{Y}_{j}^{\prime} \mathbf{u}_{j} \\
\mathbf{X}_{j}^{\prime} \mathbf{u}_{j}
\end{array}\right]\right)
\end{aligned}
$$

This bias does not disappear even in the limit as $n \rightarrow \infty$, so that OLS estimators are also asymptotically biased. Again, although $\operatorname{plim}\left(\frac{1}{n} \mathbf{X}_{j}^{\prime} \mathbf{u}_{j}\right)=\mathbf{0}, \operatorname{plim}\left(\frac{1}{n} \mathbf{Y}_{j}^{\prime} \mathbf{u}_{j}\right) \neq \mathbf{0}$, and hence this bias does not also disappear in the probability limit.

$$
\begin{aligned}
& \operatorname{plim}\left(\hat{\boldsymbol{\delta}}_{\text {jOLS }}\right)=\boldsymbol{\delta}_{j}+\operatorname{plim}\left(\left[\mathbf{Z}_{j}^{\prime} \mathbf{Z}_{j}\right]^{-1} \mathbf{Z}^{\prime} \mathbf{u}_{j}\right) \\
& =\boldsymbol{\delta}_{j}+\operatorname{plim}\left(\left[\begin{array}{cc}
\mathbf{Y}_{j}^{\prime} \mathbf{Y}_{j} & \mathbf{Y}_{j}^{\prime} \mathbf{X}_{j} \\
\mathbf{X}_{j}^{\prime} \mathbf{Y}_{j} & \mathbf{X}_{j}^{\prime} \mathbf{X}_{j}
\end{array}\right]^{-1}\left[\begin{array}{c}
\mathbf{Y}_{j}^{\prime} \mathbf{u}_{j} \\
\mathbf{X}_{j}^{\prime} \mathbf{u}_{j}
\end{array}\right]\right) \neq \boldsymbol{\delta}_{j} .
\end{aligned}
$$

This means that both parts of $\hat{\boldsymbol{\delta}}_{\text {joLS }}$ are inconsistent, implying the OLS estimators of simultaneous equation system are inconsistent.

There is, however, one case where OLS estimates are consistent. In a recursive model where the $\mathbf{B}$ matrix of the structural equations is triangular (upper or lower) and the $\boldsymbol{\Sigma}$ matrix is diagonal, that is, all the disturbance terms are uncorrelated to each other, all of the structural equations in the system can be estimated by OLS. Under these special assumptions of the recursive model the OLS estimators of the structural equation will have the desirable properties of consistency, asymptotic normality and efficiency (Johnston, 1984; Greene, 2003). 


\subsubsection{Indirect Least Squares}

Indirect least squares (ILS) is a feasible estimation technique for an equation that is just-identified. In a just-identified case the structural parameters are uniquely determined from the reduced-form parameters, so that the reduced-form parameters can be used to infer the estimated structural parameters indirectly. The approach involves two steps. The first step consists of estimating the matrix of reduced-form parameters by the application of OLS to each of the reduced-form equation. The second step consists of obtaining the estimates of the structural parameters from the algebraic relations existing between the structural and reduced-form parameters.

This method starts by estimating the reduced-form equation given in equation (3.19), which is:

$$
\underset{n \times G}{\mathbf{Y}} \underset{G \times G}{\mathbf{B}}+\underset{n \times K}{\mathbf{X}} \underset{K \times G}{\boldsymbol{\Gamma}}=\underset{n \times G}{\mathbf{U}}
$$

This may be written as:

$$
\underset{n \times G}{\mathbf{Y}}=\underset{n \times K}{\mathbf{X}} \underset{K \times G}{\boldsymbol{\Pi}}+\underset{n \times G}{\mathbf{V}}
$$

where

and

$$
\begin{gathered}
\Pi=-\boldsymbol{\Gamma}(\mathbf{B})^{-1} \\
\mathbf{V}=\mathbf{U}(\mathbf{B})^{-1}
\end{gathered}
$$

Applying OLS to the first part of equation (3.26) gives the estimator of the matrix of the reduced-form parameters $\Pi$ which is given by:

$$
\hat{\mathbf{\Pi}}=\left(\mathbf{X}^{\prime} \mathbf{X}\right)^{-1} \mathbf{X}^{\prime} \mathbf{Y}
$$

This estimator is equivalent to estimating each equation of the reduced-form separately via least squares, since each column of $\hat{\boldsymbol{\Pi}}$ is the common matrix of $\left(\mathbf{X}^{\prime} \mathbf{X}\right)^{-1} \mathbf{X}^{\prime} \mathbf{Y}$ weights times the column of the $\mathbf{Y}$ matrix corresponding to the dependent variable in that 
particular reduced-form equation. It yields the set of estimated reduced-form parameters for the first step ILS (Johnston, 1984; Intriligator, 1978).

To start the second ILS step, consider the equation given in equation (3.20):

$$
\mathbf{y}_{j}=\mathbf{Y}_{j} \boldsymbol{\beta}_{j}+\mathbf{X}_{j} \gamma_{j}+\mathbf{u}_{j} .
$$

For convenience, let us assume we are interested to estimate the first structural equation $(\mathrm{j}=1)$ assuming that it is just-identified. Then equation (3.28) becomes:

$$
\mathbf{y}_{1}=\mathbf{Y}_{1} \boldsymbol{\beta}_{1}+\mathbf{X}_{1} \boldsymbol{\gamma}_{1}+\mathbf{u}_{1}
$$

The dimensions and the definitions of the vectors and the matrices of equation (3.29) are the same as those in equation (3.20).

Rewriting equation (3.29) gives:

$$
\left[\begin{array}{lll}
\mathbf{y}_{1} & \mathbf{Y}_{1} & \mathbf{X}_{1}
\end{array}\right]\left[\begin{array}{c}
1 \\
-\boldsymbol{\beta}_{1} \\
-\boldsymbol{\gamma}_{1}
\end{array}\right]=\mathbf{u}_{1}
$$

or, mor fully:

$$
\left[\begin{array}{lllll}
\mathbf{y}_{1} & \mathbf{Y}_{1} & \mathbf{Y}_{1}^{*} & \mathbf{X}_{1} & \mathbf{X}_{1}^{*}
\end{array}\right]\left[\begin{array}{c}
1 \\
-\boldsymbol{\beta}_{1} \\
\mathbf{0} \\
-\boldsymbol{\gamma}_{1} \\
\mathbf{0}
\end{array}\right]=\mathbf{u}_{1}
$$

Where $\mathbf{Y}_{1}^{*}$ and $\mathbf{X}_{1}^{*}$ are matrices of observations on $\mathrm{G} 2$ endogenous and $\mathrm{K} 2$ predetermined variables which are excluded from equation $j(j=1$ in this case).

Using equation (3.26), the relations between the structural and reduced-form parameters can be written as;

$$
\Pi \mathbf{I B}=-\Gamma
$$


For the first equation $(\mathrm{j}=1)$, involving the first columns of $\mathbf{B}$ and $\boldsymbol{\Gamma}$, using the normalization and zero restrictions, this can be rewritten as:

$$
\boldsymbol{\Pi}\left[\begin{array}{c}
1 \\
-\beta_{1} \\
0
\end{array}\right]=\left[\begin{array}{l}
\boldsymbol{\gamma}_{1} \\
\mathbf{0}
\end{array}\right]
$$

In order to multiply out equation (3.30), $\Pi$ should be partitioned conformably into six sub-matrices as follows:

$$
\begin{aligned}
& {\left[\begin{array}{lll}
\boldsymbol{\pi}_{j} & \underline{\boldsymbol{\Pi}}_{j} & \overline{\boldsymbol{\Pi}}_{j} \\
\boldsymbol{\pi}_{j}^{*} & \underline{\boldsymbol{\Pi}}_{j}^{*} & \overline{\boldsymbol{\Pi}}_{j}^{*}
\end{array}\right]} \\
& 1 \text { G1-1 G2 }
\end{aligned}
$$

The columns of this partitioned matrix is divided to correspond to one dependent variable, G1-1 explanatory endogenous variables, and G2 excluded endogenous variables (in this case into $\mathbf{y}_{1}, \mathbf{Y}_{1}$, and $\mathbf{Y}_{1}^{*}$, respectively).

Plaguing the partitioned $\mathbf{\Pi}$ matrix into equation (3.30) gives:

$$
\left[\begin{array}{ccc}
\boldsymbol{\pi}_{j} & \underline{\boldsymbol{\Pi}}_{j} & \overline{\boldsymbol{\Pi}}_{j} \\
\boldsymbol{\pi}_{j}^{*} & \underline{\boldsymbol{\Pi}}_{j}^{*} & \overline{\boldsymbol{\Pi}}_{j}^{*}
\end{array}\right]\left[\begin{array}{c}
1 \\
-\boldsymbol{\beta}_{1} \\
0
\end{array}\right]=\left[\begin{array}{l}
\boldsymbol{\gamma}_{1} \\
\mathbf{0}
\end{array}\right]
$$

Writing out the resulting two sets of equations, where the elements of $\boldsymbol{\Pi}$ are replaced by the estimator $\hat{\boldsymbol{\Pi}}$ in (3.27) and the structural parameters of the equation are replaced by their estimators $\hat{\boldsymbol{\beta}}_{1}$ and $\hat{\boldsymbol{\gamma}}_{1}$ :

$$
\begin{array}{lr}
\hat{\boldsymbol{\pi}}_{1}-\underline{\boldsymbol{\Pi}}_{1} \boldsymbol{\beta}_{1}=\boldsymbol{\gamma}_{1} & (\mathrm{~K} 1 \text { equations }) \\
\hat{\boldsymbol{\pi}}_{1}^{*}-\underline{\boldsymbol{\Pi}}_{1}^{*} \boldsymbol{\beta}_{1}=\mathbf{0} & \text { (K2 equations) }
\end{array}
$$


If the equation to be estimated is just-identified, then $\underline{\hat{\mathbf{I}}}_{1}^{*}$ is a square matrix and assuming that it is nonsingular; the second part of equation (3.32) can be solved for $\boldsymbol{\beta}_{1}$ as:

$$
\hat{\boldsymbol{\beta}}_{1}=\left(\underline{\boldsymbol{\Pi}}_{1}^{*}\right)^{-1} \hat{\boldsymbol{\pi}}_{1}^{*} .
$$

It is also possible to obtain $\hat{\gamma}_{1}$ by combining equation (3.33) and the first part of equation (3.32) as follows:

$$
\hat{\boldsymbol{\gamma}}_{1}=\hat{\boldsymbol{\pi}}_{1}-\underline{\hat{\mathbf{\Lambda}}}_{1}\left(\underline{\hat{\mathbf{\Lambda}}}_{1}^{*}\right)^{-1} \hat{\boldsymbol{\pi}}_{1}
$$

Thus, the indirect least square estimators may be written as:

$$
\left[\begin{array}{l}
\hat{\boldsymbol{\beta}}_{1} \\
\hat{\boldsymbol{\gamma}}_{1}
\end{array}\right]_{I L S}=\left[\begin{array}{l}
\left(\hat{\hat{\boldsymbol{\Pi}}}_{1}^{*}\right)^{-1} \hat{\boldsymbol{\pi}}_{1}^{*} \\
\hat{\boldsymbol{\pi}}_{1}-\underline{\hat{\boldsymbol{\Pi}}}_{1}\left(\underline{\hat{\boldsymbol{\Pi}}}_{1}^{*}\right)^{-1} \hat{\boldsymbol{\pi}}_{1}^{*}
\end{array}\right]
$$

Like the OLS estimators, the ILS estimators are generally biased, but unlike the former the latter are consistent estimators, where:

$$
\operatorname{plim}\left[\begin{array}{l}
\hat{\boldsymbol{\beta}}_{1} \\
\hat{\boldsymbol{\gamma}}_{1}
\end{array}\right]=\left[\begin{array}{l}
\boldsymbol{\beta}_{1} \\
\boldsymbol{\gamma}_{1}
\end{array}\right]
$$

As summarized in equation (3.35), the ILS estimators are obtained as continuous functions of the reduced-form estimators. Since the reduced-form estimators are consistent, from the least squares consistency theorem, and continuous functions of consistent estimators are also consistent, ILS estimators are consistent. 


\subsubsection{Two-Stage Least Squares}

The technique of two-stage least squares (2SLS) is the most important and widely used method for estimating simultaneous equations models. Unlike the OLS and ILS estimators, which are defined only for just-identified equations, 2SLS estimator can be used to estimate either an over-identified or a just identified equation from a system of simultaneous equations. In the case of just-identified equations, it also turns out that 2SLS estimates are identical with the ILS estimates given in equation (3.35).

Suppose the structural equation to be estimated is again, as given in (3.29), of the form

$$
\mathbf{y}_{1}=\mathbf{Y}_{1} \boldsymbol{\beta}_{1}+\mathbf{X}_{1} \gamma_{1}+\mathbf{u}_{1}
$$

As previously stated, the problem in applying OLS directly to estimate this equation is that the embedding of the equation in simultaneous equation system makes the explanatory endogenous variables in $\mathbf{Y}_{1}$ correlated with $\mathbf{u}_{1}$, even in the probability limit.

If these variables could be replaced by related variables that are uncorrelated with the stochastic disturbance term, the resulting estimator would be consistent. The technique of 2SLS consists of replacing $\mathbf{Y}_{1}$ by a computed matrix $\hat{\mathbf{Y}}_{1}$, which hopefully is purged of the stochastic element, and then performing OLS of $\mathbf{y}_{1}$ on $\hat{\mathbf{Y}}_{1}$ and $\mathbf{X}_{1}$. The matrix in $\hat{\mathbf{Y}}_{1}$ is computed in the first stage by regressing each variable in $\mathbf{Y}_{1}$ on all the predetermined (exogenous) variables in the complete model using the reduced-form and replacing the actual observations on the $\mathbf{y}_{1}$ variables by the corresponding regression values. 
To develop the computation of these estimates, let us consider the reduced-form, which is also given in the first part of equation (3.26):

$$
\underset{n \times G}{\mathbf{Y}}=\underset{n \times K}{\mathbf{X}} \underset{K \times G}{\mathbf{I}}+\underset{n \times G}{\mathbf{V}}
$$

and the least-squares estimator of the reduced-form coefficient $\Pi$, which is also given in equation (3.27) is:

$$
\hat{\mathbf{\Pi}}=\left(\mathbf{X}^{\prime} \mathbf{X}\right)^{-1} \mathbf{X}^{\prime} \mathbf{Y}
$$

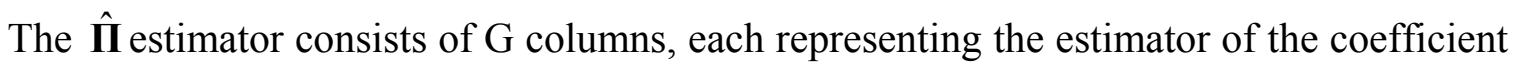
in one such regression. The estimates of the endogenous variable are obtained from the estimated $\hat{\boldsymbol{\Pi}}$ and data on all exogenous variables of the model $\mathrm{X}$ as

$$
\begin{aligned}
\hat{\mathbf{Y}} & =\mathbf{X} \hat{\mathbf{\Pi}}=\mathbf{X}\left(\mathbf{X}^{\prime} \mathbf{X}\right)^{-1} \mathbf{X}^{\prime} \mathbf{Y} \\
& =\left[\begin{array}{lll}
\hat{\mathbf{y}}_{1} & \hat{\mathbf{Y}}_{1} & \hat{\mathbf{Y}}_{1}^{*}
\end{array}\right]=\mathbf{X} \hat{\mathbf{\Pi}}=\mathbf{X}\left(\mathbf{X}^{\prime} \mathbf{X}\right)^{-1} \mathbf{X}^{\prime} \mathbf{Y}(\hat{\mathbf{Y}} \text { is partitioned }) \\
& =\mathbf{X}\left(\mathbf{X}^{\prime} \mathbf{X}\right)^{-1} \mathbf{X}^{\prime}\left[\begin{array}{lll}
\mathbf{y}_{1} & \mathbf{Y}_{1} & \mathbf{Y}_{1}^{*}
\end{array}\right] \quad(\mathbf{Y} \text { is partitioned })
\end{aligned}
$$

Thus $\hat{\mathbf{Y}}_{1}$ can be expressed as the linear combination of the actual $\mathbf{Y}_{1}$ as:

$$
\hat{\mathbf{Y}}_{1}=\mathbf{X}\left(\mathbf{X}^{\prime} \mathbf{X}\right)^{-1} \mathbf{X}^{\prime} \mathbf{Y}_{1}
$$

In the second stage, $\mathbf{Y}_{1}$ is replaced in equation (3.36) by $\hat{\mathbf{Y}}_{1}$ and the least-squares estimator of the resulting equation is the two-stage least-squares (2SLS) estimator, of the form:

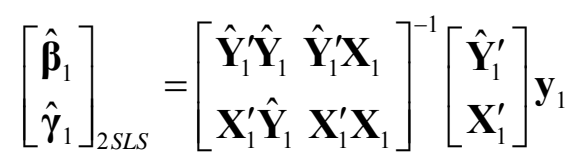


Equation (3.38) indicates that it yields estimators of all coefficients of one equation of the system, given data on the dependent endogenous variables $\mathbf{y}_{1}$, data on the predetermined (exogenous) variables $\mathbf{X}_{1}$, and the estimated values of the explanatory endogenous variables $\mathbf{Y}_{1}$, that is $\hat{\mathbf{Y}}_{1}$. Since $\hat{\mathbf{Y}}_{1}$ are determined themselves from the estimated reduced-form coefficients and data on all predetermined (exogenous) variables of the system, the 2SLS estimator, thus depends on all exogenous variables, not just those included in the equation to be estimated.

For the actual estimation there is no need to compute the regression values in $\hat{\mathbf{Y}}_{1}$ explicitly. An alternative expression for the 2 SLS estimator can be derived by only involving the matrices of actual observations. Combining equations (3.37), (3.38), and (3.39) gives

$$
\begin{aligned}
\mathbf{Y} & =\mathbf{X}\left(\mathbf{X}^{\prime} \mathbf{X}\right)^{-1} \mathbf{X}^{\prime} \mathbf{Y}+\mathbf{V} \\
& =\hat{\mathbf{Y}}+\mathbf{V}
\end{aligned}
$$

By partitioning $\mathbf{Y}$ and $\mathbf{V}$ and using equation (3.40), the matrix $\mathbf{Y}_{1}$ can be written as;

$$
\mathbf{Y}_{1}=\hat{\mathbf{Y}}_{1}+\mathbf{V}_{1} \rightarrow \hat{\mathbf{Y}}_{1}=\mathbf{Y}_{1}-\mathbf{V}_{1}
$$

where $\mathbf{V}_{1}$ is an $\mathrm{n} \times(\mathrm{G} 1-1)$ matrix of reduced-form residuals with the usual properties of

$$
\hat{\mathbf{Y}}_{1}^{\prime} \mathbf{V}_{1}=\mathbf{0} \text { and } \mathbf{X}^{\prime} \mathbf{V}_{1}=\mathbf{0}
$$

Hence

$$
\begin{aligned}
\hat{\mathbf{Y}}_{1}^{\prime} \hat{\mathbf{Y}}_{1}= & \hat{\mathbf{Y}}_{1}^{\prime}\left(\mathbf{Y}_{1}-\mathbf{V}_{1}\right) \quad \text { (substitution from equation(3.43)) } \\
& \left.=\hat{\mathbf{Y}}_{1}^{\prime} \mathbf{Y}_{1} \quad \text { (because } \hat{\mathbf{Y}}_{1}^{\prime} \mathbf{V}_{1}=\mathbf{0}\right) \\
& =\mathbf{Y}_{1}^{\prime} \mathbf{X}\left(\mathbf{X}^{\prime} \mathbf{X}\right)^{-1} \mathbf{X}^{\prime} \mathbf{Y}_{1} \text { (substitution from equation(3.40)) }
\end{aligned}
$$

Similarly, 


$$
\begin{aligned}
\hat{\mathbf{Y}}_{1}^{\prime} \mathbf{X}_{1}= & \left(\mathbf{Y}_{1}-\mathbf{V}_{1}\right)^{\prime} \mathbf{X}_{1} \quad \text { (substitution from equation (3.43)) } \\
& =\mathbf{Y}_{1}^{\prime} \mathbf{X}_{1} \quad\left(\text { because } \mathbf{X}^{\prime} \mathbf{V}_{1}=\mathbf{0}\right)
\end{aligned}
$$

Now the equation for 2SLS can be written in terms of the actual observations as:

$$
\left[\begin{array}{l}
\hat{\boldsymbol{\beta}}_{1} \\
\hat{\boldsymbol{\gamma}}_{1}
\end{array}\right]_{2 S L S}=\left[\begin{array}{cc}
\mathbf{Y}_{1}^{\prime} \mathbf{X}\left(\mathbf{X}^{\prime} \mathbf{X}\right)^{-1} \mathbf{X}^{\prime} \mathbf{Y}_{1} & \mathbf{Y}_{1}^{\prime} \mathbf{X}_{1} \\
\mathbf{X}_{1}^{\prime} \mathbf{Y}_{1} & \mathbf{X}_{1}^{\prime} \mathbf{X}_{1}
\end{array}\right]^{-1}\left[\begin{array}{c}
\mathbf{Y}_{1}^{\prime} \mathbf{X}\left(\mathbf{X}^{\prime} \mathbf{X}\right)^{-1} \mathbf{X}^{\prime} \mathbf{Y} \\
\mathbf{X}_{1}^{\prime}
\end{array}\right] \mathbf{y}_{1}
$$

Using equations (3.43) and (3.40) $\mathbf{V}_{1}$ can be expressed as:

$$
\begin{aligned}
\mathbf{V}_{1}= & \mathbf{Y}_{1}-\hat{\mathbf{Y}}_{1}^{\prime} \\
& =\mathbf{Y}-\mathbf{X}\left(\mathbf{X}^{\prime} \mathbf{X}\right)^{-1} \mathbf{X}^{\prime} \mathbf{Y}_{1} \\
& =\left[\mathbf{I}-\mathbf{X}\left(\mathbf{X}^{\prime} \mathbf{X}\right)^{-1} \mathbf{X}^{\prime}\right] \mathbf{Y}_{1} \\
& =\mathbf{M} \mathbf{Y}_{1}
\end{aligned}
$$

where $\mathbf{M}$ is both symetric and idemponent

Thus,

$$
\mathbf{X}\left(\mathbf{X}^{\prime} \mathbf{X}\right)^{-1} \mathbf{X}^{\prime}=(\mathbf{I}-\mathbf{M}) \rightarrow \mathbf{M X}=\mathbf{0}
$$

and using this relation the 2 SLS can also be expressed as:

$$
\left[\begin{array}{l}
\hat{\boldsymbol{\beta}}_{1} \\
\hat{\boldsymbol{\gamma}}_{1}
\end{array}\right]_{2 S L S}=\left[\begin{array}{ll}
\mathbf{Y}_{1}^{\prime}(\mathbf{I}-\mathbf{M}) \mathbf{Y}_{1} & \mathbf{Y}_{1}^{\prime}(\mathbf{I}-\mathbf{M}) \mathbf{X}_{1} \\
\mathbf{X}_{1}^{\prime}(\mathbf{I}-\mathbf{M}) \mathbf{Y}_{1} & \mathbf{X}_{1}^{\prime}(\mathbf{I}-\mathbf{M}) \mathbf{X}_{1}
\end{array}\right]^{-1}\left[\begin{array}{r}
\mathbf{Y}_{1}^{\prime}(\mathbf{I}-\mathbf{M}) \mathbf{Y} \\
\mathbf{X}_{1}^{\prime}(\mathbf{I}-\mathbf{M})
\end{array}\right] \mathbf{y}_{1}
$$

Like the OLS estimators, the 2SLS estimators are biased but unlike the former the latter are generally consistent. The source of inconsistent estimators in the case of OLS was the inclusion of $\mathbf{Y}_{1}$ as explanatory variables in the model. Since $\mathbf{Y}_{1}$ are replaced by linear combinations of exogenous variables $\left(\hat{\mathbf{Y}}_{1}\right)$, the explanatory variables in the case of 2SLS are either exogenous $\left(\mathbf{X}_{1}\right)$ or linear combinations of exogenous variables $\left(\hat{\mathbf{Y}}_{1}\right)$ which are not correlated with the disturbance terms, even in the probability limit, that ensure 
consistency. To show this, consider equation (3.36), which may be written, after $\mathbf{Y}_{1}$ are replaced by $\hat{\mathbf{Y}}_{1}$, as:

$$
\begin{aligned}
\mathbf{y}_{1} & =\hat{\mathbf{Y}}_{1} \boldsymbol{\beta}_{1}+\mathbf{X}_{1} \boldsymbol{\gamma}_{1}+\mathbf{u}_{1} \\
& =\hat{\mathbf{Z}}_{1} \boldsymbol{\delta}_{1}+\mathbf{u}_{1}
\end{aligned}
$$

where

$$
\hat{\mathbf{Z}}_{1}=\left[\begin{array}{ll}
\hat{\mathbf{Y}}_{1} & \mathbf{X}_{1}
\end{array}\right] .
$$

The 2SLS estimator is thus

$$
\hat{\boldsymbol{\delta}}_{12 \mathrm{SLS}}=\left[\begin{array}{l}
\hat{\boldsymbol{\beta}}_{1} \\
\hat{\gamma}_{1}
\end{array}\right]_{2 S L S}=\left(\hat{\mathbf{Z}}_{1}^{\prime} \hat{\mathbf{Z}}_{1}\right)^{-1} \hat{\mathbf{Z}}_{1}^{\prime} \mathbf{y}_{1}
$$

Combining equations (3.47) and (3.48) gives:

$$
\hat{\boldsymbol{\delta}}_{12 S L S}=\boldsymbol{\delta}_{1}+\left(\hat{\mathbf{Z}}_{1}^{\prime} \hat{\mathbf{Z}}_{1}\right)^{-1} \hat{\mathbf{Z}}_{1}^{\prime} \mathbf{u}_{1}
$$

Taking expectations

$$
\mathrm{E}\left(\hat{\boldsymbol{\delta}}_{12 S L S}\right)=\boldsymbol{\delta}_{1}+\mathrm{E}\left[\left(\hat{\mathbf{Z}}_{1}^{\prime} \hat{\mathbf{Z}}_{1}\right)^{-1} \hat{\mathbf{Z}}_{1}^{\prime} \mathbf{u}_{1}\right]
$$

Since the second term on the right-hand side of equation (3.49) in general does not vanish, the 2SLS estimator is generally biased. That is:

$$
\mathrm{E}\left(\hat{\boldsymbol{\delta}}_{12 S L S}\right) \neq \boldsymbol{\delta}_{1} .
$$

The 2SLS estimator, however, is consistent which can be proved as:

$$
\begin{aligned}
& \hat{\boldsymbol{\delta}}_{12 S L S}=\boldsymbol{\delta}_{1}+\left(\frac{1}{n} \hat{\mathbf{Z}}_{1}^{\prime} \hat{\mathbf{Z}}_{1}\right)^{-1}\left(\frac{1}{n} \hat{\mathbf{Z}}_{1}^{\prime} \mathbf{u}_{1}\right) \\
& \operatorname{plim} \hat{\boldsymbol{\delta}}_{12 S L S}=\boldsymbol{\delta}_{1}+\operatorname{plim}\left(\frac{1}{n} \hat{\mathbf{Z}}_{1}^{\prime} \hat{\mathbf{Z}}_{1}\right)^{-1} \operatorname{plim}\left(\frac{1}{n} \hat{\mathbf{Z}}_{1}^{\prime} \mathbf{u}_{1}\right) \\
& \operatorname{plim} \hat{\boldsymbol{\delta}}_{12 S L S}=\boldsymbol{\delta}_{1}+\mathbf{Q}^{-1} \operatorname{plim}\left(\frac{1}{n} \hat{\mathbf{Z}}_{1}^{\prime} \mathbf{u}_{1}\right)
\end{aligned}
$$


where

$\operatorname{plim}\left(\frac{1}{n} \hat{\mathbf{Z}}_{1}^{\prime} \hat{\mathbf{Z}}_{1}\right)^{-1}=\mathbf{Q}^{-1}$ is a nonsingular matrix if $\Pi_{1}$ has full column rank, which, in turn, will be true if the equation is identified.

Thus, the 2SLS estimator is consistent if

$$
\operatorname{plim}\left(\frac{1}{n} \hat{\mathbf{Z}}_{1}^{\prime} \mathbf{u}_{1}\right)=\operatorname{plim} \frac{1}{n}\left[\begin{array}{c}
\hat{\mathbf{Y}}_{1}^{\prime} \mathbf{u}_{1} \\
\mathbf{X}_{1}^{\prime} \mathbf{u}_{1}
\end{array}\right]=\mathbf{0}
$$

$\operatorname{plim} \frac{1}{n}\left[\mathbf{X}_{1}^{\prime} \mathbf{u}_{1}\right]=\mathbf{0}$, because $\mathbf{X}_{1}$ are exogenous and hence they are not correlated with the stochastic disturbance term in the probability limit. It can also be shown by direct substitution that $\operatorname{plim} \frac{1}{n}\left[\hat{\mathbf{Y}}_{1}^{\prime} \mathbf{u}_{1}\right]=\mathbf{0}$ as:

$\operatorname{plim} \frac{1}{n}\left[\hat{\mathbf{Y}}_{1}^{\prime} \mathbf{u}_{1}\right]=p \lim \frac{1}{n}\left(\mathbf{Y}_{1}^{\prime} \mathbf{X}\left(\mathbf{X}^{\prime} \mathbf{X}\right)^{-1} \mathbf{X}^{\prime} \mathbf{u}_{1}\right)=\left(\frac{\mathbf{Y}_{1}^{\prime} \mathbf{X}}{n}\right)\left(\frac{\left(\mathbf{X}^{\prime} \mathbf{X}\right)^{-1}}{n}\right)\left(\frac{\mathbf{X}^{\prime} \mathbf{u}_{1}}{n}\right)$

The third part on the right converges to zero, whereas the other two converge to finite matrices and hence their product converges to zero, that is:

$$
\operatorname{plim} \frac{1}{n}\left[\hat{\mathbf{Y}}_{1}^{\prime} \mathbf{u}_{1}\right]=\mathbf{0}
$$

Thus, 2SLS produces consistent estimator

$$
\operatorname{plim} \hat{\boldsymbol{\delta}}_{12 S L S}=\boldsymbol{\delta}_{1} .
$$

The 2SLS estimator can also be treated as an instrumental variable (IV) estimator. Consider once again the $\mathrm{jth}$ structural equation $(\mathrm{j}=1)$, which may be written as

$$
\mathbf{y}_{1}=\mathbf{Y}_{1} \boldsymbol{\beta}_{1}+\mathbf{X}_{1} \boldsymbol{\gamma}_{1}+\mathbf{u}_{1}=\mathbf{Z}_{1} \boldsymbol{\delta}_{1}+\mathbf{u}_{1}
$$

where 


$$
\mathbf{Z}_{1}=\left[\begin{array}{ll}
\mathbf{Y}_{1} & \mathbf{X}_{1}
\end{array}\right] \text { and } \boldsymbol{\delta}_{1}=\left[\begin{array}{l}
\boldsymbol{\beta}_{1} \\
\boldsymbol{\gamma}_{1}
\end{array}\right]
$$

Pre-multiplying equation (3.51) by $\mathbf{Z}_{1}^{\prime}$ gives:

$$
\mathbf{Z}_{1}^{\prime} \mathbf{y}_{1}=\mathbf{Z}_{1}^{\prime} \mathbf{Z}_{1} \boldsymbol{\delta}_{1}+\mathbf{Z}_{1}^{\prime} \mathbf{u}_{1} .
$$

In context of simultaneous equations system, it is not possible to drop the last term from equation (3.52) because the explanatory endogenous variables in $\mathbf{Z}_{1}$ are not statistically independent of $\mathbf{u}_{1}$, even in the probability limit. Suppose, however, that there exists a set of variables (the same number as in $\mathbf{Z}_{1}$ ) that are correlated with $\mathbf{Z}_{1}$ but uncorrelated with $\mathbf{u}_{1}$. Let the data on these instrumental variables be summarized by an $n \times$ (G1 -1 $+\mathrm{K} 1$ ) matrix $\mathbf{D}_{1}$, where $\mathbf{D}_{1}$ is assumed to satisfy the requirements for an IV estimator,

$$
\left\{\begin{array}{ll}
\operatorname{plim}\left(\frac{1}{n} \mathbf{D}_{1}^{\prime} \mathbf{D}_{1}\right)=\boldsymbol{\Sigma}_{D D} & \text { a finite symetric positive definite matrix } \\
\operatorname{plim}\left(\frac{1}{\mathrm{n}} \mathbf{D}_{1}^{\prime} \mathbf{Z}_{1}\right)=\boldsymbol{\Sigma}_{D Z} & \text { a finite nonsingular matrix } \\
\operatorname{plim}\left(\frac{1}{\mathrm{n}} \mathbf{D}_{1}^{\prime} \mathbf{u}_{1}\right)=\mathbf{0} &
\end{array}\right\}
$$

Then pre-multiplying equation (3.51) by the transpose of this matrix gives:

$$
\mathbf{D}_{1}^{\prime} \mathbf{y}_{1}=\mathbf{D}_{1}^{\prime} \mathbf{Z}_{1} \boldsymbol{\delta}_{1}+\mathbf{D}_{1}^{\prime} \mathbf{u}_{1}
$$

Since it is assumed that the instrumental variables are uncorrelated with the disturbance term, the last term from equation (3.54) can be dropped and the resulting equation can be solved for the instrumental variable estimator as:

$$
\hat{\boldsymbol{\delta}}_{1 \mathrm{IV}}=\left(\mathbf{D}_{1}^{\prime} \mathbf{Z}_{1}\right)^{-1} \mathbf{D}_{1}^{\prime} \mathbf{y}_{1} .
$$


Note that the instrumental variable estimator is a function of the matrix of data on the instrumental variables $\left(\hat{\boldsymbol{\delta}}_{1}\left(\mathbf{D}_{1}\right)\right)$, and hence it depends on the choice of the instruments and the data on these instruments. As discussed above, in the case of 2SLS method the explanatory endogenous variables are replaced by their estimated values $\hat{\mathbf{Y}}_{1}$, which can serve as instrumental variable. Thus, since the included exogenous variables $\mathbf{X}_{1}$ can be used as their own instrumental variables, the choice of instrumental variables for 2SLS is given by $\hat{\mathbf{Z}}_{1}=\left[\begin{array}{ll}\hat{\mathbf{Y}}_{1} & \mathbf{X}_{1}\end{array}\right]$. Thus, in the case of 2SLS approach, $\mathbf{D}_{1}=\left[\begin{array}{ll}\hat{\mathbf{Y}}_{1} & \mathbf{X}_{1}\end{array}\right]=\hat{\mathbf{Z}}_{1}$. Then substituting $\mathbf{D}_{1}$ by $\left[\begin{array}{ll}\hat{\mathbf{Y}}_{1} & \mathbf{X}_{1}\end{array}\right]$ and $\mathbf{Z}_{1}$ by $\left[\begin{array}{ll}\mathbf{Y}_{1} & \mathbf{X}_{1}\end{array}\right]$ in equation (3.54) yields:

$$
\begin{aligned}
& \hat{\boldsymbol{\delta}}_{1 \mathrm{IV}}=\left(\left[\begin{array}{ll}
\hat{\mathbf{Y}}_{1} & \mathbf{X}_{1}
\end{array}\right]^{\prime}\left[\begin{array}{ll}
\mathbf{Y}_{1} & \mathbf{X}_{1}
\end{array}\right]\right)^{-1}\left[\begin{array}{ll}
\hat{\mathbf{Y}}_{1} & \mathbf{X}_{1}
\end{array}\right]^{\prime} \mathbf{y}_{1} \\
& {\left[\begin{array}{l}
\hat{\boldsymbol{\beta}}_{1} \\
\hat{\boldsymbol{\gamma}}_{1}
\end{array}\right]_{I V}=\left[\begin{array}{ll}
\hat{\mathbf{Y}}_{1}^{\prime} \mathbf{Y}_{1} & \hat{\mathbf{Y}}_{1}^{\prime} \mathbf{X}_{1} \\
\mathbf{X}_{1}^{\prime} \mathbf{Y}_{1} & \mathbf{X}_{1}^{\prime} \mathbf{X}_{1}
\end{array}\right]^{-1}\left[\begin{array}{l}
\hat{\mathbf{Y}}_{1}^{\prime} \\
\mathbf{X}_{1}^{\prime}
\end{array}\right] \mathbf{y}_{1}}
\end{aligned}
$$

By the proof from equation (3.43), however, $\hat{\mathbf{Y}}_{1}^{\prime} \mathbf{Y}_{1}=\hat{\mathbf{Y}}_{1}^{\prime} \hat{\mathbf{Y}}_{1}$ and $\hat{\mathbf{Y}}_{1}^{\prime} \mathbf{X}_{1}=\mathbf{Y}_{1} \mathbf{X}_{1}$. Thus equations (3.56a) and (3.41) are identical, which indicates that 2SLS is in fact an IV estimator with $\hat{\mathbf{Y}}_{1}$ as the instrument for $\mathbf{Y}_{1}$.

Using the IV approach, it is possible to develop another useful formulation for 2SLS estimator. To do this notes that:

$$
\hat{\mathbf{Y}}_{1}=\mathbf{X}\left(\mathbf{X}^{\prime} \mathbf{X}\right)^{-1} \mathbf{X}^{\prime} \mathbf{Y}_{1} \text { (from equation (3.40)) }
$$

and that $\mathbf{X}_{1}=\mathbf{X}\left(\mathbf{X}^{\prime} \mathbf{X}\right)^{-1} \mathbf{X}^{\prime} \mathbf{X}_{1}$ (derived by dropping $\mathbf{X}_{1}^{*}$ from the first and last expression of the following relations:

$$
\left.\left[\begin{array}{ll}
\mathbf{X}_{1} & \mathbf{X}_{1}^{*}
\end{array}\right]=\mathbf{X}=\mathbf{X}\left(\mathbf{X}^{\prime} \mathbf{X}\right)^{-1} \mathbf{X}^{\prime} \mathbf{X}=\mathbf{X}\left(\mathbf{X}^{\prime} \mathbf{X}\right)^{-1} \mathbf{X}^{\prime}\left[\begin{array}{ll}
\mathbf{X}_{1} & \mathbf{X}_{1}^{*}
\end{array}\right]\right) .
$$


These two equalities can be combined to give:

$$
\mathbf{D}_{1}=\hat{\mathbf{Z}}_{1}=\left[\begin{array}{ll}
\hat{\mathbf{Y}}_{1} & \mathbf{X}_{1}
\end{array}\right]=\mathbf{X}\left(\mathbf{X}^{\prime} \mathbf{X}\right)^{-1} \mathbf{X}^{\prime}\left[\begin{array}{ll}
\mathbf{Y}_{1} & \mathbf{X}_{1}
\end{array}\right]=\mathbf{X}\left(\mathbf{X}^{\prime} \mathbf{X}\right)^{-1} \mathbf{X}^{\prime} \mathbf{Z}_{1}
$$

which is the matrix of estimated values obtained from a regression of all explanatory variables $\mathbf{Z}_{1}$ (both endogenous and exogenous) on all exogenous variables $\mathbf{X}$. As this is the instrumental variables data matrix used in 2SLS, the 2SLS estimator can be expressed as

$$
\hat{\boldsymbol{\delta}}_{12 S L S}=\left[\mathbf{Z}_{1}^{\prime} \mathbf{X}\left(\mathbf{X}^{\prime} \mathbf{X}\right)^{-1} \mathbf{X}^{\prime}\right]^{-1} \mathbf{Z}_{1}^{\prime} \mathbf{X}\left(\mathbf{X}^{\prime} \mathbf{X}\right)^{-1} \mathbf{X}^{\prime} \mathbf{y}_{1}
$$

The expression for the 2SLS estimator in equation (3.56b) involves only actual data matrices. In this respect, the 2SLS estimator can be interpreted as the estimator that resulted from a procedure when all the exogenous variables are used as instrumental variable and the GLS estimator is used. Thus, pre-multiplying equation (3.51) by $\mathbf{X}^{\prime}$ :

$$
\mathbf{X}^{\prime} \mathbf{y}_{1}=\mathbf{X}^{\prime} \mathbf{Z}_{1} \boldsymbol{\delta}_{1}+\mathbf{X}^{\prime} \mathbf{u}_{1}
$$

where

$$
\operatorname{Var}-\operatorname{Cov}\left(\mathbf{X}^{\prime} \mathbf{u}_{1}\right)=\mathrm{E}\left(\mathbf{X}^{\prime} \mathbf{u}_{1} \mathbf{u}_{1}^{\prime} \mathbf{X}\right)=\sigma_{1}^{2}\left(\mathbf{X}^{\prime} \mathbf{X}\right)
$$

Using the inverse of this variance-covariance matrix for the GLS estimator gives:

$$
\hat{\boldsymbol{\delta}}_{12 S L S}=\left[\mathbf{Z}_{1}^{\prime} \mathbf{X}\left(\mathbf{X}^{\prime} \mathbf{X}\right)^{-1} \mathbf{X}^{\prime}\right]^{-1} \mathbf{Z}_{1}^{\prime} \mathbf{X}\left(\mathbf{X}^{\prime} \mathbf{X}\right)^{-1} \mathbf{X}^{\prime} \mathbf{y}_{1}
$$

As the expressions in equation (3.56c) and (3.56b) are identical, the 2SLS estimator has the additional interpretation as the GLS estimator of the equation after having used all exogenous variables as instrumental variables. 
For the 2SLS instrumental variables (IV) estimator to be consistent, the three conditions in equation (3.53) should be fulfilled. The first two conditions are generally assumed will be fulfilled. The third condition can be given as:

$$
\operatorname{plim}\left(\frac{1}{n} \mathbf{D}_{1}^{\prime} \mathbf{u}_{1}\right)=\operatorname{plim}\left[\begin{array}{l}
\operatorname{plim}\left(\frac{1}{n} \hat{\mathbf{Y}}_{1}^{\prime} \mathbf{u}_{1}\right) \\
\operatorname{plim}\left(\frac{1}{n} \mathbf{X}_{1}^{\prime} \mathbf{u}_{1}\right)
\end{array}\right]=\mathbf{0}
$$

The proof for equation (3.57) is given in equation (3.50). Thus, the 2SLS estimator which is an IV estimator will be consistent and have asymptotic covariance matrix:

$$
\begin{aligned}
\operatorname{Asy} \cdot \operatorname{Var}\left(\hat{\mathbf{\delta}}_{12 \mathrm{SL}(\mathrm{IV})}\right)= & \frac{\boldsymbol{\sigma}_{1}^{2}}{n} \operatorname{plim}\left[\left(\frac{1}{n} \mathbf{D}_{1}^{\prime} \mathbf{Z}_{1}\right)\left(\frac{1}{n} \mathbf{D}_{1}^{\prime} \mathbf{D}_{1}\right)^{-1}\left(\frac{1}{n} \mathbf{Z}_{1}^{\prime} \mathbf{D}_{1}\right)\right]^{-1} \\
& =\frac{\boldsymbol{\sigma}_{1}^{2}}{n}\left[\boldsymbol{\Sigma}_{\mathrm{DZ}}^{-1} \boldsymbol{\Sigma}_{\mathrm{DD}} \boldsymbol{\Sigma}_{\mathrm{ZD}}^{-1}\right] .
\end{aligned}
$$

The variance $\boldsymbol{\sigma}_{1}^{2}$ can be consistently estimated as:

$$
\hat{\boldsymbol{\sigma}}_{1}^{2}=\frac{1}{n} \hat{\mathbf{u}}_{1}^{\prime} \hat{\mathbf{u}}_{1}=\frac{1}{n}\left(\mathbf{y}_{1}-\mathbf{Z}_{1} \hat{\boldsymbol{\delta}}_{12 \mathrm{SLS}(\mathrm{IV})}\right)^{\prime}\left(\mathbf{y}_{1}-\mathbf{Z}_{1} \hat{\boldsymbol{\delta}}_{12 \mathrm{SLS}(\mathrm{IV})}\right)
$$

A degrees of freedom of correction for the denominator, $\mathrm{n}-\mathrm{G} 1-\mathrm{K} 1+1$, is some times suggested. Asymptotically, the correction is immaterial, although its beneficial in small samples remains to be settled (Greene, 2003). The resulting estimator, although is not unbiased, as it would be in the classical regression model, is consistent and asymptotically distributed with the estimated variance matrix given in equation (3.58) after $\boldsymbol{\sigma}_{1}^{2}$ is replaced by its estimated value, $\hat{\boldsymbol{\sigma}}_{1}^{2}$, from equation (3.59). This estimator can be used to construct asymptotic tests of hypotheses and interval estimates for structural parameters. While these tests apply asymptotically, finite-sample approximations are 
frequently used in applications of the 2SLS approach for given $n, \mathbf{D}_{1}$ and $\mathbf{Z}_{1}$ in equation (3.58) and with $\boldsymbol{\sigma}_{1}^{2}$ estimated by $\hat{\boldsymbol{\sigma}}_{1}^{2}$ in equation (3.59).

Application of 2SLS approach to equation in medium-size and large-size econometric models may some times be difficult. The difficulty arises because the number of predetermined variables in such models may become large relative to the number of observations. Suppose, for example, $\mathrm{K}=\mathrm{n}$, that is, the number of predetermined/ exogenous variables becomes as large as the number of observations. Then, the $\mathbf{X}$ matrix becomes square and in the absence of exact linear relations between the predetermined variables, it is nonsingular. In that case, the relation in equation (3.40) reduces to:

$$
\begin{aligned}
\hat{\mathbf{Y}}_{1} & =\mathbf{X}\left(\mathbf{X}^{\prime} \mathbf{X}\right)^{-1} \mathbf{X}^{\prime} \mathbf{Y}_{1} \\
& =\mathbf{X X}^{-1}\left(\mathbf{X}^{\prime}\right)^{-1} \mathbf{X}^{\prime} \mathbf{Y}_{1} \\
& =\mathbf{Y}_{1}
\end{aligned}
$$

This result would imply that 2SLS is equivalent to OLS. The consistency requirement is not fulfilled since the matrix of the instrumental variables is now $\mathbf{D}_{1}=\left[\begin{array}{ll}\mathbf{Y}_{1} & \mathbf{X}_{1}\end{array}\right]$ and $\operatorname{plim} \frac{1}{n}\left[\hat{\mathbf{Y}}_{1}^{\prime} \mathbf{u}_{1}\right] \neq \mathbf{0}$ which leads to $\operatorname{plim}\left(\frac{1}{n} \mathbf{D}_{1}^{\prime} \mathbf{u}_{1}\right) \neq \mathbf{0}$. Besides, when $\mathrm{K}>\mathrm{n}$, the $\mathbf{X}^{\prime} \mathbf{X}$ matrix is of the order $\mathrm{K} \times \mathrm{K}$ and of rank $\mathrm{n}$. Thus, it is singular and the inverse $\left(\mathbf{X}^{\prime} \mathbf{X}\right)^{-1}$ does not exist.

Moreover, although the 2SLS estimator is asymptotically efficient with the class of all estimators that use the same a priori restrictions for a single equation, but it is not 
asymptotically efficient relative to the full-information technique of three-stage least squares (Intriligator, 1978). The three-stage least squares technique is discussed later in this chapter.

\subsubsection{System of Equations Method}

The estimators considered so far are essentially limited-information estimators in the sense that complete information on all the other structural equations in the model is not taken into account in the estimation of each structural equation separately. System of equations method estimates all the identified structural equations together as a set, instead of estimating the structural equations of each equation separately. These methods are also called full-information methods because they utilize knowledge of all the restriction in the entire system when estimating the structural parameters. In principle information on the complete structure, if correct, will yield estimators with greater asymptotic efficiency (smaller asymptotic variance-covariance matrix) than that attainable by single-equation (limited-information) methods. There are three major systems of equations (fullinformation) methods: three-stage least squares (3SLS), full-information maximum likelihood (FIML), and generalized method of moments (GMM).

\subsubsection{Three-Stage Least Squares}

The three-stage least squares (3SLS), as its name implies, can be computed in three stages and since the first two stages are those of the 2SLS, it is considered as an extension of the 2SLS. Actually, the structure of 3SLS is based on an alternative interpretation of 2SLS: Consider, for example, a single equation of the model. If this single equation is multiplied through by a matrix of observations on all the exogenous variables in the model, applying the generalized-least-squares (GLS) to this new 
transformed relationship creates the 2SLS estimates. Now if all the equations in the model are transformed in this way, stacked one on top of the other and then this stack is rewritten as a single, very large equation, applying GLS to this very giant equation should produce 2SLS estimates of each of the component equations. Note, however, that these estimates can be different from the standard 2SLS estimates because the variancecovariance matrix of this giant equation may not be diagonal (the off-diagonal elements do not vanish), and hence the asymptotic efficiency of the 2SLS estimates the giant equation can be improved by taking explicit account of the inter-equation correlations. This improvement in asymptotic efficiency is incorporated in the 3SLS estimator. The initial development of the 3SLS is based on the works of Zellner and Theil (1962).

In order to develop the 3SLS estimator, consider again the general linear model containing $\mathrm{G}$ jointly dependent endogenous variables and $\mathrm{K}$ predetermined variables given in equation (3.19):

$$
\underset{n \times G}{\mathbf{Y}} \underset{G \times G}{\mathbf{B}}+\underset{n \times K}{\mathbf{X}} \underset{K x G}{\boldsymbol{\Gamma}}=\underset{n \times G}{\mathbf{U}}
$$

The jth equation, which is also given in equation (3.20) can be written as:

$$
\mathbf{y}_{j}=\mathbf{Y}_{j} \boldsymbol{\beta}_{j}+\mathbf{X}_{j} \boldsymbol{\gamma}_{j}+\mathbf{u}_{j}, \quad j=1,2, \ldots G
$$

where $\mathbf{y}_{j}$ is $\mathrm{n} \times 1$ column vector of data on the dependent endogenous variable (the one on which this equation has been normalized), $\mathbf{Y}_{j}$ is the $\mathrm{n} \times(\mathrm{G} 1-1)$ matrix of data on the G1 - 1 include right-hand side endogenous variable, $\boldsymbol{\beta}_{j}$ is $(\mathrm{G} 1-1) \times 1$ column vector of non-zero coefficients on $\mathbf{Y}_{j} \mathbf{X}_{j}$ is $\mathrm{n} \times \mathrm{K} 1$ matrix of observation on the predetermined variables, $\boldsymbol{\gamma}_{j}$ is $\mathrm{K} 1 \mathrm{x} 1$ column vector of coefficients on $\mathbf{X}_{j}$, and $\mathbf{u}_{j}$ is $\mathrm{n} \times 1$ vector of disturbance terms. Rewriting equation (3.60): 


$$
\mathbf{y}_{j}=\mathbf{Z}_{j}^{\prime} \boldsymbol{\delta}_{j}+\mathbf{u}_{j}
$$

where

$$
\mathbf{Z}_{j}=\left[\begin{array}{ll}
\mathbf{Y}_{j} & \mathbf{X}_{j}
\end{array}\right] \text { and } \quad \boldsymbol{\delta}_{j}=\left[\begin{array}{c}
\boldsymbol{\beta}_{j} \\
\boldsymbol{\gamma}_{j}
\end{array}\right]
$$

Thus all $\mathrm{G}$ equations of the system can be written as:

$$
\underset{\operatorname{Gn} \times 1}{\mathbf{y}}=\underset{\operatorname{Gn} \times K^{\oplus} K^{\oplus} \times 1}{\mathbf{\delta}}+\underset{G n \times 1}{\mathbf{u}},
$$

with the following stacked vectors and matrix

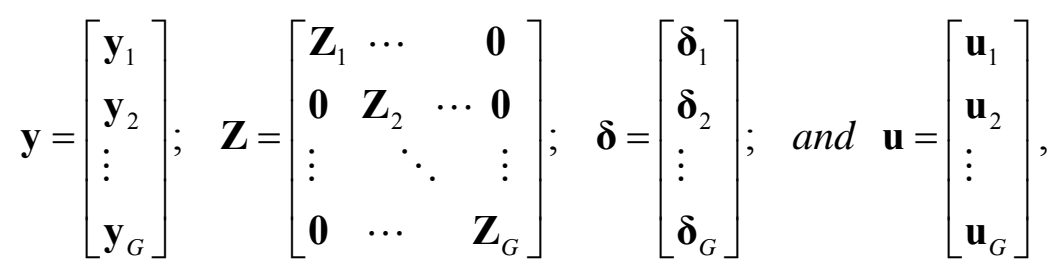

where $\mathrm{K}^{\oplus}=\sum_{j=1}^{G}\left(G 1_{j}-1+K 1_{j}\right)$ is the total number of parameters to be estimated.

The assumptions on the stochastic disturbance term for simultaneous equations system can be stated as:

$$
\begin{aligned}
& \mathrm{E}(\mathbf{u})=\mathbf{0} \\
& \operatorname{Cov}(\mathbf{u})=\mathrm{E}\left(\mathbf{u u}^{\prime}\right)=\left[\begin{array}{llll}
\boldsymbol{\sigma}_{11} \mathbf{I}_{n} & \boldsymbol{\sigma}_{12} \mathbf{I}_{n} & \cdots & \boldsymbol{\sigma}_{1 G} \mathbf{I}_{n} \\
\boldsymbol{\sigma}_{21} \mathbf{I}_{n} & \boldsymbol{\sigma}_{22} \mathbf{I}_{n} \cdots & \boldsymbol{\sigma}_{2 G} \mathbf{I}_{n} \\
\vdots & \vdots & & \vdots \\
\boldsymbol{\sigma}_{G 1} \mathbf{I}_{n} & \boldsymbol{\sigma}_{G 2} \mathbf{I}_{n} & \cdots & \boldsymbol{\sigma}_{G G} \mathbf{I}_{\mathrm{n}}
\end{array}\right]=\boldsymbol{\Sigma} \otimes \mathbf{I}_{n},
\end{aligned}
$$

where $\boldsymbol{\Sigma} \otimes \mathbf{I}_{n}$ is the Kronecker product of these matrices.

In equation (3.63), the variance-covariance matrix has been partitioned into blocks, where each block is a diagonal matrix made up of an element of the $\boldsymbol{\Sigma}$ matrix times the $\mathrm{n} \times \mathrm{n}$ identity matrix. These diagonal matrices reflect the independence among non-contemporaneous disturbances. The elements in the $\boldsymbol{\Sigma}$ matrix, however, show the 
possible correlation among contemporaneous disturbances. The zero off-diagonal elements in the $\boldsymbol{\sigma}_{22} \mathbf{I}_{n}$ matrix, for example, indicate the assumption of absence of correlation between the disturbance terms of the second equation at different observations, while the equal diagonal elements of this matrix (block) shows the constant variance of the disturbance terms of the second equation. Similarly, $\boldsymbol{\sigma}_{21} \mathbf{I}_{n}$ is a diagonal matrix which refers to the second and the first equation, where its equal diagonal elements indicate the assumption of constant variance at corresponding observation for the second and first equations, while the zero off-diagonal elements show the assumption that the disturbance terms of the respective equations are uncorrelated at different observations. Note that the Kronecker product reduces to $\boldsymbol{\sigma}_{1}^{2} \mathbf{I}$, which is the covariance matrix, in the case of single equation $(\mathrm{G}=1)$.

In equation (3.56c) it was shown that 2SLS is equivalent to using all exogenous variables as instrumental variables and estimating the resulting equation using GLS. Similarly, the 3SLS estimator is a GLS estimator of the entire system in equation (3.62) that takes explicit account of the variance-covariance matrix in equation (3.63). Thus, the equation to be estimated is obtained by pre-multiplying equation (3.62) by $\mathbf{X}^{\oplus \prime}$, where the matrix $\mathbf{X}^{\oplus^{\prime}}$ is defined by:

$$
\underset{\mathrm{GK}^{\prime} \mathrm{Gn}}{\mathbf{X}^{\prime}}=\left[\begin{array}{lccr}
\mathbf{X}^{\prime} & \mathbf{0} & \cdots & \mathbf{0} \\
\mathbf{0} & \mathbf{X}^{\prime} & \cdots & \mathbf{0} \\
\vdots & \vdots & & \vdots \\
\mathbf{0} & \mathbf{0} & \cdots & \mathbf{X}^{\prime}
\end{array}\right]=\underset{\mathrm{G} \times \mathrm{G}}{\mathbf{I}} \otimes \underset{\mathrm{K} \times \mathrm{Xn}}{\mathbf{X}^{\prime}}
$$

The resulting expression is written as:

$$
\mathbf{X}^{\oplus \prime} \mathbf{y}=\mathbf{X}^{\oplus \prime} \mathbf{Z} \boldsymbol{\delta}+\mathbf{X}^{\oplus \prime} \mathbf{u}
$$


This is equivalent to the system obtained by pre-multiplying each equation by $\mathbf{X}^{\prime}$, using all exogenous variables as instrumental variables in each equation and hence the GLS estimator of this equation is the 3SLS estimator which can be written as

$$
\begin{aligned}
\hat{\boldsymbol{\delta}}_{3 S L S} & =\left[\mathbf{Z}^{\prime} \mathbf{X}^{\oplus}\left\{\operatorname{Cov}\left(\mathbf{X}^{\oplus^{\prime}} \mathbf{u}\right)\right\}^{-1} \mathbf{X}^{\oplus} \mathbf{Z}\right]^{-1} \mathbf{Z}^{\prime} \mathbf{X}^{\oplus}\left\{\operatorname{Cov}\left(\mathbf{X}^{\oplus^{\prime}} \mathbf{u}\right)\right\}^{-1} \mathbf{X}^{\oplus \prime} \mathbf{y} \\
& =\left[\mathbf{Z}^{\prime} \mathbf{X}^{\oplus}\left\{\mathbf{X}^{\oplus^{\prime}}(\mathbf{\Sigma} \otimes \mathbf{I}) \mathbf{X}^{\oplus}\right\}^{-1} \mathbf{X}^{\oplus} \mathbf{Z}\right]^{-1} \mathbf{Z}^{\prime} \mathbf{X}^{\oplus}\left\{\mathbf{X}^{\oplus^{\prime}}(\mathbf{\Sigma} \otimes \mathbf{I}) \mathbf{X}^{\oplus}\right\}^{-1} \mathbf{X}^{\oplus^{\prime}} \mathbf{y}
\end{aligned}
$$

Note that the $\mathbf{X}^{\oplus \prime}(\boldsymbol{\Sigma} \otimes \mathbf{I}) \mathbf{X}^{\oplus}$ is derived from the expression for variance-covariance matrix given equation (3.63) as follows:

$$
\operatorname{Cov}\left(\mathbf{X}^{\oplus \prime} \mathbf{u}\right)=\mathbf{X}^{\oplus \prime} \operatorname{Cov}(\mathbf{u}) \mathbf{X}^{\oplus}=\mathbf{X}^{\oplus \prime}(\mathbf{\Sigma} \otimes \mathbf{I}) \mathbf{X}^{\oplus}
$$

and substituting $\mathbf{X}^{\oplus^{\prime}}=\mathbf{I} \otimes \mathbf{X}^{\prime}$ from equation (3.64) into this expression also gives:

$$
\operatorname{Cov}\left(\mathbf{X}^{\oplus^{\prime}} \mathbf{u}\right)=\mathbf{X}^{\oplus^{\prime}}(\mathbf{\Sigma} \otimes \mathbf{I}) \mathbf{X}^{\oplus}=\left(\mathbf{I} \otimes \mathbf{X}^{\prime}\right)(\mathbf{\Sigma} \otimes \mathbf{I})\left(\mathbf{I} \otimes \mathbf{X}^{\prime}\right)=\mathbf{\Sigma} \otimes\left(\mathbf{X}^{\prime} \mathbf{X}\right)
$$

Combining equations (3.65) and (3.66), the 3SLS estimator can be written as:

$$
\begin{aligned}
\hat{\boldsymbol{\delta}}_{3 \text { sLS }} & =\left[\mathbf{Z}^{\prime} \mathbf{X}^{\oplus}\left\{\mathbf{\Sigma} \otimes\left(\mathbf{X}^{\prime} \mathbf{X}\right)\right\}^{-1} \mathbf{X}^{\oplus} \mathbf{Z}\right]^{-1} \mathbf{Z}^{\prime} \mathbf{X}^{\oplus}\left\{\boldsymbol{\Sigma} \otimes\left(\mathbf{X}^{\prime} \mathbf{X}\right)\right\}^{-1} \mathbf{X}^{\oplus} \mathbf{y} \\
& =\left[\mathbf{Z}^{\prime} \mathbf{X}^{\oplus}\left\{\boldsymbol{\Sigma}^{-1} \otimes\left(\mathbf{X}^{\prime} \mathbf{X}\right)^{-1}\right\} \mathbf{X}^{\oplus} \mathbf{Z}\right]^{-1} \mathbf{Z}^{\prime} \mathbf{X}^{\oplus}\left\{\mathbf{\Sigma}^{-1} \otimes\left(\mathbf{X}^{\prime} \mathbf{X}\right)^{-1}\right\} \mathbf{X}^{\oplus} \mathbf{y} \\
& =\left[\mathbf{Z}^{\prime}\left(\mathbf{I} \otimes \mathbf{X}^{\prime}\right)\left\{\mathbf{\Sigma}^{-1} \otimes\left(\mathbf{X}^{\prime} \mathbf{X}\right)^{-1}\right\}\left(\mathbf{I} \otimes \mathbf{X}^{\prime}\right) \mathbf{Z}\right]^{-1} \mathbf{Z}^{\prime}\left(\mathbf{I} \otimes \mathbf{X}^{\prime}\right)\left\{\mathbf{\Sigma}^{-1} \otimes\left(\mathbf{X}^{\prime} \mathbf{X}\right)^{-1}\right\}\left(\mathbf{I} \otimes \mathbf{X}^{\prime}\right) \mathbf{y} \\
& =\left[\mathbf{Z}^{\prime}\left\{\boldsymbol{\Sigma}^{-1} \otimes \mathbf{X}\left(\mathbf{X}^{\prime} \mathbf{X}\right)^{-1} \mathbf{X}^{\prime}\right\} \mathbf{Z}\right]^{-1} \mathbf{Z}^{\prime}\left\{\boldsymbol{\Sigma}^{-1} \otimes \mathbf{X}\left(\mathbf{X}^{\prime} \mathbf{X}\right)^{-1} \mathbf{X}^{\prime}\right\} \mathbf{y}
\end{aligned}
$$

where the second equality uses $\left\{\boldsymbol{\Sigma} \otimes\left(\mathbf{X}^{\prime} \mathbf{X}\right)\right\}^{-1}=\boldsymbol{\Sigma}^{-1} \otimes\left(\mathbf{X}^{\prime} \mathbf{X}\right)^{-1}$ and the fourth equality uses $(\mathbf{A} \otimes \mathbf{B})(\mathbf{C} \otimes \mathbf{D})(\mathbf{E} \otimes \mathbf{F})=(\mathbf{A C E}) \otimes(\mathbf{B D F})$. 
Note that all the components of the 3SLS estimator, as expressed in the fourth equality in equation (3.67), other than the covariance matrix are obtained directly from the data. The remaining difficulty is to obtain and estimate the covariance matrix $(\boldsymbol{\Sigma})$. For efficient estimation any consistent estimation of $\boldsymbol{\Sigma}$ will do. Zellner and Theil (1962), the designers of the 3SLS, suggest the natural choice arising out of the 2SLS estimates. This would mean that the result of the first two stages, the 2SLS estimates, yields the information, in the residuals, needed to estimate the covariance matrix. In this respect, $\boldsymbol{\Sigma}$ is estimated as:

$$
\begin{gathered}
\hat{\boldsymbol{\Sigma}}=\left(\hat{\sigma}_{j l}\right) \text { where } \hat{\sigma}_{j l}=\frac{1}{n} \hat{\mathbf{u}}_{j}^{\prime} \hat{\mathbf{u}}_{l}=\frac{1}{n}\left(\mathbf{y}_{j}-\mathbf{Z} \boldsymbol{\delta}_{j 2 S L S}\right)^{\prime}\left(\mathbf{y}_{l}-\mathbf{Z} \boldsymbol{\delta}_{l 2 S L S}\right), \\
j, l=1,2, \ldots, G
\end{gathered}
$$

By replacing $\boldsymbol{\Sigma}$ by $\hat{\boldsymbol{\Sigma}}$ in equation (3.67), the 3SLS estimator can thus be given as:

$$
\hat{\boldsymbol{\delta}}_{3 S L S}=\left[\mathbf{Z}^{\prime}\left\{\hat{\boldsymbol{\Sigma}}^{-1} \otimes \mathbf{X}\left(\mathbf{X}^{\prime} \mathbf{X}\right)^{-1} \mathbf{X}^{\prime}\right\} \mathbf{Z}\right]^{-1} \mathbf{Z}^{\prime}\left\{\hat{\boldsymbol{\Sigma}}^{-1} \otimes \mathbf{X}\left(\mathbf{X}^{\prime} \mathbf{X}\right)^{-1} \mathbf{X}^{\prime}\right\} \mathbf{y}
$$

This estimator is obtained by using all predetermined/exogenous variables as instrumental variable and then applying GLS to the whole system, where the 2SLS estimates are used to obtain the estimate for the relevant covariance matrix. The three stages of the three-stage least squares are thus defined as follows:

1. Estimate $\Pi$ by ordinary least squares (OLS) and compute $\hat{\mathbf{Y}}_{j}$ for each equation.

2. Compute $\hat{\boldsymbol{\delta}}_{3 S L S}$ for each equation; then

$$
\hat{\sigma}_{j l}=\frac{1}{n} \hat{\mathbf{u}}_{j}^{\prime} \hat{\mathbf{u}}_{l}=\frac{1}{n}\left(\mathbf{y}_{j}-\mathbf{Z} \boldsymbol{\delta}_{j 2 S L S}\right)^{\prime}\left(\mathbf{y}_{l}-\mathbf{Z} \boldsymbol{\delta}_{l 2 S L S}\right)
$$

3. Compute the GLS estimator according to equation (3.69). 
Like the 2SLS estimator, the 3SLS estimator can also be given an instrumental variable (IV) interpretation. In this case, $\mathbf{D}$, the matrix of instrumental variable will be:

$$
\mathbf{D}=\left[\mathbf{\Sigma}^{-1} \otimes \mathbf{X}\left(\mathbf{X}^{\prime} \mathbf{X}\right)^{-1} \mathbf{X}^{\prime}\right] \mathbf{Z}
$$

Substituting this in equation (3.69) gives:

$$
\hat{\boldsymbol{\delta}}_{3 S L S(\mathrm{IV})}=\left[\mathbf{Z}^{\prime} \mathbf{D}\right]^{-1} \mathbf{D}^{\prime} \mathbf{y}=\left[\mathbf{D}^{\prime} \mathbf{Z}\right]^{-1} \mathbf{D}^{\prime}(\mathbf{Z} \boldsymbol{\delta}+\mathbf{u})=\boldsymbol{\delta}+\left[\mathbf{D}^{\prime} \mathbf{Z}\right]^{-1} \mathbf{D}^{\prime} \mathbf{u}
$$

so that

$$
\operatorname{plim} \hat{\boldsymbol{\delta}}_{3 S L S(\mathrm{IV})}=\boldsymbol{\delta}+\operatorname{plim}\left[\frac{1}{n} \mathbf{D}^{\prime} \mathbf{Z}\right]^{-1} \operatorname{plim}\left(\frac{1}{n} \mathbf{D}^{\prime} \mathbf{u}\right)
$$

Assuming the instrumental variables are asymptotically correlated with $\mathbf{Z}$ but uncorrelated with the stochastic error terms, then:

$$
\operatorname{plim}\left[\frac{1}{n} \mathbf{D}^{\prime} \mathbf{Z}\right]^{-1}=\mathbf{Q}^{-1}
$$

where $\mathbf{Q}$ exists and is nonsingular matrix

and

$$
\operatorname{plim}\left(\frac{1}{n} \mathbf{D}^{\prime} \mathbf{u}\right)=\mathbf{0}
$$

Thus

$$
\operatorname{plim} \hat{\boldsymbol{\delta}}_{3 S L S(\mathrm{IV})}=\boldsymbol{\delta}
$$


This shows that the 3SLS estimator is consistent estimator.

To derive the asymptotic covariance for the 3SLS consider equation (3.70) which can be rewritten as:

$$
\hat{\boldsymbol{\delta}}_{3 S L S(\mathrm{IV})}-\boldsymbol{\delta}=\left[\mathbf{D}^{\prime} \mathbf{Z}\right]^{-1} \mathbf{D}^{\prime} \mathbf{u}
$$

The asymptotic covariance for is given by:

$$
\begin{aligned}
\operatorname{Asy} \cdot \operatorname{Var}\left(\hat{\boldsymbol{\delta}}_{3 S L S}\right)=\lim \operatorname{Cov}\left(\hat{\boldsymbol{\delta}}_{3 S L S}\right)= & \frac{1}{n} \operatorname{plim}\left[n\left(\hat{\boldsymbol{\delta}}_{3 S L S}-\boldsymbol{\delta}_{3 S L S}\right)\left(\hat{\boldsymbol{\delta}}_{3 S L S}-\boldsymbol{\delta}_{3 S L S}\right)^{\prime}\right] \\
& =\frac{1}{n} \operatorname{plim}\left[\left\{\sqrt{n}\left(\hat{\boldsymbol{\delta}}_{3 S L S}-\boldsymbol{\delta}_{3 S L S}\right)\right\}\left\{\sqrt{n}\left(\hat{\boldsymbol{\delta}}_{3 S L S}-\boldsymbol{\delta}_{3 S L S}\right)\right\}^{\prime}\right] \\
& =\frac{1}{n} \operatorname{plim}\left[\left\{\sqrt{n}\left[\mathbf{D}^{\prime} \mathbf{Z}\right]^{-1} \mathbf{D}^{\prime} \mathbf{u}\right\}\left\{\sqrt{n}\left[\mathbf{D}^{\prime} \mathbf{Z}\right]^{-1} \mathbf{D}^{\prime} \mathbf{u}\right\}^{\prime}\right] \\
& =\frac{1}{n} \operatorname{plim}\left[\left(\frac{1}{n} \mathbf{D}^{\prime} \mathbf{Z}\right)^{-1}\left(\frac{1}{n} \mathbf{D}^{\prime} \mathbf{u} \mathbf{u}^{\prime} \mathbf{D}\right)\left(\frac{1}{n} \mathbf{Z}^{\prime} \mathbf{D}\right)^{-1}\right]
\end{aligned}
$$

since $\mathbf{D}=\left[\mathbf{\Sigma}^{-1} \otimes \mathbf{X}\left(\mathbf{X}^{\prime} \mathbf{X}\right)^{-1} \mathbf{X}^{\prime}\right] \mathbf{Z}$, this becomes:

$$
\begin{aligned}
& \text { Asy. } \operatorname{Var}\left(\hat{\boldsymbol{\delta}}_{3 S L S}\right)=\frac{1}{n} \operatorname{plim}\left\{\left(\frac{1}{n} \mathbf{Z}^{\prime}\left[\Sigma^{-1} \otimes \mathbf{X}\left(\mathbf{X}^{\prime} \mathbf{X}\right)^{-1} \mathbf{X}^{\prime}\right] \mathbf{Z}\right)^{-1}\right. \\
& \left.\cdot\left(\frac{1}{n} \mathbf{Z}^{\prime}\left[\Sigma^{-1} \otimes \mathbf{X}\left(\mathbf{X}^{\prime} \mathbf{X}\right)^{-1} \mathbf{X}^{\prime}\right][\mathbf{\Sigma} \otimes \mathbf{I}]\left[\Sigma^{-1} \otimes \mathbf{X}\left(\mathbf{X}^{\prime} \mathbf{X}\right)^{-1} \mathbf{X}^{\prime}\right] \mathbf{Z}\right)\left(\frac{1}{n} \mathbf{Z}^{\prime}\left[\Sigma^{-1} \otimes \mathbf{X}\left(\mathbf{X}^{\prime} \mathbf{X}\right)^{-1} \mathbf{X}^{\prime}\right] \mathbf{Z}\right)^{-1}\right\} \\
& =\frac{1}{n} \operatorname{plim}\left\{\left(\frac{1}{n} \mathbf{Z}^{\prime}\left[\mathbf{\Sigma}^{-1} \otimes \mathbf{X}\left(\mathbf{X}^{\prime} \mathbf{X}\right)^{-1} \mathbf{X}\right] \mathbf{Z}\right)^{-1}\left(\frac{1}{n} \mathbf{Z}^{\prime}\left[\boldsymbol{\Sigma}^{-1} \otimes \mathbf{X}\left(\mathbf{X}^{\prime} \mathbf{X}\right)^{-1} \mathbf{X}^{\prime}\right] \mathbf{Z}\right)\left(\frac{1}{n} \mathbf{Z}^{\prime}\left[\mathbf{\Sigma}^{-1} \otimes \mathbf{X}\left(\mathbf{X}^{\prime} \mathbf{X}\right)^{-1} \mathbf{X}^{\prime}\right] \mathbf{Z}\right)^{-1}\right\} \\
& =\frac{1}{n} \operatorname{plim}\left\{\left(\frac{1}{n} \mathbf{Z}^{\prime}\left[\mathbf{\Sigma}^{-1} \otimes \mathbf{X}\left(\mathbf{X}^{\prime} \mathbf{X}\right)^{-1} \mathbf{X}^{\prime}\right] \mathbf{Z}\right)^{-1}\right\}
\end{aligned}
$$


The estimator $\sqrt{n}\left(\hat{\boldsymbol{\delta}}_{3 S L S}-\boldsymbol{\delta}_{3 S L S}\right)$ is asymptotically normal provided the error terms at each observation are independently and identically (but not necessarily normally) distributed. Thus, the 3SLS estimator is asymptotically and normally distributed with mean given by the true vector of parameters and with an asymptotic covariance matrix given by equation (3.74).

The covariance matrix can once again be consistently estimated by using the 3SLS estimators instead of the 2SLS estimators in equation (3.68) as follows:

$$
\begin{gathered}
\hat{\boldsymbol{\Sigma}}=\left(\hat{\sigma}_{j l}\right) \text { where } \hat{\sigma}_{j l}=\frac{1}{n} \hat{\mathbf{u}}_{j}^{\prime} \hat{\mathbf{u}}_{l}=\frac{1}{n}\left(\mathbf{y}_{j}-\mathbf{Z} \boldsymbol{\delta}_{j 3 S L S}\right)^{\prime}\left(\mathbf{y}_{l}-\mathbf{Z} \boldsymbol{\delta}_{l 23 S L S}\right), \\
j, l=1,2, \ldots, G
\end{gathered}
$$

The fact the 3SLS is more asymptotically efficient is indicated by:

$$
\operatorname{Asy.} \operatorname{Var}\left(\hat{\boldsymbol{\delta}}_{3 S L S}\right)-\operatorname{Asy} \cdot \operatorname{Var}\left(\hat{\boldsymbol{\delta}}_{2 S L S}\right) \text { is negative definite . }
$$

Note also that if there are no restrictions on the covariance matrix, the distribution of the 3SLS estimator is identical to that of the full-information maximum-likelihood estimator (as discussed in the next subsection). In such a case, the asymptotic distribution is normal and the asymptotic covariance matrix given in equation (3.74) is asymptotically efficient in that it attains the asymptotic Cramer-Rao bound of full-information maximumlikelihood estimator (Davidson and Mackinnon, 1993).

\subsubsection{Full-Information Maximum-Likelihood}

Another full-information method of estimation is the full-information maximumlikelihood (FIML). Like the 3SLS, it is a complete system method of estimation but computationally more expensive than $3 \mathrm{SLS}$ as it involves the solution of nonlinear simultaneous equations. In the FIML method the likelihood function for the entire system 
is maximized by choice of all system parameters, subject to all a priori identifying restrictions. Like the 3SLS estimators, the resulting estimators are consistent and asymptotically efficient and they have the same asymptotic properties as 3SLS, including the same asymptotic covariance matrix. Unlike in 3SLS, it is, however, possible to use in the estimation process a wide range of a priori information, pertaining not only to each equation individually but also to several equations simultaneously, such as constraints involving coefficients of different structural equations and certain restriction on the error structure.

To develop the FIML we use notation comparable to that of 3SLS. Consider the linear simultaneous equations model given in equation (3.62). This equation, with disturbance terms that are assumed to be normally distributed, homoskedastic, and serially independent, can be written as:

$$
\underset{\operatorname{Gn} \times 1}{\mathbf{y}}=\underset{\operatorname{Gn} \times K^{\oplus} K^{\oplus} \times 1}{\mathbf{Z}}+\underset{\operatorname{Gn} \times 1}{\mathbf{u}}, \quad \mathbf{u} \sim N(\mathbf{0}, \mathbf{\Sigma} \otimes \mathbf{I})
$$

Note that since the FIML is one of maximum likelihood, it always requires the assumption of the normal distribution of the stochastic disturbances. Thus, assuming that the disturbance terms are normally distributed, the logarithm of the likelihood function of $\mathbf{u}$ is given by:

$$
\ln L(\mathbf{u})=-\frac{\mathrm{Gn}}{2} \ln 2 \pi-\frac{1}{2} \ln |\boldsymbol{\Sigma} \otimes \mathbf{I}|-\frac{1}{2}(\mathbf{y}-\mathbf{Z} \boldsymbol{\delta})^{\prime}\left|\boldsymbol{\Sigma}^{-1} \otimes \mathbf{I}\right|(\mathbf{y}-\mathbf{Z} \boldsymbol{\delta})
$$

where

$$
-\frac{1}{2} \ln |\mathbf{\Sigma} \otimes \mathbf{I}|=-\frac{1}{2} \ln |\mathbf{\Sigma}|^{n}=-\frac{\mathrm{n}}{2} \ln |\boldsymbol{\Sigma}|
$$

Thus, combining the two gives: 


$$
\ln L(\mathbf{u})=-\frac{\mathrm{Gn}}{2} \ln 2 \pi-\frac{\mathrm{n}}{2} \ln |\boldsymbol{\Sigma}|-\frac{1}{2}(\mathbf{y}-\mathbf{Z} \mathbf{\delta})^{\prime}\left|\boldsymbol{\Sigma}^{-1} \otimes \mathbf{I}\right|(\mathbf{y}-\mathbf{Z} \boldsymbol{\delta})
$$

Since the objective is to choose values of the parameters so as to maximize the likelihood of observing the values given by the endogenous variables $(\mathbf{y})$, what is needed is the likelihood function of the endogenous variable and not the that of the disturbance terms (u). It is, however, possible to get the likelihood function for $(\mathbf{y})$ from equation (3.76) using the following transformation (see also equations (3.8), (3.9), and (3.10))

$$
\ln L(\mathbf{y})=\ln (\mathbf{u})+\ln \left|\frac{\partial \mathbf{u}}{\partial \mathbf{y}}\right|
$$

where

$$
\left|\frac{\partial \mathbf{u}}{\partial \mathbf{y}}\right|=\left\|\begin{array}{cccc}
\mathbf{B} & \mathbf{0} & \cdots & \mathbf{0} \\
& \mathbf{B} & \cdots & \mathbf{0} \\
& & \ddots & \vdots \\
& & \cdots & \mathbf{B}
\end{array} \mid=\right\| \mathbf{B} \|^{n}
$$

Note that while the elements of the $\mathbf{B}$ matrix below the diagonal may be nonzero, reflecting lagged endogenous variables, all the element above the diagonal must be zero, reflecting the fact that future variables do not affect present ones. This helps in simplifying the expression in equation (3.77), because the zeros ensure that the determinant of the Jacobian is the nth power of the absolute value of the determinant of B. The relevant likelihood function of the vector of endogenous variables $\mathbf{y}$ is then:

$$
\ln L(\mathbf{y})=-\frac{\mathrm{Gn}}{2} \ln 2 \pi-\frac{\mathrm{n}}{2} \ln |\boldsymbol{\Sigma}|+\mathrm{n} \ln \|\mathrm{B}\|-\frac{1}{2}(\mathbf{y}-\mathbf{Z} \boldsymbol{\delta})^{\prime}\left|\boldsymbol{\Sigma}^{-1} \otimes \mathbf{I}\right|(\mathbf{y}-\mathbf{Z \delta})
$$


The fist-order conditions of maximization of this likelihood function characterize the estimators of the system parameters, as given by $\boldsymbol{\delta}$ and $\boldsymbol{\Sigma}$. It is maximized by the choice of the parameters and it is convenient to consider the choice of the elements of the covariance matrix $\boldsymbol{\Sigma}$, assuming that there are no a priori restrictions on $\boldsymbol{\Sigma}$. For convenience sake let $\boldsymbol{\sigma}^{j l}$ represent the jlth element of the inverse of the covariance matrix, $\boldsymbol{\Sigma}^{-1}$, and $\boldsymbol{\sigma}_{j l}$ those of the matrix itself. Using these notations, it is possible to rewrite the last term of equation (3.78) as follows:

$$
-\frac{1}{2}(\mathbf{y}-\mathbf{Z} \boldsymbol{\delta})^{\prime}\left|\mathbf{\Sigma}^{-1} \otimes \mathbf{I}\right|(\mathbf{y}-\mathbf{Z} \boldsymbol{\delta})=-\frac{1}{2} \sum_{j=1}^{G} \sum_{l=1}^{G} \boldsymbol{\sigma}^{j l}\left(\mathbf{y}_{j}-\mathbf{Z}_{j} \boldsymbol{\delta}_{j}\right)^{\prime}\left(\mathbf{y}_{j}-\mathbf{Z}_{j} \boldsymbol{\delta}_{j}\right)
$$

Thus,

$$
\ln L(\mathbf{y})=-\frac{\mathrm{Gn}}{2} \ln 2 \pi-\frac{\mathrm{n}}{2} \ln |\boldsymbol{\Sigma}|+\mathrm{n} \ln \|\mathrm{B}\|-\frac{1}{2} \sum_{j=1}^{G} \sum_{l=1}^{G} \boldsymbol{\sigma}^{j l}\left(\mathbf{y}_{j}-\mathbf{Z}_{j} \boldsymbol{\delta}_{j}\right)^{\prime}\left(\mathbf{y}_{j}-\mathbf{Z}_{j} \boldsymbol{\delta}_{j}\right)
$$

Then the first-order conditions for a maximum by choice of elements of $\boldsymbol{\Sigma}^{-1}$ (the inverse covariance matrix), is given by:

$$
\begin{aligned}
\frac{\partial \ln L(\mathbf{y})}{\partial \boldsymbol{\sigma}^{j l}} & =\frac{n}{2} \boldsymbol{\sigma}_{j l}-\frac{1}{2}\left(\mathbf{y}_{j}-\mathbf{Z}_{j} \boldsymbol{\delta}_{j}\right)^{\prime}\left(\mathbf{y}_{l}-\mathbf{Z}_{l} \boldsymbol{\delta}_{l}\right)=\mathbf{0} \\
& \rightarrow \hat{\boldsymbol{\sigma}}_{j l}=\frac{1}{n}\left(\mathbf{y}_{j}-\mathbf{Z}_{j} \hat{\boldsymbol{\delta}}_{j}\right)^{\prime}\left(\mathbf{y}_{l}-\mathbf{Z}_{l} \hat{\boldsymbol{\delta}}_{l}\right), \quad j, l=1,2, \ldots, G
\end{aligned}
$$

Hence, the FIML estimator of the elements of the covariance matrix is given by:

$$
\hat{\boldsymbol{\Sigma}}=\left(\hat{\boldsymbol{\sigma}}_{j l}\right),
$$

where the elements of $\left(\hat{\boldsymbol{\sigma}}_{j l}\right)$ are given by the second part of equation (3.81) above. As it was the case for the 3SLS, the second part of equation (3.81) can also be used to estimate $\boldsymbol{\Sigma} \otimes \mathbf{I}$ as: 


$$
\hat{\mathbf{\Sigma}} \otimes \mathbf{I}=\frac{1}{n}(\mathbf{y}-\mathbf{Z} \hat{\boldsymbol{\delta}})^{\prime}(\mathbf{y}-\mathbf{Z} \hat{\mathbf{\delta}}) .
$$

Combining equations (3.78) and (3.82) and replacing $\boldsymbol{\Sigma}$ by $\hat{\boldsymbol{\Sigma}}$, then gives:

$$
\begin{aligned}
& \ln L(\mathbf{y})=-\frac{\mathrm{Gn}}{2} \ln 2 \pi-\frac{\mathrm{n}}{2} \ln |\mathbf{\Sigma}|+\mathrm{n} \ln \|\mathrm{B}\|-\frac{1}{2}(\mathbf{y}-\mathbf{Z} \boldsymbol{\delta})^{\prime}\left[\frac{1}{n}(\mathbf{y}-\mathbf{Z} \hat{\mathbf{\delta}})^{\prime}(\mathbf{y}-\mathbf{Z} \hat{\mathbf{\delta}})\right]^{-1}(\mathbf{y}-\mathbf{Z} \boldsymbol{\delta}) \\
& \ln L(\mathbf{y})=-\frac{\mathrm{Gn}}{2} \ln 2 \pi-\frac{\mathrm{n}}{2} \ln |\mathbf{\Sigma}|+\mathrm{n} \ln \|\mathrm{B}\|-\frac{\mathrm{n}}{2}
\end{aligned}
$$

The FIML estimators of the parameters are obtained by maximizing the likelihood function in the second part of equation (3.83) by choice of $\boldsymbol{\delta}$ subject to all a priori restrictions. When there are no restrictions, the first-order conditions for maximization become:

$$
\frac{\partial \ln L(\mathbf{y})}{\partial \boldsymbol{\delta}}=-\left(\frac{n}{2}\right)\left(\frac{\partial \ln |\hat{\boldsymbol{\Sigma}}|}{\partial \boldsymbol{\delta}}\right)+n \frac{\partial \ln \|\mathbf{B}\|}{\partial \boldsymbol{\delta}}=\mathbf{0},
$$

where $\hat{\boldsymbol{\Sigma}}$ depends on $\hat{\boldsymbol{\delta}}$ as given in equation (3.81).

Equation (3.84) is a system of equations which are nonlinear in parameters. These firstorder conditions should be solved for the FIML estimator $\left(\hat{\boldsymbol{\delta}}_{F M L}\right)$, although it is difficult to solve. The difficulty arises because the partial derivatives of $\ln |\hat{\Sigma}|$ are nonlinear in parameters. Besides since $\|\mathbf{B}\|$ is a function of the coefficients of endogenous variables in all equations, the system of nonlinear equations in unknown parameters is difficult to solve. Consider, for example, the partial derivative with respect to, say, $\boldsymbol{\beta}_{j l}$ (the jlth element of $\|\mathbf{B}\|$ ) which can be given as:

$$
\frac{\partial \ln \|\mathbf{B}\|}{\partial \boldsymbol{\beta}_{j l}}=\boldsymbol{\beta}^{j l},
$$


where $\boldsymbol{\beta}^{j l}$ is the jlth element of $\mathbf{B}^{-1}$ (the inverse of the covariance matrix $\mathbf{B}$ ), involves all elements of $\mathbf{B}$ in nonlinear fashion. Thus, solving for FIML estimators is difficult and computationally expensive unless the model is recursive in which case the estimators are reduced to OLS. This is so because since $\mathbf{B}$ is triangular, the system can be normalized so that $\|\mathbf{B}\|$ is unity and hence $\ln \|\mathbf{B}\|$ vanishes leading to the possibility of estimating the parameters from:

$$
\frac{\partial \ln |\hat{\boldsymbol{\Sigma}}|}{\partial \boldsymbol{\delta}}=\mathbf{0}
$$

and since $\Sigma$ is diagonal, the solution for this first-order conditions give the OLS estimators.

A concentrated likelihood function for the endogenous variable can be derived. Consider equation (3.9) which can be written as with respect to $\boldsymbol{\Sigma}^{-1}$ as follows:

$$
\begin{aligned}
& L(\mathbf{B}, \boldsymbol{\Gamma}, \boldsymbol{\Sigma})=(2 \pi)^{-n G / 2}\|\mathbf{B}\|^{n}|\boldsymbol{\Sigma}|^{-n / 2} \exp \left[-\frac{1}{2} \sum_{i=1}^{n}\left(\mathbf{y}_{i}^{\prime} \mathbf{B}+\mathbf{x}_{i}^{\prime} \boldsymbol{\Gamma}\right)^{\prime} \mathbf{\Sigma}^{-1}\left(\mathbf{y}_{i}^{\prime} \mathbf{B}+\mathbf{x}_{i}^{\prime} \boldsymbol{\Gamma}\right)\right] \\
& \ln L(\mathbf{B}, \boldsymbol{\Gamma}, \boldsymbol{\Sigma})=-\frac{\mathrm{Gn}}{2} \ln 2 \pi-\frac{\mathrm{n}}{2} \ln |\boldsymbol{\Sigma}|+\mathrm{n} \ln \|\mathrm{B}\|-\frac{1}{2} \sum_{i=1}^{n}\left(\mathbf{y}_{i}^{\prime} \mathbf{B}+\mathbf{x}_{i}^{\prime} \boldsymbol{\Gamma}\right)^{\prime} \boldsymbol{\Sigma}^{-1}\left(\mathbf{y}_{i}^{\prime} \mathbf{B}+\mathbf{x}_{i}^{\prime} \boldsymbol{\Gamma}\right) \\
& \ln L(\mathbf{B}, \boldsymbol{\Gamma}, \boldsymbol{\Sigma})=-\frac{\mathrm{Gn}}{2} \ln 2 \pi-\frac{\mathrm{n}}{2} \ln |\boldsymbol{\Sigma}|+\mathrm{n} \ln \|\mathrm{B}\|-\frac{1}{2} \operatorname{tr} \boldsymbol{\Sigma}^{-1}(\mathbf{Y} \mathbf{B}+\mathbf{X} \boldsymbol{\Gamma})^{\prime}(\mathbf{Y B}+\mathbf{X} \boldsymbol{\Gamma})
\end{aligned}
$$

The partial derivative of the last part of equation (3.85) gives:

$$
\begin{aligned}
\frac{\partial L(\mathbf{B}, \boldsymbol{\Gamma}, \boldsymbol{\Sigma})}{\partial \boldsymbol{\Sigma}^{-1}} & =\frac{n}{2} \boldsymbol{\Sigma}-\frac{1}{2}(\mathbf{Y B}+\mathbf{X} \boldsymbol{\Gamma})^{\prime}(\mathbf{Y B}+\mathbf{X} \boldsymbol{\Gamma})=\mathbf{0} \\
& \rightarrow \hat{\boldsymbol{\Sigma}}=\frac{1}{n}(\mathbf{Y B}+\mathbf{X} \boldsymbol{\Gamma})^{\prime}(\mathbf{Y B}+\mathbf{X} \boldsymbol{\Gamma})
\end{aligned}
$$

Since there are no restrictions on $\boldsymbol{\Sigma}$, it is customary to replace the estimate of $\boldsymbol{\Sigma}$ from equation (3.86) in the last part of equation (3.85) and get the concentrated log-likelihood function. Let the $\ell(\mathbf{B}, \Gamma)$ denote the concentrated log-likelihood, then: 


$$
\ell(\mathbf{B}, \boldsymbol{\Gamma})=-\frac{\mathrm{Gn}}{2}(\ln 2 \pi+1)-\frac{\mathrm{n}}{2} \ln \left|\frac{1}{n}(\mathbf{Y B}+\mathbf{X} \boldsymbol{\Gamma})^{\prime}(\mathbf{Y B}+\mathbf{X} \boldsymbol{\Gamma})\right|+\mathrm{n} \ln \|\mathbf{B}\|
$$

Thus, the maximum likelihood estimates for $\mathbf{B}$ and $\boldsymbol{\Sigma}$ can be obtained by maximizing equation (3.87):

$$
\begin{aligned}
& \max _{\mathbf{B}, \boldsymbol{\Gamma}} \ell(\mathbf{B}, \boldsymbol{\Gamma})=\max _{\mathbf{B}, \boldsymbol{\Gamma}}\left\{-\frac{\mathrm{Gn}}{2}(\ln 2 \pi+1)-\frac{\mathrm{n}}{2} \ln \left|\frac{1}{n}(\mathbf{Y B}+\mathbf{X} \boldsymbol{\Gamma})^{\prime}(\mathbf{Y} \mathbf{B}+\mathbf{X} \boldsymbol{\Gamma})\right|+\mathrm{n} \ln \|\mathbf{B}\|\right\} \\
& \frac{\partial \ell(\mathbf{B}, \boldsymbol{\Gamma})}{\partial \mathbf{B}}=\mathbf{0} \Rightarrow n\left(\mathbf{B}^{\prime}\right)^{-1}-n \mathbf{Y}^{\prime}(\mathbf{Y B}+\mathbf{X} \boldsymbol{\Gamma})\left((\mathbf{Y B}+\mathbf{X} \boldsymbol{\Gamma})^{\prime}(\mathbf{Y B}+\mathbf{X} \boldsymbol{\Gamma})\right)^{-1}=\mathbf{0} \\
& \Rightarrow\left(\mathbf{B}^{\prime}\right)^{-1}-\mathbf{Y}^{\prime}(\mathbf{Y B}+\mathbf{X} \boldsymbol{\Gamma})\left((\mathbf{Y B}+\mathbf{X} \boldsymbol{\Gamma})^{\prime}(\mathbf{Y B}+\mathbf{X} \boldsymbol{\Gamma})\right)^{-1}=\mathbf{0} \\
& \frac{\partial \ell(\mathbf{B}, \boldsymbol{\Gamma})}{\partial \boldsymbol{\Gamma}}=\mathbf{0} \Rightarrow-n \mathbf{X}^{\prime}(\mathbf{Y B}+\mathbf{X} \boldsymbol{\Gamma})\left((\mathbf{Y B}+\mathbf{X} \boldsymbol{\Gamma})^{\prime}(\mathbf{Y B}+\mathbf{X} \boldsymbol{\Gamma})\right)^{-1}=\mathbf{0} \\
& \Rightarrow-\mathbf{X}^{\prime}(\mathbf{Y B}+\mathbf{X} \boldsymbol{\Gamma})\left((\mathbf{Y B}+\mathbf{X} \boldsymbol{\Gamma})^{\prime}(\mathbf{Y B}+\mathbf{X} \boldsymbol{\Gamma})\right)^{-1}=\mathbf{0}
\end{aligned}
$$

Note that the first-order conditions for maximization are nonlinear in unknown coefficients which make them to be difficult to solve.

\subsubsection{Generalized Method of Moments (GMM)}

The maximum likelihood estimator is fully efficient among consistent and asymptotically normally distributed estimators, in the context of the specified parametric model. To attain that efficiency, however, it is necessary to make possibly strong restrictive assumptions about the distribution or data generating process. An estimator which does not require these restrictive assumptions is the generalized method of moments (GMM). GMM move away from parametric assumptions toward estimators which are robust to some variations in the underlying data generating process (Greene, 2003). The essential idea of GMM is that moment conditions can be used to define model parameters, in the sense of providing a parameter-defining mapping for a model, besides their use in testing model specification. The parameter-defining mapping associates a 
parameter vector in some parameter space with each data generating process of the model. Thus, one advantage of GMM as an estimation method is that it permits models which consist of a very large number of data generating processes. Any data generating process is admissible provided it satisfies a relatively small number of restrictions or regularity conditions. The moment conditions yield a parameter-defining mapping under suitable regularity conditions, and the existence of a well-defined parameter-defining mapping, in turn, guarantees that the model parameters are asymptotically identified. The essence of GMM is that it replaces population moments by sample moments and whether or not model parameters are identified by a given sample depends on whether or not there is a unique solution to the estimator-defining equations that are the sample counterparts to the moment conditions. Although the basic idea of GMM goes back at least as far as Sargan (1958), it was first suggested under that name by Hansen (1982).

All the estimators that we have discussed so far can be obtained as GMM estimators but without imposing the assumptions of homoskedasticity on the disturbance terms in each equation. To develop the GMM method, consider equation (3.61) which can be written as follows with a small change in notation:

$$
\mathbf{y}_{j i}=\mathbf{Z}_{j i}^{\prime} \boldsymbol{\delta}_{j}+\mathbf{u}_{j i}
$$

where

$$
\mathbf{Z}_{j i}=\left[\begin{array}{ll}
\mathbf{Y}_{j i} & \mathbf{X}_{j i}
\end{array}\right]
$$

and

$$
E\left(\mathbf{u}_{j i} \mid \mathbf{X}_{i}\right)=\mathbf{0}
$$


with $\mathbf{X}_{i}$ denoting the full set of exogenous/predetermined variables in the model. The assumption $E\left(\mathbf{u}_{j i} \mid \mathbf{X}_{i}\right)=\mathbf{0}$ implies the following orthogonality conditions,

$$
\operatorname{Cov}\left(\mathbf{X}_{i}, \mathbf{u}_{j i}\right)=\mathbf{0} \quad \text { or } E\left[\mathbf{X}\left(y_{j i}-\mathbf{Z}_{j i}^{\prime} \boldsymbol{\delta}_{j}\right)\right]=\mathbf{0}
$$

If we consider all the equation jointly, then the criterion function for the GMM estimator is given by:

$$
\begin{aligned}
q= & \sum_{j=1}^{G} \sum_{l=1}^{G}\left[\frac{\mathbf{u}\left(\mathbf{Z}_{i}, \boldsymbol{\delta}_{j}\right)^{\prime} \mathbf{X}}{n}\right][\mathbf{D}]^{j l}\left[\frac{\mathbf{X}^{\prime} \mathbf{u}\left(\mathbf{Z}_{i}, \boldsymbol{\delta}_{j}\right)}{n}\right] \\
& =\sum_{j=1}^{G} \sum_{l=1}^{G} \overline{\mathbf{m}}\left(\boldsymbol{\delta}_{j}\right)^{\prime}[\mathbf{D}]^{j l} \overline{\mathbf{m}}\left(\boldsymbol{\delta}_{j}\right),
\end{aligned}
$$

where

$$
\overline{\mathbf{m}}\left(\boldsymbol{\delta}_{j}\right)=\frac{1}{n} \sum \mathbf{X}_{i}\left(y_{j i}-\mathbf{Z}_{j i} \boldsymbol{\delta}_{j}\right)
$$

and

$$
[\mathbf{D}]^{j l}=\text { block } \mathrm{jl} \text { of the weighting matrix, } \mathbf{D}^{-1}
$$

An optimal weighting matrix can be obtained by considering the asymptotic covariance matrix of the empirical moments, $\overline{\mathbf{m}}\left(\boldsymbol{\delta}_{j}\right)$. These moments are stacked in a single vector $\overline{\mathbf{m}}(\boldsymbol{\delta})$.Then, the jlth block Asy. $\operatorname{VAr}[\sqrt{n} \overline{\mathbf{m}}(\boldsymbol{\delta})]$ is given by:

$$
\begin{aligned}
& \boldsymbol{\Psi}_{j l}=\operatorname{plim}\left\{\frac{1}{n} \sum\left[\mathbf{X}_{i} \mathbf{X}_{i}^{\prime}\left(y_{j i}-\mathbf{Z}_{j i}^{\prime} \boldsymbol{\delta}_{j}\right)\left(y_{j i}-\mathbf{Z}_{j i}^{\prime} \boldsymbol{\delta}_{l}\right)\right]\right\}=\operatorname{plim}\left(\frac{1}{n} \sum_{i=1}^{n} d_{j l} \mathbf{X}_{i} \mathbf{X}_{i}^{\prime}\right) \\
& \Rightarrow \boldsymbol{\Psi}_{j l}=\left\{\begin{array}{l}
\sigma_{j l}\left[p \lim \left(\frac{\mathbf{X}^{\prime} \mathbf{X}}{n}\right)\right], \text { if the disturbancs are homoscedastic } \\
\operatorname{plim}\left[\frac{\mathbf{X}^{\prime} \mathbf{\Omega}_{\mathrm{j} 1} \mathbf{X}}{n}\right],
\end{array}\right\}
\end{aligned}
$$


Combining equations (3.91) and (3.92), then, the criterion function for GMM can be written as:

$$
q=\left[\begin{array}{c}
\mathbf{X}^{\prime}\left(\mathbf{y}_{1}-\mathbf{Z}_{1} \boldsymbol{\delta}_{1}\right) / n \\
\mathbf{X}^{\prime}\left(\mathbf{y}_{2}-\mathbf{Z}_{2} \boldsymbol{\delta}_{2}\right) / n \\
\vdots \\
\mathbf{X}^{\prime}\left(\mathbf{y}_{G}-\mathbf{Z}_{G} \boldsymbol{\delta}_{G}\right) / n
\end{array}\right]^{\prime}\left[\begin{array}{cccc}
\boldsymbol{\Psi}_{11} & \boldsymbol{\Psi}_{12} & \ldots & \boldsymbol{\Psi}_{1 G} \\
\boldsymbol{\Psi}_{21} & \boldsymbol{\Psi}_{22} & \ldots & \boldsymbol{\Psi}_{2 G} \\
\vdots & \vdots & \ldots & \vdots \\
\boldsymbol{\Psi}_{G 1} & \boldsymbol{\Psi}_{G 2} & \ldots & \boldsymbol{\Psi}_{G G}
\end{array}\right]^{-1}\left[\begin{array}{c}
\mathbf{X}^{\prime}\left(\mathbf{y}_{1}-\mathbf{Z}_{1} \boldsymbol{\delta}_{1}\right) / n \\
\mathbf{X}^{\prime}\left(\mathbf{y}_{2}-\mathbf{Z}_{2} \boldsymbol{\delta}_{2}\right) / n \\
\vdots \\
\mathbf{X}^{\prime}\left(\mathbf{y}_{G}-\mathbf{Z}_{G} \boldsymbol{\delta}_{G}\right) / n
\end{array}\right]
$$

The elements in $\boldsymbol{\Psi}_{j l}$ can be estimated with:

$$
\hat{\mathbf{\Psi}}_{j l}=\frac{1}{n} \sum_{i=1}^{n} \mathbf{X}_{i} \mathbf{X}_{i}^{\prime}\left(y_{j i}-\mathbf{Z}_{j i}^{\prime} \hat{\boldsymbol{\delta}}_{j}\right)\left(y_{j i}-\mathbf{Z}_{j i}^{\prime} \hat{\boldsymbol{\delta}}_{l}\right)
$$

where $\hat{\boldsymbol{\delta}}_{j}$ is a consistent estimator of $\boldsymbol{\delta}_{j}$ such the two-stage least squares $\left(\hat{\boldsymbol{\delta}}_{j 2 S L S}\right)$ or the three-stage least squares $\left(\hat{\boldsymbol{\delta}}_{j}\right.$ 3SLS $)$. Replacing $\boldsymbol{\Psi}_{j l}$ in equation (3.93) by $\hat{\mathbf{\Psi}}_{j l}$ from equation (3.94), then, gives:

$$
\hat{q}=\left[\begin{array}{c}
\mathbf{X}^{\prime}\left(\mathbf{y}_{1}-\mathbf{Z}_{1} \boldsymbol{\delta}_{1}\right) / n \\
\mathbf{X}^{\prime}\left(\mathbf{y}_{2}-\mathbf{Z}_{2} \boldsymbol{\delta}_{2}\right) / n \\
\vdots \\
\mathbf{X}^{\prime}\left(\mathbf{y}_{G}-\mathbf{Z}_{G} \boldsymbol{\delta}_{G}\right) / n
\end{array}\right]^{\prime}\left[\begin{array}{cccc}
\hat{\mathbf{\Psi}}_{11} & \hat{\mathbf{\Psi}}_{12} & \ldots & \hat{\mathbf{\Psi}}_{1 G} \\
\hat{\mathbf{\Psi}}_{21} & \hat{\mathbf{\Psi}}_{22} & \ldots & \hat{\mathbf{\Psi}}_{2 G} \\
\vdots & \vdots & \ldots & \vdots \\
\hat{\mathbf{\Psi}}_{G 1} & \hat{\mathbf{\Psi}}_{G 2} & \ldots & \hat{\mathbf{\Psi}}_{G G}
\end{array}\right]^{-1}\left[\begin{array}{c}
\mathbf{X}^{\prime}\left(\mathbf{y}_{1}-\mathbf{Z}_{1} \boldsymbol{\delta}_{1}\right) / n \\
\mathbf{X}^{\prime}\left(\mathbf{y}_{2}-\mathbf{Z}_{2} \boldsymbol{\delta}_{2}\right) / n \\
\vdots \\
\mathbf{X}^{\prime}\left(\mathbf{y}_{G}-\mathbf{Z}_{G} \boldsymbol{\delta}_{G}\right) / n
\end{array}\right]
$$

Differentiating equation (3.95) with respect to $\boldsymbol{\delta}_{j}$ yields the first-order conditions for GMM estimation

$$
\frac{\partial \hat{q}}{\partial \boldsymbol{\delta}_{j}}=2 \sum_{l=1}^{G}\left(\frac{\mathbf{Z}_{j} \mathbf{X}}{n}\right) \hat{\mathbf{\Psi}}^{j l}\left(\frac{\mathbf{X}^{\prime}\left(\mathbf{y}_{l}-\mathbf{Z}_{l} \boldsymbol{\delta}_{l}\right)}{n}\right)
$$

where $\hat{\boldsymbol{\Psi}}^{j l}$ represents the jlth block of the inverse matrix in the center of equation (3.95). The solution for these first-order conditions gives the GMM estimators, which can be written as: 


$$
\left[\begin{array}{c}
\hat{\boldsymbol{\delta}}_{1, G M M} \\
\hat{\boldsymbol{\delta}}_{2, G M M} \\
\vdots \\
\hat{\boldsymbol{\delta}}_{\mathrm{G}, G M M}
\end{array}\right]=\left[\begin{array}{cccc}
\mathbf{Z}_{1}^{\prime} \mathbf{X} \hat{\mathbf{\Psi}}^{11} \mathbf{X}^{\prime} \mathbf{Z}_{1} & \mathbf{Z}_{1}^{\prime} \mathbf{X} \hat{\mathbf{\Psi}}^{12} \mathbf{X}^{\prime} \mathbf{Z}_{2} & \ldots & \mathbf{Z}_{1}^{\prime} \mathbf{X} \hat{\mathbf{\Psi}}^{1 G} \mathbf{X}^{\prime} \mathbf{Z}_{G} \\
\mathbf{Z}_{2}^{\prime} \mathbf{X} \hat{\mathbf{\Psi}}^{21} \mathbf{X}^{\prime} \mathbf{Z}_{1} & \mathbf{Z}_{2}^{\prime} \mathbf{X} \hat{\mathbf{Y}}^{22} \mathbf{X}^{\prime} \mathbf{Z}_{2} & \ldots & \mathbf{Z}_{2}^{\prime} \mathbf{X} \hat{\mathbf{\Psi}}^{2 G} \mathbf{X}^{\prime} \mathbf{Z}_{G} \\
\vdots & \vdots & \ldots & \vdots \\
\mathbf{Z}_{G}^{\prime} \mathbf{X} \hat{\mathbf{\Psi}}^{G 1} \mathbf{X}^{\prime} \mathbf{Z}_{1} & \mathbf{Z}_{G}^{\prime} \mathbf{X} \hat{\mathbf{\Psi}}^{G 2} \mathbf{X}^{\prime} \mathbf{Z}_{2} & \ldots & \mathbf{Z}_{G}^{\prime} \mathbf{X} \hat{\mathbf{\Psi}}^{G G} \mathbf{X}^{\prime} \mathbf{Z}_{G}
\end{array}\right]^{-1}\left[\begin{array}{c}
\sum_{j=1}^{G} \mathbf{Z}_{1}^{\prime} \mathbf{X} \hat{\Psi}^{1 j} \mathbf{y}_{j} \\
\sum_{j=1}^{G} \mathbf{Z}_{2}^{\prime} \mathbf{X} \hat{\mathbf{Y}}^{2 j} \mathbf{y}_{j} \\
\vdots \\
\sum_{j=1}^{G} \mathbf{Z}_{G}^{\prime} \mathbf{X} \hat{\mathbf{\Psi}}^{G j} \mathbf{y}_{j}
\end{array}\right]
$$

The asymptotic covariance matrix for the GMM estimator is then calculated with $\mathrm{n}$ times the larger inverse matrix in brackets. The advantage of this estimator is that it brings efficiency in the presence of heteroskedasticity. 


\subsection{Estimating Simultaneous-Equations Models Using Panel Data}

The issues and techniques which are involved in estimating simultaneous equations models using cross-sectional data have been discussed in the preceding section of this chapter. In this section, the discussion is extended to the case of estimating simultaneous equations models using panel data. Using panel data for estimating econometric model has several advantages over cross-sectional data sets (see Hsiao, 1985, 1986; Solon, 1989; Klevmarken, 1989; Baltagi, 1995). Panel data sets, for example, usually give the researcher a large number of data points that increase the degree of freedom and reduce the collinearity among explanatory variables which in turn lead to the improvement in efficiency of econometric estimates. As panel data involve two dimensions, a cross-sectional dimension $\mathrm{n}$ and a time-series dimension $\mathrm{T}$, the computation of panel data estimators would, however, be more complicated than the analysis of cross-sectional data.

\subsubsection{Single-Equation Estimation}

Consider equation (3.51) which is the $\mathrm{jth}(\mathrm{j}=1)$ structural equation of the simultaneous equation model:

$$
\mathbf{y}_{1}=\mathbf{Y}_{1} \boldsymbol{\beta}_{1}+\mathbf{X}_{1} \gamma_{1}+\mathbf{u}_{1}=\mathbf{Z}_{1} \boldsymbol{\delta}_{1}+\mathbf{u}_{1}
$$

where

$$
\mathbf{Z}_{1}=\left[\begin{array}{ll}
\mathbf{Y}_{1} & \mathbf{X}_{1}
\end{array}\right] \text { and } \boldsymbol{\delta}_{1}=\left[\begin{array}{l}
\boldsymbol{\beta}_{1} \\
\boldsymbol{\gamma}_{1}
\end{array}\right]
$$

Like in the standard simultaneous equation model, $\mathbf{Y}_{1}$ is the set of $\mathrm{G} 1$ - 1 right-hand side endogenous variables, and $\mathbf{X}_{1}$ is the set of $\mathrm{K} 1$ included exogenous variables. Let $\mathbf{X}=\left[\begin{array}{ll}\mathbf{X}_{1} & \mathbf{X}_{1}^{*}\end{array}\right]$ be the set of all exogenous/ predetermined variables in the system. 
Note that $\mathbf{X}_{1}^{*}$ is the $\mathrm{K} 2$ excluded exogenous variables from the first equation. For this equation to be identified, then, $\mathrm{K} 2$ should be greater than or equal to G1 - 1 ( $K 2 \geq G 1-1)$. But, unlike in the standard simultaneous equation model, the panel data applications utilize a one-way or a two-way error component model for the disturbances

$$
\begin{aligned}
& \left.\mathbf{u}_{1}=\mathbf{Z}_{\mu} \boldsymbol{\mu}_{1}+\boldsymbol{\omega}_{1} \text { (one-way error component }\right) \\
& \mathbf{u}_{1}=\mathbf{Z}_{\mu} \boldsymbol{\mu}_{1}+\mathbf{Z}_{\lambda} \boldsymbol{\lambda}_{1}+\boldsymbol{\omega}_{1}(\text { two-way error component })
\end{aligned}
$$

where $\mathbf{Z}_{\mu}=\left(\mathbf{I}_{n} \otimes \mathbf{l}_{T}\right), \boldsymbol{\mu}_{1}^{\prime}=\left(\mu_{11}, \ldots, \mu_{11}\right), \boldsymbol{\omega}_{1}^{\prime}=\left(\omega_{111}, \ldots, \omega_{n T 1}\right)$, $\mathbf{Z}_{\lambda}=\left(\mathbf{t}_{n} \otimes \mathbf{I}_{T}\right)$ and $\lambda_{1}^{\prime}=\left(\lambda_{1}, \ldots, \lambda_{T}\right)$ with $\mathbf{t}_{T}$ and $\mathbf{t}_{n}$ denoting a vector of ones of size $\mathrm{T}$ and n, respectively. Following Baltagi (1995), the focus of this section will be on the oneway error component model.

Combining equations (3.98) and (3.99), the simultaneous equation model in the context of panel data, then can be written as

$$
\mathbf{y}_{1}=\mathbf{Y}_{1} \boldsymbol{\beta}_{1}+\mathbf{X}_{1} \boldsymbol{\gamma}_{1}+\mathbf{Z}_{\mu} \boldsymbol{\mu}_{\mathbf{1}}+\boldsymbol{\omega}_{1}=\mathbf{Z}_{1} \boldsymbol{\delta}_{1}+\mathbf{Z}_{\mu} \boldsymbol{\mu}_{\mathbf{1}}+\boldsymbol{\omega}_{1}
$$

where $\boldsymbol{\mu}_{1}$ and $\boldsymbol{\omega}_{1}$ are random vectors with zero means and covariance matrix:

$$
E\left(\begin{array}{l}
\boldsymbol{\mu}_{1} \\
\boldsymbol{\omega}_{1}
\end{array}\right)\left(\boldsymbol{\mu}_{1}^{\prime} \boldsymbol{\omega}_{1}\right)=\left[\begin{array}{cc}
\boldsymbol{\sigma}_{\mu_{11}}^{2} \mathbf{I}_{n} & \mathbf{0} \\
\mathbf{0} & \boldsymbol{\sigma}_{\omega_{11}}^{2} \mathbf{I}_{n T}
\end{array}\right] .
$$

Thus

$$
\begin{aligned}
\boldsymbol{\Omega}_{11} & =E\left(\mathbf{u}_{1} \mathbf{u}_{1}^{\prime}\right)=\mathbf{Z}_{\mu} E\left(\boldsymbol{\mu}_{1} \boldsymbol{\mu}_{1}^{\prime}\right) \mathbf{Z}_{\mu}^{\prime}+E\left(\boldsymbol{\omega}_{1} \boldsymbol{\omega}_{1}^{\prime}\right) \\
& =\boldsymbol{\sigma}_{\mu_{11}}^{2}\left(\mathbf{I}_{n} \otimes \mathbf{J}_{T}\right)+\boldsymbol{\sigma}_{\omega_{11}}^{2}\left(\mathbf{I}_{n} \otimes \mathbf{I}_{T}\right)=\boldsymbol{\sigma}_{\mu_{11}}^{2}\left(\mathbf{I}_{n} \otimes \mathbf{J}_{T}\right)+\boldsymbol{\sigma}_{\omega_{11}}^{2} \mathbf{I}_{n T}
\end{aligned}
$$

where $\mathbf{J}_{T}$ is a matrix of ones of dimension T. Note that $\mathbf{Z}_{\mu} \mathbf{Z}_{\mu}^{\prime}=\mathbf{I}_{n} \otimes \mathbf{J}_{T}$. Now before proceeding to the derivation of the estimators, it is helpful to define two matrices, $\mathbf{P}$ and 
$\mathbf{H}$, which are useful in transforming the structural equations. Let $\mathbf{P}$ be the matrix which averages the observations across time for each individual and $\mathbf{H}$ be the matrix which obtains the deviations from individual means. Thus,

$$
\left[\begin{array}{l}
\mathbf{P}=\mathbf{Z}_{\mu}\left(\mathbf{Z}_{\mu}^{\prime} \mathbf{Z}_{\mu}\right) \mathbf{Z}_{\mu}^{\prime}=\mathbf{I}_{n} \otimes \overline{\mathbf{J}}_{T}, \text { where } \overline{\mathbf{J}}_{T}=\mathbf{J}_{T} / T \\
\mathbf{H}=\mathbf{I}_{n T}-\mathbf{P}=\left(I_{T}-\frac{J_{T}}{T}\right) \otimes I_{n}
\end{array}\right] 1 .
$$

Transforming equation (3.98) by $\mathbf{H}$ then gives:

$$
\begin{aligned}
& \mathbf{H y} \mathbf{y}_{1}=\mathbf{H Z}_{1} \boldsymbol{\delta}_{1}+\mathbf{H u} \mathbf{u}_{1} \\
& \overline{\mathbf{y}}_{1}=\overline{\mathbf{Z}}_{1} \boldsymbol{\delta}_{1}+\overline{\mathbf{u}}_{1}, \text { for } \overline{\mathbf{y}}_{1}=\mathbf{H} \mathbf{y}_{1}, \overline{\mathbf{Z}}_{1}=\mathbf{H Z} \mathbf{Z}_{1} \text { and } \overline{\mathbf{u}}_{1}=\mathbf{H u} \mathbf{u}_{1}
\end{aligned}
$$

Within 2SLS (W2SLS) estimator for $\boldsymbol{\delta}_{1}$ can be obtained by performing 2SLS on equation (3.103) with $\overline{\mathbf{X}}=\mathbf{H X}$ as the set of instruments:

$$
\hat{\boldsymbol{\delta}}_{1, W 2 S L S}=\left(\overline{\mathbf{Z}}_{1}^{\prime} \mathbf{P}_{\bar{X}} \overline{\mathbf{Z}}_{1}\right)^{-1} \overline{\mathbf{Z}}_{1}^{\prime} \mathbf{P}_{\bar{X}} \overline{\mathbf{y}}_{1} \text {, where } \mathbf{P}_{\bar{X}}=\overline{\mathbf{X}}\left(\overline{\mathbf{X}}^{\prime} \overline{\mathbf{X}}\right)^{-1} \overline{\mathbf{X}}^{\prime}
$$

Thus, the

$$
\operatorname{var}\left(\hat{\boldsymbol{\delta}}_{1, W 2 S L S}\right)=\boldsymbol{\sigma}_{\omega_{11}}^{2}\left(\overline{\mathbf{Z}}_{1}^{\prime} \mathbf{P}_{\bar{X}} \overline{\mathbf{Z}}_{1}\right)^{-1}
$$

It is also possible to get W2SLS by performing GLS on equation (3.103) with $\overline{\mathbf{X}}=\mathbf{H X}$ as the set of instruments:

$$
\overline{\mathbf{X}} \overline{\mathbf{y}}_{1}=\overline{\mathbf{X}} \overline{\mathbf{Z}}_{1} \boldsymbol{\delta}_{1}+\overline{\mathbf{X}} \overline{\mathbf{u}}_{1}
$$

If equation (3.98) is transformed by $\mathbf{P}$ instead of $\mathbf{H}$, the Between 2SLS (B2SLS) estimator for $\boldsymbol{\delta}_{1}$ can be obtained by performing 2 SLS on the transformed equation with $\dddot{\mathbf{X}}=\mathbf{P X}$ as the set of instruments:

$$
\begin{aligned}
& \mathbf{P} \mathbf{y}_{1}=\mathbf{P Z} \mathbf{Z}_{1} \boldsymbol{\delta}_{1}+\mathbf{P} \mathbf{u}_{1} \\
& \dddot{\mathbf{y}}_{1}=\dddot{\mathbf{Z}}_{1} \boldsymbol{\delta}_{1}+\dddot{\mathbf{u}}_{1}, \quad \text { for } \dddot{\mathbf{y}}_{1}=\mathbf{P} \mathbf{y}_{1}, \dddot{\mathbf{Z}}_{1}=\mathbf{P Z} \mathbf{Z}_{1} \text { and } \dddot{\mathbf{u}}_{1}=\mathbf{P} \mathbf{u}_{1}
\end{aligned}
$$

\footnotetext{
${ }^{1} \mathbf{P}$ and $\mathbf{H}$ are idempotent, orthogonal and sum to the identity matrix.
} 
Then, the B2SLS estimator is given by

$$
\hat{\boldsymbol{\delta}}_{1, B 2 S L S}=\left(\dddot{\mathbf{Z}}_{1}^{\prime} \mathbf{P}_{\dddot{X}} \dddot{\mathbf{Z}}_{1}\right)^{-1} \dddot{\mathbf{Z}}_{1}^{\prime} \mathbf{P}_{\dddot{X}} \dddot{\mathbf{y}}_{1}, \quad \text { where } \mathbf{P}_{\dddot{X}}=\dddot{\mathbf{X}}\left(\dddot{\mathbf{X}}^{\prime} \dddot{\mathbf{X}}\right)^{-1} \dddot{\mathbf{X}}^{\prime}
$$

and its covariance, $\operatorname{var}\left(\hat{\boldsymbol{\delta}}_{1, B 2 S L S}\right)$, can be computed as:

$$
\operatorname{var}\left(\hat{\boldsymbol{\delta}}_{1, B 2 S L S}\right)=\boldsymbol{\sigma}_{1_{11}}^{2}\left(\dddot{\mathbf{Z}}_{1}^{\prime} \mathbf{P}_{\dddot{X}} \dddot{\mathbf{Z}}_{1}\right)^{-1}, \text { where } \boldsymbol{\sigma}_{1_{11}}^{2}=T \boldsymbol{\sigma}_{\mu_{11}}^{2}+\boldsymbol{\sigma}_{\omega_{11}}^{2}
$$

Using $\dddot{\mathbf{X}}=\mathbf{P X}$ as the set of instruments, the B2SLS can also be obtained by performing GLS on:

$$
\dddot{\mathbf{X}}^{\prime} \dddot{\mathbf{y}}_{1}=\dddot{\mathbf{X}}^{\prime} \dddot{\mathbf{Z}}_{1} \boldsymbol{\delta}_{1}+\dddot{\mathbf{X}}^{\prime} \dddot{\mathbf{u}}_{1}
$$

Note that $\boldsymbol{\delta}_{1}$ is the same in equation (3.105) and (3.108) and when these two equations are stacked together they form a system given by:

$$
\left(\begin{array}{c}
\overline{\mathbf{X}}^{\prime} \overline{\mathbf{y}}_{1} \\
\dddot{\mathbf{X}}^{\prime} \dddot{\mathbf{y}}_{1}
\end{array}\right)=\left(\begin{array}{c}
\overline{\mathbf{X}}^{\prime} \overline{\mathbf{Z}}_{1} \\
\dddot{\mathbf{X}}^{\prime} \dddot{\mathbf{Z}}_{1}
\end{array}\right) \boldsymbol{\delta}_{1}+\left(\begin{array}{c}
\overline{\mathbf{X}}^{\prime} \overline{\mathbf{u}}_{1} \\
\dddot{\mathbf{X}}^{\prime} \dddot{\mathbf{u}}_{1}
\end{array}\right)
$$

where

$$
E\left(\begin{array}{c}
\overline{\mathbf{X}}^{\prime} \overline{\mathbf{u}}_{1} \\
\dddot{\mathbf{X}}^{\prime} \dddot{\mathbf{u}}_{1}
\end{array}\right)=\mathbf{0} \text { and } \operatorname{var}\left(\begin{array}{l}
\overline{\mathbf{X}}^{\prime} \overline{\mathbf{u}}_{1} \\
\dddot{\mathbf{X}}^{\prime} \dddot{\mathbf{u}}_{1}
\end{array}\right)=\left[\begin{array}{cc}
\sigma_{\omega_{11}}^{2} \overline{\mathbf{X}}^{\prime} \overline{\mathbf{X}} & \mathbf{0} \\
\mathbf{0} & \sigma_{1_{11}}^{2} \dddot{\mathbf{X}} \dddot{\mathbf{X}}
\end{array}\right]
$$

Baltagi (1981) derived the error component two-stage least squares (EC2SLS) for $\boldsymbol{\delta}_{1}$ by performing GLS on equation (3.109):

$$
\hat{\boldsymbol{\delta}}_{1, E C 2 S L S}=\left[\frac{\overline{\mathbf{Z}}_{1}^{\prime} \mathbf{P}_{\bar{X}} \overline{\mathbf{Z}}_{1}}{\boldsymbol{\sigma}_{\omega_{11}}^{2}}+\frac{\dddot{\mathbf{Z}}_{1}^{\prime} \mathbf{P}_{\dddot{X}} \dddot{\mathbf{Z}}_{1}}{\boldsymbol{\sigma}_{1_{11}}^{2}}\right]^{-1}\left[\frac{\overline{\mathbf{Z}}_{1}^{\prime} \mathbf{P}_{\bar{X}} \overline{\mathbf{y}}}{\boldsymbol{\sigma}_{\omega_{11}}^{2}}+\frac{\dddot{\mathbf{Z}}_{1}^{\prime} \mathbf{P}_{\dddot{X}} \dddot{\mathbf{y}}_{1}}{\boldsymbol{\sigma}_{1_{11}}^{2}}\right]
$$

with $\operatorname{var}\left(\hat{\boldsymbol{\delta}}_{1, E C 2 S L S}\right)$ given by the fist inverted bracket in equation (3.110). This estimator turns out to be the matrix-weighted average of the Within and the Between two-stage 
least squares estimators which are given in equations (3.104) and (3.107), respectively, with the weights depending upon their respective variance-covariance matrices, that is

$$
\hat{\boldsymbol{\delta}}_{1, E C 2 S L S}=\mathbf{D}_{W} \hat{\boldsymbol{\delta}}_{1, W 2 S L S}+\mathbf{D}_{B} \hat{\boldsymbol{\delta}}_{1, B 2 S L S}
$$

with

$$
\mathbf{D}_{W}=\left[\frac{\overline{\mathbf{Z}}_{1}^{\prime} \mathbf{P}_{\bar{X}} \overline{\mathbf{Z}}_{1}}{\boldsymbol{\sigma}_{\omega_{11}}^{2}}+\frac{\dddot{\mathbf{Z}}_{1}^{\prime} \mathbf{P}_{\dddot{X}} \dddot{\mathbf{Z}}_{1}}{\boldsymbol{\sigma}_{1_{11}}^{2}}\right]^{-1}\left[\frac{\overline{\mathbf{Z}}_{1}^{\prime} \mathbf{P}_{\bar{X}} \overline{\mathbf{Z}}_{1}}{\boldsymbol{\sigma}_{\omega_{11}}^{2}}\right]
$$

and

$$
\mathbf{D}_{B}=\left[\frac{\overline{\mathbf{Z}}_{1}^{\prime} \mathbf{P}_{\bar{X}} \overline{\mathbf{Z}}_{1}}{\boldsymbol{\sigma}_{\omega_{11}}^{2}}+\frac{\dddot{\mathbf{Z}}_{1}^{\prime} \mathbf{P}_{\dddot{X}_{X}} \dddot{\mathbf{Z}}_{1}}{\boldsymbol{\sigma}_{1_{11}}^{2}}\right]^{-1}\left[\frac{\dddot{\mathbf{Z}}_{1}^{\prime} \mathbf{P}_{\overparen{X}} \dddot{\mathbf{Z}}_{1}}{\boldsymbol{\sigma}_{1_{11}}^{2}}\right] .
$$

A feasible estimate of EC2SLS can also be calculated by replacing the variance components estimates in equation (3.110) by their respective consistent estimates. Note that consistent estimates of $\boldsymbol{\sigma}_{\omega_{11}}^{2}$ and $\boldsymbol{\sigma}_{1_{11}}^{2}$ can be obtained by substituting the W2SLS and B2SLS residuals, respectively, in the usual variance formula:

$$
\begin{aligned}
& \hat{\boldsymbol{\sigma}}_{\omega_{11}}^{2}=\left(\mathbf{y}_{1}-\mathbf{Z}_{1} \hat{\boldsymbol{\delta}}_{1, W 2 S L S}\right)^{\prime} \mathbf{H}\left(\mathbf{y}_{1}-\mathbf{Z}_{1} \hat{\boldsymbol{\delta}}_{1, W 2 S L S}\right) / n(T-1) \\
& \hat{\boldsymbol{\sigma}}_{1_{11}}^{2}=\left(\mathbf{y}_{1}-\mathbf{Z}_{1} \hat{\boldsymbol{\delta}}_{1, B 2 S L S}\right)^{\prime} \mathbf{P}\left(\mathbf{y}_{1}-\mathbf{Z}_{1} \hat{\boldsymbol{\delta}}_{1, B 2 S L S}\right) / n
\end{aligned}
$$

Note that it is easy to run W2SLS and B2SLS using standard 2SLS package that computes equations (3.104) and (3.107). Then it is easy to compute the consistent covariance from equations (3.112).

\subsubsection{System of Equations Method}

The single-equation estimation method considered in the preceding subsection ignores restriction in all equations in the structural system except the one being estimated. Similar to what we have seen in the case of the standard simultaneous equations, more 
efficient estimates can be obtained by considering the additional information contained in the other equations. In this subsection we consider the system estimation method. Consider the system of identified equations:

$$
\mathbf{y}=\mathbf{Z} \boldsymbol{\delta}+\mathbf{u}
$$

where

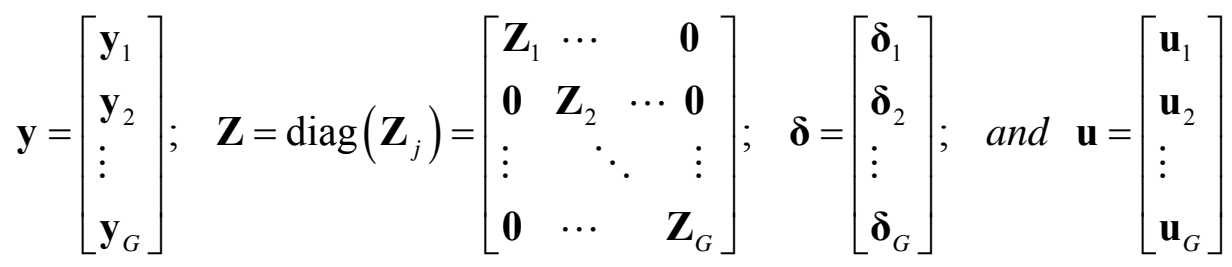

with $\mathbf{Z}_{j}=\left[\begin{array}{ll}\mathbf{Y}_{j} & \mathbf{X}_{j}\end{array}\right]$ of dimensionnT x $\left(G 1_{j}-1+K 1_{j}\right)$ for $j=1,2, \ldots, G$. For the one-way error component model, the disturbance of the jth equation $\mathbf{u}_{j}$ is given by:

$$
\mathbf{u}_{j}=\mathbf{Z}_{\mu} \boldsymbol{\mu}_{j}+\boldsymbol{\omega}_{j}
$$

where

$$
\mathbf{Z}_{\mu}=\left(\mathbf{I}_{n} \otimes \mathbf{t}_{T}\right), \boldsymbol{\mu}_{j}^{\prime}=\left(\mu_{1 j}, \mu_{2 j}, \ldots, \mu_{n j}\right) \text {, and } \boldsymbol{\omega}_{j}^{\prime}=\left(\omega_{11 j}, \omega_{11 j}, \ldots, \omega_{1 T j}, \ldots, \omega_{n 1 j}, \ldots, \omega_{n T j}\right)
$$

Thus,

$$
\boldsymbol{\Omega}_{j l}=E\left(\mathbf{u}_{j} \mathbf{u}_{l}^{\prime}\right)=\boldsymbol{\sigma}_{\mu_{j l}}^{2}\left(\mathbf{I}_{n} \otimes \mathbf{J}_{T}\right)+\boldsymbol{\sigma}_{\omega_{j l}}^{2}\left(\mathbf{I}_{n} \otimes \mathbf{I}_{T}\right)
$$

In this case, the covariance matrix between the disturbances of different equations has the same one-way error component form. But, now there are additional cross equation variances components to be estimated. When one considers the whole model, the variance-covariance matrix for the set of $\mathrm{G}$ structural equations is given by

$$
\boldsymbol{\Omega}=E\left(\mathbf{u u}^{\prime}\right)=\boldsymbol{\Sigma}_{\mu} \otimes\left(\mathbf{I}_{n} \otimes \mathbf{J}_{T}\right)+\boldsymbol{\Sigma}_{\omega} \otimes\left(\mathbf{I}_{n} \otimes \mathbf{I}_{T}\right)
$$


where $\boldsymbol{\Sigma}_{\mu}=\left[\boldsymbol{\sigma}_{\mu_{j l}^{2}}^{2}\right]$ and $\boldsymbol{\Sigma}_{\omega}=\left[\boldsymbol{\sigma}_{\omega_{j l}}^{2}\right]$ are both G x G matrices, and $\mathbf{u}^{\prime}=\left(\mathbf{u}_{1}^{\prime}, \mathbf{u}_{2}^{\prime}, \ldots, \mathbf{u}_{G}^{\prime}\right)$ is a 1 $\mathrm{x}$ nGT vector of disturbances with $\mathbf{u}_{j}$ defined in equation (3.114) for $\mathrm{j}=1,2, \ldots \mathrm{G}$. Alternatively, by replacing $\mathbf{J}_{j}$ by $T \overline{\mathbf{J}}_{j}$ and $\mathbf{I}_{T}$ by $\mathbf{E}_{T}+\overline{\mathbf{J}}_{j}$ the variance-covariance matrix can be written as:

$$
\begin{aligned}
\mathbf{\Omega}=E\left(\mathbf{u} \mathbf{u}^{\prime}\right)=\left(T \boldsymbol{\Sigma}_{\mu}\right. & \left.+\boldsymbol{\Sigma}_{\omega}\right) \otimes\left(\mathbf{I}_{n} \otimes \overline{\mathbf{J}}_{T}\right)+\boldsymbol{\Sigma}_{\omega} \otimes\left(\mathbf{I}_{n} \otimes \mathbf{E}_{T}\right) \\
& =\boldsymbol{\Sigma}_{1} \otimes \mathbf{P}+\boldsymbol{\Sigma}_{\omega} \otimes \mathbf{H}
\end{aligned}
$$

where $\boldsymbol{\Sigma}_{1}=T \boldsymbol{\Sigma}_{\mu}+\boldsymbol{\Sigma}_{\omega}$ and $\mathbf{P}=\mathbf{I}_{n} \otimes \overline{\mathbf{J}}_{T}$ and $\mathbf{H}=\mathbf{I}_{n T}-\mathbf{P}$ are as defined in equation (3.102). Equation (3.117) is the spectral decomposition of $\boldsymbol{\Omega}$, which means that

$$
\mathbf{\Omega}^{r}=\boldsymbol{\Sigma}_{1}^{r} \otimes \mathbf{P}+\boldsymbol{\Sigma}_{\omega}^{r} \otimes \mathbf{H}
$$

where $\mathrm{r}$ is an arbitrary scalar (see Baltagi, 1980; Magnus, 1982). The inverse of the variance-covariance matrix, $\boldsymbol{\Omega}^{-1}$, can be calculated by setting $\mathrm{r}=-1$ in equation (3.118). That is:

$$
\mathbf{\Omega}^{-1}=\boldsymbol{\Sigma}_{1}^{-1} \otimes \mathbf{P}+\boldsymbol{\Sigma}_{\omega}^{-1} \otimes \mathbf{H}
$$

Similarly, for $r=-1 / 2$, one gets:

$$
\mathbf{\Omega}^{-1 / 2}=\boldsymbol{\Sigma}_{1}^{-1 / 2} \otimes \mathbf{P}+\boldsymbol{\Sigma}_{\omega}^{-1 / 2} \otimes \mathbf{H}
$$

It is possible to transform the structural equations system given in equation (3.113) by $\left(\mathbf{I}_{G} \otimes \mathbf{H}\right)$ to get:

$$
\widehat{\mathbf{y}}=\widehat{\mathbf{Z}} \boldsymbol{\delta}+\widehat{\mathbf{u}}
$$


where $\widehat{\mathbf{y}}=\left(\mathbf{I}_{G} \otimes \mathbf{H}\right) \mathbf{y}, \widehat{\mathbf{Z}}=\left(\mathbf{I}_{G} \otimes \mathbf{H}\right) \mathbf{Z}$, and $\widehat{\mathbf{u}}=\left(\mathbf{I}_{G} \otimes \mathbf{H}\right) \mathbf{u}$. Now the Within 3SLS can be obtained by performing 3SLS on equation (3.121) with $\left(\mathbf{I}_{G} \otimes \hat{\mathbf{X}}\right)$ as the set of instruments, where $\widehat{\mathbf{X}}=\mathbf{H X}$ :

$$
\hat{\boldsymbol{\delta}}_{\text {W3SLS }}=\left[\hat{\mathbf{Z}}^{\prime}\left(\boldsymbol{\Sigma}_{\omega}^{-1} \otimes \mathbf{P}_{\hat{X}}\right) \hat{\mathbf{Z}}\right]^{-1}\left[\hat{\mathbf{Z}}^{\prime}\left(\boldsymbol{\Sigma}_{\omega}^{-1} \otimes \mathbf{P}_{\widehat{X}}\right) \hat{\mathbf{y}}\right]
$$

It is also possible to transform equation (3.113) by $\left(\mathbf{I}_{G} \otimes \mathbf{P}\right)$ to get:

$$
\breve{\mathbf{y}}=\breve{\mathbf{Z}} \boldsymbol{\delta}+\breve{\mathbf{u}}
$$

where $\breve{\mathbf{y}}=\left(\mathbf{I}_{G} \otimes \mathbf{P}\right) \mathbf{y}, \breve{\mathbf{Z}}=\left(\mathbf{I}_{G} \otimes \mathbf{P}\right) \mathbf{Z}$, and $\breve{\mathbf{u}}=\left(\mathbf{I}_{G} \otimes \mathbf{P}\right) \mathbf{u}$. Now, the Between 3SLS can be obtained by performing 3SLS on equation (3.123) with $\left(\mathbf{I}_{G} \otimes \breve{\mathbf{X}}\right)$ as the set of instruments, where $\breve{\mathbf{X}}=\mathbf{P X}$ :

$$
\hat{\boldsymbol{\delta}}_{\text {B3SLS }}=\left[\breve{\mathbf{Z}}^{\prime}\left(\boldsymbol{\Sigma}_{1}^{-1} \otimes \mathbf{P}_{\breve{X}}\right) \breve{\mathbf{Z}}\right]^{-1}\left[\breve{\mathbf{Z}}^{\prime}\left(\boldsymbol{\Sigma}_{1}^{-1} \otimes \mathbf{P}_{\breve{X}}\right) \breve{\mathbf{y}}\right]
$$

Pre-multiplying equation (3.121) by $\left(\mathbf{I}_{G} \otimes \widehat{\mathbf{X}}\right)$ and equation (3.123) by $\left(\mathbf{I}_{G} \otimes \breve{\mathbf{X}}\right)$ and stacking the transformed equations by recognizing that $\boldsymbol{\delta}$ is the same for both equations will give:

$$
\left(\begin{array}{c}
\left(\mathbf{I}_{G} \otimes \widehat{\mathbf{X}}^{\prime}\right) \widehat{\mathbf{y}}_{1} \\
\left(\left(\mathbf{I}_{G} \otimes \breve{\mathbf{X}}^{\prime}\right) \breve{\mathbf{y}}_{1}\right.
\end{array}\right)=\left(\begin{array}{l}
\left(\mathbf{I}_{G} \otimes \widehat{\mathbf{X}}^{\prime}\right) \widehat{\mathbf{Z}}_{1} \\
\left(\mathbf{I}_{G} \otimes \breve{\mathbf{X}}^{\prime}\right) \breve{\mathbf{Z}}_{1}
\end{array}\right) \boldsymbol{\delta}_{1}+\left(\begin{array}{c}
\left(\mathbf{I}_{G} \otimes \widehat{\mathbf{X}}^{\prime}\right) \widehat{\mathbf{u}}_{1} \\
\left(\mathbf{I}_{G} \otimes \breve{\mathbf{X}}^{\prime}\right) \breve{\mathbf{u}}_{1}
\end{array}\right)
$$

Performing GLS on equation (3.125) gives the error component three-stage least squares (EC3SLS) estimator for $\boldsymbol{\delta}$ (see Baltagi, 1981):

$$
\hat{\boldsymbol{\delta}}_{E C 3 S L S}=\left[\widehat{\mathbf{Z}}^{\prime}\left(\boldsymbol{\Sigma}_{\omega}^{-1} \otimes \mathbf{P}_{\widehat{X}}\right) \widehat{\mathbf{Z}}+\breve{\mathbf{Z}}^{\prime}\left(\boldsymbol{\Sigma}_{1}^{-1} \otimes \mathbf{P}_{\breve{X}}\right) \breve{\mathbf{Z}}\right]^{-1}\left[\widehat{\mathbf{Z}}^{\prime}\left(\boldsymbol{\Sigma}_{\omega}^{-1} \otimes \mathbf{P}_{\widehat{X}}\right) \hat{\mathbf{y}}+\breve{\mathbf{Z}}^{\prime}\left(\boldsymbol{\Sigma}_{1}^{-1} \otimes \mathbf{P}_{\breve{X}}\right) \breve{\mathbf{y}}\right](3
$$

As in EC2SLS, this estimator can also be written as a matrix-weighted combination of $\hat{\boldsymbol{\delta}}_{\text {W3SLS }}$ and $\hat{\boldsymbol{\delta}}_{\text {B3SLS }}$ as follows: 


$$
\hat{\boldsymbol{\delta}}_{E C 3 S L S}=\mathbf{D}_{W} \hat{\boldsymbol{\delta}}_{W 3 S L S}+\mathbf{D}_{B} \hat{\boldsymbol{\delta}}_{B 3 S L S}
$$

where

$$
\mathbf{D}_{W}=\left[\widehat{\mathbf{Z}}^{\prime}\left(\boldsymbol{\Sigma}_{\omega}^{-1} \otimes \mathbf{P}_{\widehat{X}}\right) \widehat{\mathbf{Z}}+\breve{\mathbf{Z}}^{\prime}\left(\boldsymbol{\Sigma}_{1}^{-1} \otimes \mathbf{P}_{\breve{X}}\right) \breve{\mathbf{Z}}\right]^{-1}\left[\widehat{\mathbf{Z}}^{\prime}\left(\boldsymbol{\Sigma}_{\omega}^{-1} \otimes \mathbf{P}_{\widehat{X}}\right) \widehat{\mathbf{y}}\right]
$$

and

$$
\mathbf{D}_{B}=\left[\widehat{\mathbf{Z}}^{\prime}\left(\boldsymbol{\Sigma}_{\omega}^{-1} \otimes \mathbf{P}_{\widehat{X}}\right) \hat{\mathbf{Z}}+\breve{\mathbf{Z}}^{\prime}\left(\boldsymbol{\Sigma}_{1}^{-1} \otimes \mathbf{P}_{\breve{X}}\right) \breve{\mathbf{Z}}\right]^{-1}\left[\breve{\mathbf{Z}}^{\prime}\left(\boldsymbol{\Sigma}_{1}^{-1} \otimes \mathbf{P}_{\breve{X}}\right) \breve{\mathbf{y}}\right]
$$

Note that consistent estimates of $\boldsymbol{\Sigma}_{\omega}$ and $\boldsymbol{\Sigma}_{1}$ can be obtained using W2SLS and B2SLS residuals with:

$$
\begin{aligned}
& \hat{\boldsymbol{\sigma}}_{\omega_{j l}}^{2}=\left(\mathbf{y}_{j}-\mathbf{Z}_{j} \hat{\boldsymbol{\delta}}_{j, W 2 S L S}\right)^{\prime} \mathbf{H}\left(\mathbf{y}_{l}-\mathbf{Z}_{l} \hat{\boldsymbol{\delta}}_{l, W 2 S L S}\right) / n(T-1) \\
& \hat{\boldsymbol{\sigma}}_{1_{j l}}^{2}=\left(\mathbf{y}_{j}-\mathbf{Z}_{j} \hat{\boldsymbol{\delta}}_{j, B 2 S L S}\right)^{\prime} \mathbf{P}\left(\mathbf{y}_{l}-\mathbf{Z}_{l} \hat{\boldsymbol{\delta}}_{l, B 2 S L S}\right) / n
\end{aligned}
$$

The EC3SLS estimator is asymptotically equivalent to the full-information maximum-likelihood estimator. When the error terms have error component structure, unlike the case in the standard simultaneous equation system, the EC3SLS does not necessarily reduce to EC2SLS, even if all the structural equations are just identified.

It is also possible to transform equation (3.113) by using $\boldsymbol{\Omega}^{-1 / 2}$ from equation (3.120) to get:

$$
\mathbf{y}^{\mp}=\mathbf{Z}^{\mp} \boldsymbol{\delta}+\mathbf{u}^{\mp}
$$

where $\mathbf{y}^{\mp}=\boldsymbol{\Omega}^{-1 / 2} \mathbf{y}, \mathbf{Z}^{\mp}=\boldsymbol{\Omega}^{-1 / 2} \mathbf{Z}$, and $\mathbf{u}^{\mp}=\boldsymbol{\Omega}^{-1 / 2} \mathbf{u}$.

Now, using $\mathbf{X}^{\mp}=\mathbf{\Omega}^{-1 / 2}\left(\mathbf{I}_{G} \otimes \mathbf{X}\right)=\left(\boldsymbol{\Sigma}_{\omega}^{-1 / 2} \otimes \mathbf{H X}\right)+\left(\boldsymbol{\Sigma}_{1}^{-1 / 2} \otimes \mathbf{P X}\right)$ (see White, 1986) as a set of optimal instruments, the 3SLS estimator of equation (3.129) becomes the efficient three-stage least squares (E3SLS) given as: 


$$
\hat{\boldsymbol{\delta}}_{E 3 S L S}=\left(\mathbf{Z}^{\mp^{\prime}} \mathbf{P}_{X^{\mp}} \mathbf{Z}^{\mp}\right)^{-1}\left(\mathbf{Z}^{\mp^{\prime}} \mathbf{P}_{X^{\mp}} \mathbf{y}^{\mp}\right)
$$

\subsection{Spatial Analysis}

The diffusion of geographic information system (GIS) technology and the associated availability of geo-coded socio-economic data sets have created the need for a specialized method to deal with the distinguishing characteristics of such geographic data. Although there are many standard statistical techniques that can handle traditional data, they cannot be used to handle spatial data. Many results from the analysis of time series data do not apply to spatial data mainly because, due to the two-directional and two-dimensional nature, dependence and heterogeneity in space are qualitatively more complex than those in time dimension. Consequently, a number of spatial data analysis techniques have been developed in the last three decades (see Cliff and Ord, 1973, 1981;

Paelink and Klaassen, 1979; Upton and Fingleton, 1985; Anselin, 1988, 1992; Getis and Ord, 1992; Anselin and Getis, 1992; Griffith, 1992; Goodchild, Haining and Wise. 1993). These works on spatial data analysis can be divided into data-driven and model-driven approaches (Anselin 1990).

In the data-driven approach, all the techniques start from the assumption of a randomized distribution of spatial pattern (that is, any observed data value could occur equally likely at each location) and the spatial pattern, spatial structure, or form for the spatial dependence are derived from the data only without pre-conceived theoretical notion. Exploratory data analysis such as indices of spatial association, point pattern analysis, spatial adaptive filtering and spatial time series analysis are some of these techniques (Cliff and Ord, 1973, 1981; Upton and Fingleton, 1985; Getis and Ord, 1992). The model-driven approach, however, starts form a theoretical specification which is 
subsequently confronted with data. Most of the techniques under this category deal with estimation and specification diagnostics in spatial models (Paelink and Klaassen, 1979; Anselin, 1988; Griffith, 1992). There is also a growing literature on the methodological issues related to model specification, estimators and test statistics geared to spatial models (see Conley, 1996; Driscoll and Kraay, 1998; Pinkse and Slade, 1998; Kelejian and Prucha, 1999, 2004; Kapoor, Kelejian and Prucha, 2004).

In the section, a brief discussion of the issues related to measuring and testing of spatial autocorrelation as well as issues associated with spatial autoregressive problem is given. But, before proceeding to this discussion, first it is important to consider the concept of spatial proximity matrices.

\subsubsection{Spatial Weights Matrix}

One of the characteristics that distinguish spatial data from that of time series data is the spatial arrangement of observations. In contrast to the unambiguous notion of shift along the time axis, there is no corresponding concept in the spatial domain, especially when observations are located irregularly in space. Yet, in parallel to time series analysis, spatial stochastic processes are categorized as spatial autoregressive (SAR) and spatial moving average (SMA) processes (Anselin, 2001). The spatial linkages or proximity of observations are measured by defining a non-stochastic (fixed) and positive $n$ by $n$ spatial weight matrix, denoted by $\mathbf{W}$, where $\mathrm{n}$ is the number of observations. The spatial weight matrix, W, represents the strength of potential interactions between locations. The specification of the weight matrix, however, involves some arbitrariness. Actually, the determination of the proper specification for the elements of a spatial weight matrix is one of the difficult and controversial methodological issues in spatial data analysis. The 
specification of the weight matrix is also one of the points of contention in the literature, because the choice of spatial weights can have a substantive impact on the results of the analysis (Abrue, de Groot and Florax, 2004)

In the presence of spatial autocorrelation, the variance-covariance matrix contains too many parameters to be estimated using only cross-sectional data. The specification of the weight matrix is necessary to deal with this identification problem ${ }^{2}$. In the lattice approach of spatial analysis, the variance-covariance structure between observations is not modeled directly, but follows from the specification of the stochastic spatial process and the choice of the spatial weights matrix. For identification reasons the spatial weights matrix must be exogenous to the model. The empirical model becomes highly non-linear if the spatial weights matrix contains any of the exogenous or endogenous variables of the model. Consequently, most spatial weights matrices are based on contiguity or distance, since these are geography-based measures that are unambiguously exogenous, despite their lesser theoretical appeal (Anselin, 2001). The derivation of spatial weights from the location and spatial arrangement of observations must be carried out by means of geographic information system, since for all but the smallest data set a visual inspection of a map is impractical. Thus, general spatial weights matrix can be defined by a symmetric binary contiguity matrix, which can be generated from the topological information given by geographic information system (GIS) based on adjacency or distance criteria.

\footnotetext{
${ }^{2}$ Two main approaches - geo-statistical and lattice - have been used to deal with identification problem. In the geo-statistical approach, all pairs of location are sorted according to the distance that separate them, and the strength of the covariance (correlation) between them is expressed as a continuous function of this distance, in a so called variogram and semi-variogram. This perspective is seldom used in empirical economic since it necessitates an underlying process that is continuous over space (Anselin, 1996). The lattice approach assumes that the unit of analysis is a discrete entity or object. Objects are linked by a spatial pattern, expressed in terms of a spatial weights matrix.
} 
Given a set of $\mathrm{n}$ observations (locations), we build an $\mathrm{n}$ by $\mathrm{n}$ positive and symmetric matrix $\mathbf{W}$, where each element $w_{i j}$ represents a measure of proximity between observation (location) i and observation (location) j. According to the adjacency criteria, $w_{i j}$ is equal to one if observation (location) $\mathrm{i}$ is adjacent to observation (location) $\mathrm{j}$, and zero otherwise. This can be written more succinctly as:

$$
w_{i j}=\left\{\begin{array}{l}
1 \text { when } i \text { and } j \text { are neighbors (adjacent) } \\
0 \text { otherwise }
\end{array}\right.
$$

According to the distance criteria, $w_{i j}$ is equal to one if location $i$ and location $j$ are within a given distance, say, d, of each other, and zero otherwise. More succinctly:

$w_{i j}=\left\{\begin{array}{l}1 \text { for } d_{i j} \leq d, \text { where } d_{i j} \text { is the distance between } i \text { and } j \text { and } d \text { is a distance cut-off value } \\ 0 \text { otherwise }\end{array}\right.$ Note that by convention, all diagonal elements of the weights matrix $\left(w_{i i}\right)$ are set to zero. Since the weights matrix is utilized in the calculations of indicators during the exploratory analysis phase, it is useful to normalize its lines (rows) so that the sum of the weights of each line (row) equals one. Thus, the elements of a row-standardized weights matrix $\left(w_{i j}^{s}\right)$ can be written as;

$$
w_{i j}^{s}=\frac{w_{i j}}{\sum_{j=1}^{n} w_{i j}} .
$$

This ensures that all weights are between zero and one which helps simplifying a lot many calculations of spatial correlation indexes and also eases interpretation of 
operations with the weights matrix as an averaging of neighboring values. It also ensures that the spatial parameters in many spatial stochastic processes are comparable between models (Anselin, 1996)

It is also possible to define a general measure of weighted spatial proximity matrix in terms of some attribute value, say, $x_{j}$, and the binary spatial weights matrix $w_{i j}$. In both the adjacency and distance criteria, for example, a non-binary spatial weights matrix can be defined in a row-standardized form as

$$
w_{i j}^{n b}=\frac{w_{i j} x_{j}}{\sum_{j=1}^{n} w_{i j} x_{j}}
$$

Where $w_{i j}$ the spatial weights matrix as is defined either for the adjacency criteria or for the distance criteria and $x_{j}$ is any attribute value of interest.

Cliff and Ord $(1973,1981)$ have generalized this geographic approach of deriving weights to the so-called Cliff-Ord weights that consists of a function of the relative length of the common border, adjusted by the inverse distance between two observations. The idea of spatial proximity matrix can also be generalized to neighbors of higher order (the neighbors of neighbors) using a criteria analogous to the one adopted for the first order spatial proximity matrix.

\subsubsection{Spatial Autocorrelation}

A fundamental aspect of the exploratory analysis is the characterization of the spatial dependency, showing how the values are correlated in space. Spatial autocorrelation is a numerical summary of the observed spatial pattern that is largely employed in spatial statistics. Spatial autocorrelation exists whenever a variable exhibits 
a regular pattern over space and its values at a set of locations depend on the values of the same variables at other locations. Positive autocorrelation occurs when features that are similar in location are also similar in attributes, and negative autocorrelation occurs when features that are close in space are dissimilar in attributes. When attributes are independent of location, zero autocorrelation occurs (Anselin, 1996; Breschi, 1998). Following Anselin (1996), the existence of spatial autocorrelation can be more formally expressed by the following moment condition:

$$
\operatorname{Cov}\left(y_{i} y_{j}\right)=E\left(y_{i} y_{j}\right)-\left(E\left(y_{i}\right)\right)\left(E\left(y_{j}\right)\right) \neq 0, \text { for } \mathrm{i} \neq \mathrm{j}
$$

where $y_{i}$ and $y_{j}$ are observations on a random variable at location $i$ and $\mathrm{j}$. There are two main reasons for the existence of spatial autocorrelation or spatial dependence between regions. First, data collected on observations associated with spatial units such as counties may contain measurement error because the administrative boundaries for data collection do not reflect the underlying process generating the sample data (Anselin, 1988). Second, location and distance are important forces at work in human geography and market activity. Local information spillovers and spillover effects ensure the spread of local shocks to neighboring regions (Topa, 2001). Tobler's (1979) first law of geography also states that everything is related to everything else, but closer things more so, suggesting spatial dependence (spatial autocorrelation) to be the rule rather than exception. Thus, spatial autocorrelation analysis can be helpful in identifying spatial pattern.

Several indexes have been proposed in the spatial statistics literature to assess the presence of spatial autocorrelation or spatial dependence. These can be categorized into 
global and local indicators of spatial autocorrelation (see Cliff an Ord, 1973, 1981; Getis, 1992; Anselin, 1995).

\subsubsection{Global Indicators of Spatial Autocorrelation}

\section{Moran's I and Geary's C}

Moran's I is the most popular and the most commonly used measure of global spatial autocorrelation. Formally this statistic is given by:

$$
I=\frac{n}{S_{0}} \frac{\sum_{i}^{n} \sum_{j}^{n} w_{i j}\left(x_{i}-\bar{x}\right)\left(x_{j}-\bar{x}\right)}{\sum_{i}^{n}\left(x_{i}-\bar{x}\right)^{2}}, \text { for } \mathrm{i} \neq \mathrm{j}
$$

where $\mathrm{n}$ is the number of observations, $w_{i j}$ is the element in the spatial weights matrix $\mathbf{W}$ corresponding to the observation pair $(\mathrm{i}, \mathrm{j}), x_{i}$ and $x_{j}$ are observations for location $\mathrm{i}$ and $\mathrm{j}$ (with mean $\bar{x}$ ) and $S_{0}$ is a scaling constant

$$
S_{0}=\sum_{i}^{n} \sum_{j}^{n} w_{i j}
$$

which is the sum of all weights.

For a row-standardized spatial weights matrix, $S_{0}=\sum_{i}^{n} \sum_{j}^{n} w_{i j}=n$, because each row sums to one and hence equation (3.134) simplifies to a ratio of a spatial cross product to a variance that can be given by:

$$
I^{*}=\frac{\sum_{i}^{n} \sum_{j}^{n} w_{i j}\left(x_{i}-\bar{x}\right)\left(x_{j}-\bar{x}\right)}{\sum_{i}^{n}\left(x_{i}-\bar{x}\right)^{2}}, \text { for } \mathrm{i} \neq \mathrm{j}
$$

where $w_{i j}$ is now a row-standardized weights matrix.

or, in matrix notation 


$$
I^{*}=\frac{(\mathbf{x}-\overline{\mathbf{x}}) \mathbf{W}(\mathbf{x}-\overline{\mathbf{x}})}{(\mathbf{x}-\overline{\mathbf{x}})^{\prime}(\mathbf{x}-\overline{\mathbf{x}})}
$$

Similarly, Moran's I for higher order proximity matrices can be calculated as:

$$
I^{(h)}=\frac{n \sum_{i}^{n} \sum_{j}^{n} w_{i j}^{(h)}\left(x_{i}-\bar{x}\right)\left(x_{j}-\bar{x}\right)}{\sum_{i}^{n}\left(x_{i}-\bar{x}\right)^{2}}, \text { for } \mathrm{i} \neq \mathrm{j}
$$

where $\mathrm{h}$ is the lag.

Moran's index basically serves as a test where the null hypothesis is the spatial independence. It is very similar but not equivalent to a correlation coefficient and it is not centered on 0 . To estimate the significance of the index it will be necessary to associate it to a statistical distribution. Generally, there are two main approaches - the normal distribution assumption and the randomization assumption ${ }^{3}$. The expected value and variance of Moran's I for the samples of $\mathrm{n}$ could then be calculated according to the assumed pattern of the spatial data distribution (Cliff and Ord, 1973, 1981; Goodchild, 1986). Accordingly, the mean and the variance under the normal assumption are given by

$$
E_{N}(I)=-\left(\frac{1}{n-1}\right)
$$

and

$$
\operatorname{Var}_{N}(I)=\left(\frac{n^{2} S_{1}-n S_{2}+3 S_{0}^{2}}{S_{0}^{2}\left(n^{2}-1\right)}\right)-\left(-\frac{1}{n-1}\right)^{2}
$$

\footnotetext{
${ }^{3}$ Under the normal assumption, it is assumed that the $\left\{x_{i}\right\}$ are the results of $\mathrm{n}$ independent drawings from a normal population (s), under the randomization assumption, however, whatever the underlying distribution of the population(s), the observed values of the index are considered relative to the set of all possible values which the index could take. There are $n$ ! (n permutation) such values and each value observed could equally likely have occurred at all locations.
} 
The mean and variance of the sampling distribution of Moran's I arising from the randomization process is given by:

$$
E_{R}(I)=-\left(\frac{1}{n-1}\right)
$$

and

$$
\operatorname{var}_{R}(I)=\frac{n\left[\left(n^{2}-3 n+3\right) S_{1}-n S_{2}+3 S_{0}^{2}\right]-b_{2}\left[\left(n^{2}-n\right) S_{1}-2 n S_{2}+6 S_{0}^{2}\right]}{S_{0}^{2}(n-1)(n-2)(n-3)}-\left(-\frac{1}{n-1}\right)^{2}
$$

where

$$
\begin{aligned}
& b_{2}=\frac{n \sum_{i}^{n}\left(x_{i}-\bar{x}\right)^{4}}{\left(\sum\left(x_{i}-\bar{x}\right)^{2}\right)^{2}}, \mathrm{~S}_{0}=\sum_{\mathrm{i}}^{\mathrm{n}} \sum_{\mathrm{j}}^{\mathrm{n}} \mathrm{w}_{\mathrm{ij}}, \mathrm{S}_{1}=\frac{1}{2} \sum \sum\left(w_{i j}+w_{j i}\right)^{2}, S_{2}=\sum_{i}^{n}\left(w_{i .}+w_{. i}\right)^{2}, \text { and } \\
& \mathrm{w}_{\mathrm{i} .}=\sum_{j}^{n} w_{i j} \text { and } w_{. j}=\sum_{i}^{n} w_{i j}
\end{aligned}
$$

Note that $w_{. i}$ is the sum of column $\mathrm{i}$ of the weights matrix which is equivalent to the expression given by $w_{. j}=\sum_{i}^{n} w_{i j}$.

From equations (3.137) and (3.139) we can see that the expected value of Moran's I is negative and it depends only on the sample size (n). The mean, however, approaches zero as the sample size increases. Whereas a Moran's I coefficient value greater than its expected value (the theoretical mean) indicates therefore positive spatial autocorrelation, a smaller value of Moran's I coefficient indicates a negative spatial autocorrelation. The test of the null hypothesis $\left(\mathrm{H}_{0}\right)$ of no spatial autocorrelation against the alternative $\left(\mathrm{H}_{1}\right)$ that the data are spatially uncorrelated, however, is typically based on the standardized statistic (z-value) that can be computed as follows: 


$$
Z_{I}=\frac{I-E(I)}{\sqrt{\operatorname{Var}(I)}}
$$

Cliff and Ord (1981) show that the z-value under the normal as well as the randomization assumptions follow asymptotically a standard normal distribution so that their significance can be judged by means of standard normal table.

The standardized Moran's I is positive when the observed value of locations within a certain distance tend to be similar, negative when they tend to be dissimilar and approximately zero when the observed values are arranged randomly and independently over space (Goodchild, 1986). This would mean that positive values (between 0 and +1 ) $\mathrm{Z}_{\mathrm{I}}$ indicate direct correlation, negative values (between -1 and 0 ) indicate inverse correlation and zero value indicates no correlation. The implicit hypothesis of the calculation of the Moran index is the stationarity of first and second order, and the index loses its validity when calculated for non-stationary data. When there is a non-stationarity of first order (trend), the neighbors will tend to have closer values than the ones more distant because each value is compared to the global average, inflating the index. The autocorrelation function continues to decay even after surpassing the distance where there are local influences if the data is non-stationary.

Another measure of spatial autocorrelation is Geary's C. Unlike Moran's I, Geary's $\mathrm{C}$ is based on the weighted sum of square difference between observations and not between each point and the global average. This statistic is defined by:

$$
C=\frac{(n-1)}{2 S_{0}} \frac{\sum_{i}^{n} \sum_{j}^{n} w_{i j}\left(x_{i}-x_{j}\right)^{2}}{\sum_{i}^{n}\left(x_{i}-\bar{x}\right)^{2}}=\frac{(n-1) \sum_{i}^{n} \sum_{j}^{n} w_{i j}\left(x_{i}-x_{j}\right)^{2}}{2 \sum_{i}^{n} \sum_{j}^{n} w_{i j}\left(\sum_{i}^{n}\left(x_{i}-\bar{x}\right)^{2}\right)}
$$


The first moment (mean) and the second moment (variance) of Geary's C for samples of size $\mathrm{n}$ could be calculated according to the assumed pattern of spatial data distribution (see Cliff and Ord, 1973, 1981). Thus, under the normality assumption the expected value and the variance are, respectively, given by:

$$
E_{N}(C)=1
$$

and

$$
\operatorname{Var}_{N}(C)=\frac{\left(2 S_{1}+S_{2}\right)(n-1)-4 S_{0}^{2}}{2(n+1) S_{0}^{2}}
$$

The corresponding expressions under the randomization assumption are as follows:

$$
E_{\mathrm{R}}(C)=1
$$

and

$$
\begin{gathered}
\operatorname{Var}_{R}(C)=\left\{(n-1) S_{1}\left[n^{2}-3 n+3-(n-1) b_{2}\right]-\frac{1}{4}(n-1) S_{2}\left[n^{2}+3 n-6-\left(n^{2}-n+2\right) b_{2}\right]\right. \\
\left.+S_{0}^{2}\left[n^{2}-3-(n-1)^{2} b_{2}\right]\right\} \div n(n-2)(n-3) S_{0}^{2}
\end{gathered}
$$

where $\mathrm{S}_{0}, \mathrm{~S}_{1}, \mathrm{~S}_{2}$, and $\mathrm{b}_{2}$ are as defined above.

A value of $\mathrm{C}$ greater than one (the theoretical mean) shows that the observed values of locations within certain distance tend to be dissimilar, whereas a value of $\mathrm{C}$ smaller than one indicates similar trends in the observed values of locations within certain distance. Like the Moran's I, Geary's C is also asymptotically normally distributed as $n$ increases.

Hence, the normal test for the null hypothesis of no spatial autocorrelation between observed values over the $\mathrm{n}$ locations can be conducted based on the standardized Geary's C. 
One of the main limitations of these measures of spatial autocorrelations is that once the weights matrix has been specified, the size and the shape of counties (locations) in the system, and the relative strength of links between counties (say, roads and rail links, for example) are completely ignored. The measures are, therefore, invariant under certain topological transformation of the underlying county (location) structure (Cliff and Ord, 1973).

\section{Spatial Association (G Statistic)}

The spatial statistic $\mathrm{G}(\mathrm{d})$ is a distance-based measure of global spatial association proposed by Getis and Ord (1992) which can be computed by defining a set of neighbors for each location as those observations that fall within a critical distance (d) from the locations. Different spatial weights matrices can be constructed for different values of the critical distance (d). The G(d) statistic is similar to the Moran's I and Geary's C in that it assesses a global pattern of clustering, summarizing into one value. But, like all distance-based measures it is only applicable to positive observations. Formally, for a given critical distance (d), G(d) is defined as

$$
G(d)=\frac{\sum_{i}^{n} \sum_{j}^{n} w_{i j}(d) x_{i} x_{j}}{\sum_{i}^{n} \sum_{j}^{n} x_{i} x_{j}}
$$

where $w_{i j}(d)$ stand for the ijth element of the symmetric (not standardized) spatial weights matrix for distance $\mathrm{d}$, and $x_{i}$ is the value observed at location $i$. Similar to Moran's I and Geary's C, the significance of this statistic can be assessed by means of a standardized z-value, $(\mathrm{Z}(\mathrm{G}))$, obtained in the usual fashion. The theoretical mean and the variance for the $G(d)$ statistic are computed under the randomization assumption and it 
can be shown that $\mathrm{Z}(\mathrm{G})$ tends to a standard normal vitiate in the limit (Getis and Ord, 1992). A high positive $Z(G)$ value indicates that the spatial pattern are dominated by clusters of high values, while a high negative $Z(G)$ value indicates that the spatial pattern is dominated by clusters of small values. This interpretation contrasts with the interpretation of Moran's I and Geary's C, where positive spatial autocorrelation refers to the clustering of either large or small values, and where negative spatial autocorrelation is a totally different concept (Anselin, 1992).

We can also use Variogram and Moran Scatter Plot as additional tools to visualize spatial dependence.

\subsubsection{Local Indicators of Spatial Association (LISA)}

The global indicators of spatial autocorrelation, such as Moran’s I, Geary’s C, and Getis and Ord's G(d), provide a unique number as a measure of spatial association for the whole data set, which is useful for the characterization of the study area as a whole. The test on the null hypothesis of no spatial autocorrelation using these measures is based on the important assumption of stationarity or structural stability over space. This may, however, be unrealistic, especially when we deal with a great number of areas (observations), in that it is very likely that different types of spatial association and that

local maximum of spatial autocorrelation will appear where the spatial dependence is stronger. Hence, it is desirable to examine the local pattern of spatial associations more carefully and some indicators have been proposed to detect these local spatial associations with allowance for local instabilities in over all spatial association (see Anselin, 1992, 1993, 1995; Getis and Ord, 1992). 
Anselin (1995) suggested local Moran's I and local Geary's C as alternative measures of spatial association for individual spatial unit or observation which have some advantages over the Gi(d) statistic suggested by Getis and Ord (1992) which is also discussed in the next subsection.. These local indicators produce a specific value for each area or observation, allowing the identification of groupings.

\section{Local Spatial Association - Local Moran}

As given in Anselin (1995), Moran's local index for each observation i can be defined by:

$$
I_{i}=Z_{i} \sum_{j \neq i}^{n} w_{i j} Z_{j}
$$

where $Z_{i}$ and $Z_{j}$ are the standardized (normalized) values (with mean zero and variance one) of the attribute and $w_{i j}$ is a row-standardized weights matrix. In that case the local Moran's I is expressed as a product of $Z_{i}$ and the average of the values of the attribute in surrounding locations. Following Anselin (1995), the average of local Moran's I is exactly the same as the corresponding global Moran's I that can be given by:

$$
I_{i}=\frac{\sum_{i}^{n} \sum_{j \neq i}^{n} w_{i j} Z_{i} Z_{j}}{S_{0}^{2} \sum_{i}^{n} \sum_{j \neq i}^{n} w_{i j}}=\frac{1}{n} \sum_{i}^{n}\left(Z_{i} \sum_{j \neq i}^{n} w_{i j} Z_{j}\right)=\frac{1}{n} \sum_{i}^{n} I_{i},
$$

where, given the row-standardized $w_{i j}$ and the normalized $Z_{i}, S_{0}^{2}=1$ and $\sum_{i}^{n} \sum_{j \neq i}^{n} w_{i j}=n$.

The statistical significance of Moran's local index is computed similar to the global index case - pseudo-significance test. For each location, the local index is calculated and by holding that index fixed the remaining values of the other locations are 
randomly permuted until a pseudo-distribution that can be used to compute the parameters of the significance is achieved. Each permutation produces a new spatial arrangement where the values are redistributed among the locations and each re-sampled data set can be selected from the population randomly. Since only one of the arrangements corresponds to the observed situation, an empirical distribution can be built for $I_{i}$. That is, by calculating the proportion of data permutations in the data set that have emulated $I_{i}$ less than or equal to or greater than the actual $I_{i}$, a p-value can be obtained. Note that $Z_{i}$ is fixed in each emulated $I_{i}$. Thus, p-value for the distribution can easily be obtained by calculating the proportion of permutations in the data set that have emulated the average $I_{i}$ less than or equal to or greater than the average attribute or observations surrounding locations $i$. The p-values obtained in such a manner can offer the basis for testing the null hypothesis of randomly distributed values over space (that is, the average of the observations surrounding location $i$ is in no extreme).

Once the significance level $\mathrm{p}$-values are obtained, the interpretation of the $I_{i}$ statistic is straightforward. Whereas a large $\mathrm{p}$-value (such as $\mathrm{p}>0.95$ ) indicates that location $\mathrm{i}$ is associated with relatively low values of surrounding locations, a small pvalue (such as $\mathrm{p}<0.005$ ) indicates that location $\mathrm{i}$ is associated with the relatively high values of the surrounding locations. Again, once the statistical significance of Moran's local index has been established, it is possible to generate a map indicating locations that present local correlation significantly from the rest of the data. Besides, together with the information from the observed values at location $\mathrm{i}$ and the other statistic, $I_{i}$ can be employed to identify the nature of spatial association that night exist.

\section{Local Spatial Association - Local Geary}


Unlike local Moran's I which provides the test of agglomerative cluster, local Geary's C offers a basis for test of extremes of dissimilarity of similarity between the ith observed value and its surrounding values. This statistic can be defined by:

$$
C_{i}=Z_{i} \sum_{j \neq i}^{n} w_{i j}\left(Z_{i}-Z_{j}\right)^{2}
$$

where all the variables on the right-hand side of the expression are as defined in equation (3.148). Note that local Geary statistic is different from Local Moran statistic in that it is a measure of the weighted sum of squared differences between the observed values at location $\mathrm{i}$, and the values at surrounding locations. The calculation of the pseudosignificance level p-value, however, is similar to that of local Moran's. Following Anselin (1995), the local Geary statistic $\left(\mathrm{C}_{\mathrm{i}}\right)$ can also be related to the global Geary's C as follows:

$$
C=\frac{(n-1)}{2 S_{0}} \frac{\sum_{i}^{n} \sum_{j}^{n} w_{i j}\left(Z_{i}-Z_{j}\right)^{2}}{\sum_{i}^{n} \sum_{j}^{n} w_{i j}}=\frac{(n-1)}{2 n^{2}} \sum_{i}^{n} \sum_{j}^{n} w_{i j}\left(Z_{i}-Z_{j}\right)^{2}=\frac{(n-1)}{2 n^{2}} \sum_{i}^{n} C_{i}
$$

where all the variables are as defined before.

Inference in the $C_{i}$ statistic is based on the computation of p-value for the individual $C_{i}$ statistic which can be done by the same permutation approach used for the $I_{i}$ statistic outlined above. The $C_{i}$ statistic allows the test of similarity ('+ +' or '- - ') and dissimilarity ('+ -' or '- +') of local pattern and once the p-values are generated, interpretation of the $C_{i}$ statistic is straightforward. Whereas a large p-value (such as $\mathrm{p}>0.95$ ) indicates a small $C_{i}$, which suggests a negative spatial association (i.e., dissimilarity) of observation i with its surrounding observations, a small p-value (such as 
$\mathrm{p}<0.005$ ) indicates a large $C_{i}$ in extremes, which in turn suggests a positive spatial association (i.e., similarity) of observation i with its surrounding observations.

Bao, Hery, Barkley and Brook (1995) have identified four categories of spatial associations between location I and nearby locations by simply combining local Moran statistic and local Geary statistic as follows:

High p-value of $I_{i}$ and low p-value of $C_{i} \rightarrow$ negative spatial association(+ -)

Low p-value of $\mathrm{I}_{\mathrm{i}}$ and low p-value of $\mathrm{C}_{\mathrm{i}} \rightarrow$ negative spatial association(- + )

High p-value of $\mathrm{I}_{\mathrm{i}}$ and high p-value of $\mathrm{C}_{\mathrm{i}} \rightarrow$ positive spatial association(- -)

Low p-value of $\mathrm{I}_{\mathrm{i}}$ and high p-value of $\mathrm{C}_{\mathrm{i}} \rightarrow$ positive spatial association $(++)$

\section{Local Spatial Association - The Gi(d) Statistic}

Getis and Ord (1992) suggested the $\mathrm{G}_{\mathrm{i}}(\mathrm{d})$ statistic as a measure of spatial association for each individual spatial unit or observation. This statistic is developed for tests of hypothesis about the spatial concentration of the sum of $\mathrm{x}$ values associated with the $\mathrm{j}$ location within d distance of the ith location. Thus, it indicates the extent to which that the ith location is surrounded by high values or low values for the variable under consideration. Formally, following Getis and Ord (1992), the $\mathrm{G}_{\mathrm{i}}(\mathrm{d})$ statistic for observation I and distance $\mathrm{d}$ can be expressed as:

$$
G_{i}(d)=\frac{\sum_{j, j \neq i}^{n} w_{i j}(d) x_{j}}{\sum_{j, j \neq i}^{n} x_{j}}
$$

Note that the value of the observation itself is not included in the statistic. This statistic is simply the ratio of the sum of the values in the surrounding locations to the sum of the values in the data set as a whole except the location under consideration. Getis 
and Ord $\left(1992\right.$, however, have suggested the so-called $G_{i}^{*}(d)$ statistic that includes the value at location $i$, which can be defined as:

$$
G_{i}^{*}(d)=\frac{\sum_{j}^{n} w_{i j}(d) x_{j}}{\sum_{j}^{n} x_{j}}
$$

The $G_{i}(d)$ statistic should be interpreted as a measure of clustering of like values around a location, irrespective of the value at that location. In contrast, the $G_{i}^{*}(d)$ statistic includes the value at the location within the measure of clustering and is thus more in accordance with the usual interpretation (Anselin, 1992).

The theoretical mean and the variance of the $G_{i}(d)$ statistic as developed in Getis and Ord (1992) can be given by;

$$
E\left(G_{i}(d)\right)=\frac{\sum_{j, j \neq i}^{n} w_{i j} E\left(x_{j}\right)}{\sum_{j, j \neq i}^{n} x_{j}}
$$

and

$$
\operatorname{Var}\left(G_{i}(d)\right)=\frac{R_{i}\left(n-1-R_{i}\right)}{(n-1)^{2}(n-2)} \frac{k_{i 2}}{k_{i 1}^{2}}
$$

where $w_{i j}$ is a symmetric binary weights matrix defined as:

$$
\begin{gathered}
w_{i j}=\left\{\begin{array}{l}
1 \text { if location } \mathrm{j} \text { is within distance } \mathrm{d} \text { from location } \mathrm{i} \\
0 \text { otherwise }
\end{array}\right. \\
R_{1}=\sum_{j, j \neq i}^{n} w_{i j}, \quad k_{i 1}=\sum_{j, j \neq i}^{n} x_{j}^{2}, \quad k_{i 2}=\sum_{j, j \neq i}^{n} x_{j}^{2} /(n-1)-k_{i 1}^{2}
\end{gathered}
$$


$\mathrm{n}$ is the number of observations, and $x_{i}$ is the observed value at location $\mathrm{i}$.

Inference for $G_{i}(d)$ and $G_{i}^{*}(d)$ statistics is based on the computation of a standardized zvalue, which asymptotically tends to a normal distribution (Anselin, 1995). The z-values for the case where $i$ is not equal to $j$ and where $i$ is equal to $j$ is allowed, respectively, can be computed as follows:

$$
Z\left(G_{i}(d)\right)=\frac{G_{i}(d)-E\left(G_{i}(d)\right)}{\sqrt{\operatorname{Var}\left(G_{i}(d)\right)}}=\frac{\sum_{j, j \neq i}^{n} w_{i j}\left(x_{j}-\bar{x}_{i}\right)}{S_{i} \sqrt{R_{i}\left(n-1-R_{i}\right) /(n-2)}}
$$

and

$$
Z\left(G_{i}^{*}(d)\right)=\frac{G_{i}^{*}(d)-E\left(G_{i}^{*}(d)\right)}{\sqrt{\operatorname{Var}\left(G_{i}^{*}(d)\right)}}=\frac{\sum_{j}^{n} w_{i j}\left(x_{j}-\bar{x}\right)}{S_{i}^{\prime} \sqrt{R_{i}^{\prime}\left(n-1-R_{i}^{\prime}\right) /(n-1)}}
$$

where

$$
\begin{aligned}
& \bar{x}_{i}=\frac{1}{n-1} \sum_{j, j \neq i}^{n} x_{j}, \bar{x}=\frac{1}{n} \sum_{j}^{n} x_{j}, \quad R_{i}=\sum_{j, j \neq i}^{n} w_{i j}(d), \quad R_{i}^{\prime}=\sum_{j}^{n} w_{i j}(d), \\
& S_{i}=\sqrt{\frac{1}{n-1} \sum_{j, j \neq i}^{n}\left(x_{j}-x_{i}\right)^{2}}, S_{i}^{\prime}=\sqrt{\frac{1}{n} \sum_{j}^{n}\left(x_{j}-\bar{x}\right)^{2}}
\end{aligned}
$$

and $x_{i}$, and $w_{i j}$ are as defined above. When the weights $\left\{w_{i j}\right\}$ are not binary, however, the z-value for $G_{i}(d)$ and $G_{i}^{*}(d)$ statistics are different and they can be defined as follows:

$$
Z\left(G_{i}(d)\right)=\frac{\sum_{j, j \neq i}^{n} w_{i j}(d)\left(x_{j}-R_{i}^{\prime} \bar{x}_{i}\right)}{S_{i} \sqrt{\left((n-1) S_{i 1}-R_{i}^{2}\right) /(n-2)}}
$$


and

$$
Z\left(G_{i}^{*}(d)\right)=\frac{\sum_{j}^{n} w_{i j}(d)\left(x_{j}-R_{i}^{\prime} \bar{x}\right)}{S \sqrt{\left(n S_{i 1}^{\prime}-1-R_{i}^{\prime 2}\right) /(n-1)}}
$$

where $R_{i}, R_{i}^{\prime}, S_{i}, S_{i}^{\prime}, x_{i}, \bar{x}_{i}, \bar{x}$ are as defined above, and $S_{i 1}=\sum_{j, j \neq i}^{n} w_{i j}^{2}$ and $S_{i 1}^{\prime}=\sum_{j}^{n} w_{i j}^{2}$.

Similar to the interpretation of the global $G$ statistics, a positive $Z\left(G_{i}(d)\right)$ indicates that location I is surrounded by relatively large values (a spatial clustering of high values), while a negative $Z\left(G_{i}(d)\right)$ indicates that location $\mathrm{i}$ is surrounded by relatively small values (a spatial clustering of low values). The interpretation for $Z\left(G_{i}^{*}(d)\right)$ is the same to that of $Z\left(G_{i}(d)\right)$, apart from the fact that $Z\left(G_{i}^{*}(d)\right)$ does not exclude the observation $\mathrm{i}$ from the index. Note that although the $G$ statistics can be used to identify spatial agglomerative pattern with high-value clusters or low-value clusters, it cannot identify the type of spatial association that results from a high-value attribute surrounded by lowvalue attributes and vice versa (i.e., negative spatial association). In this respect, the interpretation of the $\mathrm{G}$ statistic is similar to that of local Moran but different from that of local Geary. Generally, although the different statistics are developed for the test of spatial association, they are applied for different purposes. For example, while both the G statistic and local Moran allow the test of hypothesis about the spatial concentration of high-values or low-values, local Geary allow the test of similarity or dissimilarity of local pattern. One also should be cautious in interpreting the results because the analytical results may be sensitive to the number of units included and the weights matrix defined. 


\subsection{Spatial Autoregressive Process Models}

The investigation of regression residuals in the search of signs of a spatial structure is the first step in the analysis of spatial data. The usual graphical analysis tools and the residual mapping can provide the first indication that the observed values are more correlated than would be expected under random assignment. In this case, the presence of spatial clustering can be tested by using the spatial correlation tests such as Moran, Geary or G statistic, on the residuals. Although such tests can detect the presence of spatial clustering, however, they do not explain why such clustering occurs, nor do they explain which factors determine its shape and strength. In other wards, the alternative hypothesis of spatial autocorrelation is too vague to be useful in the construction of theory. Rather, spatial autoregressive process, the process that expresses how observations at each location depend on values at neighboring locations -the spatial lag, is the relevant concept that formalizes the way in which the spatial association is generated (Anselin, 1992).

Spatial dependence is one of the main problems in stochastic processes and can be caused by a variety of measurement problems that are associated with the arbitrary delineation of spatial units of observation (such as counties boundaries, census tracts), problems of spatial aggregation, and the presence of spatial externalities and spillover effects. The existence of spatial dependence would then mean that the observations contain less information than if there had been spatial independence. Consequently, hypothesis tests and the statistical properties for estimators in the standard econometric approach will not hold. Thus, in order to obtain approximately the same information as in 
the case of spatial independence, the spatial dependence needs to be explicitly quantified and modeled.

\subsubsection{Spatial Regression Models}

The explicit inclusion of spatial dependence in regression models can be done in different ways. Anselin (2003), for example, attempts to extend the earlier work on spatial dependence and he notice that "the standard taxonomy of spatial autoregressive lag and error models commonly applied in spatial econometrics (Anselin, 1988) is perhaps too simplistic and leaves out other interesting possibilities for mechanisms through which phenomena or actions at a given location affects actors or properties at other locations" In this extension, he makes a distinction between global and local range of dependence which have implications for the econometric specifications of spatially lagged dependent variable (Wy), spatially lagged explanatory variables (WX) and spatially lagged error terms $(\mathbf{W u})$. The distinction between the global and local effects models depends upon the assumption on the underlying spatial process. The spatial regression models with global effects are based on the principle that the underlying spatial process on the analyzed data is stationary. This means that the spatial autocorrelation patterns of the data sets can be captured in one parameter only. The spatial regression models with local spatial effects, however, are based on the principle that the underlying spatial process on the analyzed data is non-stationary and hence spatial autocorrelation patterns of the data cannot be captured by one parameter only. Thus, when the spatial process is non-stationary, the coefficients of regression need to reflect the spatial heterogeneity. 
The conditional autoregressive (CAR) model and the simultaneous models, spatial autoregressive (SAR) and the spatial moving average (SMA), models are the most commonly employed types of models in spatial statistics and spatial econometrics. In the conditional model, a random variable at a location is conditioned on the observations on that random variable at neighboring locations. The latter are treated as exogenous and can be exploited to construct optimal prediction for the random variable at unobserved locations (Anselin, 2003). The inverse covariance matrix for this model is constructed by $(\mathbf{I}-\rho \mathbf{D}) / \sigma^{2}$ where $\mathbf{D}$ is a binary spatial weights matrix. This type of model is appropriate for studies involving first-order dependency which are most common in spatial statistics. In the simultaneous models (SAR and SMA models), however, the focus is on the explanation of the complete interactions between all observations or locations observed simultaneously. The covariance structure in such models is compatible with the spatial ordering and the inverse covariance matrix is constructed by $(\mathbf{I}-\rho \mathbf{W})^{\prime}(\mathbf{I}-\rho \mathbf{W}) / \sigma^{2}$ where $\mathbf{W}$ is a row-standardized spatial weights matrix. The simultaneity in these models follows from the nature of dependence in space which is two-directional. As a result, each location is in turn a neighbor for its neighbors, so that the effect of the neighbors has to be treated as endogenous (Anselin, 2003). The SAR model is appropriate for studies involving first-order as well as second-order dependency which are most common in regional studies. This research also follows the regional studies tradition. In the next subsection, the spatial dependence (spatial global effects) will be incorporated into the standard simultaneous equations model (given in equation (3.19)), first in the context of cross-sectional setting and then the extension to panel data will follow. 


\subsubsection{Spatial Dependence in Cross-Sectional Models}

There are two distinct ways of incorporating spatial dependence into the standard linear regression models: as an additional explanatory variable in the form of spatially lagged dependent variables $\left(\mathbf{W}_{\mathbf{j}}\right)$ (spatial lag), or in the error structure $\left(E\left[\mathbf{u}_{i} \mathbf{u}_{j}\right] \neq \mathbf{0}\right)$ (spatial error) (Anselin, 2001).

\section{Spatial Lag Model}

The spatial lag model combines the spatial dependence in the form of a spatial lag term with the usual linear explanation of a dependent variable by a set of explanatory variables. It is similar to the inclusion of a serially autoregressive term for the dependent variable in a time series context (Anselin and Bera, 1996; LeSage, 1999). This model is more appropriate when the focus of interest is the assessment of the existence and strength of spatial interaction. Anselin (1993) referred this model as the spatial autoregressive model with substantive spatial dependence.

Formally, a spatial lag model, in the context of single equation and in a cross-sectional setting, is expressed as:

$$
\mathbf{y}=\rho \mathbf{W} \mathbf{y}+\mathbf{X} \boldsymbol{\gamma}+\mathbf{u}
$$

where $\mathbf{y}$ is an $\mathrm{n}$ by 1 vector of observations on the dependent variable, $\mathbf{W y}$ is the corresponding spatial lagged dependent variable for weights matrix $\mathbf{W}, \mathbf{X}$ is $\mathrm{n}$ by $\mathrm{K}$ matrix of observations on the explanatory variables, $\mathbf{u}$ is an $\mathrm{n}$ by 1 vector of error terms, $\rho$ is the spatial autoregressive parameter and $\gamma$ is a $\mathrm{K}$ by 1 vector of regression coefficients. The parameter $\rho$ measures the degree of spatial dependence inherent in the data. As this model combines the standard regression model with a spatially lagged 
dependent variable, it is also called a mixed regressive-spatial autoregressive model (Anselin, 1998).

The spatial single equation model in equation (3.160) can be extended to a system of spatially interrelated cross sectional equations corresponding to $\mathrm{n}$ cross sectional units. But, first note that after normalization and rearranging, the system of equations given in equation (3.19) can be rewritten as:

$$
\mathbf{Y}=\mathbf{Y B}+\mathbf{X} \boldsymbol{\Gamma}+\mathbf{U}
$$

with

$$
\mathbf{Y}=\left(\mathbf{y}_{1}, \ldots, \mathbf{y}_{G}\right) \quad \mathbf{X}=\left(\mathbf{x}_{1}, \ldots, \mathbf{x}_{K}\right) \quad \mathbf{U}=\left(\mathbf{u}_{1}, \ldots, \mathbf{u}_{G}\right)
$$

where $\mathbf{y}_{\mathrm{j}}$ is the $\mathrm{n}$ by 1 vector of cross sectional observations on the dependent variable in jth equation, $\mathbf{x}_{1}$ is an $\mathrm{n}$ by 1 vector of cross sectional observations on the lth exogenous variable, $\mathbf{u}_{\mathrm{j}}$ is an $\mathrm{n}$ by 1 vector of error terms in the jth equation, and $\mathbf{B}$ and $\boldsymbol{\Gamma}$ are correspondingly defined parameter matrices of dimension $\mathrm{G}$ by $\mathrm{G}$ and $\mathrm{K}$ by $\mathrm{G}$, respectively. B is a diagonal matrix, unlike the case in equation (3.19). Following Kelejian and Prucha (2004), the spatial lag dependent variables can be incorporated in equation (3.161) as follows:

$$
\mathbf{Y}=\mathbf{Y B}+\mathbf{X} \boldsymbol{\Gamma}+\mathbf{W Y} \boldsymbol{\Lambda}+\mathbf{U}
$$

where $\mathbf{W}$ is an $\mathrm{n}$ by $\mathrm{n}$ weights matrix of known constants, and $\boldsymbol{\Lambda}$ is a $\mathrm{G}$ by $\mathrm{G}$ matrix of parameters. Note that the spatial global spillover effects in the endogenous variables is modeled via $\mathbf{W Y}$, with $\mathbf{W} \mathbf{y}_{\mathbf{j}}$ representing the spatial lag in the jth equation for $\mathbf{j}=1, \ldots$, G. The ith element of the vector of the spatial lag, $\mathbf{W} \mathbf{y}_{\mathbf{j}}$, can be computed as:

$$
\mathbf{W} y_{i j}=\sum_{r=1}^{n} w_{i r} y_{r j}
$$


where

$$
w_{i r}=\left\{\begin{array}{l}
1 \text { when } i \text { and } r \text { are neighbors (adjacent) } \\
0 \text { otherwise }
\end{array}\right.
$$

Note that the spatial interactions in the system are determined by the nature of the $\boldsymbol{\Lambda}$ matrix. Specifying $\boldsymbol{\Lambda}$ as not a diagonal matrix of parameters allows the jth endogenous variable to depend on its spatial lag as well as on the spatial lags of the other endogenous variables in the model. If, however, there is a theoretical reason to believe that the jth endogenous variable depends either only on the spatial lags in the other endogenous variables in the model or only on its own spatial lag, then $\boldsymbol{\Lambda}$ should be specified as a diagonal matrix or as an identity matrix, respectively.

The system in equation (3.162) can be expressed in a form where its solution for the endogenous variables is clearly revealed. First, consider the following vector transformations:

$$
\begin{aligned}
\operatorname{vec}(\mathbf{Y}) & =\operatorname{vec}(\mathbf{Y B})+\operatorname{vec}(\mathbf{X} \boldsymbol{\Gamma})+\operatorname{vec}(\mathbf{W Y} \mathbf{\Lambda})+\operatorname{vec}(\mathbf{U}) \\
& =\left(\mathbf{B}^{\prime} \otimes \mathbf{I}\right) \operatorname{vec}(\mathbf{Y})+\left(\boldsymbol{\Gamma}^{\prime} \otimes \mathbf{I}\right) \operatorname{vec}(\mathbf{X})+\left(\mathbf{\Lambda}^{\prime} \otimes \mathbf{W}\right) \operatorname{vec}(\mathbf{Y})+\operatorname{vec}(\mathbf{U})
\end{aligned}
$$

Letting $\mathbf{y}=\operatorname{vec}(\mathbf{Y}), \quad \mathbf{x}=\operatorname{vec}(\mathbf{X})$, and $\mathbf{u}=\operatorname{vec}(\mathbf{U}), \quad$ it follows from equation (3.164) that

$$
\begin{aligned}
\mathbf{y} & =\left(\mathbf{B}^{\prime} \otimes \mathbf{I}\right) \mathbf{y}+\left(\boldsymbol{\Gamma}^{\prime} \otimes \mathbf{I}\right) \mathbf{x}+\left(\boldsymbol{\Lambda}^{\prime} \otimes \mathbf{W}\right) \mathbf{y}+\mathbf{u} \\
& =\left[\left(\mathbf{B}^{\prime} \otimes \mathbf{I}\right)+\left(\mathbf{\Lambda}^{\prime} \otimes \mathbf{W}\right)\right] \mathbf{y}+\left(\boldsymbol{\Gamma}^{\prime} \otimes \mathbf{I}\right) \mathbf{x}+\mathbf{u}
\end{aligned}
$$

The mixed regressive-spatial autoregressive specification given above can be interpreted in three different ways. First, in the specification given in equation (3.165), the interest is in finding out how the each of variables in $\mathbf{y}$ relate to their values in the surrounding locations (spatial own lags), the values of the other endogenous variables in the surrounding locations (cross spatial lags) and the values of the other endogenous 
variables in the respective location, while controlling for the influence of other predetermined (exogenous) variables. The second perspective is when the interest is to detect the relations between the dependent variables y and the predetermined (exogenous) variables $\mathrm{x}$, after all the spatial effects and the other endogenous variables effects are controlled for or filtered out. Formally, this can be expressed as:

$$
\begin{gathered}
\mathbf{y}-\left[\left(\mathbf{B}^{\prime} \otimes \mathbf{I}\right)+\left(\mathbf{\Lambda}^{\prime} \otimes \mathbf{W}\right)\right] \mathbf{y}=\left(\boldsymbol{\Gamma}^{\prime} \otimes \mathbf{I}\right) \mathbf{x}+\mathbf{u} \\
\left(\mathrm{I}-\left[\left(\mathbf{B}^{\prime} \otimes \mathbf{I}\right)+\left(\mathbf{\Lambda}^{\prime} \otimes \mathbf{W}\right)\right]\right) \mathbf{y}=\left(\boldsymbol{\Gamma}^{\prime} \otimes \mathbf{I}\right) \mathbf{x}+\mathbf{u}
\end{gathered}
$$

The third perspective is the interpretation of the model in its reduced form. The reduced form is nonlinear and it clearly illustrates how the expected value of the dependent variables at each location depend not only on the predetermined (exogenous) variables at the respective locations but also on the predetermined (exogenous) variables at all other locations. The reduced form is given by:

$$
\mathbf{y}=\left(\mathrm{I}-\left[\left(\mathbf{B}^{\prime} \otimes \mathbf{I}\right)+\left(\mathbf{\Lambda}^{\prime} \otimes \mathbf{W}\right)\right]\right)^{-1}\left(\boldsymbol{\Gamma}^{\prime} \otimes \mathbf{I}\right) \mathbf{x}+\left(\mathrm{I}-\left[\left(\mathbf{B}^{\prime} \otimes \mathbf{I}\right)+\left(\mathbf{\Lambda}^{\prime} \otimes \mathbf{W}\right)\right]\right)^{-1} \mathbf{u}
$$

The expected or mean value can be computed by taking expectations on both sides of equation (3.167) as follows:

$$
E[\mathbf{y}]=E\left[\left(\mathrm{I}-\left[\left(\mathbf{B}^{\prime} \otimes \mathbf{I}\right)+\left(\mathbf{\Lambda}^{\prime} \otimes \mathbf{W}\right)\right]\right)^{-1}\left(\boldsymbol{\Gamma}^{\prime} \otimes \mathbf{I}\right) \mathbf{x}\right]+E\left[\left(\mathrm{I}-\left[\left(\mathbf{B}^{\prime} \otimes \mathbf{I}\right)+\left(\mathbf{\Lambda}^{\prime} \otimes \mathbf{W}\right)\right]\right)^{-1} \mathbf{u}\right]
$$

Since the mean of the error term is assumed to be zero, this gives:

$$
E[\mathbf{y}]=E\left[\left(\mathrm{I}-\left[\left(\mathbf{B}^{\prime} \otimes \mathbf{I}\right)+\left(\boldsymbol{\Lambda}^{\prime} \otimes \mathbf{W}\right)\right]\right)^{-1}\left(\boldsymbol{\Gamma}^{\prime} \otimes \mathbf{I}\right) \mathbf{x}\right]
$$

To continue with formulating the model in form more convenient to reveal its solution for the endogenous variables, consider equation (3.165). Let: 


$$
\mathbf{B}^{*}=\left[\left(\mathbf{B}^{\prime} \otimes \mathbf{I}\right)+\left(\boldsymbol{\Lambda}^{\prime} \otimes \mathbf{W}\right)\right] \text { and } \boldsymbol{\Gamma}^{*}=\left(\boldsymbol{\Gamma}^{\prime} \otimes \mathbf{I}\right)
$$

Then, equation (3.165) can be rewritten as:

$$
\begin{aligned}
& \mathbf{y}=\mathbf{B}^{*} \mathbf{y}+\boldsymbol{\Gamma}^{*} \mathbf{x}+\mathbf{u} \\
& \mathbf{y}=\left(\mathbf{I}_{n}-\mathbf{B}^{*}\right)^{-1} \boldsymbol{\Gamma}^{*} \mathbf{x}+\left(\mathbf{I}_{n}-\mathbf{B}^{*}\right)^{-1} \mathbf{u}(\text { in reduced-form })
\end{aligned}
$$

From this general form of the spatial econometric model, various specifications can be generated. By imposing zero restrictions on various model parameters, Rey and Boarnet (2004), for example, have identified 35 different specification cases from their twoequation spatial econometric model. In order to structure the taxonomy of the spatial econometric model, they considered three dimensions of simultaneity: feedback simultaneity; spatial autoregressive lag simultaneity; and spatial cross-regressive lag simultaneity. Depending on the underlying theoretical arguments, each equation of the model contains either all or some or none of these dimensions.

The system in equation (3.162) can also be expressed more compactly by imposing exclusion restriction on the parameters of the model. Particularly, let the vectors of nonzero elements of the jth column of $\mathbf{B}, \boldsymbol{\Gamma}$, and $\boldsymbol{\Lambda}$ be $\boldsymbol{\beta}_{j}, \boldsymbol{\gamma}_{j}$, and $\boldsymbol{\lambda}_{j}$ respectively. Again, let the corresponding matrices of observations on the endogenous variables, exogenous variables, and the spatially lagged endogenous variables that appear in the jth equation be $\mathbf{Y}_{j}, \mathbf{X}_{j}$, and $\mathbf{W} \mathbf{Y}_{j}$ respectively. Then equation (3.162) can be written as:

$$
\mathbf{y}_{j}=\mathbf{Z}_{j} \boldsymbol{\delta}_{j}+\mathbf{u}_{j}
$$

where

$$
\mathbf{Z}_{j}=\left(\mathbf{Y}_{j}, \mathbf{X}_{j}, \mathbf{W} \mathbf{Y}_{j}\right) \text { and } \boldsymbol{\delta}_{j}=\left(\boldsymbol{\beta}_{j}^{\prime}, \boldsymbol{\gamma}_{j}^{\prime}, \lambda_{j}^{\prime}\right)^{\prime}
$$




\section{Spatial Error Model}

A second way to incorporate spatial autocorrelation in a regression model is to specify a spatial process for the disturbance term. The disturbance terms in a regression model can be considered to contain all ignored elements, and when spatial dependence is present in the disturbance term, the spatial effects are assumed to be a noise, or perturbation, that is, a factor that needs to be removed (Anselin, 2001). Such spatial pattern in the residuals of the regression model may lead to the discovery of additional variables that should be included in the model. Spatial dependence in the disturbance term also violates the basic OLS estimation assumption of uncorrelated errors. Hence, when the spatial dependence is ignored, OLS estimates will be inefficient, though unbiased, the student $\mathrm{t}$ - and F-statistics for tests of significance will be biased, the $\mathrm{R}^{2}$ measure will be misleading, which in turn lead to a wrong statistical interpretation of the regression mode (Anselin, 1996). More efficient estimators can be obtained by taking advantage of the particular structure of the error covariance implied by the spatial process. The disturbance term is non-spherical where the off-diagonal elements of the associated covariance matrix express the structure of spatial dependence.

A spatial dependence model is more common in social science applications using cross sectional data due to the predominance of spatial interaction and spatial externalities as well as due to the poor choice of spatial units in such applications (Anselin, 1992). The dependence in the disturbance term can be expressed either as spatial autoregressive or as a spatial moving average spatial process. The most common specification, however, is the spatial autoregressive spatial process, although most tests 
for spatial error autocorrelation are the same for either form (Anselin, 1992). The spatial dependence in the disturbance term, thus, can be expressed using matrix notation as:

$$
\mathbf{y}=\mathbf{X} \gamma+\mathbf{u}
$$

with

$$
\mathbf{u}=\rho \mathbf{W u}+\boldsymbol{\varepsilon}
$$

where $\mathbf{u}$ is assumed to follow a spatial autoregressive process, with $\rho$ as the spatial autoregressive coefficient for the error lag $\mathbf{W u}$, and $\boldsymbol{\varepsilon} \mathrm{n}$ by 1 vector of innovations or white noise error, and the other notations as defined before. Equation (3.172) is the structural form of the SAR model which expresses global spatial effects. The corresponding reduced form of the model can be specified as:

$$
\mathbf{y}=\mathbf{X} \boldsymbol{\gamma}+(\mathbf{I}-\rho \mathbf{W})^{-1} \boldsymbol{\varepsilon}
$$

with the corresponding error covariance matrix given as:

$$
E\left(\mathbf{u} \mathbf{u}^{\prime}\right)=\sigma^{2}(\mathbf{I}-\rho \mathbf{W})^{-1}\left(\mathbf{I}-\rho \mathbf{W}^{\prime}\right)^{-1}=\sigma^{2}(\mathbf{I}-\rho \mathbf{W})^{\prime}(\mathbf{I}-\rho \mathbf{W})^{-1}
$$

The structure in equation (3.174) shows that the spatial error process leads to a non-zero error covariance between every pair of observation, but decreasing in magnitude with the order of contiguity. Note also that heteroskedasticity is induced in $\mathbf{u}$, irrespective of the heteroskedasticity of $\boldsymbol{\varepsilon}$, because the inverse matrices in equation (3.174) yields nonconstant diagonal element in the error covariance matrix.

An alternative structural form, the so-called spatial Durbin or common factor model, can be generated by pre-multiplying equation (3.173) by $(\mathbf{I}-\rho \mathbf{W})$ and moving the spatial lag term to the right-hand side as:

$$
\mathbf{y}=\rho \mathbf{W} \mathbf{y}+\mathbf{X} \boldsymbol{\gamma}-\rho \mathbf{W} \mathbf{X} \boldsymbol{\gamma}+\boldsymbol{\varepsilon}
$$


This spatial model has spatially lagged exogenous variables (WX) in addition to the spatially lagged dependent variable $(\mathbf{W y})$ and a well-behaved disturbance term $\boldsymbol{\varepsilon}$. Equation (3.175), however, becomes a proper spatial error model only if a set of $\mathrm{K}$ nonlinear constraints on the parameters, the so-called common factor constraints, $\rho \cdot \gamma=-\rho \gamma$ (the product of the spatial autoregressive coefficient $\rho$ with the regression coefficient $\gamma$ should equal the negative of the coefficient of spatially lagged exogenous variables (WX), $\rho \gamma)$, are satisfied. The spatial error model can also be expressed in terms of spatially filtered variables as:

$$
(\mathbf{I}-\rho \mathbf{W}) \mathbf{y}=(\mathbf{I}-\rho \mathbf{W}) \mathbf{X} \boldsymbol{\gamma}+\boldsymbol{\varepsilon}
$$

The single equation spatial error model developed above can easily be extended to a system of spatially interrelated cross sectional equations corresponding to $\mathrm{n}$ cross sectional units. Assuming the spatial dependence in the error term, the system of simultaneous equations given in equation (3.19) can be expressed as:

$$
\mathbf{Y}=\mathbf{Y B}+\mathbf{X} \boldsymbol{\Gamma}+\mathbf{U}
$$

with

$$
\mathbf{U}=\mathbf{W U C}+\mathbf{E}
$$

where $\mathbf{W U}=\left(\mathbf{W} \mathbf{u}_{1}, \ldots, \mathbf{W u}_{\mathrm{G}}\right), \mathbf{C}=\operatorname{diag}_{\mathrm{j}=1}^{\mathrm{G}}\left(\rho_{j}\right), \mathbf{E}=\left(\boldsymbol{\varepsilon}_{1}, \ldots, \boldsymbol{\varepsilon}_{\mathrm{G}}\right)$ and the other notations as defined before. Note that $\rho_{j}$ denotes the spatial autoregressive parameter in the jth equation and since $\mathbf{C}$ is taken to be diagonal, the specification relates the disturbance vector in the jth equation only to its own spatial lag. Since it is assumed that $E(\boldsymbol{\varepsilon})=\mathbf{0}$ and $E\left(\boldsymbol{\varepsilon} \boldsymbol{\varepsilon}^{\prime}\right)=\boldsymbol{\Sigma} \otimes \mathbf{I}_{n}$, the disturbances, however, will be spatially correlated across units and across equations. 
The system in equation (3.177) and (3.178) can be expressed in a form where its solution for the endogenous variables is clearly revealed. But, first consider the following vector transformations:

$$
\begin{aligned}
\operatorname{vec}(\mathbf{Y}) & =\operatorname{vec}(\mathbf{Y B})+\operatorname{vec}(\mathbf{X} \boldsymbol{\Gamma})+\operatorname{vec}(\mathbf{U}) \\
\operatorname{vec}(\mathbf{Y}) & =\operatorname{vec}(\mathbf{Y B})+\operatorname{vec}(\mathbf{X} \boldsymbol{\Gamma})+\operatorname{vec}(\mathbf{U W C}+\mathbf{E}) \\
& =\left(\mathbf{B}^{\prime} \otimes \mathbf{I}\right) \operatorname{vec}(\mathbf{Y})+\left(\boldsymbol{\Gamma}^{\prime} \otimes \mathbf{I}\right) \operatorname{vec}(\mathbf{X})+\left(\mathbf{C}^{\prime} \otimes \mathbf{W}\right) \operatorname{vec} \mathbf{U}+\operatorname{vec} \mathbf{E}
\end{aligned}
$$

Letting $\mathbf{y}=\operatorname{vec}(\mathbf{Y}), \quad \mathbf{x}=\operatorname{vec}(\mathbf{X}), \mathbf{u}=\operatorname{vec}(\mathbf{U})$, and $\boldsymbol{\varepsilon}=\operatorname{vec}(\mathbf{E})$ it follows from equation (3.179) that

$$
\begin{aligned}
& \mathbf{y}=\left(\mathbf{B}^{\prime} \otimes \mathbf{I}\right) \mathbf{y}+\left(\boldsymbol{\Gamma}^{\prime} \otimes \mathbf{I}\right) \mathbf{x}+\left(\mathbf{C}^{\prime} \otimes \mathbf{W}\right) \mathbf{u}+\boldsymbol{\varepsilon} \\
& \text { or } \\
& \mathbf{y}=\left(\mathbf{B}^{\prime} \otimes \mathbf{I}\right) \mathbf{y}+\left(\boldsymbol{\Gamma}^{\prime} \otimes \mathbf{I}\right) \mathbf{x}+\mathbf{u}, \\
& \mathbf{u}=\left(\mathbf{C}^{\prime} \otimes \mathbf{W}\right) \mathbf{u}+\boldsymbol{\varepsilon}
\end{aligned}
$$

The system in equation (3.180) can also be rewritten more compactly in a form that can reveal its solution for the endogenous variables as follows:

$$
\begin{aligned}
& \mathbf{y}=\mathbf{B}^{* *} \mathbf{y}+\Gamma^{*} \mathbf{x}+\mathbf{u}, \\
& \mathbf{u}=\mathbf{C}^{*} \mathbf{u}+\boldsymbol{\varepsilon}
\end{aligned}
$$

where $\mathbf{B}^{* *}=\left(\mathbf{B}^{\prime} \otimes \mathbf{I}_{n}\right), \mathbf{C}^{*}=\mathbf{C}^{\prime} \otimes \mathbf{W}=\operatorname{diag}_{j=1}^{G}\left(\rho_{j} \mathbf{W}\right)$, and the other notations as before.

Further more, by imposing exclusion restriction on the system in equation (3.181), it can be expressed as:

$$
\begin{aligned}
& \mathbf{y}_{j}=\mathbf{Z}_{j} \boldsymbol{\delta}_{j}+\mathbf{u}_{j}, \\
& \mathbf{u}_{j}=\rho_{j} \mathbf{W} \mathbf{u}_{j}+\boldsymbol{\varepsilon}_{j}, \quad j=1, \ldots, G
\end{aligned}
$$

where

$$
\mathbf{Z}_{j}=\left(\mathbf{Y}_{j}, \mathbf{X}_{j}\right) \text { and } \boldsymbol{\delta}_{j}=\left(\boldsymbol{\beta}_{j}^{\prime}, \boldsymbol{\gamma}_{j}^{\prime}\right)^{\prime}
$$




\section{Spatial Autoregressive Model}

When there are no strong a priori theoretical reasons to believe that interdependences between spatial units arises either due to the spatial lags of the dependent variables or due to spatially autoregressive error terms, the standard approach is to model the system with both effects included (Anselin, 2003).The spatial lag model and the spatial error model are discussed in the above two subsections separately. By combining these two models, the spatial autoregressive model with both the spatial lag and spatial error effects can be expressed as (all notations are as defined before):

$$
\mathbf{Y}=\mathbf{Y B}+\mathbf{X} \boldsymbol{\Gamma}+\mathbf{W Y} \mathbf{\Lambda}+\mathbf{U}
$$

with

$$
\mathbf{U}=\mathbf{W U C}+\mathbf{E}
$$

Combining equation (3.170) and (3.181) gives the system in its more compact form as (all notations are as expressed before):

$$
\begin{aligned}
& \mathbf{y}=\mathbf{B}^{*} \mathbf{y}+\Gamma^{*} \mathbf{x}+\mathbf{u}, \\
& \mathbf{u}=\mathbf{C}^{*} \mathbf{u}+\boldsymbol{\varepsilon}
\end{aligned}
$$

Assuming that $\mathbf{I}_{n G}-\mathbf{B}^{*}$ and $\mathbf{I}_{n G}-\mathbf{C}^{*}$ are nonsingular matrices with $\left|\rho_{j}\right|<1, \mathbf{j}=1, \ldots, \mathrm{G}$, the system in equation (3.184) can be expressed in its reduced form as:

$$
\begin{aligned}
& \mathbf{y}=\left(\mathbf{I}_{n G}-\mathbf{B}^{*}\right)^{-1}\left(\boldsymbol{\Gamma}^{*} \mathbf{x}+\mathbf{u}\right), \\
& \mathbf{u}=\left(\mathbf{I}_{n G}-\mathbf{C}^{*}\right)^{-1} \boldsymbol{\varepsilon}
\end{aligned}
$$

Since the innovations are assumed to be independently and identically distributed, that is, $E(\boldsymbol{\varepsilon})=\mathbf{0}$ and $E\left(\boldsymbol{\varepsilon} \boldsymbol{\varepsilon}^{\prime}\right)=\boldsymbol{\Sigma} \otimes \mathbf{I}_{n}$, the means and variance covariance matrices of the disturbance terms $\mathbf{u}$, and the endogenous variables $\mathbf{y}$, are given, respectively, as follows: 


$$
\begin{aligned}
& E(\mathbf{u})=0 ; \quad E\left(\mathbf{u} \mathbf{u}^{\prime}\right)=\mathbf{\Omega}_{\mathbf{u}}=\left(\mathbf{I}_{n G}-\mathbf{C}^{*}\right)^{-1}\left(\mathbf{\Sigma} \otimes \mathbf{I}_{n}\right)\left(\mathbf{I}_{n G}-\mathbf{C}^{* \prime}\right)^{-1} \\
& E(\mathbf{y})=\left(\mathbf{I}_{n G}-\mathbf{B}^{*}\right)^{-1}\left(\boldsymbol{\Gamma}^{*} \mathbf{x}\right) ; \quad E\left(\mathbf{y y}^{\prime}\right)=\boldsymbol{\Omega}_{\mathbf{y}}=\left(\mathbf{I}_{n G}-\mathbf{B}^{*}\right)^{-1} \boldsymbol{\Omega}_{\mathbf{u}}\left(\mathbf{I}_{n G}-\mathbf{B}^{* \prime}\right)^{-1}
\end{aligned}
$$

The endogenous variables as well as the disturbances are, therefore, seen to be correlated both spatially and across equation, and furthermore will generally be hetroskedastic. In this study, the spatial units are counties and each county has only a small number of neighbors and, in turn, it is only a neighbor to a small number of counties. The weights matrix $\mathbf{W}$ is a row standardized sparse matrix and hence the row and column sums of the weights matrix is bounded in absolute values. It is also assumed that $\left(\mathbf{I}_{n}-\rho_{j} \mathbf{W}\right), \mathrm{j}=1, \ldots, \mathrm{G}$ and $\left(\mathbf{I}_{n}-\mathbf{B}^{*}\right)^{-1}$ are bounded uniformly in absolute values, which imply that $\boldsymbol{\Omega}_{\mathbf{u}}$ and $\boldsymbol{\Omega}_{\mathbf{y}}$ are also bounded uniformly as it can easily be seen from the relations in equation (3.186). Thus, the degree of correlation between the elements of $\mathbf{u}$ and $\mathbf{y}$ are limited, which is a necessary condition for all large sample analysis (see Kelejian and Prucha, 1998, 2004).

By imposing exclusion restrictions on the system in equation (3.183), the spatial autoregressive model can also be reformulated as follows (all notations are as defined before):

$$
\begin{aligned}
& \mathbf{y}_{j}=\mathbf{Z}_{j} \boldsymbol{\delta}_{j}+\mathbf{u}_{j}, \\
& \mathbf{u}_{j}=\rho_{j} \mathbf{W} \mathbf{u}_{j}+\boldsymbol{\varepsilon}_{j}, \quad j=1, \ldots, G
\end{aligned}
$$

where

$$
\mathbf{Z}_{j}=\left(\mathbf{Y}_{j}, \mathbf{X}_{j}, \mathbf{W} \mathbf{Y}_{j}\right) \text { and } \boldsymbol{\delta}_{j}=\left(\boldsymbol{\beta}_{j}^{\prime}, \boldsymbol{\gamma}_{j}^{\prime}, \boldsymbol{\lambda}_{j}^{\prime}\right)^{\prime}
$$

Following Kelejian and Prucha (2004), a set of instruments are utilized to estimate the spatial models in equations (3.171), (3.182) and (3.187) using the instrumental variable 
techniques. Let $\mathbf{N}$ denote the $\mathrm{n}$ by $\mathrm{p}$ matrix of those instruments and as suggested by Kelejian and Prucha (2004), $\mathbf{N}$ will be chosen as a subset of the linearly independent columns of $\left(\mathbf{X}, \mathbf{W X}, \ldots, \mathbf{W}^{\mathrm{s}} \mathbf{X}\right)$, where $\mathrm{s}$ is an integer such that $1 \leq s \leq 2$. It is assumed that the elements of $\mathbf{N}$ are uniformly bounded in absolute value. Besides, $\mathbf{N}$ is full column rank non-stochastic instrument matrix with the following properties:

1) $\lim _{n \rightarrow \infty} \frac{\mathbf{N}^{\prime} \mathbf{N}}{n}=\mathbf{Q}_{N N}$, where $\mathbf{Q}_{N N}$ is a finite and nonsingular matrix

2) $\lim _{n \rightarrow \infty} \frac{\mathbf{N}^{\prime} E\left(\mathbf{Z}_{j}\right)}{n}=\mathbf{Q}_{N Z_{j}}$, where $\mathbf{Q}_{N Z_{j}}$ is a finite matrix which has full column rank, $\mathrm{j}=1, \ldots, \mathrm{G}$

3) $\lim _{n \rightarrow \infty} \frac{\mathbf{N}^{\prime} \mathbf{W} E\left(\mathbf{Z}_{j}\right)}{n}=\mathbf{Q}_{N W Z_{j}}$, where $\mathbf{Q}_{N W Z_{j}}$ is a finite matrix which has full column rank, $\mathrm{j}=1, \ldots, \mathrm{G}$

4) $\mathbf{Q}_{N Z_{j}}-\rho_{j} \mathbf{Q}_{N W Z_{j}}=\lim _{n \rightarrow \infty} \frac{\mathbf{N}^{\prime}\left(\mathbf{I}_{n}-\rho_{j} \mathbf{W}\right) \mathbf{Z}_{j}}{n}$ has a full column rank, $\mathrm{j}=1, \ldots, \mathrm{G}$

5) $\lim _{n \rightarrow \infty} \frac{\mathbf{N}^{\prime}\left(\mathbf{I}_{n}-\rho_{j} \mathbf{W}\right)^{-1}\left(\mathbf{I}_{n}-\rho_{j} \mathbf{W}^{\prime}\right)^{-1} \mathbf{N}}{n}=\boldsymbol{\Phi}$, where $\boldsymbol{\Phi}$ is a finite and nonsingular matrix, $\mathrm{j}=1, \ldots, \mathrm{G}$ Assuming that the matrix of exogenous (nonstochastic) variables $\mathbf{X}$ has full column rank, properties 1 and 2 are important to ensure the consistency of the initial two stage least squares estimators. Property 2 also ensures that the instruments $\mathbf{N}$ allow the identification of the regression parameters $\boldsymbol{\delta}_{j}$ in equations (3.171), (3.182) and (3.187). Note that the 2SLS estimator for the parameters of the models in each of these equations is a generalized moments estimator corresponding to the moment conditions $E\left(\mathbf{N}^{\prime} \mathbf{u}_{j}\right)=\mathbf{0}$.

Let $\mathbf{u}_{j}\left(\underline{\boldsymbol{\delta}}_{j}\right)=\mathbf{y}_{j}-\mathbf{Z}_{j} \underline{\boldsymbol{\delta}}_{j}=\mathbf{u}_{j}+\mathbf{Z}_{j}\left(\boldsymbol{\boldsymbol { \delta }}_{j}-\underline{\boldsymbol{\delta}}_{j}\right)$, then the condition that $\mathbf{Q}_{N Z_{j}}$ has full column rank implies that $\lim _{n \rightarrow \infty} \frac{E\left(\mathbf{N}^{\prime} \mathbf{u}_{j}\left(\underline{\boldsymbol{\delta}}_{j}\right)\right)}{n}=\left[\lim _{n \rightarrow \infty} \frac{\mathbf{N}^{\prime} E\left(\mathbf{Z}_{j}\right)}{n}\right]\left(\boldsymbol{\delta}_{j}-\underline{\boldsymbol{\delta}}_{j}\right)$ is zero if and only if $\underline{\boldsymbol{\delta}}_{j}=\boldsymbol{\delta}_{j}$.Thus fulfillment of the rank condition for $\mathbf{Q}_{N Z_{j}}$ ensures that the instruments $\mathbf{N}$ 
identify the true parameter vector $\boldsymbol{\delta}_{j}, j=1, \ldots, \mathrm{G}$, and the objective function is uniquely maximized at $\underline{\boldsymbol{\delta}}_{j}=\boldsymbol{\delta}_{j}$, at least in the limit.

Properties 3 and 4 are important in ensuring the consistency of the generalized two and three stage estimators, which are based on a Cochrane-Orcutt-type transformation of the models. Property 5 is used in deriving the limiting distribution of the initial two stages least squares estimator from the untransformed model (see Kelejian and Prucha (2004) for details and proofs).

\subsubsection{Spatial Dependence in Simultaneous-Equations Panel Data Models}

When data is available across space and over time, spatial dependence can be incorporated into the standard simultaneous equations panel data models (as developed above) in a straightforward way. Spatial lag dependent variables, for example, can be incorporated into equation (3.113) as follows (all notations are as given before):

$$
\mathbf{y}=\mathbf{Z} \boldsymbol{\delta}+\mathbf{u}
$$

where

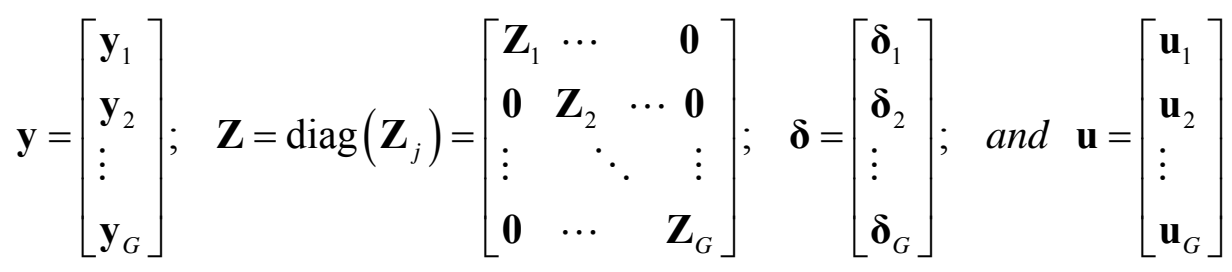

Note that $\mathbf{y}_{\mathbf{j}}$ is $\mathrm{nT} \times 1$ vector of observations on the endogenous variable in the $\mathrm{jth}$ equation, $\mathbf{Z}_{j}=\left(\mathbf{Y}_{j}, \mathbf{X}_{j}, \mathbf{W} \mathbf{Y}_{j}\right)$ a matrix of dimension nT by $\left(\mathrm{G} 1_{\mathrm{j}}-1+\mathrm{K} 1_{\mathrm{j}}+\mathrm{G} 1_{\mathrm{j}}\right)$ , $\boldsymbol{\delta}_{j}=\left(\boldsymbol{\beta}_{j}^{\prime}, \boldsymbol{\gamma}_{j}^{\prime}, \lambda_{j}^{\prime}\right)^{\prime}$ where $\boldsymbol{\beta}_{j}$ is $\mathrm{G} 1_{\mathrm{j}}-1$ by $1, \boldsymbol{\gamma}_{j}$ is $\mathrm{K} 1_{\mathrm{j}}$ by 1 , and $\boldsymbol{\lambda}_{j}$ is $\mathrm{G} 1_{\mathrm{j}}$ by 1 , and $\mathbf{u}_{j}$ is $\mathrm{nT}$ by 1 vector of disturbance in the jth equation, for $j=1,2, \ldots, G$.For the one-way error component model, the disturbance of the jth equation $\mathbf{u}_{j}$ is given by: 


$$
\mathbf{u}_{j}=\mathbf{Z}_{\mu} \boldsymbol{\mu}_{j}+\boldsymbol{\omega}_{j}
$$

where

$$
\mathbf{Z}_{\mu}=\left(\mathbf{I}_{n} \otimes \mathbf{i}_{T}\right), \boldsymbol{\mu}_{j}^{\prime}=\left(\mu_{1 j}, \mu_{2 j}, \ldots, \mu_{n j}\right) \text {, and } \boldsymbol{\omega}_{j}^{\prime}=\left(\omega_{11 j}, \omega_{11 j}, \ldots, \omega_{1 T j}, \ldots, \omega_{n 1 j}, \ldots, \omega_{n T j}\right)
$$

Thus,

$$
\boldsymbol{\Omega}_{j l}=E\left(\mathbf{u}_{j} \mathbf{u}_{l}^{\prime}\right)=\boldsymbol{\sigma}_{\mu_{j l}}^{2}\left(\mathbf{I}_{n} \otimes \mathbf{J}_{T}\right)+\boldsymbol{\sigma}_{\omega_{j l}}^{2}\left(\mathbf{I}_{n} \otimes \mathbf{I}_{T}\right)
$$

In this case, the covariance matrix between the disturbances of different equations has the same one-way error component form. But, now there are additional cross equation variances components to be estimated. When one considers the whole model, the variance-covariance matrix for the set of $\mathrm{G}$ structural equations is given by:

$$
\boldsymbol{\Omega}=E\left(\mathbf{u u}^{\prime}\right)=\boldsymbol{\Sigma}_{\mu} \otimes\left(\mathbf{I}_{n} \otimes \mathbf{J}_{T}\right)+\boldsymbol{\Sigma}_{\omega} \otimes\left(\mathbf{I}_{n} \otimes \mathbf{I}_{T}\right)
$$

where $\boldsymbol{\Sigma}_{\mu}=\left[\boldsymbol{\sigma}_{\mu_{j l}}^{2}\right]$ and $\boldsymbol{\Sigma}_{\omega}=\left[\boldsymbol{\sigma}_{\omega_{j l}}^{2}\right]$ are both G x G matrices, and $\mathbf{u}^{\prime}=\left(\mathbf{u}_{1}^{\prime}, \mathbf{u}_{2}^{\prime}, \ldots, \mathbf{u}_{G}^{\prime}\right)$ is a 1 x nGT vector of disturbances with $\mathbf{u}_{j}$ defined in equation (3.189) for $\mathrm{j}=1,2, \ldots \mathrm{G}$.

Now it is possible to transform the stacked system of equations in equation (3.188) by $\left(\mathbf{I}_{G} \otimes \mathbf{H}\right)$ and $\left(\mathbf{I}_{G} \otimes \mathbf{P}\right)$ to get, respectively,

$$
\widehat{\mathbf{y}}=\widehat{\mathbf{Z}} \boldsymbol{\delta}+\widehat{\mathbf{u}} \text { and } \breve{\mathbf{y}}=\breve{\mathbf{Z}} \boldsymbol{\delta}+\breve{\mathbf{u}}
$$

where $\widehat{\mathbf{y}}=\left(\mathbf{I}_{G} \otimes \mathbf{H}\right) \mathbf{y}, \widehat{\mathbf{Z}}=\left(\mathbf{I}_{G} \otimes \mathbf{H}\right) \mathbf{Z}$, and $\widehat{\mathbf{u}}=\left(\mathbf{I}_{G} \otimes \mathbf{H}\right) \mathbf{u} ;$ and $\breve{\mathbf{y}}=\left(\mathbf{I}_{G} \otimes \mathbf{P}\right) \mathbf{y}$,

$$
\breve{\mathbf{Z}}=\left(\mathbf{I}_{G} \otimes \mathbf{P}\right) \mathbf{Z} \text {, and } \breve{\mathbf{u}}=\left(\mathbf{I}_{G} \otimes \mathbf{P}\right) \mathbf{u} \text {. The W3SLS and B3SLS estimators can be }
$$

obtained by performing 3SLS on these transformed equations using, respectively, $\left(\mathbf{I}_{G} \otimes \widehat{\mathbf{N}}\right)$ and $\left(\mathbf{I}_{G} \otimes \breve{\mathbf{N}}\right)$ as sets of instruments, where $\widehat{\mathbf{N}}=\mathbf{H N}$ and $\breve{\mathbf{N}}=\mathbf{P N}$. 
Similarly, the system in equation (3.113) can be extended to incorporate spatial dependence in the errors as follows:

$$
\begin{gathered}
\mathbf{y}=\mathbf{Z} \boldsymbol{\delta}+\mathbf{u} \\
\mathbf{u}=\rho\left(\mathbf{I}_{T} \otimes \mathbf{W}\right) \mathbf{u}+\left(\mathbf{I}_{n} \otimes \mathbf{t}_{T}\right) \mu+\boldsymbol{\omega}
\end{gathered}
$$

where

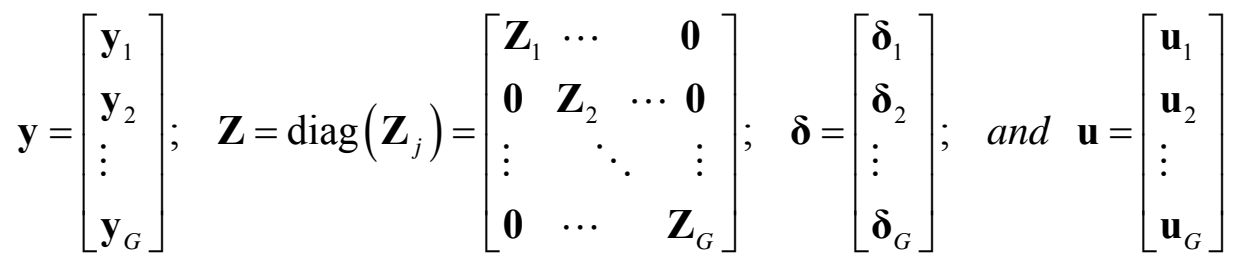

with $\mathbf{Z}_{j}=\left(\mathbf{Y}_{j}, \mathbf{X}_{j}\right), \boldsymbol{\delta}_{j}=\left(\boldsymbol{\beta}_{j}^{\prime}, \boldsymbol{\gamma}_{j}^{\prime}\right)^{\prime}$ for $\mathrm{j}=1, \ldots, \mathrm{G}$ and the other notations as given in equation (3.188).

In order to facilitate the modeling of spatial error dependence in the context of panel data, the data is arranged time wise. Apart from this difference in the arrangement of the data, the format is similar to equation (3.188) above. Now consider the jth equation of the system in equation (3.193):

$$
\begin{gathered}
\mathbf{y}_{j}=\mathbf{Z}_{j} \boldsymbol{\delta}_{j}+\mathbf{u}_{j}, \\
\mathbf{u}_{j}=\rho_{j}\left(\mathbf{I}_{T} \otimes \mathbf{W}\right) \mathbf{u}_{j}+\mathbf{v}_{j}
\end{gathered}
$$

where

$$
\mathbf{v}_{j}=\left(\mathbf{I}_{n} \otimes \mathbf{u}_{T}\right) \mu_{j}+\boldsymbol{\omega}_{j}
$$

The mean and the covariance of the innovation vector $\boldsymbol{v}_{j}$ can be given by: 


$$
\begin{aligned}
& E\left(\mathbf{v}_{j}\right)=0 \\
& E\left(\boldsymbol{v}_{j} \boldsymbol{v}_{j}^{\prime}\right)=\mathbf{\Omega}_{\mathbf{v}_{j}}=\sigma_{\mu_{j}}^{2}\left(\mathbf{J}_{T} \otimes \mathbf{I}_{n}\right)+\sigma_{\omega_{j}}^{2}\left(\mathbf{I}_{n} \otimes \mathbf{I}_{n}\right)
\end{aligned}
$$

From the second part of equation (3.194) it follows that

$$
\mathbf{u}_{j}=\left(\mathbf{I}_{T} \otimes\left(\mathbf{I}_{n}-\rho_{j} \mathbf{W}\right)^{-1}\right) \mathbf{v}_{j}
$$

Thus, the mean and the covariance of $\mathbf{u}_{j}$ can be given as follows:

$$
\begin{aligned}
E\left(\mathbf{u}_{j}\right)= & 0 \\
E\left(\mathbf{u}_{j} \mathbf{u}_{j}^{\prime}\right)= & \boldsymbol{\Omega}_{\mathbf{u}_{j}}=\left[\mathbf{I}_{T} \otimes\left(\mathbf{I}_{n}-\rho_{j} \mathbf{W}\right)^{-1}\right] \boldsymbol{\Omega}_{\mathbf{v}_{j}}\left[\mathbf{I}_{T} \otimes\left(\mathbf{I}_{n}-\rho_{j} \mathbf{W}^{\prime}\right)^{-1}\right] \\
& =\boldsymbol{\Omega}_{\mathbf{v}_{j}}\left[\mathbf{I}_{T} \otimes\left(\mathbf{I}_{n}-\rho_{j} \mathbf{W}\right)^{-1}\right]\left[\mathbf{I}_{T} \otimes\left(\mathbf{I}_{n}-\rho_{j} \mathbf{W}^{\prime}\right)^{-1}\right] \\
& =\boldsymbol{\Omega}_{\mathbf{v}_{j}}\left[\mathbf{I}_{T} \otimes\left(\mathbf{I}_{n}-\rho_{j} \mathbf{W}\right)^{-1}\left(\mathbf{I}_{n}-\rho_{j} \mathbf{W}^{\prime}\right)^{-1}\right]
\end{aligned}
$$

where $\boldsymbol{\Omega}_{\mathbf{v}_{j}}$ is as given in equation (3.196). This can easily be extended to the whole model. But, first note that the variance-covariance of the innovations for the whole model can be given by (see also equation (3.117) for the derivation and the definitions of the notations):

$$
\boldsymbol{\Omega}_{\mathbf{v}}=\boldsymbol{\Sigma}_{1} \otimes \mathbf{P}+\boldsymbol{\Sigma}_{\omega} \otimes \mathbf{H}
$$

Thus, the variance-covariance matrix for the set of $\mathrm{G}$ structural equations is computed as:

$$
E\left(\mathbf{u} \mathbf{u}^{\prime}\right)=\boldsymbol{\Omega}_{\mathbf{u}}=\boldsymbol{\Omega}_{\mathbf{v}}\left[\mathbf{I}_{T} \otimes\left(\mathbf{I}_{n}-\rho_{j} \mathbf{W}\right)^{-1}\left(\mathbf{I}_{n}-\rho_{j} \mathbf{W}^{\prime}\right)^{-1}\right], \text { for } \mathbf{j}=1, \ldots, \mathbf{G}
$$

Both spatial lag dependence and spatial error dependence can also be incorporated into the standard simultaneous equations models in the context of panel data. Recalling equations (3.188) and (3.193) the spatial autoregressive panel data model with spatial 
autoregressive disturbances can be formulated as follows (all notations and definitions are as expressed before):

$$
\begin{gathered}
\mathbf{y}=\mathbf{Z} \boldsymbol{\delta}+\mathbf{u}, \\
\mathbf{u}=\rho\left(\mathbf{I}_{T} \otimes \mathbf{W}\right) \mathbf{u}+\mathbf{v}
\end{gathered}
$$

with

$$
\mathbf{v}=\left(\mathbf{I}_{n} \otimes \mathbf{l}_{T}\right) \mu+\boldsymbol{\omega}
$$

where

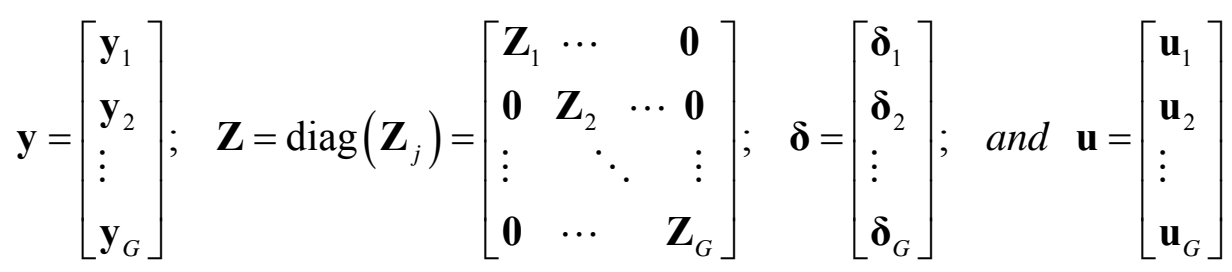

Note that $\mathbf{y}_{\mathbf{j}}$ is $\mathrm{nT} \times 1$ vector of observations on the endogenous variable in the $\mathrm{jth}$ equation, $\mathbf{Z}_{j}=\left(\mathbf{Y}_{j}, \mathbf{X}_{j}, \mathbf{W} \mathbf{Y}_{j}\right)$ a matrix of dimension nT by $\left(\mathrm{G} 1_{\mathrm{j}}-1+\mathrm{K} 1_{\mathrm{j}}+\mathrm{G} 1_{\mathrm{j}}\right)$ , $\boldsymbol{\delta}_{j}=\left(\boldsymbol{\beta}_{j}^{\prime}, \boldsymbol{\gamma}_{j}^{\prime}, \lambda_{j}^{\prime}\right)^{\prime}$ where $\boldsymbol{\beta}_{j}$ is $\mathrm{G} 1_{\mathrm{j}}-1$ by $1, \boldsymbol{\gamma}_{j}$ is $\mathrm{K} 1_{\mathrm{j}}$ by 1 , and $\boldsymbol{\lambda}_{j}$ is $\mathrm{G} 1_{\mathrm{j}}$ by 1 , and $\mathbf{u}_{j}$ is $\mathrm{nT}$ by 1 vector of disturbance in the $\mathrm{jth}$ equation, for $\mathrm{j}=1,2, \ldots, \mathrm{G}$. The variancecovariance of the innovations and the disturbances for this model are the same to those given in equations (3.199) and (3.200), respectively. 


\subsection{Estimation Issues in Spatial Simultaneous-Equations Models}

The presence of a combination of feedback simultaneity, spatial autoregressive lag simultaneity and spatial cross-regressive lag simultaneity in spatial simultaneous equations models creates a number of complications of which the questions of whether or not each equation of the model is identified, the choice of estimators and the treatment of instruments are the most important ones (Rey and Boarnet, 2004). The traditional rank and order conditions for identification, for example, are not applied if the system is expressed as (all notations are as defined before except now $\mathbf{B}$ is a matrix of coefficients whose diagonal elements are 1):

\section{$\mathbf{Y B}=\mathbf{X} \Gamma+\mathbf{W} \mathbf{Y} \boldsymbol{\Lambda}+\mathbf{U}$}

Pre- and post-multiplying the matrix of endogenous variables in equation (3.202) by two distinct coefficient matrices leads to two different reduced forms. Thus, this system as it stands does not lend itself to the application of the traditional rank and order conditions for checking identification. If the models, however, are viewed as special cases of simultaneous equations that are nonlinear in endogenous variables, identification can be checked by checking for the following necessary conditions:

1. All the endogenous variables in the model can be expressed in terms of the exogenous variables and the disturbance terms. This condition is fulfilled if matrices $\left(\mathbf{I}_{n G}-\mathbf{B}^{*}\right)$ and $\left(\mathbf{I}_{n}-\rho_{j} \mathbf{W}\right)$ are nonsingular with $\left|\rho_{j}\right|<1$, for $\mathbf{j}=1, \ldots, \mathrm{G}$.

2. The solution of the model for the endogenous variables in terms of the exogenous variables and the disturbance terms is unique. This condition is fulfilled if the 
instruments matrix $\mathbf{N}$ is selected in such away that $\lim _{n \rightarrow \infty} \frac{\mathbf{N}^{\prime} E \mathbf{Z}_{j}}{n}$ is a finite matrix which has full column rank for $\mathrm{j}=1, . ., \mathrm{G}$.

3. The number of endogenous variables appearing on the right hand side of an equation must be less than or equal to the number of exogenous and lagged endogenous variables appearing in the model but not in that equations

It is well known that the presence of endogenous variables on the right hand side of an equation in the simultaneous equations system violates the assumption of zero correlation between the regressors and the disturbance term upon which the unbiasedness or consistency of ordinary least squares (OLS) estimators are based. For the same reason, the presence of the endogenous variables in their lagged form on the right hand side of an equation leads to biased and inconsistent OLS estimates. Besides, the existence of a spatially autoregressive error term in an equation leads to the inconsistency of OLS. Hence, an alternative estimation method must be used in order to obtain unbiased and consistent estimator for the parameters of the spatial simultaneous equations models. One such an approach is the instrumental variables procedure suggested in Kelejian and Prucha (2004).

\subsubsection{Cross-Sectional Data Setting}

Kelejian and Prucha (2004) suggest limited and full information instrumental variable estimator for the parameters of a spatial simultaneous equation model and derive the limiting distribution of those estimators. In the case of limited information (single equation) estimation, they proposed a three step generalized spatial two-stage least squares (GS2SLS) procedure to estimate the unknown parameters in the jth equation of the model in equation (3.183). The first step consists of the estimation of the model 
parameter vector $\boldsymbol{\delta}_{j}$ in equation (3.187) by two-stage least squares (2SLS) using the instruments $\mathbf{N}$, where $\mathbf{N}$ is defined in reference to equation (3.187) above. The resulting 2SLS estimator is given by:

$$
\hat{\boldsymbol{\delta}}_{j}=\left(\overline{\mathbf{Z}}_{j}^{\prime} \overline{\mathbf{Z}}_{j}\right)^{-1} \overline{\mathbf{Z}}_{j}^{\prime} \mathbf{y}_{j}
$$

where $\quad \overline{\mathbf{Z}}_{j}=\mathbf{P}_{N} \mathbf{Z}_{j}=\left(\overline{\mathbf{Y}}_{j}, \mathbf{X}_{j}, \overline{\mathbf{W Y}}\right)$ with $\quad \overline{\mathbf{Y}}_{j}=\mathbf{P}_{N} \mathbf{Y}_{j}, \overline{\mathbf{W Y}}_{j}=\mathbf{P}_{N} \mathbf{W} \mathbf{Y}_{j}$, and $\mathbf{P}_{N}=\mathbf{N}\left(\mathbf{N}^{\prime} \mathbf{N}\right)^{-1} \mathbf{N}^{\prime}$ is a projection matrix. Although $\hat{\boldsymbol{\delta}}_{j}$ is consistent estimator of $\boldsymbol{\delta}_{j}$, it does not utilize information relating to the spatial correlation of the disturbance terms. These 2SLS estimates are used to compute estimates for the disturbances $\mathbf{u}_{j}$ which in turn are used to estimate the autoregressive parameter $\rho_{j}$ in the second step of the procedure. The resulting 2SLS residuals are hence given by:

$$
\tilde{\mathbf{u}}_{j}=\mathbf{y}_{j}-\mathbf{Z}_{j} \hat{\boldsymbol{\delta}}_{j} .
$$

In the second step, Kelejian and Prucha (2004) used the generalized moments procedure to estimate the spatial autoregressive parameter of the disturbances of the jth equation, for $\mathrm{j}=1, \ldots, \mathrm{G}$, of the model in equation (3.187). Note that from the relation in equation (3.187) we have:

$$
\mathbf{u}_{j}-\rho_{j} \mathbf{W} \mathbf{u}_{j}=\boldsymbol{\varepsilon}_{j}
$$

and pre-multiplication by the weights matrix $\mathbf{W}$ gives:

$$
\mathbf{W} \mathbf{u}_{j}-\rho_{j} \mathbf{W}^{2} \mathbf{u}_{j}=\mathbf{W} \boldsymbol{\varepsilon}_{j}
$$

The following three-equation system is obtained from the relationships between equations (3.205) and (3.206): 


$$
\begin{aligned}
& \frac{\boldsymbol{\varepsilon}_{j}^{\prime} \boldsymbol{\varepsilon}_{j}}{n}=\frac{\mathbf{u}_{j}^{\prime} \mathbf{u}_{j}}{n}+\rho_{j}^{2} \frac{\left(\mathbf{W} \mathbf{u}_{j}\right)^{\prime}\left(\mathbf{W} \mathbf{u}_{j}\right)}{n}-2 \rho_{j} \frac{\mathbf{u}_{j}^{\prime}\left(\mathbf{W} \mathbf{u}_{j}\right)}{n} \\
& \frac{\left(\mathbf{W} \boldsymbol{\varepsilon}_{j}\right)^{\prime}\left(\mathbf{W} \boldsymbol{\varepsilon}_{j}\right)}{n}=\frac{\left(\mathbf{W} \mathbf{u}_{j}\right)^{\prime}\left(\mathbf{W} \mathbf{u}_{j}\right)}{n}+\rho_{j}^{2} \frac{\left(\mathbf{W}^{2} \mathbf{u}_{j}\right)^{\prime}\left(\mathbf{W}^{2} \mathbf{u}_{j}\right)}{n}-2 \rho_{j} \frac{\left(\mathbf{W}^{2} \mathbf{u}_{j}\right)^{\prime}\left(\mathbf{W} \mathbf{u}_{j}\right)}{n} \\
& \frac{\boldsymbol{\varepsilon}_{j}^{\prime} \mathbf{W} \boldsymbol{\varepsilon}_{j}}{n} \\
& =\frac{\mathbf{u}_{j}^{\prime}\left(\mathbf{W} \mathbf{u}_{j}\right)}{n}+\rho_{j}^{2} \frac{\left(\mathbf{W} \mathbf{u}_{j}\right)^{\prime}\left(\mathbf{W}^{2} \mathbf{u}_{j}\right)}{n}-\rho_{j} \frac{\left(\mathbf{u}_{j}^{\prime}\left(\mathbf{W}^{2} \mathbf{u}_{j}\right)+\left(\mathbf{W} \mathbf{u}_{j}\right)^{\prime}\left(\mathbf{W} \mathbf{u}_{j}\right)\right)}{n}
\end{aligned}
$$

Taking expectations across equation (3.207):

$$
\left[\begin{array}{ll}
\frac{\boldsymbol{\varepsilon}_{j}^{\prime} \boldsymbol{\varepsilon}_{j}}{n} & =\frac{\mathbf{u}_{j}^{\prime} \mathbf{u}_{j}}{n}+\rho_{j}^{2} \frac{\left(\mathbf{W} \mathbf{u}_{j}\right)^{\prime}\left(\mathbf{W} \mathbf{u}_{j}\right)}{n}-2 \rho_{j} \frac{\mathbf{u}_{j}^{\prime}\left(\mathbf{W} \mathbf{u}_{j}\right)}{n} \\
\frac{\left(\mathbf{W} \boldsymbol{\varepsilon}_{j}\right)^{\prime}\left(\mathbf{W} \boldsymbol{\varepsilon}_{j}\right)}{n} & =\frac{\left(\mathbf{W} \mathbf{u}_{j}\right)^{\prime}\left(\mathbf{W} \mathbf{u}_{j}\right)}{n}+\rho_{j}^{2} \frac{\left(\mathbf{W}^{2} \mathbf{u}_{j}\right)^{\prime}\left(\mathbf{W}^{2} \mathbf{u}_{j}\right)}{n}-2 \rho_{j} \frac{\left(\mathbf{W}^{2} \mathbf{u}_{j}\right)^{\prime}\left(\mathbf{W} \mathbf{u}_{j}\right)}{n} \\
\frac{\boldsymbol{\varepsilon}_{j}^{\prime} \mathbf{W} \boldsymbol{\varepsilon}_{j}}{n} & =\frac{\mathbf{u}_{j}^{\prime}\left(\mathbf{W} \mathbf{u}_{j}\right)}{n}+\rho_{j}^{2} \frac{\left(\mathbf{W} \mathbf{u}_{j}\right)^{\prime}\left(\mathbf{W}^{2} \mathbf{u}_{j}\right)}{n}-\rho_{j} \frac{\left(\mathbf{u}_{j}^{\prime}\left(\mathbf{W}^{2} \mathbf{u}_{j}\right)+\left(\mathbf{W} \mathbf{u}_{j}\right)^{\prime}\left(\mathbf{W} \mathbf{u}_{j}\right)\right)}{n}
\end{array}\right]
$$

yields:

$$
\left[\begin{array}{l}
\sigma_{j}^{2} \\
\sigma_{j}^{2} \frac{\operatorname{tr}\left(\mathbf{W}^{\prime} \mathbf{W}\right)}{n} \\
\sigma_{j}^{2} \frac{\operatorname{tr}(\mathbf{W})}{n}=0
\end{array}\right]=E\left[\begin{array}{l}
\frac{\left(\mathbf{W} \mathbf{u}_{j}\right)^{\prime}\left(\mathbf{W} \mathbf{u}_{j}\right)}{n}+\rho_{j}^{2} \frac{\left(\mathbf{W}^{2} \mathbf{u}_{j}\right)^{\prime}\left(\mathbf{W}^{2} \mathbf{u}_{j}\right)}{n}-2 \rho_{j} \frac{\left(\mathbf{W} \mathbf{u}_{j}\right)^{\prime}\left(\mathbf{W} \mathbf{u}_{j}\right)}{n}-2 \rho_{j} \frac{\mathbf{u}_{j}^{\prime}\left(\mathbf{W} \mathbf{u}_{j}\right)}{n} \\
n \\
\frac{\mathbf{u}_{j}^{\prime}\left(\mathbf{W} \mathbf{u}_{j}\right)}{n}+\rho_{j}^{2} \frac{\left(\mathbf{W} \mathbf{u}_{j}\right)}{n}
\end{array}\right]
$$

and after rearranging: 


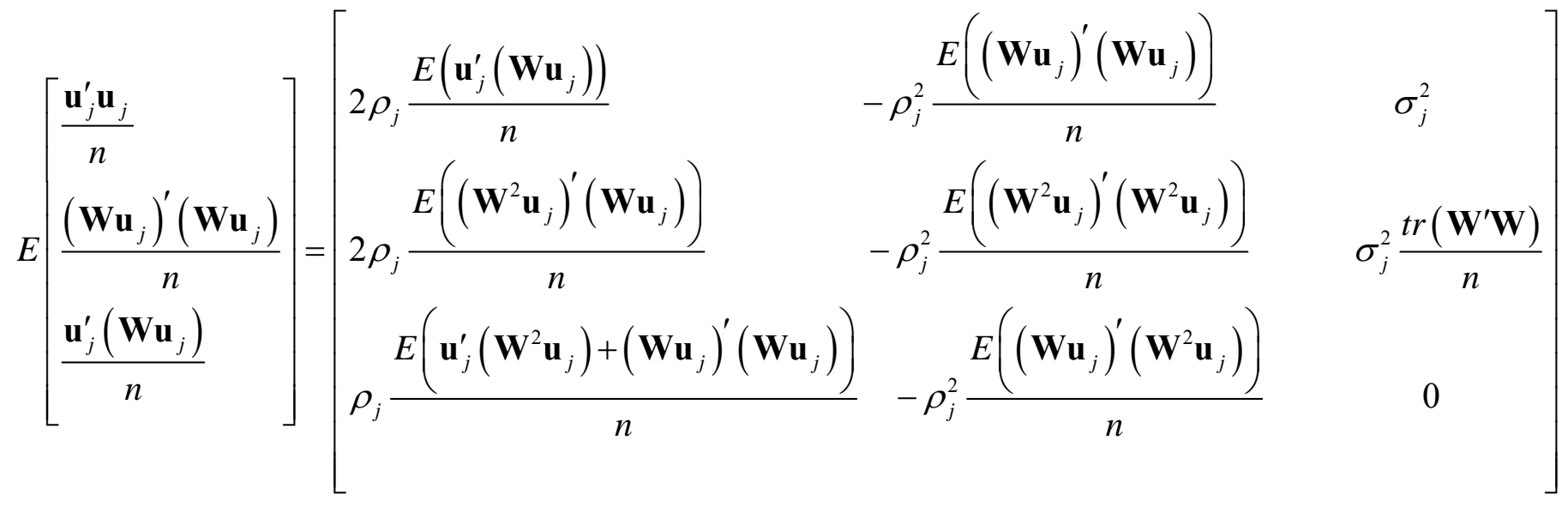

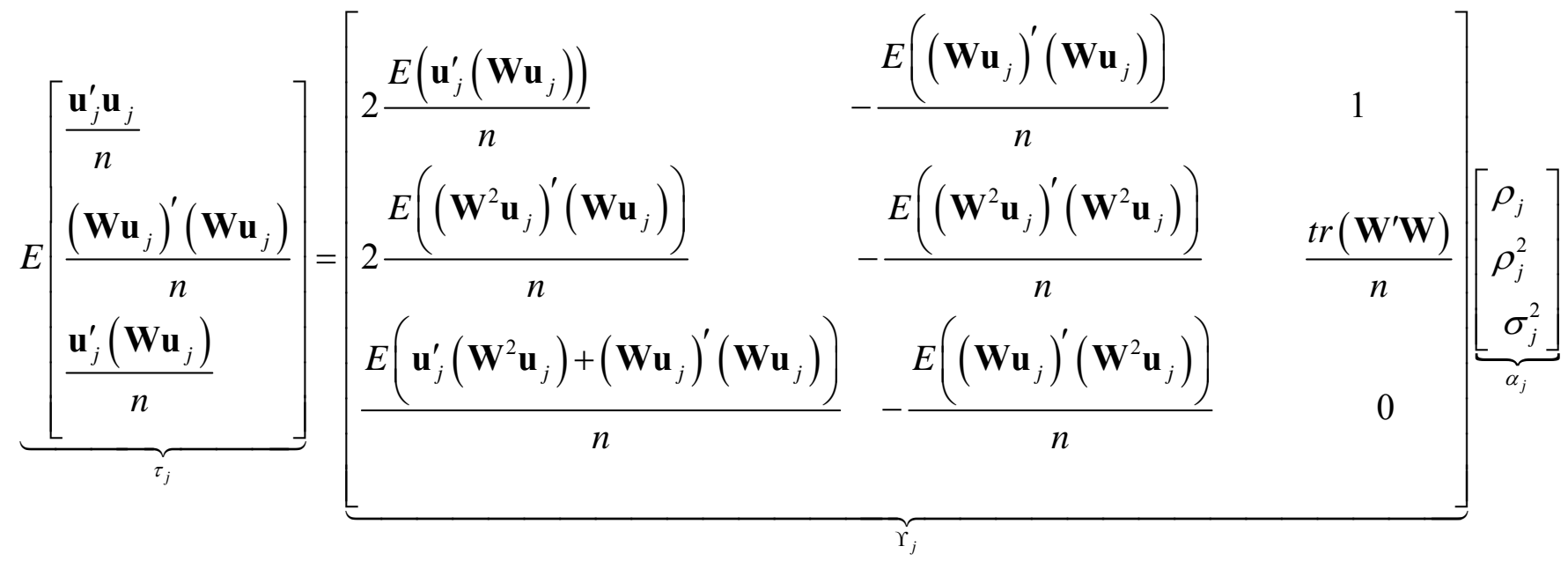

(3.211)

Thus, the system in equation (3.211) can be rewritten as $(\mathrm{j}=1, \ldots, \mathrm{G})$ :

$$
\tau_{j}=\Upsilon_{j} \boldsymbol{\alpha}_{j} \rightarrow \boldsymbol{\alpha}_{j}=\Upsilon_{j}^{-1} \tau_{j}
$$

The parameter vector $\boldsymbol{\alpha}_{j}=\left(\rho_{j}, \rho_{j}^{2}, \sigma_{j}^{2}\right)^{\prime}$ would be completely determined in terms of the relation in equation (3.212) if $\tau_{j}$ and $\Upsilon_{j}$ were known. Note that $\tau_{j}$ and $\Upsilon_{j}$ are not 
observable. Following the suggestions in Kelejian and Prucha (2004), however, the following estimators for $\tau_{j}$ and $\Upsilon_{j}$ in terms of sample moments can be defined as:

$$
\begin{aligned}
\mathbf{o}_{j} & =\frac{\left[\tilde{\mathbf{u}}_{j}^{\prime} \tilde{\mathbf{u}}_{j},\left(\mathbf{W} \tilde{\mathbf{u}}_{j}\right)^{\prime}\left(\mathbf{W} \tilde{\mathbf{u}}_{j}\right), \tilde{\mathbf{u}}_{j}^{\prime}\left(\mathbf{W} \tilde{\mathbf{u}}_{j}\right)\right]}{n}, \\
\mathbf{O}_{j} & =\frac{1}{n}\left[\begin{array}{llc}
2 \tilde{\mathbf{u}}_{j}^{\prime}\left(\mathbf{W} \tilde{\mathbf{u}}_{j}\right) & -\left(\mathbf{W} \tilde{\mathbf{u}}_{j}\right)^{\prime}\left(\mathbf{W} \tilde{\mathbf{u}}_{j}\right) & \mathrm{n} \\
2\left(\mathbf{W}^{2} \tilde{\mathbf{u}}_{j}\right)^{\prime}\left(\mathbf{W} \tilde{\mathbf{u}}_{j}\right) & -\left(\mathbf{W}^{2} \tilde{\mathbf{u}}_{j}\right)^{\prime}\left(\mathbf{W}^{2} \tilde{\mathbf{u}}_{j}\right) & \operatorname{tr}\left(\mathbf{W}^{\prime} \mathbf{W}\right) \\
\left(\tilde{\mathbf{u}}_{j}^{\prime}\left(\mathbf{W}^{2} \tilde{\mathbf{u}}_{j}\right)+\left(\mathbf{W} \tilde{\mathbf{u}}_{j}\right)^{\prime}\left(\mathbf{W} \tilde{\mathbf{u}}_{j}\right)\right) & -\left(\mathbf{W} \tilde{\mathbf{u}}_{j}\right)^{\prime}\left(\mathbf{W}^{2} \tilde{\mathbf{u}}_{j}\right) & 0
\end{array}\right]
\end{aligned}
$$

Thus, given the estimates in equation (3.213), the empirical form of the relationship in equation (3.212) can be given by:

$$
\mathbf{o}_{j}=\mathbf{O}_{j} \boldsymbol{\alpha}_{j}+\xi_{j}
$$

Since $\mathbf{o}_{j}$ and $\boldsymbol{O}_{j}$ are observable and $\boldsymbol{\alpha}_{j}$ is vector of parameters to be estimated, $\boldsymbol{\xi}_{j}$ can be viewed as a vector of regression residuals. Thus, the second step estimators of $\rho_{j}$ and $\sigma_{j}^{2}$, say, $\hat{\rho}_{j}$ and $\hat{\sigma}_{j}^{2}$, are nonlinear least squares estimators defined as the minimizer of:

$$
\left[\mathbf{o}_{j}-\mathbf{O}_{j}\left[\begin{array}{c}
\rho_{j} \\
\rho_{j}^{2} \\
\sigma_{j}^{2}
\end{array}\right]\right]^{\prime}\left[\mathbf{o}_{j}-\mathbf{O}_{j}\left[\begin{array}{c}
\rho_{j} \\
\rho_{j}^{2} \\
\sigma_{j}^{2}
\end{array}\right]\right]
$$

or

$$
\left(\hat{\rho}_{j}, \hat{\sigma}_{j}^{2}\right)=\operatorname{argmin}\left[\mathbf{o}_{j}-\mathbf{O}_{j}\left[\begin{array}{c}
\rho_{j} \\
\rho_{j}^{2} \\
\sigma_{j}^{2}
\end{array}\right]\right]^{\prime}\left[\mathbf{o}_{j}-\mathbf{O}_{j}\left[\begin{array}{c}
\rho_{j} \\
\rho_{j}^{2} \\
\sigma_{j}^{2}
\end{array}\right]\right] .
$$

In the third step of the procedure a Cochrane-Orcutt type transformation is applied to the model in equation (3.187). More specifically, let: 


$$
\mathbf{y}_{j}^{*}\left(\rho_{j}\right)=\mathbf{y}_{j}-\rho_{j} \mathbf{W} \mathbf{y}_{j} \text { and } \mathbf{Z}_{j}^{*}\left(\rho_{j}\right)=\mathbf{Z}_{j}-\rho_{j} \mathbf{W} \mathbf{Z}_{j}
$$

Then, equation (3.187) becomes:

$$
\mathbf{y}_{j}^{*}\left(\rho_{j}\right)=\mathbf{Z}_{j}^{*}\left(\rho_{j}\right) \boldsymbol{\delta}_{j}+\boldsymbol{\varepsilon}_{j}
$$

If $\rho_{j}$ were know we could perform 2SLS on equation (3.216) to obtain the generalized spatial two-stage squares (GS2SLS) estimator for $\boldsymbol{\delta}_{j}$. That is:

$$
\hat{\boldsymbol{\delta}}_{j}=\left(\overline{\mathbf{Z}}_{j}^{*}\left(\rho_{j}\right)^{\prime} \overline{\mathbf{Z}}_{j}^{*}\left(\rho_{j}\right)\right)^{-1} \overline{\mathbf{Z}}_{j}^{*}\left(\rho_{j}\right)^{\prime} \mathbf{y}_{j}^{*}\left(\rho_{j}\right)
$$

where $\overline{\mathbf{Z}}_{j}^{*}\left(\rho_{j}\right)=\mathbf{P}_{N} \mathbf{Z}_{j}^{*}\left(\rho_{j}\right)$ and $\mathbf{P}_{N}=\mathbf{N}\left(\mathbf{N}^{\prime} \mathbf{N}\right)^{-1} \mathbf{N}^{\prime}$. But, since in practical applications $\rho_{j}$ is not known, it is replaced with its estimate as defined in equation (3.215) and estimate the model in equation (3.216) using 2SLS. The resulting estimator is termed as the feasible GS2SLS and is given by:

$$
\hat{\boldsymbol{\delta}}_{j}^{F}=\left(\overline{\mathbf{Z}}_{j}^{*}\left(\hat{\rho}_{j}\right)^{\prime} \overline{\mathbf{Z}}_{j}^{*}\left(\hat{\rho}_{j}\right)\right)^{-1} \overline{\mathbf{Z}}_{j}^{*}\left(\hat{\rho}_{j}\right)^{\prime} \mathbf{y}_{j}^{*}\left(\hat{\rho}_{j}\right)
$$

where $\overline{\mathbf{Z}}_{j}^{*}\left(\hat{\rho}_{j}\right)=\mathbf{P}_{N}\left[\mathbf{Z}_{j}-\hat{\rho}_{j} \mathbf{W} \mathbf{Z}_{j}\right]$ and $\mathbf{y}_{j}^{*}\left(\hat{\rho}_{j}\right)=\mathbf{y}_{j}-\hat{\rho}_{j} \mathbf{W} \mathbf{y}_{j}$.

The three step GS2SLS procedure is applied in estimating the parameters of spatial simultaneous equation models when the spatial dependence is either spatial error dependence or both spatial error dependence and spatial lag dependence. When the spatial dependence is only spatial lag type and if the disturbances are white noise, then, the second step and consequently the third step are not required.

One of the limitations of the limited information (single equation) estimation technique is that it does not take into account the information provided by the potential cross equation correlation in the innovation vectors $\varepsilon_{j}$. In order to use the information from such cross 
equation correlations, it is important to stack the equations given in equation (3.216) as follows:

$$
\mathbf{y}^{*}(\rho)=\mathbf{Z}^{*}(\rho) \boldsymbol{\delta}+\boldsymbol{\varepsilon}
$$

where

$\mathbf{y}^{*}(\rho)=\left(\mathbf{y}_{1}^{*}\left(\rho_{1}\right)^{\prime}, \ldots, \mathbf{y}_{G}^{*}\left(\rho_{G}\right)^{\prime}\right)^{\prime}, \mathbf{Z}^{*}(\rho)=\operatorname{diag}_{j=1}^{G}\left(\mathbf{Z}_{j}^{*}\left(\rho_{j}\right)\right), \rho=\left(\rho_{1}, \ldots, \rho_{G}\right)^{\prime}$ and $\delta=\left(\delta_{1}, \ldots, \delta_{G}\right)^{\prime}$ Recall from equation (3.185) that $E(\boldsymbol{\varepsilon})=\mathbf{0}$ and $E\left(\boldsymbol{\varepsilon} \boldsymbol{\varepsilon}^{\prime}\right)=\boldsymbol{\Sigma} \otimes \mathbf{I}_{n}$. Assuming that $\rho$ and $\boldsymbol{\Sigma}$ were known, equation (3.219) could be estimated using the instrumental variable technique. In that case, the resulting systems instrumental variable estimator of $\boldsymbol{\delta}$ would be the generalized spatial three-stage least squares (GS3SLS) estimator which can be given by (all notations as defined before):

$$
\hat{\boldsymbol{\delta}}=\left(\overline{\mathbf{Z}}^{*}(\hat{\rho})^{\prime}\left(\boldsymbol{\Sigma}^{-1} \otimes \mathbf{I}_{n}\right) \overline{\mathbf{Z}}^{*}(\hat{\rho})\right)^{-1} \overline{\mathbf{Z}}^{*}(\hat{\rho})^{\prime}\left(\boldsymbol{\Sigma}^{-1} \otimes \mathbf{I}_{n}\right) \mathbf{y}^{*}(\hat{\rho})
$$

Since in practical applications $\rho$ and $\Sigma$ are not known, their estimators are required to obtain the feasible estimator for $\boldsymbol{\delta}$. The generalized moments estimators for $\rho_{j}$ and $\sigma_{j}^{2}$ are defined in equation (3.215). Note that $\sigma_{j}^{2}$ is the jth diagonal element of $\boldsymbol{\Sigma}$. Besides, a consistent estimator for $\boldsymbol{\Sigma}$ can be derived by combining equations (3.216) and (3.218) as:

$$
\hat{\boldsymbol{\sigma}}_{j l}^{2}=\frac{1}{n} \hat{\boldsymbol{\varepsilon}}_{j}^{\prime} \hat{\boldsymbol{\varepsilon}}_{l}, j, l=1, \ldots, G
$$

where $\hat{\boldsymbol{\varepsilon}}_{j}=\mathbf{y}_{j}^{*}\left(\hat{\rho}_{j}\right)-\mathbf{Z}_{j}^{*}\left(\hat{\rho}_{j}\right) \hat{\boldsymbol{\delta}}_{j}^{F}$. Then, the G by G matrix whose (j,1)th element is $\hat{\boldsymbol{\sigma}}_{j l}^{2}$ defines a consistent estimator for $\boldsymbol{\Sigma}$ denoted by $\hat{\boldsymbol{\Sigma}}$. Substituting $\boldsymbol{\Sigma}$ with $\hat{\boldsymbol{\Sigma}}$ in equation (3.220) gives the feasible generalized spatial three-stage least squares (FGS3SLS) estimator for $\boldsymbol{\delta}$. That is:

$$
\hat{\boldsymbol{\delta}}^{F}=\left(\overline{\mathbf{Z}}^{*}(\hat{\rho})^{\prime}\left(\hat{\boldsymbol{\Sigma}}^{-1} \otimes \mathbf{I}_{n}\right) \overline{\mathbf{Z}}^{*}(\hat{\rho})\right)^{-1} \overline{\mathbf{Z}}^{*}(\hat{\rho})^{\prime}\left(\hat{\boldsymbol{\Sigma}}^{-1} \otimes \mathbf{I}_{n}\right) \mathbf{y}^{*}(\hat{\rho})
$$




\subsubsection{Panel Data Setting}

The same procedure is also applicable to the panel data case with minor change in the arrangement of the data set and some changes in notations. Now, recall the spatial autoregressive panel data model with spatial autoregressive disturbances, from equation (3.201):

$$
\begin{aligned}
& \mathbf{y}=\mathbf{Z} \boldsymbol{\delta}+\mathbf{u}, \\
& \mathbf{u}=\rho\left(\mathbf{I}_{T} \otimes \mathbf{W}\right) \mathbf{u}+\mathbf{v}
\end{aligned}
$$

where

$$
\boldsymbol{v}=\left(\mathbf{I}_{n} \otimes \mathbf{I}_{T}\right) \mu+\boldsymbol{\omega} .
$$

As it is evident from equations (3.196)-(3.200), the variance-covariance matrix $\boldsymbol{\Omega}_{u}$ depends on $\rho, \boldsymbol{\sigma}_{\omega}^{2}$ and $\boldsymbol{\sigma}_{1}^{2}$. Thus, a feasible estimator for the parameter of the model requires consistent estimators of $\rho, \boldsymbol{\sigma}_{\omega}^{2}$ and $\boldsymbol{\sigma}_{1}^{2}$. To this end, generalized moments estimators of $\rho, \boldsymbol{\sigma}_{\omega}^{2}$ and $\boldsymbol{\sigma}_{1}^{2}$ are defined in the following subsection. These generalized moments estimators generalize the generalized moments estimators given in Kelejian and Prucha (2004) for the case of a single cross section. First, the generalized moments estimators of $\rho, \boldsymbol{\sigma}_{\omega}^{2}$ and $\boldsymbol{\sigma}_{1}^{2}$ in context of single equation are defined and then the procedure is generalized to system of simultaneous equations model.

Consider the jth equation of the system in (3.223). The generalized moments estimators of $\rho_{j}, \boldsymbol{\sigma}_{\omega \mathrm{j}}^{2}$ and $\boldsymbol{\sigma}_{1 j}^{2}$ are defined in terms of six moments conditions. These six moments conditions, for $T \geq 2$, are given as follows: 


$$
\begin{array}{ll}
E \frac{1}{n(T-1)} \mathbf{v}_{j}^{\prime} \mathbf{H} \mathbf{v}_{j} & =\boldsymbol{\sigma}_{\omega j}^{2} \\
E \frac{1}{n(T-1)}\left(\left(\mathbf{I}_{T} \otimes \mathbf{W}\right) \mathbf{v}_{j}\right)^{\prime} \mathbf{H}\left(\left(\mathbf{I}_{T} \otimes \mathbf{W}\right) \mathbf{v}_{j}\right) & =\boldsymbol{\sigma}_{\omega j}^{2} \frac{1}{n} \operatorname{tr}\left(\mathbf{W}^{\prime} \mathbf{W}\right) \\
E \frac{1}{n(T-1)} \mathbf{v}_{j}^{\prime} \mathbf{H}\left(\left(\mathbf{I}_{T} \otimes \mathbf{W}\right) \mathbf{v}_{j}\right) & =\mathbf{0} \\
E \frac{1}{n} \mathbf{v}_{j}^{\prime} \mathbf{P} \mathbf{v}_{j} & =\boldsymbol{\sigma}_{1 j}^{2} \\
E \frac{1}{n}\left(\left(\mathbf{I}_{T} \otimes \mathbf{W}\right) \mathbf{v}_{j}\right)^{\prime} \mathbf{P}\left(\left(\mathbf{I}_{T} \otimes \mathbf{W}\right) \mathbf{v}_{j}\right) & =\boldsymbol{\sigma}_{1 j}^{2} \frac{1}{n} \operatorname{tr}\left(\mathbf{W}^{\prime} \mathbf{W}\right) \\
E \frac{1}{n} \mathbf{v}_{j}^{\prime} \mathbf{P}\left(\left(\mathbf{I}_{T} \otimes \mathbf{W}\right) \mathbf{v}_{j}\right) & =\mathbf{0}
\end{array}
$$

Note that since $\mathbf{v}_{j}$ are not observable, $E \frac{1}{n(T-1)} \mathbf{v}_{j}^{\prime} \mathbf{H} \mathbf{v}_{j}$ and $E \frac{1}{n} \mathbf{v}_{j}^{\prime} \mathbf{P} \mathbf{v}_{j}$ do not represent the unbiased analysis of variance estimators of $\boldsymbol{\sigma}_{\omega \mathrm{j}}^{2}$ and $\boldsymbol{\sigma}_{1 j}^{2}$, respectively. The innovations $v_{j}$ can, however, be expressed in terms of the disturbances and the disturbances in turn can be substituted by their estimated values which are observable. Thus, using the relations in the jth equation of (3.223), let:

$$
\begin{aligned}
& \mathbf{v}_{j}=\mathbf{u}_{j}-\rho_{j}\left(\mathbf{I}_{T} \otimes \mathbf{W}\right) \mathbf{u}_{j} \\
& \left(\mathbf{I}_{T} \otimes \mathbf{W}\right) \mathbf{v}_{j}=\left(\mathbf{I}_{T} \otimes \mathbf{W}\right) \mathbf{u}_{j}-\rho_{j}\left(\mathbf{I}_{T} \otimes \mathbf{W}^{2}\right) \mathbf{u}_{j}
\end{aligned}
$$

Substituting the expressions in (3.225) for $\mathbf{v}_{j}$ and $\left(\mathbf{I}_{T} \otimes \mathbf{W}\right) \mathbf{v}_{j}$ into (3.224) gives the moments conditions in (3.224) in terms of the disturbances. That is: 


$$
\begin{array}{lc}
E \frac{1}{n(T-1)}\left(\mathbf{u}_{j}-\rho_{j}\left(\mathbf{I}_{T} \otimes \mathbf{W}\right) \mathbf{u}_{j}\right)^{\prime} \mathbf{H}\left(\mathbf{u}_{j}-\rho_{j}\left(\mathbf{I}_{T} \otimes \mathbf{W}\right) \mathbf{u}_{j}\right) & =\boldsymbol{\sigma}_{\omega j}^{2} \\
E \frac{1}{n(T-1)}\left(\left(\mathbf{I}_{T} \otimes \mathbf{W}\right) \mathbf{u}_{j}-\rho_{j}\left(\mathbf{I}_{T} \otimes \mathbf{W}^{2}\right) \mathbf{u}_{j}\right)^{\prime} \mathbf{H}\left(\left(\mathbf{I}_{T} \otimes \mathbf{W}\right) \mathbf{u}_{j}-\rho_{j}\left(\mathbf{I}_{T} \otimes \mathbf{W}^{2}\right) \mathbf{u}_{j}\right) & =\boldsymbol{\sigma}_{\omega j}^{2} \frac{1}{n} \operatorname{tr}\left(\mathbf{W}^{\prime} \mathbf{W}\right) \\
E \frac{1}{n(T-1)}\left(\mathbf{u}_{j}-\rho_{j}\left(\mathbf{I}_{T} \otimes \mathbf{W}\right) \mathbf{u}_{j}\right)^{\prime} \mathbf{H}\left(\left(\mathbf{I}_{T} \otimes \mathbf{W}\right) \mathbf{u}_{j}-\rho_{j}\left(\mathbf{I}_{T} \otimes \mathbf{W}^{2}\right) \mathbf{u}_{j}\right) & =\mathbf{0} \\
E \frac{1}{n}\left(\mathbf{u}_{j}-\rho_{j}\left(\mathbf{I}_{T} \otimes \mathbf{W}\right) \mathbf{u}_{j}\right)^{\prime} \mathbf{P}\left(\mathbf{u}_{j}-\rho_{j}\left(\mathbf{I}_{T} \otimes \mathbf{W}\right) \mathbf{u}_{j}\right) & =\boldsymbol{\sigma}_{1 j}^{2} \\
E \frac{1}{n}\left(\left(\mathbf{I}_{T} \otimes \mathbf{W}\right) \mathbf{u}_{j}-\rho_{j}\left(\mathbf{I}_{T} \otimes \mathbf{W}^{2}\right) \mathbf{u}_{j}\right)^{\prime} \mathbf{P}\left(\left(\mathbf{I}_{T} \otimes \mathbf{W}\right) \mathbf{u}_{j}-\rho_{j}\left(\mathbf{I}_{T} \otimes \mathbf{W}^{2}\right) \mathbf{u}_{j}\right) & =\boldsymbol{\sigma}_{1 j}^{2} \frac{1}{n} \operatorname{tr}\left(\mathbf{W}^{\prime} \mathbf{W}\right) \\
E \frac{1}{n}\left(\mathbf{u}_{j}-\rho_{j}\left(\mathbf{I}_{T} \otimes \mathbf{W}\right) \mathbf{u}_{j}\right)^{\prime} \mathbf{P}\left(\left(\mathbf{I}_{T} \otimes \mathbf{W}\right) \mathbf{u}_{j}-\rho_{j}\left(\mathbf{I}_{T} \otimes \mathbf{W}^{2}\right) \mathbf{u}_{j}\right) & =\mathbf{0}
\end{array}
$$

After rearranging, this yields a system of six equations that can be expressed as:

$$
\Upsilon_{j} \alpha_{j}-\tau_{j}=0
$$

where

$$
\tau_{j}=E\left[\begin{array}{l}
\frac{\mathbf{u}_{j}^{\prime} \mathbf{H} \mathbf{u}_{j}}{n(T-1)} \\
\frac{\left(\left(\mathbf{I}_{T} \otimes \mathbf{W}\right) \mathbf{u}_{j}\right)^{\prime} \mathbf{H}\left(\left(\mathbf{I}_{T} \otimes \mathbf{W}\right) \mathbf{u}_{j}\right)}{n(T-1)} \\
\frac{\mathbf{u}_{j}^{\prime} \mathbf{H}\left(\left(\mathbf{I}_{T} \otimes \mathbf{W}\right) \mathbf{u}_{j}\right)}{n(T-1)} \\
\frac{\mathbf{u}_{j}^{\prime} \mathbf{P} \mathbf{u}_{j}}{n} \\
\frac{\left(\left(\mathbf{I}_{T} \otimes \mathbf{W}\right) \mathbf{u}_{j}\right)^{\prime} \mathbf{P}\left(\left(\mathbf{I}_{T} \otimes \mathbf{W}\right) \mathbf{u}_{j}\right)}{n} \\
\frac{\mathbf{u}_{j}^{\prime} \mathbf{P}\left(\left(\mathbf{I}_{T} \otimes \mathbf{W}\right) \mathbf{u}_{j}\right)}{n}
\end{array}\right],
$$




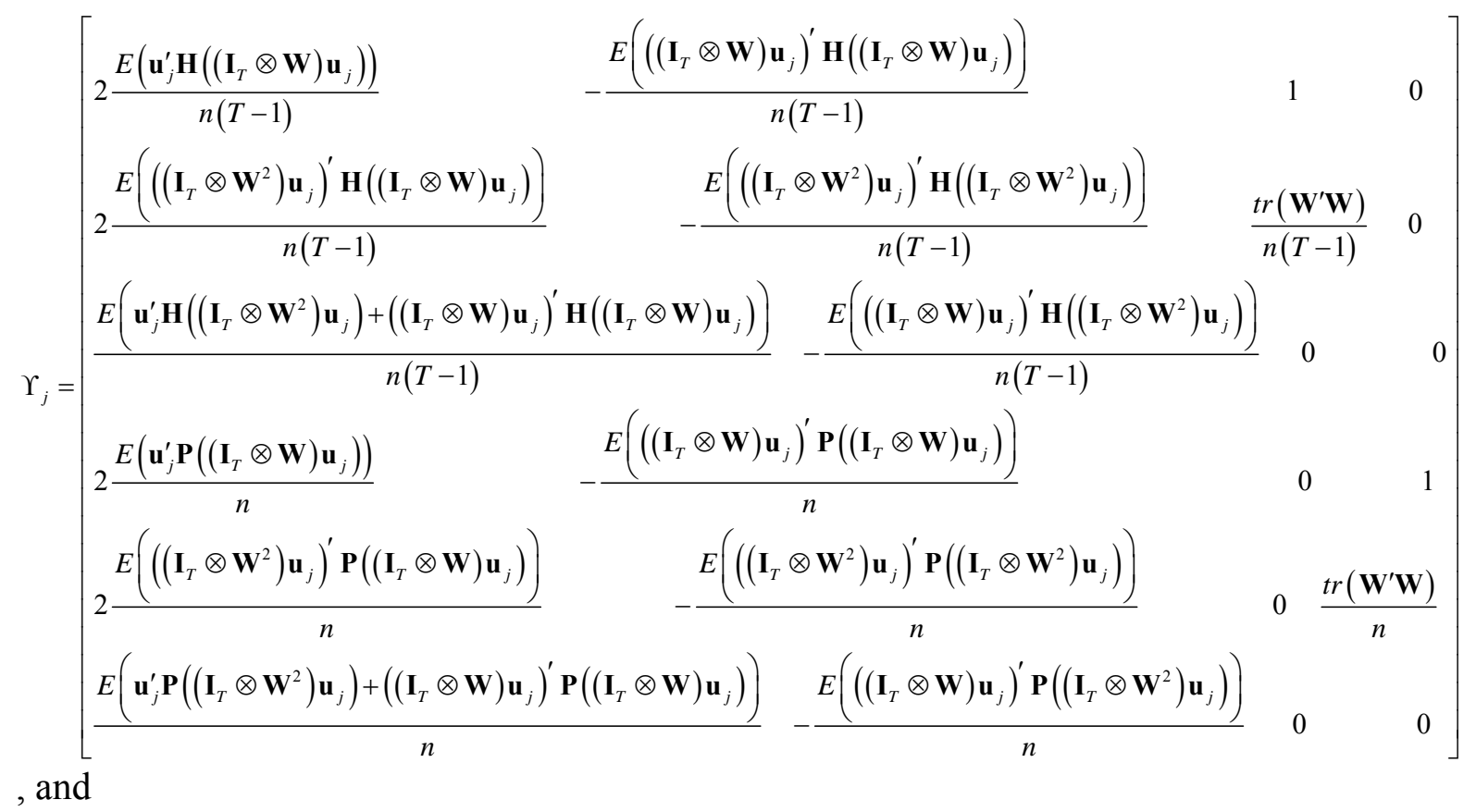

$$
\alpha_{j}=\left[\begin{array}{c}
\rho_{j} \\
\rho_{j}^{2} \\
\sigma_{\omega j}^{2} \\
\sigma_{1 j}^{2}
\end{array}\right] .
$$


The system in (3.227) consists of six equations involving the second moments of $\mathbf{u}_{j},\left(\mathbf{I}_{T} \otimes \mathbf{W}\right) \mathbf{u}_{j}$ and $\left(\mathbf{I}_{T} \otimes \mathbf{W}^{2}\right) \mathbf{u}_{j}$. However, since $\mathbf{u}_{j},\left(\mathbf{I}_{T} \otimes \mathbf{W}\right) \mathbf{u}_{j}$ and $\left(\mathbf{I}_{T} \otimes \mathbf{W}^{2}\right) \mathbf{u}_{j}$ are not observable, the generalized moments estimators are defined in terms of sample moments. These sample moments are obtained by replacing the $\mathbf{u}_{j}$ 's in (3.227) by their estimated values $\left(\tilde{\mathbf{u}}_{j}\right)$, where the estimated disturbances are computed as:

$$
\tilde{\mathbf{u}}_{j}=\mathbf{y}_{j}-\mathbf{Z}_{j} \hat{\boldsymbol{\delta}}_{j}
$$

where $\mathbf{y}_{j}$ and $\mathbf{Z}_{j}$ are defined in equation (3.201) and $\hat{\boldsymbol{\delta}}_{j}$ is an estimator of $\boldsymbol{\delta}_{j}$ obtained by estimating the regression model in equation (3.223) by two-stage least squares(2SLS) using the instruments $\mathbf{N}$. After substituting $\mathbf{u}_{j}$ by $\tilde{\mathbf{u}}_{j}$ the system in (3.227) becomes the sample analogue to (3.227) and can be expressed as:

$$
\mathbf{O}_{j} \boldsymbol{\alpha}_{j}-\mathbf{o}_{j}=\xi_{j}\left(\boldsymbol{\alpha}_{j}\right)
$$

where

$$
\mathbf{o}_{j}=E\left[\begin{array}{l}
\frac{\tilde{\mathbf{u}}_{j}^{\prime} \mathbf{H} \tilde{\mathbf{u}}_{j}}{n(T-1)} \\
\frac{\left(\left(\mathbf{I}_{T} \otimes \mathbf{W}\right) \tilde{\mathbf{u}}_{j}\right)^{\prime} \mathbf{H}\left(\left(\mathbf{I}_{T} \otimes \mathbf{W}\right) \tilde{\mathbf{u}}_{j}\right)}{n(T-1)} \\
\frac{\tilde{\mathbf{u}}_{j}^{\prime} \mathbf{H}\left(\left(\mathbf{I}_{T} \otimes \mathbf{W}\right) \tilde{\mathbf{u}}_{j}\right)}{n(T-1)} \\
\frac{\tilde{\mathbf{u}}_{j}^{\prime} \mathbf{P} \tilde{\mathbf{u}}_{j}}{n} \\
\frac{\left(\left(\mathbf{I}_{T} \otimes \mathbf{W}\right) \tilde{\mathbf{u}}_{j}\right)^{\prime} \mathbf{P}\left(\left(\mathbf{I}_{T} \otimes \mathbf{W}\right) \tilde{\mathbf{u}}_{j}\right)}{n} \\
\frac{\tilde{\mathbf{u}}_{j}^{\prime} \mathbf{P}\left(\left(\mathbf{I}_{T} \otimes \mathbf{W}\right) \tilde{\mathbf{u}}_{j}\right)}{n}
\end{array}\right],
$$




$$
\mathbf{O}_{j}=\left[\begin{array}{llll}
2 \frac{E\left(\tilde{\mathbf{u}}_{j}^{\prime} \mathbf{H}\left(\left(\mathbf{I}_{T} \otimes \mathbf{W}\right) \tilde{\mathbf{u}}_{j}\right)\right)}{n(T-1)} & -\frac{E\left(\left(\left(\mathbf{I}_{T} \otimes \mathbf{W}\right) \tilde{\mathbf{u}}_{j}\right)^{\prime} \mathbf{H}\left(\left(\mathbf{I}_{T} \otimes \mathbf{W}\right) \tilde{\mathbf{u}}_{j}\right)\right)}{n(T-1)} & 1 & 0 \\
\frac{E\left(\left(\left(\mathbf{I}_{T} \otimes \mathbf{W}^{2}\right) \tilde{\mathbf{u}}_{j}\right)^{\prime} \mathbf{H}\left(\left(\mathbf{I}_{T} \otimes \mathbf{W}\right) \tilde{\mathbf{u}}_{j}\right)\right)}{n(T-1)} & -\frac{E\left(\left(\left(\mathbf{I}_{T} \otimes \mathbf{W}^{2}\right) \tilde{\mathbf{u}}_{j}\right)^{\prime} \mathbf{H}\left(\left(\mathbf{I}_{T} \otimes \mathbf{W}^{2}\right) \tilde{\mathbf{u}}_{j}\right)\right)}{n(T-1)} & \frac{\operatorname{tr}\left(\mathbf{W}^{\prime} \mathbf{W}\right)}{n(T-1)} & 0 \\
\frac{E\left(\tilde{\mathbf{u}}_{j}^{\prime} \mathbf{H}\left(\left(\mathbf{I}_{T} \otimes \mathbf{W}^{2}\right) \tilde{\mathbf{u}}_{j}\right)+\left(\left(\mathbf{I}_{T} \otimes \mathbf{W}\right) \tilde{\mathbf{u}}_{j}\right)^{\prime} \mathbf{H}\left(\left(\mathbf{I}_{T} \otimes \mathbf{W}\right) \tilde{\mathbf{u}}_{j}\right)\right)}{n(T-1)} & -\frac{E\left(\left(\left(\mathbf{I}_{T} \otimes \mathbf{W}\right) \tilde{\mathbf{u}}_{j}\right)^{\prime} \mathbf{H}\left(\left(\mathbf{I}_{T} \otimes \mathbf{W}^{2}\right) \tilde{\mathbf{u}}_{j}\right)\right)}{n(T-1)} & 0 & 0 \\
\frac{E\left(\tilde{\mathbf{u}}_{j}^{\prime} \mathbf{P}\left(\left(\mathbf{I}_{T} \otimes \mathbf{W}\right) \tilde{\mathbf{u}}_{j}\right)\right)}{n} & -\frac{E\left(\left(\left(\mathbf{I}_{T} \otimes \mathbf{W}\right) \tilde{\mathbf{u}}_{j}\right)^{\prime} \mathbf{P}\left(\left(\mathbf{I}_{T} \otimes \mathbf{W}\right) \tilde{\mathbf{u}}_{j}\right)\right)}{n} & 0 & 1 \\
\frac{E\left(\left(\left(\mathbf{I}_{T} \otimes \mathbf{W}^{2}\right) \tilde{\mathbf{u}}_{j}\right)^{\prime} \mathbf{P}\left(\left(\mathbf{I}_{T} \otimes \mathbf{W}\right) \tilde{\mathbf{u}}_{j}\right)\right)}{n} & -\frac{E\left(\left(\left(\mathbf{I}_{T} \otimes \mathbf{W}^{2}\right) \tilde{\mathbf{u}}_{j}\right)^{\prime} \mathbf{P}\left(\left(\mathbf{I}_{T} \otimes \mathbf{W}^{2}\right) \tilde{\mathbf{u}}_{j}\right)\right)}{n} & 0 & \frac{\operatorname{tr}\left(\mathbf{W}^{\prime} \mathbf{W}\right)}{n} \\
\frac{E\left(\tilde{\mathbf{u}}_{j}^{\prime} \mathbf{P}\left(\left(\mathbf{I}_{T} \otimes \mathbf{W}^{2}\right) \tilde{\mathbf{u}}_{j}\right)+\left(\left(\mathbf{I}_{T} \otimes \mathbf{W}\right) \tilde{\mathbf{u}}_{j}\right)^{\prime} \mathbf{P}\left(\left(\mathbf{I}_{T} \otimes \mathbf{W}\right) \tilde{\mathbf{u}}_{j}\right)\right)}{n} & -\frac{E\left(\left(\left(\mathbf{I}_{T} \otimes \mathbf{W}\right) \tilde{\mathbf{u}}_{j}\right)^{\prime} \mathbf{P}\left(\left(\mathbf{I}_{T} \otimes \mathbf{W}^{2}\right) \tilde{\mathbf{u}}_{j}\right)\right)}{n} & 0 & 0
\end{array}\right],
$$

$\boldsymbol{\alpha}_{j}=\left[\begin{array}{c}\rho_{j} \\ \rho_{j}^{2} \\ \sigma_{\omega j}^{2} \\ \sigma_{1 j}^{2}\end{array}\right]$, and $\xi_{j}\left(\boldsymbol{\alpha}_{j}\right)$ is a vector of residuals.

Now, it is possible to define the unweighted and weighted generalized moments estimators of $\rho_{j}, \boldsymbol{\sigma}_{\omega \mathrm{j}}^{2}$ and $\boldsymbol{\sigma}_{1 j}^{2}$. When equal weights are given to the moments conditions, the generalized moments estimators of $\rho_{j}, \boldsymbol{\sigma}_{\omega \mathrm{j}}^{2}$ and $\boldsymbol{\sigma}_{1 j}^{2}$, say, $\hat{\rho}_{j}, \hat{\boldsymbol{\sigma}}_{\omega j}^{2}$ and $\hat{\boldsymbol{\sigma}}_{1 j}^{2}$ respectively, are defined as the unweighted nonlinear least squares estimators corresponding to (3.229). More formally, $\hat{\rho}_{j}, \hat{\boldsymbol{\sigma}}_{\omega j}^{2}$ and $\hat{\boldsymbol{\sigma}}_{1 j}^{2}$ are defined as the nonlinear least squares estimators that minimize: 


$$
\xi_{j}\left(\rho_{j}, \sigma_{\omega j}^{2}, \sigma_{1 j}^{2}\right)^{\prime} \xi_{j}\left(\rho_{j}, \sigma_{\omega j}^{2}, \sigma_{1 j}^{2}\right)=\left[\mathbf{o}_{j}-\mathbf{O}_{j}\left[\begin{array}{c}
\rho_{j} \\
\sigma_{\omega j}^{2} \\
\sigma_{1 j}^{2}
\end{array}\right]\right]^{\prime}\left[\mathbf{o}_{j}-\mathbf{O}_{j}\left[\begin{array}{c}
\rho_{j} \\
\sigma_{\omega j}^{2} \\
\sigma_{1 j}^{2}
\end{array}\right]\right]
$$

or

$$
\begin{aligned}
\left(\hat{\rho}_{j}, \hat{\sigma}_{\omega j}^{2}, \hat{\sigma}_{1 j}^{2}\right)=\operatorname{argmin}\{ & \left\{\left[\mathbf{o}_{j}-\mathbf{O}_{j}\left[\begin{array}{c}
\rho_{j} \\
\sigma_{\omega j}^{2} \\
\sigma_{1 j}^{2}
\end{array}\right]\right]^{\prime}\left[\mathbf{o}_{j}-\mathbf{O}_{j}\left[\begin{array}{c}
\rho_{j} \\
\sigma_{\omega j}^{2} \\
\sigma_{1 j}^{2}
\end{array}\right]\right],\right. \\
& \left.\rho_{j} \in(-1,1), \sigma_{\omega j}^{2} \in\left[0, c_{\omega}\right], \sigma_{1 j}^{2} \in\left[0, c_{1}\right]\right\}
\end{aligned}
$$

When the moments conditions are weighed by a weighing matrix, say, $\boldsymbol{\Phi}$, the generalized moments estimators of $\rho_{j}, \boldsymbol{\sigma}_{\omega \mathrm{j}}^{2}$ and $\boldsymbol{\sigma}_{1 j}^{2}$, however, are defined as the nonlinear least squares estimators that minimize:

$\boldsymbol{\xi}_{j}\left(\rho_{j}, \sigma_{\omega j}^{2}, \sigma_{1 j}^{2}\right)^{\prime} \tilde{\boldsymbol{\Phi}}_{j}^{-1} \boldsymbol{\xi}_{j}\left(\rho_{j}, \sigma_{\omega j}^{2}, \sigma_{1 j}^{2}\right)=\left[\mathbf{o}_{j}-\mathbf{O}_{j}\left[\begin{array}{l}\rho_{j} \\ \sigma_{\omega j}^{2} \\ \sigma_{1 j}^{2}\end{array}\right]\right]^{\prime} \tilde{\boldsymbol{\Phi}}_{j}^{-1}\left[\mathbf{o}_{j}-\mathbf{O}_{j}\left[\begin{array}{l}\rho_{j} \\ \sigma_{\omega j}^{2} \\ \sigma_{1 j}^{2}\end{array}\right]\right]$

or

$$
\begin{array}{r}
\left(\tilde{\rho}_{j}, \tilde{\sigma}_{\omega j}^{2}, \tilde{\sigma}_{1 j}^{2}\right)=\operatorname{argmin}\left\{\left[\mathbf{o}_{j}-\mathbf{O}_{j}\left[\begin{array}{c}
\rho_{j} \\
\sigma_{\omega j}^{2} \\
\sigma_{1 j}^{2}
\end{array}\right]\right]^{\prime} \tilde{\boldsymbol{\Phi}}_{j}^{-1}\left[\mathbf{o}_{j}-\mathbf{O}_{j}\left[\begin{array}{c}
\rho_{j} \\
\sigma_{\omega j}^{2} \\
\sigma_{1 j}^{2}
\end{array}\right]\right],\right. \\
\left.\rho_{j} \in(-1,1), \sigma_{\omega j}^{2} \in\left[0, c_{\omega}\right], \sigma_{1 j}^{2} \in\left[0, c_{1}\right]\right\}
\end{array}
$$

where $\tilde{\rho}_{j}, \tilde{\sigma}_{\omega j}^{2}$ and $\tilde{\sigma}_{1 j}^{2}$ are the weighted generalized moments estimators of $\rho_{j}, \boldsymbol{\sigma}_{\omega \mathrm{j}}^{2}$ and $\boldsymbol{\sigma}_{1 j}^{2}$ respectively and $\tilde{\boldsymbol{\Phi}}_{j}$ is the consistent estimator of $\boldsymbol{\Phi}_{j}$ with $\boldsymbol{\Phi}_{j}$ representing the variance covariance matrix of the sample moments at the true parameter values. Recall that in this 
research the sample moments at the true parameter values are given by the left hand side expressions on equation (3.224) with the expectations operator suppressed. Thus, $\boldsymbol{\Phi}_{j}$ can be expressed by:

$$
\boldsymbol{\Phi}_{j}=\left[\begin{array}{cc}
\frac{\boldsymbol{\sigma}_{\omega j}^{4}}{T-1} & \mathbf{0} \\
\mathbf{0} & \boldsymbol{\sigma}_{1 j}^{4}
\end{array}\right] \otimes\left[\begin{array}{ccc}
2 & 2 \operatorname{tr}\left(\frac{\mathbf{W}^{\prime} \mathbf{W}}{n}\right) & \mathbf{0} \\
2 \operatorname{tr}\left(\frac{\mathbf{W}^{\prime} \mathbf{W}}{n}\right) & 2 \operatorname{tr}\left(\frac{\mathbf{W}^{\prime} \mathbf{W} \mathbf{W}^{\prime} \mathbf{W}}{n}\right) & \operatorname{tr}\left(\frac{\mathbf{W}^{\prime} \mathbf{W}\left(\mathbf{W}^{\prime}+\mathbf{W}\right)}{n}\right) \\
\mathbf{0} & \operatorname{tr}\left(\frac{\mathbf{W}^{\prime} \mathbf{W}\left(\mathbf{W}^{\prime}+\mathbf{W}\right)}{n}\right) & \operatorname{tr}\left(\frac{\mathbf{W} \mathbf{W}+\mathbf{W}^{\prime} \mathbf{W}}{n}\right)
\end{array}\right]
$$

where $\boldsymbol{\sigma}_{\omega j}^{4}$ and $\boldsymbol{\sigma}_{1 j}^{4}$ are as defined in equation (3.230). When $\boldsymbol{\sigma}_{\omega j}^{4}$ and $\boldsymbol{\sigma}_{1 j}^{4}$ are replaced by their consistent estimators, say, $\tilde{\boldsymbol{\sigma}}_{\omega j}^{4}$ and $\tilde{\boldsymbol{\sigma}}_{1 j}^{4}$ respectively, equation (3.232) becomes :

$$
\tilde{\mathbf{\Phi}}_{j}^{-1}=\left[\begin{array}{cc}
\frac{\tilde{\boldsymbol{\sigma}}_{\omega j}^{4}}{T-1} & \mathbf{0} \\
\mathbf{0} & \tilde{\mathbf{\sigma}}_{1 j}^{4}
\end{array}\right] \otimes\left[\begin{array}{ccc}
2 & 2 \operatorname{tr}\left(\frac{\mathbf{W}^{\prime} \mathbf{W}}{n}\right) & \mathbf{0} \\
2 \operatorname{tr}\left(\frac{\mathbf{W}^{\prime} \mathbf{W}}{n}\right) & 2 \operatorname{tr}\left(\frac{\mathbf{W}^{\prime} \mathbf{W} \mathbf{W}^{\prime} \mathbf{W}}{n}\right) & \operatorname{tr}\left(\frac{\mathbf{W}^{\prime} \mathbf{W}\left(\mathbf{W}^{\prime}+\mathbf{W}\right)}{n}\right) \\
\mathbf{0} & \operatorname{tr}\left(\frac{\mathbf{W}^{\prime} \mathbf{W}\left(\mathbf{W}^{\prime}+\mathbf{W}\right)}{n}\right) & \operatorname{tr}\left(\frac{\mathbf{W} \mathbf{W}+\mathbf{W}^{\prime} \mathbf{W}}{n}\right)
\end{array}\right]
$$

$\tilde{\boldsymbol{\Phi}}_{j}$ is consistent estimator of $\boldsymbol{\Phi}_{j}$ provided the estimators for $\boldsymbol{\sigma}_{\omega \mathrm{j}}^{2}$ and $\boldsymbol{\sigma}_{1 j}^{2}$ are consistent.

Now, consider once again the jth equation of the system given in (3.223) and recall (3.198). If $\rho_{j}, \boldsymbol{\sigma}_{\omega \mathrm{j}}^{2}$ and $\boldsymbol{\sigma}_{1 j}^{2}$ were known, then the generalized spatial two-stage least squares (GS2SLS) estimator of $\boldsymbol{\delta}_{j}$ can be given by: 


$$
\begin{aligned}
\hat{\boldsymbol{\delta}}_{j}= & {\left[\overline{\mathbf{Z}}_{j}^{\prime}\left(\boldsymbol{\Omega}_{u_{j}}^{-1}\left(\rho_{j}, \boldsymbol{\sigma}_{\omega j}^{2}, \boldsymbol{\sigma}_{1 j}^{2}\right)\right) \mathbf{Z}_{j}\right]^{-1} \overline{\mathbf{Z}}_{j}^{\prime}\left(\boldsymbol{\Omega}_{u_{j}}^{-1}\left(\rho_{j}, \boldsymbol{\sigma}_{\omega j}^{2}, \boldsymbol{\sigma}_{1 j}^{2}\right)\right) \mathbf{y}_{j} } \\
= & \left\{\overline{\mathbf{Z}}_{j}^{\prime}\left[\boldsymbol{\Omega}_{\boldsymbol{v}_{j}}\left(\boldsymbol{\sigma}_{\omega j}^{2}, \boldsymbol{\sigma}_{1 j}^{2}\right)\left[\mathbf{I}_{T} \otimes\left(\mathbf{I}_{n}-\rho_{j} \mathbf{W}\right)^{-1}\left(\mathbf{I}_{n}-\rho_{j} \mathbf{W}^{\prime}\right)^{-1}\right]\right]^{-1} \overline{\mathbf{Z}}_{j}\right\}^{-1} \\
& \times \overline{\mathbf{Z}}_{j}^{\prime}\left[\boldsymbol{\Omega}_{\boldsymbol{v}_{j}}\left(\boldsymbol{\sigma}_{\omega j}^{2}, \boldsymbol{\sigma}_{1 j}^{2}\right)\left[\mathbf{I}_{T} \otimes\left(\mathbf{I}_{n}-\rho_{j} \mathbf{W}\right)^{-1}\left(\mathbf{I}_{n}-\rho_{j} \mathbf{W}^{\prime}\right)^{-1}\right]\right]^{-1} \mathbf{y}_{j} \\
= & {\left[\overline{\mathbf{Z}}_{j}^{*}\left(\rho_{j}\right)^{\prime}\left(\boldsymbol{\Omega}_{\mathbf{v}_{j}}^{-1}\left(\boldsymbol{\sigma}_{\omega j}^{2}, \boldsymbol{\sigma}_{1 j}^{2}\right)\right) \overline{\mathbf{Z}}_{j}^{*}\left(\rho_{j}\right)\right]^{-1} \overline{\mathbf{Z}}_{j}^{*}\left(\rho_{j}\right)^{\prime}\left(\mathbf{\Omega}_{\mathbf{v}_{j}}^{-1}\left(\boldsymbol{\sigma}_{\omega j}^{2}, \boldsymbol{\sigma}_{1 j}^{2}\right)\right) \mathbf{y}_{j}^{*}\left(\rho_{j}\right) }
\end{aligned}
$$

where $\overline{\mathbf{Z}}_{j}^{*}\left(\rho_{j}\right)=\mathbf{P}_{N} \mathbf{Z}_{j}^{*}\left(\rho_{j}\right)$ with $\mathbf{Z}_{j}^{*}\left(\rho_{j}\right)=\left[\mathbf{I}_{T} \otimes\left(\mathbf{I}_{n}-\rho_{j} \mathbf{W}\right)\right] \mathbf{Z}_{j}, \mathbf{P}_{N}=\mathbf{N}\left(\mathbf{N}^{\prime} \mathbf{N}\right)^{-1} \mathbf{N}^{\prime}$

, and $\mathbf{y}_{j}^{*}\left(\rho_{j}\right)=\left[\mathbf{I}_{T} \otimes\left(\mathbf{I}_{n}-\rho_{j} \mathbf{W}\right)\right] \mathbf{y}_{j}$. But, since in practical applications $\rho_{j}, \boldsymbol{\sigma}_{\omega \mathrm{j}}^{2}$ and $\boldsymbol{\sigma}_{1 j}^{2}$ are not known, their estimators as defined in (3.230) or (3.231) are used instead. The resulting estimator is termed as feasible generalized spatial two-stage least squares estimator and is given by

$$
\hat{\boldsymbol{\delta}}_{j}^{F}=\left[\overline{\mathbf{Z}}_{j}^{*}\left(\tilde{\rho}_{j}\right)^{\prime}\left(\boldsymbol{\Omega}_{\mathbf{v}_{j}}^{-1}\left(\tilde{\boldsymbol{\sigma}}_{\omega j}^{2}, \tilde{\boldsymbol{\sigma}}_{1 j}^{2}\right)\right) \overline{\mathbf{Z}}_{j}^{*}\left(\tilde{\rho}_{j}\right)\right]^{-1} \overline{\mathbf{Z}}_{j}^{*}\left(\tilde{\rho}_{j}\right)^{\prime}\left(\boldsymbol{\Omega}_{\mathbf{v}_{j}}^{-1}\left(\tilde{\boldsymbol{\sigma}}_{\omega j}^{2}, \tilde{\boldsymbol{\sigma}}_{1 j}^{2}\right)\right) \mathbf{y}_{j}^{*}\left(\tilde{\rho}_{j}\right)
$$

The variables $\mathbf{y}_{j}^{*}\left(\rho_{j}\right)$ and $\mathbf{Z}_{j}^{*}\left(\rho_{j}\right)$ can be viewed as the result of a spatial Cochrane-Orcutt type transformation of the $\mathrm{jth}$ equation of the model in (3.223). That is, premultiplication of the first and the second parts of the jth equation of (3.223) by $\mathbf{I}_{T} \otimes\left(\mathbf{I}_{n}-\rho_{j} \mathbf{W}\right)$ gives $(\mathrm{j}=1, \ldots, \mathrm{G})$ :

$$
\mathbf{y}_{j}^{*}\left(\rho_{j}\right)=\mathbf{Z}_{j}^{*}\left(\rho_{j}\right) \boldsymbol{\delta}_{j}+\mathbf{v}_{j}
$$

One of the limitations of this estimator in (3.235), however, is that it does not take into account the information provided by the potential cross equation correlation in the innovation vectors $\boldsymbol{v}_{j}$. In order to use the information from such cross equation correlations, it is important to stack the equations given in (3.236) as follows:

$$
\mathbf{y}^{*}(\rho)=\mathbf{Z}^{*}(\rho) \boldsymbol{\delta}+\mathbf{v}
$$


where

$\mathbf{y}^{*}(\rho)=\left(\mathbf{y}_{1}^{*}\left(\rho_{1}\right)^{\prime}, \ldots, \mathbf{y}_{G}^{*}\left(\rho_{G}\right)^{\prime}\right)^{\prime}, \mathbf{Z}^{*}(\rho)=\operatorname{diag}_{j=1}^{G}\left(\mathbf{Z}_{j}^{*}\left(\rho_{j}\right)\right), \rho=\left(\rho_{1}, \ldots, \rho_{G}\right)^{\prime}$ and $\delta=\left(\delta_{1}, \ldots, \delta_{G}\right)^{\prime}$ Note that the mean of the innovation vector $v$ is zero. Recall also from (3.199) that $E\left(\mathbf{v v}^{\prime}\right)=\boldsymbol{\Omega}_{\mathbf{v}}=\boldsymbol{\Sigma}_{1} \otimes \mathbf{P}+\boldsymbol{\Sigma}_{\omega} \otimes \mathbf{H}$, where $\boldsymbol{\Sigma}_{1}=T \boldsymbol{\Sigma}_{\mu}+\boldsymbol{\Sigma}_{\omega}, \quad$ with $\boldsymbol{\Sigma}_{\mu}=\left[\sigma_{\mu_{j l}}^{2}\right]$, $\boldsymbol{\Sigma}_{\omega}=\left[\sigma_{\omega_{j l}}^{2}\right]$ and hence $\boldsymbol{\Sigma}_{1}=\left[\sigma_{1_{j l}}^{2}=T \sigma_{\mu_{j l}}^{2}+\sigma_{\omega_{j l}}^{2}\right]$ all $\mathrm{G}$ by $\mathrm{G}$ matrices. Assuming that $\rho$ and $\boldsymbol{\Omega}_{\mathbf{v}}$ were known, the system in (3.237) could be estimated using instrumental variable estimation technique. The resulting systems instrumental variable estimator of $\boldsymbol{\delta}$ would be the generalized spatial three-stage least squares (GS3SLS) estimator and can be expressed as

$$
\hat{\boldsymbol{\delta}}=\left[\overline{\mathbf{Z}}^{*}(\rho)^{\prime}\left(\boldsymbol{\Omega}_{\mathbf{v}}^{-1}\left(\boldsymbol{\sigma}_{\omega}^{2}, \boldsymbol{\sigma}_{1}^{2}\right)\right) \overline{\mathbf{Z}}^{*}(\rho)\right]^{-1} \overline{\mathbf{Z}}^{*}(\rho)^{\prime}\left(\boldsymbol{\Omega}_{\mathbf{v}}^{-1}\left(\boldsymbol{\sigma}_{\omega}^{2}, \boldsymbol{\sigma}_{1}^{2}\right)\right) \mathbf{y}^{*}(\rho)
$$

However, since $\rho$ and $\boldsymbol{\Omega}_{v}$ are not known in practical applications, their estimators are required to obtain a feasible estimator for $\boldsymbol{\delta}$. The generalized moments estimator for $\rho$ can be obtained from (3.231) and since $\boldsymbol{\Omega}_{\mathbf{v}}$ is composed of $\boldsymbol{\sigma}_{\omega}^{2}$ and $\boldsymbol{\sigma}_{1}^{2}$ its estimator can also be obtained from (3.231). Besides, consistent estimators of $\boldsymbol{\sigma}_{\omega}^{2}$ and $\boldsymbol{\sigma}_{1}^{2}$ and hence of $\boldsymbol{\Omega}_{\mathbf{v}}$ can be derived by combining (3.235) and (3.236) as $(\mathrm{j}, 1=1, \ldots, \mathrm{G})$ :

$$
\begin{aligned}
& \tilde{\boldsymbol{\sigma}}_{\omega j l}^{2}=\left(\mathbf{y}_{j}^{*}\left(\tilde{\rho}_{j}\right)-\mathbf{Z}_{j}^{*}\left(\tilde{\rho}_{j}\right) \hat{\boldsymbol{\delta}}_{j}^{F}\right) \mathbf{H}\left(\mathbf{y}_{l}^{*}\left(\tilde{\rho}_{l}\right)-\mathbf{Z}_{l}^{*}\left(\tilde{\rho}_{l}\right) \boldsymbol{\delta}_{l}^{F}\right) / n(T-1) \\
& \tilde{\boldsymbol{\sigma}}_{1 j l}^{2}=\left(\mathbf{y}_{j}^{*}\left(\tilde{\rho}_{j}\right)-\mathbf{Z}_{j}^{*}\left(\tilde{\rho}_{j}\right) \hat{\boldsymbol{\delta}}_{j}^{F}\right) \mathbf{P}\left(\mathbf{y}_{l}^{*}\left(\tilde{\rho}_{l}\right)-\mathbf{Z}_{l}^{*}\left(\tilde{\rho}_{l}\right) \boldsymbol{\delta}_{l}^{F}\right) / n
\end{aligned}
$$

Then, the $G$ by $G$ matrix whose $(\mathrm{j}, 1)$ th element is $\tilde{\boldsymbol{\sigma}}_{\omega j l}^{2}$ defines a consist estimator for $\boldsymbol{\Sigma}_{\omega}$ denoted by $\tilde{\boldsymbol{\Sigma}}_{\omega}$ and the G by G matrix whose $(\mathrm{j}, 1)$ th element is $\tilde{\boldsymbol{\sigma}}_{1 j l}^{2}$ defines a consistent estimator for $\boldsymbol{\Sigma}_{1}$ denoted by $\tilde{\boldsymbol{\Sigma}}_{1}$. Thus, 


$$
\tilde{\mathbf{\Omega}}_{\mathbf{v}}=\tilde{\boldsymbol{\Sigma}}_{\omega} \otimes \mathbf{H}+\tilde{\boldsymbol{\Sigma}}_{1} \otimes \mathbf{P}
$$

Replacing $\rho$ and $\boldsymbol{\Omega}_{\mathbf{v}}$ by $\tilde{\rho}$ and $\tilde{\boldsymbol{\Omega}}_{\mathbf{v}}=\mathbf{\Omega}_{\mathbf{v}}\left(\tilde{\boldsymbol{\sigma}}_{\omega}^{2}, \tilde{\boldsymbol{\sigma}}_{1}^{2}\right)$ in (3.238), hence, yields the feasible generalized spatial three-stage least squares (FGS3SLS) estimator which can be expressed as

$$
\hat{\boldsymbol{\delta}}^{F}=\left[\overline{\mathbf{Z}}^{*}(\tilde{\rho})^{\prime}\left(\boldsymbol{\Omega}_{\mathbf{v}}^{-1}\left(\tilde{\boldsymbol{\sigma}}_{\omega}^{2}, \tilde{\boldsymbol{\sigma}}_{1}^{2}\right)\right) \overline{\mathbf{Z}}^{*}(\tilde{\rho})\right]^{-1} \overline{\mathbf{Z}}^{*}(\tilde{\rho})^{\prime}\left(\mathbf{\Omega}_{\mathbf{v}}^{-1}\left(\tilde{\boldsymbol{\sigma}}_{\omega}^{2}, \tilde{\boldsymbol{\sigma}}_{1}^{2}\right)\right) \mathbf{y}^{*}(\tilde{\rho})
$$

\subsection{Specification Tests}

Specification tests form one of the most important areas of research in econometrics (Hausman, 1978). Once the system of simultaneous equations is specified as in (3.19), there is an opportunity to test both coefficient restrictions and asymptotic orthogonality assumptions. When there are more instruments than needed to identify an equation, a test can be done to investigate whether the additional instruments are valid in the sense that they are uncorrelated with the error term. This is commonly known as test of the overidentifying restriction. To explain this, consider the jth equation of the system of simultaneous equations given in this study:

$$
\mathbf{y}_{\mathbf{j}}=\mathbf{Y}_{\mathbf{j}} \boldsymbol{\beta}_{\mathbf{j}}+\mathbf{X}_{\mathbf{j}} \gamma_{\mathbf{j}}+\mathbf{u}_{\mathbf{j}}
$$

where $\mathbf{Y}_{\mathbf{j}}$ is a vector of $G_{j}-1$ included right-hand endogenous variables, $\mathbf{X}_{\mathbf{j}}$ is a vector of $\mathrm{k}_{\mathbf{j}}$ included predetermined variables, $\mathbf{u}_{\mathbf{j}}$ is disturbance term, and the vector of $\mathrm{K}_{\mathbf{j}}$ excluded predetermined variables is given by $\mathbf{X}^{*}$.

Anderson and Rubin (1950) was the first to develop the procedure for testing the overidentifying restriction based on the asymptotic distribution of the smallest characteristic $\operatorname{root}\left(\boldsymbol{\lambda}_{\mathbf{j}}\right)$ derived from LIML estimation. Their likelihood ratio test statistic is base on $\mathrm{n}\left(\boldsymbol{\lambda}_{\mathrm{j}}-1\right)$ which under the null hypothesis is distributed as Chi-squared with $\left(\mathrm{K}_{\mathrm{j}^{-}}\right.$ 
$\left.\left(G_{j}-1\right)\right)$ degree of freedom which is equal to the number of overidentifying restrictions.. That is

$$
\mathbf{L R}=\boldsymbol{\chi}^{2}\left[\mathbf{K}_{\mathbf{j}}-\left(\mathbf{G}_{\mathbf{j}}-\mathbf{1}\right)\right]=n\left(\boldsymbol{\lambda}_{\mathbf{j}}-\mathbf{1}\right)
$$

where $\mathrm{n}$ is the sample size and all other notations are as defined above. A large value for LR is an indication that there are exogenous variables in the model that have been inappropriately omitted from the jth equation. This test statistic, however, is difficult to compute. Hausman (1983) proposed an alternative test statistic based on Lagrange multiplier principle which is asymptotically equivalent but easier to compute. This test statistic is obtained as $n R_{u}^{2}$, where $\mathrm{n}$ is the sample size and $R_{u}^{2}$ is the usual $\mathrm{R}$-squared or the uncentered R-squared of the regression of residuals from the second-stage equation on all included and excluded instruments. In other words, simply estimate equation (3.242) by 2SLS, GMM, LIML or any efficient limited-information estimator and obtain the resulting residuals, $\hat{u}_{j}$. Then, regress these on all instruments and calculate $n R_{u}^{2}$. The statistic has a limiting chi-squared distribution with $\left(\mathrm{K}_{\mathrm{j}}-\left(\mathrm{G}_{\mathrm{j}}-1\right)\right)$ degree of freedom which is equal to the number of overidentifying restrictions, under the assumed specification of the model.

One potential source of misspecification in spatial econometric models comes from spatial autocorrelation in the dependent variable or in the error term or in both. Anselin and Kelejian (1997) proposed Moran's I statistics based on residuals that are obtained from an instrumental variable (IV) procedure such as 2SLS in a general model that encompasses endogeneity due to feedback simultaneities as well as spatial autoregressive /or cross-regressive lag simultaneities. Following Anselin and Kelejian (1997), this statistic is specified as: 


$$
I^{*}=n\left(\frac{\hat{u}^{\prime} W \hat{u}}{S_{0} \hat{u}^{\prime} \hat{u}}\right)
$$

where $\mathrm{n}$ is the sample size, $\hat{u}$ is the IV residuals, $\mathrm{W}$ is the spatial weights matrix, and $\mathrm{S}_{0}$ is the usual normalizing factor given by

$$
S_{0}=\sum_{i}^{n} \sum_{j}^{n} w_{i j} .
$$

Note that for a row standardized spatial weights matrix, $\mathrm{S}_{0}$ is equal to $\mathrm{n}$ because each row sums to one. Hence equation (3.244) is simplified to:

$$
I^{*}=\left(\frac{\hat{u}^{\prime} W \hat{u}}{\hat{u}^{\prime} \hat{u}}\right)
$$

Anselin and Kelejian (1997) shows that

$$
n^{1 / 2} I^{*} \stackrel{D}{\longrightarrow} N\left[0, \phi^{2}\right]
$$

where

$$
\phi^{2}=\frac{S_{2}}{2 S_{1}^{2}}+\left(\frac{4}{S_{1}^{2} \sigma_{u}^{2}}\right) A
$$

with $\mathrm{S}_{1}$ and $\mathrm{S}_{2}$ finite constants such that

$$
\begin{gathered}
S_{1}=\lim _{n \rightarrow \infty} \sum_{i}^{n} \sum_{j}^{n} w_{i j} / n, \text { and } \\
S_{2}=\lim _{n \rightarrow \infty} \operatorname{tr}\left[\left(W+W^{\prime}\right)\left(W+W^{\prime}\right)\right] / n,
\end{gathered}
$$

$\sigma_{u}^{2}$ is error variance, and

$$
A=p \lim \left[\left(n^{-1} u^{\prime} W Z_{j}\right)\left(n\left(Z_{j}^{\prime} P_{X} Z_{j}\right)^{-1}\right)\left(n^{-1} Z_{j} W^{\prime} u^{\prime}\right)\right]
$$

Replacing $\mathrm{S}_{1}, \mathrm{~S}_{2}$, A by their finite sample counterparts, respectively

$$
\begin{gathered}
\hat{S}_{1}=\sum_{i}^{n} \sum_{j}^{n} w_{i j} / n \\
\hat{S}_{2}=\operatorname{tr}\left[\left(W+W^{\prime}\right)\left(W+W^{\prime}\right)\right] / n
\end{gathered}
$$




$$
\hat{A}=\left[\left(n^{-1} u^{\prime} W Z_{j}\right)\left(n\left(Z_{j}^{\prime} P_{X} Z_{j}\right)^{-1}\right)\left(n^{-1} Z_{j} W^{\prime} u^{\prime}\right)\right]
$$

and $\sigma_{u}^{2}$ by its consistent estimator, $\hat{\sigma}_{u}^{2}=\left(\hat{u}^{\prime} \hat{u} / n\right)$ in equation (3.246) would give a consistent estimator for $\phi^{2}$, say $\hat{\phi}^{2}$.

With $\phi^{2}$ replaced by its consistent estimator $\hat{\phi}^{2}$, an asymptotic test can be constructed such that the null hypothesis of no spatial autocorrelation may be rejected at the $\alpha$ level of significance if

$$
\left|\frac{n^{1 / 2} I^{*}}{\hat{\phi}}\right| \succ z_{\alpha}
$$

where $z_{\alpha}$ is the value of the standard normal variate corresponding to $\alpha$.

In summary, this chapter has presented the conceptual and methodological framework that helps guide the empirical analysis in this study. Particularly, the fundamental issues of simultaneous-equations model specification, the identification problem and the techniques and principles of estimation of simultaneous-equations models have been examined in light of this study. Techniques of exploratory spatial data analysis and spatial cross-sectional and spatial panel data extensions of the standard simultaneous-equation models have also been discussed and developed. Lastly, a discussion on specification tests relevant to simultaneous-equations models (both spatial and non-spatial) has been presented. Now that the foundation for simultaneous-equations modeling has been laid down, next chapter develops the theoretical and empirical models of the present study. 


\section{CHAPTER IV}

\section{METHODOLOGY AND DATA}

\subsection{Introduction}

The methodology followed in this study is an extension of the "jobs follow people, or people follow jobs" literature. The technical issues in standard (non-spatial) and spatial simultaneous equations modeling as well as estimation have been discussed in some detail in Chapter III. Against the backdrop of this theoretical framework, this chapter develops a simultaneous-equation system that expresses the interdependences among small business growth, migration behavior, local public services and median household income in a partial lag-adjustment growth-equilibrium framework. First, under the assumptions of profit maximization of firm and utility maximization of households as well as the neoclassical assumptions of equilibrium growth, a standard (non-spatial) simultaneous equations model is developed. Second, the standard (non-spatial) model is further extended to address the problems of model misspecifications that could arise from the existence of spatial dependence in the spatially lagged endogenous variables or/and the error terms. The sources of the data and variable descriptions are also presented.

The rest of the chapter is organized as follows: First, the standard (non-spatial) theoretical growth equilibrium model is discussed and developed in section 2. Section 3 presents the spatial extension of the standard model. The empirical models are specified in section 4. Finally, section 5 presents data sources and descriptions of variables. 


\subsection{Theoretical Model Development}

The relationship between economic growth and its determinants has been extensively studied in the economic literature. The issue whether regional development can be associated with population driving employment changes or employment driving population changes (do 'jobs follow people' or 'people follow jobs'?) has, for example, recently attracted considerable interest among researchers and policy makers. Empirical works on identification of the direction of causality in this 'jobs follow people' or 'people follow jobs' literature (Steinnes and Fischer, 1974) have resulted in the view that empirical models of regional development often reflect the interdependence between household residential choices and firm location choices. To account for this causation and interdependency, Carlino and Mills (1987) suggested and constructed a two-equation simultaneous system with the two partial location equations as its components. This model has subsequently been used by a number of regional science researchers in order to examine regional economic growth (see Boarnet, 1994; Duffy, 1994; Henry, Barkley, and Bao, 1997; Duffy-Dino, 1998; Barkley, Henry and Bao 1998,; Henry, Schmitt, Kritstesen, Barkley, and Bao, 1999; Edmiston, 2004). More recently, Deller, Tsai, Marcouiller, and English (2001) have expanded upon the original Carlino-Mills model to capture explicitly the role of income. According to the proposition of utility maximization in the traditional migration literature, households migrate to capture higher wages or income. The model expanded by Deller et al, (2001) is three-dimensional (jobspeople-income) and explicitly traces the role of income in regional growth process. It also explicitly captures the increasing concerns about job quality as measured by income levels those jobs can support. There have also been efforts to model the interactions 
between employment growth and human migration ( MacDonald, 1992; Clark and Murphy, 1996), per capita personal income and public expenditures (Duffy-Deno and Eberts, 1991), net migration, employment growth, and average income (earnings) (Greenwood and Hunt 1984; Greenwood et al., 1986; and Lewis, Hunt and Plantigna, 2002) in simultaneous-equations methods.

The theoretical base for the interdependencies between population (migration behavior), employment and income is the idea that households and firms are both mobile and that household location decisions maximize utility while firm location decisions maximize profits. That is, households migrate to capture higher wages or income and firms migrate to be near growing consumer markets. These actions in turn generate income to the regional (local) economy. However, according to the principle of utility maximization, household location decisions are expected to be influenced not only by the location of job opportunities and income but also by other factors such as the provision of local public goods and services, social and natural amenities (and disamenities), demographic factors, and regional location. Similarly, the location decisions of firms are expected to be influenced not only by population and income (i.e., growing consumer markets) but also by other factors such as local business climate, wage rates, tax rates, local public services, and regional location. Firm location decisions are also influenced by the substantial financial incentive that local governments offer in an effort to create jobs, spur income growth, and enhance the economic opportunities of the local population. According to the median-voter models of local fiscal behavior, local public expenditures, however, approximate the choices of the utility-maximizing median voter and so depend on income and other revenue sources such as property taxes, income taxes, 
and factors that determine consumer preferences. In this study, the "jobs versus people versus income' debate is expanded from three-dimensional into four-dimensional: 'jobs versus people (migration behavior) versus income (poverty) versus local public services'. By expanding the growth partial equilibrium model into four dimensions to explicitly trace the role of local public services in regional growth, the model in this fully captures the growth process. The complex causations and interdependencies between business growth and entrepreneurship, migration behavior, household income and wealth and local public services are given in Figure 1 as shown below.

In general, it is assumed that both households and business firms are free to migrate. Utility-maximizing households migrate in search of utility derived the consumption of market goods, amenities (both social and natural) and local public services. Profit-maximizing business firms migrate in search of lower production costs and higher consumer market demands. The determinants of the demand for local public services are based upon the principles of the median-voter models that assume that local governments use property and income taxes to collect revenues. Local fiscal behavior is influenced by the need for local governments to actively pursue policies that encourage newly locating and expanding business firms in order to create jobs, spur income growth and enhance economic opportunities to the public, provide efficient and quality public services, and balancing their budgets. Based upon these assumptions, the following central hypotheses are formulated in this research: 


\section{Figure 4 1: Cycle of Poverty}

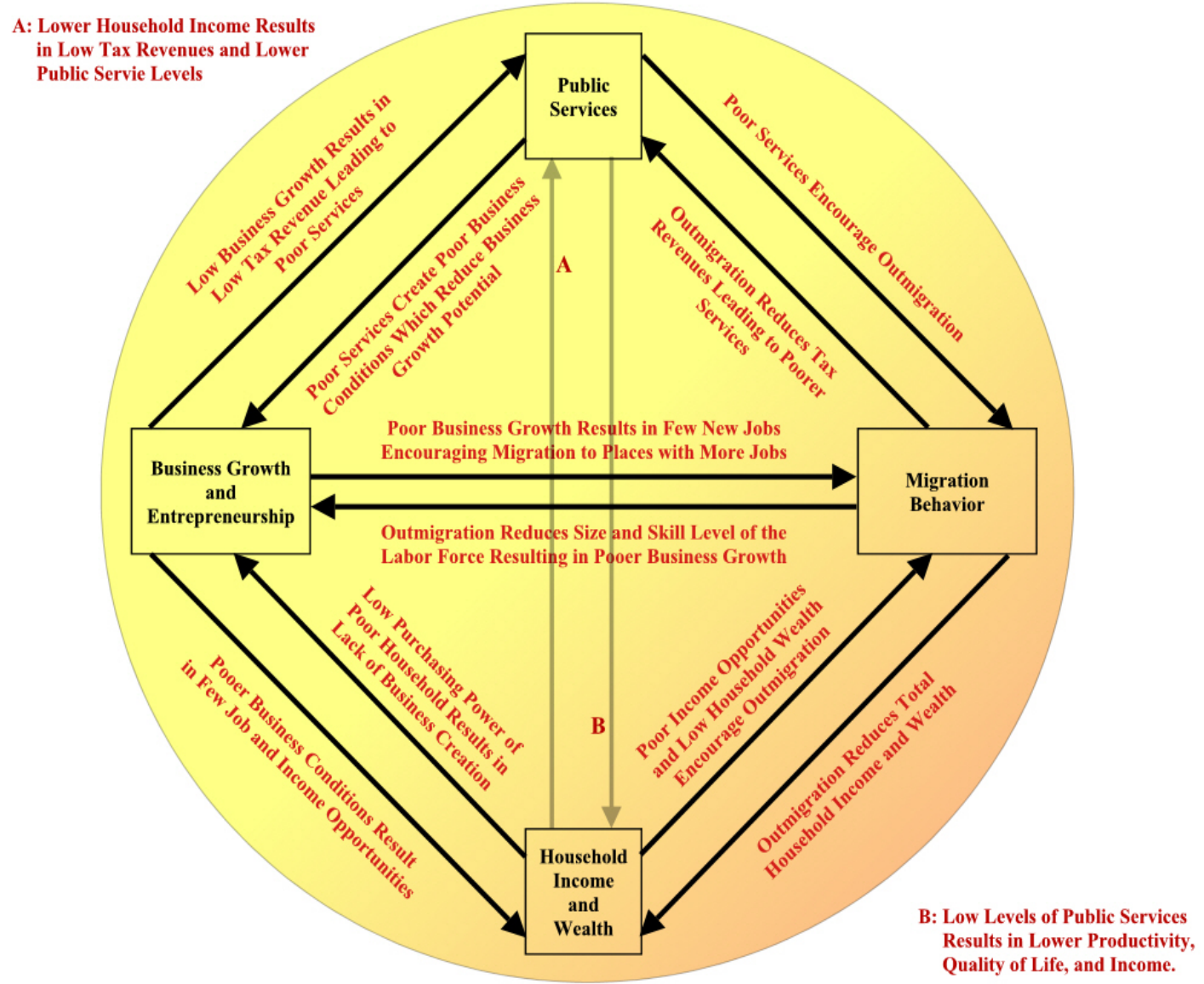

1. Business growth, migration behavior (in-migration and out-migration), household median income and local public services are interdependent and are jointly determined by regional covariates

2. Growth is conditional upon initial conditions.

3. Growth in a county is conditional upon growth in neighboring counties.

These hypotheses form the core research agenda for this study. Specifically, emphasis is put not only on examining the linkages among business growth, migration behavior, 
household median income and local public services, but also on investigating the elasticity of these variables with respect to each of the regional covariates. The elasticity analyses help to draw some policy recommendations for regional and rural development.

To test these hypotheses, a spatial simultaneous equations model of business growth, migration behavior, household median income, and local public services is used. Following the Carlino and Mills tradition and building upon Deller et al. (2001) and Lewis et al.(2002), the basic model is specified as:

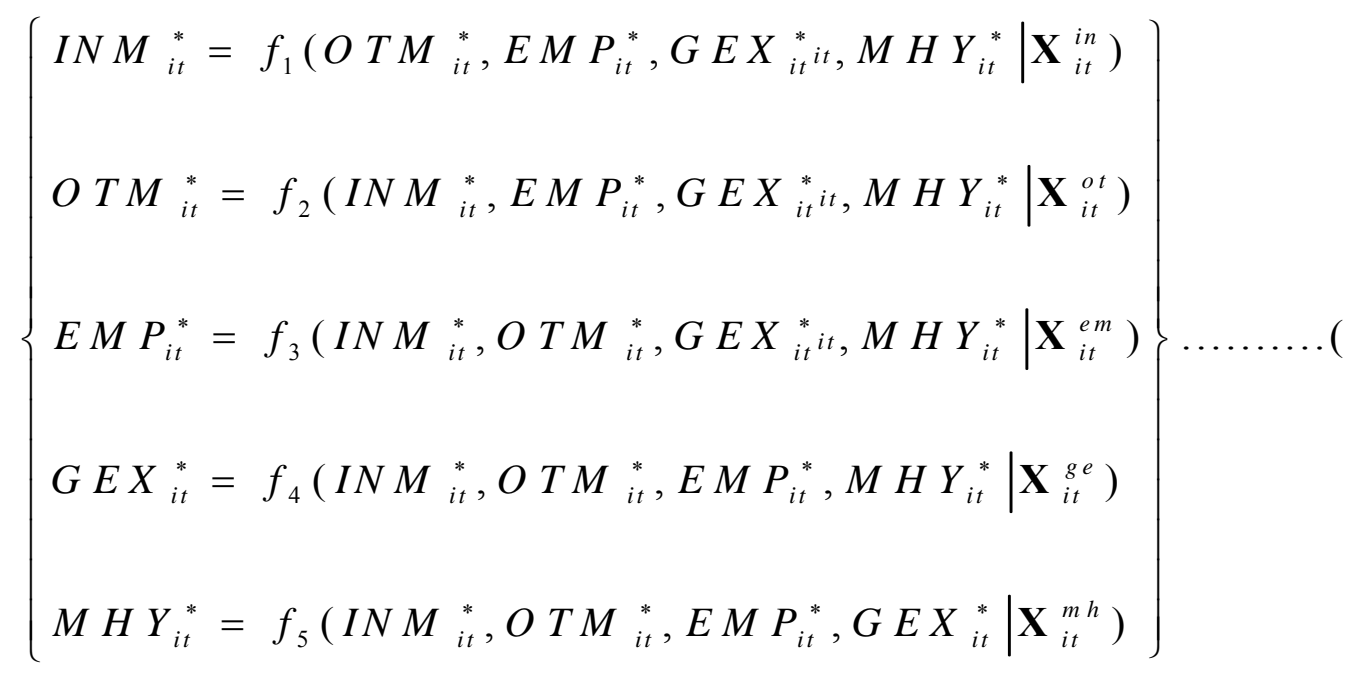

where $I N M_{i t}^{*}, O T M_{i t}^{*}, E M P_{i t}^{*}, G E X_{i t}^{*}$, and $M H Y_{i t}^{*}$ are equilibrium levels of gross in-migration, gross out-migration, private business employment, median household income and local public expenditures respectively, and $\mathrm{i}$ and $\mathrm{t}$ index county and time, respectively. The vectors of additional exogenous variables that are included in the respective equations of the system of simultaneous equations are given by $\mathbf{X}_{i t}^{i n}, \mathbf{X}_{i t}^{o t}, \mathbf{X}_{i t}^{e m}$, $\mathbf{X}_{i t}^{g e}$, and $\mathbf{X}_{i t}^{m h}$, respectively.

Migration Equations: Individuals are assumed to maximize utility, which varies over locations, and it is expected that utility for representative individuals is equalized over locations through migration. Households evaluate the earning opportunities, cost of 
living, local amenities or disamenities, local taxes, and local public services associated with alternative residential locations and move freely to reside and seek employment they wish, although there are costs associated with such moves. Thus, the probability of moving to other location, say, $\mathrm{P}(\mathrm{M})$, exceeds zero if and only if the difference between the individual's discounted utility streams in some other location $j, U_{j}(t)$ exceed the discounted utility streams in the current location $\mathrm{k}, \mathrm{U}_{\mathrm{k}}(\mathrm{t})$, that is $0<\mathrm{P}(\mathrm{M}) \leq 1$ if and only if

$$
U_{j}(t)=\int_{t=0}^{T} \boldsymbol{\Theta}_{j}(t) e^{-r t} d t-C>\int_{t=0}^{T} \boldsymbol{\Theta}_{k}(t) e^{-r t} d t=U_{k}(t)
$$

where $\Theta$ refers to the overall quality of life, $\mathrm{r}$ is the discount factor, and $\mathrm{C}$ refers to the fixed cost of migration. In equilibrium, no household can improve its well being by moving. Thus, equilibrium requires that utility be equalized at some level $U^{*}$ across all locations,

$$
U_{k}(t)=U^{*}, \quad \text { for all } \mathrm{k}
$$

The observed migration flows consist of individual decisions that are aggregated over all potential migrants. This means, net and gross migration flows are functions of the same variables that affect the behavior of individuals. Note also that (4.2) does not necessarily restrict the determinants of utility terms to be the same between gross migration flows, so different variables can have similar net-migration effects, having totally different grossmigration effects.

The equilibrium migration flow into or out of a given location, say $k$, is a function of the relative utility available in that location. This can be formalized as:

$$
M R_{k}^{*}(t)=M R\left(U_{k}(t)-U^{*}(t)\right)
$$


where $M R_{k}^{*}(t)$ is equilibrium migration flow into or out of location k at time t, $U_{k}(t)$ is utility per unit time at $\mathrm{k}$, and $U^{*}(t)$ may be interpreted as a measure of the flow of utility available elsewhere, which is the same for all locations in the system. The relative utility at a location, in turn, is dependent on the earning opportunities, cost of living, local amenities or disamenities, local taxes, and local public services in that location. This formulation leads to the first two equations in (4.1).

Business Growth (Employment) Equation: Consistent with the profit maximization assumption, business firm location is assumed to be determined by demand and cost factors. These include access to labor and output markets, local demand, the cost and availability of commercial land and labor, local taxes, and local public services. In addition, different locations are likely to have different characteristics that raise or lower firm costs of production. These could include, for example, agglomeration economies associated with dense urban settlement, transportation costs, or site specific attributes. Following Carlton (1983), Friedman, Gerlowski, and Silberman (1992), Guimaraes, Figueiredo, and Woodward (2000), and Gabe and Bell (2004), the expect profit, $\pi_{j k}$,earned by business firm $\mathrm{j}$ in county $\mathrm{k}$ can be given by:

$$
\pi_{j, k}=\boldsymbol{\beta}^{\prime} \boldsymbol{\Xi}_{j, k}+e_{j, k}
$$

where, $\boldsymbol{\beta}$ is a vector of parameters, $\boldsymbol{\Xi}_{j, k}$ is a vector of county specific attributes, and $e_{j k}$ is a random error term. Profit maximization behavior asserts that businesses will locate and invest in the county that provides the highest expected profits. Thus, business firm $\mathrm{j}$ will locate in county $\mathrm{k}$ if the expected profits in county $\mathrm{k}$ are greater than the expected profits the business could earn elsewhere. That is, 


$$
\pi_{j, k}>\pi_{j, i}, \quad \text { for all } \mathrm{i} \neq k
$$

In equilibrium, no business firm can improve its profits by moving. Thus, equilibrium requires that profits be equalized at some level $\pi^{*}$ across all locations,

$$
\pi_{j, k}=\pi^{*}, \quad \text { for all } \mathrm{k}
$$

For each business firm, the profit function can also be formulated as maximizing the following expression:

$$
\pi_{k}=p_{k} Q_{k}-\sum_{i=1}^{n} w_{i, k} X_{i, k}
$$

where $\pi_{k}$ is the profit at $\mathrm{k}, p_{k}$ is the tax inclusive price of output at $\mathrm{k}, Q_{k}$ is quantity sold at $\mathrm{k}, w_{i, k}$ is a vector of tax inclusive input prices at $\mathrm{k}$, and $x_{i \mathrm{k}}$ is a vector of inputs at $\mathrm{k}$. Using a cost function in the production of $\mathrm{Q}$ and the first order profit maximization conditions, $\pi_{k}=p_{k} Q_{k}-\sum_{i=1}^{n} w_{i, k} X_{i, k}$ can be rewritten as:

$$
\pi_{k}=\pi\left(p_{k}, w_{i, k}, C A_{k}\right)
$$

where $C A_{k}$ is a vector of other covariates that affect profits at $\mathrm{k}$, and the other notations are as defined before. Note that the cost factors include the wage rate and hence differentiating with respect to the wage rate gives the business firm's demand for labor. Thus, the demand for labor at location $\mathrm{k}$ by firm $\mathrm{j}$ can be written as:

$$
E M P_{j, k}=\operatorname{EMP}\left(p_{k}, w_{i, k}, C A_{k}\right)
$$

where $E M P_{j, k}$ is employment level at location $\mathrm{k}$ by firm $\mathrm{j}$, and the other notations are as defined above. 
In a comparative static framework, the percentage change in employment is related to the changes in the right-hand side variables as one moves from an initial equilibrium to another equilibrium position. $E M P_{j, k}^{*}$ is the level of employment when firm j's profit at location $\mathrm{k}$ is in equilibrium (i.e., $\pi_{j, k}=\pi^{*}$ ).

The observed business growths (employment expansions) consist of individual business firm decisions that are aggregated over all potential newly locating and expanding business firms. Thus, the equilibrium level of employment at location $\mathrm{k}, E M P_{k}^{*}$, is dependent on the access to labor and output markets, local demand, the cost and availability of commercial land and labor, local taxes, and local public services. This formulation leads to the third equation in (4.1).

Local Public Services Equation: Following the studies by Borcherding and Deacon (1972), and Bergstrom and Goodman (1973), the median voter model will be used to analyze the determinants of the demand for local public services or the expenditures for local public services. In this model it is assumed that utility-maximizing citizens elect government by majority rule and that the size of the public sector is the only issue to be decided. Citizens are assumed to be informed about the costs and benefits of government expenditures and hence the median voter chooses the level of spending by voting for candidates who offer him/her the most efficient set of public services and taxes. Aggregating over individual in a community, a utility function that represents community preferences can be generated.

Based on these assumptions, a theoretical model in order to derive hypotheses on the determinants of public spending on local public services is developed. The model is given by the following set of equations: 


$$
\begin{gathered}
U=U(G, \text { inctax } ; A) \\
G E X=G E X(G ; G F) \\
\operatorname{Re} v=\operatorname{Re} v(\text { inctax }, \text { Tax }, \text { Grant } ; T) \\
\operatorname{Re} v=G E X
\end{gathered}
$$

Equation (a) is the community utility function which is assumed to be strictly quasiconcave over local public services (G), community tax rate (inctax), and also may depend on socio-economic, demographic and amenity variables (A). Equation (b) is local government cost function, which depends on $\mathrm{G}$ and other local government functions (GF). Equation (c) represents local government revenue function, which is assumed to depend upon the community income tax rate (inctax), the tax base (Tax), intergovernmental grants (Grant) and other socio-economic and demographic variables (T). Equation (d) is local government budget constraint, which states that local government revenue should equal to local government expenditure. Maximizing the utility function given in (a) with respect to $G$ and inctax, subject to (b)-(d), gives a local public services demand function of the form (all notation as before)

$$
G=G(\text { inctax }, \text { Tax }, \text { Grant } ; A, G F, T) .
$$

Substituting in (b) gives the local public services expenditure demand function:

$$
G E X^{*}=G E X(\text { inctax }, \text { Tax }, \text { Grant } ; A, G F, T)
$$

This equation forms the basis for formulating the fourth equation in (4.1).

Median Household Income (Poverty) Equation: Analyses of the determinants of economic growth at the regional level have focused attention on the so-called 
convergence hypothesis predicted by the neoclassical growth model as developed by Solow (1954) and others. This model implies that the lower the starting level of per capita income, the higher the rate of growth, with the economy converging to a steady-state level as time passes. The steady-state per capita income attained depends on the propensity to save and the position of the production function, and these factors may vary across regions. The steady-state also depends on government policies, for example, with regards to public consumption spending [local public expenditure], protection of property rights, and distortions in the domestic and international markets. The concept of capital in the standard neoclassical growth model can also be made to include human capital in the form of education, experience and health (Barro, 1997). Thus, per capita incomes in the long run are determined by the levels of human and physical capital, and the underlying level of productivity, which in turn are themselves determined by the amount of social infrastructure. By social infrastructure is meant the institutions and government policies that determine the economic environment, within which individuals accumulate skills and firms accumulate capital and produce output. In this study, median household income is used as a proxy for per capita income.

Based on the conceptual frame work developed in the literature review and above, it is hypothesized that equilibrium level of median household income is determined by equilibrium migration behavior, equilibrium employment, equilibrium local government expenditure and other socio-economic, demographic and amenity variables. The general conceptual model that expresses this relationship is given by (also given by the fifth equation in (4.1)):

$$
M H Y_{i t}^{*}=f^{m h}\left(I N M_{i t}^{*}, O T M_{i t}^{*}, E M P_{i t}^{*}, G E X_{i t}^{*} \mid \mathbf{X}_{i t}^{m h}\right)
$$


where all variables are the same as defined before.

The system of equations in (4.1) captures the simultaneity nature of the interactions among migration behavior (in-migration, and out-migration), employment growth, local public services spending and median household income at equilibrium. The nature of interaction among the endogenous variables is dependent upon the initial conditions of a county. Suppose the initial conditions of a county do not favor, say, business growth. Then, poor business growth that results in few new jobs encourages outmigration from a county. At the same time, negative net migration decreases the population of county, which negatively affects employment (business growth) by decreasing the demand for goods and services and by providing smaller workforce. The resulting shifts in the county's labor demand and supply curves affect wage earnings in the county, with the ultimate results determined by the demand and supply elasticities and the degree of responsiveness of local wages to these changes. Usually the demand effect is stronger than the supply effect (Greenwood et al., 1986). Thus, the result is that a decrease in earnings results in a decrease in total household income and wealth in the county. Low household income and wealth in turn results in negative net migration. The decrease in wage earnings also results in lower tax revenue for local government that results in low and poor local public services. Low local public service provision in turn results in negative net migration.

Low business growth results in low tax revenue that leads to poor public services. Poor public services in turn create poor business conditions, which reduce business growth potential, encourage out-migration, and reduce household income and wealth. Poor business conditions result in few jobs and income opportunities that reduce 
household income and wealth, which in turn result in low demand for goods and service that farther limit business growth. The reduced household income and wealth also encourages out-migration (creates negative net migration) and low tax base that farther reduces local public services.

Negative net migration results in poor business growth, which in turn results in low tax revenue for local public sector and fewer income opportunities for households. Negative net migration also reduces median household income, and tax revenue leading to poorer public services that both further encourage out-migration. Poor public services create poor business condition, encourage out-migration, and reduce household income, which all in turn lead to poorer public services. Low household income results in lack of business creation, encourages out-migration, and leads to poorer public services, which all in turn reduce household income and wealth. The opposite set of interactions result when initial conditions of county favor business growth.

In order to reduce the effects of the large diversity found in the data used in empirical analysis, a multiplicative (log-linear) form of the model is used. Such specification also implies a constant-elasticity form for the equilibrium conditions given in (4.1). A log-linear (i.e., log-log) representation of these equilibrium conditions can thus be expressed as: 


$$
\begin{aligned}
I N M_{i t}^{*}= & \left(O T M_{i t}^{*}\right)^{a_{1}} \times\left(E M P_{i t}^{*}\right)^{b_{1}} \times\left(G E X_{i t}^{*}\right)^{c_{1}} \times\left(M H Y_{i t}^{*}\right)^{d_{1}} \times \prod_{k_{1}=6}^{K_{1}}\left(\mathrm{X}_{k_{1} i t}^{i n}\right)^{x_{1 k_{1}}} \\
\rightarrow & \ln \left(I N M_{i t}^{*}\right)=a_{1} \ln \left(O T M_{i t}^{*}\right)+b_{1} \ln \left(E M P_{i t}^{*}\right)+c_{1} \ln \left(G E X_{i t}^{*}\right) \\
& +d_{1} \ln \left(M H Y_{i t}^{*}\right)+\sum_{k_{1}=6}^{K_{1}} x_{1 k_{1}} \ln \left(\mathrm{X}_{k_{1} i t}^{i n}\right)
\end{aligned}
$$

where $a_{i}, b_{i}, c_{i}, d_{i}$ for $i=1,2,3,4$ are the exponents on the endogenous variables, $x_{i k_{j}}$ for $i, j=1, \ldots, 5$ are vectors of exponents on the exogenous variables, $\Pi$ is the product operator, and $K_{i}$ for $i=1, . ., 5$ are the number of exogenous variables in the in-migration, out-migration, employment growth, local public expenditure, and median household income equations respectively. The log-linear specification has an advantage of yielding 
a log-linear reduced form for estimation, where the estimated coefficients represent elasticities. Duffy-Deno (1998) and MacKinnon, White, and Davidson, 1983) also showed that, compared to a linear specification, a log-linear specification is more appropriate for models involving population and employment densities.

The various literatures (Edmiston, 2004; Hamalainen and Bockerman, 2004; Aronsson, Lundberg, and Wikstrom, 2001; Deller et al., 2001; Henry et al., 1999; DuffyDeno, 1998; Barkley et al., 1998; Henry et al., 1997; Boarnet, 1994; Duffy, 1994, Carlino and Mills, 1987; Mills and Price, 1984) suggest that in-migration, out-migration, employment, local public expenditure and median household income likely adjust to their equilibrium levels with a substantial lags (i.e., initial conditions). Following the previous literature a distributed lag adjustment is introduced and the corresponding partial-adjustment process for each of the equations given in (4.1) is of the form:

$$
\begin{aligned}
\frac{I N M_{i t}}{I N M_{i t-1}} & =\left(\frac{I N M_{i t}^{*}}{I N M_{i t-1}}\right)^{\eta_{i n}} \\
\ln \left(I N M_{i t}\right)-\ln \left(I N M_{i t-1}\right) & =\eta_{i n} \ln \left(I N M_{i t}^{*}\right)-\eta_{i n} \ln \left(I N M_{i t-1}\right) \\
\frac{O T M_{i t}}{O T M_{i t-1}} & =\left(\frac{O T M_{i t}^{*}}{O T M_{i t-1}}\right)^{\eta_{t o t}} \\
\ln \left(O T M_{i t}\right)-\ln \left(O T M_{i t-1}\right) & =\eta_{o t} \ln \left(O T M_{i t}^{*}\right)-\eta_{o t}\left(O T M_{i t-1}\right) \\
\frac{E M P_{i t}}{E M P_{i t-1}} & =\left(\frac{E M P_{i t}^{*}}{E M P_{i t-1}}\right)^{\eta_{e m}} \\
\ln \left(E M P_{i t}\right)-\ln \left(E M P_{i t-1}\right) & =\eta_{e m} \ln \left(E M P_{i t}^{*}\right)-\eta_{e m}\left(E M P_{i t-1}\right) \\
\frac{G E X_{i t}}{G E X_{i t-1}} & =\left(\frac{G E X_{i t}^{*}}{G E X_{i t-1}}\right)^{\eta_{g e}}
\end{aligned}
$$




$$
\begin{aligned}
\ln \left(G E X_{i t}\right)-\ln \left(G E X_{i t-1}\right) & =\eta_{g e} \ln \left(G E X_{i t}^{*}\right)-\eta_{g e}\left(G E X_{i t-1}\right) \\
\frac{M H Y_{i t}}{M H Y_{i t-1}} & =\left(\frac{M H Y_{i t}^{*}}{M H Y_{i t-1}}\right)^{\eta_{m h}} \\
\ln \left(M H Y_{i t}\right)-\ln \left(M H Y_{i t-1}\right) & =\eta_{m h} \ln \left(M H Y_{i t}^{*}\right)-\eta_{m h} \ln \left(M H Y_{i t-1}\right)
\end{aligned}
$$

where the subscript t-1 refers to the indicated variable lagged one period, one decade in this study, and $\eta_{i n}, \eta_{o t}, \eta_{e m}, \eta_{g e}$, and $\eta_{m h}$ are the speed of adjustment parameters that represent, respectively, the rate at which in-migration, out-migration, employment, local public expenditure and median household income adjust to their respective desired equilibrium levels. They are interpreted as the shares or proportions of the respective equilibrium rate of growth that were realized each period

Solving equations (4.3a)-(4.3e) for the equilibrium values gives:

$$
\begin{aligned}
\ln \left(I N M_{i t}^{*}\right)= & \frac{1}{\eta_{i n}}\left(\ln \left(I M_{i t}\right)-\ln \left(I N M_{i t-1}\right)+\eta_{i n} \ln \left(I N M_{i t-1}\right)\right) \\
= & \frac{1}{\eta_{i n}} I N M R_{i t}+\ln \left(I N M_{i t-1}\right) \\
\ln \left(O T M_{i t}^{*}\right)= & \frac{1}{\eta_{o t}}\left(\ln \left(O T M_{i t}\right)-\ln \left(O T M_{i t-1}\right)+\eta_{o t} \ln \left(O T M_{i t-1}\right)\right) \\
= & \frac{1}{\eta_{o t}} O T M R_{i t}+\ln \left(O T M_{i t-1}\right) \\
\ln \left(E M P_{i t}^{*}\right)= & \frac{1}{\eta_{e m}}\left(\ln \left(E M P_{i t}\right)-\ln \left(E M P_{i t-1}\right)+\eta_{e m} \ln \left(E M P_{i t-1}\right)\right) \\
& =\frac{1}{\eta_{e m}} E M P R_{i t}+\ln \left(E M P_{i t-1}\right)
\end{aligned}
$$




$$
\begin{aligned}
\ln \left(G E X_{i t}^{*}\right)= & \frac{1}{\eta_{g e}}\left(\ln \left(G E X_{i t}\right)-\ln \left(G E X_{i t-1}\right)+\eta_{g e} \ln \left(G E X_{i t-1}\right)\right) \\
& =\frac{1}{\eta_{g e}} G E X R_{i t}+\ln \left(G E X_{i t-1}\right) \\
\ln \left(M H Y_{i t}^{*}\right)= & \frac{1}{\eta_{m h}}\left(\ln \left(M H Y_{i t}\right)-\ln \left(M H Y_{i t-1}\right)+\eta_{m h} \ln \left(M H Y_{i t-1}\right)\right) \\
& =\frac{1}{\eta_{m h}} M H Y R_{i t}+\ln \left(M H Y_{i t-1}\right)
\end{aligned}
$$

where INMR, OTMR, EMPR, GEXR, and MHYR denote the gross in-migration growth rate, gross out-migration growth rate, employment growth rate, local public expenditure growth rate and median household income growth rate, respectively. ${ }^{4}$

Substituting from equations (4.4a)-(4.4e) into equations (4.2a)-(4.2e) gives:

${ }^{4}$ The growth rate from period $t-1$ to period $t$ in a time series observation, say, $y_{t}$ can be denoted by $g_{t}$, where

$$
g_{t}=\frac{y_{t}}{y_{t-1}}-1
$$

Now, if $\mathrm{x}$ is a small number, then $\ln (1+x) \approx x$. Therefore, if $g_{t}$ is small, $\ln \left(1+g_{t}\right) \approx g_{t}$ or $\ln \left(\frac{y_{t}}{y_{t-1}}\right) \approx g_{t}$ or $\ln \left(y_{t}\right)-\ln \left(y_{t-1}\right)$. 
Gross In-migration Growth Rate Equation:

$$
\begin{aligned}
& \frac{1}{\eta_{i n}} I N M R_{i t}+\ln \left(I N M_{i t-1}\right)=a_{1}\left(\frac{1}{\eta_{o t}} O T M R_{i t}+\ln \left(O T M_{i t-1}\right)\right)+b_{1}\left(\frac{1}{\eta_{e m}} E M P R_{i t}+\ln \left(E M P_{i t-1}\right)\right) \\
& +c_{1}\left(\frac{1}{\eta_{g e}} G E X R_{i t}+\ln \left(G E X_{i t-1}\right)\right)+d_{1}\left(\frac{1}{\eta_{m h}} M H Y R_{i t}+\ln \left(M H Y_{i t-1}\right)\right)+\sum_{k_{1}=6}^{K_{1}} x_{1 k_{1}} \ln \left(\mathrm{X}_{k_{1} i t}^{i n}\right) \\
& I N M R_{i t}=\eta_{i n}\left\{a_{1}\left(\frac{1}{\eta_{o t}} O T M R_{i t}+\ln \left(O T M_{i t-1}\right)\right)+b_{1}\left(\frac{1}{\eta_{e m}} E M P R_{i t}+\ln \left(E M P_{i t-1}\right)\right)\right. \\
& \left.+c_{1}\left(\frac{1}{\eta_{g e}} G E X R_{i t}+\ln \left(G E X_{i t-1}\right)\right)+d_{1}\left(\frac{1}{\eta_{m h}} M H Y R_{i t}+\ln \left(M H Y_{i t-1}\right)\right)+\sum_{k_{1}=6}^{K_{1}} X_{1 k_{1}} \ln \left(\mathrm{X}_{k_{1} i t}^{i n}\right)-\ln \left(I N M_{i t-1}\right)\right\} \\
& \quad I_{N M R_{i t}=} \beta_{11} O T M R_{i t}+\beta_{12} E M P R_{i t}+\beta_{13} G E X R_{i t}+\beta_{14} M H Y R_{i t}+\gamma_{11} \ln \left(I N M_{i t-1}\right)+\gamma_{12} \ln \left(O T M_{i t-1}\right) \\
& \quad \gamma_{13} \ln \left(E M P_{i t-1}\right)+\gamma_{14} \ln \left(G E X_{i t-1}\right)+\gamma_{15} \ln \left(M H Y_{i t-1}\right)+\sum_{k_{1}=6}^{K_{1}} \gamma_{1 k_{1}} \ln \left(\mathrm{X}_{k_{1} i t}^{i n}\right)
\end{aligned}
$$

Gross Out-Migration Growth Rate Equation:

$$
\begin{gathered}
\frac{1}{\eta_{o t}} \text { OTMR }_{i t}+\ln \left(O T M_{i t-1}\right)=a_{2}\left(\frac{1}{\eta_{i n}} I N M R_{i t}+\ln \left(I N M_{i t-1}\right)\right)+b_{2}\left(\frac{1}{\eta_{e m}} E M P R_{i t}+\ln \left(E M P_{i t-1}\right)\right) \\
+c_{2}\left(\frac{1}{\eta_{g e}} G E X R_{i t}+\ln \left(G E X_{i t-1}\right)\right)+d_{2}\left(\frac{1}{\eta_{m h}} M H Y R_{i t}+\ln \left(M H Y_{i t-1}\right)\right)+\sum_{k_{2}=6}^{K_{2}} x_{2 k_{2}} \ln \left(\mathrm{X}_{k_{2} i t}^{i n}\right) \\
\begin{aligned}
& O T M R_{i t}= \eta_{o t}\left\{a_{2}\left(\frac{1}{\eta_{i n}} I N M R_{i t}+\ln \left(I N M_{i t-1}\right)\right)+b_{2}\left(\frac{1}{\eta_{e m}} E M P R_{i t}+\ln \left(E M P_{i t-1}\right)\right)\right. \\
&\left.+c_{2}\left(\frac{1}{\eta_{g e}} G E X R_{i t}+\ln \left(G E X_{i t-1}\right)\right)+d_{2}\left(\frac{1}{\eta_{m h}} M H Y R_{i t}+\ln \left(M H Y_{i t-1}\right)\right)+\sum_{k_{2}=6}^{K_{2}} x_{2 k_{2}} \ln \left(\mathrm{X}_{k_{2} i t}^{i n}\right)-\ln \left(O T M_{i t-1}\right)\right\} \\
& \quad+\gamma_{23} \ln \left(E M P_{i t-1}\right)+\gamma_{24} \ln \left(G E X_{i t-1}\right)+\gamma_{25} \ln \left(M H Y_{i t-1}\right)+\sum_{k_{2}=6}^{K_{2}} \gamma_{2 k_{2}} \ln \left(\mathrm{X}_{k_{2} i t}^{i n}\right)
\end{aligned} \\
\text { OTMR }_{i t}=\beta_{21} I N M R_{i t}+\beta_{22} E M P R_{i t}+\beta_{23} G E X R_{i t}+\beta_{24} M H Y R_{i t}+\gamma_{21} \ln \left(I N M_{i t-1}\right)+\gamma_{22} \ln \left(O T M_{i t-1}\right)
\end{gathered}
$$


Business (Employment) Growth Rate Equation:

$$
\begin{gathered}
\frac{1}{\eta_{e m}} E M P R_{i t}+\ln \left(E M P_{i t-1}\right)=a_{3}\left(\frac{1}{\eta_{i n}} I N M R_{i t}+\ln \left(I N M_{i t-1}\right)\right)+b_{3}\left(\frac{1}{\eta_{o t}} O T M R_{i t}+\ln \left(O T M_{i t-1}\right)\right) \\
+c_{3}\left(\frac{1}{\eta_{g e}} G E X R_{i t}+\ln \left(G E X_{i t-1}\right)\right)+d_{3}\left(\frac{1}{\eta_{m h}} M H Y R_{i t}+\ln \left(M H Y_{i t-1}\right)\right)+\sum_{k_{3}=6}^{K_{3}} x_{3 k_{3}} \ln \left(X_{k_{3 i t}}^{i n}\right) \\
E M P R_{i t}=\eta_{e m}\left\{a_{3}\left(\frac{1}{\eta_{i n}} I N M R_{i t}+\ln \left(I N M_{i t-1}\right)\right)+b_{3}\left(\frac{1}{\eta_{o t}} O T M R_{i t}+\ln \left(O T M_{i t-1}\right)\right)\right. \\
\left.+C_{3}\left(\frac{1}{\eta_{g e}} G E X R_{i t}+\ln \left(G E X_{i t-1}\right)\right)+d_{3}\left(\frac{1}{\eta_{m h}} M H Y R_{i t}+\ln \left(M H Y_{i t-1}\right)\right)+\sum_{k_{3}=6}^{K_{3}} x_{3 k_{3}} \ln \left(\mathrm{X}_{k_{3 i t}}^{i n}\right)-\ln \left(E M P_{i t-1}\right)\right\} \\
E M P R_{i t}=\beta_{31} I N M R_{i t}+\beta_{32} O T M R_{i t}+\beta_{33} G E X R_{i t}+\beta_{34} M H Y R_{i t}+\gamma_{31} \ln \left(I N M_{i t-1}\right)+\gamma_{32} \ln \left(O T M_{i t-1}\right) \\
+\gamma_{23} \ln \left(E M P_{i t-1}\right)+\gamma_{34} \ln \left(G E X_{i t-1}\right)+\gamma_{35} \ln \left(M H Y_{i t-1}\right)+\sum_{k_{3}=6}^{K_{3}} \gamma_{3 k_{3}} \ln \left(X_{k_{3 i t}}^{i n}\right)
\end{gathered}
$$

Local Government Expenditure Growth Rate Equation:

$$
\begin{aligned}
& \frac{1}{\eta_{g e}} G E X R_{i t}+\ln \left(G E X_{i t-1}\right)=a_{4}\left(\frac{1}{\eta_{i n}} I N M R_{i t}+\ln \left(I N M_{i t-1}\right)\right)+b_{4}\left(\frac{1}{\eta_{o t}} O T M R_{i t}+\ln \left(O T M_{i t-1}\right)\right) \\
& +c_{4}\left(\frac{1}{\eta_{e m}} E M P R_{i t}+\ln \left(E M P_{i t-1}\right)\right)+d_{4}\left(\frac{1}{\eta_{m h}} M H Y R_{i t}+\ln \left(M H Y_{i t-1}\right)\right)+\sum_{k=1}^{K_{4}} x_{4 k} \ln \left(\mathrm{X}_{k i t}^{i n}\right) \\
& G E X R_{i t}=\eta_{g e}\left\{a_{4}\left(\frac{1}{\eta_{i n}} I_{N M R_{i t}}+\ln \left(I N M_{i t-1}\right)\right)+b_{4}\left(\frac{1}{\eta_{o t}} O T M R_{i t}+\ln \left(O T M_{i t-1}\right)\right)\right. \\
& \left.+c_{4}\left(\frac{1}{\eta_{e m}} E M P R_{i t}+\ln \left(E M P_{i t-1}\right)\right)+d_{4}\left(\frac{1}{\eta_{m h}} M H Y R_{i t}+\ln \left(M H Y_{i t-1}\right)\right)+\sum_{k_{4}=6}^{K_{4}} x_{4 k_{4}} \ln \left(\mathrm{X}_{k_{4} i t}^{i n}\right)-\ln \left(G E X_{i t-1}\right)\right\} \\
& \begin{array}{c}
G \mathrm{XR}_{i t}=\beta_{41} I N M R_{i t}+\beta_{42} O T M R_{i t}+\beta_{43} E M P R_{i t i t}+\beta_{44} M H Y R_{i t}+\gamma_{41} \ln \left(I N M_{i t-1}\right)+\gamma_{42} \ln \left(O T M_{i t-1}\right) \\
+\gamma_{43} \ln \left(E M P_{i t-1}\right)+\gamma_{44} \ln \left(G E X_{i t-1}\right)+\gamma_{45} \ln \left(M H Y_{i t-1}\right)+\sum_{k_{4}=6}^{K_{4}} \gamma_{4 k_{4}} \ln \left(\mathrm{X}_{k_{4} i t}^{\text {in }}\right)
\end{array}
\end{aligned}
$$


Median Household Income Growth Rate Equation:

$$
\begin{aligned}
& \frac{1}{\eta_{m h}} M H Y R_{i t}+\ln \left(M H Y_{i t-1}\right)=a_{5}\left(\frac{1}{\eta_{i n}} I N M R_{i t}+\ln \left(I N M_{i t-1}\right)\right)+b_{5}\left(\frac{1}{\eta_{o t}} O T M R_{i t}+\ln \left(O T M_{i t-1}\right)\right) \\
& +c_{5}\left(\frac{1}{\eta_{e m}} E M P R_{i t}+\ln \left(E M P_{i t-1}\right)\right)+d_{5}\left(\frac{1}{\eta_{g e}} G E X R_{i t}+\ln \left(G E X_{i t-1}\right)\right)+\sum_{k_{5}=1}^{K_{5}} x_{5 k_{5}} \ln \left(\mathrm{X}_{k_{5} i t}^{i n}\right) \\
& M H Y R_{i t}=\eta_{m h}\left\{a_{5}\left(\frac{1}{\eta_{i n}} I N M R_{i t}+\ln \left(I N M_{i t-1}\right)\right)+b_{5}\left(\frac{1}{\eta_{o t}} O T M R_{i t}+\ln \left(O T M_{i t-1}\right)\right)\right. \\
& \left.+c_{5}\left(\frac{1}{\eta_{e m}} E M P R_{i t}+\ln \left(E M P_{i t-1}\right)\right)+d_{5}\left(\frac{1}{\eta_{g e}} G E X R_{i t}+\ln \left(G E X_{i t-1}\right)\right)+\sum_{k_{5}=6}^{K_{5}} x_{5 k_{5}} \ln \left(\mathrm{X}_{k_{5} i t}^{i n}\right)-\ln \left(M H Y_{i t-1}\right)\right\} \\
& M H Y R_{i t}=\beta_{51} I_{N M R_{i t}}+\beta_{52} O T M R_{i t}+\beta_{53} E M P R_{i t}+\beta_{54} G E X R_{i t}+\gamma_{51} \ln \left(I N M_{i t-1}\right)+\gamma_{52} \ln \left(O T M_{i t-1}\right) \\
& +\gamma_{53} \ln \left(E M P_{i t-1}\right)+\gamma_{54} \ln \left(G E X_{i t-1}\right)+\gamma_{55} \ln \left(M H Y_{i t-1}\right)+\sum_{k_{5}=6}^{K_{5}} \gamma_{5 k_{5}} \ln \left(X_{k_{s} i t}^{i n}\right)
\end{aligned}
$$

Equations (4.5a)-(4.5e) are the structural equations of the basic simultaneous-equations model which constitute the basis for the empirical work reported in this study. Thus, the general form of the model to be estimated and extended (to accommodate spatial effect) in subsequent sections can be given by: 


$$
\begin{aligned}
& \left(I N M R_{i t}=\alpha_{1}+\beta_{11} O T M R_{i t}+\beta_{12} E M P R_{i t}+\beta_{13} G E X R_{i t}+\beta_{14} M H Y R_{i t}+\gamma_{11} \ln \left(I N M_{i t-1}\right)+\gamma_{12} \ln \left(O T M_{i t-1}\right)\right. \\
& +\gamma_{13} \ln \left(E M P_{i t-1}\right)+\gamma_{14} \ln \left(G E X_{i t-1}\right)+\gamma_{15} \ln \left(M H Y_{i t-1}\right)+\sum_{k_{1}=6}^{K_{1}} \gamma_{1 k_{1}} \ln \left(X_{k_{1} i}^{i n}\right)+u_{i t}^{i n} \\
& O T M R_{i t}=\alpha_{2}+\beta_{21} I N M R_{i t}+\beta_{22} E M P R_{i t}+\beta_{23} G E X R_{i t}+\beta_{24} M H Y R_{i t}+\gamma_{21} \ln \left(I N M_{i t-1}\right)+\gamma_{22} \ln \left(O T M_{i t-1}\right) \\
& +\gamma_{23} \ln \left(E M P_{i t-1}\right)+\gamma_{24} \ln \left(G E X_{i t-1}\right)+\gamma_{25} \ln \left(M H Y_{i t-1}\right)+\sum_{k_{2}=6}^{K_{2}} \gamma_{2 k_{2}} \ln \left(X_{k_{2} i t}^{\text {in }}\right)+\mathrm{u}_{\mathrm{it}}^{\mathrm{ot}} \\
& E M P R_{i t}=\alpha_{3}+\beta_{31} I N M R_{i t}+\beta_{32} O T M R_{i t}+\beta_{33} G E X R_{i t}+\beta_{34} M H Y R_{i t}+\gamma_{31} \ln \left(I N M_{i t-1}\right)+\gamma_{32} \ln \left(O T M_{i t-1}\right) \\
& +\gamma_{23} \ln \left(E M P_{i t-1}\right)+\gamma_{34} \ln \left(G E X_{i t-1}\right)+\gamma_{35} \ln \left(M H Y_{i t-1}\right)+\sum_{k_{3}=6}^{K_{3}} \gamma_{3 k_{3}} \ln \left(X_{k_{3} i t}^{\text {in }}\right)+u_{i t}^{\mathrm{em}} \\
& +\gamma_{43} \ln \left(E M P_{i t-1}\right)+\gamma_{44} \ln \left(G E X_{i t-1}\right)+\gamma_{45} \ln \left(M H Y_{i t-1}\right)+\sum_{k_{4}=6}^{K_{4}} \gamma_{4 k_{4}} \ln \left(X_{k_{4} i t}^{i n}\right)+u_{i t}^{g e} \\
& M H Y R_{i t}=\alpha_{5}+\beta_{51} I N M R_{i t}+\beta_{52} O T M R_{i t}+\beta_{53} E M P R_{i t}+\beta_{54} G E X R_{i t}+\gamma_{51} \ln \left(I N M_{i t-1}\right)+\gamma_{52} \ln \left(O T M_{i t-1}\right) \\
& +\gamma_{53} \ln \left(E M \mathbb{P}_{i t-1}\right)+\gamma_{54} \ln \left(G E X_{i t-1}\right)+\gamma_{55} \ln \left(M H Y_{i t-1}\right)+\sum_{k_{5}=6}^{K_{5}} \gamma_{5 k_{5}} \ln \left(X_{k_{5} i t}^{i n}\right)+u_{i t}^{\text {mh }}
\end{aligned}
$$

where $\alpha, \beta$, and $\gamma$ are unobserved parameters and $u_{i t}^{i n}, u_{i t}^{o t}, u_{i t}^{e m}, u_{i t}^{g e}$ and $u_{i t}^{m h}$ are disturbances. Note that the speed of adjustment parameters $\{\eta\}$ become embedded in the coefficient parameters, $\beta$ and $\gamma$.

Following the approaches developed in chapter III of this study, a more compact form of equation (4.6a) can be given by:

$$
\mathbf{y}_{j}=\mathbf{Y}_{j} \boldsymbol{\beta}_{j}+\mathbf{X}_{j} \gamma_{j}+\mathbf{u}_{j}, \quad j=1,2, \ldots, 5
$$


where $\mathbf{y}_{j}$ is $\mathrm{n} \times 1$ column vector of data on the dependent endogenous variable, $\mathbf{Y}_{j}$ is the $\mathrm{n} \times 4$ matrix of data on the 4 included right-hand side endogenous variable, $\boldsymbol{\beta}_{j}$ is $4 \times 1$ column vector of non-zero coefficients on $\mathbf{Y}_{j}, \mathbf{X}_{j}$ is n x K1 matrix of observation on the predetermined variables, including a constant term, $\gamma_{j}$ is K1 $\mathrm{x} 1$ column vector of coefficients on $\mathbf{X}_{j}$ (note $\alpha_{j}$ is included in $\gamma_{j}$ ), and $\mathbf{u}_{j}$ is $\mathrm{n} \mathrm{x} 1$ vector of disturbance terms. Rewriting equation (4.7) as:

$$
\mathbf{y}_{j}=\mathbf{Z}_{j}^{\prime} \boldsymbol{\delta}_{j}+\mathbf{u}_{j}
$$

where

$$
\mathbf{Z}_{j}=\left[\begin{array}{ll}
\mathbf{Y}_{j} & \mathbf{X}_{j}
\end{array}\right] \text { and } \quad \boldsymbol{\delta}_{j}=\left[\begin{array}{l}
\boldsymbol{\beta}_{j} \\
\boldsymbol{\gamma}_{j}
\end{array}\right]
$$

Thus, all 5 equations of the system can be written as:

$$
\underset{5 \mathrm{n} \times 1}{\mathbf{y}}=\underset{5 \mathrm{n} \times \mathrm{K}^{\oplus} \mathrm{K}^{\oplus} \times 1}{\mathbf{Z}}+\underset{5 \mathrm{n} \times 1}{\mathbf{u}}
$$

with the following stacked vectors and matrix

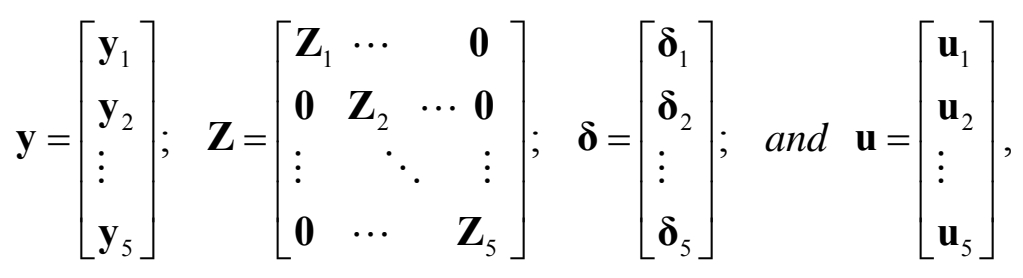

where $\mathrm{K}^{\oplus}=\sum_{j=1}^{5}\left(G 1_{j}-1+K 1_{j}\right)$ is the total number of parameters to be estimated.

The assumptions on the stochastic disturbance term for simultaneous equations system can be stated as: 


$$
\begin{aligned}
& \mathrm{E}(\mathbf{u})=\mathbf{0} \\
& \operatorname{Cov}(\mathbf{u})=\mathrm{E}\left(\mathbf{u u}^{\prime}\right)=\left[\begin{array}{llll}
\boldsymbol{\sigma}_{11} \mathbf{I}_{n} & \boldsymbol{\sigma}_{12} \mathbf{I}_{n} & \cdots & \boldsymbol{\sigma}_{1 G} \mathbf{I}_{n} \\
\boldsymbol{\sigma}_{21} \mathbf{I}_{n} & \boldsymbol{\sigma}_{22} \mathbf{I}_{n} \cdots & \boldsymbol{\sigma}_{2 G} \mathbf{I}_{n} \\
\vdots & \vdots & & \vdots \\
\boldsymbol{\sigma}_{51} \mathbf{I}_{n} & \boldsymbol{\sigma}_{52} \mathbf{I}_{n} \cdots & \boldsymbol{\sigma}_{55} \mathbf{I}_{n}
\end{array}\right]=\boldsymbol{\Sigma} \otimes \mathbf{I}_{n},
\end{aligned}
$$

where $\boldsymbol{\Sigma} \otimes \mathbf{I}_{n}$ is the Kronecker product of these matrices.

\subsection{Spatial Models}

Models such as (4.6a) are estimated using data collected for cross sectional observations on aggregate spatial units such as counties. Such data sets, however, are likely to exhibit a lack of independence in the form of spatial autocorrelation. Spatial autocorrelation or spatial dependence refers to the statistical property where the dependent variable or error term at one location is correlated with observations on the dependent variable or error term at other locations (Anselin, 1988). Expenditure spillover effects are, for example, wide spread feature of local public service as a result of policy interdependence among local jurisdictions. Commuters, for example, use public transportation, roads, recreation and cultural facilities in their working communities. Air pollution controls and sewage treatment enhance the environmental quality of neighboring jurisdictions, and educational and job training expenditures may lead to productivity gain in workplaces outside the community. Population movement and business growth also show spatial interdependences. In its 2000 report on the state-tostate migration flows between 1995 and 2000, the Bureau of the Census states that the largest migrations were to nearby or neighboring states. New York's largest migration inflows, for example, were from New Jersey and its largest migration outflows were to New Jersey and vice versa. Similarly, there were large flows between other neighboring 
states. Migration behavior of businesses also shows similar pattern. In their study of the out migration of businesses from Minnesota, Karvel, Musil and Sebatian (1998) showed that the second most important reason for business out-migration decisions were the incentives that were provided by neighboring state or local governments. Mathur (2005) also showed that higher bankruptcy exemptions in neighboring states lower the probability of starting a business in the state of residence. Similarly, studies have shown that regional income growth is space dependent (Krugman, 1991a). Factors that explain economic convergence such as technological diffusion and labor mobility have a strong geographical dimension. Theories of new economic geography stress on the role played by geographic spillovers in spatial and growth mechanisms. Economic activities performances of neighboring regions are, therefore, similar and not randomly and spatially distributed on an economic integrated regional space. The presence of spatial spillover demands the explicit modeling of the spatial interactions, by taking into account that local jurisdictions make their decisions simultaneously, and each local jurisdiction takes its neighbors' behavior into account when setting its own decisions. The problem of spatial interdependence results from model misspecification (Anselin, 1988).

Following the theoretical framework developed in chapter III of this study, equation (4.7a) can be extended to accommodate the spatial interdependences as follows:

$$
\mathbf{y}_{j}=\mathbf{Z}_{j} \boldsymbol{\delta}_{j}+\mathbf{u}_{j}, \mathbf{j}=1, \ldots, 5
$$

where

$$
\mathbf{Z}_{j}=\left(\mathbf{Y}_{j}, \mathbf{X}_{j}, \mathbf{W} \mathbf{Y}_{j}\right) \text { and } \boldsymbol{\delta}_{j}=\left(\boldsymbol{\beta}_{j}^{\prime}, \boldsymbol{\gamma}_{j}^{\prime}, \boldsymbol{\lambda}_{j}^{\prime}\right)^{\prime}
$$

and $\mathbf{Y}_{j}, \mathbf{X}_{j}$, and $\mathbf{W} \mathbf{Y}_{j}$ are the corresponding matrices of observations on the endogenous variables, exogenous variables, and the spatially lagged endogenous variables that appear 
in the jth equation, respectively, and $\lambda_{j}$ is a vector of coefficients on the spatially dependent variables $\left(\mathbf{W} \mathbf{Y}_{j}\right)$.

The complete economic model which corresponds to the spatial lag model given in equation (4.7a) looks as follows:

$$
\begin{aligned}
&{I N M R_{i t}=}_{=} \beta_{11} O T M R_{i t}+\beta_{12} E M P R_{i t}+\beta_{13} G E X R_{i t}+\beta_{14} M H Y R_{i t}+\lambda_{11} \mathbf{W}\left(O T M R_{i t}\right)+\lambda_{12} \mathbf{W}\left(E M P R_{i t}\right) \\
&+ \lambda_{13} \mathbf{W}\left(G E X R_{i t}\right)+\lambda_{14} \mathbf{W}\left(M H Y R_{i t}\right)+\gamma_{11} \ln \left(I N M_{i t-1}\right)+\gamma_{12} \ln \left(O T M_{i t-1}\right)+\gamma_{13} \ln \left(E M P_{i t-1}\right) \\
&+\gamma_{14} \ln \left(G E X_{i t-1}\right)+\gamma_{15} \ln \left(M H Y_{i t-1}\right)+\sum_{k_{1}=6}^{K_{1}} \gamma_{1 k_{1}} \ln \left(\mathrm{X}_{k_{i t}}^{i n}\right) \\
& \text { OTMR }_{i t}= \beta_{21} I N M R_{i t}+\beta_{22} E M P R_{i t}+\beta_{23} G E X R_{i t}+\beta_{24} M H Y R_{i t}+\lambda_{21} \mathbf{W}\left(I N M R_{i t}\right)+\lambda_{22} \mathbf{W}\left(E M P R_{i t}\right) \\
&+\lambda_{23} \mathbf{W}\left(G E X R_{i t}\right)+\lambda_{24} \mathbf{W}\left(M H Y R_{i t}\right)+\gamma_{21} \ln \left(I N M_{i t-1}\right)+\gamma_{22} \ln \left(O T M_{i t-1}\right)+\gamma_{23} \ln \left(E M P_{i t-1}\right) \\
&+\gamma_{24} \ln \left(G E X_{i t-1}\right)+\gamma_{25} \ln \left(M H Y_{i t-1}\right)+\sum_{k_{2}=6}^{K_{2}} \gamma_{2 k_{2}} \ln \left(X_{k_{i 2}}^{o t}\right)
\end{aligned}
$$

$$
\begin{aligned}
E M P R_{i t}= & \beta_{31} I N M R_{i t}+\beta_{32} O T M R_{i t}+\beta_{33} G E X R_{i t}+\beta_{34} M H Y R_{i t}+\lambda_{31} \mathbf{W}\left(I N M R_{i t}\right)+\lambda_{32} \mathbf{W}\left(\text { OTMR }_{i t}\right) \\
& +\lambda_{33} \mathbf{W}\left(G E X R_{i t}\right)+\lambda_{34} \mathbf{W}\left(M H Y R_{i t}\right)+\gamma_{31} \ln \left(I N M_{i t-1}\right)+\gamma_{32} \ln \left(O T M_{i t-1}\right)+\gamma_{23} \ln \left(E M P_{i t-1}\right) \\
& +\gamma_{34} \ln \left(G E X_{i t-1}\right)+\gamma_{35} \ln \left(M H Y_{i t-1}\right)+\sum_{k_{3}=6}^{K_{3}} \gamma_{3 k_{3}} \ln \left(\mathrm{X}_{k_{3} i t}^{e m}\right)
\end{aligned}
$$

$$
\begin{aligned}
\text { GEXR }_{i t}= & \beta_{41} I N M R_{i t}+\beta_{42} O T M R_{i t}+\beta_{43} E M P R_{i t i t}+\beta_{44} M H Y R_{i t}+\lambda_{41} \mathbf{W}\left(I N M R_{i t}\right)+\lambda_{42} \mathbf{W}\left(O T M R_{i t}\right) \\
& +\lambda_{43} \mathbf{W}\left(E M P R_{i t}\right)+\lambda_{44} \mathbf{W}\left(M H Y R_{i t}\right)+\gamma_{41} \ln \left(I N M_{i t-1}\right)+\gamma_{42} \ln \left(O T M_{i t-1}\right)+\gamma_{43} \ln \left(E M P_{i t-1}\right) \\
& +\gamma_{44} \ln \left(G E X_{i t-1}\right)+\gamma_{45} \ln \left(M H Y_{i t-1}\right)+\sum_{k_{4}=6}^{K_{4}} \gamma_{4 k_{4}} \ln \left(X_{k_{4}}^{g e}\right) \\
M_{H Y R_{i t}=}= & \beta_{51} I N M R_{i t}+\beta_{52} O T M R_{i t}+\beta_{53} E M P R_{i t}+\beta_{54} G E X R_{i t}+\lambda_{51} \mathbf{W}\left(I N M R_{i t}\right)+\lambda_{52} \mathbf{W}\left(O T M R_{i t}\right) \\
& +\lambda_{53} \mathbf{W}\left(E M P R_{i t}\right)+\lambda_{54} \mathbf{W}\left(G E X R_{i t}\right)+\gamma_{51} \ln \left(I N M_{i t-1}\right)+\gamma_{52} \ln \left(O T M_{i t-1}\right)+\gamma_{53} \ln \left(E M P_{i t-1}\right) \\
& +\gamma_{54} \ln \left(G E X_{i t-1}\right)+\gamma_{55} \ln \left(M H Y_{i t-1}\right)+\sum_{k_{5}=6}^{K_{5}} \gamma_{5 k_{5}} \ln \left(X_{k_{i} t}^{m h}\right)
\end{aligned}
$$


Spatial autocorrelation could be in the error term. Thus, a second way to incorporate spatial autocorrelation in a regression model is to specify a spatial process for the disturbance term. The disturbance terms in a regression model can be considered to contain all ignored elements, and when spatial dependence is present in the disturbance term, the spatial effects are assumed to be a noise, or perturbation, that is, a factor that needs to be removed (Anselin, 2001). For example, any spatially auto-correlated variable that has an influence on $y$ and is omitted from the model will lead to a spatial dependence in the residual. Such spatial pattern in the residuals of the regression model may lead to the discovery of additional variables that should be included in the model. Local jurisdictions may also be subjected to shocks that affect their expenditure decisions, and are spatially auto-correlated - such as common shocks to income and tax base, that may result from central government regional policies or intermediate level of government fiscal policies. Spatial dependence in the disturbance term also violates the basic OLS estimation assumption of uncorrelated errors. Hence, when the spatial dependence is ignored, OLS estimates will be inefficient, though unbiased, the student t- and F-statistics for tests of significance will be biased, the $\mathrm{R}^{2}$ measure will be misleading, which in turn lead to a wrong statistical interpretation of the regression mode (Anselin, Bera, Florax and Yoon, 1996). More efficient estimators can be obtained by taking advantage of the particular structure of the error covariance implied by the spatial process. The disturbance term is non-spherical where the off-diagonal elements of the associated covariance matrix express the structure of spatial dependence. The spatial dependence in the disturbance term, thus, can be expressed using matrix notation as (all notations as defined before): 


$$
\begin{aligned}
& \mathbf{y}_{j}=\mathbf{Z}_{j} \boldsymbol{\delta}_{j}+\mathbf{u}_{j}, \\
& \mathbf{u}_{j}=\rho_{j} \mathbf{W} \mathbf{u}_{j}+\boldsymbol{\varepsilon}_{j}, \quad j=1, \ldots, 5
\end{aligned}
$$

where

$$
\mathbf{Z}_{j}=\left(\mathbf{Y}_{j}, \mathbf{X}_{j}\right) \text { and } \boldsymbol{\delta}_{j}=\left(\boldsymbol{\beta}_{j}^{\prime}, \boldsymbol{\gamma}_{j}^{\prime}\right)^{\prime}
$$

and the corresponding economic model is given as:

$$
\begin{aligned}
& \begin{array}{rl}
\operatorname{INMR}_{i t}=\beta_{11} & O T M R_{i t}+\beta_{12} E M P R_{i t}+\beta_{13} G E X R_{i t}+\beta_{14} M H Y R_{i t}+\gamma_{11} \ln \left(I N M_{i t-1}\right)+\gamma_{12} \ln (O T M \\
& +\gamma_{13} \ln \left(E M P_{i t-1}\right)+\gamma_{14} \ln \left(G E X_{i t-1}\right)+\gamma_{15} \ln \left(M H Y_{i t-1}\right)+\sum_{k_{1}=6}^{K_{1}} \gamma_{1 k_{1}} \ln \left(\mathrm{X}_{k_{i t}}^{i n}\right)+\mathrm{u}_{i t}^{i n}
\end{array} \\
& \text { where } \mathbf{u}_{i t}^{\text {in }}=\rho_{1} \mathbf{W} \mathbf{u}_{i t}^{\text {in }}+\varepsilon_{i t}^{i n} \\
& O T M R_{i t}=\beta_{21} I N M R_{i t}+\beta_{22} E M P R_{i t}+\beta_{23} G E X R_{i t}+\beta_{24} M H Y R_{i t}+\gamma_{21} \ln \left(I N M_{i t-1}\right)+\gamma_{22} \ln \left(O T M_{i t-1}\right) \\
& +\gamma_{23} \ln \left(E M P_{i t-1}\right)+\gamma_{24} \ln \left(G E X_{i t-1}\right)+\gamma_{25} \ln \left(M H Y_{i t-1}\right)+\sum_{k_{2}=6}^{K_{2}} \gamma_{2 k_{2}} \ln \left(\mathrm{X}_{k_{2} i t}^{o t}\right)+\mathrm{u}_{i t}^{o t} \\
& \text { where } \mathbf{u}_{i t}^{o t}=\rho_{2} \mathbf{W} \mathbf{u}_{i t}^{o t}+\varepsilon_{i t}^{o t} \\
& E M P R_{i t}=\beta_{31} I N M R_{i t}+\beta_{32} O T M R_{i t}+\beta_{33} G E X R_{i t}+\beta_{34} M H Y R_{i t}+\gamma_{31} \ln \left(I N M_{i t-1}\right)+\gamma_{32} \ln \left(O T M_{i t-1}\right) \\
& +\gamma_{23} \ln \left(E M P_{i t-1}\right)+\gamma_{34} \ln \left(G E X_{i t-1}\right)+\gamma_{35} \ln \left(M H Y_{i t-1}\right)+\sum_{k_{3}=6}^{K_{3}} \gamma_{3 k_{3}} \ln \left(\mathrm{X}_{k_{3} i t}^{e m}\right)+\mathrm{u}_{i t}^{e m} \\
& \text { where } \mathbf{u}_{i t}^{e m}=\rho_{3} \mathbf{W} \mathbf{u}_{i t}^{e m}+\varepsilon_{i t}^{e m} \\
& G E X R_{i t}=\beta_{41} I N M R_{i t}+\beta_{42} O T M R_{i t}+\beta_{43} E M P R_{i t i t}+\beta_{44} M H Y R_{i t}+\gamma_{41} \ln \left(I N M_{i t-1}\right)+\gamma_{42} \ln \left(O T M_{i t-1}\right) \\
& +\gamma_{43} \ln \left(E M P_{i t-1}\right)+\gamma_{44} \ln \left(G E X_{i t-1}\right)+\gamma_{45} \ln \left(M H Y_{i t-1}\right)+\sum_{k_{4}=6}^{K_{4}} \gamma_{4 k_{4}} \ln \left(\mathrm{X}_{k_{4} i t}^{g e}\right)+\mathrm{u}_{i t}^{g e} \\
& \text { where } \mathbf{u}_{i t}^{g e}=\rho_{4} \mathbf{W} \mathbf{u}_{i t}^{g e}+\varepsilon_{i t}^{g e} \\
& M H Y R_{i t}=\beta_{51} I N M R_{i t}+\beta_{52} O T M R_{i t}+\beta_{53} E M P R_{i t}+\beta_{54} G E X R_{i t}+\gamma_{51} \ln \left(I N M_{i t-1}\right)+\gamma_{52} \ln \left(O T M_{i t-1}\right) \\
& +\gamma_{53} \ln \left(E M P_{i t-1}\right)+\gamma_{54} \ln \left(G E X_{i t-1}\right)+\gamma_{55} \ln \left(M H Y_{i t-1}\right)+\sum_{k_{5}=6}^{K_{5}} \gamma_{5 k_{5}} \ln \left(\mathrm{X}_{k_{5 i t}}^{m h}\right)+\mathrm{u}_{i t}^{m h} \\
& \text { where } \mathbf{u}_{i t}^{m h}=\rho_{5} \mathbf{W} \mathbf{u}_{i t}^{m h}+\varepsilon_{i t}^{m h}
\end{aligned}
$$


When there are no strong a priori theoretical reasons to believe that interdependences between spatial units arises either due to the spatial lags of the dependent variables or due to spatially autoregressive error terms, the standard approach is to model the system with both effects included (Anselin, 2003). This study also follows this approach and the spatial autoregressive model with both the spatial lag and spatial error effects can be expressed as (all notations as defined before):

$$
\begin{aligned}
& \mathbf{y}_{j}=\mathbf{Z}_{j} \boldsymbol{\delta}_{j}+\mathbf{u}_{j}, \\
& \mathbf{u}_{j}=\rho_{j} \mathbf{W} \mathbf{u}_{j}+\boldsymbol{\varepsilon}_{j}, \quad j=1, \ldots, 5
\end{aligned}
$$

where

$$
\mathbf{Z}_{j}=\left(\mathbf{Y}_{j}, \mathbf{X}_{j}, \mathbf{W} \mathbf{Y}_{j}\right) \text { and } \boldsymbol{\delta}_{j}=\left(\boldsymbol{\beta}_{j}^{\prime}, \boldsymbol{\gamma}_{j}^{\prime}, \boldsymbol{\lambda}_{j}^{\prime}\right)^{\prime}
$$

and the corresponding economic model is as given below. 


$$
\begin{aligned}
& \left(I N M R_{i t}=\beta_{11} O T M R_{i t}+\beta_{12} E M P R_{i t}+\beta_{13} G E X R_{i t}+\beta_{14} M H Y R_{i t}+\lambda_{11} \mathbf{W}\left(O T M R_{i t}\right)+\lambda_{12} \mathbf{W}\left(E M P R_{i t}\right)\right. \\
& +\lambda_{13} \mathbf{W}\left(G E X R_{i t}\right)+\lambda_{14} \mathbf{W}\left(M H Y R_{i t}\right)+\gamma_{11} \ln \left(I N M_{i t-1}\right)+\gamma_{12} \ln \left(O T M_{i t-1}\right)+\gamma_{13} \ln \left(E M P_{i t-1}\right) \\
& +\gamma_{14} \ln \left(G E X_{i t-1}\right)+\gamma_{15} \ln \left(M H Y_{i t-1}\right)+\sum_{k_{1}=6}^{K_{1}} \gamma_{1 k_{1}} \ln \left(\mathrm{X}_{k_{1} i t}^{i n}\right)+\mathrm{u}_{i t}^{i n} \\
& \text { where } \mathrm{u}_{i t}^{\text {in }}=\rho_{1} \mathbf{W} \mathrm{u}_{i t}^{\text {in }}+\varepsilon_{i t}^{\text {in }} \\
& O T M R_{i t}=\beta_{21} I N M R_{i t}+\beta_{22} E M P R_{i t}+\beta_{23} G E X R_{i t}+\beta_{24} M H Y R_{i t}+\lambda_{21} \mathbf{W}\left(I N M R_{i t}\right)+\lambda_{22} \mathbf{W}\left(E M P R_{i t}\right) \\
& +\lambda_{23} \mathbf{W}\left(G E X R_{i t}\right)+\lambda_{24} \mathbf{W}\left(M_{H Y R_{i t}}\right)+\gamma_{21} \ln \left(I N M_{i t-1}\right)+\gamma_{22} \ln \left(O T M_{i t-1}\right)+\gamma_{23} \ln \left(E M P_{i t-1}\right) \\
& +\gamma_{24} \ln \left(G E X_{i t-1}\right)+\gamma_{25} \ln \left(M H Y_{i t-1}\right)+\sum_{k_{2}=6}^{K_{2}} \gamma_{2 k_{2}} \ln \left(\mathrm{X}_{k_{2} i t}^{o t}\right)+\mathrm{u}_{i t}^{0 t} \\
& \text { where } \mathrm{u}_{i t}^{0 t}=\rho_{2} \mathbf{W} \mathrm{u}_{i t}^{0 t}+\varepsilon_{i t}^{o t} \\
& E M P R_{i t}=\beta_{31} I N M R_{i t}+\beta_{32} O T M R_{i t}+\beta_{33} G E X R_{i t}+\beta_{34} M H Y R_{i t}+\lambda_{31} \mathbf{W}\left(I N M R_{i t}\right)+\lambda_{32} \mathbf{W}\left(O T M R_{i t}\right) \\
& +\lambda_{33} \mathbf{W}\left(G E X R_{i t}\right)+\lambda_{34} \mathbf{W}\left(M_{H Y R_{i t}}\right)+\gamma_{31} \ln \left(I N M_{i t-1}\right)+\gamma_{32} \ln \left(O T M_{i t-1}\right)+\gamma_{23} \ln \left(E M P_{i t-1}\right) \\
& +\gamma_{34} \ln \left(G E X_{i t-1}\right)+\gamma_{35} \ln \left(M H Y_{i t-1}\right)+\sum_{k_{3}=6}^{K_{3}} \gamma_{3 k_{3}} \ln \left(\mathrm{X}_{k_{3} i t}^{e m}\right)+\mathrm{u}_{i t}^{e m} \\
& \text { where } \mathrm{u}_{i t}^{e m}=\rho_{3} \mathbf{W} \mathrm{u}_{i t}^{e m}+\varepsilon_{i t}^{e m} \\
& G E X R_{i t}=\beta_{41} I N M R_{i t}+\beta_{42} O T M R_{i t}+\beta_{43} E M P R_{i t i t}+\beta_{44} M H Y R_{i t}+\lambda_{41} \mathbf{W}\left(I N M R_{i t}\right)+\lambda_{42} \mathbf{W}\left(O T M R_{i t}\right) \\
& +\lambda_{43} \mathbf{W}\left(E M P R_{i t}\right)+\lambda_{44} \mathbf{W}\left(M_{H Y R_{i t}}\right)+\gamma_{41} \ln \left(I N M_{i t-1}\right)+\gamma_{42} \ln \left(O T M_{i t-1}\right)+\gamma_{43} \ln \left(E M P_{i t-1}\right) \\
& +\gamma_{44} \ln \left(G E X_{i t-1}\right)+\gamma_{45} \ln \left(M H Y_{i t-1}\right)+\sum_{k_{4}=6}^{K_{4}} \gamma_{4 k_{4}} \ln \left(\mathrm{X}_{k_{4} i t}^{g e}\right)+\mathrm{u}_{i t}^{g e} \\
& \text { where } \mathrm{u}_{i t}^{g e}=\rho_{4} \mathbf{W} \mathrm{u}_{i t}^{g e}+\varepsilon_{i t}^{g e} \\
& M H Y R_{i t}=\beta_{51} I{ } M R_{i t}+\beta_{52} O T M R_{i t}+\beta_{53} E M P R_{i t}+\beta_{54} G E X R_{i t}+\lambda_{51} \mathbf{W}\left(I N M R_{i t}\right)+\lambda_{52} \mathbf{W}\left(O T M R_{i t}\right) \\
& +\lambda_{53} \mathbf{W}\left(E M P R_{i t}\right)+\lambda_{54} \mathbf{W}\left(G E X R_{i t}\right)+\gamma_{51} \ln \left(I N M_{i t-1}\right)+\gamma_{52} \ln \left(O T M_{i t-1}\right)+\gamma_{53} \ln \left(E M P_{i t-1}\right) \\
& +\gamma_{54} \ln \left(G E X_{i t-1}\right)+\gamma_{55} \ln \left(M H Y_{i t-1}\right)+\sum_{k_{5}=6}^{K_{5}} \gamma_{5 k_{5}} \ln \left(\mathrm{X}_{k_{5} i t}^{m h}\right)+\mathrm{u}_{i t}^{m h} \\
& \text { where } \mathrm{u}_{i t}^{m h}=\rho_{5} \mathbf{W} \mathrm{u}_{i t}^{m h}+\varepsilon_{i t}^{m h}
\end{aligned}
$$




\subsection{Empirical Models}

The models outlined above and summarized in (4.6a) and (4.7c) are estimated using both county-level cross-sectional and panel data for Appalachian region for the 1980-2000 periods. The systems of equations are specified in log-linear form as follows:

a) Standard Simultaneous equations Model

$$
\begin{aligned}
& \int I N M R_{i t}=\alpha_{1}+\beta_{11} O T M R_{i t}+\beta_{12} E M P R_{i t}+\beta_{13} G E X R_{i t}+\beta_{14} M H Y R_{i t}+\gamma_{11} \ln \left(I N M_{i t-1}\right)+\gamma_{12} \ln \left(O T M_{i t-1}\right) \\
& +\gamma_{13} \ln \left(E M P_{i t-1}\right)+\gamma_{14} \ln \left(G E X_{i t-1}\right)+\gamma_{15} \ln \left(M H Y_{i t-1}\right)+\sum_{k_{1}=6}^{K_{1}} \gamma_{1 k_{1}} \ln \left(X_{k_{1} t}^{i n}\right)+u_{i t}^{i n} \\
& O T M R_{i t}=\alpha_{2}+\beta_{21} I N M R_{i t}+\beta_{22} E M P R_{i t}+\beta_{23} G E X R_{i t}+\beta_{24} M H Y R_{i t}+\gamma_{21} \ln \left(I N M_{i t-1}\right)+\gamma_{22} \ln \left(O T M_{i t-1}\right) \\
& +\gamma_{23} \ln \left(E M P_{i t-1}\right)+\gamma_{24} \ln \left(G E X_{i t-1}\right)+\gamma_{25} \ln \left(M H Y_{i t-1}\right)+\sum_{k_{2}=6}^{K_{2}} \gamma_{2 k_{2}} \ln \left(X_{k_{2} i t}^{\text {in }}\right)+\mathrm{u}_{\mathrm{it}}^{\mathrm{ot}} \\
& E M P R_{i t}=\alpha_{3}+\beta_{31} I N M R_{i t}+\beta_{32} O_{T T M R}+\beta_{33} G E X R_{i t}+\beta_{34} M H Y R_{i t}+\gamma_{31} \ln \left(I N M_{i t-1}\right)+\gamma_{32} \ln \left(O T M_{i t-1}\right) \\
& +\gamma_{23} \ln \left(E M P_{i t-1}\right)+\gamma_{34} \ln \left(G E X_{i t-1}\right)+\gamma_{35} \ln \left(M H Y_{i t-1}\right)+\sum_{k_{3}=6}^{K_{3}} \gamma_{3 k_{3}} \ln \left(\mathrm{X}_{k_{3} i t}^{i n}\right)+\mathrm{u}_{\mathrm{it}}^{\mathrm{em}} \\
& G E X R_{i t}=\alpha_{4}+\beta_{41} I N M R_{i t}+\beta_{42} O T M R_{i t}+\beta_{43} E M P R_{i t i t}+\beta_{44} M H Y R_{i t}+\gamma_{41} \ln \left(I N M_{i t-1}\right)+\gamma_{42} \ln \left(O T M_{i t-1}\right) \\
& +\gamma_{43} \ln \left(E M P_{i t-1}\right)+\gamma_{44} \ln \left(G E X_{i t-1}\right)+\gamma_{45} \ln \left(M H Y_{i t-1}\right)+\sum_{k_{4}=6}^{K_{4}} \gamma_{4 k_{4}} \ln \left(\mathrm{X}_{k_{4} i t}^{\text {in }}\right)+\mathrm{u}_{\mathrm{it}}^{\mathrm{ge}} \\
& M H Y R_{i t}=\alpha_{5}+\beta_{51} I N M R_{i t}+\beta_{52} O T M R_{i t}+\beta_{53} E M P R_{i t}+\beta_{54} G E X R_{i t}+\gamma_{51} \ln \left(I N M_{i t-1}\right)+\gamma_{52} \ln \left(O T M_{i t-1}\right) \\
& +\gamma_{53} \ln \left(E M P_{i t-1}\right)+\gamma_{54} \ln \left(G E X_{i t-1}\right)+\gamma_{55} \ln \left(M H Y_{i t-1}\right)+\sum_{k_{5}=6}^{K_{5}} \gamma_{5 k_{5}} \ln \left(\mathrm{X}_{k_{5} i t}^{i n}\right)+\mathrm{u}_{\mathrm{it}}^{\mathrm{mh}}
\end{aligned}
$$


b) Spatial Autoregressive Model

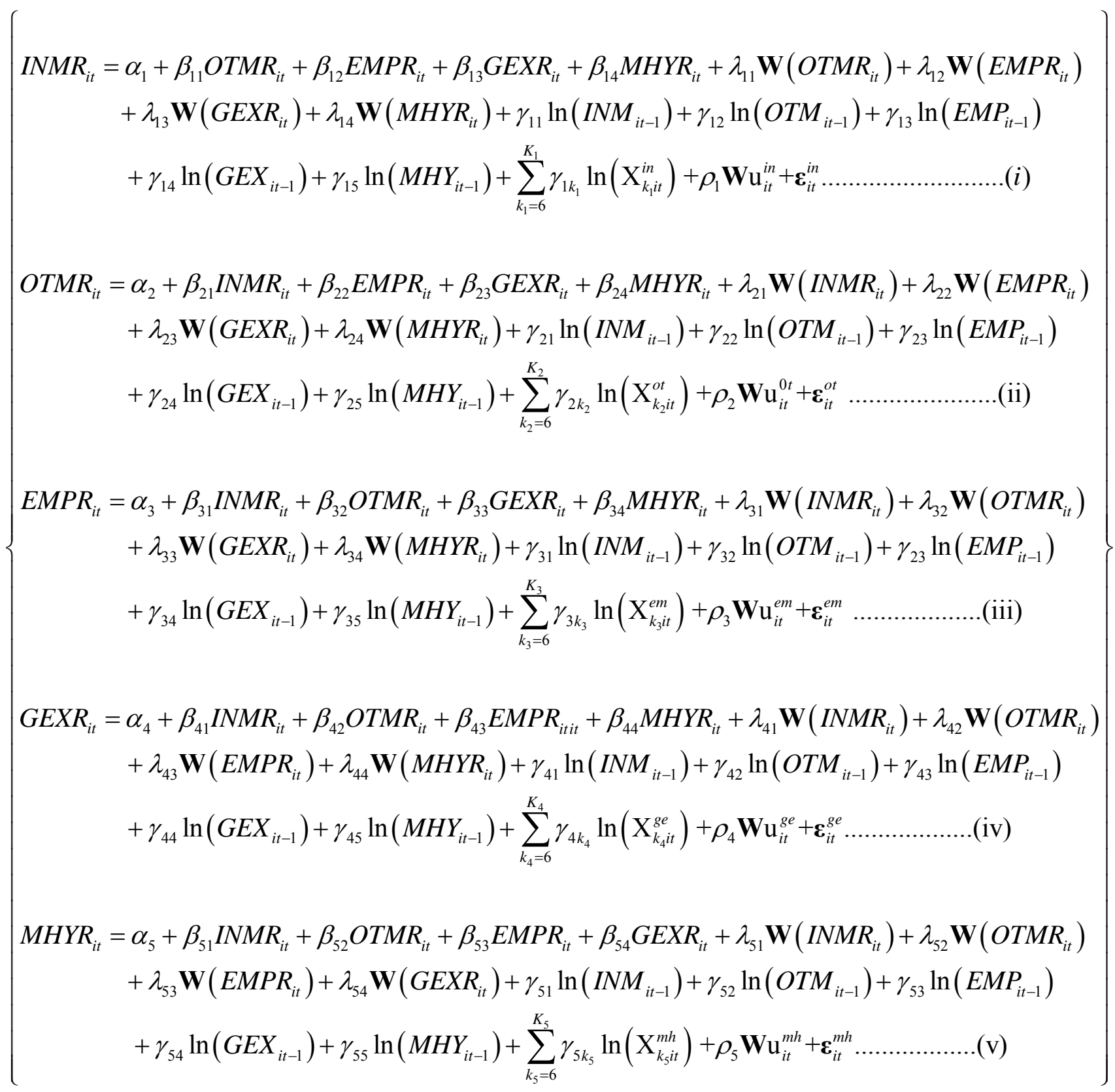

where $\alpha, \beta, \gamma, \lambda$, and $\rho$ are unobserved parameters, $u_{i t}^{i n}, u_{i t}^{o t}, u_{i t}^{e m}, u_{i t}^{g e}$ and $u_{i t}^{m h}$ are vectors of disturbances, and $\boldsymbol{\varepsilon}_{i t}^{i n}, \boldsymbol{\varepsilon}_{i t}^{o t}, \boldsymbol{\varepsilon}_{i t}^{e m}, \boldsymbol{\varepsilon}_{i t}^{g e}$, and $\boldsymbol{\varepsilon}_{i t}^{m h}$ are vectors of innovations. $K_{j}, j=1,2, \ldots, 5$ represent the number of exogenous variables included in the jth equation. 


\subsection{Definition of Variables, Sources of Data and Descriptive Statistics}

\subsubsection{Definition of Variables}

\section{a) Dependent Variables}

The dependent variables used in the empirical analysis include growth rate of employment, growth rate of gross in-and out-migration, growth rate of median household income and growth rate of direct local government expenditures.

Growth Rate of Employment (EMPR): The growth rate of employment was measured by the log-difference between the end and initial levels of employment for a given period. It is used as a proxy for the growth rate of small business. The justification for this measure is based on the results from empirical studies that indicate that newly created jobs are generated by new businesses that start small (Acs and Audretsch, 2001; Audretsch et al., 2000, 2001; Carree and Thurik, 1998, 1999; Wennekers and Thurik, 1999; Fritsch and Falck, 2003).

Growth Rate of Gross In-Migration (INMGR): The growth rate of gross inmigration is measured by the log-difference between the levels of gross in-migration into a given county end and at the beginning of a given period.

Growth Rate of Gross Out-Migration (OTMGR): The growth rate of gross outmigration is measured by the log-difference between the levels of gross out-migration away from a given county at the end and beginning of a given period. The models employed in this study attempt to explain gross in- and gross out-migration growth rates without the explicit introduction of an individual decision functions. Rather, the growth rate of gross in- and gross out-migration are related to a number of aggregate variables. 
Growth Rate of Median Household Income (MHYR): The log-difference between the end and the initial levels of median household income in a given county was used to measure the growth rate of median household income. Median household income is a good measure of average regional/county income because, unlike the mean income, it does not suffer from extreme values.

Growth Rate of Direct Local Government Expenditures (DGEXR): Similarly, the growth rate of direct local government expenditures per capita is measured by the logdifference between the end and the initial levels of per capita local government expenditures.

The spatial lag of the Growth Rate of Employment (WEMPR), Growth Rate of Gross In-Migration (WINMGR), Growth Rate of Gross Out-Migration (WOTMGR), Growth Rate of Median Household Income (WMHYR), and Growth Rate of Direct Local Government Expenditures (WDGEXR) were included on the right hand side of each equation of (4.8b). These spatially lagged endogenous variables are created by multiplying each of the dependent variables by a row standardized queen-based contiguity spatial weights matrix.

\section{b) Independent Variables}

A number of independent variables are used in the empirical analysis. These variables include demographic, human capital, labor market, housing, industry structure, and amenity and policy variables. In line with the literature, the initial values of the independent variable are used in the analysis. This type of formulation also reduces the problem of endogeneity. All the independent variables are in log form except those that can take 
negative or zero values. The descriptions of each of the independent variables of the models are given below.

Equations (i) and (ii) in both (4.8a) and (4.8b) contain vectors $X_{k_{1} i t}^{\text {in }}$ and $X_{k_{2} i t}^{\text {ot }}$, for $k_{1}=6, \ldots, K_{1}$, and $k_{2}=6, \ldots, K_{2}$, that include exogenous variables, which are believed to affect gross in-migration into and gross out-migration from a county, respectively. These include: county unemployment rate (UNEMP), county area (AREA), county initial population size (POPs), percentage of owner occupied dwelling (OWHU), median contract rent of housing cost (MCRH), Natural Amenity Index (NAIX), and local public expenditures per capita per unit of personal income tax per capita (EXTAX).

The county unemployment rate (UNEMP) indicates the extent of economic distress in the county and it is expected to exert a negative influence on net migration. POPs is included to account for the positive impacts of the potential spillover effects and good economic opportunities that are associated with larger population areas on net migration. OWHU is included to measure community stability and neighborhood quality which are potential attractions to migrants. $\mathrm{MCRH}$ is included to account for the potential impacts the cost of renter occupied housing on in-migration. To account for the differential impact of the quality of places on migration behavior, NAIX is included in both equations. How much of the tax paid is put back in the form of local public service may be more important in influencing migration behavior than the absolute amount of tax paid. EXTAX is included in both equations to account for this type of differential effects on migration behavior.

Equation (iii) in both (4.8a) and (4.8b) includes a vector of control variables $\left(\mathrm{X}_{k_{3} i t}^{e m}\right)$ for $k_{3}=6, \ldots, K_{3}$, which consists of, among others, human capital, agglomeration 
effects, unemployment, and other regional socio-economic variables that are assumed to influence county employment growth (business growth) rate. Human capital is measured as the percentage of adults (over 23 years old) with college degrees and above (POPCD), and the percentage of adults (over 23 years old) with high school diploma or higher (POPHD) and it is expected that educational attainment to be positively associated with employment growth (business growth). To control for agglomeration effects from both the supply and demand sides, the percentage population between 25 and 44 of age (POP25-44) is included and it is expected that agglomeration effects to have a positive impact on employment growth (business growth). The proportion of female household header families (FHHF) is included control for the effect of local labor market characteristics on employment. County unemployment rate (UNEMP) is also included in the vector of exogenous variables as a measure of local economic distress. Although high county unemployment rate is normally associated with poor economic environment, it may provide an incentive for individuals to form new business that can employ not only the owners, but also others. Thus, it cannot be told whether the impact of UNE on employment growth is positive or negative in a priori. Establishment density (ESBd), which is the total number of private sector establishments in the county divided by the total county's population, is included to capture the degree of competition among firms and crowding of businesses relative to the population. The coefficient on ESBd is expected to be negative. Vector $\mathrm{X}_{k_{3} i}^{e m}$ also includes OWHU to capture the effects of the availability of resources to finance businesses and create jobs on employment growth in the county. The percentage of owner-occupied dwellings is expected to be positively associated with employment growth in the county. Also included in $X_{k_{3} i t}^{e m}$ are property tax 
per capita ( PCPTAX), percentage of private employment in manufacturing (MANU), percentage of employment in wholesale and retail trade (WHRT), social capital index (SCIX), NAIX, and highway density (HWD).

The vector of exogenous variables $\left(\mathrm{X}_{k_{4}}^{g e}\right), k_{4}=6, \ldots, K_{4}$ in equation (iv) in both (4.8a) and (4.8b) contains, among others, POPd, POP $^{2}$, FHHF, POPHD, UNEMP, MANU, WHRT, and SCIX.

Equation (v) in both (4.8a) and (4.8b) also contains a vector of exogenous variables $\left(\mathbf{X}_{k_{s i t}}^{m h}\right)$, which includes POPs, percentage of school age population (POP5-17), Serious Crime per 100,000 population (SCRM), Direct Federal Expenditure and grants per capita (FFEG). Per Capita personal Income Tax (PCTAX), Per Capita Long-Term Outstanding Debt (PCLD), and Per Capita Long-Term Debt (LTD).

The initial levels of employment (EMPt-1), gross in-migration (INMGt-1), gross out-migration (OTMGt-1), median household income (MHYt-1) and direct local government expenditures per capita (DGEXt-1) were also included in each equation of (4.8a) and (4.8b). These variables are treated as predetermined variables because their values are given at the beginning of each period and hence are not affected by the endogenous variables.

\subsubsection{Sources of Data}

The data used in this study draws are mainly collected from County and City Data Book, County Business Patterns, Bureau of Economic Analysis, Bureau of Labor Statistics, Current Population Survey Reports, U. S. Counties, and U.S. Bureau of the Census, The specific data for each variable are collected as shown in the table below. 
Table 4.5.2a: Variable Descriptions and Data Sources

\begin{tabular}{|c|c|c|}
\hline \multicolumn{2}{|c|}{ Variable Code Variable Description } & Source \\
\hline \multicolumn{3}{|c|}{ Endogenous Variables } \\
\hline EMPR & Growth Rate of Employment & Computed \\
\hline INMGR & Growth Rate of Gross In-Migration & Computed \\
\hline OTMGR & Growth Rate of Gross Out-Migration & Computed \\
\hline MHYR & Growth Rate of Median Household Income & Computed \\
\hline DGEXR & Growth Rate of Direct Local Government Expenditures Per Capita & Computed \\
\hline \multicolumn{3}{|c|}{ Spatially lagged Endogenous Variables } \\
\hline WEMPR & Spatial Lag of EMPR & Computed \\
\hline WINMGR & Spatial Lag of INMGR & Computed \\
\hline WOTMGR & Spatial Lag of OTMGR & Computed \\
\hline WMHYR & Spatial Lag of MHYR & Computed \\
\hline WDGEXR & Spatial Lag of DGEXR & Computed \\
\hline \multicolumn{3}{|c|}{ Initial Condition Variables } \\
\hline EMPt-1 & Employment 1980, 1990, 2000 & County \& City Data Book \\
\hline INMGt-1 & In-migration 1983, 1990, 2000 & Internal Revenue Service \\
\hline OTMGt-1 & out-migration 1983, 1990, 2000 & Internal Revenue Service \\
\hline MHYt-1 & Median Household income 1979, 1989, 2000 & BEA \\
\hline DGEXt-1 & Direct general exp. Per capita 1982, 1992, 2002 & U.S. Bureau of the Census \\
\hline \multicolumn{3}{|c|}{ Regional and Policy Variables } \\
\hline AREA & Land area in square miles 1980 & U.S. Bureau of the Census \\
\hline POPs & Population 1980,1990 & U.S. Bureau of the Census \\
\hline POP5-17 & Percent of population between $5-17$ years 1980, 1990 & U.S. Bureau of the Census \\
\hline POP25-44 & Percent of population between $25-44$ years old 1980, 1990 & U.S. Bureau of the Census \\
\hline FHHF & percent of female householder, family householder, 1980, 1990 & County \& City Data Book \\
\hline SCRM & Serious crime per 100,000 pop. 1980,1990 & County \& City Data Book \\
\hline POPHD & Persons 25 years and over, \% high school or higher, 1980, 1990 & County \& City Data Book \\
\hline POPCD & Persons 25 years and over, \% Bachelor's degree or above, 1980, 1990 & County \& City Data Book \\
\hline OWHU & Owner-Occupied Housing Unit in percent, 1980, 1990 & U.S. Bureau of the Census \\
\hline $\mathrm{MCRH}$ & Median Contract rent of specified renter-occupied 19980, 1990 & U.S. Bureau of the Census \\
\hline UNEMP & Unemployment rate 1980,1990 & Bureau of Labor Statistics \\
\hline MANU & $\%$ employed in manufacturing 1980,1990 & County \& City Data Book \\
\hline WHRT & $\%$ employed in wholesale and retail trade 1980, 1990 & County \& City Data Book \\
\hline DFEG & Direct Federal Expenditures and Grants per Capita, 1983, 1990 & County \& City Data Book \\
\hline PCTAX & Per capital local tax 1980, 1990 & County \& City Data Book \\
\hline PCPTAX & Property tax per capita 1980,1990 & County \& City Data Book \\
\hline PCTD & Total Debt Outstanding per capita 1982, 1990 & County \& City Data Book \\
\hline LTD & Long-term debt, utility 1982, 1990 & County \& City Data Book \\
\hline SCIX & Social Capital Index 1987, 1997 & Rupasingha et al, 2006* \\
\hline NAIX & Natural Amenities Index 1980, 1990 & USDA \\
\hline HWD & Highway Density 1980, 1990 & US Highway Authority \\
\hline ESBd & Establishment density 1980, 1990 & County Business Pattern \\
\hline EXPTAX & General expenditure/ total tax 1980,1990 & Computed \\
\hline
\end{tabular}

* I thank Anil Rupasingha, Stephan J. Goetz and David Freshwater for allowing me to use their data set on social capital index for U. S. counties 


\subsubsection{Descriptive Statistics}

Tables 4.5.2b, 4.5.2c, and 4.5.2d present the descriptive statistics for Appalachia counties for 1980-1990, 1990-2000, and 1980-2000 periods, respectively.

Table 4.5.2b: Descriptive Statistics for Appalachia Counties, 1980-1990.

\begin{tabular}{|c|c|c|c|c|c|}
\hline Variable & Variable Description & Mean & Std Dev & Minimum I & Maximum \\
\hline EMPR & Growth Rate of Employment & 0.17738 & 0.27769 & -1.11305 & 1.30846 \\
\hline INMGR & Growth Rate of Gross In-Migration & -0.09866 & 0.36722 & -3.87267 & 1.44365 \\
\hline OTMGR & Growth Rate of Gross Out-Migration & -0.13212 & 0.22534 & -1.39099 & 0.59843 \\
\hline MHYR & Growth Rate of Median Household Income & 0.48556 & 0.12818 & 0.042537 & 0.8413 \\
\hline DGEXR & Growth Rate of Direct Local Government Expenditures Per Capita & 0.66384 & 0.20775 & -0.27187 & 1.49325 \\
\hline WEMPR & Spatial Lag of EMPR & 0.18525 & 0.13323 & -0.32181 & 0.62858 \\
\hline WINMGR & Spatial Lag of INMGR & -0.10052 & 0.18898 & -1.33175 & 0.44524 \\
\hline WOTMGR & Spatial Lag of OTMGR & -0.13074 & 0.12333 & -0.53841 & 0.19502 \\
\hline WMHYR & Spatial Lag of MHYR & 0.4864 & 0.088406 & 0.22941 & 0.70964 \\
\hline WDGEXR & Spatial Lag of DGEXR & 0.66848 & 0.093982 & 0.42664 & 0.95991 \\
\hline AREA & Land Area in Square Miles & 6.00594 & 0.76791 & 0.83291 & 7.27219 \\
\hline POPs & Population & 10.28041 & 0.94001 & 7.98514 & 14.18721 \\
\hline POP2 & Population-Squared & 106.5683 & 19.78781 & 63.76253 & 201.2769 \\
\hline POP5-17 & Percent of Population between $5-17$ years & 3.08638 & 0.097505 & 2.48372 & 3.30813 \\
\hline POP25-44 & Percent of population between $25-44$ years old & 3.26112 & 0.07749 & 2.85977 & 3.62103 \\
\hline FHHF & percent of Female Householder, Family Householder & 2.19815 & 0.18039 & 1.7134 & 3.07215 \\
\hline SCRM & Serious Crime per 100,000 Pop. & 2193.043 & 1410.51 & 0 & 8329 \\
\hline POPHD & Persons 25 Years and Over, \% high school or higher & 3.88069 & 0.22374 & 3.22884 & 4.39174 \\
\hline POPCD & Persons 25 Years and Over, \% Bachelor's degree or above & 2.0926 & 0.37868 & 1.02985 & 3.59229 \\
\hline OWHU & Owner-Occupied Housing Unit in Percent & 4.32536 & 0.068858 & 4.01096 & 4.45318 \\
\hline $\mathrm{MCRH}$ & Median Contract Rent of Specified Renter-Occupied & 4.70784 & 0.26485 & 3.89182 & 5.48894 \\
\hline UNEMP & Unemployment Rate & 2.1016 & 0.32516 & 1.03513 & 3.17018 \\
\hline MANU & \% Employed in Manufacturing & 30.19625 & 12.11241 & 2.38955 & 61.54639 \\
\hline WHRT & \% Employed in Wholesale and Retail Trade & 16.54802 & 3.31096 & 6.7223 & 25.24811 \\
\hline DFEG & Direct Federal Expenditures and Grants per Capita & 7.42292 & 0.41464 & 6.45363 & 10.105 \\
\hline PCTAX & Per Capital Local Tax & 5.13622 & 0.62646 & 2.958 & 6.40228 \\
\hline PCPTAX & Property Tax per Capita & 4.80801 & 0.66627 & 2.83321 & 6.39526 \\
\hline PCTD & Total Debt Outstanding per Capita & 618.9139 & 817.6579 & 0 & 8770 \\
\hline LTD & Long-Term Debt, Utility & 4635.421 & 12347.1 & 0 & 134368 \\
\hline SCIX & Social Capital Index & -0.58184 & 0.91079 & -3.19681 & 2.03804 \\
\hline NAIX & Natural Amenities Index & 0.14333 & 1.15867 & -3.72 & 3.55 \\
\hline HWD & Highway Density & 0.67484 & 0.4084 & -0.34252 & 2.36665 \\
\hline ESBs & Establishment Density & 2.6477 & 0.32883 & 0.66964 & 3.89906 \\
\hline EXPTAX & General Expenditure/ Total Tax & 1.07349 & 0.46437 & -0.8322 & 2.24636 \\
\hline EMPt-1 & Initial Level of Employment & 8.64911 & 1.2794 & 5.15906 & 13.30679 \\
\hline INMGt-1 & Initial Level of Gross In-Migration & 7.1862 & 0.96288 & 4.84419 & 10.33634 \\
\hline OTMGt-1 & Initial Level of Gross Out-Migration & 7.16981 & 0.95204 & 4.98361 & 10.7377 \\
\hline MHYt-1 & Initial Level of Median Household Income & 9.45834 & 0.1985 & 8.80583 & 10.02447 \\
\hline DGEXt-1 & Initial Level of Direct Local Gov't Expenditures per Capita & 6.56192 & 0.28627 & 5.92693 & 7.48549 \\
\hline
\end{tabular}
Note: All variables except SCRM, PCTD, LTD, SCIX and NAIX are in log form 
Table 4.5.2c: Descriptive Statistics for Appalachia Counties, 1990-2000.

\begin{tabular}{|c|c|c|c|c|c|}
\hline Variable & Description & Mean & Std Dev & Minimum & Maximum \\
\hline EMPR & Growth Rate of Employment & 0.17672 & 0.24499 & -0.69448 & 1.7868 \\
\hline INMGR & Growth Rate of Gross In-Migration & 0.096241 & 0.24922 & -0.92655 & 1.08588 \\
\hline OTMGR & Growth Rate of Gross Out-Migration & 0.096679 & 0.22048 & -1.09537 & 0.99832 \\
\hline MHYR & Growth Rate of Median Household Income & 0.47743 & 0.30826 & -0.49426 & 1.39569 \\
\hline DGEXR & Growth Rate of Direct Local Government Expenditures Per Capita & 0.61617 & 0.44636 & -0.54832 & 4.95896 \\
\hline WEMPR & Spatial Lag of EMPR & 0.17629 & 0.13013 & -0.12982 & 0.84378 \\
\hline WINMGR & Spatial Lag of INMGR & 0.094796 & 0.22541 & -0.45875 & 0.80957 \\
\hline WOTMGR & Spatial Lag of OTMGR & 0.092459 & 0.15939 & -0.33829 & 0.57753 \\
\hline WMHYR & Spatial Lag of MHYR & 0.47791 & 0.16818 & 0.076696 & 1.00418 \\
\hline WDGEXR & Spatial Lag of DGEXR & 0.61467 & 0.17942 & 0.1598 & 1.83703 \\
\hline AREA & Land Area in Square Miles & 6.00903 & 0.74824 & 1.09861 & 7.27656 \\
\hline POPs & Population & 10.29714 & 0.94766 & 7.87664 & 14.10553 \\
\hline POP2 & Population-Squared & 106.9271 & 19.95609 & 62.04143 & 198.9659 \\
\hline POP5-17 & Percent of Population between $5-17$ years & 2.92443 & 0.12003 & 2.17475 & 3.22287 \\
\hline POP25-44 & Percent of population between $25-44$ years old & 3.37993 & 0.077483 & 2.78501 & 3.74479 \\
\hline $\mathrm{FHHF}$ & percent of Female Householder, Family Householder & 2.32185 & 0.20314 & 1.81143 & 3.18787 \\
\hline SCRM & Serious Crime per 100,000 Pop. & 2284.809 & 1561.256 & 0 & 8487 \\
\hline POPHD & Persons 25 Years and Over, \% high school or higher & 4.10041 & 0.1706 & 3.56953 & 4.4682 \\
\hline POPCD & Persons 25 Years and Over, \% Bachelor's degree or above & 2.26938 & 0.40654 & 1.30833 & 3.7305 \\
\hline OWHU & Owner-Occupied Housing Unit in Percent & 4.32524 & 0.076094 & 3.86703 & 4.47278 \\
\hline MCRH & Median Contract Rent of Specified Renter-Occupied. & 5.64139 & 0.20586 & 4.94164 & 6.35784 \\
\hline UNEMP & Unemployment Rate & 2.15356 & 0.34816 & 1.22378 & 3.24649 \\
\hline MANU & \% Employed in Manufacturing & 26.24019 & 11.29556 & 2.2 & 53.6 \\
\hline WHRT & \% Employed in Wholesale and Retail Trade & 18.82775 & 3.53195 & 8.7 & 27.7 \\
\hline DFEG & Direct Federal Expenditures and Grants per Capita & 7.98688 & 0.3758 & 6.98286 & 10.1766 \\
\hline PCTAX & Per Capital Local Tax & 5.91452 & 0.52985 & 4.50736 & 7.42253 \\
\hline PCPTAX & Property Tax per Capita & 5.5236 & 0.61602 & 3.91202 & 7.36265 \\
\hline PCTD & Total Debt Outstanding per Capita & 1180.022 & 2271.215 & 0 & 30332 \\
\hline LTD & Long-Term Debt, Utility & 11728.35 & 71189.12 & 0 & 1368142 \\
\hline SCIX & Social Capital Index & -0.59298 & 0.95959 & -2.5266 & 5.64457 \\
\hline NAIX & Natural Amenities Index & 0.14333 & 1.15867 & -3.72 & 3.55 \\
\hline HWD & Highway Density & 0.69039 & 0.40412 & -0.33914 & 2.63189 \\
\hline ESBs & Establishment Density & 2.92833 & 0.3351 & 1.87398 & 4.09316 \\
\hline EXPTAX & General Expenditure/ Total Tax & 0.8429 & 0.51449 & -0.98373 & 2.60823 \\
\hline EMPt-1 & Initial Level of Employment & 8.82649 & 1.25425 & 5.42054 & 13.38131 \\
\hline INMGt-1 & Initial Level of Gross In-Migration & 7.08755 & 1.00192 & 4.54329 & 10.51994 \\
\hline OTMGt-1 & Initial Level of Gross Out-Migration & 7.03768 & 0.97551 & 4.49981 & 10.54952 \\
\hline MHYt-1 & Initial Level of Median Household Income & 9.9439 & 0.2261 & 9.05894 & 10.68093 \\
\hline DGEXt-1 & Initial Level of Direct Local Gov't Expenditures per Capita & 7.22576 & 0.27948 & 6.49224 & 8.10832 \\
\hline
\end{tabular}

Note: All variables except SCRM, PCTD, LTD, SCIX and NAIX are in log form 
Table 4.5.2d: Descriptive Statistics for Appalachia Counties, 1980- 2000.

\begin{tabular}{|c|c|c|c|c|c|}
\hline Variable & Description & Mean & Std Dev & Minimum & Maximum \\
\hline EMPR & Growth Rate of Employment & 0.1771 & 0.2617 & -1.113 & $3 \quad 1.7868$ \\
\hline INMGR & Growth Rate of Gross In-Migration & -0.001 & 0.3284 & -3.873 & 1.4437 \\
\hline OTMGR & Growth Rate of Gross Out-Migration & -0.018 & 0.2505 & -1.391 & 0.9983 \\
\hline MHYR & Growth Rate of Median Household Income & 0.4815 & 0.236 & -0.494 & 1.3957 \\
\hline DGEXR & Growth Rate of Direct Local Government Expenditures Per Capita & 0.64 & 0.3487 & -0.548 & 4.959 \\
\hline WEMPR & Spatial Lag of EMPR & 0.1765 & 0.1176 & -0.163 & 0.6445 \\
\hline WINMGR & Spatial Lag of INMGR & $-3 E-04$ & 0.1393 & -0.846 & 0.4664 \\
\hline WOTMGR & Spatial Lag of OTMGR & -0.014 & 0.1139 & -0.364 & 0.3976 \\
\hline WMHYR & Spatial Lag of MHYR & 0.4804 & 0.1 & 0.1452 & 0.982 \\
\hline WDGEXR & Spatial Lag of DGEXR & 0.6487 & 0.1639 & 0.2188 & 1.6378 \\
\hline AREA & Land Area in Square Miles & 6.0075 & 0.7577 & 0.8329 & 7.2766 \\
\hline POPs & Population & 10.289 & 0.9433 & 7.8766 & 14.187 \\
\hline POP2 & Population-Squared & 106.75 & 19.861 & 62.041 & 201.28 \\
\hline POP5-17 & Percent of Population between $5-17$ years & 3.0054 & 0.136 & 2.1748 & 3.3081 \\
\hline POP25-44 & Percent of population between $25-44$ years old & 3.3205 & 0.0976 & 2.785 & 3.7448 \\
\hline FHHF & percent of Female Householder, Family Householder & 2.26 & 0.2017 & 1.7134 & 3.1879 \\
\hline SCRM & Serious Crime per 100,000 Pop. & 2238.9 & 1487.6 & 0 & 8487 \\
\hline POPHD & Persons 25 Years and Over, \% high school or higher & 3.9906 & 0.2272 & 3.2288 & 4.4682 \\
\hline POPCD & Persons 25 Years and Over, \% Bachelor's degree or above & 2.181 & 0.4025 & 1.0299 & 3.7305 \\
\hline OWHU & Owner-Occupied Housing Unit in Percent & 4.3253 & 0.0725 & 3.867 & 4.4728 \\
\hline $\mathrm{MCRH}$ & Median Contract Rent of Specified Renter-Occupied. & 5.1746 & 0.5238 & 3.8918 & 6.3578 \\
\hline UNEMP & Unemployment Rate & 2.1276 & 0.3377 & 1.0351 & 3.2465 \\
\hline MANU & $\%$ Employed in Manufacturing & 28.218 & 11.87 & 2.2 & 61.546 \\
\hline WHRT & \% Employed in Wholesale and Retail Trade & 17.688 & 3.6063 & 6.7223 & 27.7 \\
\hline DFEG & Direct Federal Expenditures and Grants per Capita & 7.7049 & 0.4858 & 6.4536 & 10.177 \\
\hline PCTAX & Per Capital Local Tax & 5.5254 & 0.6984 & 2.958 & 7.4225 \\
\hline PCPTAX & Property Tax per Capita & 5.1658 & 0.7344 & 2.8332 & 7.3627 \\
\hline PCTD & Total Debt Outstanding per Capita & 899.47 & 1728.8 & 0 & 30332 \\
\hline LTD & Long-Term Debt, Utility & 8181.9 & 51182 & 0 & $1 \mathrm{E}+06$ \\
\hline SCIX & Social Capital Index & -0.587 & 0.935 & -3.197 & 5.6446 \\
\hline NAIX & Natural Amenities Index & 0.1433 & 1.158 & -3.72 & 3.55 \\
\hline HWD & Highway Density & 0.6826 & 0.4061 & -0.343 & 2.6319 \\
\hline ESBs & Establishment Density & 2.788 & 0.3603 & 0.6696 & 4.0932 \\
\hline EXPTAX & General Expenditure/ Total Tax & 0.9582 & 0.5032 & -0.984 & 2.6082 \\
\hline EMPt-1 & Initial Level of Employment & 8.7378 & 1.2692 & 5.1591 & 13.381 \\
\hline INMGt-1 & Initial Level of Gross In-Migration & 7.1369 & 0.9833 & 4.5433 & 10.52 \\
\hline OTMGt-1 & Initial Level of Gross Out-Migration & 7.1038 & 0.9655 & 4.4998 & 10.738 \\
\hline MHYt-1 & Initial Level of Median Household Income & 9.7011 & 0.3228 & 8.8058 & 10.681 \\
\hline DGEXt-1 & Initial Level of Direct Local Gov't Expenditures per Capita & 6.8938 & 0.4362 & 5.9269 & 8.1083 \\
\hline
\end{tabular}
Note: All variables except SCRM, PCTD, LTD, SCIX and NAIX are in log form

In summary, this chapter has developed the basic spatial and non-spatial models which are foundations for the empirical work reported in this study. The descriptions of the variables, the sources of the data, and descriptive statistics are also presented. Next 
chapter presents results from non-spatial regression analysis, exploratory spatial data analysis as well as spatial regression analysis, in the context of both cross-sectional and panel data setting. 


\section{CHAPTER V}

\section{EMPIRICAL RESULTS AND ANALYSES}

\subsection{Introduction}

The results of the empirical estimation of the growth equilibrium models that are developed in chapter four are presented in this chapter. Both single equation and system of equations methods of estimation are employed to estimate the standard as well as the spatial simultaneous equations models. Both cross-sectional and panel data from 418 Appalachian counties for 1980-2000 are utilized for the empirical estimations of the models. In the standard (non-spatial) simultaneous equations model, the estimation for cross-sectional analyses is carried in EViews using standard built-in functionalities. The estimation of the standard simultaneous panel data model and both the spatial crosssectional and spatial panel simultaneous equations models, however, required the development of special programs (see Figure 5.1). The codes for these programs are written in TSP. The spatial regression analyses are preceded by exploratory data analyses which aim at identifying spatial pattern/or spatial clustering in the data sets. In this respect, ArcGIS and GeoDa are used to calculate Moran's I of Global Spatial Autocorrelation and Local Indicators of Spatial Association (LISA) for the endogenous variables of the models.

The rest of the chapter is organized as follows. First, the results and analyses of the standard (non-spatial) simultaneous equilibrium growth models are presented in section 5.2. The results of exploratory spatial data analyses and the analyses and discussions of the results of the spatial simultaneous equilibrium growth models estimations are presented in section 5.3. 
Figure 5.1: EMPIRICAL MODELS ETIMATION STRATEGIES

\section{EMPIRICAL MODELS ETIMATION STRATEGIES}

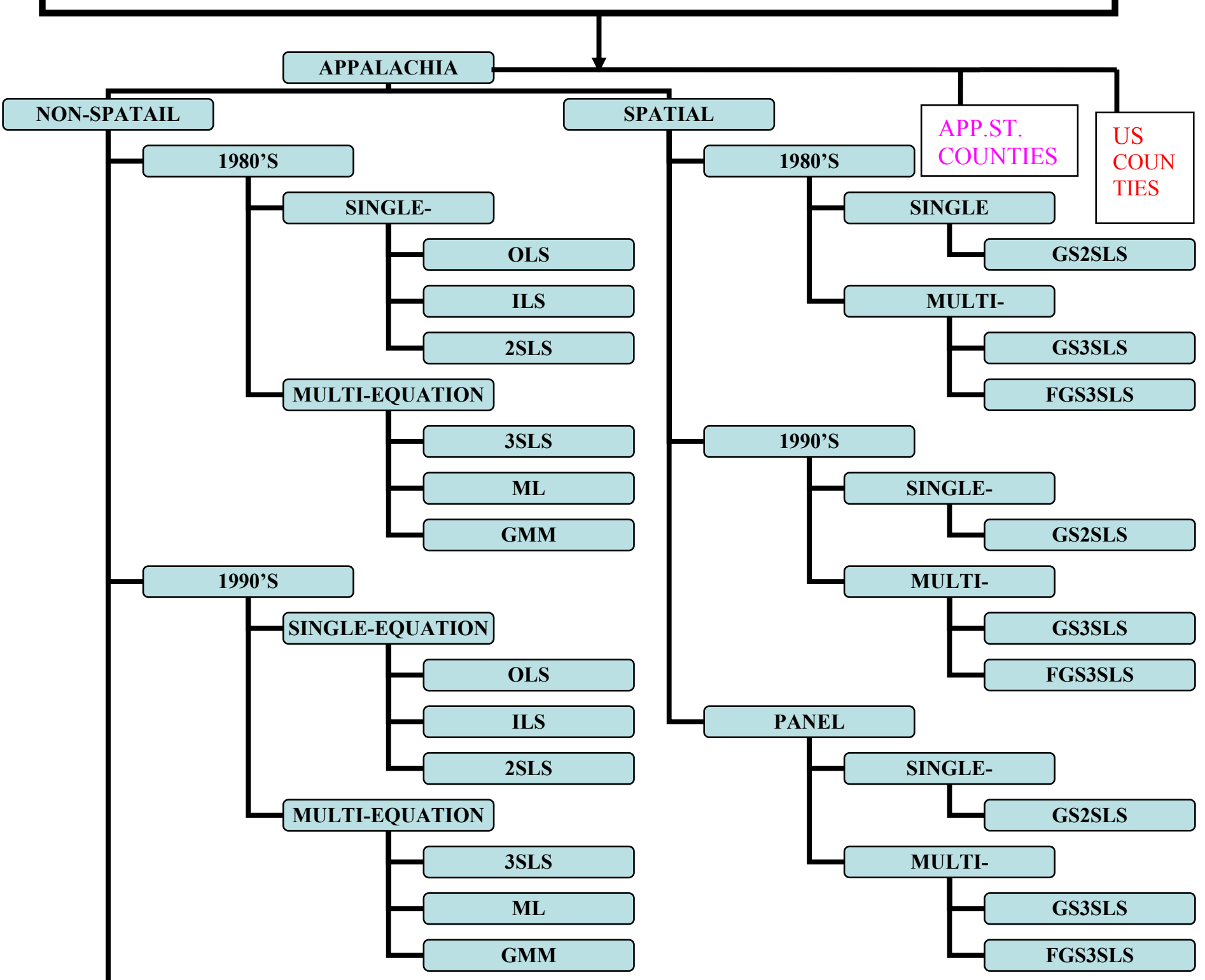

\section{PANEL}

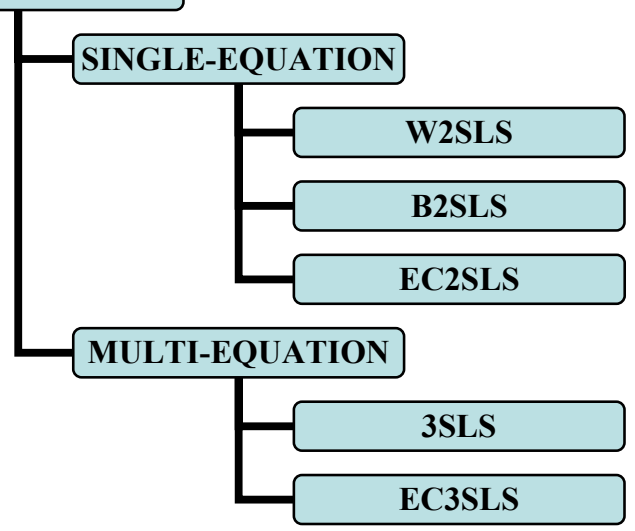




\subsection{Empirical Estimation: Standard (Non-Spatial) Simultaneous Equations Equilibrium Growth Model}

Both cross-sectional and panel data from the 418 Appalachia counties are used for the empirical implementation of the system of equations given in (4.8a). One cross section (1990-2000) and one panel (1980-2000) with two periods are considered. The endogenous variables of the system are the rate of growth expressed as the logdifferences between the value of the respective variable at the end and the beginning of each period. The exogenous variables of the system, on the other hand, are beginning period values (expressed in log terms). In line with the theoretical and econometric discussions of Chapter III of this study, the system is estimated using both Limited Information (Single-Equation) and Full Information (System of Equations) methods, in both cross-sectional and panel data settings. The Single-Equation methods are essentially inefficient in the sense that one equation at a time is estimated without utilizing complete information in all the other equations in the system. Besides, the OLS method does not give consistent parameter estimates because the right-hand side endogenous variables are correlated with the residuals. The reasons why the Single-Equation methods are not appropriate for simultaneous equations estimation is also mathematically and theoretically developed in Chapter III. Although inefficient, it is useful, however, to report the Limited Information parameter estimates along with those from the FullInformation methods that give consistent and efficient estimates. To this end, the Limited Information methods (OLS, Weighted L.S and Weighted 2SLS) parameter estimates along with the Full Information methods (3SLS, ML and GMM) parameter estimates for the five equations of the system are given in the Appendix. 
Based on the theoretical and econometric discussions of Chapter III, the estimators are arranged from the least efficient (OLS) to the most efficient (GMM), in the case of cross sectional analysis from left to right along the tables. Inspection of the empirical results also show this general tendency - efficiency, in terms of keeping the expected signs and increases in the level of significance, tend to increase from left to right for most of the variables of the model. Since the main purpose of the study is to test and establish quantitative relationships (interdependences) among the endogenous variables of the model and also to investigate the regional variables that jointly determine the endogenous variables, only GMM estimates (for the cross-sectional analysis) and E3SLS estimates (for the panel data analysis) will be discussed and analyzed in the remaining part of this section.

\subsubsection{Cross-Sectional Results Analysis: 1990-2000}

Generalized Method of Moments is the most efficient among the Full-Information method of estimating system of equations. It is robust estimator, in the sense that, unlike maximum likelihood estimation, it does not require information on the exact distribution of the disturbances. In the cross-section setting, White's heteroskedasticity consistent covariance matrix is used as weighting matrix in estimating the coefficients of the model. The GMM estimates of (4.8a) for the 1990-2000 Appalachian data sets are given Table

\section{$5.2 .1 \mathrm{a}$}

\subsubsection{Employment (Business) Growth Rate}

The growth rate in private employment (EMPR), which is the proxy for the rate of growth in small business, is regressed on the endogenous variables of the model and on a set of county-level conditioning variables related to labor market characteristics, industry 
structure, such as the proportion, demographic variables, policy variables, amenity and accessibility index variables, as well as the initial employment condition.

The results indicate some level of positive feedback simultaneities between EMPR and the endogenous variables. Particularly, the rate of growth in employment is positively and significantly affected by the rate of growth in median household income (MHYR) at the county-level during the study period. This is consistent with economic theory and empirical findings in the literature (Armington and Acs, 2002). Increases in median household income tend to increase regional wealth and consumer demands for goods and services increases as wealth increases. The growth of the market demand in turn encourages the formation small businesses. Increases in median household income could also lead to capital formation in the form of household savings that finance new firm formation.

\begin{tabular}{|c|c|c|c|c|c|c|c|c|c|c|}
\hline \multicolumn{11}{|c|}{ Table 5.2.1a: GMM Estimation Results, APPALACHIA, 1990_RATE } \\
\hline & \multicolumn{2}{|c|}{\begin{tabular}{|c|} 
EMP Equation \\
\end{tabular}} & \multicolumn{2}{|c|}{ INMG Equation } & \multicolumn{2}{|c|}{ OTMG Equation } & \multicolumn{2}{|c|}{ MHY Equation } & \multicolumn{2}{|c|}{ DGEX Equation } \\
\hline VARIABLE & Coeff. & $t$-stat. & Coeff. & t-stat & Coeff. & t-stat & Coeff. & $t$-stat & Coeff. & t-stat. \\
\hline CONSTANT & -1.7322 & -2.0558 & -2.6027 & -7.2264 & 0.6620 & 1.0485 & 2.5183 & 5.6891 & 2.9336 & 8.1579 \\
\hline \begin{tabular}{|l|} 
EMPR \\
\end{tabular} & & & 0.2655 & 3.4808 & 0.3234 & 5.2570 & 0.2817 & 13.2984 & -0.0297 & -0.4595 \\
\hline INMGR & 0.0286 & 1.2484 & & & -0.0269 & 1.2574 & -0.0636 & -5.3808 & -0.0432 & -1.8787 \\
\hline OTMGR & 0.0985 & 1.6387 & 0.0411 & 0.5860 & & & 0.0064 & 0.2863 & 0.1746 & 3.4933 \\
\hline MHYR & 1.2508 & 7.9395 & 0.7274 & 3.8102 & 0.0691 & 0.4714 & & & -0.2154 & -1.7574 \\
\hline DGEXR & 0.0225 & 0.2682 & -0.2300 & -2.3736 & -0.0290 & 0.3615 & -0.0240 & -0.7478 & & \\
\hline
\end{tabular}

Note: A coefficient is considered as statistically significant at 10 percent, 5 percent and 1 percent levels ,if $1.65 \leq \mid$ t-stat. $|\leq 1.98,1.98<|$ t-stat. $\mid \leq 2.58$, and $\mid$ t-stat. $\mid>2.58$, respectively. 
The formation and expansion of businesses creates employment opportunity and income for the new and the expanding entrepreneurs. These increases in labor and entrepreneurial incomes, in turn, feed back into the MHYR equation and further leads to an increase in median household income. This is shown by the positive and highly significant coefficient estimate on the EMPR in the MHYR equation.

To control for agglomeration effects, the model includes measure of population statistics such as the percentage of population between 25 and 44 years old (POP25_44). The results show that POP25_44 has positive and significant effects on EMPR. This result is consistent with the literature (Acs and Armington, 2004a) which indicates that a growing population increases the demand for consumer goods and services, as well as the pool of potential entrepreneurs which encourage business formation. This result is important from a policy perspective because it indicates that counties with high population concentration are benefiting from the resulting agglomerative and spillover effects that lead to localization of economic activities, in line with Krugman's (1991a, 1991b) argument on regional spillover effects. Consistent with the theoretical expectations, the results also show initial human capital endowment as measured by the percentage of adults (over 23 years old) with college degree (POPCD) is positive and statistically significant at one percent level. Highly educated people in most case have more access to research and development facilities, and perhaps a good insight to the business world and thus a clear idea about the present and the future needs of the market. As Christensen (2000) contends, entrepreneurs with good education are also more likely to know how to transform innovative ideas into marketable products. Thus, people with more educational attainment tend to establish businesses, and to be more successful when 
they do, more often than those with less educational attainments. This result is also consistent with Acs and Armington's (2004b) findings which indicates that the agglomerative effects that contribute to new firm formation could come from the supply factors related to the quality of local labor market and business climate. More educated people would mean more human capital embodied in their general and specific skills, for implementing new ideas for creating and growing new businesses. One possible implication of these findings is that regions or counties with different levels of human capital endowment and different propensities of locally available knowledge to spill over and stimulate new firm formation tend to have different rates of new firm formation, survival and growth. The percent of female householder families (FHHF) is another conditioning demographic variable included in the model. Female householder families tend to have low labor participation rate. Although insignificant, our results show that FHHF has negative impact on EMPR, consistent with theoretical expectations and empirical findings. FHHF affects both the supply-side (as source of labor input) and the demand-side (as source of demand for consumer goods) of the market.

The coefficient on the variable representing the percentage of home owned by their occupants (OWHU) is positive, although insignificant. This result indicates that high home ownership is positively associated with business formation in Appalachia. This is consistent with theoretical expectation that high home ownership is an indication that there is a capacity to finance new business by potential entrepreneurs, either by using the house as collateral for loan or as indication of availability of personal financial resources to start new businesses. 


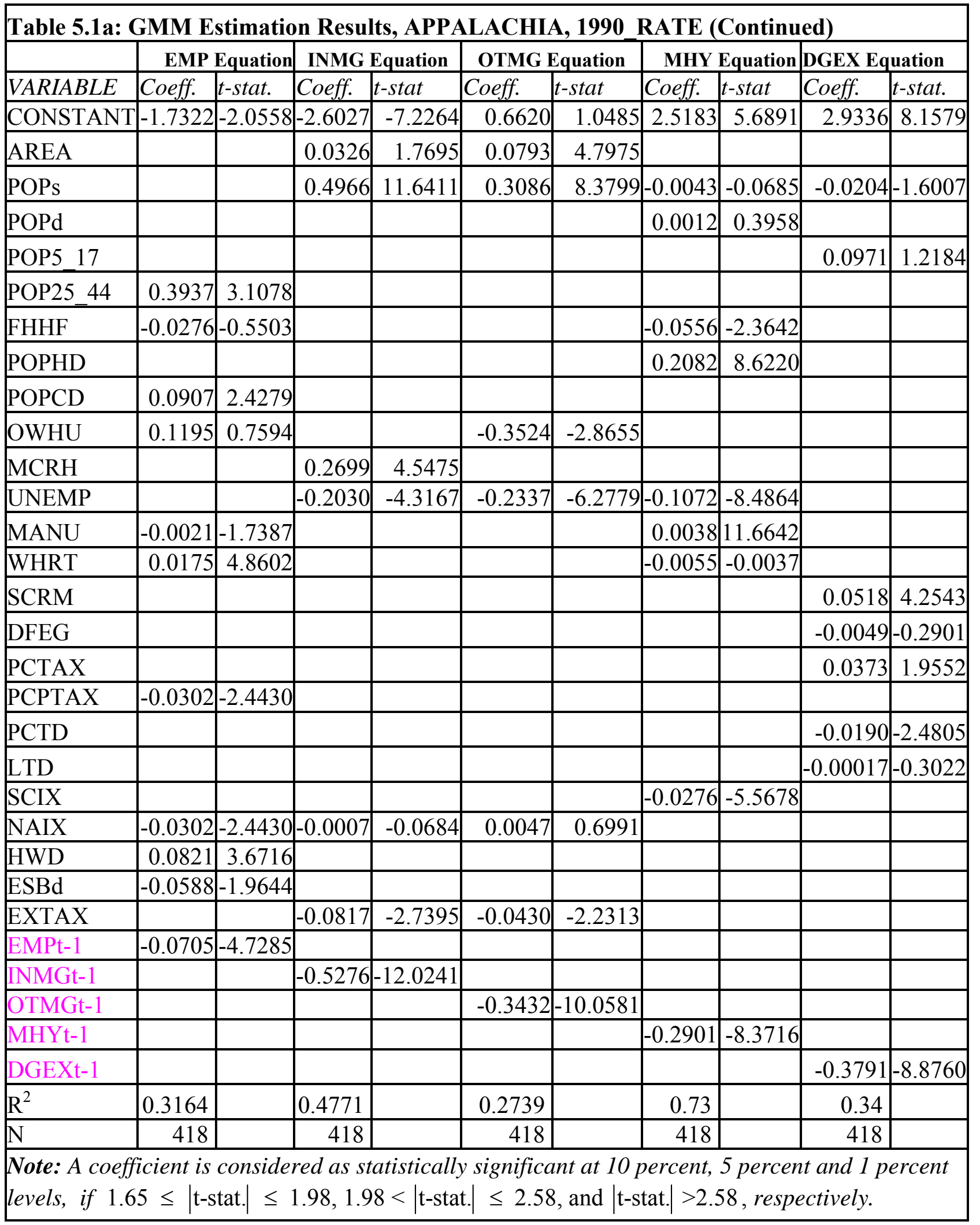


The percentage of people employed in manufacturing (MANU) and the percentage of people employed in wholesale and retail trade (WHRT) are included in the EMPR equation to control for the influence of sectoral concentration of employment on the overall employment of business growth rate. The coefficient on MANU is negative and statistically significant at ten percent level, indicating an inverse relationship between growths in over all employment or business expansion and manufacturing employment. This is not unrealistic finding when we consider the fact that manufacturing has been declining in relative terms during the 1990's as a result of industrial restructuring. The coefficient on WHRT, on the other hand, is positive and significant at the one percent level, indicating the positive role played by the service sector in expanding employment and business in Appalachia during the study period. This is not also unrealistic because the 1980's industrial restructuring has led to a shift from manufacturing into services, encouraging service sector employment growth.

The coefficient on the per capita property income tax (PCPTAX) is negative and significant at almost the 5 percent level. Note that property tax has both direct cost and input mix effects which have opposing effects on employment and business expansion. Property tax could be levied on land or on capital or on both. The direct cost effect on location decision is negative. Once location is determined, the input mix effect could, however, be in the opposite direction. An increase in property tax in capital could push existing firms towards land and labor-intensive industries, expanding employment opportunities. Similarly, an increase in property tax on land could push existing firms towards capital and labor-intensive industries, again, expanding employment opportunities. Thus, in a priori, the impact of property tax on business growth and 
employment is at best ambiguous. The negative coefficient in this study is an indication that the negative direct cost effect dominates the input mix effect, indicating per capita property income taxes have been associated with low business formation and employment growth rate in Appalachia during the study period. This result is also consistent with empirical results in the literature (Luce, 1994).

The coefficient on the natural amenity index (NAIX) is positive, but statistically insignificant. This result is consistent with McGranahan (1993) who found weaker overall association between natural amenities and employment change. High-way density (HWD) is included in the EMPR equation to measure the influence of accessibility to business and employment growth. The positive and statistically significant coefficient on HWD shows a positive association between the concentration of roads and employment growth. This result suggests that Appalachian counties with higher road densities show increases in the growths of employment, compared to counties with low road densities, during the study period. This finding is consistent with both theory and empirical findings (see Carlino and Mills, 1987).

Establishment density (ESBd), which is the total number of private sector establishments in the county divided by the total county's population, is included in our model to capture the degree of competition among firms and crowding of businesses relative to the population. The coefficient on ESBd is negative and significant indicating that Appalachia region has reached the threshold where competition among firms for consumer demands crowds businesses. According to the results, high ESBd is associated with low growth in employment (business growth), indicating that firms tend not to locate near each other possibly due to high competition for local demand. 
Finally, the elasticity of EMPR with respect to the initial employment level (EMPt-1) is negative and statistically significant indicating convergence in the sense that counties with initial low level of employment at the beginning of the period (1980) tend to show higher rate of growth of business than counties with high initial level of employment conditional on the other explanatory variables in the model. This result supports prior results of rural renaissance in the literature (Deller et al., 2001; Lunderberg, 2003).

\subsubsection{Gross In-Migration Growth Rate}

The results from the INMGR equation also indicate that the growth rate of gross in-migration into a county is strongly dependent on the growth rates of employment, median household income and direct local government expenditures. These interdependences are explained by the highly statistically significant coefficients on the endogenous variables of the model. The coefficient on the EMPR in the INMGR equation, for example, is positive and significant at the one percent level. The coefficient on INMR in the EMPR equation is also positive, although not significant. These indicate that counties with high levels of in-migration are favorable for small business growth and the growth in small business further encourages in-migration into the counties. But note that the attractive effect of business growth (employment) is more than the effect of gross in-migration on employment as indicated by the level of the coefficients on the respective variables. This result is consistent with the Todaro-thesis of rural-urban migration. A single job opening encourages more than one migrant. The results also support previous findings from the human- capital-based migration researches where migration is viewed as an investment and that real income and the probability of employment as important 
determinants of interregional migration (Greenwood and Hunt, 1989; Lundberg, 2003). Although one would expect in-migrants and out-migrants to have different characteristics which might lead to have a situation in which counties with high/low gross in-migration growth rates are also counties with high/low gross out-migration growth rates, the results in Table 5.1 do not establish that relationship. The feedback simultaneity between gross out-migration and gross in-migration is not statistically significant.

The existence of strong interdependence between gross in-migration rate and median household income growth rate is reflected by the statistically significant coefficients on the variables in the respective equations. Gross in-migration growth rate in a given county is positively and significantly affected by the growth rate of median household income in that county. This result is consistent with theoretical expectations in that growing income counties can support large market demand for business expansion that can encourage in-migrants who look for the newly crated jobs. Besides, growing income counties can support a lager tax bases that enable local governments to raise enough finance to provide quality public services. These taxes could capitalize into local amenities that attract new residents. The result also supports previous empirical findings by Greenwood (1975, 1976), and Lundberg (2003) who analyzed the relationship between interregional migration and the growth of median income.

Consistent with theoretical expectations, the results in Table 5.1 also indicate a strong negative interdependence between gross in-migration growth rate (INMGR) and the growth rate in local public expenditures (DGEXR). The coefficient on DGEXR in the INMGR equation is negative and statistically significant at the 5 percent level. This result supports previous migration researches in both the Tiebout (1956) and non-Tiebout 
tradition Local government expenditures that are financed through higher taxes, particularly property taxes, tend to deter in-migration and encourage out-migration. The property taxes have their deterrent effects on in-migration through changes in employment as discussed above. Previous studies by Mead (1982) and Schachter and Athaus (1989) have also generated similar results. The implications of this finding is that many poorer communities in Appalachian region which are forced to levy higher taxes to finance local public services at a certain level would not be able to attract people and even loose people. As the counties/communities continue to lose people, the per capita tax price of local public service for the remaining population increases which further leads to deterioration in the respective communities.

The population size (POPs) at the initial period has a positive and strong effect on in-migration into a given county. The positive and statistically significant coefficient on POPs is an indication that people migrate to areas (counties) with high concentration of population. Note also that the coefficient on POPs in the out-migration equation is positive and statistically significant at one per cent level, indicating that counties with high population concentration encourage out-migration and vice versa. These two results suggest that Appalachian counties with higher initial population sizes were both destinations and sources of migrants during the study period. This situation is possible because out-migrants and in-migrants could be people with different labor market characteristics.

County unemployment rate (UNE) is included in the vector of exogenous variables as a measure of local economic distress. The results suggest that high unemployment rate in a given county is associated with low gross in-migration growth 
rate in that county. This result is consistent with the theoretical expectations and empirical results in the migration literature. Economic theory postulates that job seekers are expected to move from high - unemployment regions where they cannot find a job to low-unemployment regions where the prospects of finding employment are more favorable. Research results from a number of studies have also supported this proposition (Carlino and Mills, 1987; Gabriel et al., 1995; Hunt, 1993; Herzog, Schlottman and Boehm, 1993; Hamalainen and Bockerman, 2004).

The coefficient on the MCRH (Median Contract Rent of Specified RenterOccupier) is positive and statistically significant at the one percent level. This is not consistent with the theoretical expectations. One would normally expect that an increase in the cost of rental housing would discourage in-migration by increasing the cost of migration. But it is important to look at MCRH as representing both the availability as well as the cost of rental housing. The expectation that increases in the cost of rental housing to discourage in-migration is based on the assumption that enough rental housing is available in all potential in-migration regions. The availability and the cost (affordability) of rental housing have opposing effects on in-migration. The result in this study suggests that the positive effect of availability dominates the negative effect of rental cost. This observation gives support to the results in Hamalainen and Bockerman, (2004) that suggested a lack of rental housing in potential in-migration regions deter outmigration from high unemployment regions.

The coefficient on the natural amenity index (NAIX) failed to be significant and showed unexpected sign. This result might suggest that Appalachia was not a destination for amenity-based migration. The coefficient on EXTAX is statistically significant 
showed unexpected sign. The EXTAX variable is derived by dividing the per capita local government expenditures by the per capita income taxes. Normally, one would expect high local expenditures on public services to encourage in-migration. But this out come is sensitive to the nature of government spending. High per capita spending in education, health and crime prevention induces in-migration. One possible explanation of the unexpected sign could, thus, be that although overall EXTAX could be high, per capita spending on those public services which induce in-migration might actually be low.

Finally, the coefficient on INMGt-1 is negative and statistically significant indicating convergence in the sense that counties with initial low level of in-migration at the beginning of the period (1990) tend to show higher rate of growth of INMG than counties with high initial gross in-migration conditional on the other explanatory variables in the model.

\subsubsection{Gross Out-Migration Growth Rate}

The results from the out-migration equation also show similar trends. The feedback simultaneities, however, are not strong. Only EMPR shows statistically significant effect on OTMGR. The coefficients on INMGR and DGEX are negative but statistically insignificant. The coefficient on MHYR is positive but also insignificant.

Similar to the case of in-migration growth rate equation, the coefficients on initial population size (POPs) and county area (AREA) are positive and statistically significant at one percent level. This result indicates that counties with high initial population sizes have experienced high growth in out-migration rate.

The impact of home ownership on out-migration is negative and significant which is consistent with the theoretical expectations. Normally, one would expect that owing a 
house to decrease the propensity to migrate due to the transaction cost and liquidity of real estate in location of economic distress. Investing in own housing may also reflect a decision to stay in the area of current residence for long. The estimated results also show a positive and statistically significant (at the one per cent level) coefficient on OWHU. This result indicates that home ownership is negatively associated with out-migration in Appalachia during the study period.

The coefficient on UNEMP shows an unanticipated sign and yet statistically significant at the one percent level. Normally, one would expect that people to move away from high-unemployment counties to low-unemployment counties. The result in Table 5.1, however, suggests that the growth rate of out-migration (OTMGR) in a given county is negatively associated with the initial level of unemployment in that county. One possible explanation of this observation, similar to what Lansing and Mueller (1967) have argued, is that unemployment tends to be highest in the least mobile groups in the labor force. It should also be noted that prospective unemployment rather than the level of unemployment rate is the major determinant of migration. Besides, the lack of rental housing in the potential in-migration counties/regions could deter out-migration from the high-unemployment counties/regions.

Similar to the case in the INMGR equation, the coefficient on the NAIX neither is statically significant nor has the expected sign. Normally, one would expect NAIX to have negative influences on OTMGR. But, it is important to note that migrations are usually motivated by the altered demand for amenities that are sight-specific. In this respect, amenity data at the county level is highly aggregated and may not reflect the true interdependence between OTMGR and NAIX. 
The results in Table 5.1 also show that an increase in EXTAX discourages outmigration from a given county. This is indicated by the significant negative coefficient on the EXTAX variable. This result suggests that the more local government puts tax money back to society in the form of local public services, the more people want to stay in that jurisdiction. This has significant implications from a policy perspective because, it not only encourages people to stay but it can also encourage people to come and stay which in turn help check a declining population. Otherwise, a declining population not only increases the cost of providing local public services but also constrains the expansion and growth of small business by limiting the supply of labor and the demand for small business products. Low quality and quantity of public services also reduces the earning capacity of residents and discourages small business growth and employment. The ultimate result is the perpetuation of poverty and underdevelopment Appalachia.

Finally, the results presented in Table 5.1 indicate the existence of significant conditional convergence in the out-migration growth rate equation. This is indicated by the negative and statistically significant coefficient on the lagged dependent variable for out-migration (OTMGt-1). Conditioned upon the other exogenous variables that are included in the OTMGR equation, counties with low initial level of out-migration showed higher growths in out-migration growth rates compared to counties with higher initial levels of out-migration.

\subsubsection{Median Household Income Growth Rate}

Similar to the results in the other equations, the estimates from the MHYR equation show the existence of significant feedback simultaneity. Two of the endogenous variables have statistically significant effect on the growth rate median household income (MHYR). 
The contemporaneous effect with respect to the rate of growth in employment (EMPR) on median household income, for example, is positive and statistically significant at the one percent level. This result indicates that high growth rate in median household income is positively associated with high growth rate of employment which is consistent with the expectations of economic theory. The contemporaneous effect with respect to the growth rate of in-migration (INMGR) on the growth rate of median household income was negative and statistically significant at the one percent level. This result indicates that the growth rate of median household income in a given county is negatively associated with the growth rate of in-migration to that county. This, in turn, suggests that the average incomes of the in-migrants were lower than that of the median incomes of the nonmovers. The contemporaneous effect with respect to the growth rate of out-migration (OTMGR) on the growth rate of median household income is positive, but statistically insignificant. Although the impact would be insignificant, this result suggests that median household income decreases with out-migration. This, in turn, would mean that the average income of the out-migrants was lower than that of the median income of the nonmovers. These two results, thus, suggests, compared to the non-movers, the movers were poor. Based on these results, it is, therefore, possible for one to claim that the population movements in Appalachia during the study period were, on average, for economic reasons.

Turning to the conditioning variable in the MHYR equation, the results indicates that the rate of growth in median household income is negatively and significantly affected by the percentage of families with female family householder (FHHF), the unemployment rate (UNEMP), and the social capital index (SCIX). POPs is also 
negatively associated with MHYR, but insignificantly. Due to the beneficial effects of agglomeration economies of firm location, one would normally tend to expect that POPs to have positive effect on median household income. A growing population captures the extent to which counties are relatively attractive to migrants and a growing population increases the demand for consumer services which in turn leads to growth in business and employments, which are themselves sources of income to the county. The coefficient on the index of social capital (SCIX) is negative and significant indicating that counties with high level of social capital decrease the well-being of their communities. This result is not consistent with the expectation of economic theory. But remember that social capital index is a composite of many factors of which ethnic homogeneity, income inequality, community attachment and homeownership are the major components. These elements are more experienced in rural and small Appalachian communities where median household income is traditionally very low, compared to metropolitan communities. The negative association of social capital index and the rate of growth of median household income could be the refection of this fact in Appalachia. The negative effect of the FHHF on MHYR, however, is consistent with theoretical expectations. Although the proportion of female family householder per se is not what is important, research results show that poverty increases with an increase in the proportion of female headed householder in a community (see, for example, Levernier, Partridge and Rickman, 2000). Female headed households tend to have low human capital, low labor participation rate and hence lower income earning capacities. The negative relationship between the rate of growth in median household income and FHHF is, therefore, a reflection of this fundamental economic fact in Appalachia. 
As expected, the coefficient on the variable that measures the proportion of the population 25 years and above with high school or above diploma (POPHD) is positive and statistically significant at the one percent level. Human capital theory postulates that entrepreneurship is related to educational attainment and work experience. People with more educational attainments tend establish businesses and also have more probability of getting and securing higher paying jobs than those with low educational background. Although industrial restructuring in the 1980's has led to a shift from manufacturing to service based industries, the process has been low in Appalachia and manufacturing remained as a major source of income compared to service industries. The positive and statistically highly significant coefficient on MANU supports this assertion. Note, however, that this does not mean that manufacturing remained as a major employer during that period. Actually, as explained above, the declining trend in manufacturing employment is supported by the results of this study.

Finally, the negative and statistically significant coefficient on MHYt-1 is an indication that there was conditional convergence with respect to the rate of growth in median household income in Appalachia during the study period. This means that counties with low initial median household income grew faster than counties with higher initial median household income.

\subsubsection{Direct Government Expenditures Growth Rate}

The growth rate of direct local government expenditures per capita (DGEXR) is regressed on the endogenous variables of the model and on a set of county-level conditioning variables related to demographic and policy environments, as well as on the initial condition of direct local government expenditures. 
Similar to the results in the other equations, the estimates from the DGEXR equation show the existence of significant feed-back simultaneity. Three of the endogenous variables have statistically significant effect on the growth rate of direct local government expenditures per capita. The contemporaneous effect with respect to the rate of growth in out-migration (OTMGR) on direct local government expenditures per capita, for example, is positive and statistically significant at the one percent level. This result indicates that high growth rate in direct local government expenditures per capita is positively associated with high growth rate of out-migration which is consistent with expectations of economic theory. Migration has important impacts on the demand of locally provided public goods and services as well as on the revenue that support the provision of these public goods and services by changing the size and the density of population of a region or a county. Out-migration reduces the possibility of gaining economies of scale in the provision of public services. Excessive out-migration creates excess capacity and very high costs of maintaining overstock of public infrastructure, such as schools, police facilities, fire protection, etc., in the area of origin. The contemporaneous effect with respect to the growth rate of in-migration (INMGR) on the growth rate of direct local government expenditures per capita is negative and statistically significant at the ten percent level. This result indicates that the growth rate of direct local government expenditures per capita in a given county is negatively associated with the growth rate of in-migration to that county. One possible explanation for this observation is that in-migration may lead to increase in population and its density in the receiving region that enable local government to realize the advantages of economies of scale in the provision of public services. In that case, although total local government expenditures 
may increase, per capita could still decline if the advantages of economies of scale are realized. The contemporaneous effect with respect to the growth rate of employment (EMPR) on the growth rate of direct local government expenditures per capita is also negative as expected, but statistically insignificant. The coefficient on MHYR is negative and statistically significant at the ten percent level. This result is not consistent with the theoretical expectations. Increases in per capita income provide local governments with more tax revenues that support the provision of more public goods and services, which in turn lead to higher local public expenditures. The result does not give support to empirical findings in Painter and Bae (2001) that indicate a positive and significant impact of increases in per capita income on government expenditures.

As expected, the coefficient on POPs is negative, but not very significant. Economic theory postulates that the size of population plays important roles in per capita spending on non-rival goods such as transportation and communication as well as merit goods and other economic services. Although statistically speaking its impact could be not very strong, negative coefficient on POPs, thus, indicates the advantages of economies of scale in the provision of local public services in Appalachia during the study period. This result also supports empirical findings in Falch and Rastto (1997), Fay (2000), and Hashimati (2001) which show that population has negative coefficient.

The proportion of school age population denoted by POP5-17 is included in the model to control for the differential impact of population age structure on local government expenditures. As expected, the coefficient on POP5-17 is positive, although insignificant. Increases in the proportion of school age population create pressure for increase in local spending on education. 
As expected, the coefficients on SCRM (serious crime per 100,000 population), and PCTAX (per capita income tax) are all positive and statistically significant at the 1, and 10 percent levels, respectively. These results indicate that (1) increases in SCRM leads to increases in local government expenditures in the form of police and crime prevention and protection expenses; and (2) since PCTAX is one of the components of local government the revenue, increases in PCTAX would provided local government with more money to spend on local public services. The coefficient on PCTD (total debt outstanding per capita) is negative and statistically significant at the one percent level. This result is consistent with theoretical expectations in that the amount of total debt outstanding accumulated constrain local governments their capacity to further borrow apart from their obligation to pay their debts now. The effect would be to decreases in local public expenditures. One of the components of local government revue is grants-inaid from higher governments. To control for the impacts of this component, DFEG (direct federal expenditures and grants) is included in the model. Contrary to the theoretical expectations, the coefficient on DFEG is negative, although very insignificant. To control for the impacts of the ability of local government to borrow from external sources in order to finance the provision of local public services, LTD (Long-Term Debt per capita) is also included in the model. The coefficient on LTD is negative which is not consistent with theoretical expectations.

Finally, the negative and statistically significant coefficient on DGEXt-1 is an indication that there was conditional convergence with respect to the rate of growth in direct local government expenditures in Appalachia during the study period. This means that counties with low initial direct local government expenditures had higher growth in 
direct local government expenditures than counties with higher initial direct local government expenditures.

\subsubsection{Panel Results Analysis}

To control for unobserved heterogeneity and also to investigate inter-temporal changes, a panel model for two time periods is estimated. Degree of freedom and efficiency increases with the use of panel data, because panel data give the advantage of using more informative, more variable, less collinear and large sample size data for estimation. The empirical application of the panel data utilizes a one-way error component model in line with the discussions in section 3.5 of chapter III of this study. Section 3.5 of chapter III developed the details of the technical and estimation issues in simultaneous equations with one-way error component models for the disturbances. Like in the cross-sectional analysis, both single-equation and system estimation methods are applied. It was not, however, possible to use EViews for the system method of estimation. EViews has not yet developed built-in functionalities that can handle system method in the panel data setting. The estimation procedures which are detailed in section 3.5 are, therefore, coded in matrix language into programs in TSP and the programs are included this study in the appendix.

The program first defines two orthogonal and symmetric idempotent matrices, $\mathbf{P}$ and $\mathbf{H} . \mathbf{P}$ is a matrix which averages the observations across time for each individual and $\mathbf{H}$ is a matrix which obtains the deviations from the individual means. Limited information (single equation) estimates can be obtained by transforming the data and then performing single-equation method of estimation on each of the equation given in (4.6). Transforming the data by $\mathbf{H}$ and then performing 2SLS on each of the equation using 
such transformed data gives the Within 2SLS (W2SLS). Transforming the data by $\mathbf{P}$ and then performing 2SLS gives the Between 2SLS (B2SLS). It is also possible to transform the data by both $\mathbf{P}$ and $\mathbf{H}$. This uses the principle of spectral decomposition of the variance-covariance matrix of the one-way error component model (see the discussion in section 3.5 of chapter III). Performing 2SLS each of the equation using such transformed data gives the Balestera and Varadharajan-Krishnakumar (1978) generalized two-stage least squares (G2SLS). Performing 3SLS on the model using data transformed by $\mathbf{H}, \mathbf{P}$ and by both $\mathbf{H}$ and $\mathbf{P}$ gives the system estimates of W3SLS, B3SLS and E3SLS respectively. For the sake of brevity, only E3SLS are discussed here and for the purpose of comparison, the single - equation estimates (W2SLS, B2SLS, and G2SLS) are given in the appendix.

The same set of variables is used in each of the corresponding equations of the panel and the cross section models. One of the advantages of panel estimation is that it tends to give more robust and more efficient estimates. Since the models are estimated by different estimators, the cross-section by GMM and the panel data by E3SLS, direct model performance comparisons, in terms of improvements in level of significance and signs of coefficients, may not, however, be feasible in this case. Nonetheless, one can still make references to the cross-sectional model in discussing the results of the panel model. Thus, only the differences in the results between the cross-section (one-time) and the panel models are discussed here. The results of E3SLS are given in Table 5.2.3a below.

\section{EMPR Equation:}

Several variables that were not significant in the cross-section model became significant in the panel model. These are OTMGR, FHHF, and NAIX. While DGEXR was positive 
but insignificant in the cross-section model, it became negative and significant in the panel mode. Similarly, while MANU was negative and significant in the cross-section model, it became positive and significant in the panel model.

\begin{tabular}{|c|c|c|c|c|c|c|c|c|c|c|}
\hline \multirow{2}{*}{\begin{tabular}{|l} 
Table 5.2.3 \\
VARIABLE \\
\end{tabular}} & \multicolumn{2}{|c|}{ EMP Equation } & \multicolumn{2}{|c|}{ INMG Equation } & \multicolumn{2}{|c|}{ OTMG Equation } & \multicolumn{4}{|c|}{\begin{tabular}{|l|l|} 
MHY Equation & DGEX Equation \\
\end{tabular}} \\
\hline & \begin{tabular}{|l|l} 
Coeff. & $t$ \\
\end{tabular} & t-statistic. & \begin{tabular}{|l|l} 
Coeff. & $t$ \\
\end{tabular} & t-statistic & \begin{tabular}{|l|l|} 
Coeff. \\
\end{tabular} & t-statistic & \begin{tabular}{|l|l} 
Coeff. & $t$ \\
\end{tabular} & t-statistic & Coeff. & t-statistic \\
\hline CONSTANT & -0.3701 & -0.4175 & -1.2377 & -5.1248 & \begin{tabular}{|l|}
0.5571 \\
\end{tabular} & 0.9806 & 8.9924 & \begin{tabular}{|l|}
5.3002 \\
\end{tabular} & 4.6386 & 8.2433 \\
\hline EMPR & & & 1.2153 & 16.4864 & 0.8364 & 17.0651 & -0.0606 & $\mid-0.9811$ & $-0.6059 \mid$ & \begin{tabular}{|l}
-6.6038 \\
\end{tabular} \\
\hline INMGR & 0.0117 & 0.3239 & & & -0.0896 & -2.9382 & 0.0695 & 1.7022 & 0.0730 & 1.2841 \\
\hline OTMGR & 0.3251 & 5.3644 & $4-0.4941$ & $|-6.3153|$ & & & 0.3037 & 4.4018 & 0.5767 & 6.5745 \\
\hline MHYR & 0.1314 & 1.7958 & -0.6190 & -5.9594 & -0.6277 & -8.2381 & & & -0.0510 & -0.4604 \\
\hline DGEXR & $\mid-0.1919$ & -3.0747 & 70.1159 & 1.4505 & 0.0137 & 0.2272 & -0.2567 & -4.6761 & & \\
\hline
\end{tabular}

\section{INMGR Equation:}

In the INMGR equation most variables have the same signs and level of significance in the cross-section and in the panel models. Few of them, however, change both their signs and level of significance and these are OTMGR, MHYR, DGEXR and NAIX. While OTMGR was positive but insignificant in the cross-section model, it became negative and highly significant in the panel model. Similarly, while DGEXR was negative and significant in the cross-section model, it became positive but insignificant in the panel model. The same with the NAIX variable, it changed from negative but insignificant in the cross-section model to positive and significant in the panel model. The most marked different result was obtained with regards to MHYR. This variable was positive and highly significant in the cross-section model but became negative and highly significant in the panel model. EXTAX also became insignificant in the panel model. 


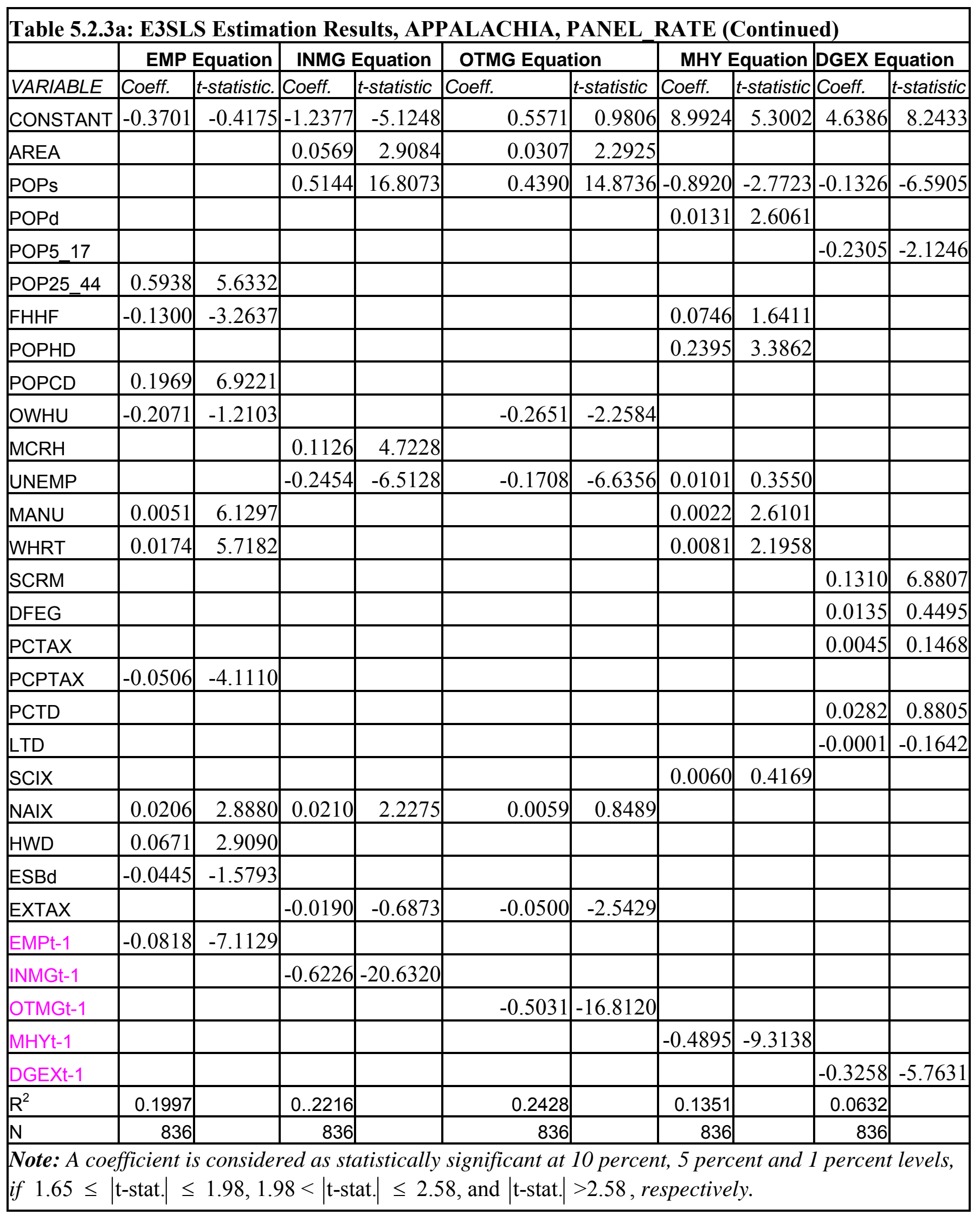




\section{OTMGR Equation:}

In the OTMGR equation, with the exception of few changes, both the cross-section and the panel model have very similar results. While INMGR became significant in the panel model, DGEXR became positive but still remains insignificant. The most marked different result was obtained with respect to MHYR, which was positive but insignificant in the cross-section model and became negative and highly significant in the panel model.

\section{MHYR Equation:}

In the MHYR equation, several variables that were statistically insignificant in the crosssection model became significant in the panel model and these are OTMGR, DGEXR, POPs, and POPs ${ }^{2}$. There are also some changes in both the signs and level significances. EMPR, FHHF, UNEMP, and SCIX changed their signs and became insignificant in the panel model. INMGR and WHRT changed both their signs and level of significance. While INMGR was negative and significant in the cross-section model, it became positive and significant in the panel model. Similarly, WHRT was negative but insignificant in the cross-section model and became positive and significant in the panel model. Since the coefficients in some of the affected control variables are very small, these changes may not have big effects in the final decisions to be reached by the two models.

\section{DGEXR Equation:}

In the DGEXR equation, EMPR and POPs were statistically insignificant in the crosssection model and became highly significant in the panel model. INMGR and PCTD changed their signs and became insignificant in the panel model. MHYR and PCTAX also became insignificant in the panel model. The most marked different result was 
obtained with respect to POP5-17. This variable changed its sign into theoretically unexpected sign and became significant in the panel model

Overall, both the cross-section and the panel data models give support to the hypotheses set in this study. The existence of feedback simultaneities among the dependent variables of the model and conditional convergence with respect to each of the dependent variables are shown by the results of both models. With panel estimation, some improvements are gained in terms of coefficient signs and robustness and efficiency of parameter estimation. Since not only the sample sizes but also the estimators are different in the two models, caution should be made not to make flat comparisons between the performances of the two models.

\subsection{Empirical Estimation: Spatial Simultaneous-Equations Equilibrium Growth Model}

The non-spatial (standard) simultaneous-equations model discussed in the previous section assumes that each of the endogenous variables of the model is only affected by the other endogenous variables and the predetermined variables in a given county, without explicitly considering possible spillover effects from neighboring counties. If spatial spillover effects exist, and if they are not accounted for, they result in model misspecifications. In that case, if the unaccounted spatial dependence is in the error term, coefficient estimates become inefficient and if the uncounted spatial dependence is in the dependent variables, coefficient estimates become biased and inconsistent. In an effort to detect whether or not spatial dependences exist in the data set, exploratory spatial data analysis is given in the next subsection. 


\subsubsection{Exploratory Spatial Data Analysis}

Section 3.6 of chapter III of this study presents a detailed discussion of the different tools of exploratory spatial data analysis. In line with that discussion, this subsection presents the results of exploratory spatial data analysis in the form of Global Moran's I statistics, Moran Scatter Plot, and LISA (Local Indicators of Spatial Association) Maps. In order to carry exploratory spatial data analysis, it is, however, important to first define a non-stochastic and positive $\mathrm{n}$ by $\mathrm{n}$ weights matrix $(\mathbf{W})$ that summarizes the linkages and measures the strength of the potential interactions between observations in our spatial data. Row-standardized Queen-based contiguity weights matrices are used in the spatial analysis of this study.

ArcGIS and GeoDa are used to undertake exploratory spatial data analysis and to do diagnostic tests for spatial dependences in the dependent variables of our model. ArcGIS is used to read the shape files of the study areas into GeoDa. The relevant Moran's I statistics, Moran Scatter Plots and LISA maps for each of the endogenous variable of the model are then created in GeoDa. It was not, however, possible to carry out diagnostic tests for spatial dependences in the residuals of our model in GeoDa, because in GeoDa, Moran's I statistics is applied to the residuals of OLS regression only. Since the model in this study contains right-hand side endogenous variables, OLS regression residual is biased and hence inappropriate for tests of spatial dependences. Besides, since the diagnostic tests for spatial dependence in the endogenous variable show the existence of spatial spillover effects, our models contain right-hand side spatial lag variables as well. Hence, residuals of OLS regressions of our model are also biased because these right-hand side spatial lag variables are correlated with the error terms. A 
test statistics that takes account of these biases is discussed in section 3.9 of chapter III of this study. ${ }^{5}$

The procedures of the test are coded into a program in TSP and subsequently incorporated into the bigger programs which are written to run the whole model. The weights matrices for this procedure as well as for the spatial regression in this study are queen-based contiguity weights matrices that are created in MATLAB using latitudes and longitudes of the county centroids. The sparse weights matrices thus created were converted into full matrices and saved as text file in MATLAB. These text files are read as inputs in TSP and a TSP program converted them into full weights matrices to make them ready for use.

The existence of spatial autocorrelation in the county-level data set for Appalachian counties for both the 1990-2000 and 1980-1990 periods is tested by means of Global Moran's I spatial autocorrelation statistics. The results of the univariate and bivariate spatial autocorrelation analysis are reported in Tables 5.3.1a and 5.3.1b for the 1990-2000 and 1980-1990 periods respectively. Moran's I spatial autocorrelation statistic is visualized by means of Moran Scatter Plot. Scatter plots which correspond to the univariate Moran's I statistics are given in the appendix.

Table 5.3.1a :Global Moran's I Statistics of Spatial Autocorrelation: Appalachia, 1990-2000

\begin{tabular}{lccccc}
\hline & EMPR & INMGR & OTMGR & MHYR & DGEXR \\
WEMPR & 0.1968 & 0.2586 & 0.2071 & 0.0319 & 0.0197 \\
WINMGR & 0.2497 & 0.3259 & 0.2575 & 0.0463 & 0.0323 \\
WOTMGR & 0.1967 & 0.2475 & 0.2116 & -0.1061 & 0.0193 \\
WMHYR & -0.0245 & -0.0198 & -0.0876 & 0.2417 & 0.0064 \\
WDGEXR & 0.0468 & 0.0586 & 0.0549 & -0.0377 & 0.0365
\end{tabular}

\footnotetext{
5 Anselin and Kelejian (1997) proposed Moran's I statistics based on residuals that are obtained from instrumental variable (IV) procedure.
} 
Table 5.3.1b :Global Moran's I Statistics of Spatial Autocorrelation: Appalachia, 1980-1990

\begin{tabular}{lccccc}
\hline & EMPR & INMGR & OTMGR & MHYR & DGEXR \\
WEMPR & 0.2106 & 0.022 & 0.1268 & 0.3541 & 0.0197 \\
WINMGR & 0.0565 & 0.2018 & 0.171 & 0.0928 & 0.0168 \\
WOTMGR & 0.1737 & 0.1342 & 0.3307 & 0.2588 & 0.0083 \\
WMHYR & 0.373 & 0.0874 & 0.2669 & 0.6428 & 0.0091 \\
WDGEXR & 0.0245 & 0.0484 & 0.0447 & 0.0025 & 0.0821
\end{tabular}

Spatial dependences in the form of spatial autoregressive lags and crossregressive lags simultaneities are detected in both periods. The strengths of the spatial interactions of each endogenous variable with respect to itself as well as with the spatial lags of the other variables are given by the numbers in the respective column. The numbers in the second column, for example, represents the strengths of the spatial interaction of EMPR with respect to its spatial lag and with respect to the spatial lags of the other endogenous variables in the model. Generally, the spatial interdependences are strong apart from the cross-regressive lags with respect to DGEXR.

A test to detect the existence of spatial dependences in the disturbances of the model in this study is also done on 2SLS regression residuals of the model given in (4.7a). The results are given in Table 5.3.1c.

Table 5.3.1c: TESTS FOR SPATIAL AUTOCORRELATION IN THE ERROR TERMS, APPALACHIA

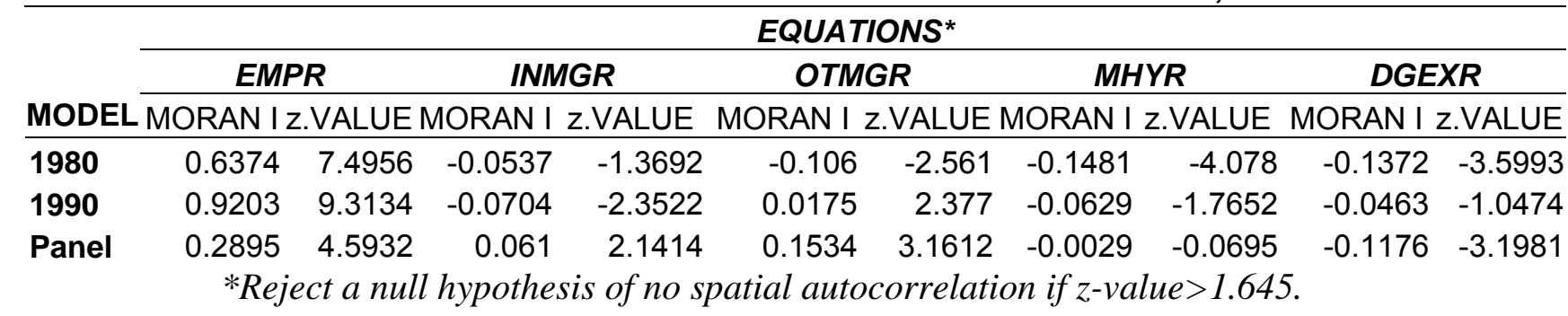

As indicated in Table 5.3.1c, spatial dependence is detected in the error terms of almost all equations of both cross-section and panel models. The existence of spatial 
dependences in the dependent variables and the error terms of the equations of the model indicate that parameter estimation without accounting for such spillover effects becomes inefficient, biased and inconsistent. In odder to accommodate such concerns, this study extended the standard (non-spatial) simultaneous-equations model given in (4.6) into a spatial simultaneous-equations model. Section 3.7 of chapter III presents the theoretical modeling issues of such extension in both the cross-section and panel data settings. The technical and methodological issues in spatial simultaneous-equations estimation are discussed and developed in section 3.8 of chapter III of this study. Based on those methodologies and estimation strategies, estimation results of the spatial models are presented in the following subsection

\subsubsection{Spatial Regression Estimation Results and Discussion}

\subsubsection{Estimation Issues}

The model given in (4.8b) is estimated using feasible generalized spatial two stage least squares (FGS2SLS) and feasible generalized spatial three stage least squares (FGS3SLS) procedures for data from Appalachian counties for 1990-2000. The model is specified in log-linear form with two modifications involving the measurement of the explanatory variables. First, the natural $\log$ formulation is dropped for the explanatory variables that can take negative or zero values. Second, one-period lagged values are used for all of the explanatory variables to avoid simultaneity bias.

\section{a) Cross-section Model}

The FGS2SLS and FGS3SLS procedures are done in a three and a four step routines, respectively. The first three steps are common for both routines. In the first step, the parameter vector consisting of betas, lambdas and gammas $\left[\beta^{\prime}, \lambda^{\prime}, \gamma^{\prime}\right]$ are 
estimated by two stage least squares (2SLS) using an instrument matrix $\mathbf{N}$ that consists of a subset of $\mathbf{X}, \mathbf{W X}, \mathbf{W}^{2} \mathbf{X}$, where $\mathbf{X}$ is the matrix that includes all control variables in the model, and $\mathbf{W}$ is the weight matrix. The disturbances for each equation in the model are computed by using the estimates for betas, lambdas and gammas from the first step. In the second step, these estimates of the disturbances are used to estimate the autoregressive parameter rho $(\rho)$ for each equation using Kelejian and Prucha's (2004) generalized moments procedure. In the third step, a Cochran-Orcutt-type transformation is done by using the estimates for rhos from the second step to account for the spatial autocorrelation in the disturbances. The FGS2SLS estimators for betas, lambdas and gammas are then obtained by estimating the transformed model using $\left[\mathbf{X}, \mathbf{W X}, \mathbf{W}^{2} \mathbf{X}\right]$ as the instrument matrix.

Although the FGS2SLS takes the potential spatial correlation into account, it does not utilize the information available across equation because it does not take into account the potential cross equation correlation in the innovation vectors $\boldsymbol{\varepsilon}_{i t}^{i n}, \boldsymbol{\varepsilon}_{i t}^{o t}, \boldsymbol{\varepsilon}_{i t}^{e m}$ and $\boldsymbol{\varepsilon}_{i t}^{m h}$. The full system information is utilized by stacking the Cochran-Orcutt-type transformed equations (from the second step) in order to estimate them jointly. Thus, in the fourth step the GS3SLS estimator of betas, lambdas, and gammas is obtained by estimating this stacked model. The FGS3SLS estimator is more efficient than FGS2SLS estimator and only the FGS3SLS estimates are reported.

\section{b) Panel Model}

The increase in the time dimension in the panel data made the estimation programs more complex. The steps are essentially similar to the cross-section case. The details, especially in the second step, however, are quite different. The fist steps, for both 
the FGS2SLS and FGS3SLS, are the same; the disturbances of each equation in the panel model are computed from first stage 2SLS estimates of betas, gammas and lambdas. Such computed disturbances are used to estimate the spatial autoregressive parameter $\rho$ and the variance components $\sigma_{w}^{2}$ and $\sigma_{1}^{2}$ using generalized moment procedure suggested by Kapoor, Kelejian and Prucha's (2003). Unlike in the cross-section case, now the generalized moments estimators of $\rho, \sigma_{w}^{2}$ and $\sigma_{1}^{2}$ are diefined in terms of six moments conditions. The second step has two parts. In the first part, initial generalized moments estimators of $\rho, \sigma_{w}^{2}$ and $\sigma_{1}^{2}$ are computed as outlined in subsection 3.8.2 of chapter III of this study. These are unweighted GM estimators. In the second part, weighted GM estimators of $\rho, \sigma_{w}^{2}$ and $\sigma_{1}^{2}$ are computed. In the third step, the data is transformed using these weighted GM estimators of the spatial autoregressive parameter $\rho$ and the variance components $\sigma_{w}^{2}$ and $\sigma_{1}^{2}$. The FGS2SLS estimators for betas, lambdas and gammas are then obtained by estimating the transformed model using $\left[\mathbf{X}, \mathbf{W X}, \mathbf{W}^{2} \mathbf{X}\right]$ as the instrument matrix. The fourth step is similar to the cross-section case.

The estimation programs for both models are written in TSP programming language and are included in the appendix. Since the results and analyses are essentially similar, for the sake of brevity, only the estimation results of the panel model are reported and discussed below.

\subsubsection{Results and Discussion}

Two-period panel data from the 418 Appalachian counties are used for the empirical implementation of the panel model. The FGS3SLS parameter estimates are presented in Table 5.3.2a. The parameter estimates are mostly consistent with the theoretical 
expectations. The coefficients on the endogenous variables in all equations of the system, with the exception of the coefficients on EMPR in the DGEXR equation and on INMGR in the MHYR equation, are statistically highly significant. This indicates the existence of very strong feedback simultaneities among the dependent variables of the spatial simultaneous equations system. The results also show strong spatial autoregressive lag and spatial cross-regressive lag simultaneities. Besides, all of the coefficients on the lagged dependent variables are statistically highly significant, indicating the existence of conditional convergence with respect to each of the endogenous variables conditional on the set of exogenous variables included in each equation of the model. In general, the above three observations support the three basic hypotheses set in this study.

\section{Employment (Business) Growth Rate:}

The results in Table 5.3.2a indicate that the growth rate of employment (EMPR) in a county is strongly dependent on the growth rates of gross in-migration (INMGR), gross out-migration (OTMGR), median household income (MHYR), and direct local government expenditures (DGEXR). Each of these variables, with the exception of DGEXR, in turn, is strongly affected by the growth rate of employment (EMPR). The coefficient on INMGR, for example, is positive and statistically significant at the one percent level. The coefficient on the EMPR in the INMGR equation is also positive and statistically significant at the one percent level. These indicate that counties with high growth rate in gross in-migration are favorable for small business growth and the growth in small business further leads to increases in the growth of gross in-migration into the counties. But note that the attractive effect of business growth (employment) rate is more than the effect of gross in-migration growth rate on employment growth rate as indicated 
by the level of the coefficients on the respective variables. This is in consistent with the Todaro-thesis of rural-urban migration. A single job opening encourages more than one migrant. Similarly, the interdependence between the growth rate of employment and the growth rate of gross out-migration is very strong.

Table5.3.2a: Feasible Generalized Spatial Three-Stage Least Squares(FGS3SLS) Estimation Results, PANEL_RATE

\begin{tabular}{|c|c|c|c|c|c|c|c|c|c|c|}
\hline \multirow[b]{2}{*}{ VARIABLE } & \multicolumn{2}{|c|}{ EMP Equation } & \multicolumn{2}{|c|}{ INMG Equat } & \multicolumn{2}{|c|}{ OTMG Equation } & \multicolumn{2}{|c|}{ MHY Equation } & \multicolumn{2}{|c|}{ DGEX Equation } \\
\hline & Coefficient & t-statistic & Coefficien & & Coefficient & & & & efficlen & t-statistic \\
\hline CONSTANT & -0.7211 & -1.4514 & -0.3951 & -1.6652 & & 7.3054 & & 6.7117 & & \\
\hline MPR & & & & & & & & & & \\
\hline NMGR & & & & & & & & & & \\
\hline DTMGR & & & & & & & & & & \\
\hline IHYR & & & -0.5 & & & & & & & \\
\hline DEEXR & & & & & & & & & & \\
\hline WEMPR & -0.6500 & & & & & & & & & 6429 \\
\hline WINMGR & & & & & & & & & & 1.6872 \\
\hline WOT & & & & & & & & & & 835 \\
\hline WMHYR & & & & & & & & & & 0.9063 \\
\hline WDGEXR & 0.1875 & 1.8502 & 0.2020 & 1.1995 & 0.2256 & 2.2746 & 0.1866 & 2.4472 & 0.4216 & 5.5303 \\
\hline
\end{tabular}

Note: A coefficient is considered as statistically significant at 10 percent, 5 percent and 1 percent levels, if $1.65 \leq \mid \mathrm{t}$-stat. $|\leq 1.98,1.98<| \mathrm{t}$-stat. $\mid \leq 2.58$, and $\mid \mathrm{t}$-stat. $\mid>2.58$, respectively.

Contrary to expectations, the coefficient on the OTMGR is positive and statistically significant at the one percent level. The coefficient on EMPR in the OTMGR equation is also positive and statistically significant at the one percent level. This means counties with high rate of growth in out-migration encourage small business growth and small business growth, in turn, encourages out-migration. Now again, the contemporaneous effects of EMPR on OTMGR is stronger than that of OTMGR on EMPR as indicated by their respective coefficients. The results also show strong positive feedback simultaneity between EMPR and MHYR. This is indicated by the positive and 
statistically significant coefficient on MHYR in the EMPR equation and the statistically significant coefficient on EMPR in the MHYR equation. These results suggest that the rate of growth of employment is positively and significantly affected by the rate of growth of median household income (MHYR) at the county-level during the study period. This is consistent with economic theory and the literature (Armington and Acs, 2002). Increases in median household income tends to increase regional wealth and as wealth increases consumer demands for goods and services increase. The growth of the market demand in turn encourages small business and firms' formation. Increases in median household income could also lead to capital formation in the form of household savings that finance new firm formation. The formation and expansion of businesses creates employment opportunity and income for the new and the expanding entrepreneurs. These increases in labor and entrepreneurial incomes, in turn, feed back into the MHYR equation and further leads to an increase in median household income. This is shown by the positive and highly significant coefficient estimate on the EMPR in the MHYR equation. This interdependence is consistent with economic theory and research results in the literature. Note, however, that the attractive effect of the rate of growth of median household income on the rate of growth of small Business growth (employment) is weaker than that of the rate of growth of small business growth on the rate of growth of median household income.

As expected, the coefficient on the rate of growth in direct local government expenditures in the EMPR equation is positive and statistically significant at the one percent level. This result is consistent with the results of many studies, which are summarized in the literature review section of this study, which show that local 
government expenditures on police, fire protection, water and sanitation infrastructure, school spending, highways, and on public health have positive effects on firm location and business expansion. One also normally expect that the gate of growth in employment to have positive effect on local public services. The coefficient on EMPR in the DGEXR, however, has the unexpected sign, though insignificant.

To control for the potential effects of spatial spillover effects on the rate of growth of employment, spatial lags of the endogenous variables are included in the EMPR equation. The results suggest a negative and significant parameter estimate on the spatial autoregressive lag variable (WEMPR). This coefficient represents the spatial autoregressive simultaneity and indicates that the growth rate of employment in a given county tends to spillover to neighboring counties and has negative effects on their rates of growth of employment. The results also show a positive and significant parameter estimate on the spatial cross-regressive variable with respect to the rate of growth of gross out-migration (WOTMGR) indicating that an increase in the rate of growth of gross out-migration in neighboring counties tends to encourage business (employment) in a given county. This is possible because the out-migrants from neighboring counties may end up in the county providing the capital and labor that are required for business expansion. Our results also show negative, although insignificant, spatial cross-regressive effects with respect to the growth rate of gross in-migration and the growth rate of median household income. This would mean that increases in gross in-migration into and median household income in neighboring counties tend to discourage business (employment) in a given county. This is consistent with economic theory because an increase in income in neighboring counties encourages firms and people to migrate to the 
neighboring counties in search of markets and jobs respectively. But the migrating firms and people take the capital and labor as well as the skills that are necessary for business expansion out of the given county leading to the decline in employment and business growth in that county. The coefficient on DGEXR is positive and significant at the one percent level. This result suggests that increases in the rate of growth of local government expenditures in neighboring counties tend to increase the rate of growth of employment in a given county. This is possible because government expenditures, for example, in highways, crime protection, pollution control, may have positive cross border effects that could benefit firm location on the other side of the county border.

All these results are important from a policy perspective as they tend to indicate that the growth rate of employment in one county has negative spillover effects to the growth rate of employment in neighboring counties. Counties tend to be in competition in their efforts to encourage business location in their jurisdictions. The results are also important from an economic perspective because the significant spatial autoregressive lag and spatial cross-regressive lags effects indicate that EMPR does not only depend on characteristics within the county, but also on that of its neighbors. Hence, spatial effects should be tested for in empirical works involving employment growth rates, growth rate of gross in- and out-migration, growth rate of median household income, as well as growth rates in local government expenditures. The model specification in this study also incorporates spatial autoregressive error component in order to control for the effects of unobservable spatial process (effect) besides the spatial lag in the dependent variables. The results in Table 5.3.2a indicate a positive parameter estimate for rho1 indicating that random shocks into the system with respect to the growth rate of employment do not only 
affect the county where the shocks originated and its neighbors, but create positive shock waves across Appalachia.

Similar to the case of the non-spatial cross-sectional analysis, the model includes measure of population statistics such as the percentage of population between 25 and 44 years old (POP25_44) to control for agglomeration effects. The coefficient on POP25-44 remains positive and became even more significant. The results show that POP25_44 has positive and significant effects on EMPR, even after the potential spatial spillover effects are controlled for. This result is consistent with the literature (Acs and Armington, 2004a) which indicates that a growing population increases the demand for consumer goods and services, as well as the pool of potential entrepreneurs which encourage business formation. This result is important from a policy perspective. It indicates that counties with high population concentration are benefiting from the resulting agglomerative and spillover effects that lead to localization of economic activities, in line with Krugman's (1991a, 1991b) argument on regional spillover effects. Consistent with the theoretical expectations, the results also show initial human capital endowment as measured by the percentage of adults (over 23 years old) with college degree (POPCD) is positive and statistically significant at the one percent level. Highly educated people in most case have more access to research and development facilities, and perhaps a good insight to the business world and thus a clear idea about the present and the future needs of the market. As Christensen (2000) contends, entrepreneurs with good education are also more likely to know how to transform innovative ideas into marketable products. Thus, people with more educational attainment tend to establish business, and to be more successful when they do, more often than those with less educational attainments. This 
result is also consistent with Acs and Armington's (2004b) findings which indicates that the agglomerative effects that contribute to new firm formation could come from the supply factors related to the quality of local labor market and business climate. More educated people would mean more human capital embodied in their general and specific skills, for implementing new ideas for creating and growing new businesses. One possible implication of these findings is that regions or counties with different levels of human capital endowment and different propensities of locally available knowledge to spill over and stimulate new firm formation tend to have different rates of new firm formation, survival and growth. The percent of female householder families (FHHF) is another conditioning demographic variable included in the model. Female householder families tend to have low labor participation rate. The coefficient on FHHF is negative and statistically significant at the one percent level, indicating that FHHF has negative impact on EMPR. This is consistent with theoretical expectations and empirical findings. FHHF affects both the supply-side (as source of labor input) and the demand-side (as source of demand for consumer goods) of the market. Thus, this result suggests that Appalachian counties with higher proportion of female household header in their communities tend to show lower growth in business or employment.

The coefficient on the variable representing the percentage of home owned by their occupants (OWHU) is positive, although insignificant. This result indicates that high home ownership is positively associated with business formation in Appalachia. This is consistent with theoretical expectation that high home ownership is an indication that there is a capacity to finance new business by potential entrepreneurs, either by using 
the house as collateral for loan or as indication of availability of personal financial resources to start new business.

The percentage of people employed in manufacturing (MANU) and the percentage of people employed in whole sale and retail trade (WHRT) are included in the EMPR equation to control for the influence of sectoral concentration of employment on the overall employment of business growth rate. The coefficient on MANU is positive and statistically significant at the one percent level, indicating an direct relationship between growths in overall employment or business expansion and manufacturing employment at the beginning of the periods. The coefficient on WHRT is also positive and significant at the 1 percent level, indicating the positive role played by the service sector in expanding employment and business in Appalachia during the study period. Thus, these results tend to suggest that Appalachian counties who had higher proportion of their labor force employed in manufacturing and whole sale and retail trade at the beginning the periods experienced higher growth rates in overall employment. This is not unrealistic because during most of the study period Appalachia has experienced a shift from coal mining-based economic activities to manufacturing and even more to services. The coefficient on WHRT is higher and even more significant than the coefficient on MANU in the EMPR equation, indicating that the contribution of WHRT to overall employment growth was higher and more sustained than that of MANU. This, in turn may indicate that industrial restructuring might have helped the service sector to grow faster than manufacturing.

The coefficient on the per capita property income tax (PCPTAX) is negative, but not significant. Note that property tax has both direct cost and input mix effects which 
have opposing effects on employment and business expansion. Property tax could be levied on land or capital or both. The direct cost effect on location decision is negative. Once location is determined, the input mix effect could, however, be in the opposite direction. An increase in property tax in capital could push existing firms towards land and labor-intensive industries, expanding employment opportunities. Similarly, an increase in property tax on land could push existing firms towards capital and laborintensive industries, again, expanding employment opportunities. Thus, in a priori, the impact of property tax on business growth and employment is at best ambiguous. The negative coefficient in this study would be an indication that the negative direct cost effect dominates the input mix effect, indicating per capita property income taxes have been associated with lower business and employment growth rate in Appalachia during the study period. This conclusion, however, is inconclusive because the coefficient is insignificant.

The coefficient on the natural amenity index (NAIX) is positive and statistically significant at the one percent level. This result is inconsistent with McGranahan (1993) who found weaker overall association between natural amenities and employment change. High-way density (HWD) is included in the EMPR equation to measure the influence of accessibility to business and employment growth. The positive and statistically significant coefficient on HWD shows a positive association between the concentration of roads and employment growth. This result suggests that Appalachian counties with higher road densities show increases in the growths of employment, compared to counties with low road densities, during the study period. This finding is consistent with both theory and empirical findings (see Carlino and Mills, 1987). 
Table 5.3.2a: Feasible Generalized Spatial Three-Stage Least Squares(FGS3SLS) Estimation Results, PANEL_RATE (Continued)

\begin{tabular}{|l|l|l|l|l|}
\hline EMP Equation & INMG Equation & OTMG Equation & MHY Equation & DGEX Equation
\end{tabular}

VARIABLE Coefficient $t$-statistic Coefficient $t$-statistic Coefficient $t$-statistic Coefficient $t$-statistic Coefficient $t$-statistic

\begin{tabular}{|c|c|c|c|c|c|c|c|c|c|c|}
\hline & & & & & & & & & & \\
\hline AREA & & & -0.0369 & -1.5766 & -0.0041 & -0.2604 & & & & \\
\hline POPs & & & 0.5519 & 20.3534 & 0.2187 & 18.4429 & -0.1567 & -1.0471 & 0.0098 & 0.9167 \\
\hline $\mathrm{POP}^{2}$ & & & & & & & 0.0064 & 0.8912 & & \\
\hline POP5_17 & & & & & & & & & 0.1267 & 2.7955 \\
\hline POP25_44 & 0.2694 & 3.8239 & & & & & & & & \\
\hline FHHF & -0.0992 & -4.1690 & & & & & -0.0236 & -1.0391 & & \\
\hline POPHD & & & & & & & 0.3128 & 7.5692 & & \\
\hline POPCD & 0.1754 & 7.9801 & & & & & & & & \\
\hline OWHU & 0.0578 & 0.5831 & & & -0.0929 & -1.6064 & & & & \\
\hline MCRH & & & 0.1141 & 8.1934 & & & & & & \\
\hline UNEMP & & & -0.3036 & -9.3346 & -0.1679 & -8.1801 & -0.0026 & -0.1692 & & \\
\hline MANU & 0.0032 & 5.4736 & & & & & 0.0023 & 5.1125 & & \\
\hline WHRT & 0.0181 & 7.3968 & & & & & -0.0007 & -0.3755 & & \\
\hline SCRM & & & & & & & & & 0.0410 & 0.4946 \\
\hline DFEG & & & & & & & & & 0.0529 & 3.9894 \\
\hline PCTAX & & & & & & & & & 0.0486 & 4.6624 \\
\hline PCPTAX & -0.0051 & -0.6112 & & & & & & & & \\
\hline PCTD & & & & & & & & & -0.0001 & -4.6535 \\
\hline LTD & & & & & & & & & 0.0017 & 4.8203 \\
\hline SCIX & & & & & & & -0.0099 & -1.3853 & & \\
\hline NAIX & 0.0169 & 3.0763 & 0.0192 & 2.3163 & 0.0084 & 1.7953 & & & & \\
\hline HWD & 0.1808 & 6.5349 & & & & & & & & \\
\hline ESBd & -0.1162 & -4.7651 & & & & & & & & \\
\hline EXTAX & & & 0.0768 & 3.1002 & 0.0226 & 1.4816 & & & & \\
\hline EMPt-1 & -0.0873 & -9.2827 & & & & & & & & \\
\hline INMGt-1 & & & -0.6774 & -23.8148 & & & & & & \\
\hline OTMGt-1 & & & & & -0.2836 & -22.4302 & & & & \\
\hline MHYt-1 & & & & & & & -0.5228 & -18.9127 & & \\
\hline DGEXt-1 & & & & & & & & & -0.2771 & -13.3590 \\
\hline $\mathrm{RHO}$ & 0.5713 & & 0.0398 & & 0.3429 & & 0.0006 & & -0.3976 & \\
\hline SIGW & 0.0603 & & 0.0866 & & 0.0396 & & 0.0534 & & 0.1236 & \\
\hline SIG1 & 0.063 & & 0.0776 & & 0.0465 & & 0.0448 & & 0.1028 & \\
\hline$R^{2}$ & 0.2659 & & 0.1225 & & 0.1886 & & 0.1654 & & 0.1176 & \\
\hline $\mathrm{N}$ & 836 & & 836 & & 836 & & 836 & & 836 & \\
\hline
\end{tabular}

Note: A coefficient is considered as statistically significant at 10 percent, 5 percent and 1 percent levels, if $1.65 \leq \mid \mathrm{t}$-stat. $|\leq 1.98,1.98<| \mathrm{t}$-stat. $\mid \leq 2.58$, and $\mid \mathrm{t}$-stat. $\mid>2.58$, respectively. 
Establishment density (ESBd), which is the total number of private sector establishments in the county divided by the total county's population, is included in our model to capture the degree of competition among firms and crowding of businesses relative to the population. The coefficient on ESBd is negative and statistically significant at the one percent level, indicating that Appalachia region has reached the threshold where competition among firms for consumer demands crowds businesses. According to the results, high ESBd is associated with low growth in Employment (business growth), indicating that firms tend not to locate near each other possibly due to high competition for local demand.

Finally, the elasticity of EMPR with respect to the initial employment level (EMPt-1) is negative and statistically significant indicating convergence in the sense that counties with initial low level of employment at the beginning of the period tend to show higher rate of growth of business than counties with high initial levels of employment conditional on the other explanatory variables in the model. This result supports prior results of rural renaissance in the literature (Deller et al., 2001; Lunderberg, 2003).

\section{Gross In-Migration Growth Rate:}

The results from the INMGR equation also indicate that the growth rate of gross in-migration into a county is dependent on the growth rates of employment, gross outmigration, median household income and direct local government expenditures. These interdependences are explained by the statistically significant coefficients on the endogenous variables of the model. Since the interdependence between EMPR and INMGR as well as the implications of this interdependence is explained in the EMPR equation above, it is not discussed here. Suffice it to say that the results from this study 
give support to previous findings from the human-capital-based migration researches where migration is viewed as an investment and that real income and the probability of employment as important determinants of interregional migration (Greenwood and Hunt, 1989; Lundberg, 2003).

The coefficient on OTMGR in the INMGR equation is negative and statistically significant at the one percent level. The coefficient on INMGR in the OTMGR equation is also negative and statistically significant at the five percent level. These results tend to show that INMGR and OTMGR in a given county are inversely related, indicating that counties with high (low) gross in-migration growth rates are also counties with low (high) gross out-migration growth rates. This is consistent with the macroeconomic theory literature where migration is considered as an equilibrating factor in regional labor markets. This is to say that job seekers are expected to move away from highunemployment regions or counties where they cannot find jobs to low-unemployment regions or counties where the prospects for finding employment are more favorable. This finding implies that the driving force for in-migration into and out-migration from a given county is linked to the labor market characteristics of that county and in-migrant and out-migrants have the same labor market characteristics

The coefficient on the MHYR variable in the INMGR equation is negative and statistically significant at the one percent level. This indicates that gross in-migration growth rate in a given county is negatively and significantly affected by the growth rate of median household income in that county. This is contrary to theoretical expectation where migration is expected to be away from counties with low median household income growth rates to counties with relatively high median household income growth 
rates. This findings, however, is not unrealistic because it could be due to the fact that some migrant prefer low income locations. Clark and Hunter (1992), for example, found that movers in their early 20 s as well as migrants 35 years and older prefer low-income locations. Besides, as Knapp and Graves (1989) suggest, higher income locations may be associated with low amenities that discourage people from migrating in.

Consistent with theoretical expectations, the results in Table 5.3.2a also suggest a strong negative interdependence between gross in-migration growth rate (INMGR) and the growth rate of local public expenditures (DGEXR). The coefficient on DGEXR in the INMGR equation is negative and statistically significant at the one percent level. This result supports previous migration researches in both the Tiebout (1956) and non-Tiebout tradition. Local government expenditures that are financed through higher taxes, particularly property taxes, tend to deter in-migration and encourage out-migration. The property taxes have their deterrent effects on in-migration through changes in employment as discussed above, in reference to the impact of PCPTAX on EMPR. Previous studies, for example, by Mead (1982) and Schachter and Athaus (1989) have also generated similar results. The implications of this finding is that many poorer communities in Appalachian region which are forced to levy higher taxes to finance local public services at a certain level would not be able to attract people and even loose people. As the counties/communities continue to loose people, the per capita tax price of local public service for the remaining population increases which further leads to deterioration in the respective communities.

Turning to the spatial autoregressive lag and spatial cross-regressive lag effects, the coefficient on the spatial autoregressive lag variable fails to be significant indicating 
the absence of spatial autocorrelation with respect to the growth rate of gross inmigration. The coefficient on the spatial cross-regressive lag variables with respect to employment (WEMPR), however, is positive and statistically significant at the five per cent level. This indicates that the growth rate of gross in-migration into one county is positively associated with the growth rate of employment in neighboring counties. This is very interesting finding because it indicates that people commute to neighboring counties to work. But as people commute to neighboring counties to work, employment/business in those neighboring counties expands and attracts in-migrants. The flow of in-migrants into neighboring counties further leads to business/employment expansion in those counties. Since, as discussed above, the growth rates of employment in neighboring counties are inversely related, the counties whose residents are commuting to the neighboring counties for work, might face a lower growth rate in employment/business. Neither the coefficients on WINMGR, and WMHYR in the INMGR equation, nor the coefficients on WINMGR in the OTMGR equation and MHYR equation are statistically significant, indicating weak cross-regressive lags simultaneities between INMGR on one hand and OTMGR and MHYR on the other hand. The coefficient on WDGEXR is positive but insignificant.

The results in Table 5.3.2a also suggest a positive parameter estimate for rho2 indicating that random shocks into the system with respect to gross in-migration growth rate do not only affect the county where the shocks originated and its neighbors, but create positive shock waves across Appalachia.

Population size (POPs) at the initial period has a positive and strong effect on inmigration into a given county. The positive and statistically significant coefficient on 
POPs is an indication that people migrate to areas (counties) with high concentration of population. Note also that the coefficient on POPs in the out-migration equation is positive and statistically significant at the one per cent level, indicating that counties with high population concentration encourage out-migration and vice versa. These two results suggest that Appalachian counties with higher initial population sizes were both destinations and sources of migrants during the study period.

County unemployment rate (UNEMP) is included in the vector of exogenous variables as a measure of local economic distress. The results suggest that high unemployment rate in a given county is associated with low gross in-migration growth rate in that county. This result is consistent to theoretical expectation and empirical results in the migration literature. Economic theory postulates that job seekers are expected to move from high-unemployment regions where they cannot find a job to lowunemployment regions where the prospects of finding employment are more favorable. Research results from a number of studies have also supported this proposition (Carlino and Mills, 1987; Gabriel et al., 1995; Hunt, 1993; Herzog, Schlottman and Boehm, 1993; Hamalainen and Bockerman, 2004).

The coefficient on the MCRH (Median Contract Rent of Specified RenterOccupier) is positive and statistically significant at the one percent level. This is not consistent with the theoretical expectations. One would normally expect that an increase in the cost of rental housing to discourage in-migration by increasing the cost of migration. But it is important to look at MCRH as representing both the availability as well as the cost of rental housing. The expectation that increases in the cost of rental housing to discourage in-migration is based on the assumption that enough rental housing 
is available in all potential in-migration regions. The availability and the cost (affordability) of rental housing have opposing effects on in-migration. The result in this study suggests that the positive effect of availability dominates the negative effect of rental cost. This observation gives support to the results in Hamalainen and Bockerman, (2004) that suggested a lack of rental housing in potential in-migration regions deter outmigration from high unemployment regions.

Consistent with the expectations, the coefficient on the natural amenity index (NAIX) is positive and statistically significant at the five percent level. This result suggests that people tend to move to places high in natural amenities. With increases in per capita incomes, peoples' valuations over local attributes that increase quality of life also tend to increase. The result from this study is also consistent with empirical findings in the compensating differential literature, which indicate that migration to places rich in natural amenities, such as warm winter weather, cooler, less-humid summer weather, etc., have increase over the last several decades (Rappaport, 2004; Blomquist, Berger, and Hooen, 1988).

The coefficient on EXTAX is positive and statistically significant at the one percent level. The EXTAX variable is derived by dividing the per capita local government expenditures by the per capita income taxes. High taxes tend to deter inmigration. But, what might be important determinant of migration behavior is the proportion of the tax which is put back in the form of public services. EXTAX is the amount of local public service per capita that a tax payer would get per unit of income tax he/she pays. Thus, normally, one would expect that high EXTAX would encourage inmigration. 
Finally, the coefficient on INMGt-1 is negative and statistically significant indicating convergence in the sense that counties with initial low level of in-migration at the beginning of the period tend to show higher rate of growth of INMGR than counties with high initial gross in-migration conditional on the other explanatory variables in the model.

\section{Gross Out-Migration Growth Rate:}

The results from the gross out-migration growth rate equation also show very strong interdependences among the endogenous variables of the model. These strong feed-back simultaneities are indicated by the statistically significant coefficients on the respective endogenous variables. The coefficient on EMPR, for example, is positive and statistically significant at the one percent level. The coefficient on INMGR is negative and statistically significant at the five percent level. The implications of these two results are discussed in the EMPR and INMGR equations, respectively. The results also show negative and statistically significant (both at the one percent level) coefficients on MHYR and DGEXR. A negative and statistically significant coefficient on MHYR indicates that Appalachian counties that registered high median household income growth rates tend to experience relatively small gross out-migration growth rates. This is consistent with economic theory and the results of the human capital based migration literature. Economic theory postulates that economic condition affects migration behavior and the relevant income measure for a potential migrant to consider is the present discounted value of his/her stream of expected future returns, both current income level and expected future levels enter into potential migrant's present-value calculation. Thus, areas/counties with relatively high median household income growth rate are expected not only to 
attract potential in-migrants but also keep potential out-migrants from migrating out. This would imply that counties with relatively high MHYR tend to experience lower gross out-migration growth rates, other things remain constant. The result in this study also gives support to Greenwood $(1975,1976)$ who found that high income localities experienced significantly less gross out-migration.

The negative and statistically significant coefficient on DGEXR is also an indication that the growth rate of gross out-migration from a given county is inversely related to the growth rate of direct local government expenditures in that county. This is also consistent to economic the expectations of economic theory and empirical findings in the migration literature. Economic theory postulates that migration behavior is affected by the site characteristics of alternative location and that humans migrate in order to consume non-traded goods or location-specific goods such as health care, education, fire protection, crime prevention, etc. Since the provision of such site attributes are associated with the public sector, local government expenditures per capita are likely to provide indicators of the present and the expected future public service levels of a given county. Thus, counties with high rate of growth of direct local government public expenditures are expected to experience small rate of growth of gross out-migration. The result in this study also give support to the findings in Herzog and Schlottmann (1986) which concluded that local government expenditures on education, recreational accessibility and lower tax rates significantly reduce the probability of out-migration.

Turning to the spatial autoregressive lag and spatial cross-regressive lag effects, the coefficient on the spatial autoregressive lag variable is no significant which indicates the absence of spatial autocorrelation with respect to the growth rate of gross out- 
migration. This suggests that gross out-migration growth rate in one counties has no impact on gross out-migration growth rates in its neighbors. As discussed above, one of the factors that determine gross out-migration growth rate in a given county is its labor market characteristics. No feedback simultaneity between neighboring counties gross outmigration growth rate, therefore, tends to suggest that the economies of Appalachian counties are not integrated as far as their labor markets are concerned. With respect to spatial cross-regressive lags simultaneities, the results, however, show that while WEMPR and WDGEXR have strong positive effects, WMHYR had strong negative effect on OTMGR. The coefficients on WEMPR and WDGEXR, for example, are positive and statistically significant at the one and five percent levels, respectively. These results are consistent with theoretical expectations and empirical findings. As discussed above, an increase in the employment growth rate in a county induces in-migration to that county by more than the increase in the rate of growth of employment - consistent with Todaro's thesis, which is likely to increase the rate of growth of gross out-migration in neighboring counties. An increase in the rate of growth of direct local government expenditures is also likely to increase the rate of growth of gross out-migration in neighboring counties because people migrate to that county in order to consume the nontraded public goods. Contrary to theoretical expectations, the coefficient on WMHYR is negative and statistically significant at the one percent level. Macroeconomic theory postulates that humans migrate out from areas with slow rate of growth of median household income/ per capita income to areas with relatively higher rate of growth of income. Accordingly, one would expect that an increase in median household income in neighboring counties to increase the rate of growth of gross out-migration in a given 
county. The result in this study, however, does not give support to such expectations. One possible reason why this might be so is that potential migrants may still be able to benefit from the increases in neighboring counties' income by commuting a cross county borders.

The results in Table 5.3.2a also suggest a positive parameter estimate for rho3 indicating that random shocks into the system with respect to gross out-migration do not only affect the county where the shocks originated and its neighbors, but create positive shock waves across Appalachia.

Similar to the case of in-migration growth rate equation, the coefficients on initial population size (POPs) is positive and statistically significant at the one percent level. This result indicates that counties with high initial population sizes have experienced high gross out-migration growth rates.

Consistent with theoretical expectation, the impact of home ownership on gross out-migration growth rate is negative but not significant. Normally, one would expect that owing a house to decrease the propensity to migrate due to the transaction cost and liquidity of real estate in location of economic distress. Investing in own housing may also reflect a decision to stay in the area of current residence for long. Similar to the nonspatial cross-sectional analysis, the coefficient on UNEMP shows an unanticipated sign and yet statistically significant at the one percent level. Normally, one would expect that people to move away from high-unemployment counties to low-unemployment counties. The result in Table 5.3.2a, however, suggests that the growth rate of out-migration (OTMGR) in a given county is negatively associated with the initial level of unemployment in that county. One possible explanation of this observation, similar to 
what Lansing and Mueller (1967) have argued, is that unemployment tends to be highest in the least mobile groups in the labor force. It should also be noted that prospective unemployment rather than the level of unemployment rate is the major determinant of migration. Besides, the lack of rental housing in the potential in-migration counties/regions could deter out-migration from the high-unemployment counties/regions.

Contrary to theoretical expectations, the coefficient on the NAIX has the wrong sign and yet statically significant at the ten percent level.. Normally, one would expect NAIX to have negative influences on OTMGR But, it is important to note that migrants are usually motivated by the altered demand for amenities that are sight-specific. In this respect, amenity data at the county level are highly aggregated and may not reflect the true interdependence between OTMGR and NAIX. The coefficient on EXTAX has the expected sign but not significant.

Finally, the results presented in Table 5.3.2a indicate the existence of significant conditional convergence in the out-migration growth rate equation. This is indicated by the negative and statistically significant coefficient on the lagged dependent variable for out-migration (OTMGt-1). This result suggests that Appalachian counties with low initial level of out-migration showed higher growths in out-migration growth rates compared to counties with higher initial levels of out-migration, conditional upon the other exogenous variables that are included in the OTMGR equation.

\section{Median Household Income Growth Rate:}

The interdependences among the endogenous variable are also witnessed in the MHYR equation. The coefficient on EMPR is positive and statistically significant at the 
one percent level, indicating that MHYR in a given county is positively and strongly affected by the rate of growth of employment in that county. This is consistent with theoretical expectations. Higher rate of growth of employment means higher employment opportunities, which in turn provide a strong attraction for migrants that leads to net inmigration. The contemporaneous effect with respect to the rate of growth of outmigration on the rate of growth of median household income is also positive and statistically significant at the one percent level. This result suggests that median household income increases with out-migration. This is consistent with theoretical expectations. Migration from or to a given county influences labor demand as well as labor supply in that county. Out-migration from a given county, for example, decreases labor supply in that county, putting upward pressure on wages and incomes in that county, provided labor-demand function is not infinitely elastic. The results in this study also give support to empirical findings in Aronson et al. (2001), which indicate that the out-migration of unemployed persons changes the population composition such that average income increases for a given structure of wage among the employed. This, in turn, would mean that the average income of the out-migrants is lower than the median income of the non-movers. The contemporaneous effect with respect to the growth rate of in-migration on the growth rate of median household income, however, is positive but statistically insignificant. If migrants' endowments of human capital in the form of education, accumulated skills, or entrepreneurial talents are higher compared to the receiving population, then their skills, inventiveness and innovativeness would contribute to local productivity. Migrants may also own physical and financial capital that they may bring with them and invest in the receiving county. Moreover, migrants may contribute to 
the growth of markets and to the achievement of scale and agglomerations economies. Such demand effects are the sources of growth in per capita personal incomes. The results in this study, however, do not strongly show the existence of such migrantinduced labor demand shifts that offset the migrant-induced labor supply shifts in Appalachian counties during the study period.

Concerning the relationship between the rate of growth of direct local government expenditures and the rate of growth of median household income, the results show that the rate of growth in direct local government expenditures has strong negative impact on the rate of growth of median household income. This is indicated by the negative and statistically significant, at the one percent level, coefficient on DGEXR in the MHYR equation. This may seem to be inconsistent with theoretical expectations. But as discussed elsewhere in this study, the effects of government expenditure depend on the nature/type of that expenditure. Government expenditures on education, health care, fire protection, crime prevention, are more likely to increase labor productivity and hence income. On the other hand, government expenditures on unemployment insurance, welfare payments, etc. have disincentives to work and are more likely to reduce labor productivity and hence income. The results in this study reflect this reality in Appalachia. Traditionally, Appalachia has had higher than average payments from federal assistance programs such as Food Stamps, Social Security Disability Insurance (SSDI), and Temporary Assistance for Needy Families (TANF) and Supplemental Security Income (SSI) (Black and Sanders, 2004). Studies also show that income from Social Security makes up a larger portion of income in Appalachia than in the United States (Thorne, Tickamyer, and Thorne, 2004). Combining these two facts about Appalachia would 
enable one to suggest that increases in the rate of growth of local government expenditures puts downward pressure on the rate of growth of median household income, by encouraging welfare- recipient induced in-migrations, and by creating disincentive to work among the welfare recipients who have lower levels of median household income. The result in this study is also consistent with empirical findings in Dye (1980), Helms (1985) and Jones (1990) which showed that government expenditures in the form of welfare spending have negative and statistically significant impacts on per capita personal income growth rates.

The results in Table 5.3.2a also suggest a positive and statistically significant, at the one percent level, spatial autoregressive lag effect, indicating that the rate of growth of median household income in a given county is positively affected by the rate of growth of median household income in neighboring counties. This strong spatial spillover effect is an indication that there is clustering of counties in Appalachia on the bases of their growth rate of median household incomes. The exploratory spatial data analysis on the same data set, presented in the appendix, shows most of the low income counties are clustered in Central Appalachia, whereas the high income counties are clustered, mostly around big cites, in the Northern and Southern Appalachia sub regions. The spatial crossregressive lag effects with respect to WEMPR, WINMGR, and WOTMGR are not strong, indicating that WMHYR in a given county is not strongly related to WEMPR, WINMGR, and WOTMGR in neighboring counties. The spatial cross-regressive lag effect with respect to WDGEXR is, however, positive and significant. This is indicated by the positive and statistically significant, at the five percent level, coefficient on WDGEXR in the WMHYR equation. This result suggests that increases in the rate of 
growth of local government expenditures in neighboring counties tend to increase the rate of growth of median household income in a given county. This is possible because government expenditures, for example, in highways, crime protection, pollution control, may have positive cross border effects that could benefit residents on the other side of the county border. Since increases in the rate of growth of local government expenditures are associated with increases in the rate of growth of employment or business in the own county, residents from across the border could commute and work in that county. This may increase the average income of those who commute and consequently, the rate of growth of median household income in the sending county (neighboring county) may increase.

The results in Table 5.3.2a also suggest a positive but weak parameter estimate for rho4 indicating that random shocks into the system with respect to median household income do not only affect the county where the shocks originated and its neighbors, but create negative shock waves across Appalachia., though weak.

As expected, the coefficient on the variable that measures the proportion of the population 25 years and above with high school or above diploma (POPHD) is positive and statistically significant at the one percent level. This implies that Appalachian counties with higher proportion of adult residents with at least high school diplomas at the beginning of the period show subsequent growth in MHYR, compared to counties with low initial POPHD's. This result is consistent with the expectations of economic theory as well as with the empirical findings in growth literature. Human capital theory postulates that entrepreneurship is related to educational attainment and work experience. People with more educational attainment tend to found business and also have more 
probability of getting and securing higher paying jobs. The results in this study are also consistent with the empirical findings in Romer (1986), Lucas (1993), Krugman (1991a), Rauch (1993), Glaeser et al. (1995), Duffy-Deno and Eberts (1991) and Simon and Nardinelli (2002), which indicate that growth in per capita income is associated, one way or the other, with the educational and human capital endowments of a given region/area.

Although industrial restructuring has led to a shift from manufacturing to service based industries, the process has been low in Appalachia and manufacturing remained as a major source of income compared to service industries. The positive and statistically highly significant coefficient on MANU in the MHYR equation supports this assertion. Note, however, that this does not mean that manufacturing remained as a major employer during that period. Actually, as explained above, the declining trend in manufacturing employment is supported by the results of this study. As expected, the coefficient on FHHF and UNEMP in the MHYR equation have the right sings (negative), but fail to be significant.. POPs and SCIX are also negatively associated with MHYR, but fail to be significant.

Finally, the negative and statistically significant coefficient on MHYt-1 is an indication that there was conditional convergence with respect to the rate of growth in median household income in Appalachia during the study period. This means that counties with low initial median household income grew faster than counties with higher initial median household income.

\section{Direct Government Expenditures Growth Rate}

Similar to what we have in the other equations, the estimates from the DGEXR equation show the existence of significant feed-back simultaneity. Three of the 
endogenous variables have statistically significant effect on the growth rate of direct local government expenditures per capita. The contemporaneous effect with respect to the rate of growth of out-migration (OTMGR) on the rate of growth of direct local government expenditures per capita, for example, is positive and statistically significant at the one percent level. This result indicates that high growth rate in direct local government expenditures per capita is positively associated with high growth rate of gross outmigration which is consistent with the expectation of economic theory. Migrants have important impacts on the demand of locally provided public goods and services as well as on the revenue that support the provision of these public goods and services by changing the size and the density of population of a region or a county. Out-migration reduces the possibility of gaining economies of scale in the provision of public services. Excessive out-migration creates excess capacity and very high costs of maintaining overstock of public infrastructure, such as schools, police facilities, fire protection, etc., in the area of origin. The contemporaneous effect with respect to the growth rate of in-migration (INMGR) on the growth rate of direct local government expenditures per capita is negative and statistically significant at the one percent level. This result indicates that the growth rate of direct local government expenditures per capita in a given county is negatively associated with the growth rate of in-migration to that county. One possible explanation for this observation is that in-migration may lead to increase in population and its density in the receiving region that enable local government to realize the advantages of economies of scale in the provision of public services. In that case, although total local government expenditures may increase, per capita could still decline if the advantages of economies of scale are realized. The contemporaneous effect with 
respect to the growth rate of employment (EMPR) on the growth rate of direct local government expenditures per capita is also negative as expected, but statistically insignificant.

The coefficient on MHYR is negative and statistically significant at the one percent level. This result is not consistent with theoretical expectations. Increases in per capita income provide local governments with more tax revenues that support the provision of more public goods and services, which in turn lead to higher local public expenditures. In the context of Appalachia, the result from this study is not unrealistic. As discussed in the subsection on 'Median Household Income Growth Rate', to the extent welfare payments constitute the biggest of local government expenditures in Appalachia, increases in the rates of growth of median household incomes are expected to lead to decreases in the rates of direct local government expenditures.

As expected, the results in Table 5.3.2a also show the existence of strong and positive spatial autoregressive lag effect with respect to DGEXR, as indicated by the positive and statistically significant, at the one percent level, coefficient on WDGEXR in the DGEXR equation. This result shows that the rate of growth of direct local government expenditures in a given county is positively associated with the rates of growth of direct local government expenditures in neighboring counties. These interdependences could arise because (1) local governments may finance public spending through a tax on mobile capital and since the level of tax base in a jurisdiction depends both on own and on other jurisdictions' tax rates, strategic interaction results; (2) beneficial or harmful effects could spill over onto residents of neighboring counties from expenditures on local public services in a given count; and (3) imperfectly informed 
voters in a given county use the performance of other governments as a yardstick to evaluate their own governments, which, in turn, lead to local governments to react to the action of their neighbors, resulting in local governments mimicking each others' behavior. The result in this study gives support to the findings in Case, Hines and Rosen (1993), Kelejian and Robinson (1993), and Besley and Case (1995) which indicate public expenditures in a given county is positively and significantly affected by public expenditures in neighboring counties.

As it is also indicated by the Global Moran's I Statistics presented in Tables 5.3.1a \& $\mathrm{b}$, the spatial cross-regressive lag effects with respect to the other endogenous variables of the model are weak. Only WINMGR has marginally significant coefficient. This indicates that the rate of growth of direct local government expenditures per capita in a given county is not very much affected by its neighbors' EMPR, OTMGR, and MHYR.

The results in Table 5.3.2a also suggest a negative parameter estimate for rho5 indicating that random shocks into the system with respect to direct local government expenditures per capita do not only affect the county where the shocks originated and its neighbors, but create negative shock waves across Appalachia.

The proportion of school age population denoted by POP5-17 is included in the model to control for the differential impact of population age structure on local government expenditures. As expected, the coefficient on POP5-17 is positive and statistically significant. Increases in the proportion of school age population create pressure for increases in local spending on education, in the form of expanding services and cost of expanding capacity. The results in this study are also consistent with the 
empirical findings in Marlow and Shiers (1999) and Alhin and Johansson (2001) which indicate that an increase in the proportion of young people generates pressure for increases in public spending in education.

As expected, the coefficients on DFEG (direct federal expenditures and grants per capita), and PCTAX (per capita income tax per capita) and LTD (long-term debt per capita) are all positive and statistically significant at the one level. Since both DFEG is one of the components of local government revenue, it is expected to have positive effects on the rate of growth of direct local government expenditures per capita. Thus, the results in this study are consistent with the expectations of economic intuition. The results also give support to empirical finding in Fisher and Navin (1992) and Henderson (1968) which show that local public expenditure per capita is positively related to grants in-aid per capita from higher governments. Similarly, since PCTAX is also one of the components of local government the revenue, increases in PCTAX would provide local government with more money to spend on local public services. To control for the impacts of the ability of local government to borrow from external sources in order to finance the provision of local public services, LTD (Long-Term Debt per capita) is also included in the model. A positive and significant coefficient on LTD means, local governments in Appalachian counties were not constrained in their capacity to borrow from external sources in order to finance local public services. Note, however, that since the coefficient is small, the net positive effect may not be big.

The coefficient on PCTD (total debt outstanding per capita) is negative and statistically significant at the one percent level. This result is consistent with theoretical expectations in that the amount of total debt outstanding accumulated constrains local 
governments their capacity to further borrow apart from their obligation to pay their debts now. The effect would be to decreases in local public expenditures, but since the coefficient is small, the net impact may not be large.

Finally, the negative and statistically significant coefficient on DGEXt-1 is an indication that there was conditional convergence with respect to the rate of growth in direct local government expenditures in Appalachia during the study period. This means that counties with low initial direct local government expenditures had higher growth in direct local government expenditures than counties with higher initial direct local government expenditures. 


\section{CHAPTER VI}

\section{SUMMARY AND CONCLUSIONS}

\subsection{Introduction}

In an effort to analyze the interdependences among small business growth, migration behavior, local public services and median household income, this study developed a simultaneous-equation system under the assumptions of profit maximization of firm and utility maximization of households as well as the neoclassical assumption of equilibrium growth in a partial lag-adjustment growth-equilibrium framework. This model is an extension of the "jobs follow people or people follow jobs" literature and it improved previous models in the growth-equilibrium tradition by explicitly modeling local government and regional income in the growth process. It also explicitly modeled gross in-migration and gross out-migration separately in order to spell out the differential effects, which used to be glossed over under net population change in previous studies.

Test for spatial effects showed that the underlying data generating process includes spatial dimension. To incorporate these spatial spillover effects, the standard model is also further extended both in the cross sectional and panel data setting. Apart from the feedback simultaneities, the models now include spatial autoregressive lag and spatial cross-regressive lag simultaneities. The models are also tested for the presence of spatial autocorrelation in the error terms using Moran's I test. The existence of both types

of spatial dependences in all equations of the system led to the specification of the system in terms of spatial cross-sectional and spatial panel data models that incorporate both spatially autoregressive dependent variables and spatial autoregressive process in the error terms. The spatial models are estimated by Generalized Spatial Three-Stage Least 
Squares (GS3SLS) Estimator. Detailed separate computer programs are written in TSP to run the five-equation spatial simultaneous equations model in cross-sectional and panel data setting. Both the modeling and the estimation strategies are significant improvements and contributions to the existing literature in spatial econometrics. The simultaneous spatial panel data model estimation is new addition. There is no research so far which used this technique in empirical work. The implementation of the model with five-equations even in a single cross-sectional data set is a major improvement over previous efforts.

The empirical implementation of the model used county-level data from the 418 Appalachian counties for 1980-2000. Both single equations and system of equations methods of estimation are employed to estimate the standard as well as the spatial simultaneous equations models. In the standard (non-spatial) simultaneous equations model, the estimation for cross-sectional analyses is carried in EViews using standard built-in functionalities. The estimation of the standard simultaneous panel data model and both the spatial cross-sectional and spatial panel simultaneous equations models, however, required the development of special programs. The codes for these programs are written in TSP. The spatial regression analyses are preceded by exploratory data analyses which aimed at identifying spatial pattern/or spatial clustering in the data sets. In this respect, ArcGIS and GeoDa are used to calculate Moran's I of Global Spatial Autocorrelation and Local Indicators of Spatial Association (LISA) for the endogenous variables of the models. 


\subsection{Concluding Summaries of Results}

Generally, the results from these model estimations are consistent with the theoretical expectations and empirical findings in the equilibrium growth literature and provide support to the basic hypotheses of this study. First, both the spatial and nonspatial models estimates showed the existence of feedback simultaneities among the endogenous variables of the models. This is especially true for the spatial panel model where the coefficients on the endogenous variables in almost all equations of the model are statistically significant at least at the five percent levels. This indicates that the interdependences among employment growth rate, gross in-migration growth rate, gross out-migration growth rate, median household income growth rate and direct local government expenditures growth rate are very strong. The directions of causation as indicated by the signs of the coefficients are also consistent with the theoretical expectations.

Second, results from both the spatial and the non-spatial model estimations also showed the existence of conditional convergence with respect to the respective endogenous variable of each equation of the models. This is indicated by the negative and statistically highly significant coefficients on the lagged dependent variables of the models. This implied that the rates of growth of employment, gross in-migration, gross out-migration, median household income and direct local government expenditures were higher in counties that had low initial levels of employment, gross in-migration, gross out-migration, median household income and direct local government expenditures, respectively compared to counties with high initial levels of the same. 
Third, the results from the parameter estimation of spatial models and from the exploratory spatial data analysis indicated the existence of spatial autoregressive lag effects and spatial cross-regressive lag effects with respect to the endogenous variables of the models. Besides, results for Global Moran's I statistics indicated the existence of spatial spillover effect with respect to the error terms of the spatial models. These results would imply that employment growth rate, gross in-migration growth rate, gross outmigration growth rate, median household income growth rate, and direct local government growth rate in a given county are dependent on the averages of employment growth rates, gross in-migration growth rates, gross in-migration growth rates, median household income growth rates, and direct local government growth rate of neighboring counties in the study area. These results are also important from the economic and policy perspectives because they indicate that each of the dependent variables in the model is not only dependent on the characteristics of that county but also on the characteristics of those of its neighbors. Thus, spatial effects should be tested for in empirical works involving EMPR, INMGR, OTMGR, MHYR and DGEXR. The existence of spatial dependences in the error terms is an indication that random shocks into the system with respect to each of these endogenous variables do not only affect the county/counties where the shock originated and its/their neighbors, but also create shocks waves across the study area (Appalachia). This is possible because of the structure of the autoregressive error model.

The existence of spatial dependences in the dependent variables and the error terms of the models would mean, retroactively, the spatial estimation methods which account for such spatial spillover effect tend to give more consistent, efficient and 
unbiased coefficient estimates compared to the non-spatial methods that are considered in this study. The summary and conclusions in this study are, therefore, mainly based on the coefficient estimates of the spatial panel model.

In the growth rate of employment (EMPR) equation, EMPR is positively associated with the growth rates of gross in-migration, gross out-migration, median household income and direct local government expenditures. This is consistent with the theoretical expectations in that (1) in-migrants could be the sources of labor and capital for business expansion and hence employment; (2) increase in median household income could be the source of demand for new businesses and business expiation; (3) direct local government expenditures in the form of highways, crime protection, schools, and on public health could have positive effects on firm location and business expansion; and (4) a positive effect of the growth rate of gross out-migration on EMPR is possible because since OTMGR is positively and highly associated with county-population size, OTMGR might pick up the effect of population size on employment. The results also suggested a negative autoregressive lag effect indicating the growth rate of employment in a certain county tends to spillover to neighboring counties and has negative effects on their growth rates of employment. This could happen because of the competition for consumer demand. This conclusion is also supported by the negative and statistically significant coefficient on ESBd (total number of establishments per capita) variable, which indicates that Appalachian region has reached the threshold where competition among firms for consumer demands crowds businesses. The negative spatial autoregressive lag effect indicates that the competition is not confined to the home county only. Access to shopping centers across county borders makes this possibility an empirical reality. 
The results from the EMPR equation also showed that growth rate of employment in a given county is positively and highly associated with the initial levels of the proportion adult population between 25 and 44 years of age (POP25-44), the percentage of adult population with a college degree (POPCD), the proportion of civilian labor force employed in manufacturing (MANU), the proportion of the civilian labor force employed in wholesale and retail trade (WHRT), natural amenity index (NAIX), and county high way density (HWD). All these results are consistent with the theoretical expectation and empirical findings. The impact of POP25-44 associated with the agglomerative effect of population on business growth. Educational attainment is also positively associated with business growth because more educated people tend to have more access to research and development facilities, good insights to the business world and thus clear ideas about the present and the future needs of the market, which in turn enable them to establish businesses and to be successful when they do. Besides, more educated people would mean more human capital embodied in their general and specific skills, for implementing new ideas, for creating and growing new businesses. These results would suggest that Appalachian region or counties with different levels of human capital endowment and different propensities of locally available knowledge to spill over and stimulate new firm formation tend to have different rates of new firm formation, survival and growth. Although both MANU and WHRT showed positive effect on the growth rate of employment of a given county, considering their coefficients and the associated levels of significances, WHRT had more impact than MANU did. These results, nonetheless, indicate that Appalachia had experienced a shift from coal miningbased economic activities to manufacturing and even more to service-based economic 
activities during the study periods. These results also suggest that the contribution of WHRT to the overall growth rate of employment was higher and more sustained than MANU did.

Although road quality differences are not accounted for in this study, the results indicated that increases in road density had positive and significant impacts on the growth rate of employment. Transportation is a critical bottle neck in the growth and development of business activities in a given area. Cost reduction as the result of the availability of roads and the increase in consumer demand that results from increased access to shopping centers boosts businesses.

Consistent with the theoretical expectations and empirical findings, the coefficient on FHHF is negative and statistically significant at the one percent level, indicating that FHHF is negatively associated with EMPR. Thus, this result suggests that Appalachian counties with higher proportion of female household header families in their communities tended to show low growth in business or employment during the study periods. Female householder families tend to have low human capital, low income and low labor participation rate. Hence, FHHF affects both the supply-side (as source of labor input) and the demand-side (as source of demand for consumer goods) of the market.

Turning to the growth rate of gross in- migration equation, the results showed that the growth rate of gross in-migration in a given county is positively associated with the growth rate of employment in that county. Further inspection of the results showed that the attractive affects of EMPR on INMGR are stronger than the effects of INMGR on EMPR creating a Todaro type migration pattern: The coefficient on EMPR in the 
INMGR equation is greater than one which indicates that a single job opening tended to lead to more than one in-migrant., holding other things to remain constant..

The results also indicated that there existed a strong inverse relationship between the growth rate of gross in-migration and the growth rate of gross out-migration in Appalachian counties during the study periods. This would mean that job seekers in Appalachia move away from high-unemployment counties where they cannot find jobs to low-unemployment counties where the prospect for finding employment are more favorable. This finding implies that the driving force for in-migration into and outmigration from a given county is linked to the labor market characteristics of that county and in-migrant and out-migrants have the same labor market characteristics. Thus, migration acted as an equilibrating factor in Appalachia labor markets during the study periods.

The negative coefficients on the growth rate of median household income and the growth rate of direct local government expenditures per capita in the growth rate of gross in-migration equation indicate that in-migrants tended to prefer low-income and low-tax counties. Since low-income counties, however, has high propensities to levy high taxes in order to finance local public services at certain levels, the net effect depends upon the respective strengths of the marginal effects.

With respect to spatial spillover effects, the results indicated that the growth rate of gross in-migration into one county is positively associated with the growth rate of employment in neighboring counties. This finding indicates that people commute to neighboring counties to work, but as people commute to neighboring counties to work, employment/business in those neighboring counties expands and attracts in-migrants. The 
flow of in-migrants into neighboring counties further leads to business/employment expansion in those counties. Since, as discussed above, the growth rates of employment in neighboring counties are inversely related, the counties whose residents are commuting to the neighboring counties for work, might face a lower growth rate in employment/business.

Concerning the effects of exogenous variable on the growth rate of gross inmigration, the results showed that INMGR is positively associated with the initial county population size (POPs), the median cost of renter occupied housing (MCRH), natural amenity index (NAIX), and the amount of local public expenditures per unit of income tax per capita (EXTAX). All these results except for MCRH are consistent with the theoretical expectations. The positive effects of population size are through its agglomerative effects that create favorable conditions for business expansion and employment, which, in turn, attract in-migrants. The positive effect of NAIX is an indication that amenity based migrations are important in Appalachia during the study periods. The positive effect of EXTAX is also an indication that tax payers are more responsive to the amount of local public services per capita that they could get for every unit income tax they pay in Appalachia during the study periods. Finally, the positive effects of MCRH indicate that the positive effects of the availability of housing dominate the negative effects of the cost of rental housing in the migration potential destination counties. The negative effects of county unemployment rate on the growth rate of gross in-migration that this study showed is also consistent with the expectations of economic theory. Regional UNEMP represents a slack labor market and deters in-migration. Thus, 
Appalachian counties with high initial UNEMP experienced lower growth rate of inmigration during the study periods and vice versa.

The coefficients on the variables in the growth rate of gross out-migration equation were also mostly consistent with the theoretical expectations. The negative coefficient on MHYR indicates that counties with high growth rate of median household income more likely to experience lower growth rate of gross out-migration, consistent with the human capital-based migration literature. The negative coefficient on DGEXR also indicates that counties with high growth rate of direct local government expenditures per capita are more likely to experience low growth rate of gross out-migration. Thus, Appalachian counties with high local government expenditures per capita, especially on location-specific public goods such as health care, education, fire protection,, etc., are more likely to keep potential out-migrants from migrating.

Concerning the spatial autoregressive and cross-regressive lags effects, the results indicated absence of spatial autoregressive lag effect and positive spatial cross-regressive lags effects with respect to WEMPR and WDGEXR and negative spatial cross-regressive lag effect with respect to WMHYR. The absence of spatial autoregressive lag effects in both the INMGR and OTMGR equations suggests that the economies of Appalachian counties were not strongly integrated as far as their labor markets are concerned. The positive coefficients on WEMPR and WDGEXR indicate that counties surrounded by counties with high growth rates of employment and direct local government expenditures per capita are more likely to experience high growth rates of gross out-migration. The negative coefficient on WMHYR, on the other hand, is an indication that potential out- 
migrants from a given county benefit from the increases in neighboring counties' incomes by commuting across the county's borders.

The results from the median household income (MHYR) equation are also mostly consistent with the theoretical expectations. The results showed that counties with higher growth rate of employment are more likely to experience higher growth rates of median household incomes. This means that the average payments for the new jobs in a given county are more than the median household income. The results also showed that counties with higher growth rates of out-migration had higher growth rates of median household income. This is possible because out-migration from a given county tends to decrease labor supply in that county, putting an upward pressure on wages and incomes in that county. The negative coefficient on DGEXR in the MHYR equation is an indication that direct local government expenditures per capita in Appalachia are mostly concentrated on non-labor productivity enhancing expenditures such as welfare and unemployment insurance payments.

The positive coefficient on the spatial lag variable (WMHYR) indicates that there are clustering of counties in Appalachia on the bases of their growth rates of median household incomes. The results from the exploratory spatial data analysis also showed most of the low income counties are clustered in Central Appalachia, whereas the high income counties are clustered, mostly around big cites, in the Northern and Southern Appalachia sub regions. The results also showed that the growth rate of direct local government expenditures per capita (DGEXR) had positive spatial cross-regressive lag effects on the growth rates of median household income in Appalachian counties during the study periods. This is possible because government expenditures, for example, in 
highways, crime protection, pollution control, may have positive cross border effects that could benefit residents on the other side of the county border. Since increases in the rate of growth of local government expenditures are associated with increases in the rate of growth of employment or business in own county, residents from across the border could commute and work in that county. This may increase the average income of those who commute and consequently, the rate of growth of median household income in the sending county (neighboring county) may increase.

The results from the MHYR equation also indicated that Appalachian counties with high proportion of adult residents with at least high school diplomas at the beginning of the period show subsequent growth in MHYR, compared to counties with low initial POPHD's. This implies that people with more educational attainment tend to establish business and also have more probability of getting and securing higher paying jobs.

The results from DGEXR equation are also mostly consistent with the theoretical expectations. The results indicated that high growth rate of direct local government expenditures per capita is positively associated with high growth rate of gross outmigration. This is possible because migrants have important impacts on the demand of locally provided public goods and services as well as on the revenue that support the provision of these public goods and services by changing the size and the density of population of a region or a county. Out-migration reduces the possibility of gaining economies of scale in the provision of public services. Excessive out-migration creates excess capacity and very high costs of maintaining overstock of public infrastructure, such as schools, police facilities, fire protection, etc., in the area of origin. 
The results also indicate that the growth rate of direct local government expenditures per capita in a given county is negatively associated with the growth rate of gross in-migration into that county. One possible explanation for this observation is that in-migration may lead to increase in population and its density in the receiving region that enable local government to realize the advantages of economies of scale in the provision of public services. In that case, although total local government expenditures may increase, per capita could still decline if the advantages of economies of scale are realized.

The negative coefficient on MHYR in the DGEXR equation indicates that Appalachian counties with high growth rates in median household income are more likely to experience low growth of direct local government expenditure per capita. This is realistic for Appalachia because welfare payments constitute the biggest share of local government expenditures of Appalachia counties.

Concerning the spatial autoregressive lag effect, the result shows that the rate of growth of direct local government expenditures in a given county is positively associated with the rates of growth of direct local government expenditures in neighboring counties. These interdependences could arise because (1) local governments may finance public spending through a tax on mobile capital and since the level of tax base in a jurisdiction depends both on own and on other jurisdictions' tax rates, strategic interaction results; (2) beneficial or harmful effects could spill over onto residents of neighboring counties from expenditures on local public services in a given count; and (3) imperfectly informed voters in a given county use the performance of other governments as a yardstick to evaluate their own governments, which, in turn, lead to local governments to react to the 
action of their neighbors, resulting in local governments mimicking each others' behavior.

\subsection{Policy Implications}

The empirical findings in this study suggested the existence of significant feedback simultaneities among the growth rates of employment, gross in-migration, gross outmigration, median household income, and direct local government expenditures per capita in Appalachian counties during the study periods. This finding is important from economic policy perspective because it indicates that sector specific policies should be integrated and harmonized in order to achieve the desirable outcome. Under this circumstance, looking at the direct impact of a change in a given policy can not tell the whole story. What is more important is the total (direct plus indirect) impact of a change in a given policy.

The results in this study also showed the existence of spatial autoregressive lag and cross-regressive lag simultaneities among the data set with respect to the growth rates of employment, gross in-migration, gross out-migration, median household income, and direct local government expenditures per capita. These findings are also important from an economic perspective because the existence of these spatial lag effects indicates that the growth rates of employment, gross in-migration, gross out-migration, median household income, and direct local government expenditures per capita in a given county are not only dependent on the characteristics of that county, but also on that of its neighbors. This further indicates for the need to do spatial effect tests in empirical research works involving the growth rates of employment, gross in-migration, gross outmigration, median household income, and direct local government expenditures per 
capita. These findings are also important from a policy perspective as they indicate crosscounty interdependences among the growth equilibrium model endogenous variables which would necessitate economic development policy coordination at the regional level. A region, here, could be a group of counties with similar socio-economic conditions or the whole Appalachia region. Poverty reduction policies, for example, may be better coordinated among counties in Central Appalachia, where there is high concentration of poverty compared to the other sub-regions. But it is also important to note that the whole Appalachia may be affected by the ripple effect- a neighbor of my neighbor type. The weights matrix is designed to account for these types of effects.

The results in this study also support the proposition which is summarized by the cycle of poverty diagram given in chapter IV. It was also theoretically argued that one way of breaking such cycle of poverty is through the promotion of small business. As discussed above, the results in this study show a positive interdependence between the growth rate of employment (the proxy for small business growth) and the growth rate of median household income (the proxy for poverty rate). Given the fact that Appalachia is dominated by widely dispersed small communities with relatively small local and regional markets, these results are significantly important. This implies that local government actions that promote small business can have significant effects on poverty reduction. This is also supported by the simulation results in this study as shown in Table 6.1 below. A one percentage increase in direct federal expenditures and grants per capita leads to 364.79 and 1163.03 percentage increases in the growth rate of employment and the growth rate of median household income, respectively, in Appalachia during the 
study periods. ${ }^{6}$ Thus, higher governments should increase their direct expenditures in the local economies and their grants-in-aid to local governments in order to address the problems of poverty and underdevelopment in Appalachia. Similarly, a one percentage increase in road density leads to 202.73 and 181.74 percentage increases in the growth rate of employment and the growth rate of median household income, respectively, in Appalachia during the study periods. Hence, local government expenditures on road improvements and expansions should increase in order to help the local economies. The simulation results also indicate that a one percentage increase in the amount of local government expenditures per capita for every dollar paid in per capita income tax which is put back into the economy in the form of local public services, such as health care, education, etc. leads to 367.30 and 156.54 percentage increases in the growth rate of employment and the growth rate of median household income, respectively, in Appalachia during the study periods. Hence, local governments should put back more tax money into the local economies. Note, however, that the nature of local government expenditures is as important as the amount of the expenditures. Local public services which enhance labor productivity and enterprise development might have more direct bearing to poverty alleviation and underdevelopment.

Table 6.1: Simulation Results

\begin{tabular}{lccccc} 
& \multicolumn{5}{c}{ Percentage change in Mean Value of } \\
\hline \multicolumn{1}{c}{ Scenarios } & EMPR & INMGR & OTMGR & MHYR & DGEXR \\
\hline $1 \%$ increase in POPCD & -35.90 & -86.38 & 305.79 & 13.21 & 66.22 \\
$1 \%$ increase in DFEG & 364.79 & 173.45 & -3051.90 & 1163.03 & 339.30 \\
$1 \%$ increase in HWD & 202.73 & -190.50 & 429.80 & 181.74 & 129.12 \\
$1 \%$ increase in EXTAX & 367.30 & 22.04 & 39.49 & 156.54 & 147.02
\end{tabular}

\footnotetext{
${ }^{6}$ Caution should be taken in interpreting these percentage changes. Since the initial values (the solution values for the actual model) are mostly small, the big percentage changes may not be translated into big changes.
} 


\subsection{Contributions and Limitations}

\section{Contributions}

The mythology developed in this study extended the traditional two-equation growth equilibrium models in the "jobs follow people, or people follow jobs" literature. The extensions and improvements made in this study can be summarized as follows:

1. Horizontal Expansion: The roles of local government and regional incomes in the growth process are explicitly modeled by incorporating measures of local government expenditures and regional incomes into the basic model. Besides, the model in this study also explicitly modeled gross in-migration and gross out-migration separately, in order to spell out their differential effect, which used to be glossed over under net population change in previous models.

2. Spatial (Vertical) Expansion: In order to incorporate possible spatial spillover effects, the horizontally expanded standard model is further extended in the spatial sense both in cross-sectional and panel data settings. Spatial autoregressive lags and spatial cross-regressive lags dependent variables are explicitly included in the spatial models. The technical issues associated with this expansion are fully developed and discussed in chapter III of this study.

3. Programs: Since there are no commercially available statistical/econometric package that can run spatial simultaneous-equations models, this study has developed special programs to run the spatially extended models. First, a program to run spatial simultaneous equations model in cross-sectional data setting was developed in TSP. This was further extended to panel data setting. 
The empirical findings in this study also support the findings of previous studies in the equilibrium growth literature. This is expected to strengthen the existing literature in the “jobs follow people, or people follow jobs" tradition.

\section{Limitations:}

Major improvements and extensions over previous similar studies have been registered in this study. It is, however, important to note some limitations in this study. Data limitation: The number of periods in the panel analysis was limited because of data limitation for some important variable. Variables related, for example, to educational attainments, income, etc. are available only for census years. Although data for government expenditures by function are available for the earlier years, it is not available for the 1997 and 2002 census years. This has limited the analysis of this study with respect to the role of local governments. Secondary data also has its own limitations, especially at the county-level. Since a lot of data transformations and approximations have been done, the data may not represent what it purports to represent.

Computing Facilities: Comparative analyses of spatial model estimation results from Appalachian counties, Appalachian States counties, and US counties are not possible because of limitation in computing facilities. The memory required for open space to run the programs, especially the panel data, is beyond the capacity of the computer at hand. Actually, Windows operating system allocates only 2 giga bytes for any application. This system limitation limited the software package from allocating enough memory to run the programs. Thus, unlike what was planned, it was not possible to run the spatial panel model using date from Appalachian States counties and US counties. 


\subsection{Future Research}

Although this study has registered a number of improvements and extensions to previous similar studies, it can still be improved in a number of ways. With better computing facilities, the models developed in this study can easily be applied to data from the whole nation as well as from bigger regions such as Appalachian State counties. With the availability of relevant data, the spatial panel analysis could also be improved.

Writing the codes for the programs in different programming languages such as in EViews and MATLAB is another possibility of improving the estimation strategy. Writing the programs in different programming languages also helps expand the scope of its application by different users.

One section of this study has discussed the tools of exploratory spatial data analysis. These tools can be used to identify spatial processes in any human endeavor. The results from these tools can be incorporated into and integrated with the results from spatial regression analyses to give better results. Thus, another area of future research is applying these tools to sub-county-level analysis in the current study area and beyond. The outcomes of such analyses can be used to better articulate policies to fit to specific area realities. Possible areas of research using this methodology may include, mentioning but a few, water quality management, waste disposal and pollution control programs, sustainable usage of local natural resources, such as forests, minerals, land, etc. 


\section{REFERENCES}

Acs, Z. J. and C. Armington, 2004a, "The Impact of Geographic Differences in Human Capital on Service Firm Formation Rates," Journal of Urban Economics, 56: 244278.

Acs, Z. J. and C. Armington, 2004b, " New Firm Survival and Human Capital," Discussion Papers on Entrepreneurship, Growth and Public Policy, No. 1404, Group Entrepreneurship, Growth and Public Policy, MPI Jena.

Acs, Z. J. and C. Armington, 2002. "Endogenous Growth and Entrepreneurial Activity in Cities," http://webserver01.ces.census.gov/index.php/ces/1.00/cespapers/index.php/ces/1. 00/cespapers?down key=101665.

Acs, Z. J. and D. B. Audretsch, 1993, "Introduction", in Z. J. Acs amd D. B. Audretsch (eds.), Small Firms and Entrepreneurship: An East-West Perspective, Cambridge University Press: New York, USA.

Acs, Z. J. and D. B. Audretsch, 2001, "The Emergence of the Entrepreneurial Society", Presentation for the Acceptance of the 2001 International Award for Entrepreneurship and Small Business Research, 3 May, Stockholm.

Acs, Z. J. and D. B. Audretsch, 1990, Innovation and Small Firms, MIT Press, Cambridge.

Acs, Z. J. and D. B. Audretsch, 1988, "Innovation in Large and Small Firms: An Empirical Analysis,” American Economic Review, 78: 678-690.

Acs, Z. J., B. Carlsson and C. Karlsson, 1999, Entrepreneurship, Small and MediumSized Enterprises and the Macroeconomy, Cambridge University Press: Cambridge, UK.

Acs, Z. J, D. B. Audretsch and M. P. Feldman, 1994, "Research and development Spillovers and Recipient Firm Size," Review of Economics and Statistics, 76(2):336-340.

Acs, Z. J, D. B. Audretsch and M. P. Feldman, 1992, "Real Effects of Academic Research: Comment," American Economic Review, 82 (1): 363-367.

Ahlin, A. and E. Johansson, 2001, "Individual Demand for Local Public Schooling: Evidence from Swedish Survey Data," International Tax and Public Finance 8(4): 331-351.

Anselin, Luc, 1988, Spatial Econometrics: Methods, and Models, Dordrecht: Kluwer Academic. 
Anselin, Luc, 1990, "Some Robust Approaches to Testing and Estimation in Spatial Econometrics," Regional Science and Urban Economics, 20: 141-163.

Anselin, Luc, 1992, "Space and Applied Econometrics: Introduction," Spatial Issue, Regional Science and Urban Economics, 22: 307-316.

Anselin, Luc, 1993, "Discrete Space Autoregressive Models," in Michael F. Goodchild, Bradley O. Parks and T. Steyaert, eds., Environmental Modeling with GIS, pp. 454-469, Oxford: Oxford University Press.

Anselin, Luc, 1995, "Local Indicators of Spatial Association-LISA," Geographical Analysis, 27: 93-115.

Anselin, Luc, 1996, "The Moran Scatterplot as an ESDA Tool to Assess Local Instability in Spatial Association," in M. Fischer, H. Scholten and D. Unwin, eds., Spatial Analytical Perspective on GIS, pp. 111-125, London: Taylor \& Francis.

Anselin, Luc, 1998, "GIS Research Infrastructure for Spatial Analysis of Real Estate Markets," Journal of Housing Research, 9: 113-133.

Anselin, Luc, 2001, "Spatial Econometrics," in B. Baltagi, (ed.), A Companion to Theoretical Econometrics, pp. 310-330, Oxford, England: Blackwell.

Anselin, Luc, 2002, "Under the Hood: Issues in the Specification and Interpretation of Spatial Regression Models," Agricultural Economics, 27: 247-267.

Anselin, Luc, 2003, "Spatial Externalities, Spatial Multipliers and Spatial Econometrics," International Regional Science Review, 26(2): 153-166.

Anselin, Luc and Arthur Getis, 1992, "Spatial Statistical Analysis and Geographic Information Systems," The Annals of Regional Science, 26: 19-33.

Anselin, Luc and A. Bera, 1998, "Spatial Dependence in Linear Regression Models with an Introduction to Spatial Econometrics," In Handbook of Applied Economic Statistics, edited by A. Ullah and D. E. Giles, 237-289, New York: Marcel Dekker.

Anselin, Luc and A. Bera, R. Flrax and M. Yoon, 1996, "Simple Diagnostic Tests for Spatial Dependence," Regional Science and Urban Economics, 26(1): 77-104.

Anselin, Luc, and Harry H. Kelejian, 1997, "Testing for Spatial Error Autocorrelation in the Presence of Endogenous Regressors," International Regional Science Review, 20: 153-182. 
Appalachian Regional Commission, 2002, "Regional Data and Research," at www.arc.gov/index.do?nodeld=46.

Arbue, M., H.L.F. de Groot and R.J.G.M. Florax, 2004, "Space and Growth," Tinbergen Institute Discussion Paper, TI 2004-129/3.

Armington, C., and M. Odle, 1982, "Sources of Job Growth: A New Look at the Small Business Role, Washington DC: National Council for Urban Economic Development," cited in R. J. Vaughan and R. Pollard, Small Business and Economic Development, Financing Economic Development in the 1980s, edited by N. Walzer and D. Chicoine. New York: Praeger, 1986.

Armington, C., and Z. J. Acs, 2002, "The Determinants of Regional Variation in New Firm Formation," Regional Studies 36(1): 33-45.

Aronsson, T., J. Lundberg and M. Wikstrom, 2001, "Regional Income Growth and Net Migration in Sweden 1970-1995," Regional Studies, 35(1): 823-830.

Ashcroft, B., J. H. Love and E. Malloy, 1991, "New Firm Formation in the UK Counties with Special reference to Scotland," Regional Studies, 25 (5): 395-410

Audretsch D. B. and M. Fritsch, 1994, "The Geography of Firm Births in Germany," Regional Studies 28(4): 359-365.

Audretsch, D.B., M.A. Carree, A.J. van Stel and A.R. Thurik, 2000, Impeded Industrial Restructuring: The Growth Penality, Research Paper, Center for Advanced Small Business Economics, Erasmus University, Rotterdam, Netherlands.

Baicker, Katherine, 2005, “The Spillover Effects of State Spending," Journal of Public Economics, 89(3):529-544.

Baltagi, Badi. H., 1980, "On Seemingly Unrelated Regression with Error Components," Econometrica, 48: 1547-1551.

Baltagi, Badi. H, 1981, "Simultaneous Equations with Error Components," Journal of Econometrics, 17: 189-200.

Baltagi, Badi. H, 1995, Econometric Analysis of Panel Data, New York: John Wiley and Sons.

Baltagi, Badi. H, Seuck Heun Song and Won Koh, 2003, "Testing Panel Data Regression Models with Spatial Error Correlation," Journal of Econometrics, Elsevier, 117(1): 123-150. 
Barkley, D.L., M.S. Henry, and S. Bao, (1998), "The Role of Local School Quality and Rural Employment and Population Growth", Review of regional Studies, 28 (summer): 81-102.

Barro, Robert J. and Xavier Sala-i-Martin, 1992, "Convergence," Journal of Political Economy, 100, 223-251.

Barro, Robert J. and Xavier Sala-i-Martin, 2004, Economic Growth, second edition, MIT Press: Cambridge MA.

Bartik, Timothy J., 1985, "Business Location Decisions in the United States: Estimates of the Effects on Unionization, Taxes, and other Characteristics of States," Journal of Business and Economic Statistics, 3: 14-22.

Bartik, Timothy J., 1989, "Small Business Start-Ups in the United States: Estimates of the Effects of Characteristics of States," Southern Economic Journal, 55: 10041018.

Bartik, Timothy J., 1991, "Who Benefits from State and Local Economic Development Policies?” Kalamazoo, MI: W.E. Upjohn Institute of Employment Research.

Beesley, M.E. and T.T. Hamilton, 1994, "Entry Propensity, the Supply of Entrants and the Spatial Distribution of Business Units," Regional Studies, 28 (3):

Beeson, P.E. and R.W. Eberts, 1989, "Identifying Productivity and Amenity Effects in Interurban Wage Differentials," Review of Economics and Statistics, 71(3): 443452.

Benirschka, M. and J.K. Binkely, 1994, "Land Price Volatility in a Geographically Dispersed Market," American Journal of Agricultural Economics, 76: 185-195.

Bergstrom, Theodore C. and Robert P. Goodman, 1973, "Private Demands for Public Goods," American Economic Review, 63 (3): 280-296.

Bergstrom, Theodore C., Daniel L. Rubinfeld and Perry Shapiro, 1982, "Micro-Based Estimates of Demand Functions for Local School Expenditures," Econometrica, 50 (5): 1183-1205.

Besley, Timothy and Anne C. Case, 1995, "Incumbent Behavior: Vote-Seeking, TaxSetting and Yardstick Competition," American Economic Review, 85(1) ;: 25-45.

Bilger, Ursula, Joachim Genosko and Georg Hirte, 1991, "Migration and Regional Labor Market Adjustment in West Germany," in Stillwell, John and Peter Congdom (eds.), Migration Models: Macro and Micro Approaches, Belhaven Press: London, UK. 
Birch, David L., 1979, The Job Generation Process: Final Report to Economic Development, Cambridge, MA: MIT Program on Neighborhood and Regional Change.

Birch, David L., 1987, Job Generation in America: How our Smallest Companies Put the Most People to Work, New York: Free Press.

Blair, John and Robert Premus, 1987, "Major Factors in Industrial Location," Economic Development Quarterly, 1: 72-85.

Blank, R., 2000, "Fighting Poverty: Lessons from Recent U.S. History," Journal of Economic Perspectives, 14: 3-19.

Blank, R., 1993, "Why were Poverty Rates so High in the 1980s?" in Poverty and Prosperity in the USA in the Late Twentieth Century, D.B. Papadimitriou and E.N. Wolff, eds., St. Mrtin's Press, New York, NY.

Blank, R., and A. Blinder, 1986, "Macroeconomics, Income Distribution and Poverty," in Fighting Poverty: What Works and What Doesn't, S. H. Danziger and D. H. Weinberg, eds.,Cambridge, MA: Cambridge University Press.

Blank, R., and D. Card, 1993, "Poverty, Income Distribution, and Growth: Are they Still Connected?" Brookings Papers on Economic Activity 2, 258-339.

Blanchard, O. J. and F. Katz, 1992, "Regional Evolution," Brookings Papers on Economic Activity 1: 1-75.

Blanco, C., 1964, "Prospective Unemployment and Interstate Population Movements," Review of Economics and Statistics, 46 (2): 221-222.

Blomquist, G.C., M.C. Berger, and J.P. Hoehn, 1988, "New Estimates of Quality of Life in Urban Areas," American Economic Review, 78 (1): 81-107.

Boarnet, M.G., (1994), "An Empirical Model of Intra-metropolitan Population and Employment Growth," Papers in Regional Science, 73 (April): 135-153.

Borcherding, Thomas E. and Robert T. Deacon, 1972, "The Demand for the Services of Non-Federal Governments," American Economic Review, 62 (5): 891-901.

Borjas, G.J., 1996, Labor Economics, New York: McGraw Hill Companies, Inc.

Brandow Company, Inc., 2001, "Analysis of Business Formation, Survival and Attrition Rates of New and Existing Firms and Related Job Flows in Appalachia."

Brazer, H.E., 1959, City Expenditures in the United States, National Bureau Economic Research, Occasional Paper 66, New York. 
Breschi, Stefano, 1998, “Agglomeration Economies, Knowlegde Spillovers, Technological Diversity and Spatial Clustering of Innovations," Liuc Papers No. 57, Serie Economia e Impresa 15.

Brock, W. A., and D. S. Evans, 1989, "Small Business Economics," Small Business Economics 1, 7-20.

Brown, Charles, James Medoff. and Jay Hamilton, 1990, Employers: Large and Small, Cambridge: Harvard University Press.

Brueckner, Jan K., 1998, "Testing for Strategic Interaction among Local governments: The Case of Growth Controls," Journal of Urban Economics, 44(3): 438-468.

Brueckner, Jan K., 2000, "A Tiebout/Tax-Competition Model," Journal of Public Economics, 77(2):285-306.

Cain, G., 1998, "The State of the Economy and the Problem of Poverty: Implications for the Success or Failure of Welfare Reform," Discussion Paper 1183-98, Institute for Research on Poverty, University of Wisconsin.

Canning, D. and Pedroni P., 1999, "Infrastructure and long-Run Economic Growth," Paper presented at the 1999 Econometric Society Summer Meeting, Madison, Wisconsin.

Callejon, M. and A. Segarra, 2001, "Geographical Determinants of The Creation of Manufacturing Firms: The Regions of Spain,” http://www.ub.es/graap/callejon.htm

Canning, D. and Pedroni P., 1999, "Infrastructure and long-Run Economic Growth," Paper presented at the 1999 Econometric Society Summer Meeting, Madison, Wisconsin.

Carlino, O.G. and E.S. Mills, 1987, “The Determinants of County Growth," Journal of Regional Science 27(1): 39-54.

Carlton, Dennis W., (1983), "The Location and Employment Choices of New Firms: An Econometric Model with Discrete and Continuous Endogenous Variables," Review of Economics and Statistics, 65 : 440-449.

Carree, M. A., et al., 2002, "Economic Development and Business Ownership: An Analysis Using Data of 23 OECD Countries in the Period 1976-1996," Small Business Economics 3, 271-290.

Carree, M.A. andA.R. Thurik, 1998, "Small Firms and Economic Growth in Europe," Atlantic Economic Journal, 26(2): 137-146. 
Carree, M.A. andA.R. Thurik, 1999, "Industrial Structure and Economic Growth," in Audretsch, D.B. and A.R. Thurik (eds.), Innovation, Industry Evolution and Employment, Cambridge, UK: Cambridge University Press.

Case, A., H.S. Rosen and J.R. Hines, 1993, "Budget Spillovers and Fiscal Policy Interdependence: Evidence from States," Journal of Public Economics, 52: 285307.

Cebula, Richard J., 1974, "Local Government Policies and Migration: An Analysis for SMSA's in the United States, 1965-1970," Public Choice, 19, 85-93.

Chalmers, James A. and Michael J. Greenwood, 1980, "The Economics of Rural-toUrban Migration Turnaround," Social Science Quarterly, 61, 524-544.

Chalmers, James A. and Michael J. Greenwood, 1984, “The Regional Labor Market Adjustment Process: Determinants of Changes in Rates of Labor Force Participation, Unemployment, and Migration," Annals of Regional Science, 18: 117.

Charney, Alberta H., 1993, "Migration and the Public Sector: A Survey," Regional Studies, 27, 313-326.

Charney, Alberta H., 1983, "Intra-urban Manufacturing Location Decisions and Local Tax Differentials," Journal of Urban Economics, 14, 184-205.

Conley, T.G., 1996, Econometric Modeling of Cross-Sectional Dependence, Ph.D. Dissertation, Department of Economics, University of Chicago, Chicago, IL.

Conley, T.G., 1999, "GMM Estimation with Cross-Sectional Dependence," Journal of Econometrics, 92: 1-45.

Christensen, G., 2000, "Entrepreneurship Education: Involving Youth in Community Development," Nebraska Department of Education, Marketing and Entrepreneurship Education, Lincoln, NE.

Christy, R.D., M.D. Wenner and W. Dassie, 2000, "A Microenterprise-Centered Economic Development Strategy for the Rural South: Sustaining Growth with Economic Opportunity," Paper Presented at the Southern Agricultural Economics Association Annual Meeting.

Clark, D. and C.A. Murphy, 1996, "Countywide Employment and Population Growth: An Analysis of the 1980s," Journal of Regional Science 36(2): 235-256.

Clark, D. and W. Hunter, 1992, "The impact of Economic Opportunity, Amenities and Fiscal Factors on Age-Specific Migration Rates," Journal of Regional Science 32(3):349-365. 
Cliff, A. and J.K. Ord, 1973, Spatial Autocorrelation, London: Pion.

Cliff, A. and J.K. Ord, 1981, Spatial Processes: Models and Applications, London: Pion.

Cook, A.K., 1987, "Non-Metropolitan Migration: The Influence of Neglected Variables," Rural Sociology, 52: 409-418.

Cross, M., 1981, "New Firm Formation and Regional Development," Gower, Aldershot, Hants.

Curie, J. and M. Yelowitz, 2000, Are Public Housing Projects Good for Kids?” Journal of Public Economics 75(1):99-124.

Cushing, Brain, and Cyntia Rogers, 1996, "Income and Poverty in Appalachia," SocioEconomic Review of Appalachia( Papers Commissioned by Appalachia Regional Commission), Regional Research Institute, West Virginia University.

Cutler, D., and L. Katz, 1991, "Macroeconomic Performance and the Disadvantaged," Brookings Papers on Economic Activity 2,1-74.

Davidson, P., L. Lindmark and C. Olofsson, 1994, "New Firm Formation and Regional Development in Sweden," Regional Studies 28: 395-410.

Davidson, P., L. Lindmark and C. Olofsson, 1993, Business Dynamics in the Swedish Economy: A Regional Perspective, Country Report Submitted to the European Commission, Umea Business School, Umea.

Davidson, R. and J.G. Mackinnon, 1993, Estimation and Inference in Econometrics, New York: Oxford University Press.

Davis, Steven J., John Haltiwanger and Scott Schuh, 1996, Job Creation and Destruction, Cambridge,MA: MIT Press.

Davis, Steven J., John Haltiwanger and Scott Schuh, 1993, "Small Business and Job Creation: Dissecting the Myth and Reassessing the Facts," Small Business Economics 8,297-315.

Day, Kathleen M., 1992, "Interprovincial Migration and Local Public Goods," Canadian Journal of Economics, 25 (1) 123-144.

Dean, J. S. Holmes and S. Smith, 1996, "Business Networks: Growth Options Manufacturing and Service Sector Comparisions," in Gibson, B. et al., (eds.), Proceedings of the Joint SEAANZ and IIE Small Enterprise Conference, Newcastle Australia: IIE University of Newcastle. 
Deavers, Kenneth L., and Robert A. Hoppe, 1992, "Overview of the Rural Poor in the 1980s," in Cynthia Duncan (ed.), Rural Poverty in America, Westport CT: Greenwood Publishing Group Inc.

Decressin, J. and Fatas A., 1995, "Regional Labor Market Dynamics in Europe," European Economic Review 39(9): 1627-1655.

Deich, Michael, 1989, "State Taxes and Manufacturing Plant Location," In Proceedings of the Eighty-Second Annual Conference on Taxation, Columbus, $\mathrm{OH}$ : National Tax Association.

Deller, Steven C., Tsung-Hsiu Tsai, David W. Marcouiller, and Donald B.K. English, (2001), "The Role of Amenities and Quality of Life in Rural Economic Growth," Journal of American Agricultural Economics, 83(3): 352-365.

Dietz, Richard, 1998, "A Joint Model of Residential and Employment Location in Urban Areas," Journal of Urban Economics, 44: 197-215.

Dilger, Robert Jay, and Tom Stuart Witt, 1994, "West Virginia's Economic Future, “ in Dilger Robert Jay and Tom Stuart Witt (eds.), West Virginia in the 1990s: Opportunities for Economic Progress, Morgantown: West Virginia University Press.

Di Matteo, L. and R. Di Matteo, 1998, "Evidence of the Determinants of Canadian Provincial Government Health Expenditures: 1965-1991," Journal of Health Economics 17(2): 209-227.

Doreian, P., 1980, "Linear Models with Spatially Distributed Data, Spatial Disturbances or with Spatial Effects," Sociological Methods and Research, 9: 29-60.

Driscoll, John C. and Aart C. Kraay, 1998, "Consistent Covariance matrix Estimation with Spatially Dependent Panel Data," Review of Economics and Statistics, 80: 549-560.

Duffy, N.E., (1994), "The Determinants of State Manufacturing Growth Rates: A TwoDigit-Level Analysis," Journal of Regional Science, 34(May): 137-162.

Duffy-Deno, K.T., (1998), "The Effect of Federal Wilderness on County Growth in the Inter-mountain Western United States," Journal of Regional Sciences, 38(February): 109-136.

Duffy-Deno, Kevin T. and Randall W. Eberts, 1991, "Public Infrastructure and Regional Economic Development: A Simultaneous Equations Approach," Journal of Urban Economics 39: 329-343. 
Duncan, J. W., and D. P. Handler, 1994, "The Misunderstood Role of Small Business," Business Economics 29, 7-12.

Dunne, Timothy, Mark J. Roberts, and Larry Samuelson, 1989, "Growth and Failure of U.S. Manufacturing Plants," Quarterly Journal of Economics 104, 671-698.

Dye, Thomas, 1980, "Taxing, Spending and Economic Growth in the American States," Journal of Politics, 42, 1085-1107.

Edmiston, Kelly D., (2004), "The Net Effect of Large Plant Locations and Expansions on Country Employment," Journal of Regional Science, 44(2): 289-319.

Ekstrom, B. and L. S. Leistritz, 1988, Rural Community Decline and Revitalization: An Annotated Bibliography, New York: Garland Publishing Inc.

Elhorst, J. Paul, 2003, "Specification and Estimation of Spatial Panel data Models," International Regional Science Review, 26(3): 244-268.

Erickson, Rodney, 1987, "Business Climate Studies,” Economic Development Quarterly, 1: $62-71$.

Evans, D. and L. S. Leighton, 1990, "Small Business Formation By Unemployed and Employed Workers," Small Business Economics 2:319-330.

Ezzet-Lofstrom, Roxanne, 2003, "Out-Migration Decisions: The Role of Regional Amenities," Political Economy Working Paper 07/03, The University of Texas at Dallas.

Fagerberger, Verspagen and Caniels, 1997, "Technology, Growth and Unemployment across European Regions,” Regional Studies, 31(5): 457-466.

Falch, T. and J. Rattso, 1997, "Political and Economic determinants of School Spending in Federal States: Theory and Time-Series Evidence," European Journal of Political Economy 13: 299-314.

Fay, M., 2000, "Financing the Future: Infrastructure Needs in Latin America, 2000-05," Finance, Private Sector Development, and Infrastructure Sector Unit, Latin America and the Caribbean Region, World Bank.

Fields, G.S., 1979, "Place-to-Place Migration: Some New Evidence," Review of Economics and Statistics, 61: 21-32.

Fisher, Ronald, 1997, "The effects of State and Local Public Services on Economic Development," New England Economic Review, March/April: 53-67. 
Fisher, Ronald C. and John C. Navin, 1992, "State-Local Fiscal Behavior: An Analysis Interjurisdictional Differences, 1962-1987," Public Finance Quarterly, 20: 433449.

Fox, W.F., 1979, "Fiscal Differential and Industrial Location: Some Empirical Evidence," Urban Studies, 18: 105-111.

Fox, William F., Henry W. Herzog Jr., and Alan M. Schlottmann, 1989, "Metropolitan Fiscal Structure and Migration," Journal of Regional Science, 29: 523-537.

Freeman, R., 2002, “The Rising Tide Lifts.....?” in Danziger, S., and R. Haveman, (eds.), Understanding Poverty, Cambridge, MA: Harvard University Press.

Freshwater, D., 1999, "Rural America at the turn of the century," TVA Rural Studies, Staff and Contractor, Working Paper 99-12.

Friedman, Joseph, Daniel Gerlowski, and Johnathan Silberman, 1992, "What attracts Forien Multinational Corporations? Evidence from Branch Plant Location in the United States," Journal of regional Sciences, 32: 403-418.

Fritsch, M., 1992, "Regional Differences in New Firm Formation: Evidence from West Germany," Regional Studies 26:233-244.

Fritsch, M. and O. Falck, 2003, "New Firm Formation by Industry over Space and Time: A Multilevel Analysis," Discussion Paper, German Institute for Economic Research, Berlin.

Fotopoulos, G. and N. Spencer, 1999, "Spatial Variations in New Manufacturing Plant Openings: Some Empirical Evidence from Greece," Regional Studies, 33(3): 219229.

Gabe, Todd M. and Kathleen P. Bell, 2004,"Tradeoff Between Local Taxes and Government Spending as Determinants of Business Location," Journal of Regional Science 44(1): 21-41.

Gabriel, S., J. Mattey, and W. Wascher, 1995, "The Demise of California Reconsidered: Interstate Migration Over the Economic Cycle," Federal Reserve Bank of San Francisco Economic Review 2, 30-45.

Garofoli, G., 1994, "New Firm Formation and Regional Development: The Italian Case," Regional Studies 28:381-393.

Gaygisiz, Esma and Miyase Yesim Koksal, 2003, Regional Variation in New Firm Formation in Turkey: Cross-Sectional and Panel Data Evidence, ERC Working Papers in Economics, 03/08. 
Gerdtham, U-G., J. Sogaard, F. Jonsson and F. Andersson, 1992, "An Econometric Analysis of Health care Expenditure: A Cross-Section of OECD Countries," Journal of Health Economics, 11: 63-84.

Getis, Arthur and J. Keith Ord, 1992, "The Analysis of Spatial Association by Use of Distance Statistics," Geographic Analysis, 24(3): 189-206.

Glaeser, E.L., J.A. Scheinkman and A. Shleifer, 1995, "Economic Growth in a CrossSection of Cities," Journal of Monetary Economics 36(1): 117-143.

Goodchild, M.F., R. Haining and S. Wise, 1992, "Integrating GIS and Spatial Data Analysis: Problems and Possibilities," International Journal of Geographic Information System, 6(5): 407-423

Gottschalk, P. and S. Danziger, 1985, "A Framework for Evaluating the Effects of Economic Growth and Transfers on Poverty," The American Economic Review 75(1), 153-161.

Graves, P. and P. Linnemann, 1979, "Household migration: Theoretical and Empirical Results," Journal of Urban Economics, 3: 383-404.

Greene, William H., 2003, Econometric Analysis, $5^{\text {th }}$ ed., Upper Saddle River N.J.: Prentice Hall.

Greenwood, Michael J., 1975, "Research on Internal Migration in the United States: A Survey," Journal of Economic Literature, 13: 397-433.

Greenwood, Michael J., 1976, "A Simultaneous Equation Model of White and NonWhite Migration and Urban Change," Economic Inquiry, 14: 1-15.

Greenwood, Michael J.,, 1985, "Human Migration: Theory, Models, and Empirical Studies," Journal of Regional Sciences, 25: 521-544.

Greenwood, Michael J.,, 1993, “Migration: A Review,” Regional Studies, 27: 295-296.

Greenwood, Michael. J. and Gary.L. Hunt, 1984 "Migration and Interregional Employment Redistribution in the United States," American Economic Review 74(5): 957-969.

Greenwood, Michael. J. and Gary.L. Hunt, 1989, "Jobs versus Amenities in the Analysis of Metropolitan Migration," Journal of Urban Economics, 25: 1-16.

Greenwood, Michael J., Gary L. Hunt and John M. McDowel, 1986, "Migration and Employment Change: Empirical Evidence on Spatial and Temporal Dimensions of The linkage," Journal of Regional Science 26(2): 223-234. 
Griffith, Daniel A., 1992, "Simplifying the Normalizing Factor in Spatial Autoregressions for Irregular Lattices," Papers in Regional Science, 71(1): 71-86.

Gudgin, G., `1978, Industrial Location Process and Regional Employment Growth, Saxon House, Farnborough, Hants.

Guesnier, B, 1994, "Regional Variation in New Firm Formation in France," Regional Studies 28(4): 347-358.

Guimaraes, Paulo, Octavio Figueiredo, and Douglas Woodward, (2000), "Agglomeration and the Location of Foreign Direct Investment in Portugal," Journal of Urban Economics, 47: 115-135.

Hagemann, R.P. and G. Nicoletti, 1989, "aging Population: Economic Effects and Implications for Public Finance,” OECD, Working Papers, No. 61.

Hamalainen, Kari, and Petri Bockerman, (2004), "Regional Labor Market Dynamics, Housing, and Migration,” Journal of Regional Science, 44(3): 543-568.

Hansen, N.M., 1965, "The Structure and Determinants of Local Public Investment Expenditures," this Review, May, 150-162.

Hansen, L.P., 1982, "Large Sample Properties of Generalized Method of Moments Estimators, Econometrica, 50:1029-1054.

Harrison, B., 1994, "The Myth of Small Firms as the Predominant Job Generators," Economic Development Quarterly, 8 (1): 3-18.

Harrison, B. and S. Kanter, 1978, "The Political Economy of States: Job-Creation and Business Incentives," American Institute of Planning Journal, 425-435.

Hart, M. and G. Gudgin, 1994, "Spatial Variations in New Firm Formation in the Republic of Ireland, 1980-1990,” Regional Studies 28(4): 367-380.

Hashmati, A., 2001, "On the Causality between GDP and Health Care Expenditure in the Augmented Solow Growth Models," Swedish Working Paper Series in Economics and Finance, 423.

Haurin, D.R. and R.J. Haurin, 1988, "Net Migration, Unemployment and the Business Cycle," Journal of Regional Science, 28: 239-253.

Hausman, J., 1978, “Specification Tests in Econometrics,” Econometrica, 46: 1251-1271.

Hausman, J., 1983, "Specification and Estimation of Simultaneous Equations Models," in Z. Griliches and m. Intriligator, eds., Handbook of Econometrics, Amsterdam: North Holland. 
Haveman, R. and J. Schwabish, 2000, "Has Macroeconomic Performance Regained its Anti-Poverty Bite?” Contemporary Economic Policy, 18,415-427.

Hayness, Ada F., 1997, Poverty in Central Appalachia: Underdevelopment and Explotiation, New York: Garland Publishing, Inc.

Hawley, A. H., 1957, "Metropolitan Population and Municipal Government Expenditures in Central Cities," in P.K. Hatt and A.J.Reiss, Jr. (eds.), Cities and Society, Revised, Glencoe, III: Free Press.

Heller, P., R. Hemming and P. Kalvert, 1986, "Aging and Social Policy in the Major Industrial Countries, 1980-2025,” IMF Paper, No. 47.

Helms, L. Hay, 1985, "The Effects of State and Local Taxes on Economic Growth: A Time Series-Cross Sectional Approach," Review of Economics and Statistics, 67: 574-582.

Henderson, James M., 1968, "Local Government Expenditures: A Social Welfare Analysis," Review of Economics and Statistics, 50 (2): 156-163.

Henry, M.S., D.L. Barkley, and S. Bao, (1997), “The Hinterland's Stake in Metropolitan Growth: Evidence from Selected Southern Regions," Journal of Regional Science, 37(August): 479-501.

Henry, Mark .S., B. Schmitt, K Kristensen, D.L. Barkley, and S. Bao, (1999), "Extending Carlino-Mills Models to Examine Urban Size and Growth Impacts on Proximate Rural Areas," Growth and Change, 30(Summer): 526-548.

Henry, Mark S., B. Schmitt and V. Piguet, 2001, "Spatial Econometric Models for Simultaneous Systems: Application to Rural Community Growth in France," International Regional Science Review, 24(2): 171-193.

Herzog, Henry W. Jr., and Alan M. Schlottmann, 1993, "Valuing Amenities and Disamenties of Urban Scale: Can Bigger be Better?" Journal of Regional Science, 33: $145-166$.

Herzog, Henry W. Jr., and Alan M. Schlottmann, 1986, "State and Local Tax Deductibility and Metropolitan Migration,” National Tax Journal, 39: 189-200.

Herzog, Henry W. Jr., and Alan M. Schlottmann and Thomas P. Boehm, 1993, "Migration and Spatial Job Search: A Survey of Empirical Findings," Regional Studies, 27: 327-340.

Highfield, R. and R. Smiley, 1987, "New Business Starts and Economic Activity: An Empirical Investigation,” International Journal of Industrial Organization 5. 
Hirsch, W.Z., 1959, "Expenditure Implications of Metropolitan Growth and Consolidation," this Review, August, 232-241.

Hoehm, John P., Mark C. Berger and Glenn C. Blomquist, 1987, “A Hedonic Model of Interregional Wages, Rent, and Amenity Values," Journal of Regional Science, 27: 605-620.

Hsiao, C., 1985, "Benefits and Limitations of Panel Data," Econometric Reviews, 4 :121174.

Hsiao, C, 1986, Analysis of Panel Data, Cambridge: Cambridge University Press.

Hunt, Gary L., 1993, "Equilibrium and Disequilibrium in Migration Modeling," Regional Studies, 27: 341-349.

Intriligator, Michael D., 1978, Econometric Models, Techniques, and Applications, Englewood Cliffs, N.J.: Prentice Hall.

Izraeli, O., 1987, "The Effects of Environmental Attributes on Earnings and House Values across SMSAs," Journal of Urban Economics, 22:361-376.

Jaffe, A. B., 1989, "Real Effects of Academic Research," American Economic Review, 79(5): 957-970.

Jones, Bryan D., 1990, "Public Policies and Economic Growth in the American States," The Journal of Politics 52(1): 219-233.

Jones, Bryan D. and Arnold Vedlitz, 1988, "Higher Education Policies and Economic Growth in the American States," Economic Development Quarterly, 2: 78-87.

Johnson, P. and S. Parker, 1996, "Spatial Variations in the Determinants and Effects of Firm Births and Deaths," Regional Studies 30(7): 676-688.

Johnston, J., 1984, Econometric Methods, $3^{\text {rd }}$ ed., Singapore: McGraw-Hill.

Kangasharju, A., 2000, "Regional Variations in Firm Formation: Panel and Cross-Section Data Evidence from Finland," Regional Science 79:355-373.

Kapoor, Mudit, Harry H. Kelejian and Ingram R. Prucha, 2004, “ Panel Data Models with Spatially Correlated Error Components, Journal of Econometrics, forthcoming.

Karlsson, C., L. Lindmark and C. Olofsson, 1993, " Regional characteristics, Business Dynamics and Economic Development," in Karlsson, C., B. Johannisson, and D. Storey (eds.), Small Business Dynamics: International, National and regional Perspectives, New York: Routledge. 
Karvel, George R., Thomas A. Musil and Richard Sebastian, 1998, "Minnesota Business Migration: Relocation, Expansion, and Formation in Border States," American Experiment Quarterly, Summer: 67-85.

Keeble, D. and S. Walker, 1994, "New Firms, Small Firms and Dead Firms: Spatial Pattern and Determinants in the United Kingdom," Regional Studies 28(4): 411427.

Keeble, D. P. Tyler, G. Broom and J. Lewis, 1992, "Business Success in the Countryside: The Performance of Rural Enterprise," Department of Environment, HMSO, London.

Kelejian, Harry H. and D.P. Robinson, 1993 "A suggested Method of Estimation for Spatial Interdependent Models with Autocorrelated Errors, and an Application to a County Expenditure Model," Papers in Regional Sciences, 72 (3):297-312.

Kelejian, Harry H. and Ingram R. Prucha, 1998, "A Generalized Two-Stage Least Squares Procedure for Estimating a Spatial Autoregressive Model with Spatial Autoregressive Disturbances," Journal of Real Estate, Finance and Economics, 17(1): 99-121.

Kelejian, Harry H. and Ingram R. Prucha, 1999, "A Generalized Moments Estimator for the Autoregressive Parameter in a Spatial Model," International Economic Review, 40: 509-533.

Kelejian, Harry H. and Ingram R. Prucha, 2002, "2SLS and OLS in a Spatial Autoregressive Model with Equal Spatial Weights," Regional Science and Urban Economics, 32:691-707.

Kelejian, Harry H. and Ingram R. Prucha, 2004, "Estimation of Simultaneous Systems of Spatially Interrelated Cross Sectional Equations," Journal of Econometrics, 118:27-50.

Kieschnick, M. D., 1981, "Taxes and Growth: Business Incentives and economic Development," Washington, D.C., Council of State Planning Agencies.

Klevmarken, N.A., 1989, "Panel Studies: What Can We Learn from Them? Introduction," European Economic Review, 33: 375-377.

Kmenta, Jan, 1997, Elements of Econometrics, $2^{\text {nd }}$ ed., Ann Arbor: The University of Michigan Press.

Krugman, P., 1991a, "Increasing Returns and Economic Geography," Journal of Political Economy, 99: 483-499.

Krugman, P., 1991b, Geography and Trade, MIT Press, Cambridge, MA. 
Land, K. and G. Deane, 1992, "On the large Sample Estimation of Regression Models with Spatial of Network-Effects Terms: A Two Stage Least Squares Approach," in P. Marsden (ed..), Sociological Methodology, pp.221-248, San Francisco: Jossey-Bass.

Lansing, J. B. and E. Mueller, 1967, "The Effects of Geographic Mobility on Income," Journal of Human Resources 2, No. 4: 449-460.

Lee, Lung-Fei, 2003, "Best Spatial Two-Stage Least Squares Estimator for a Spatial Autoregressive Model with Autoregressive Disturbances," Econometric Reviews, 22, 307-335.

LeSage, J.P., 1999, "Spatial Econometrics, University of Toledo, available at http://www.rri.wvu.edu/WebBook/LeSage/spatial/spatial.html.

Leu, Robert E., 1986, "The Public-Private Mix and International Health Care Costs," in Culyer, A. J. and B. Jonsson (eds.), Public and Private Health Services, Basil Blackwell, Oxford, pp. 41-66.

Lewis, David J., Gray L. Hunt and Andrew J. Plantigna, 2002, “ Does Public Land Policy Affect Local Wage Growth," Growth and Change 34(1): 64-86.

Liedholm, Carl, and Donald Mead, 1987, "Small-Scale Industries in Developing Countries: Empirical Evidence and Policy Implications," Michigan State University International Development Papers, Number 9.

Liu, Ben-Chieh, 1977, "Local Government Finance and Metropolitan Employment Growth: A Simultaneous Equation Approach," Southern Economic Journal, 43, $1379-1385$.

Lloyd, P. E. and C. M. Mason, 1984, "Spatial Variation in New Firm Formation in the United Kingdom: Comparative Evidence from Merseyside, Greater Manchester and South Hampshire," Regional Studies, 18: 207-220.

Loveman, G. and W. Sengenberger, 1991, "The Re-emergence of Small-Scale Production: An International Comparison," Small Business Economics, 3(1), 1-39.

Lucas, Robert E. Jr., 1988, "On the Mechanics of Economic Development," Journal of Monetary Economic, 22 (July): 3-42.

Lucas, Robert E. Jr., 1993, “Making Miracle,” Econometrica, 61: 251-272.

Lundberg, J. 2003, "On the Determinants of Average Income Growth and Net Migration at the Municipal Level in Sweden," Review of Regional Studies, 33(2): 229-253. 
MacDonald, John F., 1992, "Assessing the Development Status of Metropolitan Areas," in Edwin S.Mills and John F. MacDonald (ed.), Sources of Metropolitan Growth, New Brunswick, NJ: Center for Urban Policy Research.

Mackinnon, J.G., H. White, and R. Davidson, (1983), "Tests for Model Specification in the Presence of Alternative Hypotheses: Some Further Results," Journal of Econometrics, 21: 53-70.

Maddala, G.S., 1986, Econometrics, Singapore: McGraw-Hill.

Maggar, Sally W., 1990, "Schooling, Work Experience and Gender: The Social Reproduction of Poverty in Mining Regions," Research Paper 9014, Regional Research Institute, Morgantown: West Virginia University Press.

Magnus, J.R., 1982, "Multivariate Error Components Analysis of Linear and Non-Linear Regression Models by Maximum Likelihood,“ Journal of Econometrics, 19: 239285.

Markusen, A. R. and M. B. Teitz, 1985, "The World of Small: Turbulence and Survival," in D. Storey (ed.), Small Firms in Regional Economic Development, Cambridge: Cambridge University Press.

Marlow, M. L. and A.F. Shiers, 1999, "Do Law Enforcement Expenditures Crowd-Out Public Education Expenditures?" Applied Economics 31(2): 255-266.

Marshall, A., 1920, Principles of Economics, $8^{\text {th }}$ edition, Macmillan, London.

Mason, C.M., 1994, "Spatial Variations in Enterprise: The Geography of New Firm Formation," in Burrows, R. (ed.), Deciphering the Enterprise Culture, Routledge, London.

Mathur Aparna, 2005, "A Spatial Model of the Impact of Bankruptcy Law on Entrepreneurship," http://www.aeaweb.org/annual_mtg_papers/2005/0107_0800_0402.pdf

Mathur, Vijay K. and Sheldon H. Stein, 1991, "Migration and Population Growth," Land Economics, 67:292-298.

Mead, A. C., 1982, "A Simultaneous Equations Model of Migration and Economic Change in Non-metropolitan Areas," Journal of Regional Science, 22, 513-327.

Miller, J., 1990, "Survival and Growth of Independent Firms and Corporate Affiliates in Metro and Non-metro America," Rural Development Research Report No. 74, Washington DC: U.S. Department of Agriculture/Economic Research Service. 
Mills, E.S., and R. Price, (1984), "Metropolitan Suburbanization and central City Problems," Journal Urban Economics, 15: 1-17.

Milne, W.J., 1991, "The Human Capital Model and its Econometric Estimation," in Stillwell, J. and P. Congdon (eds.), Migration Models: Macro and Micro Approaches, Belhaven Press, London.

Molho, Ian, 1986, "Theories of Migration: A Review," Scottish Journal of Political Economy, 33: 396-419.

Muth, Richard F., 1968, "Differential Growth among U.S. Cities," In Quirk, J.P. and A.M. Zarley (eds.), Papers in Quantitative Economics, Lawrence: University Press of Kansas.

Newhouse, J.P., 1987, "Cross National Differences in Health Spending: What Do They Mean?" Journal of Health Economics, 6, 159-162.

Newman, Robert, 1983, "Industry Migration and Growth in the South," Review of Economics and Statistics, 65: 76-86.

Obermiller, Phillip J. and Steven R. Howe, 2004, "Moving Mountains: Appalachian Migration Pattern, 1995-2002," Journal of Appalachian Studies, 10(3):359-371.

O'Farrell, P.N. and R. Crouchley, 1984, “An Industrial and Spatial Analysis of New Firm Formation in Ireland," Regional Studies, 18:221-236.

Ohls, James C. and Terence J. Wales, 1972, "Supply and Demand for State and Local Services," Review of Economics and Statistics, 54(4):424-430.

Olvey, L.D., 1972, "Regional Growth and Interregional Migration: Their Patter of Interaction," Review of Regional Studies, 2(2): 139-163.

Olvey, L.D., 1872, “Migration, Chicken or Egg?" Southern Economic Journal, 37: 295306.

O’Neill, K., 1993, "Focus of Growth-Fostering Small Enterprise," Keynote Address in Proceedings of the Joint SEAANZ and IIE Small Enterprise Conference, Newcastle Australia: IIE University of Newcastle Press.

Pack, Janet R., 1973, "Determinants of Migration to Central Cities," Journal of Regional Science, 13(3): 249-260.

Paelinck, J. and L. Klaassen, 1979, Spatial Econometrics, Farnborough: Saxon House.

Pagano, P. and F. Schivardi, 2001, “Firm Size Distribution and Growth,” Banka d'Italia Working Paper 394. 
Painter, G. and K. Bae, 2001, "The Changing Determinants of State Expenditure in the United States: 1965-1992,” Public Finance and Management 1(4): 370-392.

Papke, Leslie E., 1991, "Interstate Business Tax Differential and New Firm Location: Evidence from Panel Data," Journal of Public Economics, 45(1):47-68.

Persky, J.J. and J.F. Kain, 1970, "Migration, Employment and Race in the Deep South," Southern Economic Journal, 36(3):268-276.

Persson, J., 1997, "Convergence across the Swedish Counties, 1911-1993," European Economic Review 41: 1834-1852.

Petrin, T., 1992, "Partnership and institution building as a factor in rural development," Paper presented at the Sixth Session of the FAO/ECA Working Party on Women and the Agricultural Family in Rural Development, Innsbruck, Austria, 13-16 October.

Pinkse, Joris and Margaret.E. Slade, 1998, "Contracting in Space: An Application of Spatial Statistics for Discrete-Choice Models," Journal of Econometrics, 85: $125-$ 154.

Pissarides, C.A. and J. Wadsworth, 1989, "Unemployment and the Interregional Mobility of Labor,” Economic Journal, 99(397):739-755.

Pollard, Kelvin M., 2003, "Appalachia at the Millennium: An Overview of Results from Census 2000," Washington, D.C.: Population Reference Bureau, at www.arc.gov/index.do?nodleld $=1847$

Powers, E. T., 1995, “Growth and Poverty Revisited,” Economic Contemporary, Federal Reserve Bank of Cleaveland.

Pulver, G.C., 1989, “Developing a Community Perspective on Rural Economic Development Policy,” Journal of Community Development Society 20:1-4.

Raimondos, P., 1993, “On the Todaro Paradox,” Economic Letters, 42: 261-267.

Randolph, S., Z. Bogetic and D. Hefley, 1996, "Determinants of Public Expenditure on Infrastructure, Transportation and Communication," Policy Research Working Paper 1661, World Bank.

Rauch, J.E., 1993, "Productivity Gains from Geographic Concentration of Human Capital: Evidence from Cities," Journal of Urban Economics, 34: 380-400.

Rey, Sergio J. anfd Marlon G. Boarnet, 2004, "A Taxonomy of Spatial Econometric Models for Simultaneous Equations Systems," in L. Anselin, R.J.G.M. Florax and 
S.J. Rey, Advances in Spatial Econometrics: methodology, Tools and Applications, Berlin: Springer.

Reynolds, P.D., 1991, "Regional Characteristics Affecting Business Growth: Assessing Strategies Promoting Regional Economic Well-Being," Paper Submitted to the Ford Foundation, Grant No. 900-013.

Reynolds, P.D., 1994, "Autonomous Firm Dynamics and Economic Growth in the United States, 1986-1990," Regional Studies 28(4): 429-442.

Reynolds, P.D., 1999, "Creative Destruction," in Acs, et al.,(eds.), Entrepreneurship, Small and Medium-Sized Enterprises and the Macroeconmy, Cambridge University Press: Cambridge, U.K.

Reynolds, P.D., B. Miller and W.R. Maki, 1994, "Explaining Regional Variation in Business Births and Deaths: U.S. 1976-1988," Small Business Economics, 7(4): 387-407.

Roback, Jennifer, 1982, "Wages, Rents and Quality of Life," Journal of Political Economy, 90: 1257-1278.

Romer, Paul M., 1986, “Increasing returns and Long Run Growth,” Journal of Political Economy, 94 (5): 1002-1037.

Romer, Paul M., 1990, "Endogenous Technical Change," Journal of Political Economy, 98: S71-S102.

Rosen, Sherwin, 1979, "Wage-Based Indexes of Urban Quality of Life," in Mieszkowski Peter and Mahlon Straszheim (eds.), Current Issues in Urban Economics, Baltimore: Johns Hopkins University Press.

Rosenzweig, Mark R., 1988, “ Labor Markets in Low-Income Countries," in Chenery, Hollis B. and T. N. Srinivasan (eds.), Handbook of Development Economics, Vol 1, Amsterdam: North-Holland.

Rupasingha, A. and S.J. Goetz, 2003, "County Ammenties and Net Migration," Paper Presented in WRSA Annual Meeting, Rio Rico, Arizona, Feb 26-March 1, 2003.

Sanz, Ismael and Fracisco J. Velazquez, 2002, "Determinants of the Composition of Government Expenditure By Functions," Working Paper 13/2002, European Economy Group.

Sargan, J.D., 1958, "The Estimation of Economic Relationships using Instrumental Variables, Econometrica, 26: 393-415. 
Schachter, Joseph and Paul G. Althaus, 1989, "An Equilibrium Model of Gross Migration,” Journal of Regional Science, 29: 143-160.

Schaeffer, P. E, and S.R. Mack, 1997, "An appraisal of the conceptual foundations of the new International Division of Labor," Journal of Planning Literature 12 (1, August): $3-15$.

Schneider, Mark, 1987, "Local Budgets and the Maximization of Local Property Wealth in the System of Suburban Government," Journal of Politics, 49: 1104-1116.

Simon, C. and C. Nardinelli, 1996, "The Talk of Town: Human Capital Information and the Growth of English Cities, 1861-1961," Exploration in Economic History, 33(3): 384-413.

Simon, C. and C. Nardinelli, 2002, "Human Capital and the Rise of American Cities, 1900-1990," Regional Science and Urban Economics, 32: 59-96.

Sjaastad, Larry A., 1962, "The Costs and Returns of Human Migration," Journal of Political Economy, 70: 80-93.

Snyder, J.M. and I. Yachovelev, 2000, "Political and Economic Determinants of Government Spending on Social Protection Programs," Working Paper, Massachusetts Institute of Technology, April, 2000.

Solon, G.S., 1989, "The Value of Panel Data in Economic Research," in D. Kasprzyk, G.J. Duncan, G. Kalton and M.P. Singh, eds., Panel Surveys, New York: John Wiley and Sons, 486-496.

Sommers, Paul M. and Daniel Suits, 1973, "Analysis of Net Interstate Migration," Southern Economic Journal, 40: 193-201.

Steinnes, Donald N. and Walter D. Fisher, 1974, "An Econometric Model of Intra-urban Location,” Journal of Regional Science 14: 65-80.

Storey, D.J., 1982, Entrepreneurship and the New Firm, Croom Helm, London.

Storey, D.J., 1991, "The Birth of New Firms: Does Unemployment Matters? A Review of Evidence," Small Business Economics, 3(3): 167-178.

Storey, D.J, 1994, Understanding the Small Business Sector, London: Routledge.

Storey, D.J. and S. Johnson, 1987, Job Generation and Labor Market Change, Macmillan, London.

Storey, D.J. and A.M. Jones, 1987, "New Firm Formation: Labor Market Approach to Industrial Entry," Scottish Journal of Political Economy, 34: 37-51. 
Tiebout, Charles M., 1956, "A Pure Theory of Location Expenditure," Journal of Political Economy, 64: 415-424.

Thurik, A. R. and S. Wennekers, 1999, "Linking Entrepreneurship and Economic Growth," Small Business Economics 13(1): 27-55.

Tobler, Waldo, 1979, "Cellular Geography," in S. Gale and G. Olsson (eds), Philosophy in Geography, pp. 379-386, Dordrecht, the Netherlands: Reidel.

Topa, Giorgio, 1996, "Social Interactions, Local Spillovers and Unemployment," Ph.D. Dissertation, Department of Economics, University of Chicago, Chicago IL.

Uhlaner, L.M., A.R. Thurik and J. Hutjes, 2002, "Post-Materialism: A Cultural Factor Influencing Entrepreneurial Activity across Nations," ERIM Report ERS-200262-STR, Rotterdam: Erasmus Research Institute for Management.

Upton, G. J. and Fingleton, 1985, Spatial Data Analysis by Example, Volume 1: Point Pattern and Quantitative Data, New York: Wiley.

U.S. Small Business Administration (SBA), 1998, The State of Small Business: A Report of the President, Washington D.C.: U.S. Government Printing Press

U.S. Small Business Administration (SBA), 1999, The State of Small Business: A Report of the President, Washington D.C.: U.S. Government Printing Press.

Voslee, W.B., 1994, Entrepreneurship and economic growth, Pretoria: HSRC Publisher.

Wheaton, W.C.,1975, "Consumer Mobility and Community Tax Bases: The Financing of Local Public Goods," Journal of Public Economics, 4: 377-384.

Wennekers, S and A.R.Thurik, 1999, "Linking Entrepreneurship and Economic Growth," Small Business Economics 13(1): 27-55.

Wennekers, A.R.M., L.M. Uhlaner and A.R. Thurik, 2002, "Entrepreneurship and its Conditions: A Macro Perspectives," International Journal of Entrepreneurship Education, 1(1): 25-64.

White, Nancy C. and Thomas A. Knapp, 1994, "A Dynamic Model of Migration with Public Sector Attributes," Journal of the Regional Science Association International, 7(3): 331-340.

Whittington, R.C., 1984, "Regional Bias in New Firm Formation in the UK," Regional Studies, 18(3). 
Williams, J.D., 1981, “The Non-Changing Determinants of Migration,” Rural Sociology, 46: $183-202$.

Woods, Lawrence E. and Gregory A. Bischak, 2000, "Progress and Challenges in Reducing Economic Distress in Appalachia: An Analysis of National and Regional Trends since 1960," Washington, D.C.: Appalachia Regional Commission.

Woods, M. D., V.J. Frye and S.R. Ralstin, 1999, "Blueprints for your community's future: Creating a strategic plan for local economic development," Oklahoma Cooperative Extension Service, Oklahoma State University.

Wooldridge, Jeffrey M., 2002, Econometric Analysis of Cross-Section and Panel Data, MIT Press: Cambridge MA.

Zellner, A., and H. Theil, "Three Stage Least Squares: Simultaneous Estimation of Simultaneous Equations," Econometrica, 30: 63-68. 
Appendix 1: CROSS SECTIONAL AND PANEL DATA NON-SPATIAL MODEL ESTIMATION RESULTS FOR APPALACHIAN COUNTIES, APPALCHIAN STATES COUNTIES AND US COUNTIES

TABLE A: NON-SPATIAL MODEL ESTIMATION RESULTS, LEVEL: APPALACHIA, 1980S

\begin{tabular}{|c|c|c|c|c|c|c|c|c|c|c|}
\hline \multirow[b]{3}{*}{ Equation } & \multirow[b]{3}{*}{ Variables } & \multicolumn{5}{|c|}{ SINGLE-EQUATION } & \multicolumn{4}{|c|}{ MULTI-EQUATION } \\
\hline & & \multicolumn{2}{|l|}{ OLS } & \multicolumn{2}{|c|}{ Weighted LS } & Weighted 2SLS & $3 S L S$ & \multicolumn{2}{|c|}{$\frac{\mathrm{ML}}{\text { Coefficient z-Statistic }}$} & \multirow[t]{2}{*}{ GMM } \\
\hline & & Coefficient & t-Statistic & Coefficient & t-Statistic & Coefficient t-Statistic & Coefficient t-Statistic & Coefficient 2 & z-Statistic & \\
\hline \multirow{16}{*}{ 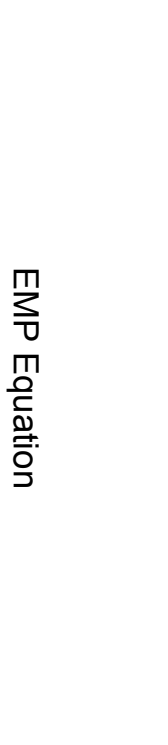 } & constant & -0.862627 & -0.599633 & -0.862627 & -0.611449 & 1.4052210 .887333 & 0.5517280 .363375 & 1.171761 & 0.606354 & 1.1035950 .857615 \\
\hline & INMG90 & 0.366949 & 5.155272 & 0.366949 & 5.256864 & 0.4669082 .405454 & $0.433288 \quad 2.30815$ & 0.631079 & 2.443757 & 0.2977941 .891065 \\
\hline & OTMG90 & -0.139192 & -1.690525 & -0.139192 & -1.723839 & $-0.143445-0.6377$ & $-0.091571-0.42261$ & -0.306 & -1.06777 & 0.0329820 .185938 \\
\hline & MHY90 & 0.14856 & 1.388191 & 0.14856 & 1.415547 & $-0.232746-1.60163$ & $-0.279928-1.99718$ & -0.34176 & -2.33477 & $-0.41882-3.18942$ \\
\hline & DGEX90 & 0.035497 & 0.74815 & 0.035497 & 0.762893 & 0.0683090 .862667 & 0.0907581 .176417 & 0.08875 & 0.93808 & $-0.01207-0.20133$ \\
\hline & POP24-44 & 0.305305 & 1.756481 & 0.305305 & 1.791095 & 0.4494622 .374391 & 0.3997022 .237827 & 0.447887 & 2.015779 & 0.6198524 .285912 \\
\hline & $\mathrm{FHHF}$ & 0.043491 & 0.580018 & 0.043491 & 0.591448 & $-0.031402-0.38232$ & $-0.031183-0.38931$ & -0.01236 & -0.11567 & $-0.09214-1.31392$ \\
\hline & POPCD & 0.089752 & 1.822945 & 0.089752 & 1.858868 & 0.1070051 .905096 & 0.1213792 .3048 & 0.137468 & 2.662576 & 0.1465383 .204918 \\
\hline & OWHU & -0.361453 & -1.446736 & -0.361453 & -1.475246 & $-0.28035-1.05746$ & $-0.002409-0.00962$ & -0.05421 & -0.19382 & 0.1443970 .667847 \\
\hline & MANU & 0.002176 & 1.655709 & 0.002176 & 1.688337 & 0.0033481 .883962 & $0.003092 \quad 1.805$ & 0.003211 & 2.065186 & 0.0041722 .774712 \\
\hline & WHRT & 0.008758 & 1.732787 & 0.008758 & 1.766934 & 0.0044840 .821039 & 0.0041320 .781491 & 0.004735 & 0.761169 & 0.0036610 .877574 \\
\hline & РСРТАХ & -0.063768 & $3-2.652075$ & -0.063768 & -2.704338 & $-0.03003-1.04715$ & $-0.035051-1.31079$ & -0.03182 & -1.02258 & $-0.01816-0$. \\
\hline & NAIX & 0.010122 & 1.014524 & 0.010122 & 1.034517 & 0.0094120 .841407 & & 205 & 0.01 & $694 \quad 1.1$ \\
\hline & HWD & 0.023228 & 0.706875 & 0.023228 & 0.720805 & 0.0372611 .099256 & 0.0394081 .224964 & 0.036527 & 0.920192 & 0.0654242 .520863 \\
\hline & ESBd & 0.114247 & 2.073581 & 0.114247 & 2.114444 & 0.1749452 .699135 & 0.1948273 .219267 & 0.172254 & 2.870422 & 0.2398744 .043696 \\
\hline & EMP80 & 0.739369 & 26.66256 & 0.739369 & 27.18798 & 0.69530117 .52952 & 0.68908418 .18648 & 0.705604 & 17.13308 & 0.70659320 .58704 \\
\hline \multirow{10}{*}{ 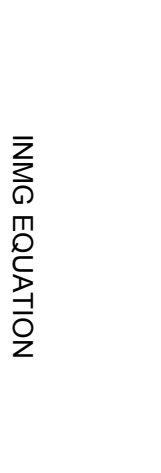 } & Constant & -3.524356 & -4.449699 & -3.524356 & -4.514979 & 0.9770790 .834812 & 1.0096540 .989218 & 4.065538 & 2.230955 & 1.0729861 .328989 \\
\hline & EMP90 & 0.066527 & 3.169826 & 0.066527 & 3.21633 & 0.0464811 .668637 & 0.0429851 .598259 & -0.04303 & -1.16296 & 0.039371 .638735 \\
\hline & OTMG90 & 0.934814 & 24.62477 & 0.934814 & 24.98603 & 1.10214518 .58335 & 1.21447926 .47208 & 1.03614 & 9.097465 & 1.17486131 .97474 \\
\hline & MHY90 & 0.519657 & 6.987232 & 0.519657 & 7.089739 & $0.112143 \quad 0.97876$ & 0.1443471 .449335 & -0.36786 & -2.02206 & 0.1323141 .651602 \\
\hline & DGEX90 & -0.118652 & -4.05581 & -0.118652 & -4.115311 & $-0.170194-3.87014$ & $-0.19753-4.56714$ & -0.04443 & -0.68372 & $-0.17433-4.91191$ \\
\hline & AREA & 0.033071 & 2.783574 & 0.033071 & 2.824411 & 0.0265522 .073003 & $0.014362 \quad 1.3074$ & 0.022583 & 1.445442 & 0.0229931 .98641 \\
\hline & POPs & -0.160994 & -4.060415 & -0.160994 & -4.119984 & $-0.255422-4.97712$ & $-0.288189-6.11623$ & -0.13122 & -1.65035 & $-0.29031-7.33643$ \\
\hline & MCRH & -0.067318 & $3-1.441699$ & -0.067318 & -1.462849 & $-0.039743-0.78911$ & $-0.032871-1.25285$ & 0.109428 & 1.8333 & $-0.05964-2.37412$ \\
\hline & UNEMP & 0.089606 & 2.737403 & 0.089606 & 2.777562 & $0.019033 \quad 0.47941$ & 0.0344730 .920601 & -0.16956 & -3.09981 & 0.0375291 .045568 \\
\hline & NAIX & 0.019855 & 2.917768 & 0.019855 & 2.960574 & 0.0225533 .136536 & 0.0209983 .411919 & 0.032601 & 3.332312 & 0.0220714 .135119 \\
\hline
\end{tabular}


EXPTAX

INMG80

Constant

EMP90

INMG90

MHY90

DGEX90

AREA

POPS

OWHU

UNEMP

NAIX

EXPTAX

OTMG80

Constant

EMP90

INMG90

OTMG90

DGEX90

POPS

POPs2

FHHF

POPHD

UNEMP

MANU

WHRT

SCIX

MHY80

Constant

EMP90

INMG90

OTMG90

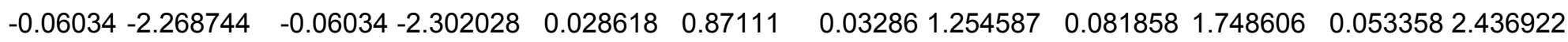

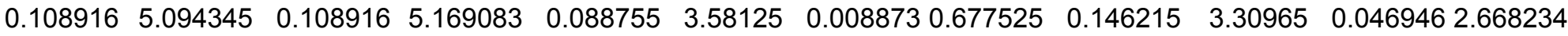

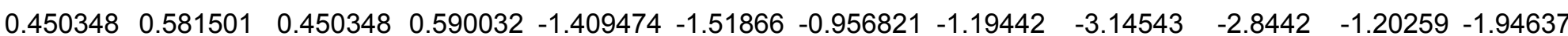

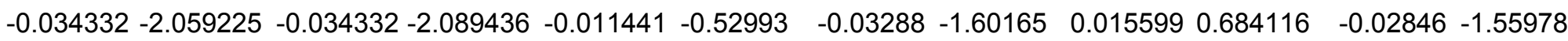

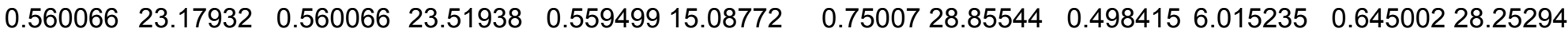

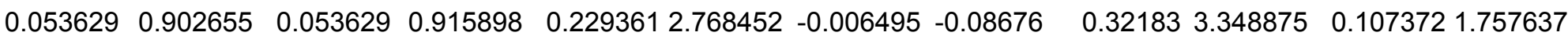
$\begin{array}{lllllllllll}0.049336 & 2.177536 & 0.049336 & 2.209482 & 0.053202 & 1.552148 & 0.1299913 .958652 & 0.019882 & 0.488615 & 0.117305 & 4.17395\end{array}$ $\begin{array}{llllllllllll}0.023868 & 2.444506 & 0.023868 & 2.480369 & 0.025043 & 2.36031 & -0.005335 & -0.58752 & 0.026699 & 2.204905 & 0.004849 & 0.442108\end{array}$ $\begin{array}{llllllllllll}0.227577 & 7.394188 & 0.227577 & 7.502666 & 0.221862 & 6.478625 & 0.2492737 .879492 & 0.21673 & 5.394581 & 0.242155 & 9.06655\end{array}$

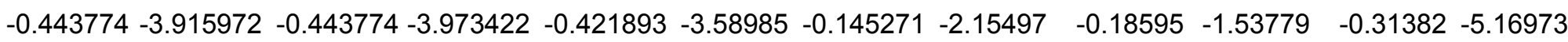

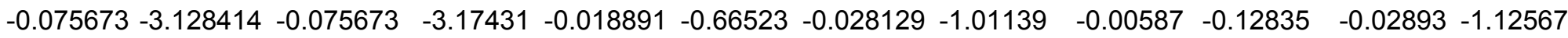

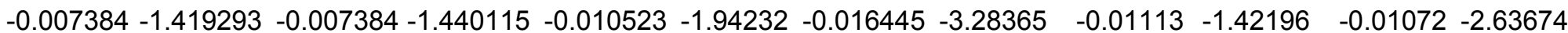

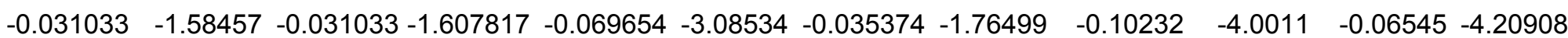

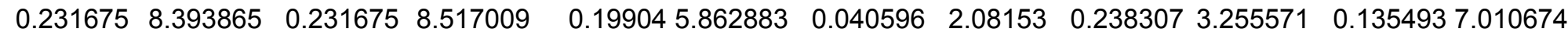

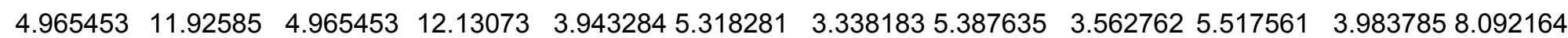

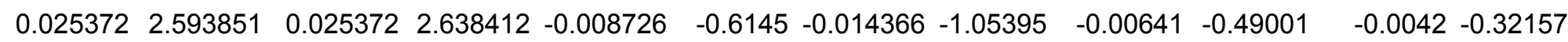

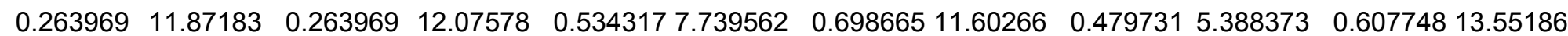

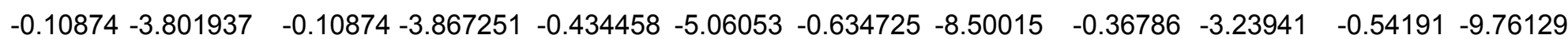

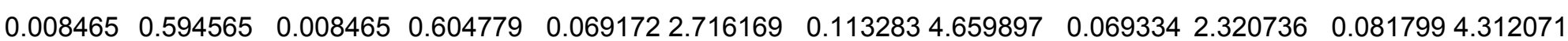

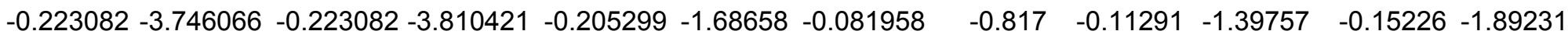

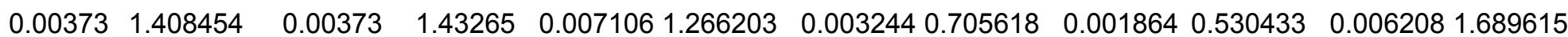

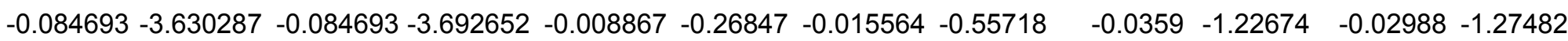
$\begin{array}{lllllllllll}0.123618 & 4.053891 & 0.123618 & 4.123533 & 0.090669 & 2.370076 & 0.0980913 .149009 & 0.135652 & 3.70373 & 0.085078 & 3.358541\end{array}$

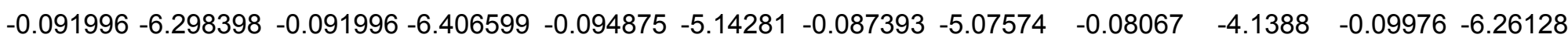

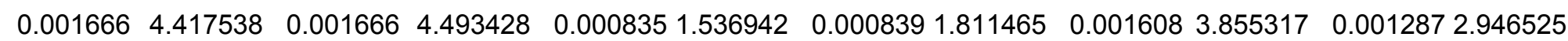

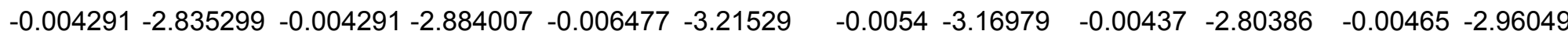

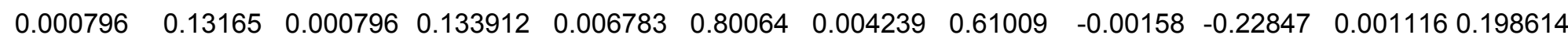

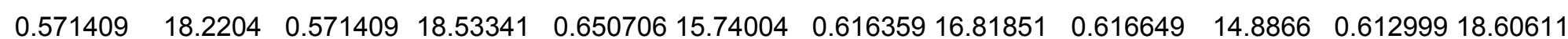
$\begin{array}{llllllllllll}3.611378 & 3.780126 & 3.611378 & 3.840315 & 3.285226 & 2.632519 & 3.082159 & 2.519943 & 3.100028 & 2.249353 & 1.448782 & 1.43813\end{array}$

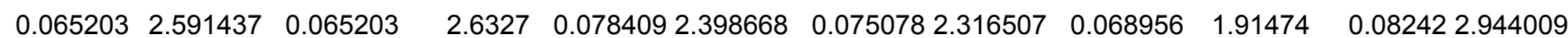

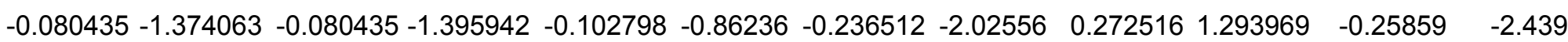

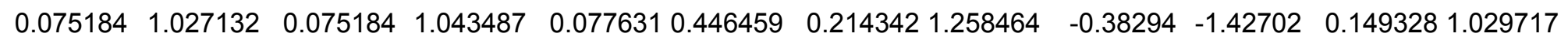




\begin{tabular}{|c|c|c|c|c|c|c|c|c|}
\hline MHY90 & -0.087797 & -1.047179 & $-0.087797-1.063853$ & $-0.049829-0.46232$ & 0.0029150 .027521 & -0.09855 & -0.8311 & 0.1240861 .333806 \\
\hline SCRM & 1.84E-05 & 2.007263 & 1.84E-05 2.039224 & 2.69E-05 1.261895 & 3.13E-05 1.504517 & 2.25E-05 & 2.089672 & 4.85E-05 2.529489 \\
\hline DCTAX & 0.049484 & 1.831663 & $0.049484 \quad 1.860828$ & 0.0370691 .220926 & 0.0363331 .234177 & 0.06339 & 2.085699 & 0.0002710 .010491 \\
\hline PCTD & $-1.72 \mathrm{E}-05$ & -1.385136 & $-1.72 \mathrm{E}-05-1.407191$ & $-1.78 \mathrm{E}-05-1.44109$ & $-1.52 E-05-1.27438$ & $-1.23 E-05$ & -0.7287 & $-3.20 \mathrm{E}-05-3.71377$ \\
\hline LTD & 4.62E-07 & 0.517079 & 4.62E-07 0.525312 & 3.43E-07 0.380241 & 3.73E-07 0.426645 & 4.39E-07 & 0.311012 & 4.34E-07 0.87438 \\
\hline
\end{tabular}




\begin{tabular}{|c|c|c|c|c|c|c|c|c|c|c|c|c|c|}
\hline \multirow[b]{3}{*}{ Equation } & \multirow[b]{3}{*}{ Variables } & \multicolumn{6}{|c|}{ SINGLE-EQUATION } & \multicolumn{6}{|c|}{ MULTI-EQUATION } \\
\hline & & \multicolumn{2}{|c|}{ OLS } & \multicolumn{2}{|c|}{ Weighted OLS } & \multicolumn{2}{|c|}{ Weighted 2SLS } & \multicolumn{2}{|c|}{ 3SLS } & \multicolumn{2}{|c|}{$\mathrm{ML}$} & \multicolumn{2}{|c|}{ GMM } \\
\hline & & & & & & & & & & & & & \\
\hline \multirow{16}{*}{ 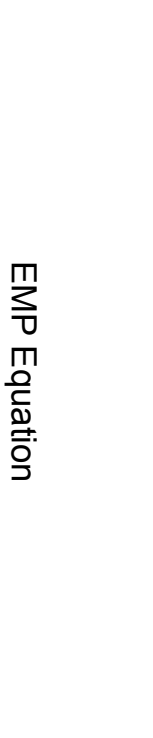 } & constant & & -1.353854 & & -1.380533 & & & & & & & & \\
\hline & INMG90 & 0.204982 & 3.262223 & 0.204982 & 3.32651 & 0.157921 & 1.087173 & 0.142246 & 1.011192 & 0.031246 & 0.165643 & 0.225605 & 1.760854 \\
\hline & OTMG90 & -0.005606 & -0.072042 & -0.005606 & -0.073462 & 0.145876 & 0.807166 & 0.181103 & 1.040233 & 0.230943 & 1.048846 & 0.045547 & 0.286453 \\
\hline & MHY90 & 0.21951 & 1.808715 & $5 \quad 0.21951$ & 1.844358 & -0.166984 & -1.08878 & -0.15802 & -1.05625 & -0.02211 & -0.13711 & -0.11993 & $3-0.96$ \\
\hline & DGEX90 & -0.009226 & -0.324228 & $3-0.009226$ & -0.330617 & 0.061922 & 1.045312 & 0.079248 & & & & & \\
\hline & POP24-44 & 0.195417 & 1.14269 & 0.195417 & 1.165208 & 0.319081 & 1.788659 & 0.196365 & & & & 0.219042 & \\
\hline & FHHF & 0.090898 & 1.377154 & 0.090898 & 1.404292 & -0.033614 & -0.42825 & -0.016753 & -0.21685 & 0.043877 & 0.451318 & -0.02901 & -0.59 \\
\hline & POPCD & -0.003761 & -0.082677 & -0.003761 & -0.084306 & 0.028532 & 0.607 & .00751 & -0 & 0.011046 & 67 & -0.0 & \\
\hline & OWHU & -0.2 & -1.209456 & -0.255236 & -1.23329 & -0.0 & -0.256 & 0538 & & & & & \\
\hline & MANU & 000499 & 0.36929 & 0.000499 & 0.376567 & 0.002296 & 1.45211 & & & & & 804 & \\
\hline & WHRT & .017706 & 4.109737 & 0.017706 & 4.190725 & 0.015982 & 3.622842 & & 3.4761 & 0.0 & & & \\
\hline & PCPTAX & -0.046447 & -2.200944 & -0.046447 & -2.244317 & -0.033965 & -1.50901 & -0.029476 & -1.38612 & -0.00826 & -0.27206 & -0.01064 & -0.62 \\
\hline & NAIX & 0.01322 & 1.436101 & 0.01322 & 1.464402 & 0.01184 & 1.230182 & 0.015067 & 1.665858 & 0.016965 & 51.8 & 0.015755 & 1.88 \\
\hline & HWD & -0.008431 & -0.261618 & -0.008431 & -0.266773 & -0.009047 & -0.25777 & 0.002978 & 0.088927 & 0.023416 & 0.531173 & -0.02344 & -0.8 \\
\hline & ESBd & 0.14897 & 3.194304 & 0.14897 & 3.257252 & 0.19098 & 3.24515 & 0.197496 & 3.547164 & 0.214495 & 3.964761 & 0.159049 & 2.961 \\
\hline & EMP80 & 0.783906 & 32.79527 & 0.783906 & 33.44155 & 0.733107 & 18.71227 & 0.72631 & 19.22636 & 0.743088 & $\begin{array}{l}39.57317 \\
\end{array}$ & 0.75903 & 19.70259 \\
\hline \multirow{10}{*}{ 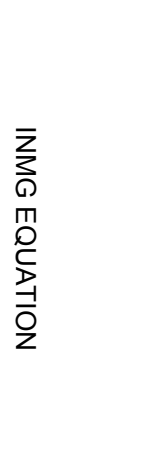 } & Constant & 1.708445 & 1.986829 & 1.708445 & 2.015977 & & 2.103809 & & & & & & \\
\hline & EMP90 & -0.012184 & -0.550599 & -0.012184 & -0.558676 & -0.029049 & -0.97226 & 0.007997 & 0.274642 & -0.01417 & -0.33887 & 0.021188 & \\
\hline & OTMG90 & 0.907416 & 21.19863 & 0.907416 & 21.50963 & 0.712938 & 6.168648 & 0.919645 & 9.002504 & 0.617059 & 2.990221 & 0.812549 & 9.558395 \\
\hline & MHY90 & 0.052301 & 0.516566 & 0.052301 & 0.524145 & 0.016209 & 0.134163 & 0.00324 & 0.029312 & 0.026507 & 0.218418 & -0.03823 & -0.34743 \\
\hline & DGEX90 & -0.094921 & -4.567342 & -0.094921 & -4.634349 & -0.190273 & -5.08353 & $3-0.188565$ & -5.2204 & -0.19514 & -3.78412 & -0.19121 & -6.28672 \\
\hline & AREA & 0.009651 & 0.686873 & 0.009651 & 0.69695 & -0.027468 & -1.5635 & $5 \quad 0.012829$ & 0.792124 & 0.021499 & 1.092307 & 0.012689 & 0.977849 \\
\hline & POPs & -0.32619 & -8.304605 & -0.32619 & -8.426439 & -0.241534 & -4.75424 & -0.322376 & -6.56629 & -0.26551 & -3.53166 & -0.29123 & -6.84865 \\
\hline & MCRH & -0.009863 & -0.125155 & -0.009863 & -0.126991 & 0.10806 & 1.204338 & $\begin{array}{ll}3 & 0.071697\end{array}$ & 0.959899 & 0.095622 & 1.425108 & 0.136815 & 2.077656 \\
\hline & UNEMP & & -0.740905 & & -0.751775 & -0.030732 & -0.95869 & -0.034815 & & -0.07718 & $\begin{array}{ll}3 & -2.49939\end{array}$ & -0.03877 & -1.59175 \\
\hline & NAIX & 0.024849 & 3.554792 & 0.024849 & 3.606943 & 0.023063 & 3.140757 & 0.02258 & 3.357223 & 0.014471 & 2.420677 & 0.011731 & 1.889077 \\
\hline
\end{tabular}




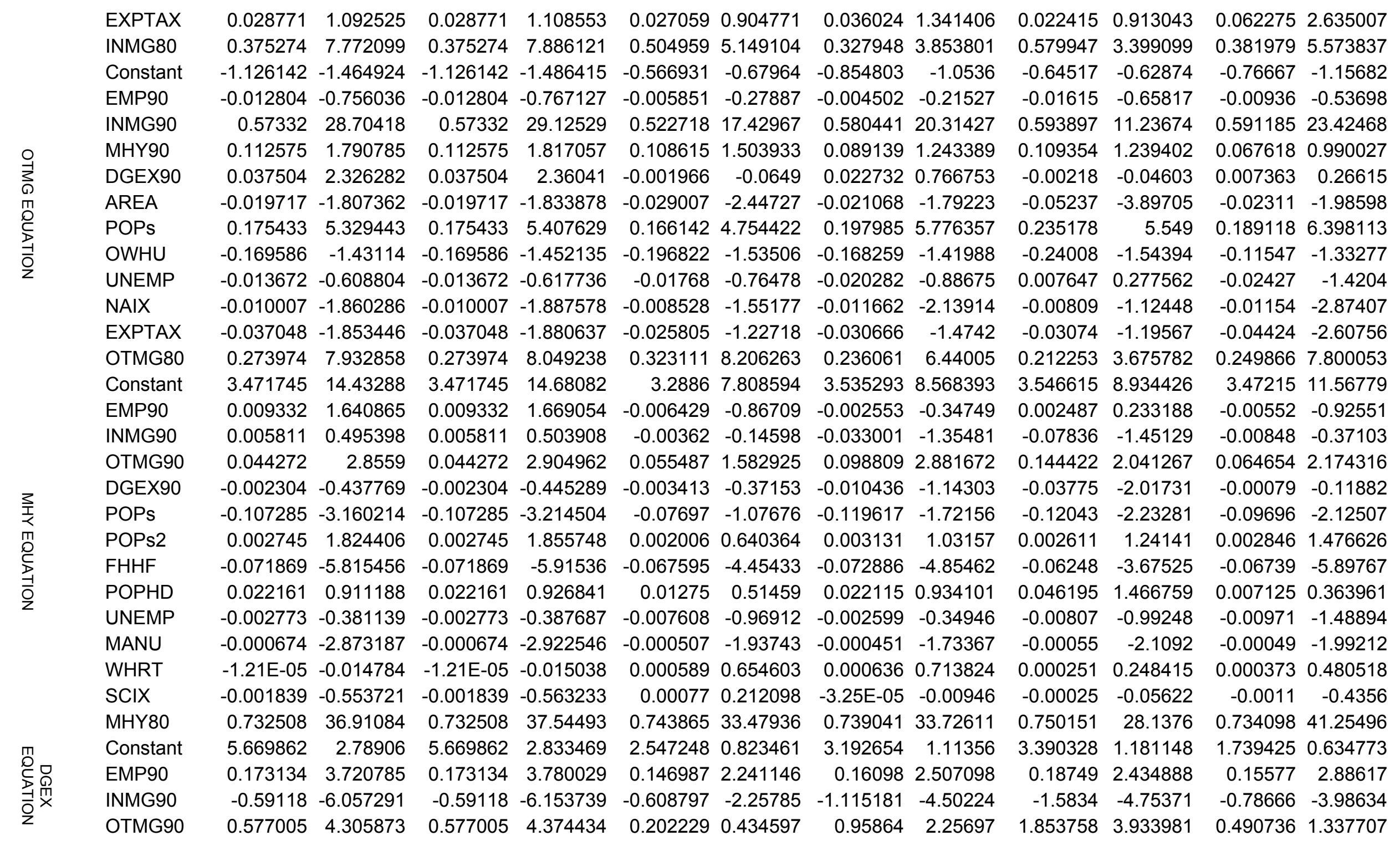




\begin{tabular}{|c|c|c|c|c|c|c|c|c|c|c|c|c|}
\hline MHY90 & -0.041203 & -0.239184 & -0.041203 & -0.242992 & 0.354704 & 1.396554 & 0.39058 & 1.642059 & 0.303385 & 1.158091 & 0.386727 & 1.800997 \\
\hline POPs & -0.319542 & -4.076895 & -0.319542 & -4.14181 & -0.060375 & -0.3044 & -0.296349 & -1.61451 & -0.65037 & -3.60842 & -0.09162 & -0.51254 \\
\hline SCRM & 3.72E-05 & 2.571174 & 3.72E-05 & 2.612114 & 0.000201 & 3.247282 & 0.000148 & 2.654631 & 1.46E-05 & 0.829818 & 0.000115 & 2.299068 \\
\hline РСТАХ & 0.138823 & 2.649309 & 0.138823 & 2.691493 & 0.061149 & 0.945055 & 0.04197 & 0.736022 & 0.077222 & 1.30565 & 0.053913 & 1.298917 \\
\hline PCTD & $-2.69 E-06$ & -0.262383 & $-2.69 E-06$ & -0.26656 & 3.88E-06 & 0.318992 & $-1.48 E-06$ & -0.13907 & -7.48E-06 & -0.86421 & $2.21 \mathrm{E}-06$ & 0.41842 \\
\hline LTD & 1.35E-07 & 0.420642 & 1.35E-07 & 0.42734 & $-1.63 E-07$ & -0.41807 & $-9.72 E-08$ & -0.28564 & $2.20 \mathrm{E}-08$ & 0.021475 & 5.80E-08 & 0.291944 \\
\hline
\end{tabular}




\begin{tabular}{|c|c|c|c|c|c|c|c|c|c|c|c|c|c|}
\hline \multirow[b]{3}{*}{ Equation } & \multirow[b]{3}{*}{ Variables } & \multicolumn{6}{|c|}{ SINGLE-EQUATION } & \multicolumn{6}{|c|}{ MULTI-EQUATION } \\
\hline & & \multicolumn{2}{|c|}{ OLS } & \multicolumn{2}{|c|}{ Weighted OLS } & \multicolumn{2}{|c|}{ Weighted 2SLS } & \multicolumn{2}{|c|}{ 3SLS } & \multicolumn{2}{|c|}{$\mathrm{ML}$} & \multicolumn{2}{|c|}{ GMM } \\
\hline & & Coefficient & t-Statistic & Coefficient & t-Statistic & Coefficient & t-Statistic & Coefficient & $\mathrm{t}$-Statistic & Coefficient & z-Statistic & Coefficient & t-Statistic \\
\hline \multirow{16}{*}{ 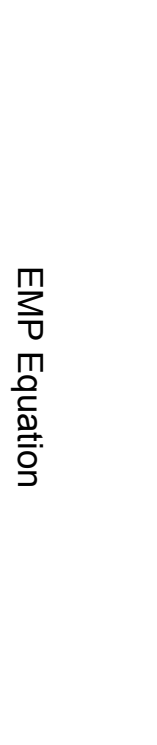 } & constant & & $3-5.923521$ & -3.405448 & -5.967238 & -0.998877 & -1.25422 & -0.49588 & $8 \quad-0.67108$ & $3 \quad-0.91632$ & -1.30868 & $8 \quad-0.68835$ & $5-0.89081$ \\
\hline & INMG90 & 0.302083 & 3.905537 & 0.302083 & 6.956501 & 0.780797 & 5.265965 & 1.076592 & 27.872718 & 0.690464 & 3.734971 & 0.877449 & \\
\hline & OTMG90 & -0.08532 & -1.753999 & -0.08532 & -1.766944 & -0.538235 & -3.43163 & -0.811431 & $1-5.61741$ & -0.42066 & -2.19339 & -0.65713 & $\begin{array}{ll}3 & -5.1572\end{array}$ \\
\hline & MHY90 & 0.134613 & 32.404574 & 0.134613 & 2.42232 & -0.115947 & -1.52064 & -0.254052 & -3.58667 & -0.24249 & -3.25715 & -0.1206 & -1.70003 \\
\hline & DGEX90 & 0.095254 & 3.558438 & 0.095254 & 3.5847 & 0.10536 & 2.167183 & 0.188652 & 4.135167 & 0.10247 & 2.273074 & 0.134772 & 23.137925 \\
\hline & POP24-44 & 0.226813 & 2.265275 & 0.226813 & 2.281993 & 0.21205 & 1.73 & 0.199236 & & 0.366436 & & 0.183301 & \\
\hline & FHHF & 0.011288 & 0.358914 & 0.011288 & 0.361563 & 0.024018 & 0.65 & 0.017971 & 10.5 & -0.00335 & -0.0847 & 0.024819 & \\
\hline & POPCD & 0.098156 & 3.52014 & 0.098156 & 3.54612 & 0.104186 & 3.263395 & 0.087091 & 13.04115 & 0.114713 & 4.374 & 0.054635 & 51.9 \\
\hline & OWHU & 6417 & 2.67183 & 0.196417 & 2.691549 & 0.152337 & 1.811201 & 0.228144 & 3.01 & 0.269714 & 3.53 & 0.106912 & 21.69 \\
\hline & MANU & 762 & 0.901453 & 0.000762 & 0.908106 & -0.000327 & -0.29271 & -0.000293 & -0.2 & 0.001384 & 1.61 & -0.00153 & $3-1$. \\
\hline & WHRT & 0.005037 & 1.756058 & 0.005037 & 1.769018 & -0.000176 & & 0.001258 & & 0.005381 & & -0.00065 & \\
\hline & РСРТАХ & -0.078669 & $9-4.906136$ & -0.078669 & -4.942344 & -0.048847 & -2.26836 & -0.061851 & $1 \quad-3.23792$ & -0.04223 & -2.38818 & -0.04774 & $-2.4 \mathrm{c}$ \\
\hline & NAIX & 007253 & $\begin{array}{l}3.229258 \\
\end{array}$ & 0.007253 & 1.23833 & -0.007424 & -0.98763 & -0.004594 & -0.67823 & 0.002118 & 0.27713 & 0.000475 & 0.07 \\
\hline & HWD & 055907 & 3.151229 & 0.055907 & 3.174485 & 0.068393 & 3.648476 & 0.0992 & 5.816282 & 0.085206 & 4.261689 & 0.056003 & 33.6 \\
\hline & ESBd & 9805 & 3.731505 & 0.119805 & 3.759044 & 0.099085 & 2.449205 & 0.086824 & & 0.118169 & 3.913806 & 0.026904 & \\
\hline & EM & 780071 & 48.8884 & 0.780071 & 49.2492 & & & 0.774231 & 138.4 & 897 & 42. & 0631 & 140.3 \\
\hline \multirow{10}{*}{ 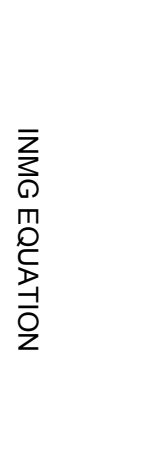 } & Constant & -2.637499 & $9-6.424735$ & -2.637499 & -6.460199 & -0.656634 & -1.2028 & -0.895985 & $5 \quad-1.81406$ & 0.752936 & 1.26491 & -0.44392 & $2 \quad-0.90178$ \\
\hline & EMP90 & 0.082663 & 36.978773 & 0.082663 & 7.017295 & 0.068013 & 4.55 & 0.057081 & 4.00 & 659 & 0.425877 & 0.091754 & 46.366396 \\
\hline & OTMG90 & 0.953372 & 51.65836 & 0.953372 & 51.94351 & 0.990653 & 41.46084 & 1.012474 & 451. & 295 & 34.2 & 0182 & \\
\hline & MHY90 & 0.394613 & 39.913383 & 0.394613 & 9.968103 & 0.207274 & 3.684817 & 0.238652 & 24.782946 & -0.02456 & -0.4003 & 0.213934 & $4 \quad 4.285005$ \\
\hline & DGEX90 & -0.096905 & $5-6.228072$ & -0.096905 & -6.26245 & -0.123786 & -5.75092 & -0.129081 & $1-6.09388$ & -0.07254 & -2.90194 & -0.1399 & $9-6.77101$ \\
\hline & AREA & 0.043362 & 6.553516 & 0.043362 & 6.589691 & 0.041504 & 6.121676 & 0.036947 & 6.253611 & 0.038345 & 5.891861 & 0.041934 & \\
\hline & POPs & -0.14454 & -6.57658 & -0.14454 & -6.612881 & -0.156149 & -5.87647 & -0.139703 & -5.67876 & -0.06164 & -2.1285 & -0.22256 & $6 \quad-8.69346$ \\
\hline & MCRH & -0.004102 & $2-0.147664$ & -0.004102 & -0.148479 & 0.020385 & 0.688394 & 0.007259 & 90.443387 & 0.121089 & 4.374747 & 0.022057 & $7 \quad 1.314112$ \\
\hline & UNEMP & 0.015873 & 30.867943 & 0.015873 & 0.872734 & -0.02811 & | -1.30917 & -0.019681 & $\begin{array}{ll}1-1.03997\end{array}$ & -0.12655 & -5.52012 & 0.010005 & 50.529336 \\
\hline & NAIX & 0.030576 & 7.584261 & 0.030576 & 7.626124 & 0.030637 & 7.502779 & 0.020311 & $1 \quad 5.6844$ & 0.026985 & 5.86768 & 0.02446 & 67.360847 \\
\hline
\end{tabular}




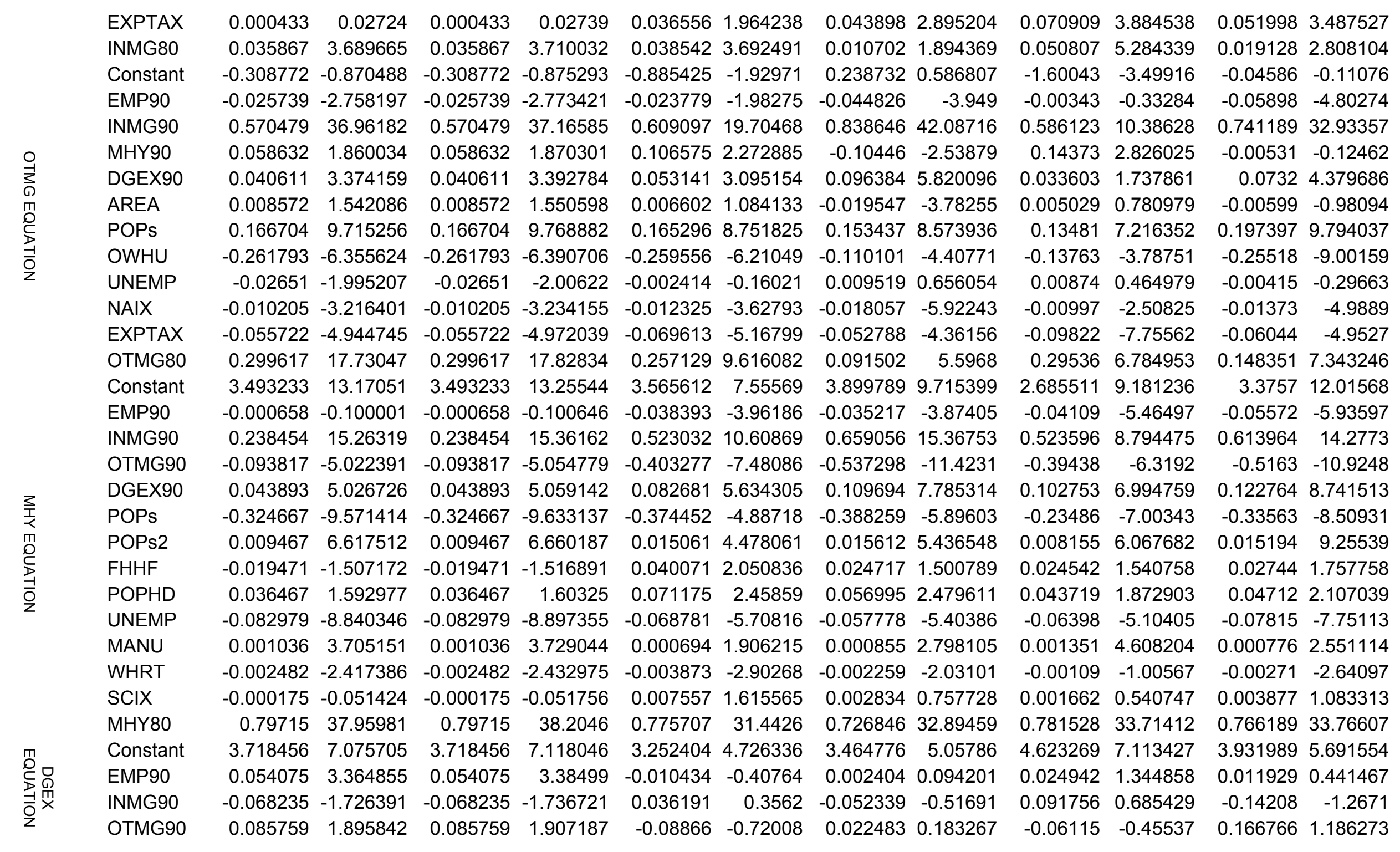




\begin{tabular}{|c|c|c|c|c|c|c|c|c|c|c|c|c|}
\hline MHY90 & & & -0.080665 & & & & & & & & & \\
\hline POPs & -0.079485 & -2.899095 & -0.079485 & -2.916443 & 0.028765 & 0.580859 & -0.003883 & -0.07869 & -0.04928 & & & \\
\hline CR & & & & & & 98 & -05 & & & 3.35 & 4.6 & \\
\hline CTAX & 769 & 6.286764 & 0.11 & 6.324 & 0.1 & & & $5.3 \varepsilon$ & & 9.20 & & \\
\hline CTD & I.38E-08 & -0.002429 & $-1.38 \mathrm{E}-08$ & -0.002443 & $.50 \mathrm{E}-06$ & 0.254074 & $E-06$ & 0.34 & 1.93E-06 & 245 & 8.93E-06 & 1.652177 \\
\hline rD & & 0.280764 & & 0.28 & -3.92 & 391 & -08 & -0.65064 & 2.96E-09 & -0.0 & $-8.22 \mathrm{E}-08$ & -2.31465 \\
\hline
\end{tabular}


Table D: NON-SPATIAL MODEL ESTIMATION RESULTS, LEVEL: APP.ST, 1990 S

SINGLE-EQUATION

MULTI-EQUATION Weighted OLS

Weighted 2SLS $M L$

GMM

Equation Variables Coefficient t-Statistic Coefficient t-Statistic Coefficient t-Statistic Coefficient t-Statistic Coefficient z-Statistic Coefficient t-Statistic

\begin{tabular}{|c|c|c|c|c|c|c|c|c|c|c|c|c|}
\hline constant & 825287 & -2.794949 & .825287 & -2.815576 & -0.692916 & & & & & & & \\
\hline & 160781 & 2.29968 & 0781 & 2.316652 & .083975 & 549 & 232 & & & & & \\
\hline & .087261 & -0.7 & .087261 & -0.768298 & 0.125 & 1.03 & & & & 18 & & \\
\hline $\mathrm{HHF}$ & 0.020681 & 0.655098 & 0.020681 & 0.659933 & -0.043834 & -1.23558 & 459 & 5066 & 336 & -1.2 & 146 & -1.2 \\
\hline & 0426 & 389 & 50426 & 9694 & 99279 & 585 & 688 & 3011 & 472 & 423 & & 015 \\
\hline $\mathrm{H}$ & 0.01618 & 5.865044 & 0.01618 & 5.908329 & 0.016805 & 6.011299 & 15889 & 5.741225 & 0.014974 & & & 7.311611 \\
\hline CPTA & 0.059107 & -4.136154 & -0.059107 & -4.16668 & -0.050358 & -3.09471 & 36033 & -2.29551 & -0.00206 & -0.09994 & 4186 & -3.04881 \\
\hline Al & 84E-05 & 0.0 & 7.84E-05 & 285 & -0.0 & -0. & 305 & 0.8 & 661 & 011 & & -0.14694 \\
\hline & 11 & -0.3 & 11 & 537 & & & & & & & & 799 \\
\hline $\mathrm{SE}$ & 437 & 2.13 & 0.05 & 146 & 0.067472 & & 442 & & & & & 944 \\
\hline 0 & 796362 & 54.33143 & 0.796362 & 54.73241 & 0.780129 & 36.1 & & 6069 & 0.787567 & 81 & & 37.82132 \\
\hline & -0.061643 & -5.77119 & -0.061643 & -5.803046 & 1571 & 3087 & 241 & 512 & & 742 & & -7.25649 \\
\hline & 0.041472 & 5.749212 & 0.041472 & 5.780947 & 0.037346 & 4.722678 & 8891 & 5.394865 & -0.0477 & -0.97513 & 86 & 5.622186 \\
\hline (1) & -0.19629 & -8.853606 & -0.19629 & -8.902477 & -0.220461 & -7.20466 & 1756 & -8.21743 & 0.923534 & 2.174833 & & -9.23627 \\
\hline $\mathrm{CRH}$ & 0.107778 & -2.443179 & -0.107778 & -2.456665 & -0.023138 & -0.45234 & 7924 & -0.28901 & 0.326342 & 1.353887 & -0.01061 & -0.43984 \\
\hline & 010172 & 0.598834 & 0.010172 & 0.602139 & 0.025144 & 1.270338 & & 1.211349 & -0.49465 & -2.61109 & 0.031586 & 1.90099 \\
\hline AIX & & 7.737021 & .032144 & & & & & & & & 0.028767 & 367419 \\
\hline
\end{tabular}




\begin{tabular}{|c|c|c|c|c|c|c|c|c|c|c|c|c|c|}
\hline \multirow{15}{*}{ 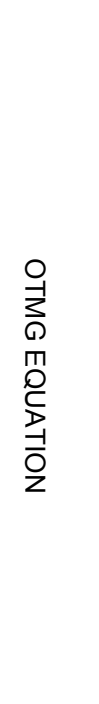 } & EXPTAX & 0.035836 & 2.235282 & 0.035836 & 2.24762 & 0.04842 & 2.594062 & 0.050559 & 2.946652 & -0.13808 & -1.14763 & 0.085849 & 5.219985 \\
\hline & INMG80 & 0.15982 & 6.236196 & 0.15982 & 6.270619 & -0.024002 & -0.45415 & -0.032624 & -1.0214 & 2.923333 & 2.802356 & 0.017142 & 0.591974 \\
\hline & Constant & 0.499968 & 1.214222 & 0.499968 & 1.220924 & 0.566339 & 1.255898 & 0.887285 & 2.04657 & 1.221531 & 2.450846 & 0.173304 & 0.427137 \\
\hline & EMP90 & 0.003816 & 0.407174 & 0.003816 & 0.409422 & -0.00376 & -0.32138 & -0.018116 & -1.56623 & -0.01268 & -1.03445 & 0.000258 & 0.025123 \\
\hline & INMG90 & 0.63397 & 50.08466 & 0.63397 & 50.36112 & 0.600834 & 29.88728 & 0.785401 & 55.28882 & 0.602628 & 26.96538 & 0.696877 & 42.73458 \\
\hline & MHY90 & -0.093476 & -2.423064 & -0.093476 & -2.436439 & -0.127223 & -2.89743 & -0.218423 & -5.10008 & -0.08162 & -1.75128 & -0.11661 & -2.70853 \\
\hline & DGEX90 & 0.029306 & 3.620962 & 0.029306 & 3.640949 & 0.042447 & 2.588292 & 0.10402 & 6.638915 & 0.013195 & 0.676284 & 0.071029 & 4.971044 \\
\hline & AREA & -0.018864 & -3.258804 & -0.018864 & -3.276792 & -0.019931 & -3.36809 & -0.028203 & -5.03042 & -0.01208 & -1.90808 & -0.01919 & -3.11407 \\
\hline & POPs & 0.116264 & 6.161606 & 0.116264 & 6.195617 & 0.120618 & 5.882783 & 0.170831 & 8.815682 & 0.167091 & 7.202049 & 0.139749 & 8.071337 \\
\hline & OWHU & -0.017322 & -0.360258 & -0.017322 & -0.362247 & 0.028416 & 0.569241 & -0.001274 & -0.03989 & -0.2069 & -4.3218 & 0.018353 & 0.454514 \\
\hline & UNEMP & -0.034591 & -2.688705 & -0.034591 & -2.703546 & -0.046325 & -3.4814 & -0.027957 & -2.14091 & -0.04032 & -2.83368 & -0.03878 & -3.18892 \\
\hline & NAIX & -0.016812 & -5.247229 & -0.016812 & -5.276193 & -0.015719 & -4.81216 & -0.022092 & -6.92519 & -0.01314 & -3.23616 & -0.01961 & -6.8253 \\
\hline & EXPTAX & -0.049931 & -4.005035 & -0.049931 & -4.027142 & -0.037309 & -2.78131 & -0.037815 & -2.87463 & -0.02475 & 5031 & 188 & 1964 \\
\hline & OTMG80 & 0.271924 & 14.52415 & 0.271924 & 14.60432 & 0.309772 & 13.7782 & 9121 & 7.878216 & 0.25 & 571 & 457 & 672 \\
\hline & Constant & 3.282564 & 24.62244 & 3.282564 & 24.78122 & 2.269875 & 9.430995 & 2.369497 & 10.16069 & 3.376337 & 18.97937 & 2.5 & 3541 \\
\hline \multirow{17}{*}{ 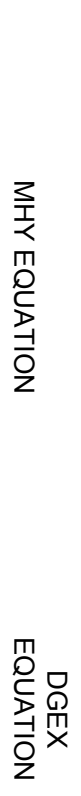 } & EMP90 & 0.015378 & 4.227216 & 0.015378 & 4.254476 & -0.00316 & -0.55823 & -0.000917 & -0.16612 & 0.012198 & 2.058938 & -0.00477 & -0.95281 \\
\hline & INMG90 & 0.037357 & 4.535826 & 0.037357 & 4.565076 & 0.00808 & 0.415501 & -0.012634 & -0.66195 & -0.0122 & 8349 & -0. & 9636 \\
\hline & OTMG90 & 0.001647 & 0.164968 & 0.001647 & 0.166032 & 0.016484 & 0.686849 & 0.04257 & 1.811737 & 0.028657 & 1.039827 & 0.0 & 2.79877 \\
\hline & DGEX90 & -0.005134 & -1.710529 & -0.005134 & -1.721559 & -0.022632 & -3.01925 & -0.027095 & -3.65618 & -0.06511 & -6.06541 & 2376 & -3.49844 \\
\hline & POPs & -0.041486 & -2.29093 & -0.041486 & -2.305704 & 0.147691 & 3.564763 & 0.13108 & 3.294888 & -0.03415 & -1.69214 & 0.137107 & 3.503616 \\
\hline & POPs2 & -0.000251 & -0.333355 & -0.000251 & -0.335504 & -0.0075 & -4.22545 & -0.007125 & -4.18789 & 0.00039 & 0.507341 & -0.00771 & -4.57581 \\
\hline & FHHF & -0.096977 & -16.65674 & -0.096977 & -16.76415 & -0.083039 & -10.4911 & -0.087295 & -11.377 & -0.08203 & -13.6609 & -0.09963 & -13.9573 \\
\hline & POPHD & 0.045631 & 2.649293 & 0.045631 & 2.666378 & 0.028519 & 1.357628 & 0.035686 & 1.813116 & 0.082095 & 4.658284 & 0.028559 & 1.590336 \\
\hline & UNEMP & -0.007697 & -1.642911 & -0.007697 & -1.653506 & -0.020218 & -3.74529 & -0.020104 & -3.98613 & -0.01568 & -3.17212 & -0.0182 & -3.69601 \\
\hline & MANU & -0.000355 & -2.189597 & -0.000355 & -2.203717 & -0.000556 & -2.96074 & -0.000531 & -2.92935 & -0.00056 & -3.1538 & -0.0 & 1055 \\
\hline & WHRT & -0.000492 & -0.901409 & -0.000492 & -0.907222 & -0.000609 & -0.97675 & -0.000814 & -1.35378 & -0.00057 & -0.99157 & -0.00019 & -0.32899 \\
\hline & SCIX & 0.001477 & 0.828753 & 0.001477 & 0.834098 & 0.005248 & 2.495427 & 0.004761 & 2.428346 & 0.005034 & 2.685856 & 0.002727 & 1.534091 \\
\hline & MHY80 & 0.717739 & 68.73188 & 0.717739 & 69.17511 & 0.74956 & 60.28487 & 0.74853 & 62.02277 & 0.742177 & 52.3183 & 0.72385 & 62.77548 \\
\hline & Constant & 1.569265 & 1.366681 & 1.569265 & 1.374859 & -0.207593 & -0.14223 & -0.181713 & -0.13794 & 1.787078 & 1.195281 & 0.332604 & 0.25445 \\
\hline & EMP90 & 0.136672 & 4.549223 & 0.136672 & 4.576445 & 0.103987 & 2.371063 & 0.121233 & 2.912805 & 0.093434 & 2.172711 & 0.111168 & 3.606066 \\
\hline & INMG90 & -0.378336 & -5.279534 & -0.378336 & -5.311127 & -0.511075 & -3.17136 & -1.060026 & -7.13806 & -0.54555 & -2.36106 & -0.5295 & -5.12347 \\
\hline & OTMG90 & 0.243171 & 2.774961 & 0.243171 & 2.791566 & 0.244546 & 1.067192 & 0.927671 & 4.424662 & 0.357827 & 1.376707 & 0.425575 & 2.513359 \\
\hline
\end{tabular}




\begin{tabular}{|c|c|c|c|c|c|c|c|c|c|c|c|c|}
\hline MHY90 & & 1.255659 & & & & & & & & & & \\
\hline POPs & 0.091023 & -1.808784 & -0.091023 & -1.819608 & 0.02976 & 0.294382 & -0.118778 & -1.27366 & -0.0049 & -0.05717 & -0.07041 & \\
\hline CRM & & -0.0482 & $-3.7^{\prime}$ & $-0.04 \varepsilon$ & 3.1 & & & & -06 & 0.6 & & \\
\hline CTAX & 387 & 2.582405 & 387 & 2.597858 & 0.077329 & & & & 5956 & 993 & & 972 \\
\hline CTD & 44E-06 & 1.19776 & & 1.204927 & 6.93E-06 & 82 & & 0.816625 & $-4.68 E-06$ & 595 & & 2064 \\
\hline rD & 08 & 2.312386 & 8.0 & 2.32 & 7.78E-08 & & & 1.855678 & $5.90 \mathrm{E}$ & 53 & & 958 \\
\hline
\end{tabular}




\begin{tabular}{|c|c|c|c|c|c|c|c|c|c|c|c|c|c|}
\hline \multirow[b]{3}{*}{ Equation } & \multirow[b]{3}{*}{ Variables } & \multicolumn{6}{|c|}{ SINGLE-EQUATION } & \multicolumn{6}{|c|}{ MULTI-EQUATION } \\
\hline & & \multicolumn{2}{|c|}{ OLS } & \multicolumn{2}{|c|}{ Weighted OLS } & \multicolumn{2}{|c|}{ Weighted 2SLS } & \multicolumn{2}{|c|}{ 3SLS } & \multicolumn{2}{|c|}{$\mathrm{ML}$} & \multicolumn{2}{|c|}{ GMM } \\
\hline & & Coefficient & t-Statistic & Coefficient & t-Statistic & Coefficient & t-Statistic & Coefficient & t-Statistic & Coefficient & z-Statistic & Coefficient & t-Statistic \\
\hline \multirow{16}{*}{ 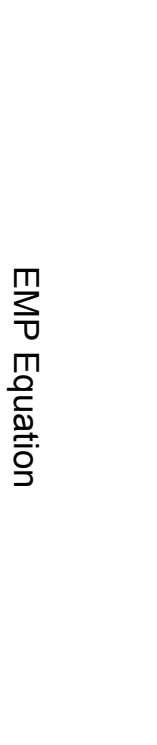 } & constant & & -10.34562 & -3.355221 & -10.37242 & -0.19711 & -0.41518 & -0.134107 & -0.31404 & -0.48453 & -1.10521 & 0.730544 & \\
\hline & INMG90 & 0.226226 & 13.77264 & 0.226226 & 13.80832 & 0.753702 & 10.0984 & 0.975987 & 14.71629 & 1.138702 & 11.45285 & 0.952986 & \\
\hline & OTMG90 & 0.021173 & 1.036231 & 0.021173 & 1.038916 & -0.508284 & $4-6.31881$ & -0.726912 & -10.1186 & -0.88814 & -8.65378 & -0.79057 & -10.4635 \\
\hline & MHY90 & 0.261947 & 7.962078 & 0.261947 & 7.982706 & -0.050931 & $1-0.92035$ & -0.133862 & -2.71904 & -0.10947 & -2.04077 & -0.12204 & -2.40675 \\
\hline & DGEX90 & 0.012967 & 0.878457 & 0.012967 & 0.880733 & -0.012963 & $3-0.50821$ & 0.073274 & 3.121987 & 0.083358 & 2.713132 & 0.047471 & 132 \\
\hline & POP24-44 & 0045 & -2.04 & -0.100045 & -2.05 & 0.032231 & & 16949 & & -0.00033 & & 0.1381 & \\
\hline & FHHF & 0.116844 & 7.135057 & 0.116844 & 7.153542 & 0.1012 & 25.0 & 0.069494 & & 441 & & 0.040444 & \\
\hline & POPCD & 0.085662 & 5.07817 & 0.085662 & 5.091326 & 0.101 & 4.782064 & .11118 & 6.179783 & 0.117601 & 7.114 & 0.063237 & 3.12 \\
\hline & OWHU & 0.214277 & 4.652313 & 0.214277 & 4.664365 & 0.076 & 1.225988 & 0.151721 & 5371 & 0.134631 & 4.431 & -0.06137 & $-0.7 \varepsilon$ \\
\hline & MANU & 817 & 3.21494 & 0.001817 & 3.22 & -0.0007 & $4 \quad-0.82074$ & 0094 & 1.26 & 02374 & & -0.00126 & \\
\hline & & 0.003938 & 2.4377 & 0.003938 & 2.44 & & & -0.000391 & & 0075 & & -0.00032 & -0.16 \\
\hline & РСРТАХ & -0.084711 & -9.311528 & $3-0.084711$ & - -9.335651 & -0.025784 & $4-2.02566$ & -0.045242 & -4.21964 & -0.05398 & -5.70415 & -0.00752 & -0.65123 \\
\hline & NAIX & .00211 & -0.935082 & -0.00211 & - -0.937504 & -0.014722 & -4.64012 & -0.011785 & -4.13377 & -0.01264 & -4.44011 & -0.008 & -2.7 \\
\hline & HWD & 06009 & 7.29504 & 0.06009 & & 0.063223 & 6.327789 & 0.041763 & & 1076 & 1.1 & 0.056958 & 2037 \\
\hline & ESBd & 2935 & 3.536813 & 0.072935 & & 0.08 & & 1442 & & 2436 & & -0.02464 & \\
\hline & & 74648 & 78.59777 & 0.774648 & 78.8 & 0.797446 & 58.4 & 903 & 64. & 666 & & 0.864917 & 438 \\
\hline \multirow{10}{*}{ 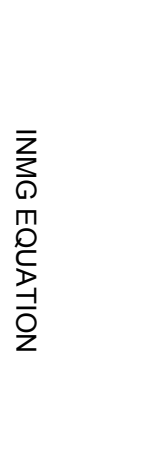 } & Constant & -2.154768 & -6.219887 & -2.154768 & -6.231961 & -0.420013 & -0.89897 & -1.339763 & -3.22681 & 0.128148 & 0.277392 & -0.60943 & -1.54832 \\
\hline & EMP90 & 509 & 8.120629 & 0.094509 & 8.136392 & 0.036896 & 2.5 & 1077 & 772 & -0.07686 & -4 & 0.050697 & \\
\hline & OTMG90 & 0.895899 & 48.79143 & 0.895899 & 48.88614 & 0.934 & 35. & 6712 & 47.7526 & 0.77 & 29. & 4298 & \\
\hline & MHY90 & .37844 & 10.98494 & 0.37844 & 11.00627 & 0.182524 & 3.700605 & 0.240613 & 5.58912 & -0.04747 & -0.95321 & 0.230796 & 5.642473 \\
\hline & DGEX90 & -0.15127 & -11.045 & -0.15127 & -11.06644 & -0.17933 & -10.0476 & -0.167152 & -9.94929 & -0.13299 & -7.07185 & -0.21623 & -13.4624 \\
\hline & AREA & 0.007775 & 1.304297 & 0.007775 & 1.306829 & -0.007241 & & -0.023672 & -4.37172 & & & -0.01009 & \\
\hline & POPs & -0.103695 & -5.144271 & -0.103695 & -5.154257 & & -2.12659 & 0.000134 & 0.005851 & & & -0.09748 & -4.50919 \\
\hline & MCRH & -0.084799 & -3.231177 & -0.084799 & -3.237449 & -0.023956 & -0.81612 & 0.037708 & 2.286416 & 0.176296 & 7.872432 & 0.025577 & 1.522993 \\
\hline & UNEMP & 0.119105 & 12.70045 & 50.119105 & 12.7251 & 0.106193 & 10.75956 & 0.09423 & 11.00886 & 0.044543 & 4.945737 & 0.098276 & 11.08761 \\
\hline & NAIX & 0.022001 & 9.586949 & 0.022001 & 9.605558 & 0.022526 & 9.454048 & 0.011329 & 5.3528 & 0.016657 & 7.152791 & 0.015109 & 7.683512 \\
\hline
\end{tabular}




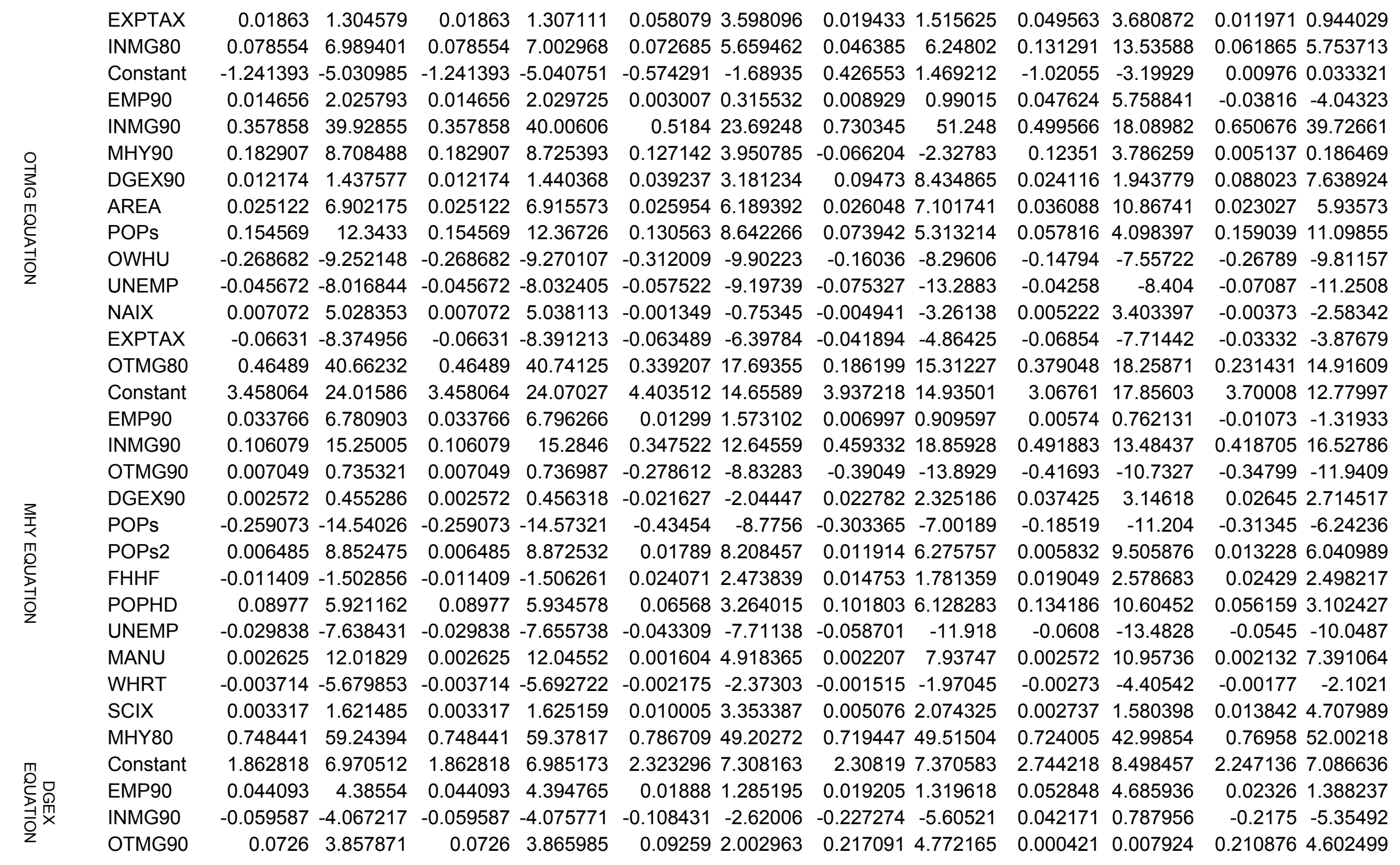




\begin{tabular}{|c|c|c|c|c|c|c|c|c|c|c|c|c|}
\hline MHY90 & 0.05566 & 2.389271 & & & & & & & & & & \\
\hline POPs & 0.073386 & -4.485423 & -0.073386 & -4.494858 & -0.03907 & & -0.048545 & & -0.10423 & & & \\
\hline OP5-1 & & 0.305527 & 0.010424 & & & & & & & & & \\
\hline & & & & & & & & & & & & \\
\hline FEG & & 0.24 & 57 & & 11 & & -0.0 & & & & & \\
\hline CTAX & 263 & 6.57 & .062263 & & & & & & & & & \\
\hline CTD & -07 & -0.30 & $-5.18 E-07$ & & & 0.05 & -1.38 & & -3.3 & & -07 & -0 . \\
\hline & & & & & & & & & & & & -0.14264 \\
\hline & .713607 & 49.45151 & 0.713607 & 49.55553 & 0.66968 & 40.0362 & 0.660773 & 40.43177 & 0.718879 & 54.25291 & 0.68849 & 31.27765 \\
\hline
\end{tabular}


Table F:NON-SPATIAL MODEL ESTIMATION RESULTS, LEVEL: US COUNTIES, $1990 \mathrm{~S}$

SINGLE-EQUATION

MULTI-EQUATION

OLS Weighted OLS Weighted 2SLS

3SLS $M L$

GMM

Equation Variables Coefficient t-Statistic Coefficient t-Statistic Coefficient t-Statistic Coefficient t-Statistic Coefficient z-Statistic Coefficient t-Statistic

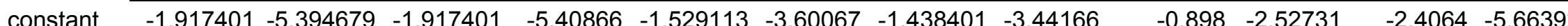

$\begin{array}{llllllll}0.200382 & 8.950064 & 0.200382 & 8.973258 & 0.10989 & 1.839866 & 0.023185 & 0.395176\end{array}$

$0.19527 \quad 4.467087$

$-0.04349-0.67799$

$\begin{array}{lllllllll}\text { OTMG90 } & 0.037444 & 1.379283 & 0.037444 & 1.382857 & 0.154572 & 2.204468 & 0.241314 & 3.505637\end{array}$

0.0322870 .662471

0.3118844 .057491

MHY90

$\begin{array}{llllll}0.163814 & 4.162118 & 0.163814 & 4.172905 & -0.013906 & -0.30802\end{array}$

0.0944712 .131613

$0.076062 \quad 1.80773$

0.1213072 .477969

$\begin{array}{lllllllll}\text { DGEX90 } & 0.062096 & 5.137991 & 0.062096 & 5.151306 & 0.09184 & 4.434315 & 0.047256 & 2.305119\end{array}$

0.0518522 .497779

0.0674913 .169819

$\begin{array}{lllllllll}\text { POP24-44 } & 0.005022 & 0.088909 & 0.005022 & 0.089139 & 0.176765 & 2.936799 & 0.005563 & 0.095705\end{array}$

$-0.00067-0.01406$

0.02190 .304338

FHHF

$-0.00177-0.103984$

$-0.044473-2.50154-0.028507-1.62712$

$-0.02811-1.77974$

$-0.02042-1.03276$

POPCD

$\begin{array}{lllll}0.063954 & 3.565017 & 0.063954 & 3.574256\end{array}$

0.1014035 .462922

0.0659353 .669506

$0.066155 \quad 3.963976$

0.0457262 .228363

OWHU

$\begin{array}{llll}-0.034966 & -0.733243 & -0.034966 & -0.735143\end{array}$

0.087831 .569003

$\begin{array}{ll}0.007969 & 0.1469\end{array}$

$-0.05188-1.23031$

0.1084331 .773683

MANU

$\begin{array}{lllll}0.001981 & 3.262915 & 0.001981 & 3.271371\end{array}$

0.003344 .699736

$0.003207 \quad 4.58892$

0.0018732 .749451

0.0030343 .954514

PCPTAX

$0.010685 \quad 6.896774$

$\begin{array}{llll}0.010685 & 6.914647 & 0.011559 & 7.273427\end{array}$

0.010636 .794878

0.0097387 .197448

0.0095695 .36431

NAIX

$-0.008118-3.452619$

$-0.02981-3.521629$

$\begin{array}{lllll}-0.031205 & -3.2413 & -0.024544 & -2.62233\end{array}$

$-0.01684-1.70451$

$-0.05221-5.38644$

HWD

ESBd $\begin{array}{lllll}0.000371 & 0.042142 & 0.000371 & 0.042251\end{array}$

$-0.008636-3.40116$

$-0.003181-1.28678$

$-0.00417-1.5684$

$-0.01046-4.27141$

EMP80 $\begin{array}{lllll}0.060319 & 3.235163 & 0.060319 & 3.243547\end{array}$

.010378 1.138644

0.0013320 .150208

$0.001833 \quad 0.188827$

$-0.02115-2.33602$

$0.766638 \quad 80.78194$

$0.766638 \quad 80.99129$

0.75111556 .17823

0.0908944 .324263

$0.056143 \quad 3.47962$

0.78288967 .95472

0.1523255 .181827

\section{EMP90}

$-0.545065-1.685518$

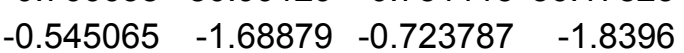

0.75265357 .28586

$-1.89727-3.71412$

$\begin{array}{llllllll}0.014862 & 1.635938 & 0.014862 & 1.639113 & -0.038853 & -3.19019 & -0.026485 & -2.18825\end{array}$

OTMG90

0.89866158 .18745

$0.898661 \quad 58.3004$

0.67737121 .62011

0.7757126 .65067

$-0.0791-5.74785$

0.2761787 .017098

$0.760101 \quad 47.061$

$-0.38533-1.06893$

$-0.03765-2.95348$

$\begin{array}{lllllllll}\text { MHY90 } & 0.188023 & 5.200194 & 0.188023 & 5.210288 & 0.16889 & 3.794755 & 0.13208 & 3.134377\end{array}$

DGEX90

$-0.212187-16.2497$

0.1997643 .601106

0.80248416 .26763

AREA

POPS

$M C R H$

$\begin{array}{llllll}-0.022221 & -5.091319 & -0.022221 & -5.101201 & -0.021498 & -4.59786\end{array}$

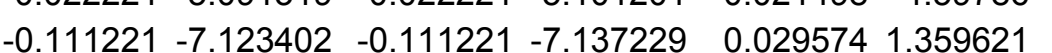

$-0.016732-3.74899$

$-0.21357-13.6518$

0.1553013 .801986

$-0.0219-4.32952$

$-0.22723-17.5368$

$-0.00982-2.00758$

$0.236886 \quad 8.287574$

UNEMP

$\begin{array}{lllll}0.020371 & 0.691159 & 0.020371 & 0.692501\end{array}$

0.1242013 .790623

0.098573 .424998

0.2326655 .897033

$0.003366 \quad 0.153131$

NAIX

$\begin{array}{lllll}0.012755 & 6.729935 & 0.012755 & 6.742998\end{array}$

$\begin{array}{llll}0.047464 & 4.767161 & 0.052222 & 5.448462\end{array}$

$-0.01281-1.03522$

0.1142463 .921838

0.060455 .784057

0.0169118 .896568 


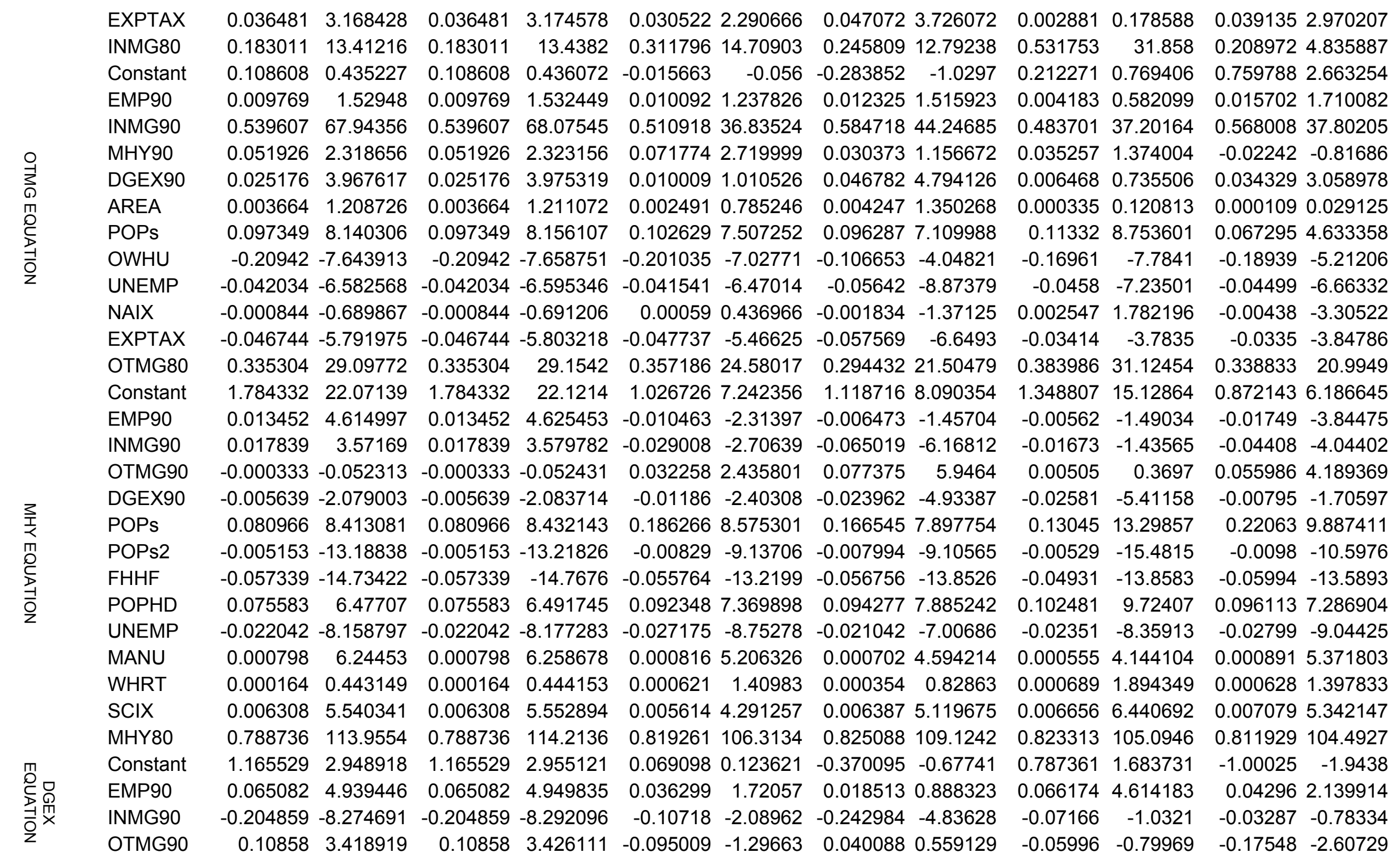




\begin{tabular}{|c|c|c|c|c|c|c|c|c|c|c|c|c|}
\hline & & & & & & & & & & & & \\
\hline POPs & -0.026502 & -1.238887 & -0.026502 & & & & & & & & & \\
\hline POP5-1 & & -0.94 & -0.0 & -0.9 & & & $-0 . c$ & -0.4 & 098 & 23 & & \\
\hline SCRM & & & & & & & & & & & & \\
\hline FEG & & 35 & 0.0 & & & & & & & & & \\
\hline CTAX & & 3.445825 & 0.04 & 3.453073 & & & & & & & & \\
\hline CTD & $-5.24 E-06$ & -4.290194 & $-5.24 \mathrm{E}-06$ & -4.299218 & $-5.19 E-06$ & -4.16008 & -5.63 & & -6.1 & -7.63456 & -7.9 & -5.69154 \\
\hline TD & -08 & 3.39 & & & & & & & & 447 & & \\
\hline & .742497 & 38.93568 & 0.742497 & 39.01758 & 0.725831 & 34.97459 & 0.692455 & 4.15963 & 0.736143 & 34.84405 & 7 & ד \\
\hline
\end{tabular}


Table G:NON-SPATIAL MODEL RESULTS, RATE, APPALACHIA, 1990

\begin{tabular}{|c|c|c|c|c|c|c|c|c|c|c|c|c|c|}
\hline \multirow{3}{*}{$\begin{array}{l}\text { Equati } \\
\text { on } \\
\end{array}$} & \multirow{3}{*}{ Variables } & \multicolumn{6}{|c|}{ SINGLE-EQUATION } & \multicolumn{6}{|c|}{ MULTI-EQUATION } \\
\hline & & \multicolumn{2}{|c|}{ OLS } & \multicolumn{2}{|c|}{ Weighted OLS } & \multicolumn{2}{|c|}{ Weighted 2SLS } & \multicolumn{2}{|c|}{ 3SLS } & \multicolumn{2}{|l|}{ ML } & \multicolumn{2}{|l|}{ GMM } \\
\hline & & Coefficient & t-Statistic & Coefficient & t-Statistic & Coefficient & t-Statistic & Coefficient & t-Statistic & Coefficient & z-Statistic & Coefficient & t-Statistic \\
\hline \multirow{16}{*}{$\begin{array}{l}\text { EMP } \\
\text { Equati } \\
\text { on }\end{array}$} & constant & -1.711706 & \begin{tabular}{|l|l|}
$j$ & -1.367227 \\
\end{tabular} & -1.711706 & \begin{tabular}{|l|}
1.39417 \\
\end{tabular} & -1.2193 & \begin{tabular}{|l|l|}
-0.95896 \\
\end{tabular} & -2.726414 & \begin{tabular}{|l}
-2.85296 \\
\end{tabular} & -1.77423 & -2.45593 & -1.73219 & -2.05581 \\
\hline & INMG90 & 0.019318 & .610754 & 0.019318 & 0.622789 & 0.033887 & 0.928499 & 0.109817 & 3.117227 & 0.117432 & 1.925455 & 0.028631 & 1.24838 \\
\hline & OTMG90 & 0.011374 & $0.20217 \varepsilon$ & 0.011374 & 0.206 & 128718 & 1.642303 & 0.193913 & $2.76185 €$ & 0.266669 & 5722 & 0.098457 & 1.638707 \\
\hline & MHY90 & 1.081128 & 9.602786 & 1.081128 & 9.792022 & 1.314638 & 7.683974 & 1.257389 & 8.29183 & 0.832393 & 5185 & 1.250831 & 7.939504 \\
\hline & DGEX90 & 0.006127 & 0.118144 & 0.006127 & 0.120473 & -0.007686 & -0.07379 & -0.062894 & $-0.6268 \mathrm{~s}$ & 0.104726 & 0.718483 & 0.022519 & 0.268154 \\
\hline & POP24-44 & 0.499257 & 3.067066 & 0.499257 & 3.127507 & 391 & 2.017925 & 0.720992 & 5.451632 & 0.505648 & 9659 & 0.393721 & 07833 \\
\hline & FHHF & -0.006531 & -0.091664 & -0.006531 & -0.09347 & -0.012959 & -0.18058 & 0.07767 & 1.242522 & 0.019203 & 36405 & -0.02757 & 55034 \\
\hline & POPCD & 0.140685 & 3.119583 & 0.140685 & 3.181058 & 0.083282 & 1.667144 & 0.108293 & 2.769468 & 0.122176 & 3.406739 & 0.090716 & 2.42792 \\
\hline & OWHU & 0.061184 & 0.24893 & 0.061184 & 0.253835 & 0.073963 & 0.299261 & 0.041081 & $0.22777 \varepsilon$ & -0.00403 & -0.03936 & 0.119479 & 0.759398 \\
\hline & MANU & -0.000494 & -0.369513 & -0.000494 & -0.376795 & -0.002432 & -1.58281 & -0.00183 & -1.3948 & 0.002453 & 2.864122 & -0.00213 & -1.73874 \\
\hline & WHRT & 0.019329 & 4.109664 & 0.019329 & 4.19065 & 0.016649 & 3.449935 & 0.016003 & 3.784386 & 0.009714 & 2.894067 & 0.017512 & 4.860183 \\
\hline & PCPTAX & -0.045556 & -2.344662 & -0.045556 & -2.390867 & -0.040976 & -2.05437 & $0.00740 \Omega$ & 0.48995 & 0.008441 & 8775 & -0.03016 & -2.44302 \\
\hline & NAIX & $7.50 \mathrm{E}-05$ & 0.007461 & 7.50E-05 & 0.007608 & -0.005115 & -0.48994 & 0.010851 & 1.36032 & 0.0124 & 0816 & 0.009772 & 1.258885 \\
\hline & HWD & 059704 & 761173 & 0.059704 & 1.795879 & 0.084779 & 2.367273 & 0.061024 & 2.21350 & 0.021599 & 1.273484 & 0.082095 & 3.671592 \\
\hline & ESBd & -0.100796 & -2.260251 & -0.100796 & -2.304792 & .106986 & -2.36687 & -0.011717 & -0.34003 & 0.033163 & 1.563469 & -0.05883 & -1.9644 \\
\hline & EMP80 & -0.077309 & -5.165817 & -0.077309 & -5.267616 & -0.06 & -4.18261 & -0.093849 & -6.33736 & -0.09068 & 7608 & 7047 & -4.72846 \\
\hline \multirow{10}{*}{$\begin{array}{l}\text { INMG } \\
\text { EQUATI } \\
\text { ON }\end{array}$} & Cons & -3.638803 & -11.83852 & -3.638803 & -12 & -3.58 & -9.27557 & -3.120407 & $-8.9972 \varepsilon$ & -2.44985 & 1021 & 271 & 2641 \\
\hline & EMP90 & 0.10589 & 2.211061 & 0.10589 & 2.243 & 0.32 & 3.415962 & 0.633445 & 6.908797 & 2.56597 & 4565 & 548 & 849 \\
\hline & OTMG90 & 0.416669 & 7.480022 & 0.416669 & 7.58 & -0.04 & -0.47901 & -0.416308 & 943 & $-1.2 \varepsilon$ & 008 & 147 & 953 \\
\hline & MHY90 & 1.103992 & 8.60562 & 1.103992 & 8.73 & & 3.83234 & 0.600503 & 2.643926 & -2.09603 & 2428 & 0.727382 & 0203 \\
\hline & DGEX90 & -0.103386 & -1.919616 & -0.103386 & -1.947778 & -0.21( & -1.85898 & -0.085312 & -0.7626 & -0.20091 & 2571 & -0.22996 & -2.37357 \\
\hline & AREA & -0.004681 & -0.262077 & -0.004681 & -0.265922 & 0.037319 & 1.779432 & 0.059994 & 3.02263 & 0.08496 & 8888 & 0.032563 & 1.769543 \\
\hline & POPs & 0.604383 & 20.15674 & 0.604383 & 20.45246 & 607578 & 17.12282 & 0.659455 & 19.5918 & 0.610802 & 5643 & 0.496596 & 11.6411 \\
\hline & MCRH & 0.405683 & $6.10783 \mathrm{~s}$ & 0.405683 & 6.197446 & 409991 & 5.501826 & 0.210561 & 3.49828 & 0.030906 & 1.149689 & 0.26988 & 4.5475 \\
\hline & UNEMP & -0.099027 & -2.303570 & -0.099027 & -2.337375 & -0.198649 & -3.71564 & -0.255704 & -5.14297 & -0.25497 & -3.47237 & -0.20302 & -4.31667 \\
\hline & NAIX & 0.000715 & 0.068826 & 0.000715 & 0.069836 & 0.007712 & 0.649658 & 0.004477 & 0.39131 & -0.01455 & -1.20827 & -0.00065 & -0.0684 \\
\hline
\end{tabular}




\begin{tabular}{|c|c|c|c|c|c|c|c|c|c|c|c|c|c|}
\hline & EXPTAX & -0.054992 & -1.627617 & -0.054992 & -1.651495 & -0.090088 & -2.39672 & -0.057497 & -1.65991 & -0.12697 & -2.83429 & -0.08167 & -2.73952 \\
\hline & INMG80 & -0.65668 & -22.40149 & -0.65668 & -22.73014 & -0.66264 & -19.2383 & -0.683693 & -20.8907 & -0.47076 & -4.5315 & -0.52764 & -12.0241 \\
\hline & Constant & -0.487759 & -0.624526 & -0.487759 & -0.633688 & -0.010328 & -0.01139 & 0.444851 & 0.6253 & -0.31867 & -0.8174 & 0.66199 & 1.048459 \\
\hline & EMP90 & 0.043131 & 1.178106 & 0.043131 & 1.195389 & 0.263287 & 3.666933 & 0.49483 & .362228 & 1.416194 & 3.829679 & 0.323431 & 5.257001 \\
\hline & INMG90 & 0.1511 & 6.210992 & 0.1511 & .302111 & 0.004549 & 0.141312 & 0.146655 & 4.98673 & -0.28674 & -2.60546 & -0.02686 & -1.2574 \\
\hline & MHY90 & 0.487872 & 5.167759 & 0.487872 & 5.243574 & 0.205421 & 1.188502 & 0.076181 & 0.47313 & -1.37566 & -2.47366 & 0.069065 & 0.471385 \\
\hline & DGEX90 & 0.043078 & 1.039256 & .043078 & & .08281 & 0.962229 & 036891 & .438509 & -0.1659 & -0.6159 & 0.02902 & -0.36146 \\
\hline & AREA & & & & & 6471 & 3.947198 & 73028 & 969693 & .054657 & 173 & 316 & \\
\hline & POPs & & 906 & & 75 & 73 & 8.278436 & & 13.2043 & .350891 & 469 & 308621 & 8.37989 \\
\hline & OWHU & 433 & 2389 & & 14 & & -1.35274 & & 87 & .19287 & 536 & 0.35236 & -2.86549 \\
\hline & VEMP & 3066 & -5.662395 & 3066 & .745466 & 1528 & -5.40972 & 0.228825 & 6.53791 & .15981 & 79903 & .23367 & -6.27791 \\
\hline & NAIX & 7513 & 0.93382 & & 947519 & & 0.711263 & .003469 & .407383 & -0.00928 & 1.38897 & .00467 & 0.699106 \\
\hline & EXPTAX & .021386 & -0.902974 & .021386 & .916222 & .024687 & -0.96743 & 0.009624 & -0.41422 & -0.0705 & 3.12139 & 0.04296 & -2.23125 \\
\hline & OTMG80 & -0.336704 & -10.12368 & 0.336704 & -10.27221 & -0.343697 & -9.36122 & -0.466329 & -14.507 & -0.27941 & -4.70161 & -0.34317 & -10.0581 \\
\hline \multirow{14}{*}{ 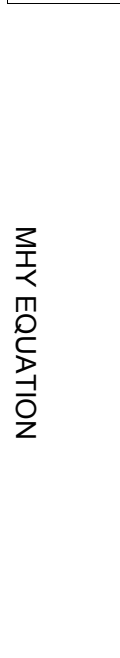 } & Constant & 3.430301 & 7.71966 & 3.430301 & 7.852277 & 2.849655 & 3.720383 & 2.376946 & .869865 & 2.557874 & 4.076893 & 2.5182 & 5.689111 \\
\hline & EMP90 & 7112 & 10.08712 & 12 & 41 & 229416 & 7.71458 & 33208 & .42811 & 537579 & 7.1545 & 0.2817 & 13.29835 \\
\hline & INMG90 & 0.007683 & 0.648044 & 0.007683 & 0.659177 & 0.047239 & -3.08696 & -0.060115 & -4.08493 & -0.10209 & -2.89142 & -0.06362 & -5.38082 \\
\hline & OTMG90 & 0.079915 & 4.07961 & 0.079915 & 4.149695 & 0.005929 & 0.204938 & -0.039952 & -1.44525 & -0.17643 & -2.63008 & 0.00640 & 0.286269 \\
\hline & DGEX90 & -0.014339 & -0.756397 & .014339 & .769391 & 0.012565 & 0.317628 & 0.013703 & 0.351568 & -0.07536 & -0.8012 & -0.02397 & -0.74779 \\
\hline & POPs & -0.051491 & -0.798629 & .051491 & .812349 & -0.018296 & -0.13666 & 0.05322 & 0.497331 & 0.042397 & 1.02043 & -0.00428 & -0.06848 \\
\hline & POPs2 & 02793 & 16398 & 2793 & 41 & & 0.229627 & 088 & -0.21416 & 0.000697 & 0.352061 & 0.0011 & 0.39582 \\
\hline & FHHF & -0.080286 & -3.175247 & .080286 & 229795 & -0.058843 & -2.04461 & 6684 & 31 & -0.05409 & -2.5775 & -0.0556 & -2.36416 \\
\hline & POPHD & 251982 & 786305 & 251982 & 20067 & 268 & 7.412893 & 193534 & 1228 & 0.094361 & 3.210856 & 0.20822 & 1998 \\
\hline & NEMP & 5406 & -8.751033 & .135406 & 901369 & 2297 & 61782 & -0.100531 & -7.10707 & -0.09169 & -3.76475 & -0.10724 & -8.48643 \\
\hline & IANU & 3578 & 901778 & 3578 & & 3441 & 76414 & 03064 & 7.36622 & 138 & 2.821583 & 0.003817 & 11.66419 \\
\hline & WHRT & 0.001217 & 0.732654 & & & & & 00306 & & -0.00276 & -2.2619 & $-5.53 E-06$ & -0.00369 \\
\hline & SCIX & 0.02569 & -4.278367 & .02569 & .351865 & -0.032174 & .14518 & -0.017433 & -2.85159 & -0.00457 & -1.06271 & -0.02757 & -5.56782 \\
\hline & IHY80 & -0.357359 & -9.855869 & -0.357359 & 0.02518 & 127 & -8.18979 & -0.303337 & -8.38473 & -0.28621 & -4.23061 & -0.2901 & -8.37157 \\
\hline \multirow{4}{*}{ 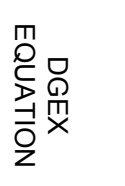 } & Constant & 2.761791 & 5.811178 & 2.761791 & 5.903707 & 3.033987 & 6.055482 & 3.025486 & 6.191218 & 2.787259 & 3.858494 & 2.93361 & 8.157871 \\
\hline & EMP90 & -0.000272 & -0.006696 & -0.000272 & -0.006803 & -0.059969 & -0.78177 & -0.102226 & -1.34337 & -0.06069 & -0.20799 & -0.02973 & -0.45945 \\
\hline & INMG90 & -0.056556 & -2.090736 & -0.056556 & -2.124026 & -0.04943 & -1.56898 & -0.055412 & -1.76042 & -0.02339 & -0.31196 & -0.04323 & -1.87872 \\
\hline & OTMG90 & 0.092074 & 1.964145 & 0.092074 & 1.99542 & 0.155705 & 2.472033 & 0.241083 & 3.882745 & 0.164776 & 1.180296 & 0.17462 & 3.493331 \\
\hline
\end{tabular}




\begin{tabular}{|c|c|c|c|c|c|c|c|c|c|c|}
\hline MHY90 & -0.118639 & -1.254317 & -0.118639 & -1.274289 & -0.10875 & -0.74492 & $\begin{array}{l}-0.115191-0.79441 \\
\end{array}$ & -0.02398 & -0.06855 & $-0.21539-1.75737$ \\
\hline POPs & -0.003185 & -0.239351 & -0.003185 & -0.243162 & -0.019443 & -1.26818 & $-0.033064-2.17759$ & -0.00778 & -0.2748 & $-0.02043-1.60074$ \\
\hline OP5-17 & 0.008465 & 0.077877 & 0.008465 & 0.079117 & 0.011407 & 0.103421 & 0.0262140 .244449 & 0.002946 & 0.020594 & 0.0970961 .218408 \\
\hline CRM & $2.44 \mathrm{E}-05$ & 2.81487 & $2.44 \mathrm{E}-05$ & 2.85969 & 4.71E-05 & 3.230234 & E-05 3.921 & 2.64 & 2.40 & 326 \\
\hline FEG & 0.01653 & 0.686925 & 0.01653 & 0.697863 & 0.006961 & 0.280495 & 0.0054170 .226891 & 0.020462 & 0.582522 & $488-0.29012$ \\
\hline CTAX & 0.043444 & 1.969823 & 0.043444 & 2.001188 & 0.030342 & 1.289566 & 0.0345511 .507017 & 0.034842 & 1.218662 & 3331.955211 \\
\hline CTD & $-1.61 \mathrm{E}-05$ & -1.304564 & $-1.61 \mathrm{E}-05$ & -1.325337 & $-1.36 \mathrm{E}-05$ & -1.10104 & $-1.22 \mathrm{E}-05 \quad-1.0221$ & $-1.40 \mathrm{E}-05$ & -0.88162 & $-1.90 E-05-2.48047$ \\
\hline TD & $4.72 \mathrm{E}-07$ & 0.527365 & $4.72 \mathrm{E}-07$ & 0.535762 & $1.12 \mathrm{E}-07$ & 0.120561 & 1.07E-07 0.119032 & 3.07E-07 & 0.207403 & $-1.73 E-07-0.30219$ \\
\hline GEX80 & -0.368603 & -8.6622 & -0.368603 & -8.800125 & -0.370191 & -8.71827 & $-0.356032-8.49822$ & -0.36456 & -7.59991 & $-0.37907 \quad-8.87604$ \\
\hline
\end{tabular}


Table H: NON-SPATIAL MODEL RESULTS, RATE, APPALACHIA, 1980

\begin{tabular}{|c|c|c|c|c|c|c|c|c|c|c|c|c|c|}
\hline \multirow[b]{3}{*}{ Equation } & \multirow[b]{3}{*}{ Variables } & \multicolumn{6}{|c|}{ SINGLE-EQUATION } & \multicolumn{6}{|c|}{ MULTI-EQUATION } \\
\hline & & \multicolumn{2}{|c|}{ OLS } & \multicolumn{2}{|c|}{ Weighted OLS } & \multicolumn{2}{|c|}{ Weighted 2SLS } & \multicolumn{2}{|c|}{$3 S L S$} & \multicolumn{2}{|c|}{$\mathrm{ML}$} & \multicolumn{2}{|c|}{ GMM } \\
\hline & & Coefficient & & Coefficient $t$ & t-Statistic C & & t-Statistic & Coefficient & t-Statistic & & & & \\
\hline \multirow{16}{*}{ 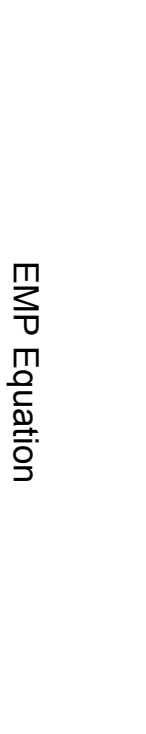 } & constant & -1.711706 & -1.367227 & -1.711706 & -1.39417 & -1.2193 & -0.95896 & -2.726414 & -2.85296 & & -2.45593 & -1.73219 & \\
\hline & INMG90 & 0.019318 & 0.610754 & 0.019318 & 0.622789 & 0.033887 & 0.928499 & 0.109817 & 3.117227 & 0.117432 & 1.925455 & 0.028631 & 1.24838 \\
\hline & OTMG90 & 0.011374 & 0.202178 & 0.011374 & 0.206162 & 0.1287 & 1.642303 & 193913 & & 266669 & 2.555722 & & \\
\hline & MHY90 & 081128 & 9.602786 & 1.08 & 9.792022 & 1.314 & & 1.257389 & & & & & \\
\hline & DGEX90 & 0.006127 & 0.118144 & 0.006127 & 0.120473 & -0.007686 & -0.07379 & -0.062894 & -0.62689 & & & & \\
\hline & POP24-44 & 0.499257 & 3.067066 & 0.499257 & 3.127507 & 0.343391 & 2.017925 & 0.720992 & 5.451632 & 0.505648 & 3.629659 & 0.393721 & \\
\hline & FHHF & -0.006531 & -0.091664 & -0.006531 & -0.09347 & -0.012959 & -0.18058 & 0.07767 & & & 0.536405 & -0.02757 & -0.55034 \\
\hline & POPCD & 0.140685 & 3.119583 & 0.140685 & 3.181058 & 0.083282 & & & & & & & \\
\hline & & & 0.24893 & & & & & & & & & & \\
\hline & MANU & -0.000494 & -0.369513 & -0.000494 & -0.376795 & -0.002432 & & -0.00183 & & & & & \\
\hline & & 0.019329 & 4.109664 & 0.019329 & 4.19065 & 0.016649 & & 0.016003 & & & & & \\
\hline & $\mathrm{PCl}$ & -0.0 & -2.3 & -0.0 & -2.3 & & & & & & & & \\
\hline & $\mathrm{NAI}$ & & & & & & & & & & & & \\
\hline & HWD & & 173 & & & & & & & & & & \\
\hline & & & & & & & & & & & & & \\
\hline & & & & & & & & & & & & & \\
\hline \multirow{10}{*}{ 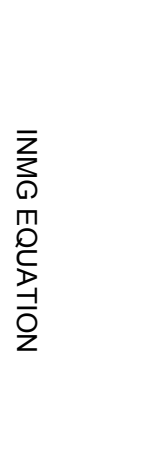 } & & -3.6 & -11 & -3.6 & & & & & & & & & \\
\hline & EMP90 & & 2.211061 & & 2.243499 & 0.328559 & 3.415962 & 0.633445 & 6.90 & & & & \\
\hline & OTMG90 & 0.416669 & 7.480022 & 0.416669 & 7.589759 & -0.043242 & -0.47901 & -0.416308 & -5.40943 & -1.28141 & -3.41008 & 0.041147 & 0.585953 \\
\hline & MHY90 & 1.103992 & 8.60562 & 1.103992 & 8.73187 & 0.90766 & 3.83234 & 0.600503 & 2.643926 & & -2.22428 & 0.727382 & \\
\hline & DGEX90 & -0.103386 & -1.919616 & -0.103386 & -1.947778 & -0.210554 & -1.85898 & -0.085312 & -0.76264 & -0.20091 & -0.42571 & -0.22996 & -2.37357 \\
\hline & AREA & -0.004681 & -0.262077 & -0.004681 & -0.265922 & 0.037319 & 1.779432 & 0.059994 & 3.022639 & 0.08496 & 3.548888 & 0.032563 & 1.769543 \\
\hline & POPs & 0.604383 & 20.15674 & 0.604383 & 20.45246 & 0.607578 & 17.12282 & 0.659455 & 19.59188 & 0.610802 & 5.315643 & 0.496596 & 11.6411 \\
\hline & MCRH & 0.405683 & 6.107839 & 0.405683 & 6.197446 & 0.409991 & 5.501826 & 0.210561 & 3.498282 & 0.030906 & 1.149689 & 0.26988 & 4.5475 \\
\hline & UNEMP & -0.099027 & -2.303579 & -0.099027 & -2.337375 & -0.198649 & & -0.255704 & & & & -0.20302 & -4.31667 \\
\hline & NAIX & 0.000715 & 0.068826 & 0.000715 & 0.069836 & 0.007712 & 0.649658 & 0.004477 & 0.391311 & -0.01455 & -1.20827 & -0.00065 & -0.0684 \\
\hline
\end{tabular}




\begin{tabular}{|c|c|c|c|c|c|c|c|c|c|c|c|c|c|}
\hline \multirow{14}{*}{ 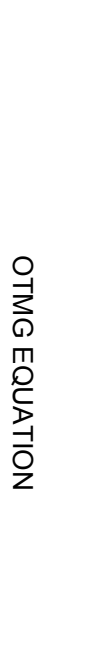 } & EXPTAX & -0.054992 & -1.627617 & -0.054992 & -1.651495 & -0.090088 & -2.39672 & -0.057497 & -1.65991 & -0.12697 & -2.83429 & -0.08167 & -2.73952 \\
\hline & INMG80 & -0.65668 & -22.40149 & -0.65668 & -22.73014 & -0.66264 & -19.2383 & -0.683693 & -20.8907 & -0.47076 & -4.5315 & -0.52764 & -12.0241 \\
\hline & Constant & -0.487759 & -0.624526 & -0.487759 & -0.633688 & -0.010328 & -0.01139 & 0.444851 & 0.6253 & -0.31867 & -0.8174 & 0.66199 & 1.048459 \\
\hline & EMP90 & 0.043131 & 1.178106 & 0.043131 & 1.195389 & 0.263287 & 3.666933 & 0.49483 & 7.362228 & 1.416194 & 3.829679 & 0.323431 & 5.257001 \\
\hline & INMG90 & 0.1511 & 6.210992 & 0.1511 & 6.302111 & 0.004549 & 0.141312 & -0.146655 & -4.98673 & -0.28674 & -2.60546 & -0.02686 & -1.2574 \\
\hline & MHY90 & 0.487872 & 5.167759 & 0.487872 & 5.243574 & 0.205421 & 1.188502 & -0.076181 & -0.47313 & -1.37566 & -2.47366 & 0.069065 & 0.471385 \\
\hline & DGEX90 & 0.043078 & 1.039256 & 0.043078 & 1.054503 & 0.08281 & 0.962229 & 0.036891 & 0.438509 & -0.1659 & -0.6159 & -0.02902 & -0.36146 \\
\hline & AREA & 0.051332 & 3.555007 & 0.051332 & 3.607161 & 0.06471 & 3.947198 & 0.073028 & 4.969693 & 0.054657 & 4.004173 & 0.079316 & 4.797523 \\
\hline & POPs & 0.308321 & 8.865906 & 0.308321 & 8.995975 & 0.320173 & 8.278436 & 0.444553 & 13.2043 & 0.350891 & 5.437469 & 0.308621 & 8.37989 \\
\hline & OWHU & -0.129433 & -0.792389 & -0.129433 & -0.804014 & -0.247046 & -1.35274 & -0.425694 & -2.9987 & -0.19287 & -3.10536 & -0.35236 & -2.86549 \\
\hline & UNEMP & -0.183066 & -5.662395 & -0.183066 & -5.745466 & -0.211528 & -5.40972 & -0.228825 & -6.53791 & -0.15981 & -3.79903 & -0.23367 & -6.27791 \\
\hline & NAIX & 0.007513 & 0.93382 & 0.007513 & 0.947519 & 0.006517 & 0.711263 & 0.003469 & 0.407383 & -0.00928 & -1.38897 & 0.00467 & 0.699106 \\
\hline & EXPTAX & -0.021386 & -0.902974 & -0.021386 & -0.916222 & -0.024687 & -0.96743 & -0.009624 & -0.41422 & -0.0705 & -3.1 & -0.04296 & -2.23125 \\
\hline & OTMG80 & -0.336704 & -10.12368 & -0.336704 & -10.2 & -0.343697 & -9.36122 & -0.466329 & -14.507 & -0.27941 & -4.70 & -0.34317 & -10.0581 \\
\hline \multirow{13}{*}{ 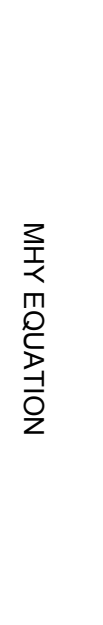 } & Constant & 3.43 & 7.71 & 3.43 & 7.85 & 2.849655 & 3.720383 & 2.37 & 3.869865 & 2.557874 & $4.07 €$ & 297 & 5.689111 \\
\hline & EMP90 & 0.157112 & 10.08712 & 0.157112 & 10.2 & 0.229416 & 7.71458 & 208 & 13.42811 & 0.537579 & 545 & 171 & 13.29835 \\
\hline & INMG90 & 0.007683 & 0.648044 & 0.007683 & 0.65 & -0.047239 & -3.08696 & -0.06 & -4.08493 & 0209 & -2.8 & $-0 .($ & -5.38082 \\
\hline & OTMG90 & 0.079915 & 4.07961 & 0.079915 & 4.149695 & 0.005929 & 0.204938 & -0.039952 & -1.44525 & -0.17643 & -2.63008 & 0.006405 & 0.286269 \\
\hline & DGEX90 & -0.014339 & -0.756397 & -0.014339 & -0.769391 & 0.012565 & 0.317628 & 0.013703 & 0.351568 & -0.07536 & -0.8012 & -0.02397 & -0.74779 \\
\hline & POPs & -0.051491 & -0.798629 & -0.051491 & -0.812349 & -0.018296 & -0.13666 & 0.053226 & 0.497331 & 0.042397 & 1.02043 & -0.00428 & -0.06848 \\
\hline & POPs2 & 0.002793 & 0.916398 & 0.002793 & 0.932141 & 0.00146 & 0.229627 & -0.001088 & -0.21416 & 0.000697 & 0.352061 & 0.00116 & 0.395829 \\
\hline & FHHF & -0.080286 & -3.175247 & -0.080286 & -3.229795 & -0.058843 & -2.04461 & -0.056684 & -2.12731 & -0.05409 & -2.5775 & -0.05561 & -2.36416 \\
\hline & POPHD & 0.251982 & 7.786305 & 0.251982 & 7.920067 & 0.264268 & 7.412893 & 0.193534 & 6.501228 & 0.094361 & 3.210856 & 0.208222 & 8.621998 \\
\hline & UNEMP & -0.135406 & -8.751033 & -0.135406 & -8.901369 & -0.132297 & -7.61782 & -0.100531 & -7.10707 & -0.09169 & -3.76475 & -0.10724 & -8.48643 \\
\hline & MANU & 0.003578 & 8.901778 & 0.003578 & 9.054703 & 0.003441 & 7.676414 & 0.003064 & 7.36622 & 0.001138 & 2.821583 & 0.003817 & 11.66419 \\
\hline & WHRT & 0.001217 & 0.732654 & 0.001217 & 0.745241 & 0.00048 & 0.257194 & -0.00306 & -1.7783 & -0.00276 & -2.2619 & $-5.53 E-06$ & -0.00369 \\
\hline & SCIX & -0.0 & -4.278367 & -0.02569 & -4.35 & -0.032174 & -4.14518 & -0.01 & -2.85159 & 0457 & -1.06271 & -0.02757 & -5.56782 \\
\hline \multirow{5}{*}{ 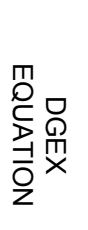 } & MHY80 & -0.357359 & -9.855869 & -0.357359 & -10.02518 & -0.33127 & -8.18979 & -0.303337 & -8.38473 & -0.28621 & -4.23061 & -0.29014 & -8.37157 \\
\hline & Constant & 2.761791 & 5.811178 & 2.761791 & 5.903707 & 3.033987 & 6.055482 & 3.025486 & 6.191218 & 2.787259 & 3.858494 & 2.933612 & 8.157871 \\
\hline & EMP90 & -0.000272 & -0.006696 & -0.000272 & -0.006803 & -0.059969 & -0.78177 & -0.102226 & -1.34337 & -0.06069 & -0.20799 & -0.02973 & -0.45945 \\
\hline & JIVIGY0 & -0.056556 & -2.090736 & -0.056556 & -2.124026 & -0.04943 & -1.56898 & -0.055412 & -1.76042 & -0.02339 & -0.31196 & -0.04323 & -1.87872 \\
\hline & OTMG90 & 0.092074 & 1.964145 & 0.092074 & 1.99542 & 0.155705 & 2.472033 & 0.241083 & 3.882745 & 0.164776 & 1.180296 & 0.174625 & 3.493331 \\
\hline
\end{tabular}




\begin{tabular}{|c|c|c|c|c|c|c|c|c|c|c|c|c|}
\hline & & & & & & & & & & & & \\
\hline OPs & & -0.239351 & & -0.243162 & -0.019443 & & & & & & & \\
\hline & 0.008465 & 0.077877 & 0.008465 & & & & & & & & & \\
\hline $\mathrm{CR}$ & & 2.81 & & & & & & & & & & \\
\hline FEG & & 0.686 & & & & & & & & & & \\
\hline CTAX & 44 & 1.96 & & 88 & & & & & & & & \\
\hline CTD & & -1.304564 & -1.61 & -1.325337 & -1.36 & -1.10104 & -1.2 & & -1.4 & -0.8 & -05 & 047 \\
\hline תז & $.72 \mathrm{E}-07$ & 0.527365 & 4.72E-07 & 0.535762 & 1.12E-07 & 0.120561 & E-07 & 032 & & 0.20 & -1.73 & -0 \\
\hline GEX8C & 0.368603 & -8.6622 & -0.368603 & -8.800125 & -0.370191 & -8.71827 & -0.356032 & -8.49822 & -0.36456 & -7.59991 & -0.37907 & $-8.876 c$ \\
\hline
\end{tabular}


Table I:: NON-SPATIAL MODEL RESULTS, RATE, APPALACHIA, 1980

\begin{tabular}{|c|c|c|c|c|c|c|c|c|c|}
\hline \multirow{3}{*}{ Equation } & \multirow[b]{3}{*}{ Variables } & \multicolumn{6}{|c|}{ SINGLE-EQUATION } & \multirow{2}{*}{\multicolumn{2}{|c|}{$\begin{array}{l}\text { MULTI-EQUATION } \\
\text { EC3SLS }\end{array}$}} \\
\hline & & \multicolumn{2}{|c|}{ W2SLS } & \multicolumn{2}{|l|}{ B2SLS } & \multicolumn{2}{|l|}{ EC2SLS } & & \\
\hline & & Coefficient & t-Statistic & Coefficient & t-Statistic & Coefficient & t-Statistic & Coefficient & t-Statistic \\
\hline \multirow{15}{*}{$\begin{array}{l}\text { EMP } \\
\text { Equation }\end{array}$} & INMG & 0.359751 & 2.42211 & 0.141423 & 0.976803 & 0.242059 & 1.61325 & 0.233448 & 1.59541 \\
\hline & OTMG & $-5.58 \mathrm{E}-03$ & -0.03147 & 0.237049 & 1.44214 & 0.110066 & 0.61122 & 0.152661 & 0.88782 \\
\hline & MHY & -0.78777 & -5.80386 & -0.340607 & -3.88869 & -0.56193 & -5.0683 & -0.34733 & -3.8552 \\
\hline & DGEX & 0.177614 & 3.17974 & 0.087403 & 1.58429 & 0.224156 & 4.05043 & 0.219288 & 4.16816 \\
\hline & POP25-44 & 0.713542 & 4.74954 & 0.518565 & 3.73625 & 0.614005 & 4.17477 & 0.263986 & 2.02492 \\
\hline & FHHF & -0.1801 & -2.98388 & -0.078936 & -1.50382 & -0.09147 & -1.7135 & -0.06109 & -1.295 \\
\hline & POPCD & 0.147994 & 3.62763 & 0.138503 & 3.78932 & 0.15429 & 3.7479 & 0.075418 & 2.12708 \\
\hline & OWHU & -0.05223 & -0.25933 & 0.102694 & 0.931571 & 0.368897 & 2.46554 & 0.156392 & 1.38822 \\
\hline & MANU & $4.04 \mathrm{E}-03$ & 3.39127 & $6.66 \mathrm{E}-03$ & 4.43578 & $3.42 \mathrm{E}-03$ & 2.80181 & $3.23 \mathrm{E}-03$ & 2.66596 \\
\hline & WHRT & $5.32 \mathrm{E}-03$ & 1.48423 & 0.018805 & 4.73728 & $5.73 \mathrm{E}-03$ & 1.59668 & $9.46 \mathrm{E}-03$ & 2.8134 \\
\hline & РСРТАХ & 0.016051 & 0.858284 & -0.054145 & -2.647 & -0.01119 & -0.6812 & -0.04844 & -3.1435 \\
\hline & NAIX & 0.010547 & 1.3749 & 0.0187 & 2.27321 & $7.62 \mathrm{E}-03$ & 0.98606 & 0.011691 & 1.59697 \\
\hline & HWD & 0.060132 & 2.26679 & -0.013061 & -0.49043 & 0.059234 & 2.22406 & $-1.38 \mathrm{E}-03$ & -0.0576 \\
\hline & ESBd & 0.135542 & 2.8753 & 0.21557 & 4.56345 & 0.14852 & 3.06391 & 0.190945 & 4.18494 \\
\hline & EMPt-1 & 700847 & 21.5838 & 0.675061 & 22.1107 & 0.694823 & 20.9121 & 0.679769 & 21.7865 \\
\hline \multirow{9}{*}{$\begin{array}{l}\text { INMG } \\
\text { EQUATION }\end{array}$} & EMP & $-6.71 E-03$ & -0.32793 & 0.032501 & 1.4787 & 0.010742 & 0.52482 & 0.018535 & 0.93855 \\
\hline & OTMG & 1.13662 & 29.8662 & 0.97591 & 16.8001 & 1.03384 & 25.0071 & 1.13816 & 35.2017 \\
\hline & MHY & 0.016954 & 0.221386 & 0.246925 & 8.78393 & 0.211417 & 7.8629 & 0.254439 & 9.9139 \\
\hline & DGEX & -0.07324 & -2.23005 & -0.232543 & -8.23003 & -0.16446 & -5.3045 & -0.19661 & -6.4197 \\
\hline & AREA & 0.032855 & 3.55489 & $2.33 \mathrm{E}-03$ & 0.211884 & 0.016041 & 1.69438 & $2.94 \mathrm{E}-03$ & 0.35154 \\
\hline & POP & -0.27817 & -8.66029 & -0.201365 & -4.40964 & -0.21784 & -6.7455 & -0.24997 & -8.5741 \\
\hline & MCRH & 0.056782 & 1.35551 & 0.013468 & 0.645788 & $-7.50 \mathrm{E}-04$ & -0.0411 & -0.01755 & -1.2023 \\
\hline & UNEMP & -0.01798 & -0.78297 & 0.037313 & 1.41907 & $2.26 \mathrm{E}-03$ & 0.09586 & 0.021059 & 0.93138 \\
\hline & NAIX & 0.019567 & 3.82272 & 0.031685 & 5.72597 & 0.026174 & 5.05953 & 0.023639 & 5.07293 \\
\hline
\end{tabular}




\begin{tabular}{|c|c|c|c|c|c|c|c|c|c|}
\hline \multirow{27}{*}{\begin{tabular}{|l} 
\\
\\
OTMG \\
EQUATION \\
MHY \\
EQUATION \\
DGEX \\
EQUATION
\end{tabular}} & EXPTAX & 0.030164 & 1.67576 & 0.048074 & 2.56692 & 0.022908 & 1.60626 & 0.014306 & 1.11732 \\
\hline & INMGt-1 & 0.106629 & 4.54565 & 0.141228 & 4.96946 & 0.127269 & 5.00469 & 0.051395 & 2.98742 \\
\hline & EMP & $1.35 \mathrm{E}-03$ & 0.093717 & -0.035736 & -2.29404 & -0.01022 & -0.6804 & $-8.50 \mathrm{E}-03$ & -0.593 \\
\hline & INMG & 0.567954 & 24.7065 & 0.499312 & 19.6427 & 0.536286 & 21.2085 & 0.693976 & 35.554 \\
\hline & MHY & 0.094489 & 1.83859 & 0.229469 & 6.79061 & 0.188432 & 5.38659 & 0.014885 & 0.500 \\
\hline & DGEX & 0.020956 & 0.824471 & $-2.49 \mathrm{E}-04$ & -0.01104 & $5.65 \mathrm{E}-03$ & 0.23204 & 0.065164 & 2.7957 \\
\hline & AREA & $2.10 \mathrm{E}-03$ & 0.299278 & 0.015456 & 2.07199 & 0.013928 & 1.94601 & $5.52 \mathrm{E}-03$ & 0.8508 \\
\hline & POP & 0.160381 & 7.07798 & 0.311886 & 11.001 & 0.200591 & 8.56436 & 0.219441 & 10.721 \\
\hline & OWHU & -0.23045 & -2.77715 & -0.723797 & -12.9048 & -0.54656 & -9.9754 & -0.30352 & -6.752 \\
\hline & UNEMP & -0.04237 & -2.64597 & -0.014427 & -0.83071 & -0.03294 & -2.0013 & -0.02145 & -1.401 \\
\hline & NAIX & $-7.26 \mathrm{E}-03$ & -1.93455 & $-7.71 \mathrm{E}-03$ & -1.92627 & $-6.62 \mathrm{E}-03$ & -1.6684 & -0.01316 & -3.699 \\
\hline & EXPTAX & -0.04777 & -3.85704 & $-5.86 \mathrm{E}-03$ & -0.48106 & -0.04039 & -3.9416 & -0.0258 & -2.785 \\
\hline & OTMGt-1 & 0.257227 & 9.69027 & 0.195752 & 9.0649 & 0.243616 & 10.0222 & 0.09475 & 5.3032 \\
\hline & EMP & 0.021412 & 0.024609 & -0.039921 & -2.14328 & -0.02823 & -2.3981 & -0.0308 & -2.637 \\
\hline & OTMG & 0.107978 & 0.033025 & 0.304896 & 4.41877 & 0.334658 & 7.33464 & 0.472469 & 11.127 \\
\hline & INMG & 0.025945 & $5.81 \mathrm{E}-03$ & -0.228626 & -2.52095 & -0.28602 & -4.9633 & -0.45276 & -8.46 \\
\hline & DGEX & 0.033847 & 0.02454 & 0.092355 & 3.177 & 0.102607 & 5.45054 & 0.156005 & 8.3786 \\
\hline & POP & -0.56717 & -0.06827 & 0.618454 & 19.149 & 0.629611 & 25.3988 & 0.630145 & 25.073 \\
\hline & POP2 & 0.021475 & 0.058222 & -0.029319 & -12.3436 & $-2.44 \mathrm{E}-03$ & -18.2 & -0.01154 & -18.00 \\
\hline & FHHF & -0.155 & -0.08967 & 0.017838 & 0.477245 & $-5.81 \mathrm{E}-03$ & -0.2682 & -0.02521 & -1.277 \\
\hline & POPHD & 0.221522 & 0.10078 & 0.134411 & 2.41697 & 0.184527 & 5.7693 & 0.192065 & 6.8793 \\
\hline & UNEMP & -0.04011 & -0.04144 & -0.095949 & -4.44813 & -0.08611 & -6.3791 & -0.07794 & -6.037 \\
\hline & MANU & $1.61 \mathrm{E}-03$ & 0.060283 & $1.02 \mathrm{E}-03$ & 1.32708 & $5.03 \mathrm{E}-04$ & 1.22009 & $5.63 \mathrm{E}-04$ & 1.5249 \\
\hline & WHRT & $-7.23 \mathrm{E}-04$ & $-7.22 \mathrm{E}-03$ & $-3.61 \mathrm{E}-03$ & -1.45767 & $-4.45 \mathrm{E}-03$ & -3.0936 & $-3.32 \mathrm{E}-03$ & -2.573 \\
\hline & SCIX & -0.01974 & -0.04509 & $-2.50 \mathrm{E}-04$ & -0.02552 & $-1.85 \mathrm{E}-03$ & -0.3137 & $-8.47 \mathrm{E}-03$ & -1.622 \\
\hline & MHYt-1 & 0.446519 & 0.213352 & 0.582956 & 21.1429 & 0.557953 & 28.8697 & 0.517837 & 27.651 \\
\hline & EMP & 0.133346 & 3.34241 & -0.030595 & -0.467 & 0.072541 & 1.52029 & 0.051354 & 1.1068 \\
\hline
\end{tabular}




\begin{tabular}{|l|r|r|r|r|r|r|r|r|}
\hline OTMG & -0.04544 & -0.25978 & 0.244669 & 1.26862 & 0.22063 & 1.10135 & 0.383922 & 2.00575 \\
\hline INMG & -0.3795 & -1.49952 & -1.06206 & -3.4404 & -0.88189 & -3.1926 & -1.15777 & -4.405 \\
\hline MHY & 0.164668 & 0.877538 & 0.31315 & 3.87911 & 0.351471 & 5.32272 & 0.384511 & 6.12255 \\
\hline POP & 0.072341 & 0.687193 & 0.601487 & 3.64518 & 0.336181 & 3.23176 & 0.461044 & 4.70338 \\
\hline POP5-17 & 0.144679 & 0.905601 & 0.199894 & 1.25766 & 0.322597 & 2.97487 & 0.197491 & 1.94281 \\
\hline SCRM & $1.70 \mathrm{E}-04$ & 6.28187 & $2.89 \mathrm{E}-04$ & 5.18503 & $2.35 \mathrm{E}-04$ & 6.41528 & $2.47 \mathrm{E}-04$ & 7.1981 \\
\hline DFEG & -0.06221 & -1.82387 & -0.048177 & -1.11468 & -0.06374 & -1.7768 & -0.05735 & -1.7874 \\
\hline PCTAX & $6.05 \mathrm{E}-03$ & 0.16378 & 0.098166 & 1.86557 & $-3.17 \mathrm{E}-03$ & -0.0869 & 0.014283 & 0.44059 \\
\hline PCTD & $3.46 \mathrm{E}-06$ & 0.104477 & $2.61 \mathrm{E}-05$ & 0.888044 & $2.15 \mathrm{E}-05$ & 0.61967 & $2.66 \mathrm{E}-05$ & 0.83676 \\
\hline LTD & $-3.78 \mathrm{E}-08$ & -0.05236 & $-9.01 \mathrm{E}-07$ & -1.41235 & $-6.10 \mathrm{E}-07$ & -0.8045 & $-7.05 \mathrm{E}-07$ & -1.0149 \\
\hline DGEXt-1 & 0.497698 & 8.10095 & 0.382818 & 4.64688 & 0.491643 & 8.44002 & 0.427713 & 7.76678 \\
\hline
\end{tabular}




\begin{tabular}{|c|c|c|c|c|c|c|c|c|c|c|c|c|c|}
\hline \multirow{3}{*}{$\begin{array}{l}\text { Equati } \\
\text { on }\end{array}$} & \multirow{3}{*}{ Variables } & \multicolumn{6}{|c|}{ SINGLE-EQUATION } & \multicolumn{6}{|c|}{ MULTI-EQUATION } \\
\hline & & \multicolumn{2}{|l|}{ OLS } & \multicolumn{2}{|c|}{ Weighted OLS } & \multicolumn{2}{|c|}{ Weighted 2SLS } & \multicolumn{2}{|c|}{ 3SLS } & \multicolumn{2}{|l|}{$\mathrm{ML}$} & \multicolumn{2}{|l|}{ GMM } \\
\hline & & Coefficient & t-Statistic & Coefficient & t-Statistic & Coefficient & t-Statistic & Coefficient & t-Statistic & Coefficient & z-Statistic & Coefficient & t-Statistic \\
\hline \multirow{16}{*}{$\begin{array}{l}\text { EMP } \\
\text { Equati } \\
\text { on }\end{array}$} & constant & -2.691683 & -5.918264 & -2.691683 & -5.961942 & -2.219214 & -4.45975 & -2.417136 & -6.3368 & -3.11234 & -8.13743 & -2.23952 & -6.38072 \\
\hline & INMG90 & -0.003843 & -0.27433 & -0.003843 & -0.276354 & -0.003896 & -0.24936 & 0.025415 & 1.673815 & -0.03084 & -1.50376 & -0.00488 & -0.41144 \\
\hline & OTMG90 & 0.107891 & 3.03252 & 0.107891 & 3.054901 & 0.15688 & 2.626021 & 0.145095 & 2.743366 & 0.07229 & 1.145847 & 0.178091 & 3.687054 \\
\hline & MHY90 & 0.871105 & 12.44886 & 0.871105 & 12.54073 & 1.4076 & 10.97676 & 1.530557 & 14.40047 & 0.424343 & 2.651422 & 1.324862 & 12.91356 \\
\hline & DGEX90 & 0.064283 & 2.146721 & 0.064283 & 2.162564 & -0.00331 & -0.049 & -0.133822 & -2.05841 & 0.044125 & 0.571203 & -0.02896 & 7456 \\
\hline & POP24-44 & 0.542713 & 6.218404 & 0.542713 & 6.264297 & 718 & 3.46807 & 0.556245 & 50403 & 45 & 603 & 065 & 572 \\
\hline & FHHF & -0.05488 & -1.784528 & -0.05488 & -1.797698 & & 54033 & -0.063613 & 368 & 06 & & 176 & 428 \\
\hline & POPCD & 0.153956 & 6331 & 0.153956 & 6.010363 & & 0381 & 0.088935 & & & & & 223 \\
\hline & OWHU & 0.255257 & 3.501468 & 0.255257 & 3.527309 & & 3.760558 & 0.07596 & 7353 & & & 685 & 785 \\
\hline & MANU & -0.001106 & -1.34041 & -0.001106 & -1.350302 & 985 & 22822 & -0.002539 & 1267 & 53 & & & 152 \\
\hline & WHRT & 0.016408 & 6.059747 & 0.016408 & 6.104469 & 052 & 4.945058 & 0.01253 & 38301 & 24 & & 085 & 8887 \\
\hline & PCPTAX & -0.051109 & -4.080186 & -0.051109 & -4.110299 & -0.06 & 20724 & -0.009445 & -0.9469 & 014 & 075 & 011 & 8884 \\
\hline & NAIX & 0.003918 & 0.663012 & 0.003918 & 0.667905 & 409 & .64597 & 0.001771 & 0.404415 & 0.01 & 211 & $E-05$ & 8781 \\
\hline & HWD & 0.08916 & 4.930852 & 0.08916 & 4.967243 & 171 & 5.071197 & -0.003788 & 5388 & 936 & & 114 & 3321 \\
\hline & ESBd & -0.072572 & -2.828229 & -0.072572 & -2.849101 & -0.076206 & -2.80947 & 0.027281 & 04489 & 0.026402 & 042 & -0.0633 & 1454 \\
\hline & EMP80 & -0.060793 & -7.39129 & -0.060793 & -7.445839 & -0.043212 & -4.58793 & -0.049583 & 6.00427 & -0.07875 & -7.23341 & -0.03704 & -4.40598 \\
\hline \multirow{11}{*}{$\begin{array}{l}\text { INMG } \\
\text { EQUATI } \\
\text { ON }\end{array}$} & Constant & -3.996693 & -20.49751 & -3.996693 & -20.61065 & -4.55938 & -15.7145 & -3.696249 & -14.7046 & -2.17539 & -3.01201 & -3.59068 & -13.5143 \\
\hline & EMP90 & 0.196806 & 5.73229 & 0.196806 & 5.763931 & 0.629468 & 6.785373 & 1.086016 & 12.46646 & 3.455138 & 7.853774 & 0.834933 & 10.43032 \\
\hline & OTMG90 & 0.409403 & 9.657773 & 0.409403 & 9.711082 & -0.577052 & -6.11326 & -1.00695 & -14.2865 & -2.20626 & -6.56036 & -0.60252 & -8.56111 \\
\hline & MHY90 & 1.087363 & 11.88945 & 1.087363 & 11.95507 & 1.15833 & 4.761981 & 0.646625 & 2.75139 & -2.52746 & -2.92199 & 0.93392 & 4.225503 \\
\hline & DGEX90 & -0.066754 & -1.821503 & -0.066754 & -1.831557 & -0.105259 & -1.05509 & 0.063405 & 0.645709 & -0.18609 & -0.57815 & -0.17623 & -1.8471 \\
\hline & AREA & 0.002215 & 0.193131 & 0.002215 & 0.194197 & 0.050779 & 3.180921 & 0.043835 & 2.944008 & 0.028863 & 1.645577 & 0.014155 & 0.847937 \\
\hline & POPs & 0.669869 & 39.56639 & 0.669869 & 39.78479 & 0.739404 & 31.72912 & 0.806136 & 37.14325 & 1.02124 & 11.8626 & 0.738643 & 25.64509 \\
\hline & MCRH & 0.546085 & 12.1532 & 0.546085 & 12.22029 & & 9.723149 & 0.24457 & 6.107792 & -0.01047 & -0.40684 & 0.372219 & 8.683186 \\
\hline & UNEMP & -0.209027 & -7.365833 & -0.209027 & -7.406491 & & -7.01086 & -0.312777 & -7.47021 & & -6.00757 & -0.30638 & 3912 \\
\hline & NAIX & 0.009711 & 1.358759 & 0.009711 & 1.366259 & & 1.631101 & 0.017729 & 1.991349 & 0.010211 & 0.720227 & 0.01815 & 2.397136 \\
\hline & EXPTAX & -0.068395 & -2.951281 & -0.068395 & -2.967571 & -0.160725 & -5.263 & -0.096821 & -3.67973 & -0.34287 & -6.09076 & -0.12849 & -5.04906 \\
\hline
\end{tabular}




\begin{tabular}{|c|c|c|c|c|c|c|c|c|c|c|c|c|c|}
\hline & INMG80 & 0.762135 & -48.00913 & -0.762135 & -48.27413 & -0.821931 & -37.6792 & -0.826413 & -39.8299 & -0.87572 & -12.1217 & -0.78532 & -27.7503 \\
\hline \multirow{12}{*}{$\begin{array}{l}\text { OTMG } \\
\text { EQUATI } \\
\text { ON }\end{array}$} & Constant & 0.251923 & -0.864997 & -0.251923 & -0.869771 & 0.622493 & 1.547599 & -0.486724 & -1.87683 & -0.33469 & -1.50104 & 0.822571 & 2.731496 \\
\hline & MP90 & 0.132824 & 6.038522 & 0.132824 & 6.071853 & 0.424919 & 8.017006 & 0.644939 & 13.41998 & 1.021738 & 8.232277 & 0.608801 & 13.08112 \\
\hline & INMG90 & 0.093883 & 8.726393 & 0.093883 & 8.774561 & -0.003713 & -0.26719 & -0.072857 & -5.73322 & -0.03206 & -1.27586 & -0.02687 & -2.37538 \\
\hline & MHY90 & 0.502791 & 8.592265 & 0.502791 & 8.639693 & 0.034549 & -0.22705 & -0.14802 & -1.1125 & -0.75728 & -2.89593 & -0.20559 & -1.61878 \\
\hline & DGEX90 & .006517 & 0.274432 & 0.006517 & 0.275947 & .005592 & 0.096509 & 0.026043 & 0.462687 & -0.12765 & -1.30893 & -0.06692 & -1.17326 \\
\hline & AREA & 0.02558 & 3.190685 & 0.02558 & 3.208297 & 77726 & 426 & 16791 & 2.01264 & & 1.43927 & 0.034814 & 3.488495 \\
\hline & POPs & 232445 & 3.09785 & 0.232445 & 13.17015 & 438 & 1.74397 & 408045 & 3.82435 & 342171 & 1.49455 & & 17.36029 \\
\hline & OWHU & 865 & .769871 & 365 & -2.78516 & 0077 & -4.3904 & .184886 & 3.98162 & 0.09996 & 5.66276 & 459 & -6.99124 \\
\hline & UNEMP & 096513 & -5.298332 & 513 & 327578 & .163982 & 0.34722 & 341 & -6.53194 & -0.18051 & 6.02285 & 241 & -6.94375 \\
\hline & NAIX & 005143 & 1.09555 & 5143 & 1.101598 & .007718 & .407881 & 648 & .336597 & -0.00293 & -0.61001 & 0.005152 & 1.254165 \\
\hline & EXPTAX & .023738 & -1.780372 & .023738 & .790199 & 0.025981 & -1.73665 & 319 & -3.42697 & .12021 & -7.4165 & -0.03199 & -2.79227 \\
\hline & OTMG80 & .236621 & -13.36975 & .236621 & 13.44355 & -0.2618 & -12.2713 & 0.391448 & -23.2811 & -0.29405 & -11.1041 & -0.32068 & -18.4113 \\
\hline & Constant & 2.323685 & 8.967019 & 2.323685 & 9.024844 & 2.59097 & 6.03241 & 2.229442 & 6.280775 & 2.882722 & .986065 & 2.380618 & 7.85793 \\
\hline & EMP90 & 0.154155 & 14.60505 & 0.154155 & 14.69924 & 0.26584 & 11.82635 & 0.367064 & 19.80984 & 0.471249 & 13.45006 & 0.328838 & 17.54434 \\
\hline & INMG90 & -0.001286 & -0.227895 & -0.001286 & -0.229365 & -0.020296 & -3.00751 & -0.022694 & -3.45019 & -0.02232 & -1.82156 & -0.02619 & -5.13704 \\
\hline & OTMG90 & 0.088483 & 6.472767 & 0.088483 & 6.514508 & -0.01983 & -0.82543 & 0.070511 & -3.12934 & -0.14566 & -3.47482 & -0.04855 & -2.46893 \\
\hline & DGEX90 & 0.009852 & 0.825512 & 0.009852 & 0.830835 & 0.052095 & 1.877403 & 0.062774 & 2.315568 & -0.03566 & -0.8017 & 0.055133 & 2.412685 \\
\hline & POPs & .202332 & -6.171022 & -0.202332 & -6.210817 & -0.24755 & -3.46133 & -0.209532 & -3.53567 & -0.11524 & -4.62207 & -0.22325 & -4.54554 \\
\hline & POPs2 & 0.009242 & 6.13183 & 0.009242 & 6.171372 & 0.011622 & 3.521031 & 0.010363 & 3.787887 & 0.006878 & 5.759517 & 0.010668 & 4.743507 \\
\hline & FHHF & -0.003566 & -0.277697 & -0.003566 & -0.279487 & 017725 & 1.148509 & 0.026211 & .918091 & .021473 & 2.03632 & 0.015637 & 1.256082 \\
\hline & POPHD & 177061 & 7.85267 & 0.177061 & 7.903309 & 0.183433 & 7.318854 & 0.098824 & 5.204577 & 0.031822 & 1.955493 & 0.122231 & 6.385879 \\
\hline & UNEMP & .117215 & -12.31474 & -0.117215 & -12.39416 & 0.111521 & -10.0677 & -0.064032 & -7.68905 & -0.11236 & -8.71386 & -0.09565 & -11.5161 \\
\hline & MANU & 0.001667 & 5.70287 & 0.001667 & 5.739646 & 0.002181 & 6.549691 & 0.002396 & 7.934792 & 0.000719 & 3.157158 & 0.00237 & 7.848016 \\
\hline & WHRT & 000712 & 0.66031 & 0.000712 & 0.664568 & 0.000421 & -0.33256 & -0.001564 & -1.39414 & -0.00324 & -3.82976 & -0.00168 & -1.52547 \\
\hline & SCIX & 5514 & 5.814263 & .018514 & -5.851757 & .017681 & -4.38141 & 0.010984 & -3.62461 & 0.001276 & 0.584503 & -0.0177 & -5.97361 \\
\hline & MHY80 & .132301 & -5.930739 & -0.132301 & -5.968984 & -0.153426 & -6.30507 & -0.121147 & -6.29652 & -0.20115 & -8.36776 & -0.1247 & -6.41253 \\
\hline \multirow{6}{*}{$\begin{array}{l}\text { DGEX } \\
\text { EQUATI } \\
\text { ON }\end{array}$} & Constant & 2.791241 & 10.55278 & 2.791241 & 10.61592 & 3.219019 & 11.11188 & 2.983545 & 10.43451 & 3.329753 & 11.67636 & 3.391916 & 11.82499 \\
\hline & EMP90 & 046989 & 1.856522 & .046989 & 1.867631 & .028624 & -0.53883 & -0.079391 & -1.50102 & -0.04253 & -0.50007 & -0.0557 & -1.06164 \\
\hline & INMG90 & & -1.232091 & -0.016037 & -1.239463 & -0.009841 & -0.69344 & 0.002777 & 0.196195 & .005675 & .347425 & .012481 & 0.987368 \\
\hline & OTMG90 & 0.060451 & 1.850672 & 0.060451 & 1.861747 & 0.169206 & 3.246646 & & 3.984408 & 0.246163 & .196415 & 0.177772 & 3.319965 \\
\hline & MHY90 & -0.0387 & -0.580486 & -0.0387 & -0.583959 & 0.044773 & -0.33301 & 0.033854 & 0.253762 & 0.075018 & 0.442118 & -0.08018 & -0.636 \\
\hline & POPs & -0.008326 & -1.084011 & -0.008326 & -1.090498 & -0.031004 & -3.23419 & -0.0437 & -4.59342 & -0.01283 & -1.31363 & -0.04683 & -5.6721 \\
\hline
\end{tabular}




\begin{tabular}{|l|r|r|r|r|r|r|r|r|r|r|r|r|}
\hline POP5-17 & -0.069047 & -1.148481 & -0.069047 & -1.155353 & -0.040841 & -0.63198 & 0.03285 & 0.517354 & -0.19929 & -3.59657 & -0.05984 & -0.8829 \\
\hline SCRM & $2.13 \mathrm{E}-05$ & 4.764197 & $2.13 \mathrm{E}-05$ & 4.792706 & $4.80 \mathrm{E}-05$ & 6.162574 & $5.59 \mathrm{E}-05$ & 7.266346 & $2.33 \mathrm{E}-05$ & 5.10424 & $6.03 \mathrm{E}-05$ & 8.61872 \\
\hline DFEG & 0.004522 & 0.346929 & 0.004522 & 0.349005 & -0.008306 & -0.6115 & 0.001052 & 0.079048 & -0.00581 & -0.35207 & -0.01158 & -1.05037 \\
\hline PCTAX & 0.100332 & 6.541673 & 0.100332 & 6.580818 & 0.089467 & 5.227802 & 0.101123 & 5.98409 & 0.082586 & 5.817318 & 0.079482 & 4.178838 \\
\hline PCTD & $3.23 \mathrm{E}-07$ & 0.05712 & $3.23 \mathrm{E}-07$ & 0.057462 & $-2.80 \mathrm{E}-07$ & -0.0485 & $2.35 \mathrm{E}-06$ & 0.415598 & $2.64 \mathrm{E}-06$ & 0.53684 & $1.86 \mathrm{E}-06$ & 0.32282 \\
\hline LTD & $1.92 \mathrm{E}-08$ & 0.284541 & $1.92 \mathrm{E}-08$ & 0.286244 & $-2.26 \mathrm{E}-08$ & -0.32407 & $-5.39 \mathrm{E}-08$ & -0.78845 & $-4.82 \mathrm{E}-08$ & -0.25251 & $-4.42 \mathrm{E}-08$ & -1.49777 \\
\hline DGEX80 & -0.36583 & -14.18509 & -0.36583 & -14.26997 & -0.391556 & -14.4927 & -0.398141 & -14.8066 & -0.35777 & -15.6153 & -0.37405 & -11.2325 \\
\hline
\end{tabular}




\begin{tabular}{|c|c|c|c|c|c|c|c|c|c|c|c|c|c|}
\hline \multirow{3}{*}{$\begin{array}{l}\text { Equati } \\
\text { on }\end{array}$} & \multirow{3}{*}{ Variables } & \multicolumn{6}{|c|}{ SINGLE-EQUATION } & \multicolumn{6}{|c|}{ MULTI-EQUATION } \\
\hline & & \multicolumn{2}{|l|}{ OLS } & \multicolumn{2}{|c|}{ Weighted OLS } & \multicolumn{2}{|c|}{ Weighted 2SLS } & \multicolumn{2}{|c|}{ 3SLS } & \multicolumn{2}{|l|}{$\mathrm{ML}$} & \multicolumn{2}{|l|}{ GMM } \\
\hline & & Coefficient & t-Statistic & Coefficient & t-Statistic & Coefficient & t-Statistic & Coefficient & t-Statistic & Coefficient & z-Statistic & Coefficient & t-Statistic \\
\hline \multirow{16}{*}{$\begin{array}{l}\text { EMP } \\
\text { Equati } \\
\text { on }\end{array}$} & constant & -1.743654 & -3.301508 & -1.743654 & -3.325874 & 1.744599 & 1.969584 & 1.649072 & 2.87199 & 2.512154 & 4.475733 & 1.601761 & 3.196563 \\
\hline & INMG90 & 0.204211 & 4.789543 & 0.204211 & 4.82489 & 0.429479 & 3.866844 & 0.352918 & 3.732291 & -0.46366 & -0.93383 & 0.448158 & 6.196739 \\
\hline & OTMG90 & 0.125555 & 2.72469 & 0.125555 & 2.744799 & 0.276382 & 3.218533 & 0.492866 & 6.466036 & 0.571983 & 2.128983 & 0.341721 & 4.266814 \\
\hline & MHY90 & -0.01253 & -0.47671 & -0.01253 & -0.480228 & -0.182574 & -2.57222 & -0.261169 & -4.9422 & -0.36356 & -4.03871 & -0.25127 & -5.28901 \\
\hline & DGEX90 & 0.016559 & 1.152568 & 0.016559 & 1.161074 & -0.233107 & -3.88947 & -0.384007 & -7.2597 & -0.76367 & -4.23453 & -0.24738 & -5.33939 \\
\hline & POP24-44 & 0.629012 & 6.705213 & 0.629012 & 6.754698 & 0.16 & 1.185539 & 0.130859 & 1.607902 & 0.052037 & 233 & 4104 & \\
\hline & FHHF & -0.052737 & -1.903567 & -0.052737 & -1.917616 & -0.05 & -1.54265 & 0.010653 & 171 & 83 & & 433 & \\
\hline & POPCD & 0.207488 & 518513 & 0.207488 & 7.574001 & 0.1 & 3.685638 & 0.07047 & 437 & 569 & & 851 & \\
\hline & OWHU & -0.009015 & -0.111417 & -0.009015 & -0.112239 & -0.3 & -3.26477 & -0.305518 & -3.40133 & & & 948 & \\
\hline & MANU & 0.001281 & 318847 & 0.001281 & 1.32858 & 0.001095 & 0.840842 & 0.00059 & 0.663797 & 0.000488 & 208 & 983 & \\
\hline & WHRT & 0.023718 & 8.312443 & 0.023718 & 8.37379 & 0.02728 & 7.374235 & 0.012656 & 4.622964 & 0.008823 & 938 & 344 & 514 \\
\hline & PCPTAX & -0.035296 & -2.569957 & -0.035296 & -2.588923 & 0.042743 & -2.54763 & -0.046952 & -4.01766 & -0. & 378 & 316 & 7665 \\
\hline & NAIX & 0.007334 & 1.208853 & 0.007334 & 1.217775 & 0.001262 & 0.171958 & -0.00021 & 3611 & -0.0 & 895 & 0.001174 & 14492 \\
\hline & HWD & -0.012852 & -0.677223 & -0.012852 & -0.682221 & -0.050296 & -2.03129 & -0.004631 & -0.25118 & 0.019112 & 1.059207 & -0.0252 & 3864 \\
\hline & ESBd & -0.09147 & -3.480569 & -0.09147 & -3.506256 & -0.061731 & -1.78706 & 0.003652 & 0.161826 & 0.033681 & 519 & -0.06532 & -2.73951 \\
\hline & EMP80 & -0.062684 & -7.285654 & -0.062684 & -7.339424 & -0.063134 & -5.13508 & -0.058628 & -5.35985 & -0.12293 & -5.22069 & -0.04288 & 59741 \\
\hline \multirow{11}{*}{$\begin{array}{l}\text { INMG } \\
\text { EQUATI } \\
\text { ON }\end{array}$} & Constant & -0.375939 & -1.675686 & -0.375939 & -1.684935 & 0.094613 & 0.347908 & 0.43087 & 1.902434 & 0.361192 & 0.809296 & 0.575001 & 2.794551 \\
\hline & EMP90 & 0.133091 & 6.462161 & 0.133091 & 6.497831 & 0.288869 & 6.224593 & 0.213044 & 4.922202 & -0.15873 & -1.28638 & 0.209141 & 4.835611 \\
\hline & OTMG90 & 0.799843 & 36.44423 & 0.799843 & 36.6454 & 0.576848 & 13.68835 & 0.698156 & 17.16868 & 0.45351 & 6.612292 & 0.675031 & 15.9209 \\
\hline & MHY90 & -0.010453 & -0.538967 & -0.010453 & -0.541942 & -0.102291 & -1.89844 & -0.030623 & -0.63313 & -0.06687 & -0.6296 & -0.07792 & -1.71673 \\
\hline & DGEX90 & -0.021236 & -2.079827 & -0.021236 & -2.091307 & -0.075734 & -2.20158 & -0.071342 & -2.10577 & 0.20802 & 0.943298 & -0.10167 & -3.19183 \\
\hline & AREA & 0.017004 & 2.361953 & 0.017004 & 2.374991 & 0.010087 & 1.221073 & 0.012995 & 1.688174 & 0.034209 & 2.334321 & 0.011419 & 1.581825 \\
\hline & POPs & 0.052779 & 3.010675 & 0.052779 & 3.027293 & 0.086278 & 4.113211 & -0.031461 & -1.75054 & -0.105 & -3.438 & -0.01563 & -0.92932 \\
\hline & MCRH & 0.093046 & 2.355122 & 0.093046 & 2.368122 & 0.02935 & 0.646387 & -0.024647 & -0.66536 & 0.000361 & 0.095964 & -0.02198 & -0.64284 \\
\hline & UNEMP & -0.037138 & -2.149898 & -0.037138 & -2.161765 & -0.054788 & -2.8513 & 0.003028 & 0.181059 & 0.000564 & 0.029015 & -0.03614 & -2.26287 \\
\hline & NAIX & 0.003777 & 0.881076 & 0.003777 & 0.885939 & 0.006475 & 1.385448 & 0.003197 & 0.696731 & 0.009742 & 1.328147 & 0.008264 & 1.946384 \\
\hline & EXPTAX & 0.003137 & 0.243341 & 0.003137 & 0.244684 & -0.002178 & -0.13444 & 0.000998 & 0.069749 & 0.033701 & 1.504319 & -0.00635 & -0.48465 \\
\hline
\end{tabular}




\begin{tabular}{|c|c|c|c|c|c|c|c|c|c|c|c|c|c|}
\hline & & .100516 & -5.272433 & 100516 & 301536 & -0.143061 & -6.30521 & -0.002485 & -0.12898 & 0.063557 & 1.867569 & -0.02807 & \\
\hline & Constant & & 1.028596 & 0.25363 & 1.034274 & 0.063148 & 0.191367 & -0.670333 & -2.20609 & -0.70162 & -0.90317 & 0.180431 & 0.638551 \\
\hline & MP90 & & & & & & & .273647 & 6.965209 & & 1.24618 & & 320097 \\
\hline & UG90 & & & 2357 & & & 0.39103 & 43284 & 2.43237 & 877621 & 7.876632 & 611276 & 9002 \\
\hline & HY9O & & 879006 & 047 & .883858 & 619 & 364 & .027545 & 660214 & 0.117411 & 601309 & -0.0048 & 1751 \\
\hline & DGEX90 & 782 & 1.179063 & 782 & 571 & 058 & 0.90552 & 0.12369 & 4.11039 & -0.42769 & 1.01341 & .071649 & .610956 \\
\hline & AREA & .005852 & -0.854774 & -0.005852 & -0.859493 & 573 & -1.38894 & 0.011522 & -1.61071 & -0.06899 & -2.31533 & 0.00647 & -0.9211 \\
\hline & POPs & 0.172797 & 9.880649 & 172797 & 9.935188 & 1469 & 10.39178 & 0.135861 & 7.141585 & 0.21153 & 3.773922 & 0.141947 & 7.639053 \\
\hline & OWHU & .130634 & -2.247717 & -0.130634 & 124 & -0.106889 & -1.37962 & 0.035227 & .500947 & 0.023621 & 1.462864 & -0.14604 & -2.38494 \\
\hline & UNEMP & .067937 & -4.400354 & -0.067937 & 4643 & 6907 & -3.81756 & 0.035287 & -2.15144 & -0.01202 & -0.32298 & -0.00878 & -0.54577 \\
\hline & NAIX & .002553 & 0.666199 & 002553 & 0.669876 & & 0.336899 & -0.000251 & -0.06254 & & -1.4245 & -0.00192 & -0.52797 \\
\hline & EXPTAX & 0.026455 & 2.328652 & 0.026455 & 2.341506 & 0.002946 & 0.1941 & 0.001928 & 0.139503 & -0.07345 & -1.613 & 0.020291 & 1.549624 \\
\hline & OTMG80 & -0.180328 & -9.758595 & -0.180328 & -9.812461 & -0.223934 & -9.74056 & -0.116524 & -5.60302 & -0.13756 & -2.02343 & -0.14276 & -6.93717 \\
\hline & Constant & & 13.94575 & 9.263127 & 14.0 & & 9.140645 & 10.54656 & 10.34501 & 9.596556 & 11.68736 & 11.08795 & 14.11606 \\
\hline & VP90 & -0.0076 & -0.245015 & -0.007622 & -0.246595 & -0.185026 & -2.55082 & 43 & 4094 & -0.41177 & -2.6634 & -0.30411 & -4.84384 \\
\hline & JMG90 & j24557 & 0.543828 & 0.024557 & 0.547335 & 0.080701 & 0.796741 & 0.001067 & -0.01083 & 0.101399 & .218594 & 0.061285 & 0.766284 \\
\hline & TMG90 & & 0.26 & 721 & 638 & 641 & 69 & 227298 & 2877 & 2625 & .656011 & 0.179369 & 2.457491 \\
\hline & DGEX90 & 591 & 237 & 5591 & .434018 & 727 & 312 & .182916 & -3.28081 & -0.27964 & -1.36393 & -0.12925 & -2.73388 \\
\hline & POPs & 83 & -0.779991 & 383 & -0.785021 & 204 & -1.39284 & -0.406102 & 1347 & 1034 & -2.34236 & -0.48616 & -3.8861 \\
\hline & POPs2 & 952 & 0.712525 & 2952 & 712 & & 1.308578 & 918 & 5583 & 734 & 53 & 0.021425 & 3.807014 \\
\hline & FHHF & & 1.096828 & 3293 & 1.10 & 93 & 829 & 644 & 0.050483 & 668 & 995 & -0.00779 & -0.25862 \\
\hline & POPHD & 05 & 099221 & 605 & & 572 & 626 & 136 & 7646 & 482 & 0.42 & 984 & -0.1175 \\
\hline & UNEMP & & 549274 & & 16 & & 19 & 822 & 7214 & 0.0 & 0.0 & 108 & 0.398809 \\
\hline & MANU & & .314649 & 285 & 78 & & & & 4652 & & -1.6 & 0058 & -0.52893 \\
\hline & WHRT & 000685 & -0.22249 & 685 & -0.22 & & 948 & 456 & 8327 & 663 & 0.206719 & 0.007346 & 2.093 \\
\hline & SCIX & & 565863 & & & & 326 & & 0.300564 & & & 0.00713 & 0.945939 \\
\hline & MHY80 & & -14.75376 & 068 & -14.8489 & 968 & & 9788 & -14.0287 & -0.78211 & -8.23297 & -0.79235 & -15.1537 \\
\hline & Constant & 3.595848 & 5.761564 & 95848 & 5.79604 & & 6.306849 & & 7.52333 & 4.7837 & 4.165257 & 3.525295 & 6.417735 \\
\hline & EMP90 & 0.055117 & 0.937709 & 055117 & 0.94332 & 0.268919 & -2.18738 & .603143 & -5.24755 & -0.71523 & -2.93621 & -0.41604 & -4.80011 \\
\hline & INMG90 & 196279 & -2.218751 & -0.196279 & -2.232028 & -0.267563 & -1.52643 & -0.798108 & -4.73957 & -1.90611 & -1.99239 & 0.36978 & -2.42189 \\
\hline & OTMG90 & 0.207116 & 2.169278 & 0.207116 & 2.182259 & 0.282011 & 1.869625 & 1.022079 & 6.957195 & 1.071938 & 2.13854 & 0.610226 & 4.773711 \\
\hline & MHY90 & 0.032133 & 0.63152 & 0.032133 & 0.635299 & -0.007078 & -0.07117 & 0.233596 & -2.49106 & -0.52383 & -2.50146 & -0.22419 & -2.83782 \\
\hline & POPs & -0.072578 & -4.144961 & -0.072578 & -4.169764 & -0.095961 & -4.35044 & -0.135086 & -6.36852 & -0.20027 & -3.50703 & -0.09034 & -5.33196 \\
\hline
\end{tabular}




\begin{tabular}{|c|c|c|c|c|c|c|c|c|c|c|c|c|}
\hline POP5-17 & -0.094469 & -0.776258 & -0.094469 & -0.780903 & 0.003547 & 0.027869 & -0.150098 & -1.3584 & -0.29508 & -3.07636 & 0.064852 & \\
\hline SCRM & 1.28E-05 & 1.490139 & 1.28E-05 & 1.499056 & 4.24E-05 & 2.77752 & $4.09 \mathrm{E}-05$ & 2.820269 & $2.20 \mathrm{E}-08$ & 0.005187 & & 15202 \\
\hline DFEG & & & 0.002981 & & 1217 & -1.18783 & & & & & & 282 \\
\hline CTAX & & & & & & 2.8 & & & & & & \\
\hline PCTD & 9.07E-07 & & & & & & & & $-2.57 \mathrm{E}-06$ & & & 039 \\
\hline LTD & $4.23 E-08$ & 0.999467 & 4.23 & 1.00 & 3.6 & 0.823 & -08 & 1.16 & $2.88 \mathrm{E}-08$ & 0.527471 & $4.32 \mathrm{E}-08$ & 2.744631 \\
\hline DGEX80 & -0.395797 & -6.340638 & .395797 & -6.37858 & -0.465404 & -6.99908 & -0.223757 & -3.70274 & -0.0794 & -2.07689 & -0.33577 & -7.24849 \\
\hline
\end{tabular}


Table L: NON-SPATIAL MODEL ESTIMATION RESULTS, RATE, US COUNTIES, 1980 SINGLE-EQUATION

\begin{tabular}{|c|c|c|c|c|c|c|c|c|c|c|c|c|c|}
\hline \multirow{3}{*}{$\begin{array}{l}\text { Equati } \\
\text { on }\end{array}$} & \multirow{3}{*}{ Variables } & \multicolumn{6}{|c|}{ SINGLE-EQUATION } & \multicolumn{6}{|c|}{ MULTI-EQUATION } \\
\hline & & \multicolumn{2}{|c|}{ OLS } & \multicolumn{2}{|c|}{ Weighted OLS } & \multicolumn{2}{|c|}{ Weighted 2SLS } & \multicolumn{2}{|c|}{$3 S L S$} & \multicolumn{2}{|l|}{$\mathrm{ML}$} & \multicolumn{2}{|l|}{ GMM } \\
\hline & & Coefficient & t-Statistic & Coefficient & t-Statistic & Coefficient & t-Statistic & Coefficient & t-Statistic & Coefficient & & Coefficient & t-Statistic \\
\hline \multirow{16}{*}{\begin{tabular}{|l|} 
EMP \\
Equati \\
on
\end{tabular}} & constant & -2.35162 & -8.761901 & -2.35162 & -8.7846 & -2.713634 & -9.66729 & -3.313182 & 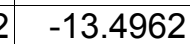 & -3.11265 & -12.2604 & -2.23773 & -6.1541 \\
\hline & INMG90 & 0.013225 & 1.356196 & 0.013225 & 1.35971 & -0.045054 & -3.268 & -0.013288 & -0.97954 & -0.16495 & -8.34357 & -0.07233 & -5.35325 \\
\hline & OTMG90 & 0.151369 & 7.068176 & 0.151369 & 7.086487 & 0.040425 & 0.993723 & 0.092396 & 2.348774 & -0.14361 & -2.69123 & 0.155426 & 3.422551 \\
\hline & MHY90 & 0.816124 & 19.89932 & 0.816124 & 19.95087 & 1.233761 & 12.85316 & 1.525121 & 17.00351 & -0.02154 & -0.13998 & 1.322245 & 13.25525 \\
\hline & DGEX90 & 0.028999 & 1.504282 & 028999 & 508179 & .088595 & 1.64295 & 0.007163 & 0.1347 & 0.30 & 4.373698 & -0.04907 & \\
\hline & POP24-44 & 323264 & & & 844 & 43 & 7.36066 & 0.372353 & 1228 & 0.45 & & 0.358 & \\
\hline & FHHF & 0.019406 & 872 & & & & 1.344435 & 0.069102 & 011 & 728 & 707 & -0.01 & \\
\hline & POPCD & 0.149715 & & & & & 52 & 0.097844 & 712 & 92 & & 0.062 & \\
\hline & OWHU & 0.34394 & 243 & & & & 7.346 & 0.377168 & & 09 & & 0.213 & \\
\hline & MANU & -0.000865 & & 365 & & 16 & -2.1 & -0.001915 & & 99 & & -0.00 & \\
\hline & WHRT & 0.015739 & & & 323 & & 10.13551 & 0.014152 & 6713 & 0.02 & 267 & 0.016499 & \\
\hline & PCPTAX & -0.056837 & 34829 & 0.056837 & -7.367327 & -0.062341 & -7.772 & -0.040328 & 36842 & -0.0 & 9473 & -0.01568 & 7995 \\
\hline & NAIX & 0.007063 & 56282 & 063 & 3.164459 & & 2.053879 & 0.001524 & 156 & 0.02 & 7568 & 0.007227 & 34696 \\
\hline & HWD & 0.06055 & 7.074147 & & 7.092474 & & 3.465489 & 0.037624 & 2963 & 0.09 & 732 & 0.024055 & 44882 \\
\hline & ESBd & -0.209988 & -12.4332 & -0.20 & -12.46541 & 341 & -11.6305 & -0.126224 & 7705 & $-0.1 \varepsilon$ & 084 & -0.20 & 165 \\
\hline & EMP80 & .024246 & -5.145485 & -0.024246 & -5.158816 & 113 & -2.36145 & -0.017754 & 1084 & -0.04217 & 406 & -0.00627 & 885 \\
\hline & Constant & -3.415823 & -26.78226 & -3.415823 & -26.83425 & -3.823065 & -20.4439 & -3.412179 & -20.734 & -1.7615 & 63108 & -3.02534 & -16.088 \\
\hline & EMP90 & 0.267827 & 11.51545 & 0.267827 & 11.5378 & 0.512445 & 8.365517 & 0.794704 & 13.32011 & 2.68727 & 13.47025 & 0.824176 & 11.82623 \\
\hline & OTMG90 & 0.427665 & 15.24726 & 0.427665 & 15.27686 & -0.525956 & -8.51513 & -0.969753 & -19.4907 & -1.65336 & -11.2807 & -0.65765 & -11.2654 \\
\hline & MHY90 & 0.544717 & 9.543827 & 544717 & 9.562353 & 0.349957 & 2.302572 & -0.02511 & -0.16927 & -3.14396 & -7.50513 & -0.00912 & -0.06597 \\
\hline & DGEX90 & -0.061128 & 0765 & -0.061128 & -2.355328 & 0.210402 & 2.366105 & 0.420645 & 4.81917 & 0.097201 & 1395 & 0.355897 & 3.661664 \\
\hline & AREA & 0.0027 & 3924 & 0.0027 & 0.38467 & 0.01 & 1.829974 & 0.031867 & 3.559119 & 0.082564 & 7.420316 & 0.03982 & 3.580919 \\
\hline & POPs & 603691 & & & & & 42.80513 & 0.719129 & 6115 & 0.822 & 328 & 0.587932 & 23.59861 \\
\hline & MCRH & 0.353164 & & 164 & & 0.285373 & 7.888631 & 0.084163 & 5014 & -0.03612 & -1.56748 & 0.116474 & \\
\hline & UNEMP & 0.01596 & & & 1.373231 & -0.007395 & -0.47685 & -0.028586 & -1.91022 & & & -0.04714 & -2.90965 \\
\hline & NAIX & 0.036677 & & & 12.75408 & & 14.76567 & 0.06242 & 3491 & 0.056755 & 11.24515 & 0.040211 & 3167 \\
\hline & EXPTAX & 0.056757 & 3.60025 & 0.056757 & 3.607239 & 0.037587 & 1.933782 & 0.084726 & 5.069199 & -0.04724 & -1.84713 & 0.0403 & 2.514641 \\
\hline
\end{tabular}




\begin{tabular}{|c|c|c|c|c|c|c|c|c|c|c|c|c|c|}
\hline & & .667161 & -53.06874 & -0.667161 & 53.17175 & -0.689291 & -41.7355 & 0.706427 & -45.1231 & -0.77445 & -20.8263 & -0.58294 & -22.2839 \\
\hline & Constant & .257326 & 1.700918 & 0.257326 & 1.704219 & -0.049976 & -0.27188 & 0.372294 & -2.67688 & -0.04247 & -0.3499 & 0.009421 & 0.059861 \\
\hline & EMP90 & 9516 & 622 & & .27807 & 289847 & 9.064824 & 0.41106 & 3.33363 & 51232 & 13.709 & & 3.60277 \\
\hline & INMG90 & 2345 & & & 56172 & .040453 & .79629 & 1622 & & 9065 & 5.34434 & 9685 & 009 \\
\hline & MHY90 & & & & .95244 & 11372 & .44765 & .229858 & & & .47826 & & \\
\hline & DGEX90 & 268 & 8728 & 14268 & 95057 & 130692 & 858586 & 0.20747 & .592018 & 010186 & 143171 & 359 & 3.38874 \\
\hline & AREA & 908 & 263 & 08 & 133271 & 344 & 992918 & .025731 & 538712 & 036427 & 8.83625 & .030369 & .081652 \\
\hline & POPs & 0.258455 & 24.85054 & 455 & 24.89878 & 292423 & 25.47192 & 0.370918 & 6.72067 & .331908 & 0.87964 & .324965 & 4.83633 \\
\hline & OWHU & 208421 & 161006 & .208421 & 6.172965 & .291311 & -7.69424 & .252044 & 9.29298 & 0.14576 & -9.25249 & 0.29247 & -9.5517 \\
\hline & UNEMP & -0.038198 & -5.554143 & -0.038198 & -5.564924 & -0.039376 & -4.78491 & 0.048957 & -6.24245 & -0.11717 & -10.3281 & -0.06587 & -7.00262 \\
\hline & NAIX & 0.022316 & 13.78292 & 0.022316 & 13.80967 & 0.022819 & 12.60999 & 0.026489 & 5.41228 & 0.017807 & 9.624005 & 0.018997 & 0.91655 \\
\hline & EXPTAX & 00683 & 0.952801 & 0.00683 & 0.95465 & 0.021 & 2.702336 & 0.035854 & .902184 & -0.03882 & -4.19603 & 0.012611 & 1.73243 \\
\hline & OTMG80 & 0.251784 & -22.59381 & -0.251784 & -22.63767 & -0.285701 & -22.783 & 0.361539 & -32.8316 & 0.31368 & -18.8151 & -0.32211 & -22.737 \\
\hline & Constant & 2.225229 & 19.09744 & 2.225229 & 19.1407 & 2.8778 & 5.24497 & 2.709009 & 5.9597 & .567182 & 19.15112 & 2.644178 & 13.78 \\
\hline & EMP90 & 0.155667 & 24.75072 & 0.155667 & 24.8068 & 0.216818 & 6.90423 & & 24.9 & .400741 & 23.00275 & 0.299806 & 21.40515 \\
\hline & INMG90 & 0.007172 & 1.835149 & 0.007172 & 1.839 & -0.019067 & -3.35213 & -0.024466 & -4.39172 & -0.01212 & -1.60085 & -0.02287 & -4.34656 \\
\hline & OTMG90 & .08604 & 10.22199 & 0.08604 & 10.24515 & 0.018366 & 1.113015 & -0.008868 & -0.55278 & -0.05381 & -2.54892 & -0.00101 & -0.0596 \\
\hline & DGEX90 & 0.050837 & 6.628076 & 0.050837 & 6.643094 & 0.149125 & 6.61083 & 0.143294 & 6.479543 & -0.02231 & -0.75706 & 0.137411 & 5.541196 \\
\hline & POPs & 811 & -8.112486 & 8811 & 3.130867 & -0.26447 & -8.02215 & -0.23384 & -7.90369 & -0.05822 & -6.3109 & 0.18695 & -5.50249 \\
\hline & POPs2 & 006124 & 8.909455 & 0.006124 & 8.929642 & 0.012942 & 8.351643 & 0.011772 & 8.486162 & 0.003968 & 887888 & .009402 & 5.892044 \\
\hline & FHHF & -0.007865 & -1.110175 & -0.007865 & -1.11269 & -0.009822 & -1.26087 & -0.026442 & -3.66792 & -0.02315 & -5.07384 & -0.01186 & -1.47717 \\
\hline & POPHD & 173856 & 12.75329 & 3856 & 12.78219 & 162959 & 10.61758 & 0.092519 & 7.186248 & 0.048136 & 5.045101 & .112716 & 8.308883 \\
\hline & UNEMP & .041347 & 1.19647 & .041347 & 11.22184 & .034143 & -7.70344 & 0.020545 & -5.55487 & -0.0463 & $\mid-12.055$ & 0.03177 & -7.63243 \\
\hline & MANU & 02708 & 177 & 708 & 14.0836 & 3239 & 51 & 002284 & 0.54303 & 92 & 6.698088 & .002474 & 10.86519 \\
\hline & WHRT & 0.001118 & 827084 & -0.001 & .831224 & 0.000965 & 1.275612 & 1615 & -2.30925 & 0.00406 & -9.23436 & -0.00124 & -1.63981 \\
\hline & SCIX & -0.0068 & 0916 & -0.0068 & 160321 & & -3.68888 & 0.000307 & 0.187752 & .007944 & 6.503966 & 0.001482 & 0.809109 \\
\hline & MHY80 & -0.19283 & -16.36828 & -0.19283 & -16.40537 & 2438 & -15.12 & -0.157651 & -13.3503 & -0.20459 & -16.4154 & -0.18526 & -14.0602 \\
\hline & Constant & 2.013476 & 12.92716 & 2.013476 & 12.95435 & 2.489062 & 13.95861 & 2.347439 & 13.85539 & 2.29987 & 13.11714 & 2.213493 & 12.86091 \\
\hline & EMP90 & -0.00438 & -0.291999 & -0.00438 & -0.292613 & -0.067194 & -2.05181 & -0.095559 & -2.95593 & -0.00947 & -0.20439 & -0.13088 & -3.86779 \\
\hline & INMG90 & -0.018884 & -2.210095 & -0.018884 & -2.214744 & -0.003771 & -0.32597 & 0.026904 & 2.348064 & 001271 & 0.11886 & .028227 & 1.886754 \\
\hline & OTMG90 & 0.032998 & 1.772378 & 0.032998 & 1.776106 & 0.109046 & 3.35244 & 0.162848 & 5.09617 & .099881 & 2.716662 & .157536 & 4.76098 \\
\hline & MHY90 & 0.179916 & 4.853126 & 0.179916 & 4.863334 & 0.146012 & 1.767721 & 0.297397 & 3.688254 & 0.034634 & 0.326459 & 0.261085 & 3.196907 \\
\hline & POPs & -0.004877 & -1.30118 & -0.004877 & -1.303917 & -0.029609 & -5.55207 & -0.046488 & -8.89061 & -0.00773 & -1.68008 & -0.04249 & -7.77094 \\
\hline
\end{tabular}




\begin{tabular}{|c|c|c|c|c|c|c|c|c|c|c|c|c|}
\hline POP5-17 & 0.006824 & 0.206228 & 0.006824 & 0.206662 & 0.04948 & 1.398665 & 0.099153 & 3.019743 & -0.0486 & -1.53624 & 0.161418 & 4.786219 \\
\hline SCRM & 1.35E-05 & 5.98075 & 1.35E-05 & 5.99333 & $3.75 \mathrm{E}-05$ & 8.165581 & $5.03 \mathrm{E}-05$ & 11.28731 & $1.55 \mathrm{E}-05$ & 6.545988 & 4.66E-05 & 9.48553 \\
\hline DFEG & -7.24E-05 & -0.008686 & $-7.24 \mathrm{E}-05$ & -0.008704 & -0.008161 & 4055 & -0.005938 & -0.74474 & 0.005566 & & & \\
\hline PCTAX & 0.08 & & & & 0.0 & & & & & 981 & & 643 \\
\hline PCTD & 26E-07 & 0.544847 & $9.26 \mathrm{E}-07$ & $0.545 \mathrm{c}$ & $1.21 \mathrm{E}-06$ & 0.692084 & & & $8.38 \mathrm{E}$ & & & 0.252645 \\
\hline LTD & 9.63E-09 & 0.410859 & 9.63E-09 & 0.411 & $6.42 \mathrm{E}-10$ & 0.026663 & -4.67 & -2.10321 & $3.80 \mathrm{E}$ & 0.065383 & $-8.10 \mathrm{E}-10$ & -0.07208 \\
\hline DGEX80 & -0.28374 & -19.70607 & -0.28374 & -19.74752 & -0.32653 & -20.2461 & -0.318669 & -19.9257 & -0.27931 & -23.091 & -0.32436 & -16.0956 \\
\hline
\end{tabular}




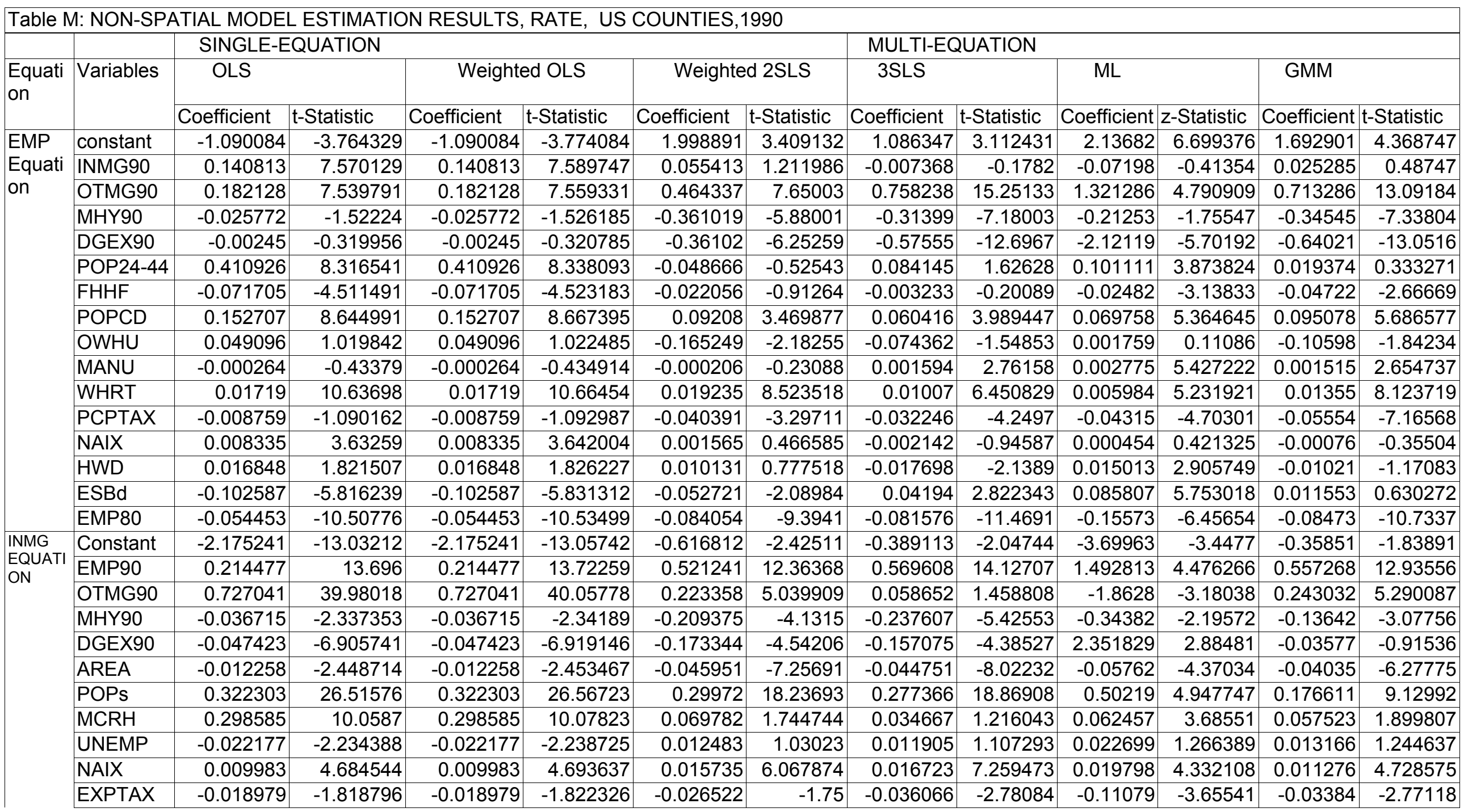




\begin{tabular}{|c|c|c|c|c|c|c|c|c|c|c|c|c|c|}
\hline & & -0.37086 & -29.20191 & -0.37086 & -29.2586 & -0.338899 & -20.7264 & 0.310946 & -21.2672 & -0.37765 & & & -10.4658 \\
\hline & Constant & -0.348376 & -2.35363 & -0.348376 & -2.358198 & -1.744472 & -7.11784 & 0.920858 & -5.20117 & -0.32817 & -0.75122 & -0.91872 & -3.53934 \\
\hline & EMP90 & 0.1314 & & 0.1314 & & 0.454262 & 488 & & 2.80672 & 636788 & 3666 & & 3.32947 \\
\hline & INMG90 & & 452 & 028 & & 95367 & -2.58139 & 277325 & -8.5903 & 37237 & 3.74869 & & 3173 \\
\hline & IHY90 & 4068 & & 04068 & & & & & 3.65221 & 0857 & & & \\
\hline & DGEX90 & 021211 & 827719 & 021211 & 835149 & 212672 & 657816 & 281994 & 374707 & 535526 & 2.065162 & 3516 & 0.63366 \\
\hline & AREA & 018628 & 657628 & .018628 & 666669 & .036243 & -5.59323 & .033411 & -6.614 & 3724 & -3.23093 & 0.02966 & -4.759 \\
\hline & POPs & 0.221622 & 18.25347 & 221622 & 18.28891 & 267151 & 13.64429 & 0.296765 & 9.13862 & 289541 & 5.5066 & 232487 & 2.84201 \\
\hline & OWHU & .044623 & .261989 & .044623 & 1.264439 & 0.279467 & 1.817053 & 0.008345 & 0.21456 & 0.12399 & -3.45254 & .012949 & .224852 \\
\hline & UNEMP & 0.010572 & -1.23516 & -0.010572 & -1.237557 & -0.039514 & -3.03895 & 0.015783 & -1.54886 & 0.00107 & 0.090884 & -0.01187 & -1.11289 \\
\hline & NAIX & 017344 & 11.0488 & 0.017344 & 11.07025 & 0.020176 & 8.43604 & 0.014408 & 7.158776 & 0.012323 & 3.479788 & .013772 & 6.717693 \\
\hline & EXPTAX & 0.038536 & 4.788105 & 0.038536 & 4.797399 & 8395 & -4.66975 & 0.061959 & -5.28422 & -0.07903 & -3.35664 & -0.06215 & -5.14459 \\
\hline & OTMG80 & -0.226302 & -17.5074 & -0.226302 & -17.54139 & 0.259905 & -11.8942 & 0.285068 & -17.0732 & -0.2826 & -3.81894 & -0.21247 & -10.7699 \\
\hline & Constant & 809024 & 29.86909 & 8.809024 & 29.93677 & 166 & 20.64306 & 9.468654 & 24.62761 & 8.837036 & 18.90417 & 9.451152 & 26.12309 \\
\hline & EMP90 & .001654 & -0.096668 & -0.001654 & -0.096887 & 0.106891 & -2.47059 & -0.358616 & -9.74559 & -0.18336 & -1.50198 & -0.27285 & -7.08131 \\
\hline & INMG90 & .006959 & -0.407208 & -0.006959 & -0.40813 & -0.017595 & -0.53 & -0.099968 & -3.16521 & 0.026032 & 0.583526 & -0.06087 & -1.9838 \\
\hline & OTMG90 & 0.037421 & 1.600363 & 0.037421 & 1.603989 & 0.068966 & 1.394 & 0.349677 & 7.519064 & 0.227761 & 0.91696 & 0.273575 & 6.027217 \\
\hline & DGEX90 & -0.011168 & -1.500705 & -0.011168 & -1.504105 & -0.152303 & -4.10534 & -0.335263 & -9.6096 & -0.25822 & -0.68156 & -0.28208 & -8.49977 \\
\hline & POPs & 03269 & 90774 & 3269 & 0.090979 & 5761 & -1.29746 & -0.287236 & -4.6294 & -0.0521 & -1.31367 & -0.23305 & -4.19131 \\
\hline & POPs2 & .000514 & .309231 & .000514 & .309931 & 0.003449 & .002697 & 0.011326 & 3.921309 & .001248 & 0.78389 & .009222 & 3.598359 \\
\hline & FHHF & 0.024954 & 1.496404 & 24954 & 1.499795 & 0.022882 & 1.279862 & 0.036093 & 2.27152 & .023679 & 388134 & .025834 & 1.621705 \\
\hline & POPHD & 0.042876 & 855855 & 042876 & 0.857794 & 0.044962 & .831272 & 0.00539 & 129776 & .058897 & 1.229188 & .067639 & .585544 \\
\hline & UNEMP & 0.036977 & 225514 & 6977 & 3.232822 & 0808 & 3.213237 & 0.069951 & .529671 & 0.069866 & 5.879559 & 5.45E-02 & .265985 \\
\hline & MANU & .000267 & 502257 & 0267 & .503394 & 553 & 0.814732 & $3.4 \mathrm{C}$ & .057964 & -0.0017 & -3.11527 & .000237 & .398243 \\
\hline & WHRT & 00051 & 321728 & & 57 & 59 & 2.107246 & 712 & 4.148187 & 8.63E-05 & -0.05679 & .006075 & 3.594406 \\
\hline & SCIX & & 2.541 & & & & 1.733366 & 133 & 6.108622 & 0.021912 & 4.73 & 8763 & 4.667085 \\
\hline & MHY80 & & -29.9042 & 865617 & -29.97195 & 8736 & -26.4257 & -0.742436 & -25.9945 & -0.82279 & -23.2319 & -0.79961 & -27.7219 \\
\hline & Constant & 3.535218 & 8.365241 & 535218 & 8.382836 & 3.401189 & 6.470703 & 1.910175 & 5.452722 & 1.638308 & 9.669365 & 2.461079 & 6.804866 \\
\hline & EMP90 & -0.009336 & .236274 & -0.009336 & -0.236771 & -0.168289 & -1.98327 & 0.890995 & -13.2041 & -0.2839 & -3.95915 & -0.49502 & -7.47003 \\
\hline & INMG90 & -0.30391 & -7.507722 & -0.30391 & -7.523514 & -0.321896 & -4.31164 & -0.507456 & -7.45278 & -0.00653 & -0.08324 & -0.2486 & -3.06998 \\
\hline & OTMG90 & 0.238821 & 4.306638 & 0.238821 & 4.315697 & 0.380406 & 3.949957 & 1.228237 & 14.93192 & 0.616004 & 7.092959 & 0.687696 & 8.629765 \\
\hline & MHY90 & 0.067694 & -1.861633 & -0.067694 & -1.865548 & -0.19277 & -2.69203 & -0.368727 & -6.00981 & -0.08058 & -1.3029 & -0.31962 & -5.34371 \\
\hline & POPs & -0.076735 & -7.422003 & -0.076735 & -7.437614 & -0.081835 & -5.14193 & -0.114544 & -8.78014 & -0.07097 & -7.28905 & $-9.23 E-02$ & -6.65943 \\
\hline
\end{tabular}




\begin{tabular}{|c|c|c|c|c|c|c|c|c|c|c|c|c|}
\hline POP5-17 & -0.012783 & -0.162901 & -0.012783 & -0.163243 & 0.022565 & 0.28199 & 5.04E-02 & 1.051759 & -0.06285 & -5.31568 & 3.75E-02 & 0.683123 \\
\hline SCRM & 1.21E-05 & 2.115401 & 1.21E-05 & 2.11985 & 4.73E-06 & 0.413744 & 8.91E-06 & 1.000082 & $-1.44 \mathrm{E}-06$ & -2.76157 & $2.74 \mathrm{E}-06$ & 0.267875 \\
\hline DFEG & 0.014149 & 0.509055 & 0.014149 & 0.510126 & 0.0261 & 0.787416 & 0.017177 & 0.905617 & 0.005319 & 2.510517 & -8.83E-03 & -0.41958 \\
\hline PCTAX & 0.043232 & 1.677591 & 0.043232 & 1.68112 & 0.027167 & 0.907805 & $-1.19 \mathrm{E}-02$ & -0.6522 & -0.01562 & -4.17606 & $-3.18 E-02$ & -1.67872 \\
\hline PCTD & $-1.85 \mathrm{E}-06$ & -0.73384 & $-1.85 \mathrm{E}-06$ & -0.735383 & $-2.69 \mathrm{E}-06$ & -1.04929 & $-8.51 \mathrm{E}-07$ & -0.60352 & -7.73E-08 & -0.38874 & $-3.44 E-06$ & -2.07713 \\
\hline LTD & 7.64E-08 & 2.753099 & 7.64E-08 & 2.75889 & 8.16E-08 & 2.917555 & 1.10E-08 & 0.682452 & $4.35 \mathrm{E}-10$ & 0.185986 & 6.04E-08 & 2.464347 \\
\hline DGEX80 & -0.342691 & -8.688564 & -0.342691 & -8.706839 & -0.317192 & -7.50484 & -0.01432 & -0.48475 & -0.00594 & -2.04379 & -0.079 & -2.64564 \\
\hline
\end{tabular}


Appendix 2: Estimation Results of the Spatial Simultaneous Equations Models

Table c11: Generalized Spatial Two-Stage Least Squares(GS2SLS) Estimation Results, 1980, LEVEL

\begin{tabular}{|c|c|c|c|c|c|c|c|c|c|c|}
\hline \multirow[b]{2}{*}{ VARIABLE } & \multicolumn{2}{|c|}{ EMP Equation } & \multicolumn{2}{|c|}{ INMG Equation } & \multicolumn{2}{|c|}{ OTMG Equation } & \multicolumn{2}{|c|}{ MHY Equation } & \multicolumn{2}{|c|}{ DGEX Equation } \\
\hline & Coeff. & $t$-stat. & Coeff. & $t$-stat. & Coeff. & t-stat. & Coeff. & $t$-stat. & Coeff. & t-stat. \\
\hline CONSTANT & -5.2483 & -1.1774 & 1.0102 & 0.6364 & 1.4781 & 1.4912 & 3.9316 & 4.8037 & 0.9785 & 0.7157 \\
\hline EMPt & & & 0.0268 & 0.1150 & -0.0176 & -0.8011 & 0.0030 & 0.2356 & 0.0864 & 2.7279 \\
\hline INMGt & 0.4635 & 2.5474 & & & 0.5975 & 16.0428 & 0.4035 & 7.7939 & 0.0326 & 0.2872 \\
\hline OTMGt & -0.0975 & -0.4770 & 0.0501 & 20.3886 & & & -0.2835 & -4.6133 & -0.1234 & -0.8895 \\
\hline MHYt & 0.0705 & 0.3980 & 0.1080 & 1.7146 & 0.2110 & 2.4343 & & & 0.0441 & 0.4207 \\
\hline DGEXt & 0.3036 & 3.0049 & 0.0492 & -2.6908 & 0.0620 & 1.6772 & 0.0521 & 2.2065 & & \\
\hline WEMPt & -0.5126 & -4.6257 & 0.0341 & 0.1438 & 0.0229 & 0.7806 & -0.0238 & -1.3674 & -0.0147 & -0.3495 \\
\hline WINMGt & -0.2159 & -0.4860 & 0.1184 & 6.4975 & -0.4576 & -4.6684 & -0.0419 & -0.5304 & -0.2550 & -1.4950 \\
\hline WOTMGt & 0.7674 & 1.6295 & 0.1189 & -6.2242 & 0.6636 & 6.6501 & 0.0569 & 0.7260 & 0.2430 & 1.4149 \\
\hline WMHYt & 0.1740 & 0.5145 & 0.1099 & -1.9963 & -0.0970 & -1.0355 & 0.1103 & 1.7826 & -0.0474 & -0.3373 \\
\hline WDGEXt & 0.3153 & 1.2236 & 0.0656 & 1.4390 & -0.1359 & -2.5208 & -0.0306 & -0.8498 & 0.3510 & 4.5708 \\
\hline AREA & & & 0.0118 & 1.2775 & 0.0274 & 2.5777 & & & & \\
\hline POPs & & & 0.0462 & -2.4209 & 0.2223 & 6.5247 & -0.2743 & -2.7766 & -0.0303 & -0.5094 \\
\hline POPd & & & & & & & 0.0089 & 1.9966 & & \\
\hline POP5_17 & & & & & & & & & 0.1139 & 1.0232 \\
\hline POP25_44 & 0.2394 & 0.9646 & & & & & & & & \\
\hline FHHF & 0.1294 & 1.1850 & & & & & -0.0356 & -1.1998 & & \\
\hline POPHD & & & & & & & 0.1000 & 2.6860 & & \\
\hline POPCD & 0.0875 & 1.6459 & & & & & & & & \\
\hline OWHU & -0.3591 & -1.4587 & & & -0.4935 & -3.9741 & & & & \\
\hline MCRH & & & 0.0451 & 0.4298 & & & & & & \\
\hline UNEMP & & & 0.0364 & 0.7969 & -0.0090 & -0.3207 & -0.0943 & -5.6072 & & \\
\hline MANU & 0.0018 & 1.0309 & & & & & 0.0012 & 2.6801 & & \\
\hline WHRT & 0.0010 & 0.1745 & & & & & -0.0053 & -2.9203 & & \\
\hline SCRM & & & & & & & & & 0.0000 & 2.7259 \\
\hline DFEG & & & & & & & & & -0.0072 & -0.2853 \\
\hline PCTAX & & & & & & & & & 0.0112 & 0.3915 \\
\hline PCPTAX & -0.0986 & -3.0926 & & & & & & & & \\
\hline PCTD & & & & & & & & & -0.0001 & -2.3808 \\
\hline LTD & & & & & & & & & 0.0000 & \begin{tabular}{|l|}
1.2739 \\
\end{tabular} \\
\hline SCIX & & & & & & & 0.0039 & 0.5092 & & \\
\hline NAIX & 0.0039 & 0.3621 & 0.0065 & 2.0832 & -0.0078 & -1.4177 & & & & \\
\hline HWD & -0.0013 & -0.0377 & & & & & & & & \\
\hline ESBd & 0.1359 & 2.3135 & & & & & & & & \\
\hline EXTAX & & & 0.0290 & 0.4084 & -0.0287 & -1.2980 & & & & \\
\hline
\end{tabular}




\begin{tabular}{|l|c|c|c|c|c|c|c|c|c|c|}
\hline EMPt-1 & 0.6382 & 15.5783 & & & & & & & & \\
\hline INMGt-1 & & & 0.0220 & 2.7883 & & & & & & \\
\hline OTMGt-1 & & & & & 0.1584 & 4.8011 & & & & \\
\hline MHYt-1 & & & & & & & 0.6117 & 16.8665 & & \\
\hline DGEXt-1 & & & & & & & & & 0.5693 & 11.6839 \\
\hline RHO & 0.9256 & & -0.503 & & -0.2561 & & -0.0271 & & -0.3201 & \\
\hline SIG & 0.4831 & & 0.0212 & & 0.0128 & & 0.0052 & & 0.0337 & \\
\hline AD.R2 & 0.9638 & & 0.9839 & & 0.9874 & & 0.8965 & & 0.6208 & \\
\hline F-STATISTIC & 552.68 & 0 & 1572.29 & 0 & 2035.02 & 0 & 198.534 & 0 & 43.0875 & 0 \\
\hline N & 418 & & 418 & & 418 & & 418 & & 418 & \\
\hline ETA (n) & 0.3618 & & 0.9780 & & 0.8416 & & 0.3883 & & 0.4307 & \\
\hline
\end{tabular}

* Figures in the t-statistics column are p-values 
Table c12: Generalized Spatial Two-Stage Least Squares(GSSLS) Estimation Results, 1990, LEVEL

\begin{tabular}{|c|c|c|c|c|c|c|c|c|c|c|}
\hline \multirow{2}{*}{ VARIABLE } & \multicolumn{2}{|c|}{ EMP Equation } & \multicolumn{2}{|c|}{ INMG Equation } & \multicolumn{2}{|c|}{ OTMG Equation } & \multicolumn{2}{|c|}{ MHY Equation } & \multicolumn{2}{|c|}{ DGEX Equation } \\
\hline & Coeff. & t-stat. & Coeff. & t-stat. & Coeff. & t-stat. & Coeff. & t-stat. & Coeff. & t-stat. \\
\hline CONSTANT & 2.9630 & 0.7523 & 1.2469 & 1.3950 & 0.7582 & 0.7682 & 3.3561 & 6.9924 & 5.9911 & 1.7428 \\
\hline EMPt & & & -0.0313 & -1.6910 & -0.0084 & -0.3978 & -0.0024 & -0.3217 & 0.2175 & 3.8023 \\
\hline INMGt & 0.0912 & 0.6477 & & & 0.5589 & 15.6497 & -0.0418 & -1.7535 & -0.8464 & -4.2610 \\
\hline OTMGt & 0.2368 & 1.4468 & 0.5712 & 9.4612 & & & 0.0933 & 3.0264 & 0.7042 & 2.5388 \\
\hline MHYt & -0.0983 & -0.5705 & 0.0072 & 0.0946 & 0.1160 & 1.5681 & & & 0.1167 & 0.5334 \\
\hline DGEXt & 0.0359 & 0.5376 & -0.0844 & -3.5440 & 0.0326 & 1.1529 & -0.0023 & -0.2655 & & \\
\hline WEMPt & -0.3351 & -3.2446 & -0.1318 & -4.6225 & -0.0361 & -1.2224 & -0.0170 & -1.7349 & -0.2680 & -3.3580 \\
\hline WINMGt & 0.1004 & 0.3163 & 0.8455 & 12.3969 & -0.3412 & -4.2297 & 0.0212 & 0.7052 & 0.4862 & 1.9415 \\
\hline WOTMGt & 0.2747 & 0.7637 & -0.7031 & -8.8866 & 0.5802 & 6.1739 & -0.0108 & -0.3222 & -0.1630 & -0.5652 \\
\hline WMHYt & -0.2792 & -0.8467 & -0.0568 & -0.5909 & -0.0776 & -0.7473 & 0.0180 & 0.4667 & -0.2499 & -0.7962 \\
\hline WDGEXt & 0.1102 & 0.7279 & 0.0155 & 0.3933 & -0.0797 & -1.9025 & -0.0125 & -0.8369 & 0.3625 & 3.0777 \\
\hline AREA & & & 0.0040 & 0.3619 & -0.0215 & -1.8221 & & & & \\
\hline POPs & & & -0.1077 & -3.1425 & 0.1890 & 5.2564 & -0.0973 & -1.6472 & -0.2636 & -2.1417 \\
\hline POPd & & & & & & & 0.0029 & 1.1155 & & \\
\hline POP5_17 & & & & & & & & & -0.4342 & -2.3412 \\
\hline POP25_44 & 0.2673 & 1.4733 & & & & & & & & \\
\hline FHHF & 0.0093 & 0.0979 & & & & & -0.0813 & -5.4949 & & \\
\hline POPHD & & & & & & & 0.0304 & 1.1280 & & \\
\hline POPCD & 0.0110 & 0.2267 & & & & & & & & \\
\hline OWHU & -0.2311 & -1.0283 & & & -0.1754 & -1.3733 & & & & \\
\hline $\mathrm{MCRH}$ & & & 0.0721 & 1.2863 & & & & & & \\
\hline UNEMP & & & 0.0290 & 1.4132 & -0.0138 & -0.6030 & -0.0068 & -0.8433 & & \\
\hline MANU & 0.0023 & 1.3637 & & & & & -0.0004 & -1.3650 & & \\
\hline WHRT & 0.0185 & 4.0703 & & & & & 0.0004 & 0.4761 & & \\
\hline SCRM & & & & & & & & & 0.8595 & 2.6449 \\
\hline DFEG & & & & & & & & & -0.0370 & -0.6495 \\
\hline PCTAX & & & & & & & & & 0.1075 & 1.9536 \\
\hline РСРТАХ & -0.0214 & -0.7958 & & & & & & & & \\
\hline PCTD & & & & & & & & & 0.3902 & 1.6108 \\
\hline LTD & & & & & & & & & -0.0089 & -1.6074 \\
\hline SCIX & & & & & & & -0.0016 & -0.4559 & & \\
\hline NAIX & -0.0021 & -0.2100 & 0.0089 & 1.8080 & -0.0084 & -1.5471 & & & & \\
\hline HWD & -0.0043 & -0.1235 & & & & & & & & \\
\hline ESBd & 0.1880 & 3.4494 & & & & & & & & \\
\hline EXTAX & & & 0.0283 & 1.5186 & -0.0232 & -1.1321 & & & & \\
\hline EMPt-1 & 0.6817 & 17.7764 & & & & & & & & \\
\hline INMGt-1 & & & 0.5487 & 11.0993 & & & & & & \\
\hline
\end{tabular}




\begin{tabular}{|l|c|c|c|c|c|c|c|c|c|c|}
\hline OTMGt-1 & & & & & 0.2626 & 6.3711 & & & & \\
\hline MHYt-1 & & & & & & & 0.7391 & 34.0631 & & \\
\hline DGEXt-1 & & & & & & & & & 0.3826 & 3.9519 \\
\hline RHO & 0.9256 & & 0.0592 & & -0.1708 & & 0.1265 & & -0.2464 & \\
\hline SIG & 0.4544 & & 0.01017 & & 0.01233 & & 0.0014 & & 0.1176 & \\
\hline AD.R2 & 0.9711 & & 0.9893 & & 0.9876 & & 0.9574 & & 0.3916 & \\
\hline F-STATISTIC* & 701633 & 0 & 2408.44 & 0 & 2076.87 & 0 & 522.475 & 0 & 19.234 & 0 \\
\hline N & 418 & & 418 & & 418 & & 418 & & 418 & \\
\hline ETA $(\eta)$ & 0.3183 & & 0.4513 & & 0.7375 & & 0.2609 & & 0.6174 & \\
\hline
\end{tabular}

* Figures in the t-statistics column are p-values 


\begin{tabular}{|c|c|c|c|c|c|c|c|c|c|c|}
\hline \multicolumn{11}{|c|}{ Table c13 Generalized Spatial Two-Stage Least Squares(GS2SLS) Estimation Results, PANEL, LEVEI } \\
\hline \multirow[b]{2}{*}{ VARIABLE } & \multicolumn{2}{|c|}{ EMP Equation } & \multicolumn{2}{|c|}{ INMG Equation } & \multicolumn{2}{|c|}{ OTMG Equation } & \multicolumn{2}{|c|}{ MHY Equation } & \multicolumn{2}{|c|}{ DGEX Equation } \\
\hline & Coeff. & t-stat. & Coeff. & $t$-stat. & Coeff. & $t$-stat. & Coeff. & $t$-stat & Coeff. & t-stat. \\
\hline CONSTANT & 3.1046 & 1.6256 & 1.6495 & 2.5354 & -0.2969 & -0.5248 & 4.8095 & 7.5325 & 6.3365 & 3.8218 \\
\hline EMPt & & & -0.0011 & -0.0531 & -0.0061 & -0.4160 & -0.0044 & -0.4470 & 0.0917 & 2.4269 \\
\hline INMGt & 0.2580 & 1.8844 & & & 0.5500 & 20.7287 & 0.1675 & 4.2590 & -0.3201 & -2.1779 \\
\hline OTMGt & 0.1234 & 0.7862 & 1.0381 & 25.0647 & & & -0.0683 & -1.3947 & 0.1019 & 0.4610 \\
\hline MHYt & -0.4849 & -4.7766 & 0.1341 & 1.8893 & 0.1793 & 4.8797 & & & -0.0441 & -0.3522 \\
\hline DGEXt & 0.1416 & 2.4375 & -0.1879 & -6.4112 & 0.0174 & 0.7051 & 0.0461 & 2.9492 & & \\
\hline WEMPt & -0.1812 & -2.7376 & 0.0563 & 1.9729 & -0.0682 & -3.0305 & -0.0084 & -0.5731 & -0.1389 & -2.2541 \\
\hline WINMGt & 0.1577 & 0.6133 & 0.6722 & 9.4036 & -0.0608 & -0.8632 & \begin{tabular}{|l|}
-0.0279 \\
\end{tabular} & -0.6109 & 0.5077 & 2.9141 \\
\hline WOTMGt & 0.1064 & 0.4024 & -0.7075 & -8.3701 & 0.1481 & 1.8919 & 0.0272 & 0.5350 & -0.3006 & -1.5051 \\
\hline WMHYt & -0.4279 & -2.3677 & -0.2507 & -4.1638 & -0.0641 & -1.2350 & 0.0452 & 1.3806 & -0.3728 & -2.9786 \\
\hline WDGEXt & 0.3291 & 2.7922 & 0.1573 & 3.6453 & 0.0146 & 0.4090 & -0.0219 & -0.9921 & 0.4139 & 5.0488 \\
\hline AREA & & & 0.0182 & 2.1203 & 0.0063 & 0.8795 & & & & \\
\hline POPs & & & -0.1782 & -5.1467 & 0.2048 & 8.6262 & -0.3119 & -3.1296 & -0.0096 & -0.1008 \\
\hline POPd & & & & & & & 0.0116 & 2.6227 & & \\
\hline POP5_17 & & & & & & & & & -0.0921 & -0.7608 \\
\hline POP25_44 & 0.5725 & 3.9578 & & & & & & & & \\
\hline FHHF & -0.0542 & -1.1902 & & & & & -0.0652 & -3.4664 & & \\
\hline POPHD & & & & & & & 0.1802 & 6.8130 & & \\
\hline POPCD & 0.1379 & 3.7541 & & & & & & & & \\
\hline OWHU & -0.0046 & -0.0253 & & & -0.3692 & -4.0623 & & & & \\
\hline MCRH & & & 0.0434 & 1.6070 & & & & & & \\
\hline UNEMP & & & 0.0112 & 0.4844 & -0.0289 & -1.7367 & -0.0568 & -4.9741 & & \\
\hline MANU & 0.0044 & 3.8282 & & & & & 0.0017 & 4.9222 & & \\
\hline WHRT & 0.0108 & 3.2272 & & & & & 0.0001 & 0.1158 & & \\
\hline SCRM & & & & & & & & & 0.1185 & 4.4423 \\
\hline DFEG & & & & & & & & & -0.0644 & -2.2835 \\
\hline PCTAX & & & & & & & & & 0.1023 & 3.0043 \\
\hline PCPTAX & -0.0220 & -0.9636 & & & & & & & & \\
\hline PCTD & & & & & & & & & 0.4044 & 2.1113 \\
\hline LTD & & & & & & & & & -0.0085 & -1.9411 \\
\hline SCIX & & & & & & & -0.0131 & -2.6979 & & \\
\hline NAIX & 0.0142 & 1.9986 & 0.0184 & 3.6273 & -0.0080 & -2.0228 & & & & \\
\hline HWD & 0.0311 & 1.2841 & & & & & & & & \\
\hline ESBd & 0.1836 & 4.3038 & & & & & & & & \\
\hline EXTAX & & & 0.0458 & 3.1160 & -0.0445 & $\mid-4.0813$ & & & & \\
\hline
\end{tabular}




\begin{tabular}{|l|c|c|c|c|c|c|c|c|c|c|}
\hline EMPt-1 & 0.6641 & 22.5431 & & & & & & & & \\
\hline INMGt-1 & & & 0.0965 & 4.1965 & & & & & & \\
\hline OTMGt-1 & & & & & 0.2338 & 9.4888 & & & & \\
\hline MHYt-1 & & & & & & & 0.5702 & 38.7790 & & \\
\hline DGEXt-1 & & & & & & & & & 0.3917 & 8.2492 \\
\hline RHO & 0.8132 & & -0.5506 & & -0.0624 & & -0.0912 & & -0.261 & \\
\hline SIGV & 0.4513 & & 0.0217 & & 0.0123 & & 0.0059 & & 0.0953 & \\
\hline SIG1 & 0.5585 & & 0.0257 & & 0.0137 & & 0.9281 & & 0.0944 & \\
\hline AD.R2 & 0.9711 & & 0.9797 & & 0.9863 & & 597.502 & 0 & 0.4351 & \\
\hline F-STATISTIC* & 1404.79 & 0 & 2456.46 & 0 & 3680.14 & 0 & 2.989 & 0 & 44.8068 & 0 \\
\hline N & 836 & & 836 & & 836 & & 836 & & 836 & \\
\hline ETA ( $\eta$ ) & 0.3359 & & 0.9035 & & 0.7662 & & 0.4298 & & 0.6083 & \\
\hline
\end{tabular}

* Figures in the t-statistics column are p-values 


\begin{tabular}{|c|c|c|c|c|c|c|c|c|c|c|}
\hline \multicolumn{11}{|c|}{$\begin{array}{l}\text { Table c14: Feasible Generaliz } \\
\text { Results, 1980_LEVEL }\end{array}$} \\
\hline \multirow[b]{2}{*}{ VARIABLE } & \multicolumn{2}{|c|}{ EMP Equation } & \multicolumn{2}{|c|}{ INMG Equation } & \multicolumn{2}{|c|}{ OTMG Equation } & \multicolumn{2}{|c|}{ MHY Equation } & \multicolumn{2}{|c|}{ DGEX Equation } \\
\hline & Coeff. & t-stat. & Coeff. & t-stat. & Coeff. & $t$-stat. & Coeff. & t-stat. & Coeff. & t-stat. \\
\hline CONSTANT & $\begin{array}{c}- \\
2.3857 \\
\end{array}$ & -0.3079 & -0.1112 & -0.1731 & -0.1231 & -0.0493 & 2.8334 & 6.4398 & 1.7655 & 2.0280 \\
\hline EMPt & & & 0.0022 & 0.1238 & -0.7775 & -5.8151 & -0.0032 & -0.3826 & 0.1064 & 5.3859 \\
\hline INMGt & 1.4269 & 6.2063 & & & 1.9768 & 9.8298 & 0.5419 & 17.0331 & -0.0336 & -0.4554 \\
\hline OTMGt & $\begin{array}{c}- \\
0.5213 \\
\end{array}$ & -2.3100 & 0.9985 & 31.5446 & & & -0.4426 & -11.6211 & -0.0092 & -0.1038 \\
\hline MHYt & $\begin{array}{c}- \\
0.5466 \\
\end{array}$ & -0.9942 & 0.3398 & 4.9766 & -0.6937 & -0.9916 & & & 0.0380 & 0.5638 \\
\hline DGEXt & 0.8932 & 2.1365 & -0.1428 & -4.4157 & 0.9228 & 1.7325 & 0.0905 & 5.9080 & & \\
\hline WEMPt & $\begin{array}{c}- \\
0.3750 \\
\end{array}$ & -3.0048 & 0.0071 & 0.3193 & 0.6701 & 4.4157 & -0.0228 & -1.9994 & -0.0282 & -1.0227 \\
\hline WINMGt & 0.3691 & 0.8244 & 0.8162 & 10.6789 & -1.7679 & -5.7487 & -0.1707 & -3.3974 & -0.1550 & -1.3873 \\
\hline WOTMGt & 0.0890 & 0.1844 & -0.7845 & -10.2182 & 0.9062 & 3.5456 & 0.1772 & 3.5493 & 0.1664 & 1.4763 \\
\hline WMHYt & 0.0296 & 0.0773 & -0.3038 & -4.2319 & 0.7412 & 1.1078 & 0.1626 & 4.0756 & -0.1363 & -1.4879 \\
\hline WDGEXt & 0.2641 & 0.9438 & 0.1104 & 2.5609 & -0.8827 & -1.7532 & -0.0553 & -2.3643 & 0.4121 & 8.3612 \\
\hline AREA & & & 0.0125 & 1.7076 & -0.0055 & -0.2087 & & & & \\
\hline POPs & & & -0.0813 & -2.7498 & 0.1107 & 4.8023 & -0.0867 & -2.1716 & & \\
\hline POPd & & & & & & & 0.0014 & 0.8328 & & \\
\hline POP5_17 & & & & & & & & & 0.0955 & 1.3383 \\
\hline POP25_44 & 0.0831 & 0.3999 & & & & & & & & \\
\hline FHHF & $\begin{array}{c}- \\
0.0163 \\
\end{array}$ & -0.1993 & & & & & -0.0272 & -1.5226 & & \\
\hline POPHD & & & & & & & 0.0953 & 4.2784 & & \\
\hline POPCD & 0.0510 & 1.1336 & & & & & & & & \\
\hline OWHU & $\begin{array}{c}- \\
0.1464 \\
\end{array}$ & -0.4421 & & & -0.2010 & -2.8894 & & & & \\
\hline MCRH & & & -0.0001 & -0.0002 & & & & & & \\
\hline UNEMP & & & 0.0575 & 2.4383 & 0.0526 & 0.9504 & -0.1070 & -9.9570 & & \\
\hline MANU & 0.0011 & 0.8361 & & & & & 0.0010 & \begin{tabular}{|l|}
3.5134 \\
\end{tabular} & & \\
\hline WHRT & 0.0002 & 0.0403 & & & & & -0.0048 & -4.3671 & & \\
\hline SCRM & & & & & & & & & 0.1532 & 2.5652 \\
\hline DFEG & & & & & & & & & 0.0017 & 0.1088 \\
\hline PCTAX & & & & & & & & & 0.0306 & 1.6928 \\
\hline РСРТAX & \begin{tabular}{|c|}
- \\
0.0310 \\
\end{tabular} & -1.2375 & & & & & & & & \\
\hline PCTD & & & & & & & & & -0.1848 & -2.2942 \\
\hline LTD & & & & & & & & & 0.0083 & 1.3973 \\
\hline
\end{tabular}




\begin{tabular}{|c|c|c|c|c|c|c|c|c|c|c|}
\hline ScIX & & & & & & & 0.0040 & 0.9004 & & \\
\hline NAIX & $\begin{array}{c}- \\
0.0007 \\
\end{array}$ & -0.0530 & 0.0141 & 3.5161 & -0.0118 & -0.6504 & & & & \\
\hline HWD & 0.0109 & \begin{tabular}{|l|}
0.3107 \\
\end{tabular} & & & & & & & & \\
\hline ESBd & 0.0759 & 1.4502 & & & & & & & & \\
\hline EXTAX & & & 0.0014 & 0.0792 & -0.0092 & -0.2098 & & & & \\
\hline EMPt-1 & 0.2140 & 3.4313 & & & & & & & & \\
\hline INMGt-1 & & & 0.0470 & 3.5787 & & & & & & \\
\hline OTMGt-1 & & & & & 0.0486 & 2.6524 & & & & \\
\hline MHYt-1 & & & & & & & 0.5723 & 25.3694 & & \\
\hline DGEXt-1 & & & & & & & & & 0.5135 & 16.9347 \\
\hline RHO & 0.9256 & & -0.503 & & -0.2561 & & -0.0271 & & -0.3201 & \\
\hline SIG & 0.4831 & & 0.0212 & & 0.0128 & & 0.0052 & & 0.0337 & \\
\hline $\mathrm{R}^{2}$ & 0.9583 & & 0.9838 & & 0.9847 & & 0.8795 & & 0.6314 & \\
\hline $\mathrm{N}$ & 418 & & 418 & & 418 & & 418 & & 418 & \\
\hline $\operatorname{ETA}(\eta)$ & 0.7860 & & 0.9530 & & 0.9514 & & 0.4277 & & 0.4865 & \\
\hline
\end{tabular}




\begin{tabular}{|c|c|c|c|c|c|c|c|c|c|c|}
\hline \multicolumn{11}{|c|}{ Table c15: Feasible Generalized Spatial Three-Stage Least Squares(FGS3SLS) Estimation } \\
\hline \multirow[b]{2}{*}{ VARIABLE } & \multicolumn{2}{|c|}{ EMP Equation } & \multicolumn{2}{|c|}{ INMG Equation } & \multicolumn{2}{|c|}{ OTMG Equation } & \multicolumn{2}{|c|}{ MHY Equation } & \multicolumn{2}{|c|}{ DGEX Equation } \\
\hline & Coeff. & $t$-stat. & Coeff. & t-stat. & Coeff. & t-stat. & Coeff. & $t$-stat. & Coeff. & $t$-stat. \\
\hline CONSTANT & \begin{tabular}{|l|}
9.3389 \\
\end{tabular} & \begin{tabular}{|l|}
2.4342 \\
\end{tabular} & 1.4574 & 2.9211 & 3.4531 & 0.8349 & \begin{tabular}{|l|}
3.4200 \\
\end{tabular} & 14.8378 & 4.1346 & 2.5985 \\
\hline EMPt & & & -0.0029 & -0.2849 & -0.6650 & -4.1990 & -0.0011 & -0.2590 & 0.2406 & 7.6888 \\
\hline INMGt & 0.5281 & 3.7094 & & & 1.6740 & 6.8210 & -0.0761 & -5.7956 & -1.5524 & -14.9068 \\
\hline OTMGt & 0.0745 & 0.5493 & 0.6542 & 20.0951 & & & 0.1327 & 7.8495 & 1.6213 & 12.0257 \\
\hline MHYt & -0.4639 & -2.1048 & 0.0074 & 0.1819 & $\mid-0.7178$ & -1.2705 & & & 0.2919 & 2.6190 \\
\hline DGEXt & 0.2196 & 2.3865 & -0.1217 & -9.3843 & 0.5149 & 2.1484 & -0.0093 & -1.8953 & & \\
\hline WEMPt & -0.4216 & $|-5.0545|$ & -0.1201 & -7.5254 & 0.3596 & 1.9221 & $\mid-0.0177$ & -3.2025 & -0.2097 & -4.6573 \\
\hline WINMGt & 0.5118 & 1.7931 & 0.7920 & 20.7316 & -1.1567 & -2.8914 & 0.0443 & 2.6120 & 0.9590 & 6.8567 \\
\hline WOTMGt & 0.0090 & 0.0283 & -0.6671 & -14.9687 & 0.5795 & 1.4585 & $|-0.0329|$ & -1.7269 & -0.7190 & -4.4648 \\
\hline WMHYt & -0.6352 & -2.1369 & -0.0453 & -0.8322 & 0.6071 & 0.9492 & 0.0124 & 0.5688 & -0.2655 & -1.5551 \\
\hline WDGEXt & 0.0967 & 0.7603 & 0.0313 & 1.4091 & -0.5939 & -2.3073 & -0.0100 & -1.1848 & 0.3690 & 5.6746 \\
\hline AREA & & & 0.0179 & 3.0890 & -0.0851 & -1.8206 & & & & \\
\hline POPs & & & -0.1500 & $\mid-7.8371$ & 0.1967 & 6.2379 & -0.1025 & -5.0282 & $\mid-0.4778$ & -7.9809 \\
\hline POPd & & & & & & & \begin{tabular}{|l|}
0.0027 \\
\end{tabular} & 3.2614 & & \\
\hline POP5_17 & & & & & & & & & -0.4299 & -4.6184 \\
\hline POP25_44 & 0.2623 & 2.3511 & & & & & & & & \\
\hline FHHF & -0.0054 & -0.0987 & & & & & -0.0819 & -10.1231 & & \\
\hline POPHD & & & & & & & \begin{tabular}{|l|}
0.0462 \\
\end{tabular} & 3.1324 & & \\
\hline POPCD & 0.0008 & 0.0281 & & & & & & & & \\
\hline OWHU & -0.3430 & -1.4091 & & & $|-0.3643|$ & -0.5095 & & & & \\
\hline MCRH & & & 0.0696 & 2.4178 & & & & & & \\
\hline UNEMP & & & 0.0089 & 0.8252 & 0.0849 & 1.1658 & -0.0073 & -1.6501 & & \\
\hline MANU & 0.0024 & 2.1701 & & & & & -0.0003 & -2.0855 & & \\
\hline WHRT & 0.0152 & 5.1067 & & & & & \begin{tabular}{|l|}
0.0004 \\
\end{tabular} & 0.8053 & & \\
\hline SCRM & & & & & & & & & 0.2038 & 2.6023 \\
\hline DFEG & & & & & & & & & 0.0218 & 0.7825 \\
\hline PCTAX & & & & & & & & & 0.0782 & 2.8166 \\
\hline РСРTAX & -0.0132 & -0.8256 & & & & & & & & \\
\hline PCTD & & & & & & & & & -0.0367 & -0.6730 \\
\hline LTD & & & & & & & & & 0.0006 & 0.3400 \\
\hline$S C I X$ & & & & & & & -0.0028 & -1.4606 & & \\
\hline NAIX & -0.0090 & -0.8581 & 0.0083 & 3.2655 & -0.0478 & -1.6560 & & & & \\
\hline HWD & 0.0360 & 1.3883 & & & & & & & & \\
\hline ESBd & 0.1618 & 4.2528 & & & & & & & & \\
\hline
\end{tabular}




\begin{tabular}{|l|c|c|c|c|c|c|c|c|c|c|}
\hline EXTAX & & & 0.0295 & 3.0699 & -0.0492 & -0.8034 & & & & \\
\hline EMPt-1 & 0.4677 & 11.9749 & & & & & & & & \\
\hline INMGt-1 & & & 0.4660 & 17.7094 & & & & & & \\
\hline OTMGt-1 & & & & & 0.3345 & 2.3134 & & & & \\
\hline MHYt-1 & & & & & & & 0.7373 & 60.7852 & & \\
\hline DGEXt-1 & & & & & & & & & 0.4685 & 10.3542 \\
\hline RHO & 0.9256 & & 0.0592 & & -0.1708 & & 0.1265 & & -0.2464 & \\
\hline SIG & 0.4544 & & 0.01017 & & 0.01233 & & 0.0014 & & 0.1176 & \\
\hline$R^{2}$ & 0.9694 & & 0.9851 & & 0.9864 & & 0.9534 & & 0.3691 & \\
\hline$N$ & 418 & & 418 & & 418 & & 418 & & 418 & \\
\hline ETA $(n)$ & 0.5323 & & 0.5340 & & 0.6655 & & 0.2627 & & 0.5315 & \\
\hline
\end{tabular}




\begin{tabular}{|c|c|c|c|c|c|c|c|c|c|c|}
\hline \multicolumn{11}{|c|}{$\begin{array}{l}\text { Table c16: Feasible Generali } \\
\text { PANEL_LEVEL }\end{array}$} \\
\hline \multirow[b]{2}{*}{ VARIABLE } & \multicolumn{2}{|c|}{ EMP Equation } & \multicolumn{2}{|c|}{ INMG Equation } & \multicolumn{2}{|c|}{ OTMG Equation } & \multicolumn{2}{|c|}{ MHY Equation } & \multicolumn{2}{|c|}{ DGEX Equation } \\
\hline & Coeff. & t-stat. & Coeff. & t-stat. & Coeff. & t-stat. & Coeff. & t-stat. & Coeff. & t-stat. \\
\hline CONSTANT & 0.6066 & 0.4511 & -1.0794 & -2.2240 & 0.4862 & 1.0461 & 4.2692 & 13.8212 & 4.3895 & 6.2253 \\
\hline EMPt & 0.4785 & 4.7812 & -0.0586 & -5.2079 & 0.0508 & 5.9168 & -0.0005 & -0.0666 & 0.0244 & 1.5807 \\
\hline INMGt & -0.0458 & -0.4260 & & & 0.7615 & 92.0929 & 0.3327 & 13.4175 & -0.0474 & -0.7593 \\
\hline OTMGt & -0.3066 & -4.3163 & 1.0964 & 88.4238 & & & -0.2494 & -8.7671 & 0.0714 & 0.9107 \\
\hline MHYt & 0.1248 & 3.0333 & 0.2489 & 8.9042 & -0.0007 & -0.0307 & & & -0.3438 & -4.8223 \\
\hline DGEXt & -0.2342 & -5.0031 & -0.0643 & -2.6391 & 0.0067 & 0.3874 & 0.0576 & 5.4442 & & \\
\hline WEMPt & -0.2091 & -1.1494 & 0.0461 & 2.2960 & -0.0191 & -1.1746 & -0.0283 & -2.3730 & -0.0932 & -3.6969 \\
\hline WINMGt & 0.4980 & 2.7156 & 0.3685 & 6.4315 & -0.3396 & -7.4906 & -0.0700 & -1.8124 & 0.2386 & 2.3429 \\
\hline WOTMGt & -0.2317 & -1.5705 & -0.4197 & -7.0536 & 0.4717 & 10.0199 & 0.1000 & 2.5897 & -0.1552 & -1.5715 \\
\hline WMHYt & 0.2502 & 2.4484 & 0.0012 & 0.0233 & -0.0634 & -1.4504 & -0.0458 & -1.4297 & -0.0710 & -0.7712 \\
\hline WDGEXt & & & 0.0245 & 0.5878 & -0.0027 & -0.0820 & 0.0452 & 1.9865 & 0.3475 & 6.7389 \\
\hline AREA & & & -0.0371 & -4.3350 & 0.0202 & 2.8061 & & & & \\
\hline POPs & & & -0.1036 & -8.2389 & 0.1177 & 13.6608 & -0.0870 & -1.9746 & -0.0006 & -0.0167 \\
\hline POPd & & & & & & & 0.0028 & 1.3568 & & \\
\hline POP5_17 & & & & & & & & & 0.1884 & 2.9596 \\
\hline POP25_44 & 0.2672 & 2.9050 & & & & & & & & \\
\hline FHHF & -0.0055 & -0.1884 & & & & & -0.0502 & -4.9470 & & \\
\hline POPHD & & & & & & & 0.1729 & 7.1760 & & \\
\hline POPCD & 0.0481 & 2.0168 & & & & & & & & \\
\hline OWHU & 0.1820 & 1.8425 & & & -0.0704 & -3.4412 & & & & \\
\hline MCRH & & & -0.0264 & -5.7028 & & & & & & \\
\hline UNEMP & & & -0.0136 & -0.4945 & 0.0260 & 1.2118 & -0.0620 & -6.2551 & & \\
\hline MANU & 0.0007 & 1.3309 & & & & & 0.0014 & 5.3244 & & \\
\hline WHRT & 0.0015 & 0.6019 & & & & & -0.0011 & -1.3583 & & \\
\hline SCRM & & & & & & & & & 0.2208 & 1.8191 \\
\hline DFEG & & & & & & & & & -0.0457 & -3.7615 \\
\hline PCTAX & & & & & & & & & 0.1316 & 7.4610 \\
\hline PCPTAX & -0.0505 & -3.7054 & & & & & & & & \\
\hline PCTD & & & & & & & & & -0.0085 & -0.0640 \\
\hline LTD & & & & & & & & & -0.0003 & -0.1042 \\
\hline SCIX & & & & & & & -0.0269 & -8.0201 & & \\
\hline NAIX & 0.0103 & 1.2937 & 0.0347 & 11.2454 & -0.0281 & -13.3797 & & & & \\
\hline HWD & 0.0413 & 1.6546 & & & & & & & & \\
\hline ESBd & 0.2027 & 6.8527 & & & & & & & & \\
\hline EXTAX & & & 0.0192 & 1.8793 & -0.0333 & -4.0278 & & & & \\
\hline
\end{tabular}




\begin{tabular}{|l|c|c|c|c|c|c|c|c|c|c|}
\hline EMPt-1 & 0.6579 & 33.3948 & & & & & & & & \\
\hline INMGt-1 & & & 0.0157 & 5.2123 & & & & & & \\
\hline OTMGt-1 & & & & & 0.0911 & 13.8780 & & & & \\
\hline MHYt-1 & & & & & & & 0.5243 & 43.1608 & & \\
\hline DGEXt-1 & & & & & & & & & 0.5029 & 19.2674 \\
\hline RHO & 0.8132 & & -0.5506 & & -0.0624 & & -0.0912 & & -0.261 & \\
\hline SIGV & 0.4513 & & 0.0217 & & 0.0123 & & 0.0059 & & 0.0953 & \\
\hline SIG1 & 0.5585 & & 0.0257 & & 0.0137 & & 0.9281 & & 0.0944 & \\
\hline$R^{2}$ & 0.9721 & & 0.9745 & & 0.9864 & & 0.9254 & & 0.4416 & \\
\hline N & 836 & & 836 & & 836 & & 836 & & 836 & \\
\hline ETA $(n)$ & 0.3421 & & 0.9843 & & 0.9089 & & 0.4757 & & 0.4971 & \\
\hline
\end{tabular}




\begin{tabular}{|c|c|c|c|c|c|c|c|c|c|c|}
\hline \multirow[b]{2}{*}{ VARIABLE } & \multicolumn{2}{|c|}{ EMPR Equation } & \multicolumn{2}{|c|}{ INMGR Equation } & \multicolumn{2}{|c|}{ OTMGR Equation } & \multicolumn{2}{|c|}{ MHYR Equation } & \multicolumn{2}{|c|}{ DGEXR Equation } \\
\hline & Coeff. & t-stat. & Coeff. & t-stat. & Coeff. & $t$-stat. & Coeff. & t-stat. & Coeff. & $t$-stat. \\
\hline CONSTANT & -1.4899 & -1.1713 & -4.0797 & -10.8967 & 3.2583 & 3.8109 & 3.0777 & 5.2685 & 1.9222 & 3.8132 \\
\hline EMPR & & & 0.2817 & 3.2108 & 0.1040 & 1.4333 & 0.1798 & 6.7990 & -0.0017 & -0.0226 \\
\hline INMGR & 0.0097 & 0.2496 & & & 0.0439 & 1.3412 & -0.0289 & -2.0050 & -0.0724 & -2.2165 \\
\hline OTMGR & 0.1273 & 1.5645 & 0.0157 & 0.1913 & & & 0.0253 & 0.9319 & 0.1961 & 3.1007 \\
\hline MHYR & 1.2547 & 6.8401 & 0.7082 & 2.8820 & -0.0083 & -0.0417 & & & 0.0901 & 0.4759 \\
\hline DGEXR & -0.0197 & -0.2100 & -0.1289 & -1.2023 & 0.2361 & 2.7720 & 0.0492 & 1.3126 & & \\
\hline WEMPR & -0.6483 & -3.1787 & -0.0730 & -0.4519 & 0.0613 & 0.4844 & -0.1132 & -2.2718 & -0.3329 & -2.7545 \\
\hline WINMGR & -0.0950 & -0.9213 & 0.3213 & 4.4689 & 0.0439 & 0.7571 & 0.0013 & 0.0582 & 0.0974 & 1.8311 \\
\hline WOTMGR & 0.2124 & 1.1811 & 0.5025 & 3.6673 & 0.6882 & 7.3433 & 0.0007 & 0.0165 & -0.1846 & -1.9528 \\
\hline WMHYR & 0.6303 & 1.6985 & 0.5507 & 1.9485 & -0.0534 & -0.2375 & 0.5890 & 7.8206 & 0.1084 & 0.4751 \\
\hline WDGEXR & -0.0863 & -0.3446 & 0.2391 & 1.2812 & -0.3531 & -2.4673 & -0.0170 & -0.2798 & 0.6971 & 6.0751 \\
\hline AREA & & & 0.0270 & 1.3780 & 0.1007 & 6.8346 & & & & \\
\hline POPs & & & 0.5866 & 17.0253 & 0.1063 & 4.9408 & -0.1734 & -1.8389 & -0.0207 & -1.5034 \\
\hline POPd & & & & & & & 0.0086 & 1.9086 & & \\
\hline POP5_17 & & & & & & & & & 0.0486 & 0.4616 \\
\hline POP25_44 & 0.4030 & 2.1278 & & & & & & & & \\
\hline $\mathrm{FHHF}$ & 0.0565 & 0.6613 & & & & & -0.0829 & -3.5711 & & \\
\hline POPHD & & & & & & & 0.1807 & 5.6973 & & \\
\hline POPCD & 0.0890 & 1.8682 & & & & & & & & \\
\hline OWHU & 0.0267 & 0.1115 & & & -0.6244 & -3.4192 & & & & \\
\hline MCRH & & & 0.4698 & 6.6798 & & & & & & \\
\hline UNEMP & & & -0.1022 & -2.0829 & -0.1416 & -3.6947 & -0.1017 & -6.5120 & & \\
\hline MANU & -0.0015 & -0.9987 & & & & & 0.0018 & 4.4008 & & \\
\hline WHRT & 0.0147 & 3.1392 & & & & & 0.0016 & 0.9672 & & \\
\hline SCRM & & & & & & & & & 0.3541 & 2.9218 \\
\hline DFEG & & & & & & & & & -0.0008 & -0.0330 \\
\hline PCTAX & & & & & & & & & 0.0388 & 1.9760 \\
\hline PCPTAX & -0.0413 & -1.9151 & & & & & & & & \\
\hline PCTD & & & & & & & & & -0.2266 & -1.1084 \\
\hline LTD & & & & & & & & & 0.0038 & 0.3920 \\
\hline SCIX & & & & & & & -0.0215 & -3.2616 & & \\
\hline NAIX & -0.0056 & -0.5463 & 0.0005 & 0.0486 & 0.0057 & 0.6436 & & & & \\
\hline HWD & 0.0778 & 2.1332 & & & & & & & & \\
\hline ESBd & -0.0871 & -1.9906 & & & & & & & & \\
\hline EXTAX & & & -0.0505 & -1.4015 & 0.0599 & 2.3518 & & & & \\
\hline EMPt-1 & -0.0688 & -3.9868 & & & & & & & & \\
\hline
\end{tabular}




\begin{tabular}{|l|c|c|c|c|c|c|c|c|c|c|}
\hline INMGt-1 & & & -0.6646 & -20.0184 & & & & & & \\
\hline OTMGt-1 & & & & & -0.1615 & -6.8919 & & & & \\
\hline MHYt-1 & & & & & & & -0.2579 & -6.7765 & & \\
\hline DGEXt-1 & & & & & & & & & -0.2970 & -6.9300 \\
\hline RHO & 0.6782 & & -0.1103 & & -0.2831 & & -0.5283 & & -0.5276 & \\
\hline SIG & 0.0657 & & 0.053 & & 0.0293 & & 0.0056 & & 0.0311 & \\
\hline AD.R2 & 0.4501 & & 0.6004 & & 0.3886 & & 0.7684 & & 0.3542 & \\
\hline$N$ & 418 & & 418 & & 418 & & 418 & & 418 & \\
\hline
\end{tabular}


Table 5.2e: Generalized Spatial Two-Stage Least Squares(GS2SLS) Estimation Results, 1990_RATE

\begin{tabular}{|c|c|c|c|c|c|c|c|c|c|c|}
\hline \multirow[b]{2}{*}{ VARIABLE } & \multicolumn{2}{|c|}{ EMPR Equation } & \multicolumn{2}{|c|}{ INMGR Equation } & \multicolumn{2}{|c|}{ OTMGR Equation } & \multicolumn{2}{|c|}{ MHYR Equation } & \multicolumn{2}{|c|}{ DGEXR Equation } \\
\hline & Coeff. & t-stat. & Coeff. & $t$-stat. & Coeff. & t-stat. & Coeff. & $t$-stat. & Coeff. & $t$-stat \\
\hline CONSTANT & 0.5469 & 0.3966 & -0.5031 & -3.4203 & 0.4464 & 0.6177 & 10.6135 & 5.5109 & 4.0414 & 3.1984 \\
\hline EMPR & & & 0.0302 & 1.3353 & 0.1726 & 3.2980 & 0.0876 & 0.8391 & -0.1808 & -1.0919 \\
\hline INMGR & 1.5961 & 2.7680 & & & 0.5825 & 1.9309 & 1.0150 & 2.0181 & -1.5190 & -1.7113 \\
\hline OTMGR & 0.5489 & 3.6228 & 0.0576 & 1.9434 & & & -0.0422 & -0.3188 & 0.8211 & 3.7961 \\
\hline MHYR & -0.0066 & -0.0618 & -0.0011 & -0.0438 & -0.1130 & -1.8898 & & & 0.1977 & 1.2632 \\
\hline DGEXR & -0.1925 & -2.7786 & -0.0181 & -1.3061 & 0.1436 & 3.9178 & -0.0495 & -0.9078 & & \\
\hline WEMPR & -1.1507 & -4.4764 & 0.0279 & 0.7359 & 0.0119 & 0.1092 & 0.0551 & 0.3355 & 0.0606 & 0.1977 \\
\hline WINMGR & -2.0268 & -2.6784 & 1.0520 & 23.8811 & -0.0451 & -0.1278 & -1.2434 & -2.1839 & 1.4677 & 1.4531 \\
\hline WOTMGR & 0.9409 & 2.1377 & -0.0184 & -0.3043 & $|-0.0636|$ & -0.3528 & 0.3166 & 1.2187 & -0.7316 & -1.5531 \\
\hline WMHYR & -0.0313 & -0.1376 & -0.0006 & -0.0235 & -0.0943 & -1.1448 & 0.2634 & 2.6510 & -0.2763 & -1.4219 \\
\hline WDGEXR & -0.2879 & -1.7994 & -0.0031 & -0.1351 & -0.0432 & -0.6687 & -0.0149 & -0.1395 & 0.2074 & 1.1706 \\
\hline AREA & & & 0.0120 & 2.1006 & 0.0153 & 0.9419 & & & & \\
\hline POPs & & & 0.0430 & 3.2346 & 0.0666 & 2.7101 & -0.2603 & -0.7952 & -0.1318 & -3.3772 \\
\hline POPd & & & & & & & 0.0122 & 0.7886 & & \\
\hline POP5_17 & & & & & & & & & -0.1648 & -0.8026 \\
\hline POP25_44 & 0.4830 & 2.5327 & & & & & & & & \\
\hline FHHF & 0.0154 & 0.1669 & & & & & -0.0433 & -0.5808 & & \\
\hline POPHD & & & & & & & -0.1895 & -1.1117 & & \\
\hline POPCD & 0.0659 & 1.2291 & & & & & & & & \\
\hline OWHU & -0.3685 & -1.5061 & & & 0.0163 & 0.1076 & & & & \\
\hline $\mathrm{MCRH}$ & & & 0.0609 & 2.5936 & & & & & & \\
\hline UNEMP & & & 0.0003 & 0.0230 & -0.0409 & -1.3534 & 0.0394 & 0.8275 & & \\
\hline MANU & 0.0022 & 1.2981 & & & & & 0.0009 & 0.6085 & & \\
\hline WHRT & 0.0278 & 5.1615 & & & & & -0.0013 & -0.2075 & & \\
\hline SCRM & & & & & & & & & 0.1081 & 3.4139 \\
\hline DFEG & & & & & & & & & 0.0540 & 0.7597 \\
\hline PCTAX & & & & & & & & & 0.1362 & 2.1619 \\
\hline PCPTAX & -0.0092 & -0.3691 & & & & & & & & \\
\hline PCTD & & & & & & & & & -0.0527 & -0.1661 \\
\hline LTD & & & & & & & & & 0.0073 & 1.0321 \\
\hline SCIX & & & & & & & 0.0783 & 3.7541 & & \\
\hline NAIX & 0.0051 & 0.4567 & -0.0010 & -0.4129 & -0.0026 & -0.3679 & & & & \\
\hline HWD & 0.0734 & 1.8183 & & & & & & & & \\
\hline ESBd & 0.0601 & 1.0300 & & & & & & & & \\
\hline EXTAX & & & -0.0009 & -0.1056 & 0.0462 & 1.9884 & & & & \\
\hline
\end{tabular}




\begin{tabular}{|l|c|c|c|c|c|c|c|c|c|c|}
\hline EMPt-1 & -0.1101 & -4.7024 & & & & & & & & \\
\hline INMGt-1 & & & -0.0506 & -3.3607 & & & & & & \\
\hline OTMGt-1 & & & & & -0.0845 & -2.9968 & & & & \\
\hline MHYt-1 & & & & & & & -0.8076 & -6.8048 & & \\
\hline DGEXt-1 & & & & & & & & & -0.4353 & -3.5435 \\
\hline RHO & 0.8746 & & -0.2589 & & 0.0507 & & -0.2177 & & -0.1531 & \\
\hline SIG & 0.0982 & & 0.0031 & & 0.0222 & & 0.0571 & & 0.1832 & \\
\hline AD.R2 & 0.2511 & & 0.9653 & & 0.5168 & & 0.4385 & & 0.0808 & \\
\hline F-STATISTIC* & 7.6389 & 0 & 659.015 & 0 & 28.6687 & 0 & 20.6384 & 0 & 4.773 & 0 \\
\hline NR ${ }^{2} \sim \chi^{*}$ & 58.7375 & 0.0170 & 43.1942 & 0.4201 & 28.9533 & 0.9703 & 41.1697 & 0.4192 & 46.8382 & 0.2457 \\
\hline N & 418 & & 418 & & 418 & & 418 & & 418 & \\
\hline
\end{tabular}

* Figures in the t-statistics columns are p-values 


\begin{tabular}{|c|c|c|c|c|c|c|c|c|c|c|}
\hline $\begin{array}{l}\text { Table 5.2d: } \\
\text { PANEL RA }\end{array}$ & $\begin{array}{l}\text { ienerali } \\
\Gamma E\end{array}$ & zed Spa & tial Two- & -Stage Le & east Squa & $\operatorname{ares}($ GS2 & SLS) Es & timatios & n Results & \\
\hline & EMPR E & Equation & INMGR E & Equation & OTMGR & Equation & MHYR E & Equation & DGEXR & Equation \\
\hline VARIABLE & Coeff. & $t$-stat. & Coeff. & $t$-stat. & Coeff. & t-stat. & Coeff. & t-stat. & Coeff. & $t$-stat. \\
\hline CONSTANT & 0.5890 & 0.6445 & -1.5231 & -5.6154 & 0.1184 & 0.1679 & 6.6826 & 4.4581 & 2.6584 & 4.7594 \\
\hline EMPR & & & 0.6289 & 8.3585 & 0.5169 & 10.2453 & 0.0089 & 0.1448 & -0.2908 & -3.3808 \\
\hline INMGR & -0.0155 & -0.4030 & & & 0.1399 & 4.2029 & 0.0442 & 1.1343 & -0.0320 & -0.5820 \\
\hline OTMGR & 0.3466 & 5.2308 & -0.0539 & -0.6102 & & & 0.2861 & 4.1420 & 0.4020 & 4.4813 \\
\hline MHYR & 0.1994 & 2.8293 & $\mid-0.2489$ & -2.6865 & $\mid-0.3804$ & -5.4480 & & & $\mid-0.0397$ & -0.4000 \\
\hline DGEXR & -0.1570 & -2.5404 & 0.0209 & 0.2814 & 0.0265 & 0.4601 & -0.1979 & -3.6993 & & \\
\hline WEMPR & -0.8953 & -5.5882 & 0.4222 & 2.3564 & 0.9091 & 6.4546 & 0.1521 & 1.1162 & 0.0482 & 0.2607 \\
\hline WINMGR & -0.0683 & -0.6375 & -0.0731 & -0.6605 & 0.0538 & 0.5802 & -0.0836 & -0.9964 & 0.1229 & 1.0670 \\
\hline WOTMGR & 0.6985 & 4.1767 & -0.0892 & -0.5026 & -0.9319 & -6.6035 & -0.2119 & -1.5648 & -0.2279 & -1.2753 \\
\hline WMHYR & 0.4054 & 2.3047 & 0.0819 & 0.4655 & -0.2222 & -1.4835 & -0.0551 & -0.4058 & 0.0898 & 0.4918 \\
\hline WDGEXR & 0.0841 & 0.7447 & -0.0381 & -0.3544 & 0.0305 & 0.3338 & 0.2181 & 2.6159 & 0.6416 & 6.5461 \\
\hline AREA & & & 0.0397 & 2.0085 & 0.0162 & 1.1383 & & & & \\
\hline POPs & & & 0.4007 & 12.3927 & 0.3119 & 9.3692 & -0.6065 & -2.1828 & -0.0768 & -4.0340 \\
\hline POPd & & & & & & & 0.0261 & 1.9803 & & \\
\hline POP5_17 & & & & & & & & & -0.0858 & -0.7484 \\
\hline POP25_44 & 0.3164 & 2.6841 & & & & & & & & \\
\hline FHHF & -0.1833 & -4.2137 & & & & & 0.1102 & 2.3232 & & \\
\hline POPHD & & & & & & & 0.3088 & 4.0701 & & \\
\hline POPCD & 0.1725 & 5.3497 & & & & & & & & \\
\hline OWHU & -0.1818 & -1.0200 & & & -0.1351 & -0.9040 & & & & \\
\hline MCRH & & & 0.1914 & 6.7315 & & & & & & \\
\hline UNEMP & & & -0.1806 & -4.5703 & -0.1453 & -5.1803 & 0.0301 & 0.9998 & & \\
\hline MANU & 0.0031 & 3.4724 & & & & & 0.0026 & 2.8918 & & \\
\hline WHRT & 0.0200 & 6.0336 & & & & & 0.0077 & 2.0307 & & \\
\hline SCRM & & & & & & & & & 0.8600 & 4.7238 \\
\hline DFEG & & & & & & & & & 0.0314 & 0.9943 \\
\hline PCTAX & & & & & & & & & 0.0259 & 0.8921 \\
\hline PCPTAX & -0.0726 & -4.7085 & & & & & & & & \\
\hline PCTD & & & & & & & & & -0.2005 & -1.0124 \\
\hline LTD & & & & & & & & & \begin{tabular}{|l|}
0.0092 \\
\end{tabular} & 2.0026 \\
\hline$S \operatorname{SCIX}$ & & & & & & & -0.0036 & -0.2554 & & \\
\hline NAIX & 0.0192 & 2.6515 & 0.0173 & 1.8418 & 0.0101 & 1.4507 & & & & \\
\hline HWD & 0.0607 & 2.4249 & & & & & & & & \\
\hline ESBd & -0.0731 & -2.3081 & & & & & & & & \\
\hline
\end{tabular}




\begin{tabular}{|l|c|c|c|c|c|c|c|c|c|c|}
\hline EXTAX & & & 0.0297 & 1.0641 & -0.0390 & -1.8028 & & & & \\
\hline EMPt-1 & -0.0704 & -6.0744 & & & & & & & & \\
\hline INMGt-1 & & & -0.5007 & -15.4215 & & & & & & \\
\hline OTMGt-1 & & & & & -0.3556 & -10.5033 & & & & \\
\hline MHYt-1 & & & & & & & -0.4659 & -8.7279 & & \\
\hline DGEXt-1 & & & & & & & & & -0.2798 & -5.7637 \\
\hline RHO & 0.5713 & & 0.0398 & & 0.3429 & & 0.0006 & & -0.3976 & \\
\hline SIGV & 0.0603 & & 0.0866 & & 0.0396 & & 0.0534 & & 0.1236 & \\
\hline SIG1 & 0.063 & & 0.0776 & & 0.0465 & & 0.0448 & & 0.1028 & \\
\hline AD.R2 & 0.3084 & & 0.2623 & & 0.3159 & & 0.1367 & & 0.1436 & \\
\hline F-STATISTIC* & 17.5037 & 0 & 23.4083 & 0 & 23.6739 & 0 & 11.2173 & 0 & 10.9343 & 0 \\
\hline NR ${ }^{2} \sim \chi^{2} *$ & 48.2656 & 0.1735 & 31.3535 & 0.6992 & 61.3559 & 0.0528 & 56.6950 & 0.4489 & 57.2199 & 0.2553 \\
\hline N & 836 & & 836 & & 836 & & 836 & & 836 & \\
\hline
\end{tabular}

* Figures in the t-statistics columns are p-values 


\begin{tabular}{|c|c|c|c|c|c|c|c|c|c|c|}
\hline \multicolumn{11}{|c|}{$\begin{array}{l}\text { Table 5.2c: Feasible Generalized Spatial Three-Stage Least Squares(FGS3SLS) Estimation Results, } \\
\text { 1980_RATE }\end{array}$} \\
\hline \multirow[b]{2}{*}{ VARIABLE } & \multicolumn{2}{|c|}{ EMPR Equation } & \multicolumn{2}{|c|}{ INMGR Equation } & \multicolumn{2}{|c|}{ OTMGR Equation } & \multicolumn{2}{|c|}{ MHYR Equation } & \multicolumn{2}{|c|}{ DGEXR Equation } \\
\hline & Coeff. & t-stat. & Coeff. & t-stat. & Coeff. & t-stat. & Coeff. & t-stat. & Coeff. & t-stat. \\
\hline CONSTANT & -2.2600 & -3.3699 & -3.1452 & -14.0277 & 3.3321 & 11.1833 & 3.0664 & 11.4549 & 1.3311 & 6.4576 \\
\hline EMPR & & & 0.5076 & 10.3055 & 0.3155 & 7.8973 & 0.2456 & 16.6563 & -0.0923 & -2.8995 \\
\hline INMGR & 0.0546 & 4.1894 & & & -0.0841 & -5.1982 & -0.0531 & -8.1012 & -0.0587 & -3.4533 \\
\hline OTMGR & 0.1492 & 4.1030 & -0.3332 & -8.9409 & & & -0.0573 & -4.9226 & 0.4419 & 16.2803 \\
\hline MHYR & 1.3857 & 14.2202 & 0.0901 & 0.6906 & -0.5464 & -6.0955 & & & 0.2229 & 2.4946 \\
\hline DGEXR & -0.0743 & -1.4392 & 0.1073 & 1.6665 & 0.4576 & 10.3267 & 0.1293 & 6.9647 & & \\
\hline WEMPR & -0.4762 & -4.4067 & -0.2162 & -2.1354 & -0.0933 & -1.2940 & -0.1469 & -5.8085 & -0.2177 & -4.0058 \\
\hline WINMGR & -0.0651 & -1.5048 & 0.3521 & 6.6787 & 0.1259 & 4.0245 & 0.0366 & 3.6539 & 0.0516 & 1.7758 \\
\hline WOTMGR & 0.1285 & 1.1980 & 0.6927 & 8.3287 & 0.6899 & 13.2999 & 0.0755 & 4.1709 & -0.3651 & -7.8898 \\
\hline WMHYR & 0.2933 & 1.4224 & 0.7947 & 5.5266 & 0.3973 & 3.3336 & 0.6254 & 16.5490 & -0.1168 & -0.9778 \\
\hline WDGEXR & 0.1824 & 1.2979 & -0.1358 & -1.1754 & -0.5755 & -7.3757 & -0.1284 & -4.0767 & 0.8019 & 14.6862 \\
\hline AREA & & & 0.0571 & 5.6826 & 0.1034 & 15.2853 & & & & \\
\hline POPs & & & 0.4905 & 19.2751 & 0.0902 & 12.4853 & -0.1422 & -3.4368 & -0.0142 & -2.1000 \\
\hline POPd & & & & & & & 0.0074 & 3.7590 & & \\
\hline POP5_17 & & & & & & & & & 0.0782 & 1.7740 \\
\hline POP25_44 & 0.4651 & 4.5540 & & & & & & & & \\
\hline FHHF & 0.0713 & 1.6084 & & & & & -0.0867 & -8.7937 & & \\
\hline POPHD & & & & & & & \begin{tabular}{|l|}
0.1424 \\
\end{tabular} & 10.6135 & & \\
\hline POPCD & 0.0951 & 3.9664 & & & & & & & & \\
\hline OWHU & 0.1125 & 0.8643 & & & -0.6153 & -10.2276 & & & & \\
\hline MCRH & & & 0.3886 & 12.8430 & & & & & & \\
\hline UNEMP & & & -0.1606 & -5.5838 & -0.1594 & -8.9913 & -0.0956 & -13.0322 & & \\
\hline MANU & -0.0010 & -1.1439 & & & & & 0.0012 & 6.6442 & & \\
\hline WHRT & 0.0156 & 6.0998 & & & & & -0.0010 & -1.3365 & & \\
\hline SCRM & & & & & & & & & 0.0334 & 6.1320 \\
\hline DFEG & & & & & & & & & -0.0076 & -0.9215 \\
\hline PCTAX & & & & & & & & & 0.0412 & 5.1950 \\
\hline PCPTAX & -0.0476 & -4.1386 & & & & & & & & \\
\hline PCTD & & & & & & & & & -0.0165 & -2.2930 \\
\hline LTD & & & & & & & & & 0.0022 & 0.5457 \\
\hline SCIX & & & & & & & -0.0119 & -4.0568 & & \\
\hline NAIX & -0.0023 & -0.4159 & 0.0041 & 0.7407 & 0.0089 & 2.6786 & & & & \\
\hline HWD & 0.0608 & 3.4528 & & & & & & & & \\
\hline ESBd & -0.0305 & -1.3399 & & & & & & & & \\
\hline EXTAX & & & -0.0436 & -2.3838 & 0.0608 & 5.7724 & & & & \\
\hline
\end{tabular}




\begin{tabular}{|l|c|c|c|c|c|c|c|c|c|c|}
\hline EMPt-1 & -0.0754 & -7.8831 & & & & & & & & \\
\hline INMGt-1 & & & -0.5795 & -21.6473 & & & & & & \\
\hline OTMGt-1 & & & & & -0.1647 & -18.0724 & & & & \\
\hline MHYt-1 & & & & & & & -0.2556 & -15.8678 & & \\
\hline DGEXt-1 & & & & & & & & & -0.2284 & -11.8194 \\
\hline RHO & 0.6782 & & -0.1103 & & -0.2831 & & -0.5283 & & -0.5276 & \\
\hline SIG & 0.0657 & & 0.053 & & 0.0293 & & 0.0056 & & 0.0311 & \\
\hline$R^{2}$ & 0.3164 & & 0.4771 & & 0.2739 & & 0.7384 & & 0.3402 & \\
\hline$N$ & 418 & & 418 & & 418 & & 418 & & 418 & \\
\hline
\end{tabular}


Table 5.2b: Feasible Generalized Spatial Three-Stage Least Squares(FGS3SLS) Estimation Results, 1990_RATE

\begin{tabular}{|c|c|c|c|c|c|c|c|c|c|c|}
\hline \multirow[b]{2}{*}{ VARIABLE } & \multicolumn{2}{|c|}{ EMPR Equation } & \multicolumn{2}{|c|}{ INMGR Equation } & \multicolumn{2}{|c|}{ OTMGR Equation } & \multicolumn{2}{|c|}{ MHYR Equation } & \multicolumn{2}{|c|}{ DGEXR Equation } \\
\hline & Coeff. & $t$-stat. & Coeff. & t-stat. & Coeff. & t-stat. & Coeff. & t-stat. & Coeff. & t-stat. \\
\hline CONSTANT & 0.3554 & 0.6458 & -0.5504 & -8.2310 & 0.3060 & 1.6230 & 9.1442 & 9.4644 & 1.9564 & 6.0960 \\
\hline EMPR & & & 0.0231 & 2.3540 & 0.2185 & 8.0177 & 0.1381 & 2.3693 & -0.3694 & -5.5825 \\
\hline INMGR & 1.8081 & 7.4377 & & & 0.7928 & 5.2962 & 2.4913 & 9.8457 & -3.2780 & -9.3664 \\
\hline OTMGR & 0.8531 & 13.9655 & 0.0783 & 6.9164 & & & -0.3149 & -5.2680 & 1.9179 & 38.5012 \\
\hline MHYR & 0.0187 & 0.4811 & 0.0405 & 5.2199 & -0.1483 & -6.6888 & & & 0.3250 & 5.7961 \\
\hline DGEXR & -0.2586 & -11.5342 & -0.0323 & -7.5391 & 0.2440 & 24.1110 & 0.0375 & 1.7309 & & \\
\hline WEMPR & -1.0443 & -9.1809 & 0.0346 & 2.4543 & 0.0215 & 0.4436 & 0.0333 & 0.3569 & 0.1144 & 1.0103 \\
\hline WINMGR & -2.7225 & -8.7347 & 1.0353 & 56.1100 & -0.2817 & -1.5762 & -2.6358 & -9.1565 & 2.5812 & 6.3069 \\
\hline WOTMGR & 1.3404 & 6.8296 & -0.0323 & -1.1572 & -0.0253 & -0.2911 & 0.2647 & 1.7087 & -0.5485 & -2.6833 \\
\hline WMHYR & 0.0151 & 0.1870 & -0.0064 & -0.6723 & -0.0703 & -1.9629 & 0.2501 & 4.7520 & -0.2141 & -2.7541 \\
\hline WDGEXR & -0.3825 & -5.9274 & 0.0014 & 0.1812 & -0.0519 & -1.8335 & 0.0490 & 0.9588 & 0.1994 & 2.7722 \\
\hline AREA & & & 0.0112 & 5.7960 & 0.0153 & 3.8789 & & & & \\
\hline POPs & & & 0.0339 & 7.4221 & 0.0572 & 7.4926 & -0.0896 & -0.5674 & -0.0506 & -3.9516 \\
\hline POPd & & & & & & & 0.0037 & 0.5011 & & \\
\hline POP5_17 & & & & & & & & & -0.0901 & -1.7541 \\
\hline POP25_44 & 0.4502 & 5.4068 & & & & & & & & \\
\hline FHHF & 0.0917 & 2.5126 & & & & & -0.0039 & -0.1285 & & \\
\hline POPHD & & & & & & & -0.1899 & -2.5590 & & \\
\hline POPCD & 0.0916 & 3.8414 & & & & & & & & \\
\hline OWHU & -0.3220 & -3.0119 & & & 0.0106 & 0.2609 & & & & \\
\hline MCRH & & & 0.0693 & 6.9536 & & & & & & \\
\hline UNEMP & & & -0.0012 & -0.3072 & -0.0173 & -2.2448 & 0.0424 & 1.8470 & & \\
\hline MANU & 0.0024 & 3.9592 & & & & & 0.0009 & 1.1803 & & \\
\hline WHRT & 0.0266 & 13.2749 & & & & & -0.0032 & -1.0561 & & \\
\hline SCRM & & & & & & & & & 0.0052 & 5.7436 \\
\hline DFEG & & & & & & & & & 0.0644 & 4.3962 \\
\hline PCTAX & & & & & & & & & 0.1151 & 7.3746 \\
\hline PCPTAX & -0.0232 & -2.3065 & & & & & & & & \\
\hline PCTD & & & & & & & & & -0.0133 & -1.8646 \\
\hline LTD & & & & & & & & & 0.0683 & 4.6108 \\
\hline ScIX & & & & & & & 0.0738 & 7.1384 & & \\
\hline NAIX & 0.0084 & 1.7118 & 0.0011 & 1.2200 & -0.0021 & -1.2050 & & & & \\
\hline HWD & 0.0775 & 4.8122 & & & & & & & & \\
\hline ESBd & 0.0702 & 3.0330 & & & & & & & & \\
\hline EXTAX & & & 0.0026 & 0.8342 & 0.0241 & 3.5259 & & & & \\
\hline
\end{tabular}




\begin{tabular}{|l|c|c|c|c|c|c|c|c|c|c|}
\hline EMPt-1 & -0.1165 & -11.1343 & & & & & & & & \\
\hline INMGt-1 & & & -0.0382 & -7.8679 & & & & & & \\
\hline OTMGt-1 & & & & & -0.0726 & -8.8184 & & & & \\
\hline MHYt-1 & & & & & & & -0.7604 & -13.2320 & & \\
\hline DGEXt-1 & & & & & & & & & -0.2843 & -9.7675 \\
\hline RHO & 0.8746 & & -0.2589 & & 0.0507 & & -0.2177 & & -0.1531 & \\
\hline SIG & 0.0982 & & 0.0031 & & 0.0222 & & 0.0571 & & 0.1832 & \\
\hline$R^{2}$ & 0.3033 & & 0.965 & & 0.4645 & & 0.3987 & & 0.086 & \\
\hline$N$ & 418 & & 418 & & 418 & & 418 & & 418 & \\
\hline
\end{tabular}




\begin{tabular}{|c|c|c|c|c|c|c|c|c|c|c|}
\hline \multirow[b]{2}{*}{ VARIABLE } & \multicolumn{2}{|c|}{ EMPR Equation } & \multicolumn{2}{|c|}{ INMGR Equation } & \multicolumn{2}{|c|}{ OTMGR Equation } & \multicolumn{2}{|c|}{ MHYR Equation } & \multicolumn{2}{|c|}{ DGEXR Equation } \\
\hline & Coeff. & t-stat. & Coeff. & t-stat. & Coeff. & t-stat. & Coeff. & t-stat. & Coeff. & t-stat. \\
\hline CONSTANT & -0.7211 & -1.4514 & -0.3951 & -1.6652 & 1.9110 & 7.3054 & 5.2007 & 6.7117 & 1.2586 & 5.1725 \\
\hline EMPR & & & 1.1016 & 23.1230 & 0.5377 & 19.0894 & 0.1310 & 4.5632 & -0.0031 & -0.1148 \\
\hline INMGR & 0.0641 & 4.0017 & & & -0.0264 & -2.0152 & 0.0169 & 1.4344 & -0.0594 & -4.9518 \\
\hline OTMGR & 0.3717 & 8.6826 & -0.5873 & -13.8519 & & & 0.2131 & 6.1175 & 0.0613 & 2.2006 \\
\hline MHYR & 0.2127 & 5.6273 & -0.5129 & -8.3058 & -0.3917 & -11.2243 & & & -0.2373 & -7.6835 \\
\hline DGEXR & 0.2897 & 6.4475 & -0.5322 & -8.6811 & -0.3497 & -9.1948 & -0.3711 & -10.1317 & & \\
\hline WEMPR & -0.6500 & -8.6300 & 0.3043 & 2.4562 & 0.2703 & 3.4283 & -0.0058 & -0.0818 & -0.0441 & -0.6429 \\
\hline WINMGR & -0.0411 & -0.7687 & 0.0225 & 0.3250 & 0.0010 & 0.0245 & -0.0434 & -1.3105 & 0.0554 & 1.6872 \\
\hline WOTMGR & 0.4872 & 4.9039 & 0.1952 & 1.4483 & 0.0469 & 0.5525 & 0.0909 & 1.3352 & -0.0792 & -1.1835 \\
\hline WMHYR & -0.1368 & -1.3878 & -0.0576 & -0.4397 & -0.2318 & -2.6638 & 0.2394 & 3.5123 & 0.0527 & 0.9063 \\
\hline WDGEXR & 0.1875 & 1.8502 & 0.2020 & 1.1995 & 0.2256 & 2.2746 & 0.1866 & 2.4472 & 0.4216 & 5.5303 \\
\hline AREA & & & -0.0369 & -1.5766 & -0.0041 & -0.2604 & & & & \\
\hline POPs & & & 0.5519 & 20.3534 & 0.2187 & 18.4429 & -0.1567 & -1.0471 & 0.0098 & 0.9167 \\
\hline POPd & & & & & & & 0.0064 & 0.8912 & & \\
\hline POP5_17 & & & & & & & & & 0.1267 & 2.7955 \\
\hline POP25_44 & 0.2694 & 3.8239 & & & & & & & & \\
\hline FHHF & -0.0992 & -4.1690 & & & & & -0.0236 & -1.0391 & & \\
\hline POPHD & & & & & & & 0.3128 & 7.5692 & & \\
\hline POPCD & 0.1754 & 7.9801 & & & & & & & & \\
\hline OWHU & 0.0578 & 0.5831 & & & -0.0929 & -1.6064 & & & & \\
\hline MCRH & & & 0.1141 & 8.1934 & & & & & & \\
\hline UNEMP & & & -0.3036 & -9.3346 & -0.1679 & -8.1801 & -0.0026 & -0.1692 & & \\
\hline MANU & 0.0032 & 5.4736 & & & & & 0.0023 & 5.1125 & & \\
\hline WHRT & 0.0181 & 7.3968 & & & & & -0.0007 & -0.3755 & & \\
\hline SCRM & & & & & & & & & 0.0410 & 0.4946 \\
\hline DFEG & & & & & & & & & 0.0529 & 3.9894 \\
\hline PCTAX & & & & & & & & & 0.0486 & 4.6624 \\
\hline РСРТAX & -0.0051 & -0.6112 & & & & & & & & \\
\hline PCTD & & & & & & & & & -0.0001 & -4.6535 \\
\hline LTD & & & & & & & & & 0.0017 & 4.8203 \\
\hline ScIX & & & & & & & -0.0099 & -1.3853 & & \\
\hline NAIX & 0.0169 & 3.0763 & 0.0192 & 2.3163 & 0.0084 & 1.7953 & & & & \\
\hline HWD & 0.1808 & 6.5349 & & & & & & & & \\
\hline ESBd & -0.1162 & -4.7651 & & & & & & & & \\
\hline EXTAX & & & 0.0768 & 3.1002 & 0.0226 & 1.4816 & & & & \\
\hline
\end{tabular}




\begin{tabular}{|l|c|c|c|c|c|c|c|c|c|c|}
\hline EMPt-1 & -0.0873 & -9.2827 & & & & & & & & \\
\hline INMGt-1 & & & -0.6774 & -23.8148 & & & & & & \\
\hline OTMGt-1 & & & & & -0.2836 & -22.4302 & & & & \\
\hline MHYt-1 & & & & & & & -0.5228 & -18.9127 & & \\
\hline DGEXt-1 & & & & & & & & & -0.2771 & -13.3590 \\
\hline RHO & 0.5713 & & 0.0398 & & 0.3429 & & 0.0006 & & -0.3976 & \\
\hline SIGV & 0.0603 & & 0.0866 & & 0.0396 & & 0.0534 & & 0.1236 & \\
\hline SIG1 & 0.063 & & 0.0776 & & 0.0465 & & 0.0448 & & 0.1028 & \\
\hline$R^{2}$ & 0.2659 & & 0.1225 & & 0.1886 & & 0.1654 & & 0.1176 & \\
\hline$N$ & 836 & & 836 & & 836 & & 836 & & 836 & \\
\hline
\end{tabular}




\begin{tabular}{|l|c|c|c|c|c|c|c|c|c|c|c|}
\hline Table c1: Generalized Spatial Two-Stage Least Squares(GS2SLS) Estimation Results: \\
\hline APPALACHIAN STATES COUNTIES, 1980_LEVEL \\
\hline \\
\hline
\end{tabular}




\begin{tabular}{|l|c|c|c|c|c|c|c|c|c|c|}
\hline EXTAX & & & 0.0458 & 3.1160 & -0.0445 & -4.0813 & & & & \\
\hline EMPt-1 & 0.6641 & 22.5431 & & & & & & & & \\
\hline INMGt-1 & & & 0.0965 & 4.1965 & & & & & & \\
\hline OTMGt-1 & & & & & 0.2338 & 9.4888 & & & & \\
\hline MHYt-1 & & & & & & & 0.5702 & 38.7790 & & \\
\hline DGEXt-1 & & & & & & & & & 0.3917 & 8.2492 \\
\hline RHO & 0.9156 & & -0.5453 & & -0.2268 & & -0.0444 & -0.0262 & \\
\hline SIG & 0.6635 & & 0.01737 & & 0.0129 & & 0.007 & & 0.0424 & \\
\hline AD.R2 & 0.972 & & 0.9919 & & 0.9918 & & 0.9088 & & 0.6348 & \\
\hline F-STATISTIC* & 1900.13 & 0 & 8304.67 & 0 & 8303.46 & 0 & 610.392 & 0 & 116.628 & 0 \\
\hline N & 1096 & & 1096 & & 1096 & & 1096 & & 1096 & \\
\hline ETA (n) & 0.3359 & & 0.9035 & & 0.7662 & & 0.4298 & & 0.6083 & \\
\hline
\end{tabular}

* Figures in the t-statistics columns are p-values 


\begin{tabular}{|c|c|c|c|c|c|c|c|c|c|c|}
\hline \multirow{2}{*}{\multicolumn{11}{|c|}{\begin{tabular}{|l} 
Table c2: Generalized Spatial Two-Stage Least Squares(C \\
APPALACHIAN STATES COUNTIES, 1990_LEVEL \\
\end{tabular}}} \\
\hline & & & & & & & & & & \\
\hline \multirow{2}{*}{\begin{tabular}{|l|} 
VARIABLE \\
\end{tabular}} & \multicolumn{2}{|c|}{ EMPR Equation } & \multicolumn{2}{|c|}{ INMGR Equation } & \multicolumn{2}{|c|}{ OTMGR Equation } & \multicolumn{2}{|c|}{ MHYR Equation } & \multicolumn{2}{|c|}{ DGEXR Equation } \\
\hline & Coeff. & $t$-stat. & Coeff. & t-stat. & Coeff. & t-stat. & Coeff. & $t$-stat. & Coeff. & t-stat. \\
\hline CONSTANT & $|-0.6619|$ & -0.2987 & -0.7798 & -0.8436 & 1.3761 & 1.9775 & 2.2498 & 7.3742 & 1.6602 & 0.9753 \\
\hline EMPt & & & 0.0204 & 1.2656 & -0.0086 & -0.7625 & -0.0062 & -1.0860 & 0.1603 & 4.1311 \\
\hline INMGt & 0.0990 & 0.8532 & & & 0.6106 & 31.4750 & -0.0042 & -0.2335 & $|-0.6669|$ & -4.8145 \\
\hline OTMGt & 0.1906 & 1.4581 & 1.0475 & 20.0901 & & & 0.0353 & 1.6001 & 0.5245 & 2.8309 \\
\hline MHYt & -0.0398 & -0.4615 & 0.3165 & 4.6583 & -0.1189 & -2.7011 & & & 0.2149 & 1.8441 \\
\hline DGEXt & 0.0878 & 2.1673 & -0.1559 & -6.8203 & 0.0548 & 3.2181 & -0.0174 & -2.3489 & & \\
\hline WEMPt & -0.1850 & -3.0401 & 0.0195 & 0.7608 & 0.0433 & 2.1669 & -0.0080 & -1.0174 & \begin{tabular}{|l|}
-0.1008 \\
\end{tabular} & -1.7418 \\
\hline WINMGt & -0.3574 & -1.3396 & 0.2634 & 2.2411 & -0.1478 & -1.6579 & -0.0110 & -0.3005 & 0.5024 & 1.9408 \\
\hline WOTMGt & 0.5676 & 2.0634 & -0.2919 & -2.4485 & 0.2288 & 2.4791 & 0.0109 & 0.2931 & -0.3646 & -1.3285 \\
\hline WMHYt & -0.0367 & -0.1866 & -0.1109 & -1.2976 & -0.0305 & -0.4751 & -0.0051 & -0.1946 & \begin{tabular}{|l|}
-0.3448 \\
\end{tabular} & 1.8880 \\
\hline WDGEXt & 0.0463 & 0.4448 & 0.0973 & 2.1486 & -0.0530 & -1.5549 & 0.0082 & 0.5928 & 0.4922 & 5.5872 \\
\hline AREA & & & 0.0348 & 4.5215 & -0.0177 & -3.0518 & & & & \\
\hline POPs & & & -0.1880 & -6.6582 & 0.1299 & 7.0269 & 0.1597 & 4.0316 & -0.1092 & -1.3904 \\
\hline POPd & & & & & & & -0.0082 & -4.8527 & & \\
\hline POP5_17 & & & & & & & & & $-0.0523 \mid$ & -0.4915 \\
\hline POP25_44 & 0.0918 & 0.7763 & & & & & & & & \\
\hline FHHF & -0.0709 & -1.9137 & & & & & -0.0882 & -11.4588 & & \\
\hline POPHD & & & & & & & 0.0222 & 1.0667 & & \\
\hline POPCD & 0.0949 & 3.1942 & & & & & & & & \\
\hline OWHU & 0.0941 & 1.1801 & & & 0.0145 & 0.2933 & & & & \\
\hline MCRH & & & -0.0195 & -0.3899 & & & & & & \\
\hline UNEMP & & & 0.0128 & 0.6671 & -0.0457 & -3.4077 & -0.0192 & -3.5726 & & \\
\hline MANU & 0.0025 & 2.2925 & & & & & -0.0005 & -2.5132 & & \\
\hline WHRT & 0.0141 & 5.1343 & & & & & -0.0007 & -1.1277 & & \\
\hline SCRM & & & & & & & & & -0.1020 & -0.6258 \\
\hline DFEG & & & & & & & & & 0.0110 & 0.3557 \\
\hline PCTAX & & & & & & & & & 0.0994 & 2.5349 \\
\hline PCPTAX & -0.0592 & -3.5169 & & & & & & & & \\
\hline PCTD & & & & & & & & & 0.1545 & 1.2505 \\
\hline LTD & & & & & & & & & 0.0006 & 1.7017 \\
\hline SCIX & & & & & & & 0.0055 & 2.6278 & & \\
\hline NAIX & 0.0036 & 0.5902 & 0.0308 & 6.9449 & -0.0162 & -4.9356 & & & & \\
\hline HWD & -0.0028 & -0.1535 & & & & & & & & \\
\hline ESBd & 0.0654 & 1.9430 & & & & & & & & \\
\hline
\end{tabular}




\begin{tabular}{|l|c|c|c|c|c|c|c|c|c|c|}
\hline EXTAX & & & 0.0451 & 2.4576 & -0.0358 & -2.6455 & & & & \\
\hline EMPt-1 & 0.7432 & 31.0737 & & & & & & & & \\
\hline INMGt-1 & & & 0.0561 & 1.2567 & & & & & & \\
\hline OTMGt-1 & & & & & 0.2938 & 13.7298 & & & & \\
\hline MHYt-1 & & & & & & & 0.7508 & 60.0481 & & \\
\hline DGEXt-1 & & & & & & & & & 0.4924 & 6.9231 \\
\hline RHO & 0.9047 & & -0.1419 & & -0.0628 & & 0.0899 & & -0.5956 & \\
\hline SIG & 1.0844 & & 0.0261 & & 0.0141 & & 0.0021 & & 0.1383 & \\
\hline AD.R2 & 0.9797 & & 0.982 & & 0.9901 & & 9630 & & 0.4804 & \\
\hline F-STATISTIC* & 2638.89 & 0 & 3654.89 & 0 & 6719.92 & 0 & 1589.79 & 0 & 56.7181 & 0 \\
\hline N & 1096 & & 1096 & & 1096 & & 1096 & & 1096 & \\
\hline ETA $(\eta)$ & 0.2568 & & 0.9439 & & 0.7062 & & 0.2492 & & 0.5076 & \\
\hline
\end{tabular}

* Figures in the t-statistics columns are p-values 


\begin{tabular}{|c|c|c|c|c|c|c|c|c|c|c|}
\hline APPALACE & HIAN ST & ATES C & OUNTIF & $S, 1980$ & LEVEL & & 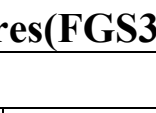 & th & & its \\
\hline & EMP Ec & quation & INMG E & quation & OTMG E & Equation & MHY Ec & quation & DGEX E & quation \\
\hline VARIABLE & Coeff. & $t$-stat. & Coeff. & $t$-stat. & Coeff. & t-stat. & Coeff. & t-stat. & Coeff. & t-stat. \\
\hline CONSTANT & 0.8912 & 1.0562 & 1.4198 & 2.2837 & -0.9352 & -2.0546 & 4.6028 & 7.9462 & 6.1495 & 4.0649 \\
\hline EMPt & & & 0.0386 & 1.9534 & -0.0050 & \begin{tabular}{|l|}
-0.3439 \\
\end{tabular} & 0.0006 & 0.0591 & 0.0976 & 2.6807 \\
\hline INMGt & 1.7993 & 5.1679 & & & 0.6891 & 33.4886 & 0.2127 & 5.5375 & -0.6413 & -4.8556 \\
\hline OTMGt & -1.3422 & -3.5921 & 1.1505 & 33.1729 & & & -0.1182 & -2.4923 & 0.4833 & 2.4460 \\
\hline MHYt & -0.9444 & -4.1957 & 0.1881 & 3.4520 & 0.0306 & 0.9581 & & & 0.0854 & 0.7467 \\
\hline DGEXt & 0.1322 & 0.9867 & -0.2441 & -8.7300 & 0.0827 & 3.6414 & 0.0687 & 4.4136 & & \\
\hline WEMPt & -0.0332 & -0.2508 & 0.0591 & 1.9658 & -0.0709 & -3.3588 & $\mid-0.0084$ & -0.6003 & -0.1176 & -1.9592 \\
\hline WINMGt & 2.9029 & 3.9196 & 0.5466 & 6.7808 & -0.2775 & -4.4247 & \begin{tabular}{|l|}
-0.0902 \\
\end{tabular} & -2.0521 & 0.7064 & 4.1954 \\
\hline WOTMGt & -2.1899 & -2.9090 & -0.5956 & -6.3462 & 0.3566 & 5.0623 & 0.0801 & 1.6335 & -0.5158 & -2.6599 \\
\hline WMHYt & -1.5843 & -3.3334 & -0.2008 & -3.1298 & 0.0411 & 0.8722 & 0.0910 & 2.9253 & -0.4413 & -3.6044 \\
\hline WDGEXX & 0.5472 & 1.8231 & 0.1643 & 3.6568 & -0.0431 & -1.3076 & -0.0451 & -2.1047 & 0.4528 & 5.6240 \\
\hline AREA & & & 0.0192 & 2.1951 & -0.0053 & -0.7745 & & & & \\
\hline POPs & & & -0.2737 & -7.8678 & 0.2136 & 9.3522 & -0.3127 & -3.4892 & -0.0780 & -0.8881 \\
\hline POPd & & & & & & & 0.0117 & 2.9566 & & \\
\hline POP5_17 & & & & & & & & & -0.1271 & -1.2016 \\
\hline POP25_44 & 0.5610 & 4.6996 & & & & & & & & \\
\hline FHHF & \begin{tabular}{|l|}
-0.0501 \\
\end{tabular} & -1.1125 & & & & & -0.0733 & -4.1209 & & \\
\hline POPHD & & & & & & & 0.1725 & 7.1751 & & \\
\hline POPCD & 0.1388 & 3.9041 & & & & & & & & \\
\hline OWHU & -0.2352 & -1.3095 & & & -0.1472 & -2.3864 & & & & \\
\hline MCRH & & & 0.0114 & 0.6424 & & & & & & \\
\hline UNEMP & & & 0.0431 & 1.9523 & -0.0336 & -2.0866 & \begin{tabular}{|l|}
-0.0513 \\
\end{tabular} & -4.7474 & & \\
\hline MANU & 0.0028 & 2.5016 & & & & & \begin{tabular}{|l|}
0.0017 \\
\end{tabular} & 5.1695 & & \\
\hline WHRT & 0.0142 & 4.0309 & & & & & 0.0005 & 0.4157 & & \\
\hline SCRM & & & & & & & & & 0.1030 & 4.4175 \\
\hline DFEG & & & & & & & & & -0.0467 & -1.9216 \\
\hline PCTAX & & & & & & & & & 0.0941 & 3.2213 \\
\hline PCPTAX & -0.0229 & -1.4616 & & & & & & & & \\
\hline PCTD & & & & & & & & & 0.4038 & 2.4088 \\
\hline LTD & & & & & & & & & -0.0089 & -2.3428 \\
\hline SCIX & & & & & & & -0.0148 & -3.3586 & & \\
\hline NAIX & 0.0131 & 1.7974 & 0.0204 & 4.1553 & -0.0137 & -3.5923 & & & & \\
\hline HWD & 0.0240 & 0.9956 & & & & & & & & \\
\hline ESBd & 0.0340 & 0.8798 & & & & & & & & \\
\hline EXTAX & & & 0.0344 & 2.5478 & -0.0463 & -4.5695 & & & & \\
\hline
\end{tabular}




\begin{tabular}{|l|c|c|c|c|c|c|c|c|c|c|}
\hline EMPt-1 & 0.8485 & 42.2464 & & & & & & & & \\
\hline INMGt-1 & & & 0.0341 & 2.3024 & & & & & & \\
\hline OTMGt-1 & & & & & 0.1133 & 6.7858 & & & & \\
\hline MHYt-1 & & & & & & & 0.5530 & 38.4415 & & \\
\hline DGEXt-1 & & & & & & & & & 0.3177 & 7.3933 \\
\hline RHO & 0.9156 & & -0.5453 & & -0.2268 & & -0.0444 & & -0.0262 & \\
\hline SIG & 0.6635 & & 0.01737 & & 0.0129 & & 0.007 & & 0.0424 & \\
\hline $\mathrm{R}^{2}$ & 0.9714 & & 0.992 & & 0.9902 & & 0.8924 & & 0.6332 & \\
\hline $\mathrm{N}$ & 1096 & & 1096 & & 1096 & & 1096 & & 1096 & \\
\hline ETA $(\mathrm{n})$ & 0.1515 & & 0.9659 & & 0.8867 & & 0.4470 & & 0.6823 & \\
\hline
\end{tabular}




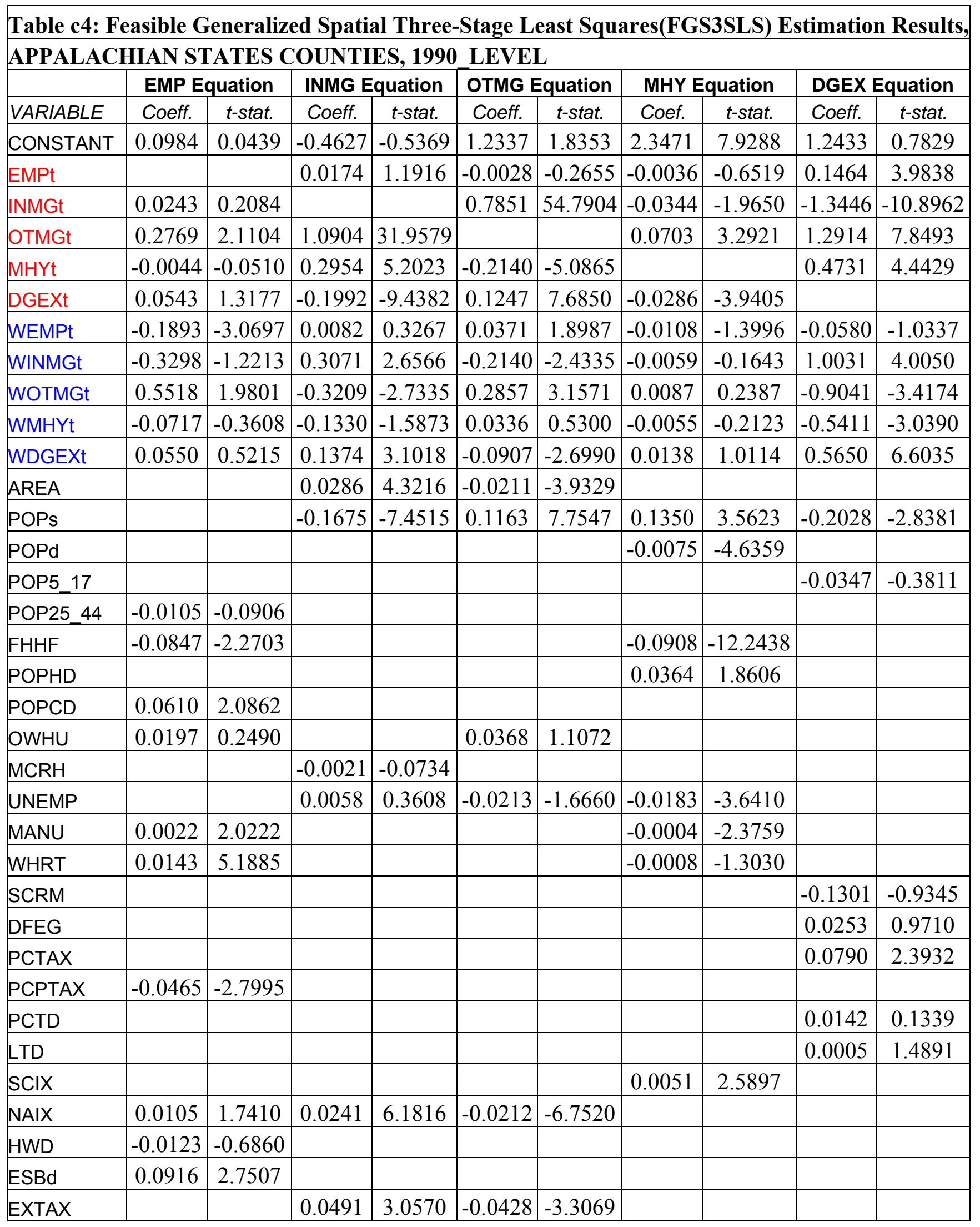




\begin{tabular}{|l|c|c|c|c|c|c|c|c|c|c|}
\hline EMPt-1 & 0.7358 & 30.6186 & & & & & & & & \\
\hline INMGt-1 & & & 0.0003 & 0.0121 & & & & & & \\
\hline OTMGt-1 & & & & & 0.1424 & 10.1943 & & & & \\
\hline MHYt-1 & & & & & & & 0.7527 & 62.3141 & & \\
\hline DGEXt-1 & & & & & & & & & 0.4319 & 6.8978 \\
\hline RHO & 0.9047 & & -0.1419 & & -0.0628 & & 0.0899 & & -0.5956 & \\
\hline SIG & 1.0844 & & 0.0261 & & 0.0141 & & 0.0021 & & 0.1383 & \\
\hline $\mathrm{R}^{2}$ & 0.9882 & & 0.9805 & & 0.9879 & & 0.9615 & & 0.4268 & \\
\hline $\mathrm{N}$ & 1096 & & 1096 & & 1096 & & 1096 & & 1096 & \\
\hline ETA $(\mathrm{n})$ & 0.2642 & & 0.9997 & & 0.8576 & & 0.2473 & & 0.5681 & \\
\hline
\end{tabular}




\begin{tabular}{|c|c|c|c|c|c|c|c|c|c|c|}
\hline \multicolumn{11}{|c|}{ APPALACHIAN STATES COUNTIES, 1980 RATE } \\
\hline \multirow[b]{2}{*}{ VARIABLE } & \multicolumn{10}{|c|}{ EMPR Equation|INMGR Equation OTMGR Equation|MHYR Equation|DGEXR Equation } \\
\hline & Coeff. & t-stat. & Coeff. & t-stat. & Coeff. & t-stat. & Coeff. & t-stat. & Coeff. & t-stat. \\
\hline CONSTANT & -2.2218 & -4.1786 & -0.6962 & -5.1962 & 1.4579 & 4.0263 & 2.0978 & 5.2192 & 2.5472 & 8.2873 \\
\hline EMPR & & & 0.1749 & 5.3504 & 0.2732 & 5.1964 & 0.2779 & 12.9137 & -0.0451 & -0.8106 \\
\hline INMGR & 0.4085 & 2.7747 & & & -0.8430 & -10.8736 & -0.2555 & -5.8895 & 0.0249 & 0.2281 \\
\hline OTMGR & 0.3041 & 4.1076 & -0.3476 & -11.2212 & & & -0.0586 & -2.0162 & 0.1602 & 2.5105 \\
\hline MHYR & 1.2787 & 9.7357 & -0.1449 & -1.6681 & -0.3454 & -2.4929 & & & -0.0717 & -0.5114 \\
\hline DGEXR & 0.0177 & 0.2534 & 0.0080 & 0.2370 & 0.0663 & 1.2349 & 0.0565 & 2.1662 & & \\
\hline WEMPR & -0.1967 & -1.2917 & -0.1291 & -2.2400 & -0.1305 & -1.5241 & -0.1792 & -4.4086 & 0.0623 & 0.6241 \\
\hline WINMGR & -0.4796 & -2.8624 & 0.9789 & 53.7869 & 0.9467 & 11.0808 & 0.2802 & 5.8234 & -0.0344 & -0.2824 \\
\hline WOTMGR & -0.1408 & -1.0126 & 0.2559 & 5.0013 & 0.7131 & 11.2305 & 0.0288 & 0.7353 & -0.2489 & -2.7818 \\
\hline WMHYR & 0.3510 & 1.1214 & 0.2915 & 2.5199 & 0.2829 & 1.6138 & 0.6302 & 8.3272 & 0.3212 & 1.5826 \\
\hline WDGEXR & 0.2183 & 1.3007 & -0.0122 & -0.2019 & -0.1469 & -1.6650 & -0.0316 & -0.7019 & 0.2690 & 2.6036 \\
\hline AREA & & & 0.0107 & 1.9078 & 0.0496 & 5.5145 & & & & \\
\hline POPs & & & 0.0975 & 6.7996 & 0.0515 & 4.0612 & -0.2404 & -3.7829 & -0.0264 & -2.7465 \\
\hline POPd & & & & & & & 0.0111 & 3.7960 & & \\
\hline POP5_17 & & & & & & & & & -0.0208 & -0.3213 \\
\hline POP25_44 & 0.2965 & 2.8768 & & & & & & & & \\
\hline FHHF & -0.0598 & -1.4966 & & & & & 0.0013 & 0.0850 & & \\
\hline POPHD & & & & & & & 0.1180 & 4.6077 & & \\
\hline POPCD & 0.1132 & 3.8970 & & & & & & & & \\
\hline OWHU & 0.2477 & 3.2233 & & & -0.2882 & -3.9827 & & & & \\
\hline $\mathrm{MCRH}$ & & & 0.1157 & 5.1181 & & & & & & \\
\hline UNEMP & & & -0.0569 & -3.8629 & -0.0627 & -2.7445 & -0.0845 & -7.9623 & & \\
\hline MANU & -0.0020 & -2.1130 & & & & & 0.0014 & 4.1323 & & \\
\hline WHRT & 0.0123 & 4.1462 & & & & & -0.0004 & -0.3645 & & \\
\hline SCRM & & & & & & & & & 0.4626 & 6.0413 \\
\hline DFEG & & & & & & & & & -0.0097 & -0.7006 \\
\hline PCTAX & & & & & & & & & 0.0854 & 4.9739 \\
\hline PCPTAX & -0.0565 & -3.6830 & & & & & & & & \\
\hline PCTD & & & & & & & & & -0.2972 & -2.2536 \\
\hline LTD & & & & & & & & & 0.0002 & 0.3262 \\
\hline ScIX & & & & & & & -0.0079 & -2.0627 & & \\
\hline NAIX & -0.0014 & -0.2155 & 0.0028 & 0.8168 & 0.0019 & 0.3533 & & & & \\
\hline HWD & 0.0899 & 4.4299 & & & & & & & & \\
\hline ESBd & -0.0941 & -3.3455 & & & & & & & & \\
\hline EXTAX & & & -0.0347 & -3.1563 & 0.0373 & 2.4989 & & & & \\
\hline
\end{tabular}




\begin{tabular}{|l|c|c|c|c|c|c|c|c|c|c|}
\hline EMPt-1 & -0.0411 & -4.0029 & & & & & & & & \\
\hline INMGt-1 & & & -0.1175 & -7.8749 & & & & & & \\
\hline OTMGt-1 & & & & & -0.0751 & -5.5674 & & & & \\
\hline MHYt-1 & & & & & & & -0.1047 & -4.1460 & & \\
\hline DGEXt-1 & & & & & & & & & $-0.3546-11.5055$ \\
\hline RHO & 0.6123 & & -0.0754 & & -0.3218 & & -0.3034 & & -0.0863 & \\
\hline SIG & 0.0746 & & 0.0154 & & 0.0371 & & 0.0096 & & 0.0438 & \\
\hline AD.R2 & 0.337 & & 0.9478 & & 0.3964 & & 0.4417 & & 0.1718 & \\
\hline F-STATISTIC* & 28.6056 & 0.000 & 1225.66 & 0.000 & 58.904 & 0.000 & 53.6867 & 0.000 & 17.7133 & 0.000 \\
\hline N & 1096 & & 1096 & & 1096 & & 1096 & & 1096 & \\
\hline
\end{tabular}

* Figures in the t-statistics columns are p-values 


\begin{tabular}{|c|c|c|c|c|c|c|c|c|c|c|}
\hline \multicolumn{11}{|c|}{ APPALACHIAN STATES COUNTIES, 1990_RATE } \\
\hline \multirow[b]{2}{*}{ VARIABLE } & \multicolumn{2}{|c|}{ EMPR Equation } & \multicolumn{4}{|c|}{ INMGR Equation OTMGR Equation } & \multicolumn{2}{|c|}{ MHYR Equation } & \multicolumn{2}{|c|}{ DGEXR Equation } \\
\hline & Coeff. & t-stat. & Coeff. & t-stat. & Coeff. & t-stat. & Coeff. & t-stat. & Coeff. & t-stat. \\
\hline CONSTANT & 0.4005 & 0.5389 & -0.1186 & -0.4424 & 0.6374 & 1.9661 & 9.3710 & 9.0076 & 3.4308 & 4.7695 \\
\hline EMPR & & & 0.2261 & 5.1193 & 0.1852 & 4.8688 & -0.1310 & -1.9737 & -0.1833 & -1.5766 \\
\hline INMGR & 0.2448 & 2.5759 & & & 0.6636 & 13.6563 & 0.0117 & 0.1264 & -0.4389 & -2.6385 \\
\hline OTMGR & 0.2996 & 3.8390 & 0.6183 & 15.6950 & & & 0.0704 & 0.8938 & 0.3863 & 2.5646 \\
\hline MHYR & -0.1309 & -2.1803 & -0.1060 & -2.2226 & 0.0255 & 0.6387 & & & 0.0330 & 0.3369 \\
\hline DGEXR & -0.1108 & -2.6132 & -0.0758 & -2.8033 & 0.0851 & 3.4948 & 0.0139 & 0.3295 & & \\
\hline WEMPR & -0.1282 & -0.6804 & 0.2267 & 1.9590 & -0.2301 & -2.3845 & 0.1379 & 0.8583 & 0.1131 & 0.3798 \\
\hline WINMGR & 0.1732 & 0.9053 & -0.1673 & -1.4325 & -0.4005 & -4.0139 & -0.1530 & -0.9507 & 0.2884 & 0.9633 \\
\hline WOTMGR & -0.3394 & -1.7410 & 0.0491 & 0.4096 & 0.5279 & 5.4440 & -0.2747 & -1.6722 & -0.3566 & -1.1354 \\
\hline WMHYR & 0.0369 & 0.4257 & 0.1337 & 2.5158 & -0.2145 & -4.5303 & 0.0860 & 1.1687 & -0.0526 & -0.3773 \\
\hline WDGEXR & 0.0935 & 0.9135 & 0.1286 & 2.0531 & -0.0950 & -1.8060 & 0.0784 & 0.9078 & 0.3849 & 2.4111 \\
\hline AREA & & & 0.0142 & 1.7412 & -0.0008 & -0.1134 & & & & \\
\hline POPs & & & 0.0744 & 3.5927 & 0.1082 & 7.2067 & -0.1382 & -0.7787 & -0.0822 & -3.7935 \\
\hline POPd & & & & & & & 0.0060 & 0.7321 & & \\
\hline POP5_17 & & & & & & & & & -0.0480 & -0.3751 \\
\hline POP25_44 & 0.3640 & 3.0128 & & & & & & & & \\
\hline FHHF & -0.0595 & -1.7914 & & & & & 0.0166 & 0.5054 & & \\
\hline POPHD & & & & & & & -0.0963 & -0.9415 & & \\
\hline POPCD & 0.1704 & 5.0411 & & & & & & & & \\
\hline OWHU & -0.2426 & -2.3303 & & & -0.0617 & -0.8389 & & & & \\
\hline MCRH & & & 0.0393 & 0.8717 & & & & & & \\
\hline UNEMP & & & -0.0470 & -2.4625 & -0.0497 & -2.7537 & 0.0125 & 0.4633 & & \\
\hline MANU & 0.0010 & 0.8750 & & & & & -0.0008 & -0.7000 & & \\
\hline WHRT & 0.0246 & 7.5613 & & & & & 0.0011 & 0.2867 & & \\
\hline SCRM & & & & & & & & & 0.2390 & 1.7311 \\
\hline DFEG & & & & & & & & & -0.0450 & -1.0629 \\
\hline PCTAX & & & & & & & & & 0.1270 & 2.6743 \\
\hline РСРТAX & -0.0496 & -3.0965 & & & & & & & & \\
\hline PCTD & & & & & & & & & -0.1969 & -1.2892 \\
\hline LTD & & & & & & & & & 0.0007 & 1.4104 \\
\hline ScIX & & & & & & & 0.0045 & 0.4537 & & \\
\hline NAIX & 0.0054 & 0.8065 & 0.0058 & 1.2264 & -0.0011 & -0.2702 & & & & \\
\hline HWD & -0.0339 & -1.5297 & & & & & & & & \\
\hline ESBd & -0.0786 & -2.6006 & & & & & & & & \\
\hline
\end{tabular}




\begin{tabular}{|l|c|c|c|c|c|c|c|c|c|c|}
\hline EXTAX & & & 0.0001 & 0.0087 & 0.0092 & 0.6258 & & & & \\
\hline EMPt-1 & -0.0641 & -6.0014 & & & & & & & & \\
\hline INMGt-1 & & & -0.1317 & -5.8972 & & & & & & \\
\hline OTMGt-1 & & & & & -0.1052 & -6.3604 & & & & \\
\hline MHYt-1 & & & & & & & -0.7886 & -13.6035 & & \\
\hline DGEXt-1 & & & & & & & & & -0.3426 & -4.1311 \\
\hline RHO & 0.3389 & & 0.02734 & & -0.2337 & & -0.0307 & & -0.2488 & \\
\hline SIG & 0.0705 & & 0.0302 & & 0.0233 & & 0.0596 & & 0.2335 & \\
\hline AD.R2 & 0.2195 & & 0.5707 & & 0.5839 & & 0.4803 & & 0.0325 & \\
\hline F-STATISTIC* & 18.9496 & 0.000 & 40.614 & 0.000 & 49.5944 & & 59.7866 & 0.000 & 5.6799 & 0.000 \\
\hline N & 1096 & & 1096 & & 1096 & & 1096 & & 1096 & \\
\hline
\end{tabular}

* Figures in the t-statistics columns are p-values 


\begin{tabular}{|c|c|c|c|c|c|c|c|c|c|c|}
\hline \multicolumn{11}{|c|}{\begin{tabular}{|l} 
Table c7: Feasible Generalized Spatial Three-Stage Le \\
APPALACHIAN STATES COUNTIES, 1980_RATE \\
\end{tabular}} \\
\hline \multirow[b]{2}{*}{ VARIABLE } & \multicolumn{2}{|c|}{ EMPR Equation } & \multicolumn{2}{|c|}{ INMGR Equation } & \multicolumn{2}{|c|}{ OTMGR Equation } & \multicolumn{2}{|c|}{ MHYR Equation } & \multicolumn{2}{|c|}{ DGEXR Equation } \\
\hline & Coeff. & t-stat. & Coeff. & t-stat. & Coeff. & t-stat. & Coeff. & t-stat. & Coeff. & t-stat. \\
\hline CONSTANT & -1.1930 & -5.7942 & -0.2591 & -4.5553 & 1.1371 & 2.4918 & 0.8537 & 8.3560 & 2.2615 & 15.4125 \\
\hline EMPR & & & 0.3773 & 26.2358 & 0.4090 & 5.8833 & 0.3750 & 44.3872 & -0.1571 & -5.7606 \\
\hline INMGR & 0.9885 & 15.4065 & & & -1.6390 & -16.2827 & -0.4186 & -23.6430 & 0.2809 & 5.3596 \\
\hline OTMGR & 0.3624 & 10.2737 & -0.3832 & -26.2421 & & & -0.1689 & -12.6388 & 0.2462 & 7.9065 \\
\hline MHYR & 2.0296 & 36.2881 & -0.7449 & -20.5559 & -1.2819 & -7.1227 & & & 0.3211 & 4.8172 \\
\hline DGEXR & -0.1499 & -4.2545 & 0.0743 & 4.5556 & 0.1827 & 2.5060 & 0.0882 & 7.0167 & & \\
\hline WEMPR & 0.0543 & 0.7647 & -0.2553 & -9.4873 & -0.4138 & -3.5893 & -0.2491 & -13.8180 & 0.1680 & 3.5099 \\
\hline WINMGR & -1.0699 & -14.6447 & 1.0167 & 130.3390 & 1.8210 & 16.4091 & 0.4593 & 23.5253 & -0.3186 & -5.4703 \\
\hline WOTMGR & -0.4489 & -6.6910 & 0.2819 & 11.6140 & 0.6043 & 7.0482 & 0.1295 & 7.2125 & -0.3066 & -7.0178 \\
\hline WMHYR & -0.2445 & -1.6443 & 0.6609 & 12.3492 & 1.0717 & 4.5525 & 0.7066 & 21.2539 & 0.0655 & 0.6708 \\
\hline WDGEXR & 0.2210 & 2.7984 & -0.0992 & -3.4460 & -0.2138 & -1.7871 & -0.1037 & \begin{tabular}{|l|}
-5.1169 \\
\end{tabular} & 0.2976 & 5.8794 \\
\hline AREA & & & 0.0062 & 2.6416 & 0.0399 & 3.4304 & & & & \\
\hline POPs & & & 0.0705 & 12.0858 & 0.0207 & 1.3417 & -0.0530 & -4.4614 & -0.0060 & -1.4907 \\
\hline POPd & & & & & & & 0.0026 & 4.8243 & & \\
\hline POP5_17 & & & & & & & & & -0.0540 & -1.7565 \\
\hline POP25_44 & 0.1497 & 4.0931 & & & & & & & & \\
\hline FHHF & -0.0504 & -3.1614 & & & & & 0.0181 & 3.2844 & & \\
\hline POPHD & & & & & & & 0.0358 & 4.1304 & & \\
\hline POPCD & 0.0305 & 2.9163 & & & & & & & & \\
\hline OWHU & 0.0420 & 1.4997 & & & -0.1574 & -1.7356 & & & & \\
\hline MCRH & & & 0.0536 & 5.9382 & & & & & & \\
\hline UNEMP & & & -0.0596 & -9.4119 & -0.1789 & -5.9444 & -0.0450 & -11.5353 & & \\
\hline MANU & -0.0013 & -3.3176 & & & & & 0.0006 & 4.6170 & & \\
\hline WHRT & 0.0086 & 7.0502 & & & & & -0.0023 & -4.8455 & & \\
\hline SCRM & & & & & & & & & 0.2069 & 9.1501 \\
\hline DFEG & & & & & & & & & 0.0007 & 0.1095 \\
\hline PCTAX & & & & & & & & & 0.0987 & 11.8826 \\
\hline РСРТAX & -0.0214 & -3.8840 & & & & & & & & \\
\hline PCTD & & & & & & & & & -0.0028 & -0.0983 \\
\hline LTD & & & & & & & & & -0.0005 & -0.1350 \\
\hline ScIX & & & & & & & 0.0027 & 2.2176 & & \\
\hline NAIX & -0.0021 & -0.8682 & 0.0022 & 1.5761 & 0.0036 & 0.5022 & & & & \\
\hline HWD & 0.0225 & 3.0271 & & & & & & & & \\
\hline ESBd & -0.0394 & -3.9420 & & & & & & & & \\
\hline EXTAX & & & -0.0223 & -4.8387 & 0.0029 & 0.1501 & & & & \\
\hline
\end{tabular}




\begin{tabular}{|l|c|c|c|c|c|c|c|c|c|c|}
\hline EMPt-1 & -0.0169 & -3.8215 & & & & & & & & \\
\hline INMGt-1 & & & -0.0802 & -13.1522 & & & & & & \\
\hline OTMGt-1 & & & & & -0.0414 & -2.5176 & & & & \\
\hline MHYt-1 & & & & & & & -0.0549 & -6.0775 & & \\
\hline DGEXt-1 & & & & & & & & & -0.3560 & -26.2269 \\
\hline RHO & 0.6123 & & -0.0754 & & -0.3218 & & -0.3034 & & -0.0863 & \\
\hline SIG & 0.0746 & & 0.0154 & & 0.0371 & & 0.0096 & & 0.0438 & \\
\hline$R^{2}$ & 0.3437 & & 0.8978 & & 0.2194 & & 0.2898 & & 0.1496 & \\
\hline$N$ & 1096 & & 1096 & & 1096 & & 1096 & & 1096 & \\
\hline
\end{tabular}


Table c8: Feasible Generalized Spatial Three-Stage Least Squares(fGS3SLS) Estimation Results, APPALACHIAN STATES COUNTIES, 1990 RATE

\begin{tabular}{|c|c|c|c|c|c|c|c|c|c|c|}
\hline \multirow[b]{2}{*}{ VARIABLE } & \multicolumn{2}{|c|}{ EMPR Equation } & \multicolumn{2}{|c|}{ INMGR Equation } & \multicolumn{2}{|c|}{ OTMGR Equation } & \multicolumn{2}{|c|}{ MHYR Equation } & \multicolumn{2}{|c|}{ DGEXR Equation } \\
\hline & Coeff. & t-stat. & Coeff. & t-stat. & Coeff. & t-stat. & Coeff. & t-stat. & Coeff. & t-stat. \\
\hline CONSTANT & 0.3869 & 0.9740 & 0.1832 & 1.3320 & -0.2707 & -1.7275 & 10.6308 & 13.7339 & 1.8265 & 5.4546 \\
\hline EMPR & & & 0.1039 & 3.0231 & 0.2674 & 8.6089 & -0.2811 & -4.6906 & -0.4665 & -6.5553 \\
\hline INMGR & 0.1239 & 2.5210 & & & 0.7055 & 30.8692 & 0.0011 & 0.0154 & -1.0213 & -11.5954 \\
\hline OTMGR & 0.5233 & 10.9257 & 0.8123 & 27.0138 & & & 0.2669 & 4.3729 & 1.2187 & 16.1288 \\
\hline MHYR & -0.1812 & -5.2003 & -0.0361 & -1.0362 & 0.0664 & 2.4028 & & & -0.1491 & -2.5738 \\
\hline DGEXR & -0.1818 & -6.8098 & -0.1551 & -7.1080 & 0.1581 & 8.7936 & -0.0457 & -1.2551 & & \\
\hline WEMPR & 0.2043 & 2.5438 & 0.0051 & 0.0720 & -0.1304 & -1.9426 & -0.0136 & -0.1252 & 0.3108 & 2.2297 \\
\hline WINMGR & -0.0651 & -0.6299 & 0.1680 & 1.8885 & -0.0775 & -0.9292 & -0.0619 & -0.4222 & 0.3522 & 1.9873 \\
\hline WOTMGR & -0.1327 & -1.1586 & -0.1283 & -1.4530 & 0.1583 & 1.8793 & -0.3315 & -2.1501 & -0.5104 & -2.7109 \\
\hline WMHYR & -0.0026 & -0.0499 & 0.0006 & 0.0154 & -0.0438 & -1.2000 & -0.2011 & -3.1118 & 0.0116 & 0.1457 \\
\hline WDGEXR & 0.1409 & 3.2693 & 0.1092 & 2.8148 & -0.0911 & -2.4673 & -0.0771 & -1.1320 & 0.6774 & 9.0265 \\
\hline AREA & & & 0.0095 & 1.6962 & -0.0077 & -1.7059 & & & & \\
\hline POPs & & & -0.0147 & -1.0934 & 0.1088 & 8.8384 & -0.2731 & -2.1349 & -0.0742 & -5.9025 \\
\hline POPd & & & & & & & 0.0121 & 2.0605 & & \\
\hline POP5_17 & & & & & & & & & 0.0874 & 1.3810 \\
\hline POP25_44 & 0.3398 & 5.4048 & & & & & & & & \\
\hline FHHF & -0.0236 & -1.3748 & & & & & -0.0313 & -1.1639 & & \\
\hline POPHD & & & & & & & -0.1179 & -1.4589 & & \\
\hline POPCD & 0.1392 & 7.0860 & & & & & & & & \\
\hline OWHU & -0.2564 & -4.8344 & & & -0.0169 & -0.5398 & & & & \\
\hline $\mathrm{MCRH}$ & & & 0.0343 & 1.5802 & & & & & & \\
\hline UNEMP & & & -0.0134 & -1.0220 & -0.0149 & -1.4645 & -0.0078 & -0.3494 & & \\
\hline MANU & 0.0018 & 3.3359 & & & & & -0.0010 & -1.1181 & & \\
\hline WHRT & 0.0195 & 10.0764 & & & & & 0.0036 & 1.2479 & & \\
\hline SCRM & & & & & & & & & 0.2393 & 3.2956 \\
\hline DFEG & & & & & & & & & -0.0103 & -0.4855 \\
\hline PCTAX & & & & & & & & & 0.0635 & 3.3627 \\
\hline PCPTAX & -0.0484 & -5.6851 & & & & & & & & \\
\hline PCTD & & & & & & & & & -0.1783 & -2.7431 \\
\hline LTD & & & & & & & & & 0.0007 & 3.1817 \\
\hline SCIX & & & & & & & 0.0016 & 0.2044 & & \\
\hline NAIX & 0.0033 & 0.7757 & 0.0055 & 1.8304 & -0.0039 & -1.7098 & & & & \\
\hline HWD & -0.0457 & -4.0720 & & & & & & & & \\
\hline ESBd & -0.0457 & -2.5074 & & & & & & & & \\
\hline
\end{tabular}




\begin{tabular}{|l|c|c|c|c|c|c|c|c|c|c|}
\hline EXTAX & & & -0.0030 & -0.2789 & 0.0154 & 1.8046 & & & & \\
\hline EMPt-1 & -0.0532 & -8.7301 & & & & & & & & \\
\hline INMGt-1 & & & -0.0311 & -2.3132 & & & & & & \\
\hline OTMGt-1 & & & & & -0.0884 & -7.0070 & & & & \\
\hline MHYt-1 & & & & & & & -0.7933 & -16.5008 & & \\
\hline DGEXt-1 & & & & & & & & & -0.1917 & -5.3064 \\
\hline RHO & 0.3389 & & 0.02734 & & -0.2337 & & -0.0307 & & -0.2488 & \\
\hline SIG & 0.0705 & & 0.0302 & & 0.0233 & & 0.0596 & & 0.2335 & \\
\hline$R^{2}$ & 0.275 & & 0.5612 & & 0.5515 & & 0.4633 & & 0.0255 & \\
\hline$N$ & 1096 & & 1096 & & 1096 & & 1096 & & 1096 & \\
\hline
\end{tabular}


Appendix 3: Global and Local Spatial Autocorrelation (Moran's I and LISA) Figure 5.3.1a: Global and Local Spatial Autocorrelation (Moran's I and LISA)

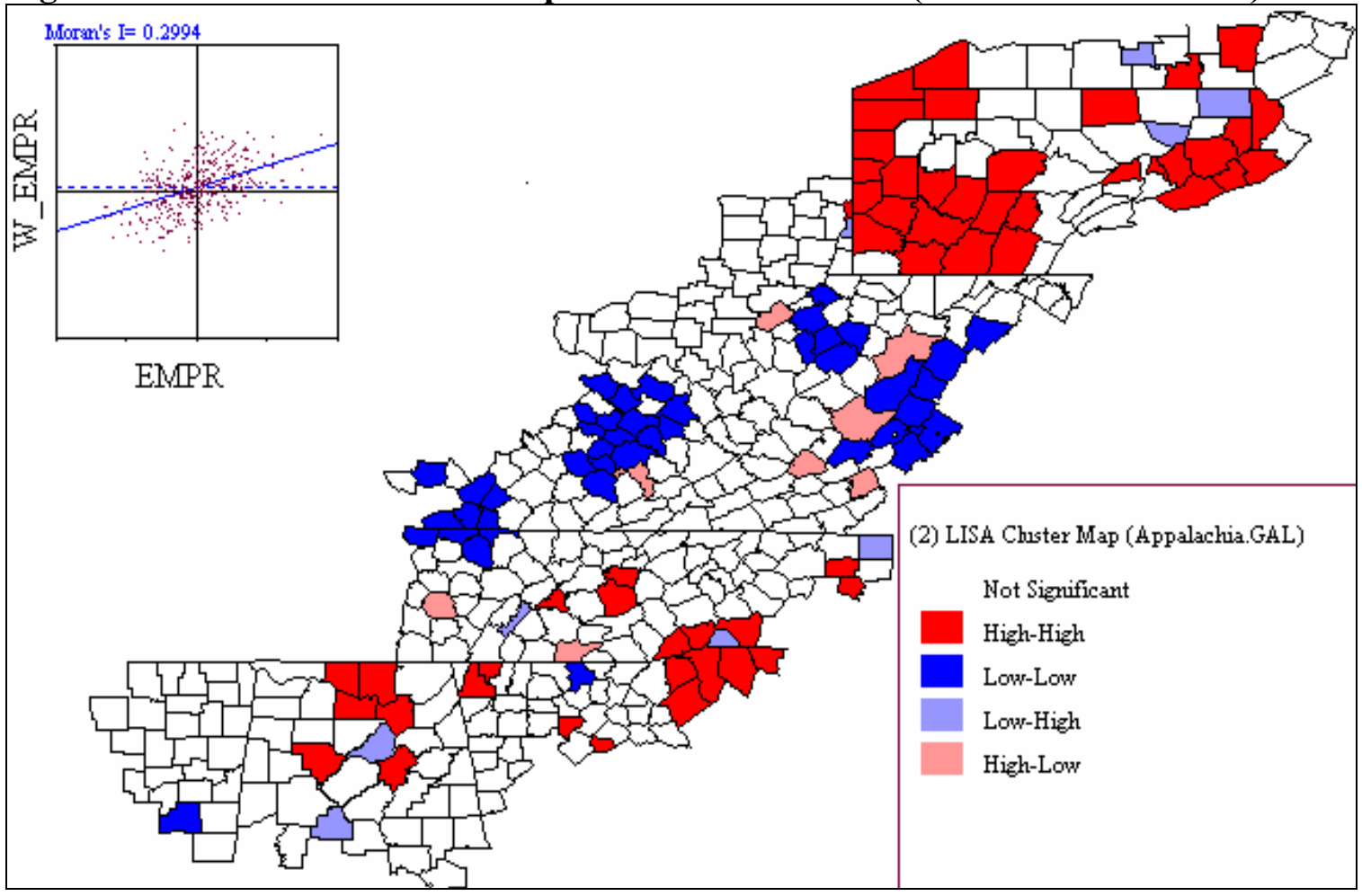

Figure 5.3.1b: Global and Local Spatial Autocorrelation (Moran's I and LISA)

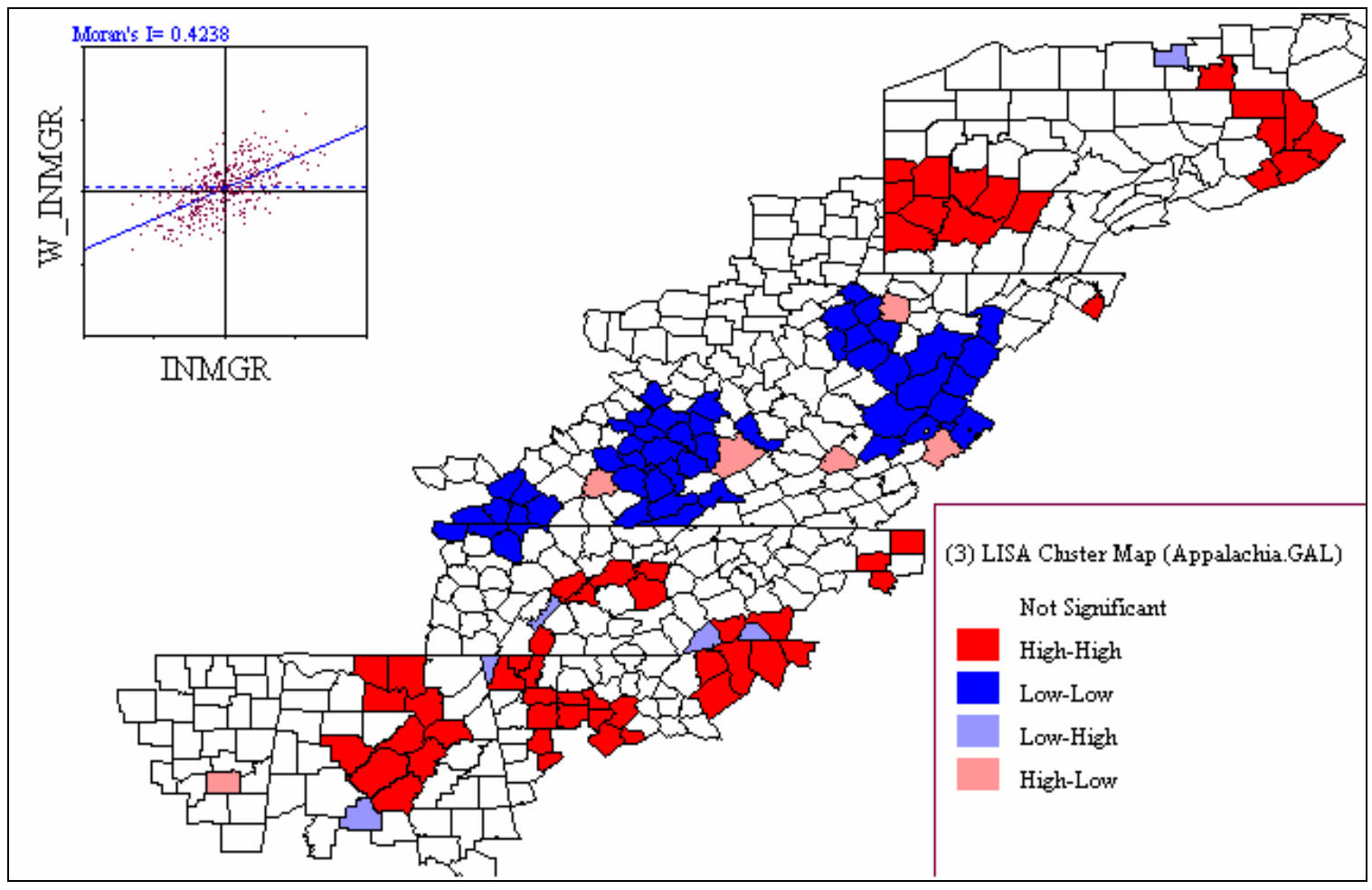


Figure 5.3.1c: Global and Local Spatial Autocorrelation (Moran's I and LISA)

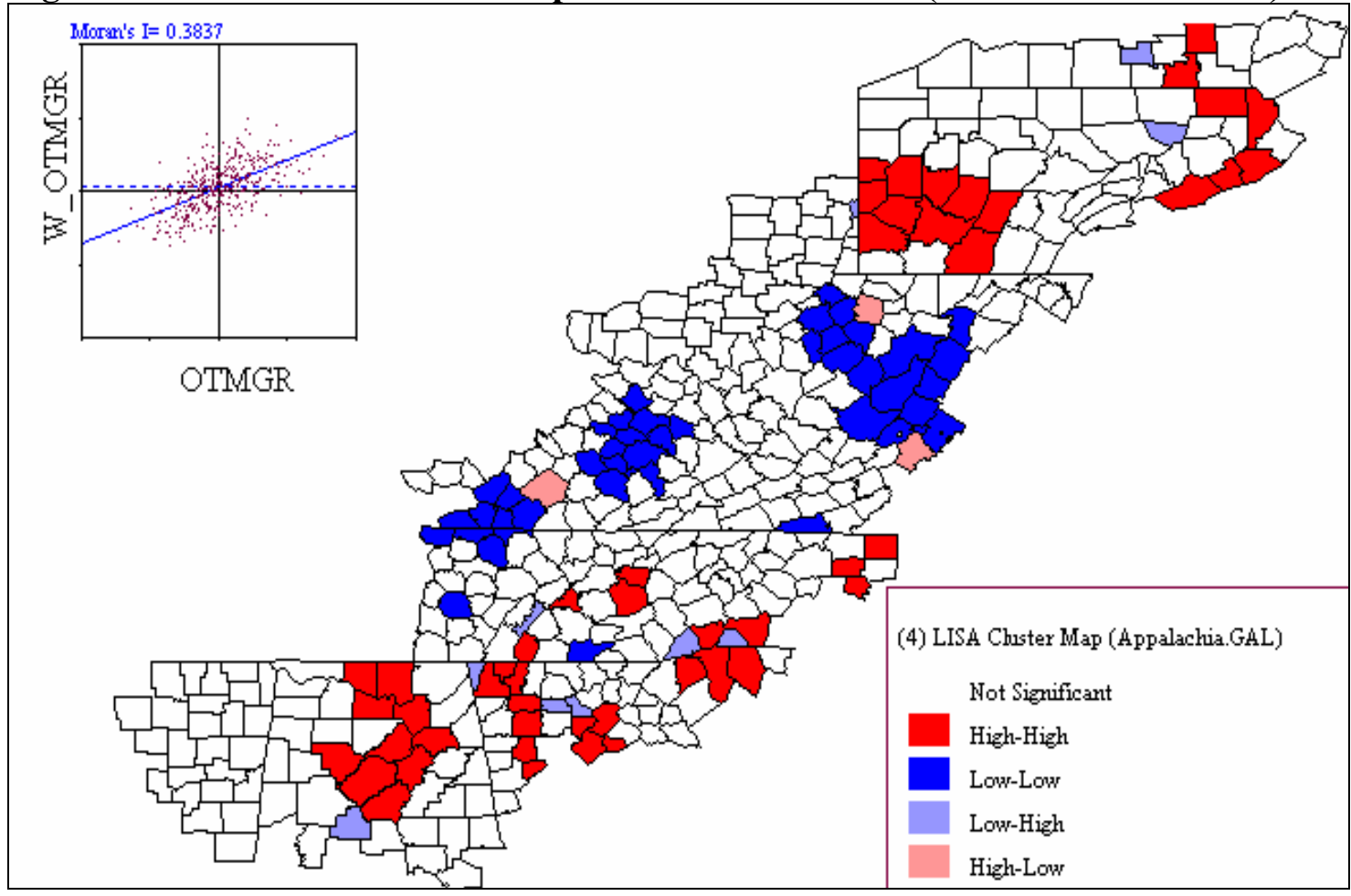

Figure 5.3.1d: Global and Local Spatial Autocorrelation (Moran's I and LISA)

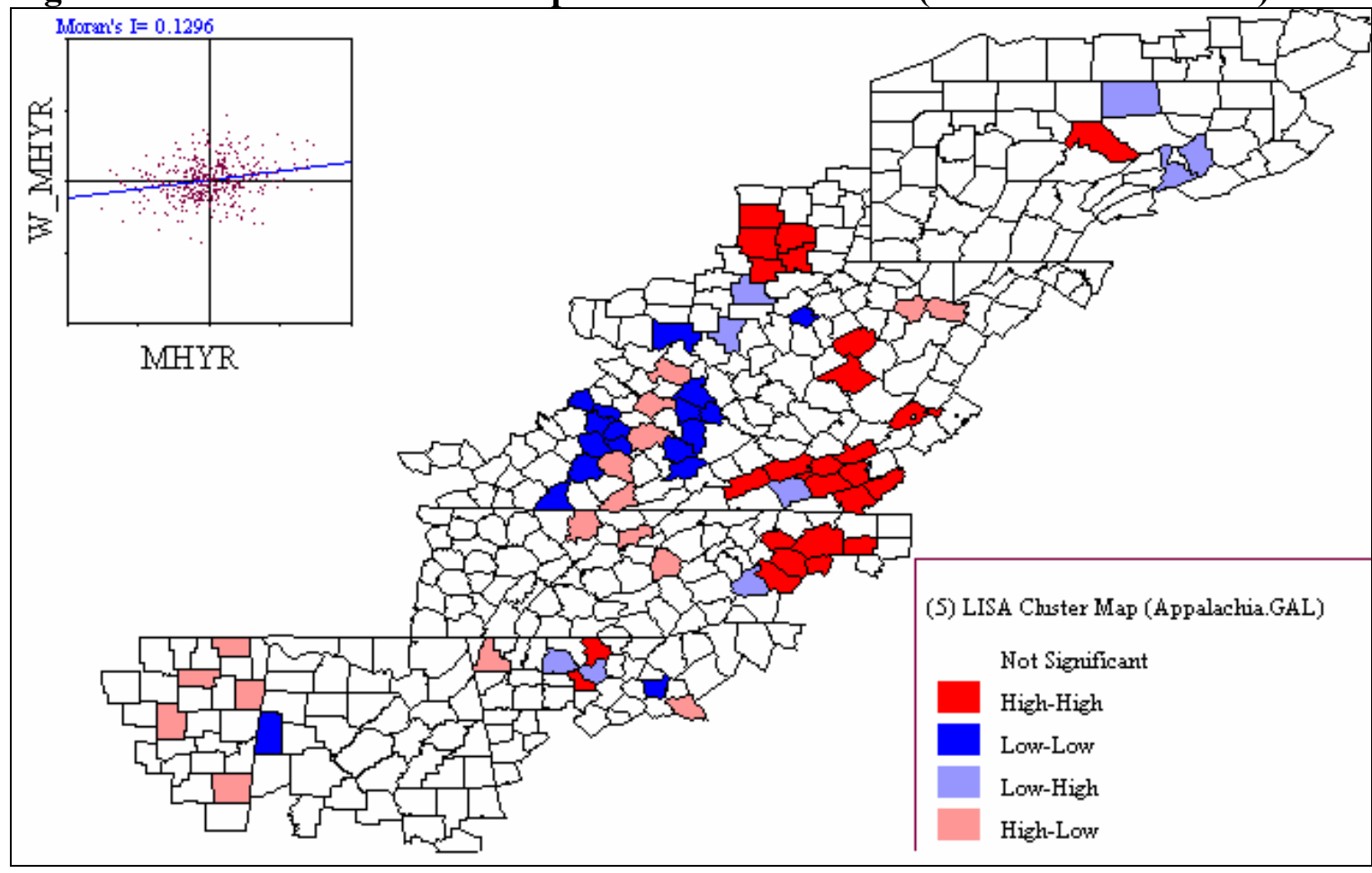


Figure 5.3.1e: Global and Local Spatial Autocorrelation (Moran's I and LISA)

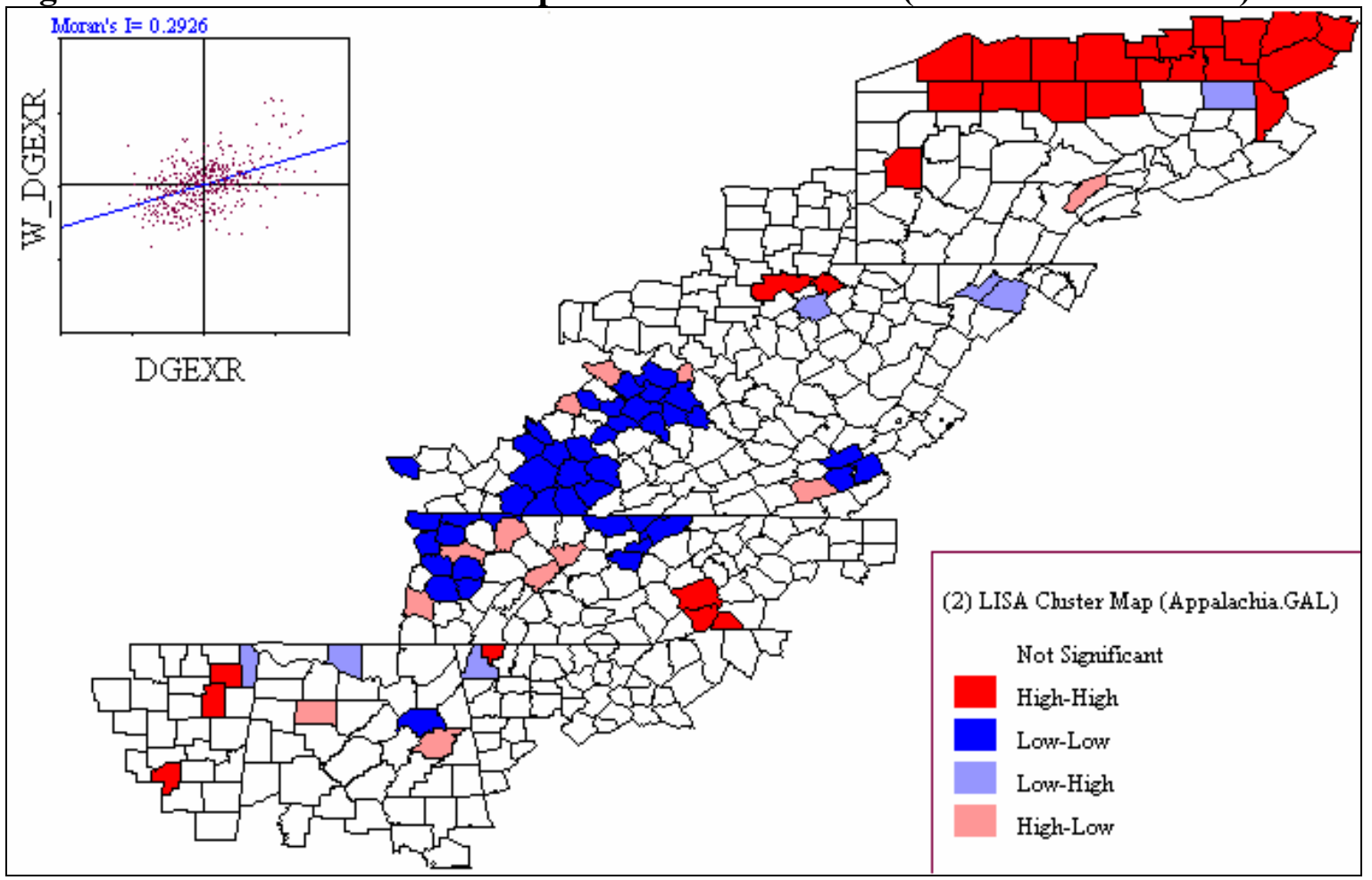

Figure 5.3.1f: Global and Local Spatial Autocorrelation (Moran's I and LISA)

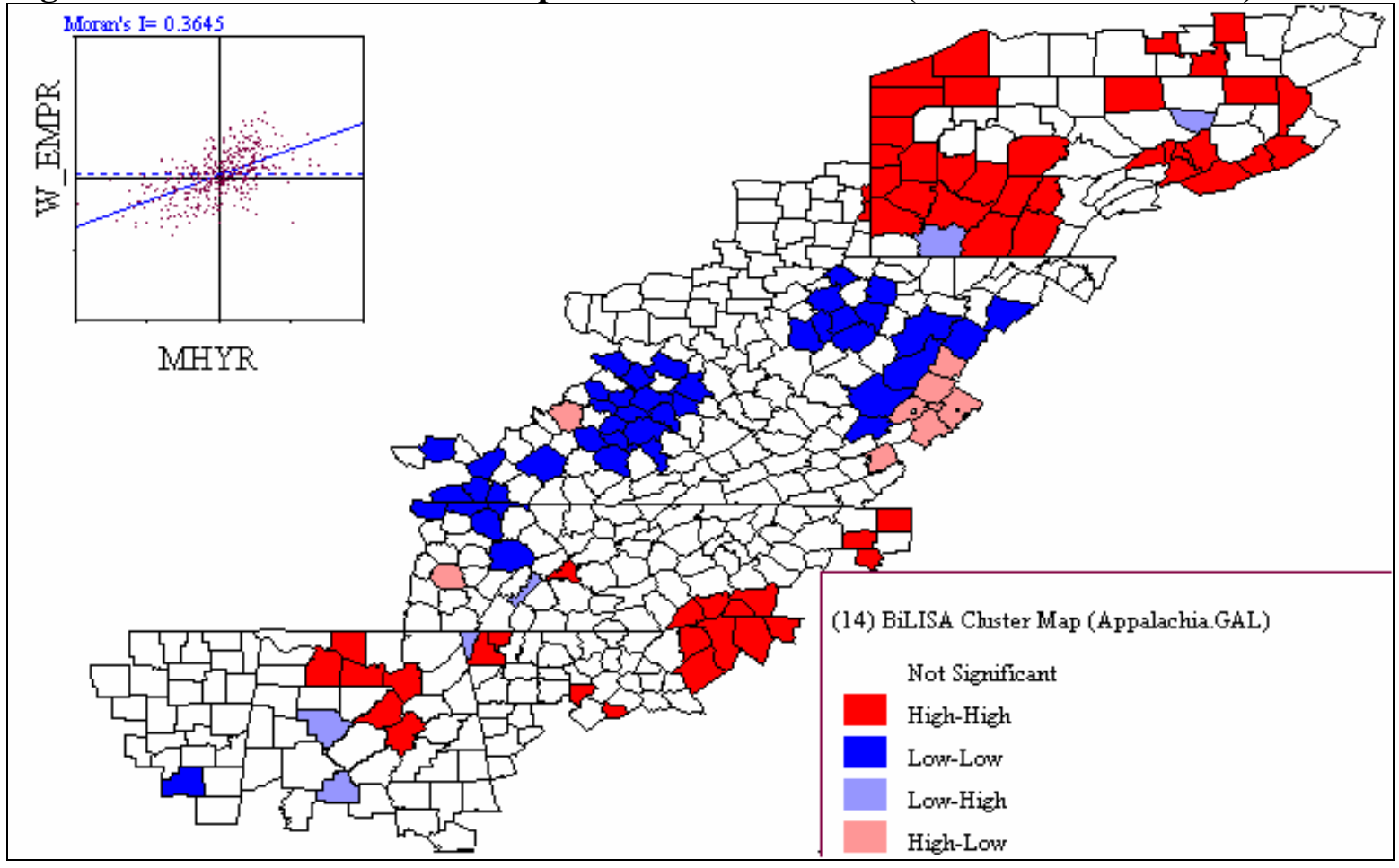




\section{Appendix 4: VITA}

\section{GEBREMESKEL H. GEBREMARIAM}

CURRICULUM VITAE

\section{MAILING ADDRESS:}

Agricultural and Resource Economics

Division of Resource Management

P. O. Box 6108

West Virginia University

Morgantown, WV 26506-6108

\section{CONTACT INFORMATION:}

Tel. 304-293-4832 ext. 4495

Fax: (304) 293-3752

E-mail: ghgebremariam@mail.wvu.edu

\section{EDUCATIONAL BACKGROUND:}

$\begin{array}{llll}\begin{array}{l}\text { Institution } \\ \text { West Virginia University }\end{array} & \begin{array}{l}\text { Period } \\ \text { Uug. 2000- } \\ \text { Aug.2006* }\end{array} & \begin{array}{l}\text { Specialization } \\ \text { Natural Resource Economics }\end{array} & \begin{array}{l}\text { Degree } \\ \begin{array}{l}\text { University of Strathclyde, } \\ \text { Glasgow, Scotland, U.K. }\end{array}\end{array} \\ \begin{array}{l}\text { University of Asmara, Asmara, } \\ \text { Eritrea }\end{array} & 1996-1997 & \begin{array}{l}\text { Industrialization, Trade and } \\ \text { Economic Policy }\end{array} & \text { MSc } \\ & \text { *I have been on leave of absence from my study between Jan. 2002- Jan 2004 } & \text { BA }\end{array}$

\section{WORK EXPERIENCE:}

\section{$\underline{\text { Position }}$}

Graduate Research Assistant

Lecturer \& Researcher

Graduate Research Assistant

Lecturer

Graduate Assistant

Research Assistant

\section{Institution}

West Virginia University, U.S.A.

University of Asmara, Eritrea

West Virginia University, U.S.A.

University of Asmara, Department of

Economics \& Finance, Eritrea

University of Asmara, Department of

Economics \& Finance, Eritrea

Asmara Chamber of Commerce, Eritrea $\underline{\text { Period }}$

Jan. 2004-Present

Jan. 2002- Jan.2004

August 2000-Jan.2002

Nov.1997 - Aug. 2000

Sept. 1995 -Sept. 1996

Nov 1993 - Sept. 1995 


\section{COURSES TAUGHT: Undergraduate}

- Principles of Microeconomics

- Intermediate Microeconomics

- Principles of Macro-economics

- Intermediate Macro-economics

- Development Economics

- Calculus for Economists

- Eritrean Economy

- International Economics

- Agricultural Economics

- International Political Economy

\section{THESIS SUPERVISION:}

I have supervised more than 30 BA theses (Senior Papers) in the Department of Economics and Finance, University of Asmara.

Acted as co-referee with Peter V. Schaeffer for Journal of Regional Science, for the manuscript entitled "The Interaction between Individuals' Destination Choice and Occupational Choice: A Simultaneous Equation Approach,” JRS, MS \#06.21.

\section{RESEARCH PAPERS}

Gebremariam, Gebremeskel H., Tesfa G. Gebremedhin, and Peter V. Schaeffer, Scientific Abstract Publication, "Modeling Small Business Growth, Migration Behavior, and Household Income in Appalachia: A Spatial Simultaneous Equations Approach," Agricultural and Resource Economics Review, Forthcoming, NAREA Annual Meeting, 11-14 June 2006, Mystic, CT.

Gebremariam, Gebremeskel H., Tesfa G. Gebremedhin, "County-Level Determinants of Local Public Services in Appalachia: A Multivariate Spatial Autoregressive Model Approach,” http://agecon.lib.umn.edu, AAEA-2006.

Gebremariam, Gebremeskel H., Tesfa G. Gebremedhin, and Peter V. Schaeffer, "An Empirical Analysis of County-Level Determinants of Small Business Growth and Poverty in Appalachia: A Spatial Simultaneous Equations Approach", Regional Research Institute, WVU, Working Paper No. 03, 2006.

Gebremariam, Gebremeskel H., Tesfa G. Gebremedhin, and Randall W. Jackson, "The Role of Small Business in Economic Growth and Poverty Alleviation in West Virginia: An Empirical Analysis," Regional Research Institute, WVU, Working Paper No. 10, 2004.

Gebremariam, Gebremeskel H., "Regional Economic Integration: A Means to a Successful Open-Export-Led Development Strategy in Post- Independence Eritrea," MSc. Thesis, University of Strathclyde, U. K., 1997.

Gebremariam, Gebremeskel H., "The Role of Trade in the Economic Development of Eritrea," BA Thesis, University of Asmara, Eritrea, 1995.

Gebremariam, Gebremeskel H., "Do Indigenous Land Tenure Systems Constrain Land Productivity? A Critical Evaluation of the Eritrean Case," West Virginia University (Unpublished Paper), 2002. 
Gebremariam, Gebremeskel H., "Testing for Structural Changes in the U. S. A. Economy due to the Oil Price Shocks of the 1970,s Using the General Regression Method," West Virginia University (Unpublished Paper), 2001.

Gebremariam, Gebremeskel H., "Using Travel Cost Methods (TCM) in Valuing Recreational Benefits of an Existing Single-site Forest: A Literature Review, West Virginia University (Unpublished Paper), 2000.

\section{CONFERENCE PRESENTATIONS:}

Gebremariam, Gebremeskel H., Tesfa G. Gebremedhin, and Peter V. Schaeffer, "Modeling Small Business Growth, Migration Behavior, and Household Income in Appalachia: A Spatial Simultaneous Equations Approach," NAREA Annual Meeting, 11-14 June 2006, Mystic, CT.

Gebremariam, Gebremeskel H., Tesfa G. Gebremedhin, "County-Level Determinants of Local Public Services in Appalachia: A Multivariate Spatial Autoregressive Model Approach," AAEA Annual Meeting, 23-26 July 2006, Long Beach, CA.

Gebremariam, Gebremeskel H., Tesfa G. Gebremedhin, and Peter V. Schaeffer, "An Empirical Analysis of County-Level Determinants of Small Business Growth and Poverty in Appalachia: A Spatial Simultaneous Equations Approach”, SAEA Annual Meeting, 5-8 February 2006, Orlando, FL.

Amarasinghe, Anura, and G.H. Gebremariam, "A Dynamic optimization Approach in Evaluating Investment Benefits for Wetland Restoration," Northeastern Agricultural and Resource Economics Association Annual Meeting, 12-15 June 2005, Annapolis, MD.

Gebremariam, Gebremeskel H., Tesfa G. Gebremedhin, and Randall W. Jackson, "The Role of Small Business in Economic Growth and Poverty Alleviation in West Virginia: An Empirical Analysis," AAEA Annual Meeting, 1-4 August 2004, Denver, CO.

\section{Awards:}

The second Thomas F. Torries Outstanding Ph.D. Research Award

Travel Award, AAEA, Paper Presentation at the 2004 AAEA Annual Meeting

\section{Computing Skills:}

Basics:

Econometric Packages:

Statistical and Mathematics Packages:

Spatial Software:
Word, Excel, PowerPoint, Access

TSP, EViews, Limped, SPSS, SAS

GiveWin, Matlab, @Risk, Maple

ArcGIS, GeoDa, Matlab 